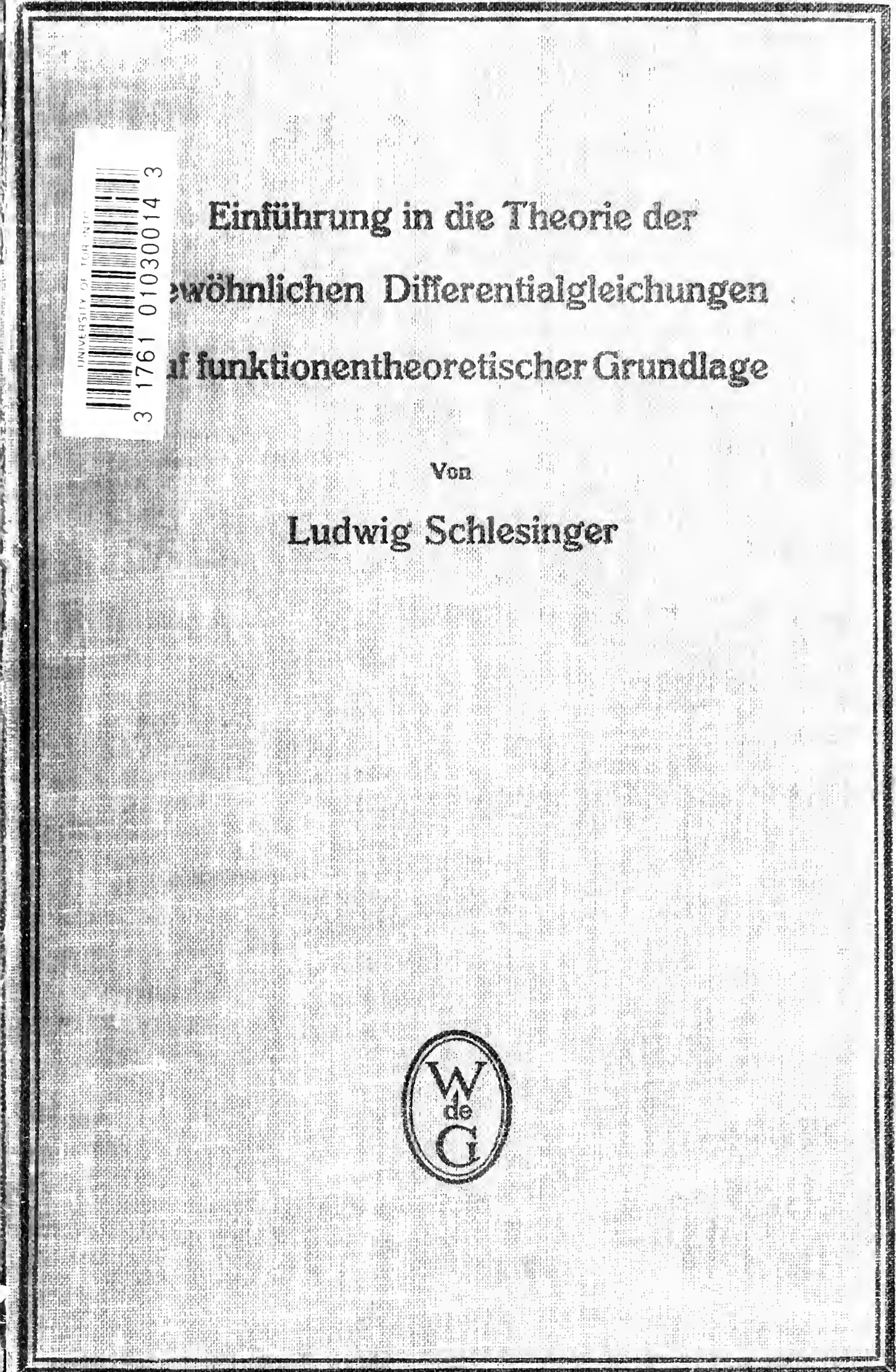





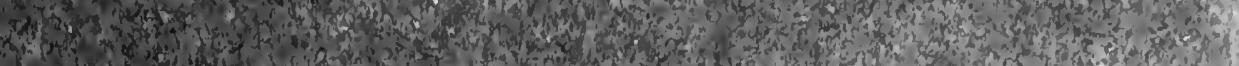
SCA A H.

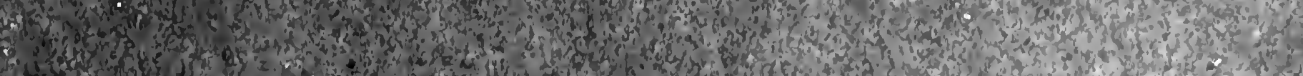

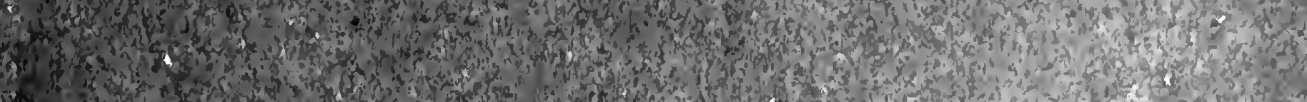

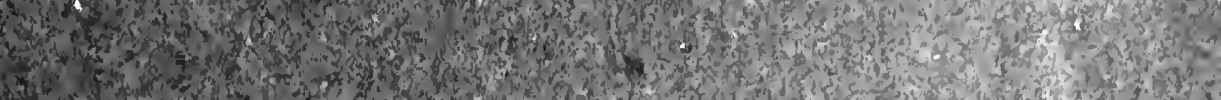

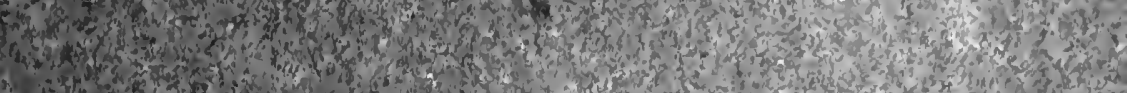
20

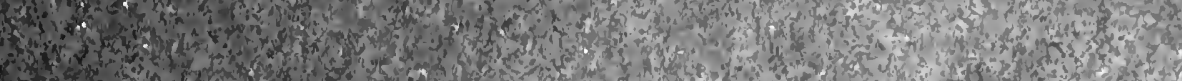

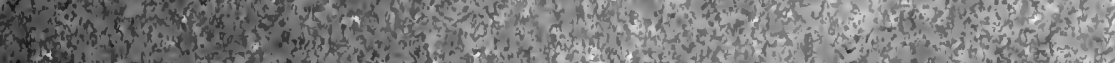

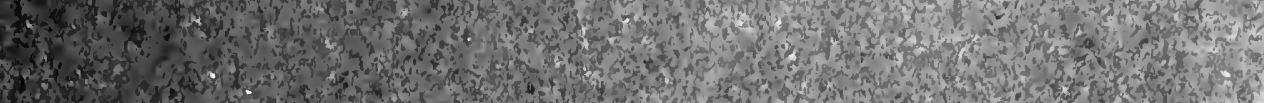
(15.

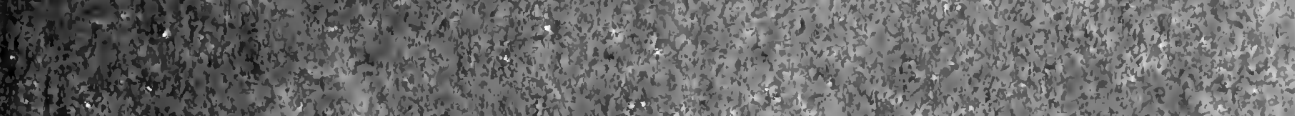

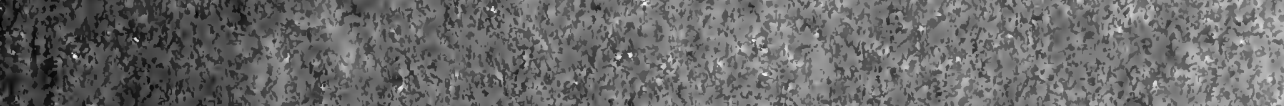

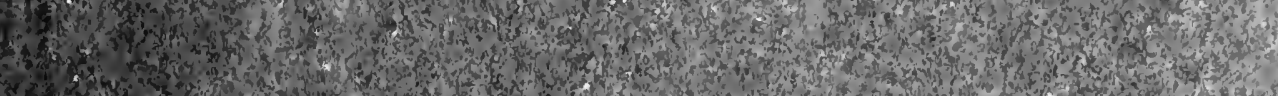
6.

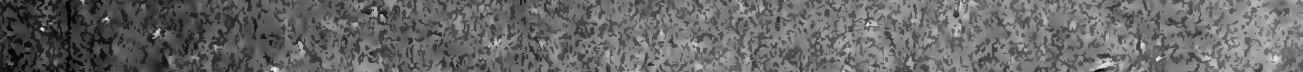

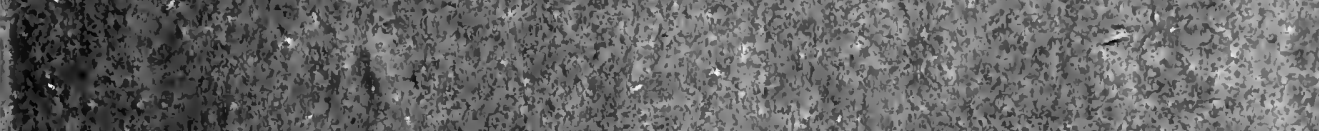
Hot

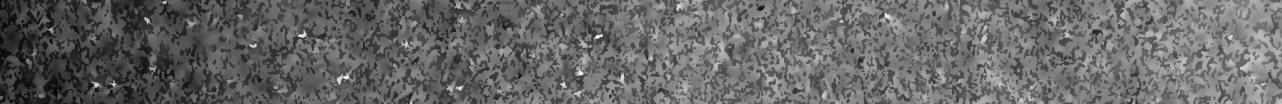

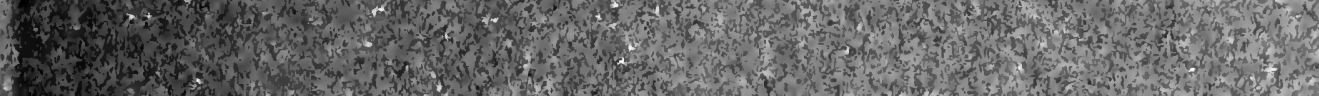

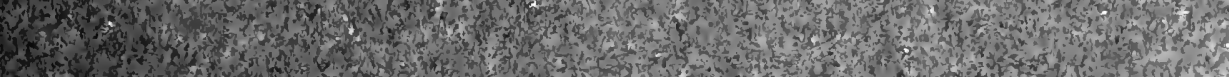

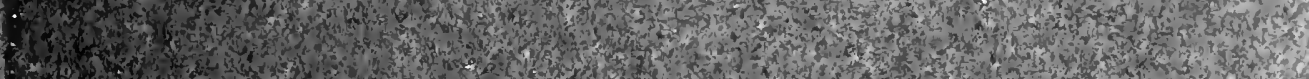

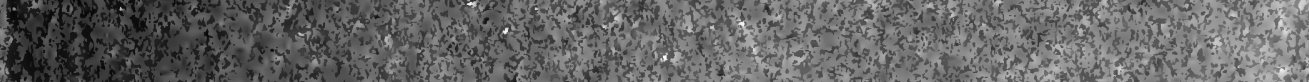

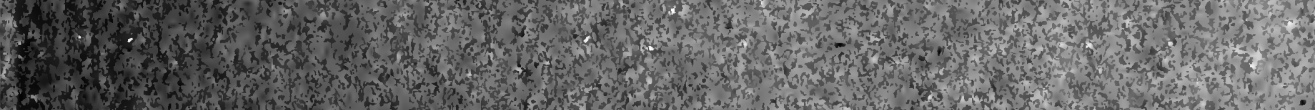

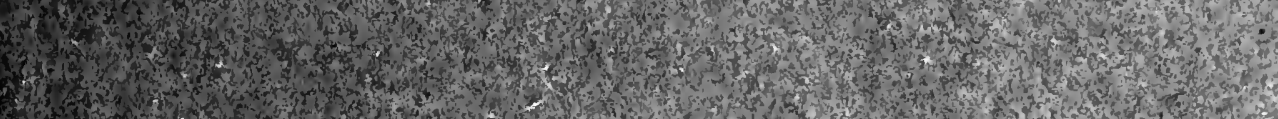

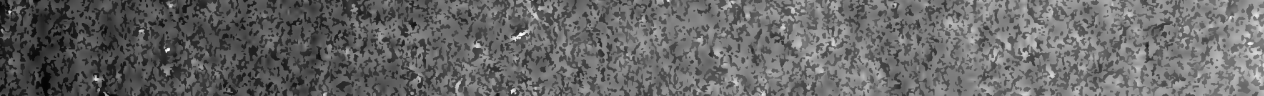
9
3

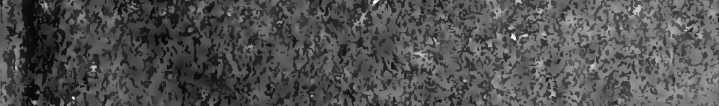
(3) Hots 3
3 10
3

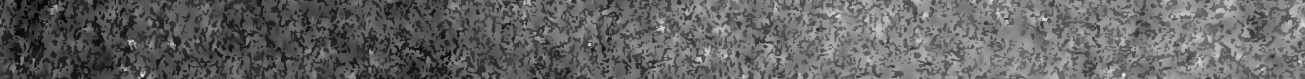

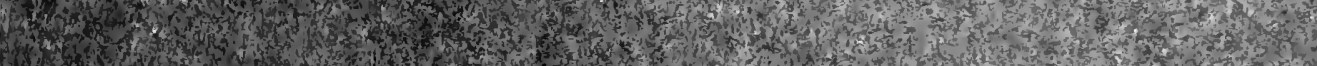

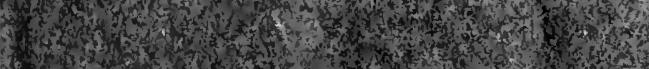





\section{Einführung in die Theorie der}

gewöhnlichen Differentialgleichungen auf funktionentheoretischer Grundlage

Ludwig Schlesinger
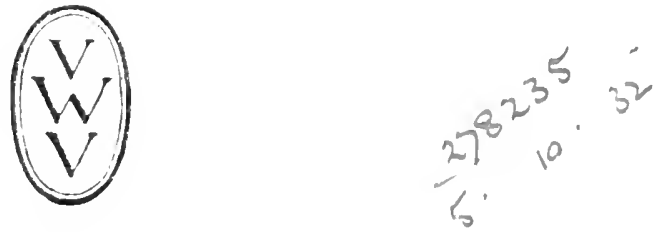

VEREINIGUNG WISSENSCHAFTLICHER VERLEGER WALTER DE GRUYTER \& CO.

IUKMALS G. J. GÖSCHEN'SCHE VERLAGSHANDI C M -

CUTTENTAG, VERLAGSBUCHHANDLUNG GEORG

REIMER KARL J. TRUBNER VEIT \& COMP

BERLIN W. 10 UND LEIPZIG 


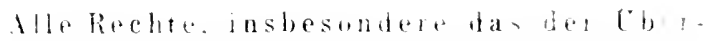
-etzung in fremde siprachen. vorbehat:er:

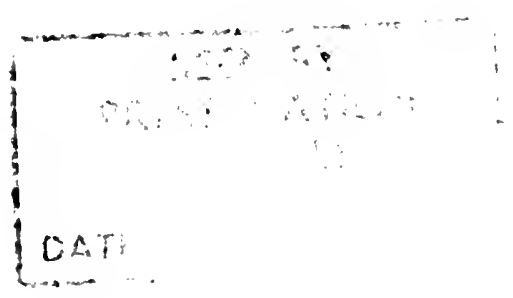

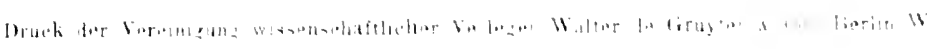




\section{Vorwort.}

D

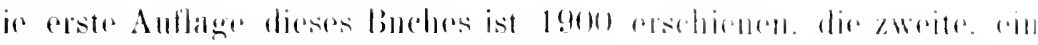

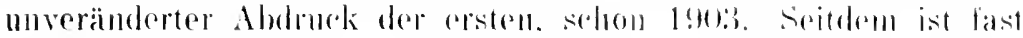
ein Menschenalter verflossen. während dessen sireh amsehringud data gemeine Interesse an dem hice behandelten liegenstande. wonigstens

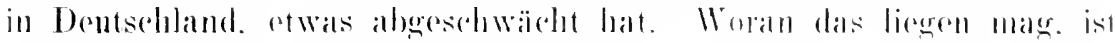

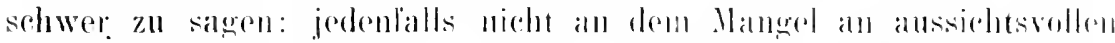

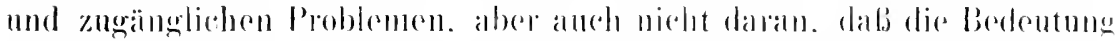
dieser Probleme tïr Theorie mod Anwendungen geringer grworden wär.

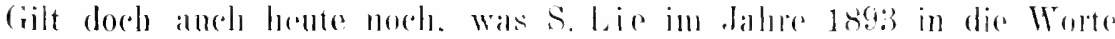
gefabt hat* ${ }^{*}$ ): .. In der gamzen modernen . Wathomatili ist dire Theorio dor Differentialeleichungen die wichtigste l)isziplin..

Bej der Bearbejtung der nemen Auflage sind die cimulsiator heibehalten worden. die lïr die Ablassung dex ersten mabgebend waren. Es heibt darïber in dem Vorwort zar resten Auflage: .. Es kommt. wiemir

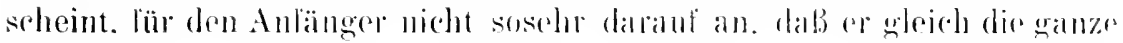
Tragweite einer bestimmten Hethode kemurnlormt. als vielunehr darant.

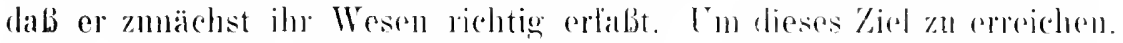
geniugt es aber. die betreffende Methode an Beispielen zn entwioknln. die einerseits so allgemein sind. dab keine do Schwierigkeiten. die durrob das Wesen jener Methode ïberwunden werden sollen. fohlt. mud andererseits so speziell. lab schwierigkeiten akzessorischor Natmr möglichst vermieden serden.."

..Der Wert einer Darstellung. we der hier rorliogenden. wirl maturgemäB nicht in ihrer durchgängigen Eigenatt zu suchen soin: latndrlt es sich ja zum Teil mm die Entwicklung von Theorion. die aubor von ihren Urhebern anch schon anderweitig vielfach behandelt worden sind. Die ersten Guellen wall ich bestrebt überall zn nennen. ron späteren Bearbeitungen sind meist nur diejenigen angefïhrt. dir mir bei der Ausarbeitung numittelbar vorgelegen haben."

..Nor zwei (iesichtspunkte möchte ich mir noch erlauben hervorzuheben. Einmal. dab ich dic Grundlage fïr einen systematischen Autban der Theorie der Differentialgleichmngen in der l'nterscheidungr zwischen

* Leipziger Berichte 18!3. Seite 5:3. 


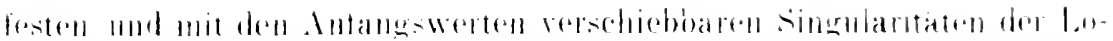

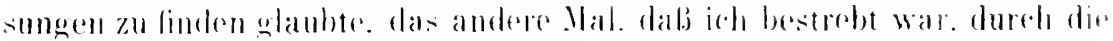

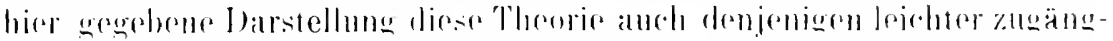

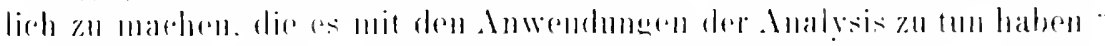

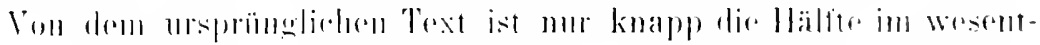

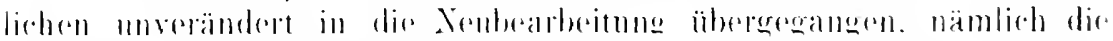

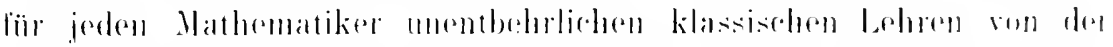

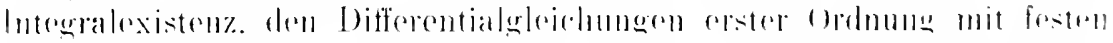

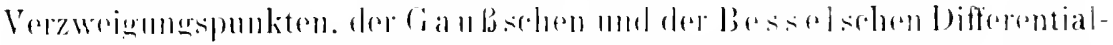

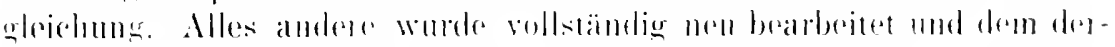

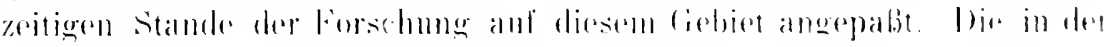

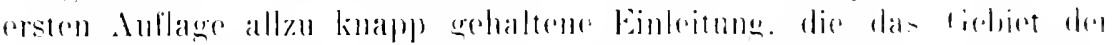

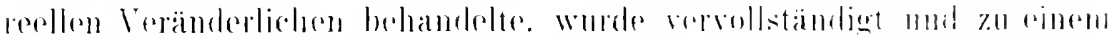
hesenderen Kapitel gestaltet. dir Theorio der linatron Differential-

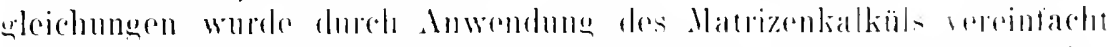

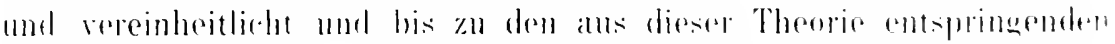

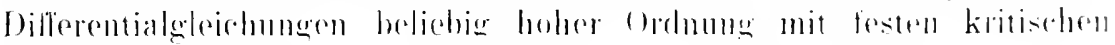

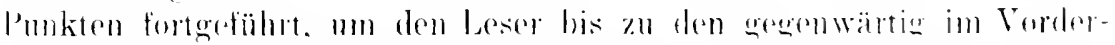
grmud dos lutereseses stehenden Problemen hinzuleiten. Auch sind bej-

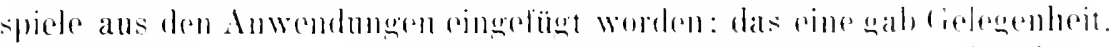

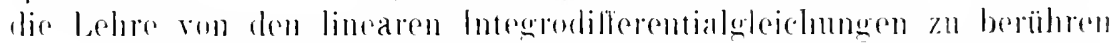

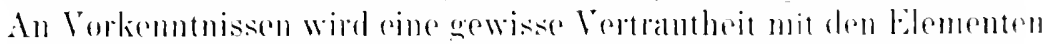

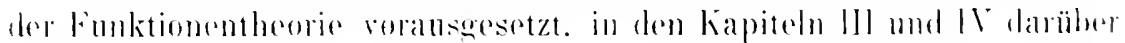

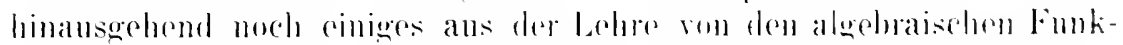

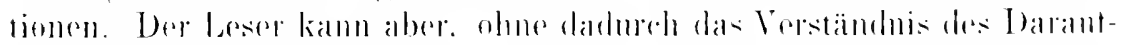

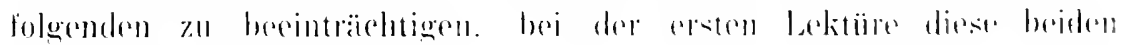

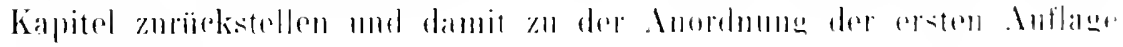
zurürckkchrem.

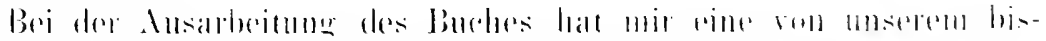

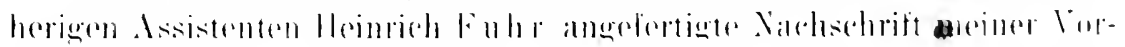

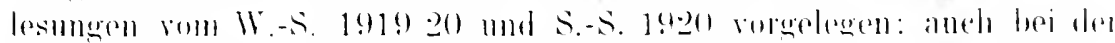

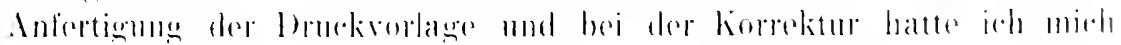

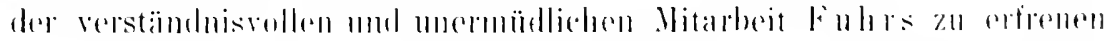

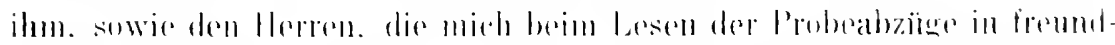

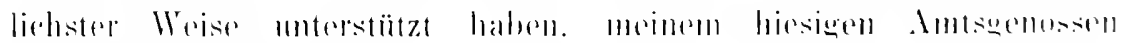

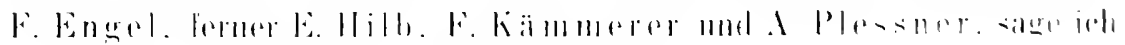

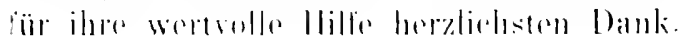

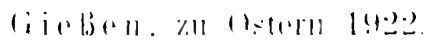

\section{Schlesinger.}




\section{Inhaltsverzeichnis.}

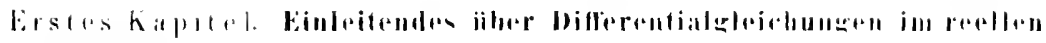

trehiel.

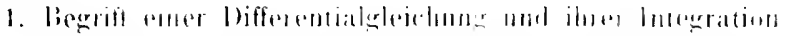

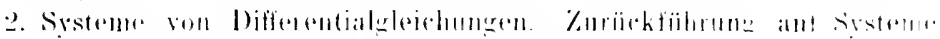

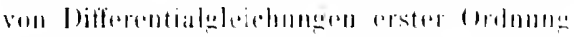

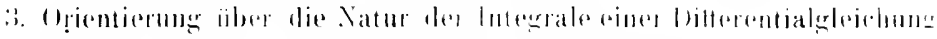

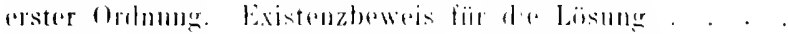

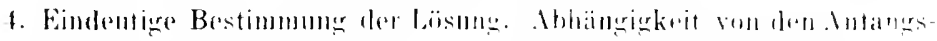

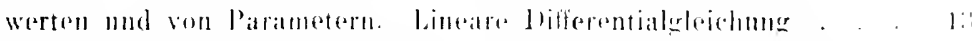

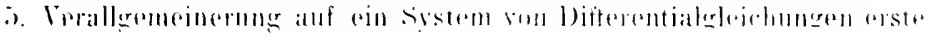

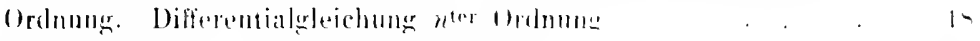

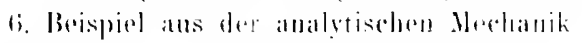

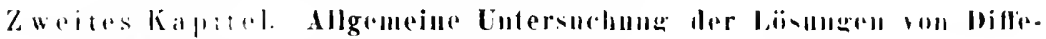
renlialegleichungen "rster Orilume.

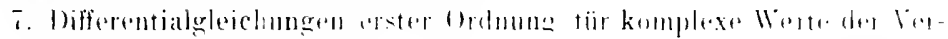

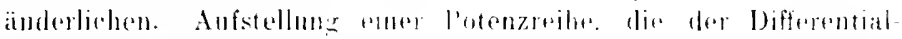
eleichung formal genizet.

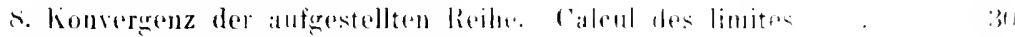

9. Lonvergenzbeweis nach Cauchy . . . . . . . . . . . . . 31

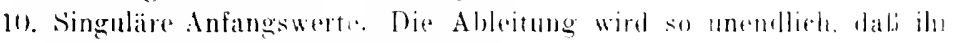
reziproker Heri lobomorph heibe. . . . . . . . . 34

11. Cntersuchung des Falles. wo die Intewralfunktion selbst unendlich wird in

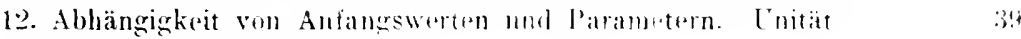

13. Analytische Fortsetzmng. . . . . . . . . . . . . . 4. 4

14. Feste und verschiehbare singuliar l'unkte. Der satz rom l'alinleve th

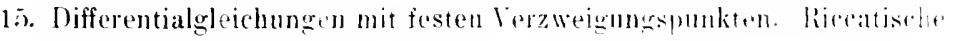

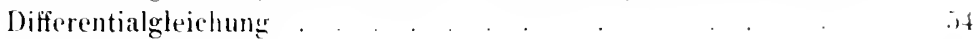

16. Form des allgemeinem Interrals . . . . . . . . . . . . . . .

17. Zusammenlangr der Ricatischen lifferentialghoichmur mit linearen homogenen Differentialghloichungeil . . . . . . . dit

lirites liapitel. Differentialgleichungen erster Ordnung. wo die Al. leitung als implizite Funktion der unabüngigen Verämerlichen gegeluen ist.

18. Begriff der Integralfunktion

19. I'ntersuchung des Falles. Wo der ligeffizient des höchsten Ponenz. der Ableitung verschwindet 


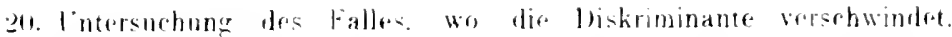
Vorberaiteneles

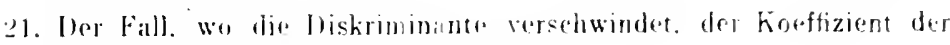
bïrelstron l'otemz der Ableitung aber nicht . . . . . . it

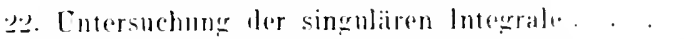

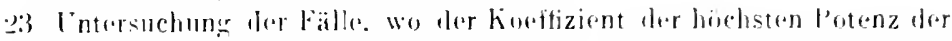
Abluitung zughoich mit der Diskriminante verschwindet. ond wo das Intergal sellost unenellich wird

-4. Ther die Theorie der singulären intexrale

Vielter hapitel. Dilferentialgleichungen mit fe-ten Verzweigungspunkton.

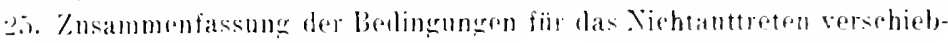
harer Verzweigungspunkte. Briot- and Bumplotertie Infferentialgleichungen

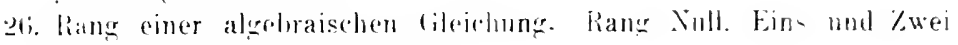

-7. Gleichungren rom liange Null

… Rileichungen rom Range Eins.

29. Briot- und Bompuetsehe Glejelsuner rom Ranere Ein-

:il. Integration dor Differentialglojchung rom liange Ein mit festen Verzwoizungspunkten.

:31. Additionstheorem der elliptisclen runktionen

3.). Cileichungen von hange Zwei. Zusammentassume der fiesultate

33. Cirschichtliches. Weitere Fragestedluneren

Fin

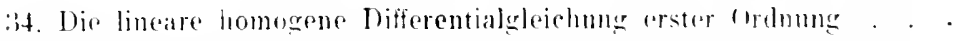

33. Sisteme won zwei linearen Differentialgheichungen Matrizankalkial

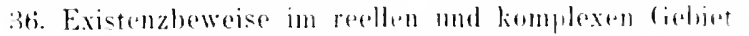

37. Methofe der sukzessiven . Ipproximationers. Melurdenteghest

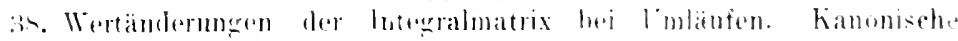
Integralmatrix

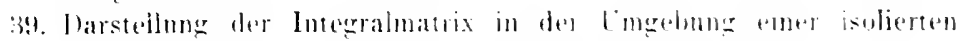

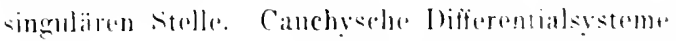

suchsteskapitel. Cntersuchune der singuliren stellum. wo lie Integrale nicht unbestimmit werden.

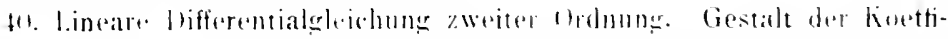

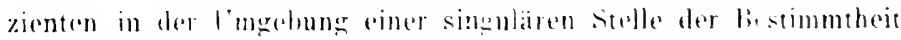

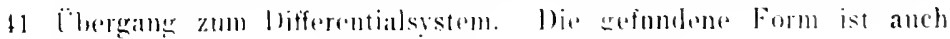
limreichend

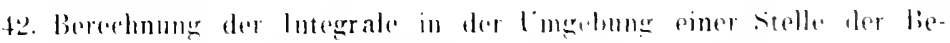

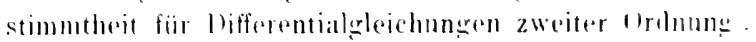

43. Beispiele. Verhalten der Integrale im l'nendliehen

44. Direkte Behandlumer der Differentialsysteme

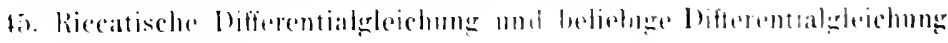
arser tromonge

46. Her Fuchseche livple

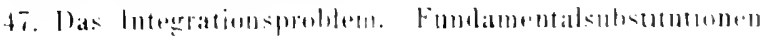




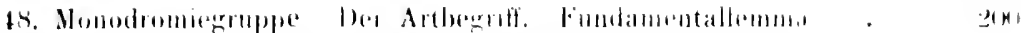

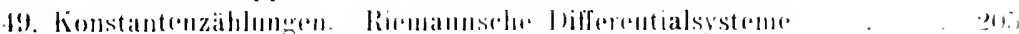

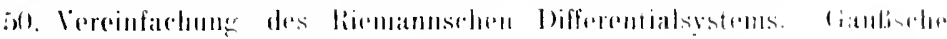
bilferentialorleichung

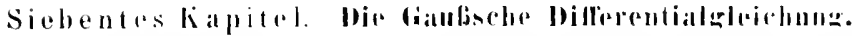

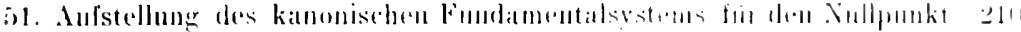

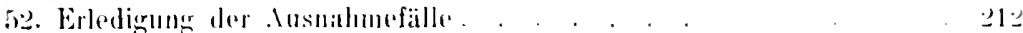

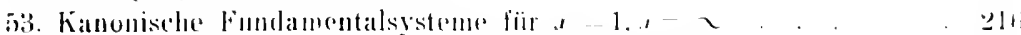

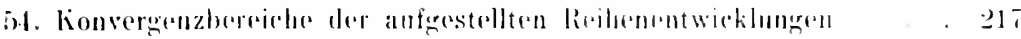

5. lestimmum der Fumdamentalsulstitutionen . . . . . . . 219

56. Multiplikator. Adjungierte Differentialgleichmer. lelentitit vol

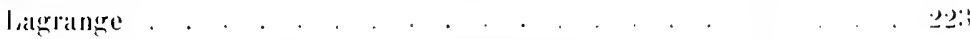

is. Integration des (ianbschen Differentialgleidung durch bestimmu

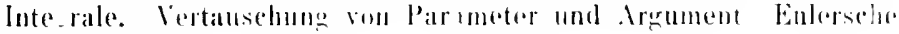

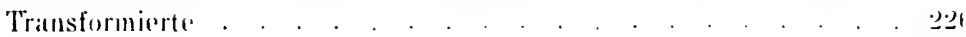

5s. Bestimmung are Dichtinkeitsfunktion mol des Integrationsweges

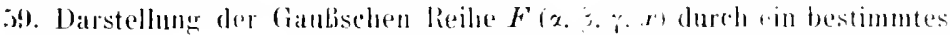
Interaral.

(bo. Darstellung des zweiten $z 1 n, r=11$ achörigen kanonischen lntegrats.

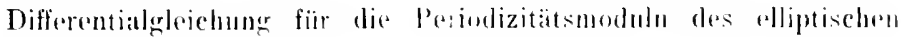

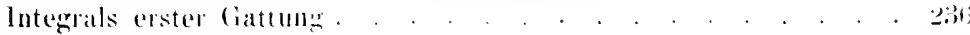

(i1. Die Klassenbeziehung

(i.2. Behandlung eines speziellon falles . . . . . . . . . . . . 24;

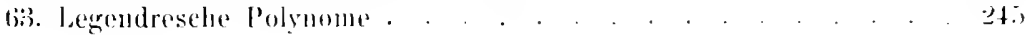

Achtes Kapitel. Intersuchung ler Integrale in der imgrhuns rine Punktes der Enlostimmtheit.

64. Differentialsysteme rom liange Eins. Cormalreiler.1.

(5). Differentialgleichung zwoiter ordunng. Riccatischro lofferentialgleichung

66. Begriff der asymptotischen Dit"stellung. Differentialıleichungen vom Range Eins. Angenäherte l)ifferentialgleichungen. . . . . . . 25i

ii. Laplacesche Differentialgleichnng. Integration durch bestimmte Integrale . . . . . . . . . . . . . . . . . . . . . 260

lis. Bestimmung der Diclitigkeitsfunktion und des lntegrationsweges

(99. Reellpositive Werte der mabhängigen Veränderlichen. Reihenentwicklung der lntegrale. tiammafunktion. . . . . . . . . . 24tit

70. Beweis der asymptotischen Iarstellung durch Intersuchung des Restgliedes . . . . . . . . . . . . . . . . . . .

71. Die Besselsche Differentialgleichung . . . . . . . . . . . 27t

7.. Darstellung der lösungen der laplaceschen Differentialgle ¿ichung durch Fakultätenreihen

Neuntes hapitel. Verallgemeinerungen. Parametrale Probleme.

73. Differentialsysteme für u lnhekamme. Integrodifferentiajgleichungatn 


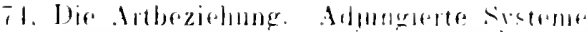

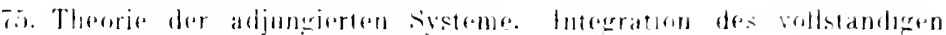
limearen Differmtialesstems. . . . . . . . . . . 2.9.

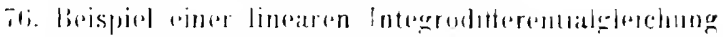

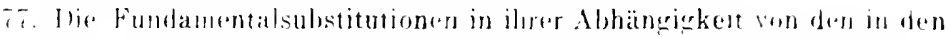

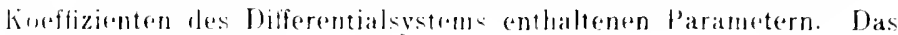
Rirmanumselar Problem.

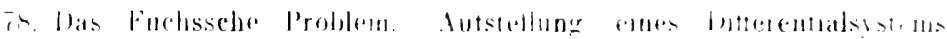
zwriten liatides. . . . . . . . . . 311:

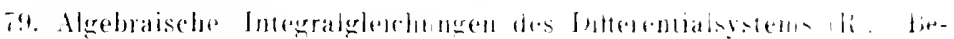
sondere fialle. . . . . . . . . . . . . . . .

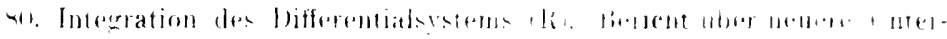
surhungen . . . . . . . . . . . . 31 . .

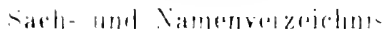


Listies liapitul.

\section{Einleitendes iiber Differentialgleichungen int reellen Gebiet.}

\section{Begriff einer Differentialgleichung und ihrer Integration.}

\%ahlreiche Anlgaben dor Anadysis. deg Geometrie, der analytischen Mechanik Pühren anf dir Aufgalu. eine Funktion von einer odre melueren veränderlichen Gröben zu bestimmen, wenn eine broinhung zwischen dieser Funktion, ihren Ablritungen mud den mabhängigen Veränderlichen gegeben ist. Eime solehe Buxirumg nemut man rime Differentialgl.iihung.

In den lustifutiones ealeuli intergals Pentesturg 1768 bis 1770, 1. Eulrei Oprea mmia, Sos. I, vol. X1-Xlll, Leipzig mod berlin

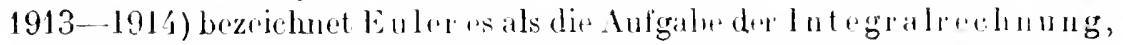
aus ciner gegobenen Boziehung zwischen den Differentialen die broinung zwischen den Gröfen selhst zu finden (Definitio I). und teilt (D)rfuntio III) die Integralrechnung in zwei Teile, von denen der arste dir Furbtionen

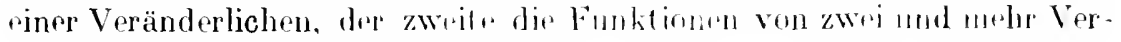
änderlichon zum Gegenstindor hat.

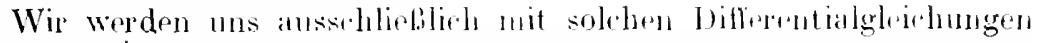
beschäftigen, in denen die Ableitungen der unbekanntm Funktion mur nach einer der Veränderlichon. von domen dir Funktion ahhängt. anfreten; man bezeichnet diese als ä"wöhnliehe Differentialglei"chugen. Für eine gewöhnliche Differentialgleiehung ist es also keineswegs ansgeschlossen, dab die zu bestimmencle Funktion von mehr als ciner Veränderlichen abhängt, viehmelı können nebst der Virändmplirhen, nach der die in der Differentialgleichung enthaltenen Ableitungen grnommen sind, in den Koeffizienten der Diflerentialgleichung noch and.r, ron jener unabhängige Veränderliche auftesten. Diese letztern werden aber dann als Konstante angesehen und zum Untersthicde von drrjinigen Veränderlichen, nach der die auftretenden Ableitungen genommen sind und die schlechthin die unabhängige Verändepliche heißcu soll, Parameter genannt. So wird uns sehr bald ( $\mathrm{Nr}$. (i) die Differentialgleichune Sehlasinger, Differentialglerichungen. 


$$
\begin{aligned}
& d r \\
& d r
\end{aligned}
$$

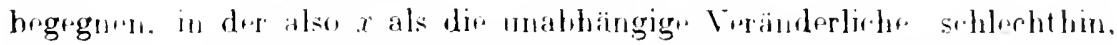

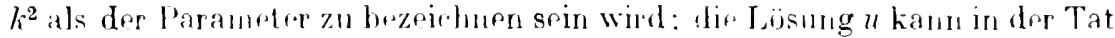

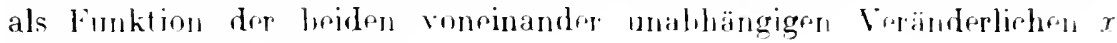
und $l^{2}$ angesohen werdent.

Wrun in einer Differmtialgleirhung partielle Wifferentialquotienten der unbekannters Funktion nark melneren vomeinander unabhängigen

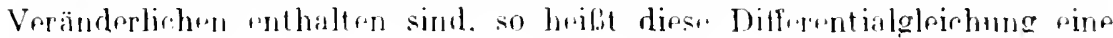
partiolle.

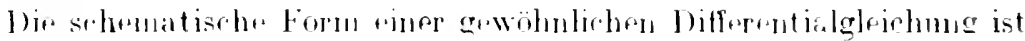

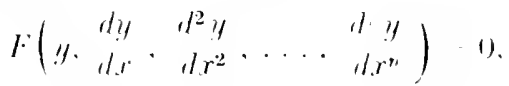

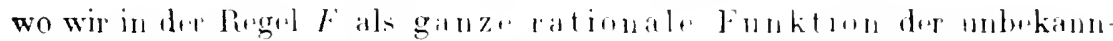

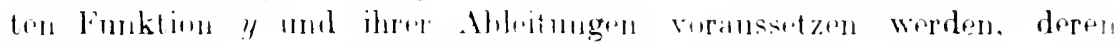

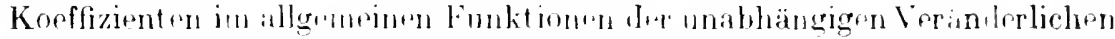

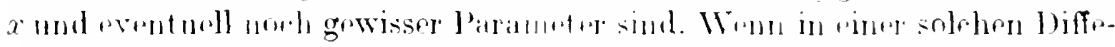

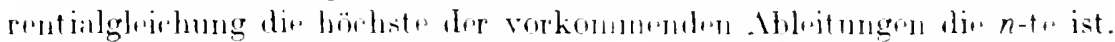

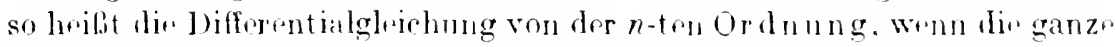
rationale lunktion $F$ and solche vou Grade $m$ ist. so ist die Jifferentialgleichung rom $m$-trin Giadn.

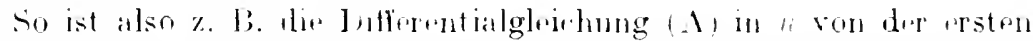

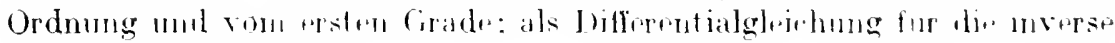
Funtion ansthriphen:

$\left(1^{\prime}\right)$

$$
\left.\left(\begin{array}{l}
d r \\
1 n
\end{array}\right)^{2}-11 \quad r^{2}\right)\left(1 \quad-l^{2} r^{2}\right) \quad 11
$$

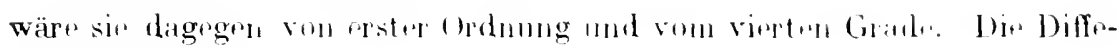
rontialgloichung

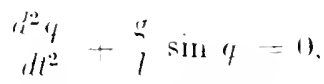

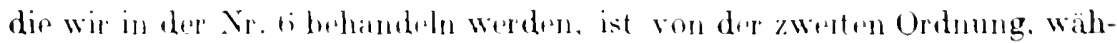
rend von riner Gradzahl nitht dir Rede soin kann. wril dir linke seite kein ganzer rationaler Ausdemek der unbekionten Funktion op und ihrer Ahloilungen ist.

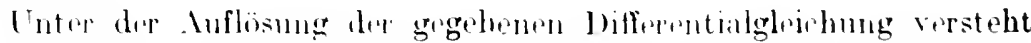
man dio bestimmung der simuthehen Funktionen, dio fiir y emgesetzt.

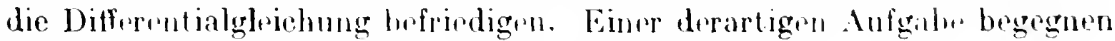
wir schon in den lidementen der lutegrallechmung. In dere Tat ist

$$
y \quad-\quad f(x) d x
$$

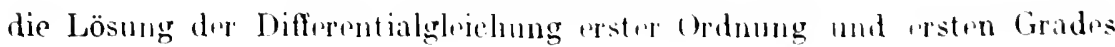




$$
\text { ily } \quad(1, r)
$$

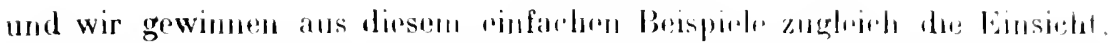

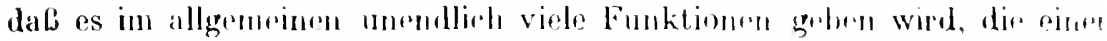

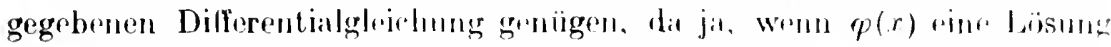
von (2) darstellt, anch $q(x)+c$, wo crine willkiirlichn Kunstante bedeutert.

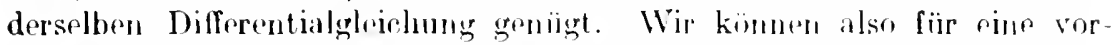

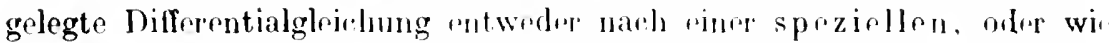
inan zu sagen pllegt, partikulären Lösung fragen oder nach der allyomeinen Iösung, d. h. der allgemeinsten Funktion, dir der Differential gleichung genügt. Wie in Fallo des gewöhulieleen Intrghals (der Yua dratur), das ja einen besonderen Fall der Lösung einer Differmitial. gleichung darstellt, bezeichnet man auch allgemein die Auflösung einet Differentialgleichung als ilure Integration, und spricht von pintiknlärem und allgemeinem Integral einer solchen fileichuns.

\section{Systeme ron Diflerentialgleichungen. Kurïcliührung auf Systeme von Differentialgleichmanen erster Ordnung.}

In dem aus den Elementen der Intrgralrechnumg entuonmenen Beispiele handelt es sich un die Auffindung einer gegebenen Finktion. für die eine Differentialgleichung gegeben ist. Oft handelt es sich aber un eine allgemeinere Aufgabe. Betrachten wir z. B. dir Bewegung eines Systems materieller Punkte unter den Einflusse grwisser Kräfte, dif auf diese Punkte einwirken. Es seien

$$
\left(x_{1}, y_{1}, z_{1}\right),\left(x_{2}, y_{2}, z_{2}\right), \ldots\left(x_{m, n}, y_{m}, z_{m}\right)
$$

die rechtwinkligen Koordinaten von $m$ solchen P'unktm. dinn hat man die Aufgabe, diese $3 m$ Koordinaten als Funktionen der Zeit $t$ zu bestimmen. Die ersten Differentialquotienten von $\left(x_{k}, y_{k}, z_{i}\right)$ nach $t$ geben di. Komponenten der Geschwindigkeit, di’ zweiten Difierentialquotienten derselben Größen die Komponenten der Beschleunigung für den Punkt $\left(x_{k}, y_{k}, z_{i}\right)$. Die mechanische Aufgabe, auf deren Lösung es ankommt, liefert eine grwisse Anzahl von Beziehungen zwischen den Koordinaten, den Geschwindigkeits- und Beschleunigungskomponenten und der Zeit $t$, die wir uns in der Regel von der Forn

$$
\begin{aligned}
& F_{\lambda}\left(x_{1}, y_{1}, z_{1}, \ldots, x_{m}, y_{m}, z_{m}:\right. \\
& \left.\frac{d x_{1}}{d t}, \frac{d y_{1}}{d t}, \begin{array}{c}
d z_{1} \\
d t
\end{array} \ldots .: \begin{array}{c}
d^{2} x_{1} \\
d t^{2}
\end{array}, \begin{array}{c}
d^{2} y_{1} \\
d t^{2}
\end{array}, \begin{array}{c}
d^{2} z_{1} \\
d t^{2}
\end{array} \ldots .\right)=0 \\
& (i=1,2, \ldots, p)
\end{aligned}
$$


denken, wo die $F_{z}$ ganzo rationale Funktionen der angedeuteten Elemente mit von $t$ abhängigen Koeffizienten bedeuten. Gewöhrlich ist die Anzahl $p$ dieser Rolationen gleich der Anzahl $3 m$ der zu bestimmrnden Koordinaten, dabei ist es nicht ausgeschlossen, daß einzelne dieser Rulationen nur dir Kondinaten, nicht aber deren Differentialquotienten enthalten, d. h. sogenannte Bedirgungegloichungen sind. Ein solches System ron Relationen bildet dann ein System von Differentialgleichungen (kürzer Differentialsystem) für dir uabekannten Funktionen. In allgeneinen können in einen Systeme von Differentialgleichungen natiirlich nicht nur die ersten und zweiten, sondern auch noch höhere Differentialquotienten der unbrkannten Funktion's auftreten. In jedem Falle ist es aber durch oin rein formalfs Verfahren müglich, ein System von Differentialglrichungen beliebig hoher Ordnung durch ein System von Dillerentialgleichungen erster Ordnung zu ersetzen, wobei allerdings die Anzahl der zu bestimmenden funktione's und darnit auch die Anzahl der Differentialgleichungen vergröbert werden muf. Setzt man z. B. in dem Fall, der Gleichungen (3)

$$
d x_{k}=\xi_{k}, \quad \begin{aligned}
& d y_{k} \\
& d t
\end{aligned} \quad 4 k, \begin{aligned}
& d z_{k} \\
& d t
\end{aligned} \quad y_{k} . \quad(k=1,2 \ldots, \ldots)
$$

so haben wir für die $6 m$ unbekannten finnktion'n

tie Glnichungen

$$
i_{k} \cdot y_{l}, z_{1} \cdot \xi_{1}, \eta_{1} \cdot \varepsilon_{k}
$$

(f $1,2, \ldots, m)$

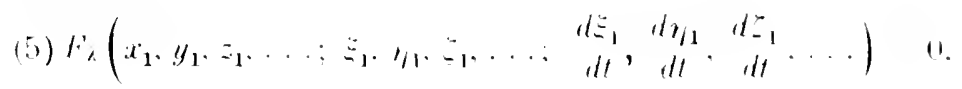

$$
\text { (1. } 1,2, \ldots, 3,3)
$$

die in Verbindung nist deng Gleichungen (4) ain System von bim Diff... rentialgleichungen erster Orduung bilden.

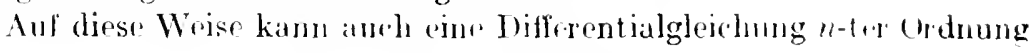

$$
f\left(y, \quad d y, \ldots d d^{n} y\right) \quad 0
$$

fur die rine unlukannte funktion $y$, durch das System

$$
\begin{aligned}
& d y^{2}=y_{1}, \quad d y_{1} \quad y_{2} \ldots d y_{n} d y^{2}=y_{n} 1 .
\end{aligned}
$$

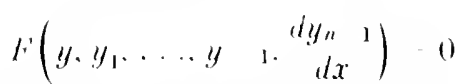

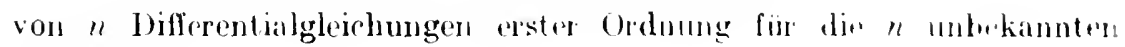
Funktionen

ersetzt werden.

$$
\|, y_{1} \cdot y_{2} \ldots \ldots, n+1
$$

lliernach besteht die allgemeinste Aufrobe der Theorie der arewohnlichen Differentialgheichungen in der Bestimmung der sambliehern 


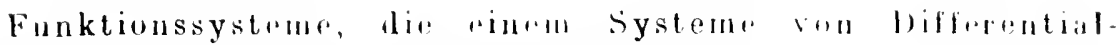

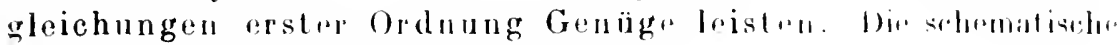
Form eines solehrn Diffurentialsystems ist

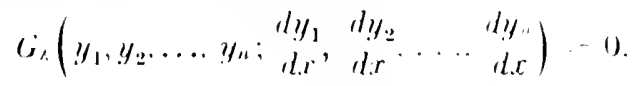

$$
\begin{aligned}
& \text { (. I. } 2 . \ldots, n \text { ) }
\end{aligned}
$$

wo wir in der Regel die ( $)$ als ganze rational. Funktionen der angednuteten Elenente, mit von $x$ abhängigen Korffrienten voranssrotzen wriden

Wenn wir in din Gleichungen (6) dir.

$$
\begin{array}{llll}
d y_{1}, & d y_{2} & & d ! n^{\prime} \\
d x & d x & \ldots & d x
\end{array}
$$

als Unbekannte ansehen, su kann nan nach den Regoln le. Eliminationstheorie das Gleichungssysten (6) durch ein ihn in algebraischen Sinm äquivalentes Systen ersetzen, das so beschaff'n ist, daß in jeder Gleichung dieses neuen Systems nur eine der Crößen (7) anftritt, das also dip Form hat

(8) $\quad F_{\lambda}\left(y_{1}, y_{2} \ldots . . y_{n}, d y\right)=0$.

wo die $F_{\lambda}$ cbenfalls ganze rational. Funktionen der angedeuteten Elemente mit von $x$ abhängigen Koeffizienten brdeuten: der Ubergang von den Gleiehungen (6) zu den Gleichungen (8) erfolgt dureh Ausführung rein rationaler Operationen.

Die allgemeinste Aufgabr, mil der wir uns zu beschäftigen haben, besteht also darin, für ein gegebenes Gleichnngssysten von der Form (8). sei es ein spezielles (partikuläres), sei es das allgemeinste Funktionssystem $y_{1}, y_{2}, \ldots, y_{n}$ zu bestimmen, das diese Gleichungen befriedigt. Man n'nnt ein solches Funktionssystem ein partikuläres beziehungsweise das allgemeine Integ'ralsyston des gegebenen Systems von Differentialgleichungen.

Bei der Darlegung der Methoden, die für die Lösung dieser Aulgabe ausgebildet worden sind. werden wir uns zumeist auf den einfachsten Fall $n=1$ beschränken, da das Wesen dieser Methoden an diesem Falle in völlig ausreichender Weise erläutert werd‘n kann, und durch diese Beschränkung eine nicht geringe Menge von Schwierigkeiten algebraischer und funktionentheoretischer Natur vermieden wird. die hei der Bebandlung der allgemeinen Aufgabe auftreten.

\section{Orientierung über die Natur der Integrale einer Differential- gleichung erster Ordnung. Existenzbeweis für die Lösung.}

Wenn $n=1$ ist, so wird das System der Gleichungen (6) zu der einon Diffe ontialgleiehung erster Ordnung 


$$
F\left(y, \begin{array}{l}
d y \\
d x
\end{array}\right) \quad 0
$$

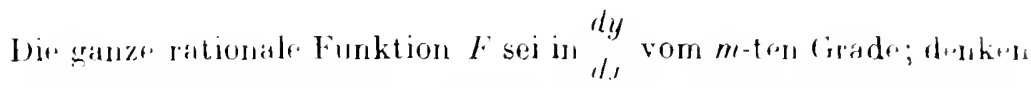
wir uns narh Potenzen dieser ciroble grenclurt, so hat de bifforentialglol chung die Citstalt:

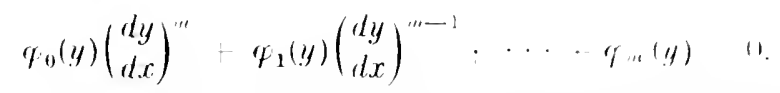

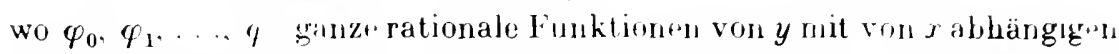
Kocffizienten bedeuten. Un vorerst Schwirigkeiten aus d.rn Weg* zu gehen, dir das Wesen der hier zu entwickelnden l'rinzipien nicht berühr.n. denken wir uns aus diesere Gleichung m-ten Grades dy ausgerechnet. Wir kommen an späterer Stelle: anl die Art und Weise, wite man siele deste Rechnung ausgeführt zu denken hat, zurïek; hiør genügt rs zu hemerkin.

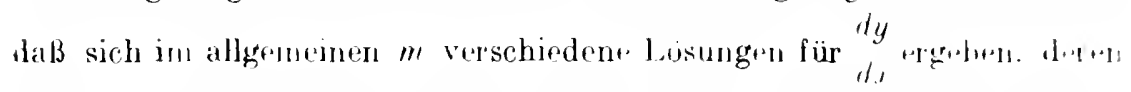
eine wir den nachfolgenden Betrachtungen zu firunde legen mud inder Furm

$$
\text { dy } \quad \text { in }(x, y)
$$

sehreiben wollen, wo $f(x, y)$ von dun koeffizionten der Glunhung "1) in algebraischer Weise abhängt.

Die Gleichung (10) hesagt, dalle der bifferentialquotitent he mulw. kannten Funktion $y$ dureh $x$ und $y$ bestimml ist. Deuten wir a und $y$ ats rechtwinklige Koordinaten in einer Ebene, so wird die unbekannte Funktion $y$ von a dureh eine Kurve dieser Ebene. eine sogenannte Integralkurve, largestellt, und die Gleichung (10) liefurt die trigonometriseh" Tangente des Winkels. unter den sich diw an jene Kurve in l'unkte $(x, y)$ gelegte Tangente zur positiven $x$-Achse meight. ausgedrückt dureh dir Koordinaten dieses Punktes. Es sei nun $\left(x_{0}, y_{0}\right)$ ein beliebiger l'unkt jemer Kurve, so kinnen wir vermöge der Gleichung (10) den Wert

$$
\left(\begin{array}{l}
d y \\
d x
\end{array}\right)_{\substack{x \\
y \\
y \\
y_{0}}}=f\left(x_{0} \cdot y_{0}\right)
$$

also dir 'langente an jene kurve in l'unkte $\left(x_{1}-y_{0}\right)$, vorausgeetet, dab $f\left(x_{0}, y_{0}\right)$ einen eindeutig bestimmten Wort hat. Diese Tangente enthält nun den dinn l'unktr $\left(x_{0}, y_{0}\right)$ unendlich benachbatten l'unkt der Kurr. mit den Kourdinaten

$$
\begin{aligned}
& x_{0}+d x=x_{1} \\
& y_{0}+d y=y_{1} .
\end{aligned}
$$

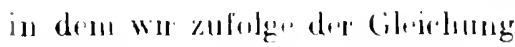




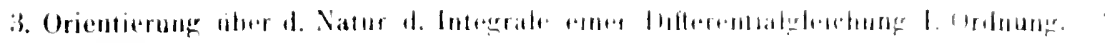

$$
\left(\begin{array}{l}
d y \\
d y
\end{array}\right)_{\substack{x \\
y}} \quad\left(x_{1} x_{1} \cdot y_{1}\right)
$$

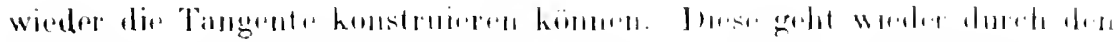

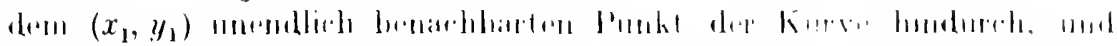

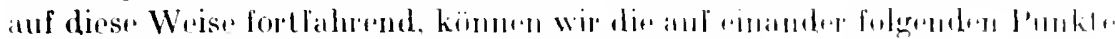

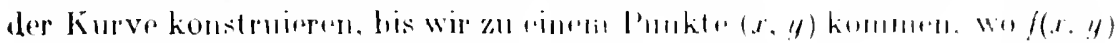

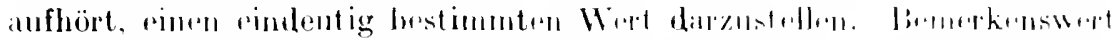

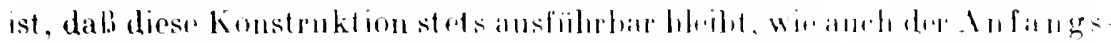

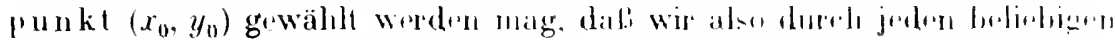

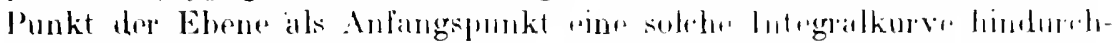

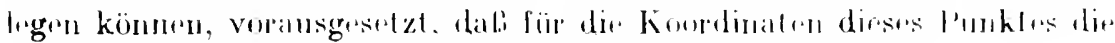
Funktion $f(x, y)$ rinen rindeutig hestimulen Wort lesil\%t

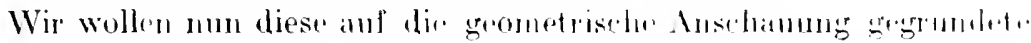

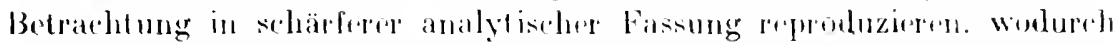

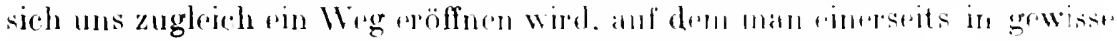
Stetigkeitseigensehaften der Integralfunktion Einsithl gowimm, andertr-

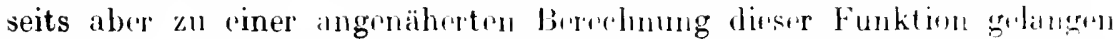
kann. Das Bedïrfnis nath solehent Nähremgeverfahren in aus den

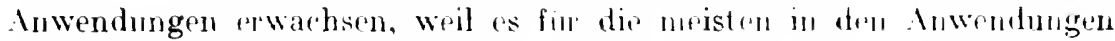

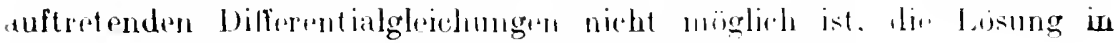
endlicher Form dureh bekanntr Funktionen dar\%ustollen.

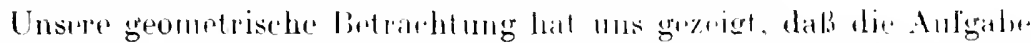
eine. Funktion zu suchen, dir der Dilferent ialgrloiehung geneiggt, noth dureh die Forderung verschärlt waden kann, dati jenr Funktion fijm ainen gat

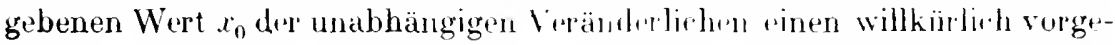
schriebenen Werl $y_{0}$ annehmensoll, d. h. dal.i man die gesucht" Funktiurt

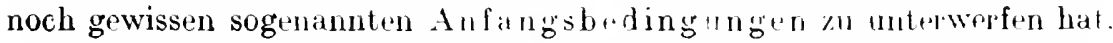
um sie genauer zu bestimmen. Nan versteht nun zunächst eim Funktion $\eta$ herzustellen, die die Anlangsbedingungrn erlüllt. also fïr $l=x_{0}$ den Wert $\eta=y_{0}$ annimmt, und dite gregelwene Differentialgleichung (10) mit einer gewissen Annäherung rrlüll. 1. h. der Gleichung

$$
d y \quad f(x, y) \cdots
$$

Genüge leistet, wo 1 eine dem absoluten butrag nath klain. Grubu bedeutet. Für die (Yaadratur. at. h. für die Differentialghrichungs

$$
d y=j(x)
$$

wo $f(x)$ von $y$ unabhängig ist, gelingt dies bukanntlich an rinfachsten in folgender Weise. IVenn etwa $f(x)$ in dem Intervalle $\left(\iota_{0} \ldots\right.$. a s) stetig Ist, sn teilt man dieses Intervall der $x$-Achse durch dit Punkte $\iota_{0}<x_{1}$ 
$<x_{2}-\ldots<x_{n-1}-a$ in $"$ Trile. ersetzt die Differentialgleichung (10a) dureh die Kette von Differenzengleichnngen

$$
\begin{array}{lll}
y_{1}-y_{0} & f\left(x_{0}\right) . \\
x_{1}-x_{0} & \\
y_{2}-y_{1} & f\left(x_{1}\right) . \\
x_{2}-r_{1} &
\end{array}
$$

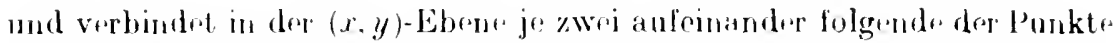
$\left(x_{0}, y_{0}\right),\left(r_{1}, y_{1}\right), \ldots .\left(x_{n-1}, y_{n-1}\right),(a, b)$ durch gerado Linien. Die so entstehende gebrodhene Linie stellt dann eine Funktion $\eta$ von $r$ dar, deren Ableitung sich mur an den Knickstellen $\left(x_{k}, y_{k}\right)$ sprungweiso ändert. Verfeinert man dir Teilung so weit, daf innerhalb rines joden Triles dir Schwankung von $\left.f(x)^{1}\right)$ kleiner als $\varepsilon$ bleiht. so befriedigt $\eta$ die Cileichung

$$
\left(10^{*} \mathrm{a}\right) \quad d \eta \quad f(x)+\mathbf{A},
$$

wo $1<\varepsilon$ ist. Es entsteht aber nun die Frage ob die Funktion, die dor angenäherten Differentialgleichung genügt, anch eine Annäherung an dir Lösung $y$ der gegehenen Differentialgleichung liefert, und wenn das der Fall ist. ob sich der Unterschied zwischen $\eta$ und $y$ beliebig verkleinern läßt. Man weiß aus don Elen,enten der Integralrechnung, wir diese Fragen zu einem kxistenzbeweis für die Lösung der Differentialgleichung (10:1 . d. h. zur Jofinition des bestimunten Integrals als

$$
!^{\prime \prime} f(x) d x=\lim _{k} \sum_{1}^{\prime \prime}\left(x-x_{k-1}\right) f\left(x_{k-1}\right)
$$

hinführen. Wir wollen hier gleich dire analogen L ntersuehungen fiit h. allgemeino Gleichung (10) in Angriff mehmen.

Es sei $f(x, y)$ in $\mathrm{d} e \mathrm{~m}$ Bereich $b$ dor $(x, y)$-Ebome eindentig. stotin und beschränkl.

$$
|f(x, y)|<x \text {. }
$$

der Anfangspunkt $\left(x_{0}, y_{0}\right)$ und das dureh die Ingleichmugen $x-x_{n}$ " $y-y_{0} \quad \therefore b$ gegubene Rechteck $R$ soien immohalh $l i$ gedegen. Theses Rechteck denken wir uns durch Parallelon zur $x$ - und $y$-Arhse in rechtrekige Zellen geteilt. Wir gehen nun von $\left(x_{0}, y_{0}\right)$ in der dured $f\left(x_{0}, y_{0}\right)$ brstimnten Richtung ans und heschreiben eine gebroehene Linir $L$, die ihre Richtung nur heim Auftreffen auf eine der Zellwände in der Weise ändert, dab sic allemal durch den Wert ron $f(x, y)$ in dem Treffpunkte

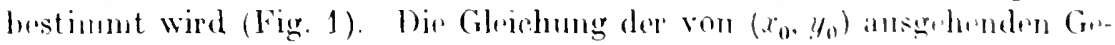
raden lautet also

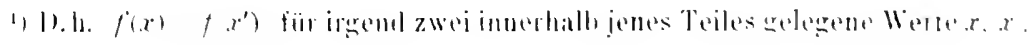




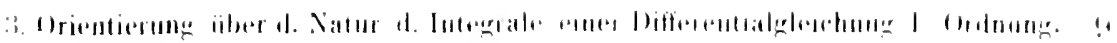

$$
\eta-y_{0}=f\left(x_{0} \cdot y_{01}\right)\left(x-i_{0}\right)
$$

Es seien $x_{1}, y_{1}$ dir Konrdinaten des Punktes, in den diusu Girade die Begremzing der zellu. trifft, in der der Punkt $\left(x_{0}, y_{0}\right)$ gelegen ist, dann ist

$$
y_{1}-y_{0}-f\left(x_{0}, y_{0}\right)\left(x_{1}-x_{11}\right)
$$

und die Gleichung des von $\left(x_{1}, y_{1}\right)$ ausgohendon St ïeks der gebrochenen Linir $I$ lauted

$$
\eta-y_{1}=f\left(x_{1}, y_{1}\right)\left(x-x_{1}\right) \text {. }
$$

Der nächste Treffpunkt dieser Geraden mit einer Zellwand sai $\left(x_{2}, y_{2}\right)$, also

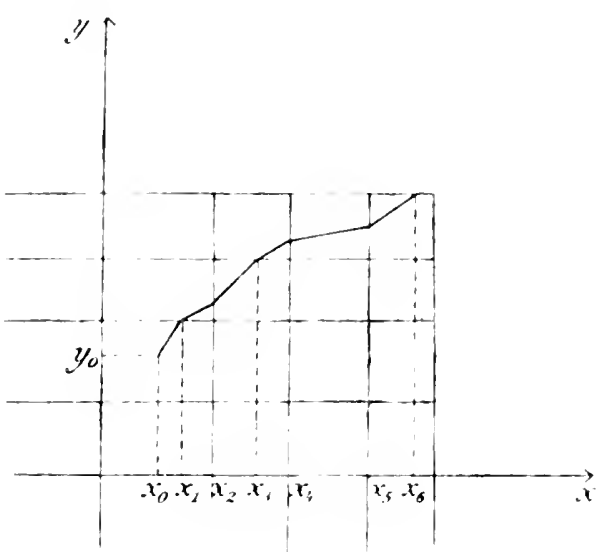

Fig. 1

$$
y_{2} \quad y_{1} \quad\left(\left(x_{1} \cdot y_{1}\right)\left(x_{2}-x_{1}\right)\right.
$$

usw. Wenn also $\left(x_{1}, y_{1}\right),\left(x_{2}, y_{2}\right),\left(x_{2}, y_{3}\right) \ldots$ die Koopliuaten dre Treffpunkte von $L$ mit den Zellwänden sind. su haben wir die Folgr von Diffa. renzrngloichungen:

$$
\begin{aligned}
& y_{1}-y_{1} \quad\left(\begin{array}{ll}
x_{1} & \left.t_{0}\right)
\end{array}\right)\left(x_{0} \cdot y_{0}\right) \\
& y_{2}-y_{1}-\left(x_{2}-t_{1}\right) !\left(x_{1} \cdot y_{1}\right), \\
& y_{3}-y_{2}=\left(x_{3} \quad x_{2}\right) f\left(x_{2} \cdot y_{2}\right),
\end{aligned}
$$

und es handelt sich nun zuvorderst darum, as so einzurichten, daß dir Linie $L$ aus dem Rechteck $\bar{R}$ nicht hinaussteigt. Wir beschränken darum $x$ auf das Intervall

(b)

$$
\left|x-x_{0}\right| \cdots A
$$

wo $A$ kleiner ist als die kleinere der beiden Gröben $a, \ldots$ und brzeirlinen das Rechteck $x-x_{0} \equiv A, y-y_{0} \mid b$ mit $R$. Wenn dann die Treffpunkte $\left(x_{0}, y_{0}\right),\left(x_{1}, y_{1}\right), \ldots\left(x_{k}, y_{k}\right)$ noch innerhalb von $R$ liegen. so sind alle $f\left(x_{0}, y_{0}\right), f\left(x_{1}, y_{1}\right), \ldots, f\left(x_{k}, y_{k}\right)$ dem absoluten Betrag' nach kloiner als $\boldsymbol{M}$, und folglich ist für das von $\left(x_{k}, y_{i}\right)$ ausgehende grradlinige St üch

von $L$

$$
\eta-y_{k}=\left(x-x_{k}\right) f\left(x_{k}, y_{k}\right), \quad x_{k} \leqq x=x_{k}+1
$$

$$
\begin{aligned}
\eta-y_{0} \mid & =\mid \stackrel{k}{\sum}\left(x_{1}-x_{2}-1\right) j\left(r_{1}-1 \cdot y_{2}-1\right)+\left(i-i_{i}\right) j\left(x_{i}, y \cdot\right) \\
& <\left|x-i_{0}\right| M .
\end{aligned}
$$

also, solange $x$ der Ungleichung (b) genügt,

$$
\left|\dot{\eta}-y_{0}\right| \leqq A M<b \text {. }
$$

Es liegt also in der Tat auch noch dieses von $\left(x_{k}, y_{k}\right)$ ausgehende St ürk von $L$ innerhalb von $F$. 
Dir dureh $L$ dargestellte linnktion $1 /-\varphi(x)$ hat aufier in den Trifl-

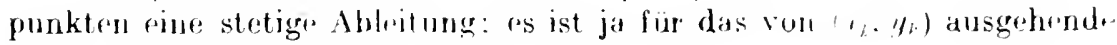
geradlinig. Stück von $l$.

$$
d_{\|} \quad f\left(x_{k}, y_{k}\right) .
$$

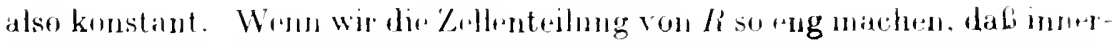
halb riner jeden Zelle die Sohwankn ng von $\left.j(x, y)^{1}\right)$ kleiner hleibt als $z$.

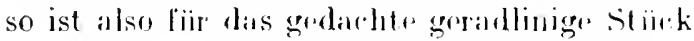

$$
f\left(x, y_{1}\right) \cdots f\left(x_{k}, y_{k}\right)
$$

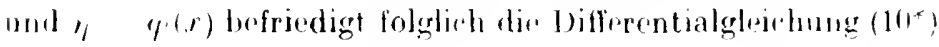

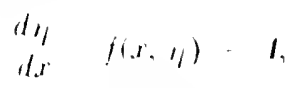

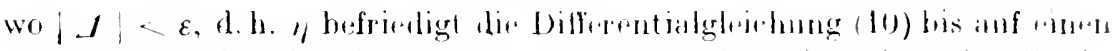

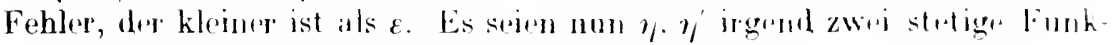
tionen mit gleichen Anfangswerten und mit. algesehen von rinzehen Aur

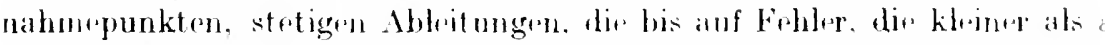

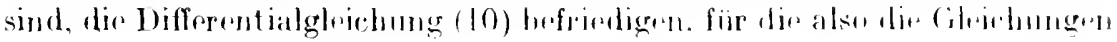

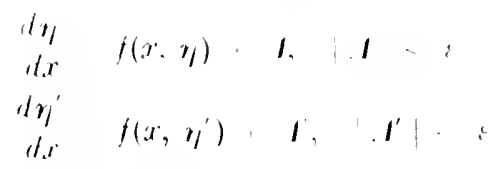

gelten. Dann folgt aus dirsin Gileichungen dureh lntegration du jat trotz der ofwa vorhandenen Ausnahmepunkte gestatlet ist mud Sub. traktionı

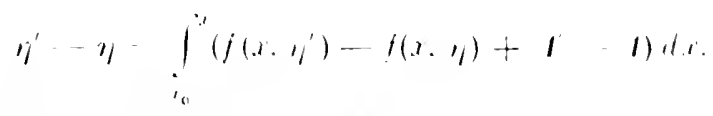

Un dirse Jifferenz abschätzen zon kömnen. noterwerfen wir dir Funtion $f(x, y)$ noch der sogenannten Lipsehitzschen Bedingung. d. h. wu setzen voratus. dat. ain positives $N$ vorhanden ist. firr das

$$
\left|f(x, y) \quad f\left(x, y^{\prime}\right)\right| \quad x\left|y-y^{\prime}\right|
$$

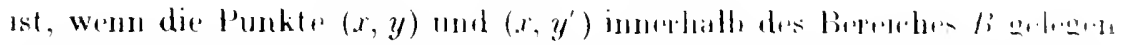

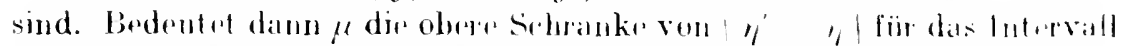

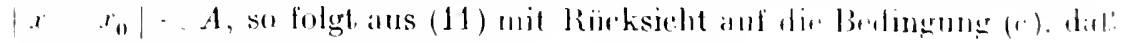

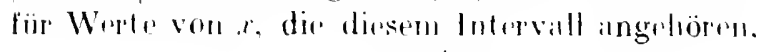

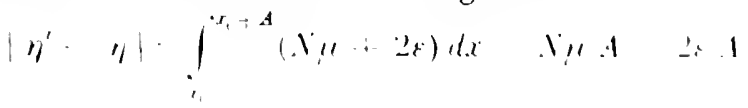

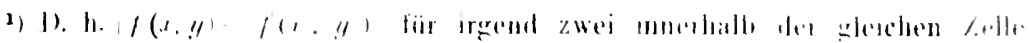
noverme l'onkte $(x, y)$ und $(x, y)$ 


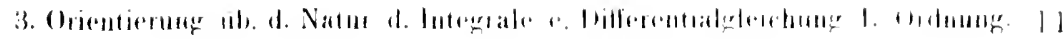

und daher

$$
11 \cdot \quad N \mid 11: 2 x+1
$$

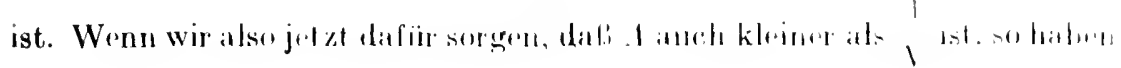
wir

$$
11 \quad 1 \quad 2 \varepsilon \cdot 1
$$

d. h. es wird der Untersehied $\eta^{\prime}-\eta$ mit $\varepsilon$ belinhele klein mad awar so, diti

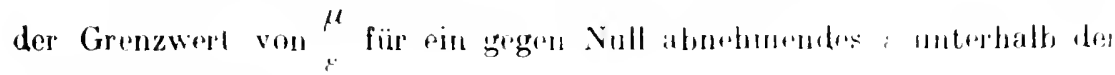
angebbaren GrößBe

verbleibt.

$$
\begin{aligned}
& 24 \\
& A N
\end{aligned}
$$

Lassen wir nummehr z gegen Null konvergiren mod betratehten di Folge der zugehërigen Funktionfln $y \quad q(x)$. w wird also dre Untersehied zwischen zwei beliebigen unter ihnen kleinm sein als: multiphiziert mil einer von $x$ unabhängigen angebbaren Größe. Diess Funktionen nähern

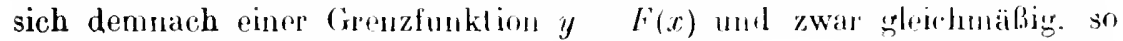
lange $x$ die Bedingung (b) trliallt, in dor jotzt a kleiner gewählt werden

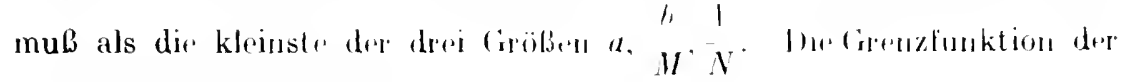
stetigen Funktion $\varphi(x)$

$$
F(x)=\lim _{\varepsilon \rightarrow 0} \varphi(x)
$$

ist folglich selbst stetig, und es is!

$$
\left.\lim _{x \rightarrow 0} \int_{x_{0}}^{x} f(x, y) d x=\int_{i_{n}}^{x} f(x, F(x)) d x^{1}\right) \text {. }
$$

1) Nähert sich die Folge $q n(x)(n=1,2,3, \ldots)$ stetiger Funktionen fiur सul gewisses Intervall $(a \ldots b)$ der Veränderlichen $x$ gleichmäbig der Cirenzfuntion $F(x)=\lim _{n \rightarrow \infty} \varphi_{n}(x)$, so gilt:

1) $\boldsymbol{H}^{\prime}(x)$ ist selbst stetig.

2) wenn $f(x, y)$ eine stetige funktion von $y$ ist. $\lim _{n \rightarrow \infty} f(x .4 n(x))=f(x . F(x) 1$.

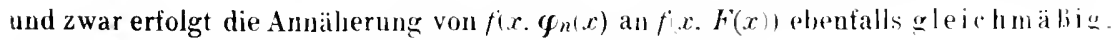
wie aus der gleichnäßigen Stetigkeit von $f(x, y)$ folgt,

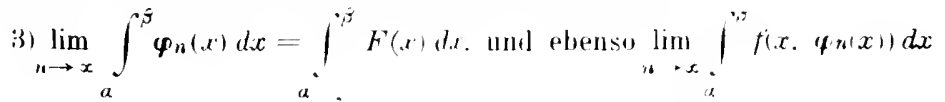
$=\int_{u}^{\dot{s}} f\left(x . F^{\prime}(x)\right) d x$, wemn das Integrationsintervall $(\alpha \ldots \beta)$ in dem Intervall $\left.a \ldots b\right)$ enthalten ist. Yergl. z. B. Osgood, Lehrbuch der Funktionentheorie I (1!12). S. !1 it 
arstes kapitel Einleitendes ibter bifferentialgleichungen im reellen Gebiet.

sus der fïr jedes $\eta$ gellenden Cileichme (10*) folgt derrel Integration die Integralebiehung

$$
\eta--y_{0}=\int_{\dot{x}_{0}}^{s}(f(x, y)+1) d x \quad, \quad \cdots
$$

lassen wir hirrin $\varepsilon$ gegen Null konvergieren. so mgibt sirh

$$
f(x)=y_{0}+\dot{f}_{x_{n}}^{* r} f(x, l(x)) d x .
$$

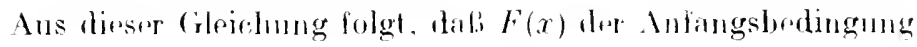

$$
F\left(x_{0}\right)=y_{0}
$$

geniigt, und dureh bifferentiation des Integrals narh der oburm Grenze. die ertanbt ist. da $F(x)$ und folglich anch $f(x, F(x))$ stretig ist.

$$
d F(x)=-j(r . F(r))
$$

so dali also $F(x)$ eine Jösung der Joifterentialgleirhung (10) darstellt. die dir Anfangshordingung, für $x=x_{0}$ den Wort $y_{0}$ anzmmolımen. erfüllt

Damit sind wir von der Frage dre angenähreten lösung unserer bifferenlialgleichung (10) ausgehend. zu rinem Existenzbewe is für dir den vorgeschriwbenen Anfangshedingungen genïginde Lösung ron (10) vorgedrungen. Fs erübrigt noch, in bezug auf diese Lösung weitrero Folgr rungen zu ziehrn; whe wir aber dazu ïhergehen, seien worh einige ge schichtliche Bemerkungen gemacht.

Jir Frage der angenäherlen Darstellung fur die Lösung riner go gebenen Differentialgleichung hat anch Euler in den Institutiones raleul integralis (vol. I, art. fisoff.) in dre hier eregebenen form behandelt. ohne sich jedoch mit der quantilaliven Ahsehätzong des begangenen Fehlers zu beschäftigen. Der erste, der versuld hat. dirs zu tun. War Canehy in seimm (182.) gehaltenen) Vorbsungen an der Pariser Feole Polytéchnique ${ }^{1}$ ). Seine Betrachtungen worden dann von R. Lipsehitz (Lehrbuch der Analysis II, Boun 1880, S. 50) vereinfacht und rersehärft. indem Lipsehitz hervorhoh, dal. die Funktion $f(x, y)$ anber den Bedingungen der Eindentigkrit und Stetigknit now der Bedingung (r) unter-

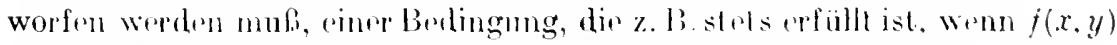
in dem Bereich $B$ eine beschänkte patioll, bhleitung nach y. Wesitzt. voranggesetzt, dal. die Begenzung von $B$ von eiture Parallelen zur y- Aehse

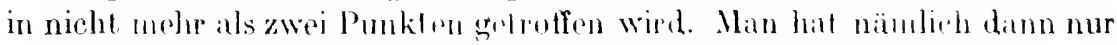

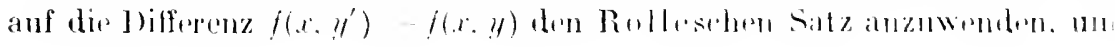

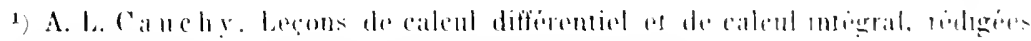

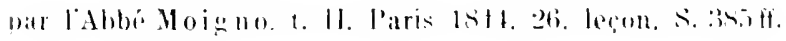




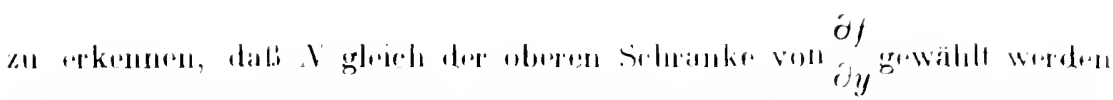

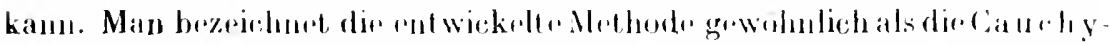

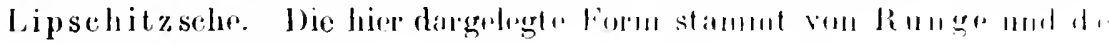
la Vallea l'oussin').

\section{Eindentige Bestimmmng der Lïsung.}

Abhängigkeit von den Anfangswerten mod ron Parametorn.

\section{Lineare Differentialgleichnng.}

Aus der Absrhätzungsformel (12) der vorigen Nummer, die liir ingend zwei stetige Funktionen gilt, die dur Differentialgleichung (10) bis auf Fehler von der Größre $\varepsilon$ genïgen, folgt nunmehr, dal.s der Lutersehied zwischen einer solchen Funktion $\eta$ mol der Lösung $F(x)$ mit denselben Anfangswert $y_{0}$ unit $\varepsilon$ beliebig kloin wird, und zwar so, lab das Virhältnis

$$
F(x)-\eta \mid
$$

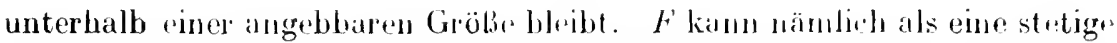
Funktion angesehen werden, dir are Dilferentialgleichmng bis and rinen Fehler von der Gröle o genügt, und damit ist zuglrieh groeigt, dals die Lösung $F$ durch ilren Aufangswert rindeutig bestimme ist. Diese Benerkung ist namentlich iür die Anwendungen von Wichtigkeit: denn wenn z. B. eine physikalisehe Aufgabe die Bestimmung einer Funktion erfordert, die die Differentialgleichung (10) befriedigt und fuir $x=x_{0}$ den Wert $y_{0}$ anninmt, so sind wir jetzt sicher, eben diese Funktion durch unsere Funktionen $\eta=\varphi(x)$ mit beliebiger Anuäherung dargestellt mnd als Grenzwert dieser Funktionen für $\varepsilon \rightarrow 0$ bestimmt zu haben.

Da aus dem Integral $y=F\left(x, y_{0}\right)$ bei geeigneter Wahl von $y_{0}$ jede Lösung unserer Differentialgleichung liervorgeht, dir für $x=x_{0}$ einen bestimmten Wert annimnt, so kamn man, indenı man $x_{0}$ fest, $y_{0}$ willkürlich läßt, die Funktion $F\left(x, y_{0}\right)$ als das allgeneine Integral der Differentialgleichung (10) ansehen; dieses hängt also von dor einen willkür lichen Konstanten $y_{0}$ ab.

Es verdient besonders hervorgehoben zu werden, dab der Satz von der eindeutigen Bestimmung des lntegrals durch die Anfangsbedingung wesentlich daran geknüpft ist, daß für $f(x, y)$ die Lipschitzsche Bedin-

1) Siehe C. Runge, Mathem. Annalen. Bd. 44 (1894). S. 4:37ff.; de la Vallée Poussin. Cours d'Analyse infinitésimale, T. 11, 2e éd., Lourain et l'aris: 1912. S. $181 \mathrm{ff}$. 
gung besteht. J)es zrigt das folgrnde von (' "a $10^{1}$ ) herrihrende Brispiel der Differentialgleichung

$$
d y=\begin{gathered}
4 y x^{3} \\
y^{2}+x^{4}
\end{gathered}
$$

wenn für $x=0$ der Anfangswert $y$ - 9 vorgeschrieben wird. Zunächst scheint der Punkt $x-0 . y=0$ eine singuläre Stelle der Funktion $f(x . y)$ zu sein: beachtrit man aber, dab

also für alle reellen Werte von $x$ und $y$

$$
y^{2}-x^{4}-2 y x^{2}-\left(y-y^{2}\right)^{2} .
$$

$$
\begin{gathered}
2 x^{2} y \\
y^{2}-x^{4}
\end{gathered}
$$

ist. so erkennt man!, dal?

$$
\begin{gathered}
4 y x^{3} \\
y^{2}+x^{3}
\end{gathered} 2 x \cdot \begin{gathered}
2 x^{2} y \\
y^{2}-x^{4}
\end{gathered}
$$

als Funktion der reallen Veräuderlichen $x, y$ fïr $x-0 . y=0$ stetig und in jedem endlichen Gebiete $|x|<a, y<b$ beschränkt ist. Die allgemeine Lösung unserer Differentialgleichung ergibt sirh. inden man $x^{4}$ - : setzt, leicht in der Form

$$
y-C=\frac{\bar{z}}{y}
$$

wo C einr wilkiirliche koustante bedeuter orler

$$
x^{4}=y^{2}+C y .
$$

Wir erkenurn daraus, dab y hei jedem Wrert der Konstanten (' fur $r=0$ den Wert $y$ Oannehmen kann. daf also durrh den Punkt $r=0 . y=0$ unendlich vielo Integralkurven hindurchgehen. Der Punkt $r=0$. y=0 ist demnach eine singuläre Stelle der Differentialgleichung, nach der buzeichnungsweise von l'uine ar pin Sattripunkt.

Das Auftreten dioses Sattelpunlits hängt nun damit zusammen, daf unsere Funlition $f(x, y)$ nicht fïr alle llerte $x, a, y, b$ die Lipschitzsche Borlingung in bezug anf y orfiillt. Narh ihr mubte für $|r|<a,\left|y^{\prime}\right|<b,\left|y^{\prime \prime}\right|<b$

$$
\begin{gathered}
4 y^{\prime \prime} x^{3}-\begin{array}{c}
4 y^{\prime} x^{3} \\
y^{\prime \prime 2}+x^{4}-y^{\prime 2}+x^{4}
\end{array} x^{\prime \prime} y^{\prime \prime}-y^{\prime}
\end{gathered}
$$

sein, wo $N$ pine endlinle, angebbare Zahl hedeutert. Dirs ist aher nicht der Fall, wenn man z. B. $y^{\prime}=0$ nimmt, da dann

$$
\begin{gathered}
4 x^{3} \\
y^{\prime \prime 2} \therefore x^{4}
\end{gathered}
$$

für hinreieh"'nd kleine Worte von $x$ und $y^{\prime \prime}$ beliwhig grof gemacht werden kann.

1) G. Pra nt). Mathem. Anmalen Bal. 37 (1890). S. 1s:2. 


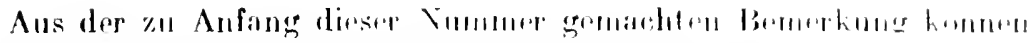

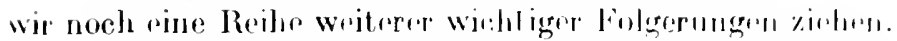

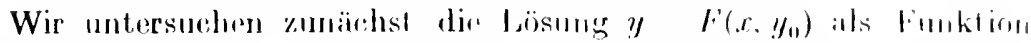

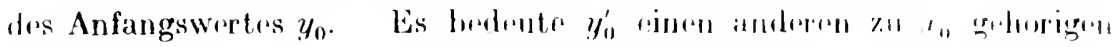

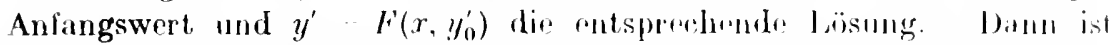
$y^{\prime}-\left(y_{11}^{\prime}-y_{0}\right)$ aine stetige Funktion von $x$ mit dem Anliangswrete $y_{10}$ firr

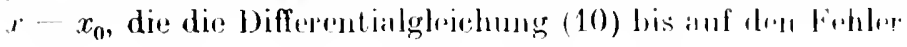

$$
f\left(x, y^{\prime}\right)-f\left(x, y^{\prime} \quad\left(y_{1}^{\prime} \quad y_{1,}\right)\right)
$$

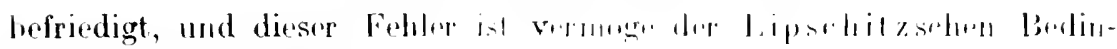
gung sicher kleiner als

$$
V: y_{11}^{\prime}-y_{0}
$$

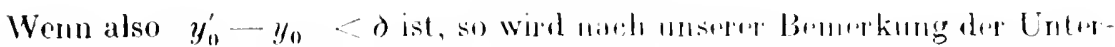

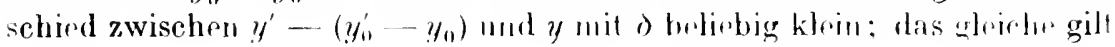

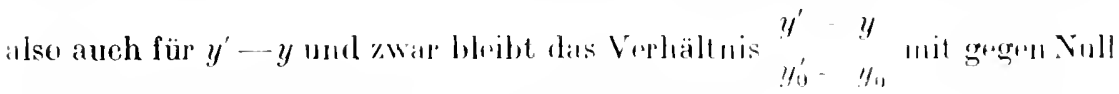
abnehmendem o mtorhalb einor angebbaren firen\%.

Dif Lösung $F\left(x, y_{0}\right)$ ist folglich rin" stetige Funktion ihres für $x=x_{0}$ vorgeseluriblenth Anfangswertes $y_{0}$.

Auf Grond dieses Ergenhisses können dir vorstohemben. hishrer aur für das Intervall $x-r_{n} \quad l$ bewiesenen Sätze, aluf all diejenigen Wertepaare $(x, y)$ aweited werlem. fïr die der Punkt $(x, y)$ inmehalb des Bereiches $B$ verbleiht. In der Tat kïmnoll wil das für $x-x_{0}=A$ erklärte Integral $F\left(x, y_{0}\right)$ ïbre dieses Intervall himaus fortsotzen, indem wir als neuen Anfangswert den Wert von $F\left(x, y_{0}\right)$ in einem der Endpunkt." dieses Intervalls wählen, und können so fortfalıen, so langt nicht die Begrenzung des Berejches $B$ erreicht wird. Dir Wreter für die dies eintritt, sind im allgemeimen singuläre Stellen dre so definierten Integralfunktion, und es ist eine der wichtigsten Aufgabrn. diese Stellen und damil den Geltungsbereich dir durch unser Verfahren gewonnenen Integralfunktion zu bestimmen. Im allgemeinen lı̈̈ngen die singulären Punkt» eines Integrals von den Infangswerten ab: so hat z. B. die Jiffermentialgleichung

$$
d y=y^{2}
$$

für die der Bereich $B$ aus allen in Endlichen golegenen Punkten der (x. $y$ )Ebene besteht, die dor Anfangshedingung $y=y_{0}$ für $x=x_{0}$ genügende Lösung

$$
y=F\left(x, \dot{y}_{0}\right)={ }_{x-x_{0}-\frac{1}{y_{0}}}
$$




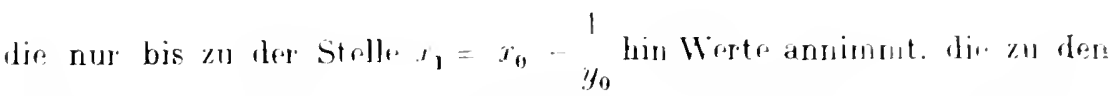

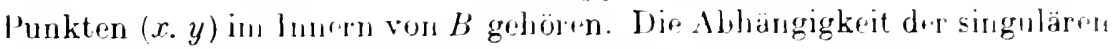
stelle $x_{1}$ von den Anfangswerten $\left(x_{0}, y_{0}\right)$ liegt hier klar zutagr.

Besonders rinfach erestaltet sich die Auffindung dre singularen stellen, wenn ihr Lage nicht von drer Wabl der Anfangswerte ahhängt: das ist z. B. offenhar der Fall fin dir gewöhulichr. Quadratu

$$
d y=f(x)
$$

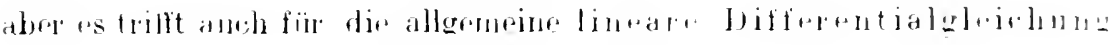

$$
d y=y \cdot g(x)+f(x)
$$

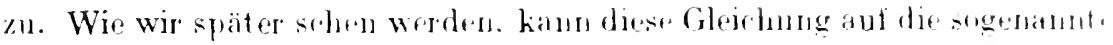
hormogene

$$
\begin{array}{ll}
d y & y \cdot n(1, r)
\end{array}
$$

zuruckgelibht werten, deren Lösung sich solort in der Form

$$
y=y_{0} \cdot e^{i i^{x}} g(x) d t
$$

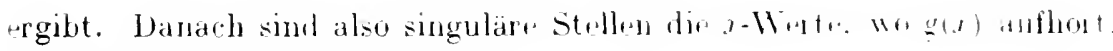
stetig und beschränkt zu scin.

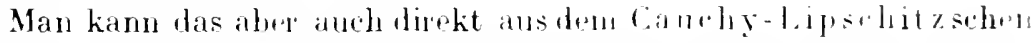
Verfahren entnelunn. Es sei nänlich für $x-x_{0}<a$ die Funktion

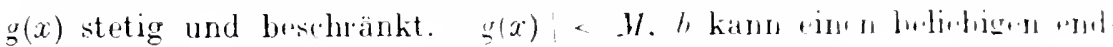
lichen Wert bedeuten; dir l.ipselistzsche bediugung

$$
\left|i(x, y)-i\left(x \cdot y^{\prime}\right)\right| \ldots x \quad y \quad y
$$

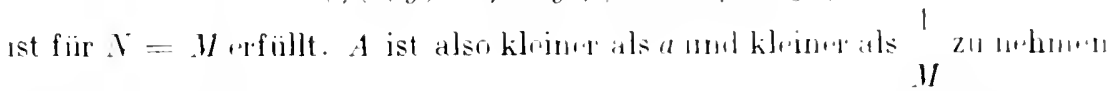

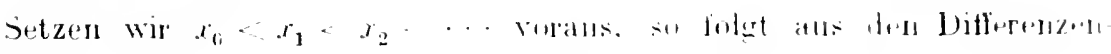
gleichungtr

$$
y_{k}-y_{k} \quad 1 \quad\left(x_{k}-x_{k-1}\right) g\left(x_{k-1}\right) y:-1 \quad \text { in } \quad 1, \ldots .
$$

dite Unglejelunng

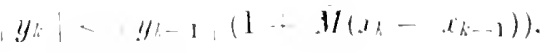

und dat fir $a>0,1+u<r^{\prime 2}$ ist.

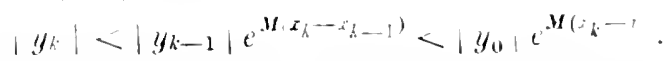

Dir $y_{5}$ bleiben also muter einer angehbaren Selaranke, so lange des fur $g(x)$ zutriffe. Ferner kann für das von $\left(x_{k}, y_{k}\right)$ atusgehenter gerathing

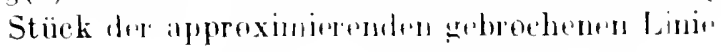

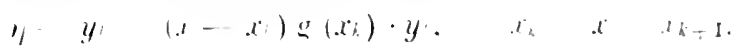




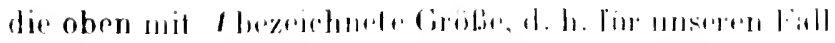

$$
1 \quad y(x) \cdot y \quad \underline{x}\left(x_{h}\right) \cdot y_{h}
$$

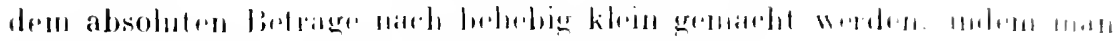

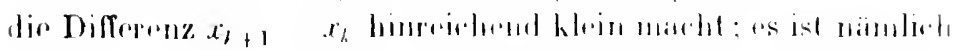

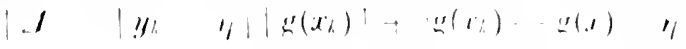

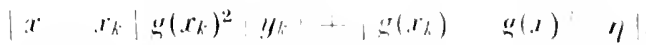

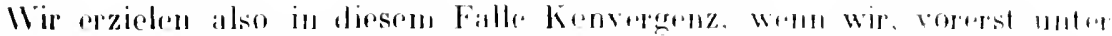

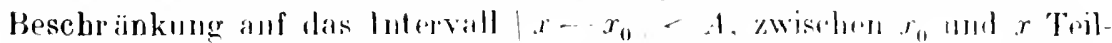

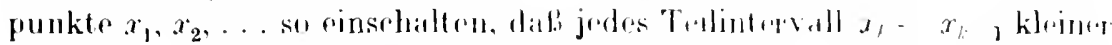

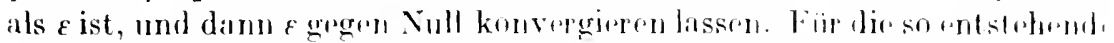
Lösung ergibt sich damn dureh Multiplikation der Givelungen

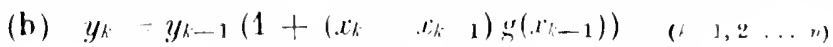

und Übrergang zur Grenze im l'unktra $=x_{n}$ der Mirt

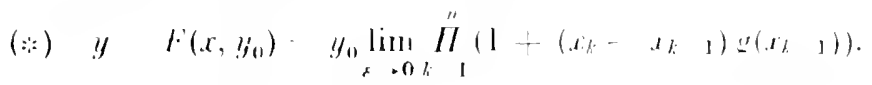

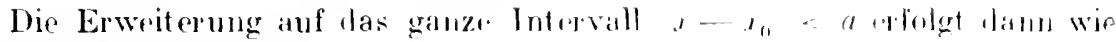
ini allgemeinen Fallı.

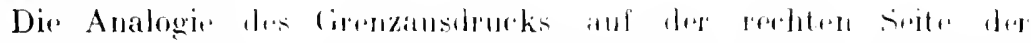

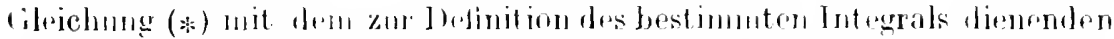
Grenzwert eimer Summe (sime uluen s. S) lällt in dir Augen. Wir wollen daher setzen

$$
\int_{i_{0}}(1+g(x) d x)=\lim _{x \rightarrow \infty} \|_{k=1}^{n}\left(1+\left(x-x_{k}-1\right) g(x-1)\right),
$$

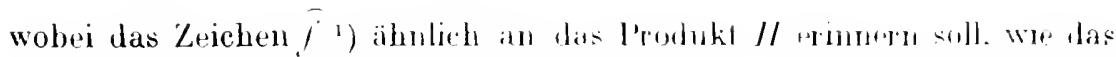

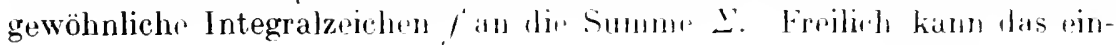

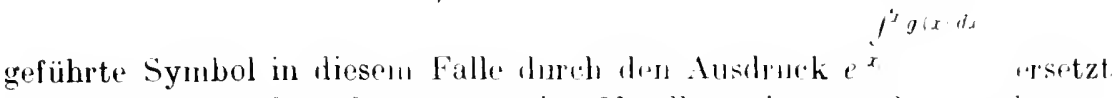
werden; wir werden aber spätor and Verallgemenurung kemmen lernen. wo eine solche Zurückführung aul bekannte Zeichen nicht mehr möglivh ist .

Hier wollen wir nur norh dru besonderen Fall anmerkin, wo $g(x)$ den konstanten Wert 1 besitzt. Tuilon wir dann das Intervall $\left(x_{0} \ldots x\right)$ in $n$ gleiche Teile und lassen $n$ ius Uneudliche wachsen. so urgiht sich

$$
\int_{x_{i}}^{x}(1+u x)=\lim _{n \rightarrow \infty}\left(1+x_{0}\right)^{\prime \prime}
$$

also die übliche Definition der Exponentialfunktion $e^{1-x}$.

Zum allgemeinen Falle der Gleichung (10) zurürkehrent. wollet wir noch untersuchen, wie die Lösumg dieser Glejehung von P'ar ametern

1) Wir lesen es: Produktinttegral. 
$\alpha, \beta \ldots$ abhängt. die in $f(x, y)$ auftreten mogral. Es saialso $f(x, y)$ eine stetige Funktion von grewissm Paranetrin $\alpha$. $\beta$..... was wir in dor Brzeichnung $f(x, y: \alpha, \beta \ldots)$ zum Ausdruck bringen. und as mögo aurh vom Anfangswert" $y_{0}$ das gejeich genten. Wird dann das Wertasystmm $(x, y, a, \beta, \ldots)$ anf einen brerech $B$ besehränkt, innrohalt dressen dir strigu. Funktion $f(x, y ; \alpha . \beta \ldots)$ noch der Lipso hit schen Berlingung in bezug auf $y$ genügt, so bohaupten wir. dal dir Lösung $y=F\left(x, y_{0}\right)$. dir ja jetzt als Funktion $F(x, \alpha, \beta \ldots \ldots)$ erschrint, auch rine stetige Funktion von $\alpha, \beta, \ldots$ sein wird. Es sej nämlich $\alpha^{\prime} . \beta^{\prime}$. . . ein Wertesysten der Parameter, das dem Bereich $B$ angehört. und für das $a^{\prime}-a_{1} \cdot \beta^{\prime}-\beta \ldots$. kleiner als $\delta$ sind, $y_{1}^{\prime}$ der diesem Wertesystem ent sprechende Anfangswert. und $y^{\prime}=F\left(x, \alpha^{\prime}, \beta^{\prime}, \ldots\right)$ das zugehörige Intrgral. Jaun hat $y^{\prime}-\left(y_{1}^{\prime}-y_{0}\right)$ lür $x=x_{0}$ den Anfangswert $y_{0}$ und hefriedigt dir. Differentialgleichung

$$
d y=f(x \cdot y ; u \cdot \beta . \cdots)
$$

bis anf den Fohller

$$
f\left(r . y^{\prime}: \iota^{\prime}, \beta^{\prime}, \cdots\right)-f\left(x \cdot y^{\prime}-\left(y_{11}^{\prime}-y_{10}\right):(\ell . \beta, \cdots)\right. \text {. }
$$

der nath unseren Voranssetzungen nit $\delta$ belinbig klein grmanht werden kann. Es kann folglich nach unserer Benerkung anch der Cntersehind zwischen $y^{\prime}-\left(y_{11}^{\prime}-y_{0}\right)$ und $y$ und d.munarh auch die Differenz $\mid y^{\prime}-y$, mit $\delta$ beliehig kenin gemacht werden.

\section{Verallgemeinerung anf ein System ron Differentialgleichungen erster Ordnung. Differentialgleichung $n^{\text {ter }}$ Ordimuns.}

Wir wenden uns nun zu den Fallo pine Systems ron $n$ Differentialgleichungen erster Ordnung und denken uns aurh hier dir dieses System darstellinden Gleichungen ( $(s)$ der Nr. 2 (S. 5) in bezug auf die Ableitungen $d y_{1}, \ldots \frac{d y_{n}}{d r}$ anfgreüst, so dal. also Glrichungen von der Form

$$
\left.d y_{2}=f_{\lambda}\left(x_{1} \cdot y_{1} \cdot y_{2} \ldots . . y_{n}\right) \quad \text { (s } 1,2, \ldots, n\right)
$$

vorliegen. J)e Cbertragung der in den Xin. 3 und 4 durchegefuhrten Betrachtung auf diese allgemeinere Aufgabe bereitet keinerlei grundsätzliche Sehwierigkeiton. so daf. wir uns danit hegnïg'n können, das für das System (13) gettrude Jigrhnis auszusprechen:

Es sei $B$ ein Bereich in Gebinte der reellon Veränderliehen $x, y_{1}, \ldots, y_{n}$, innerhalb dessen die lunktionen $f_{2}$ stetig und beschränkt sind. Es mögen ferner die Lipsehitzschen Bedingungen bestehen. d. h. e's seien positive 


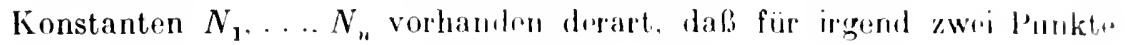
$\left(x, y_{1}, \ldots, y_{n}\right)$ und $\left(x, y_{1}^{\prime}, \ldots, y_{n}^{\prime}\right)$ von $B$

(14) $\left|f_{\lambda}\left(x, y_{1}, \cdots, y_{n}\right)\right|-f_{\lambda}\left(x, y_{1}, \cdots, y_{n}^{\prime}\right)\left|<N_{i} \sum_{i}^{\prime \prime}\right| y_{i}^{\prime}-y_{2} \mid$

ist. Ist dann $\left(x_{0}, y_{1}^{(1)}, \ldots, y_{n}^{(0)}\right)$ irgendein l'nukt ron $B$, so besitzt das System (13) stets ein System von Lösungen

$$
y_{\lambda}=F_{\lambda}\left(x ; x_{0}, y_{1}^{(0)}, \cdots, y_{n}^{(0)}\right), \quad(1=1,2, \ldots n)
$$

die für $x=x_{0}$ die Anfangswerte $y_{\lambda}=y_{\lambda}^{(1)}$ amelmen und stetig* Finnktionen der Werte $x, x_{0}, y_{1}^{(0)}, \ldots y_{n}^{(1)}$ sind. Dieses Lösungssystrul ist durch die Angabe der Anfangswrerte eindeutig bestimmt.

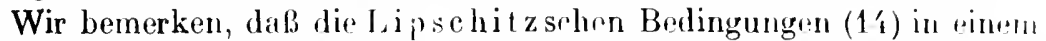
konvexen Bereiche $B^{1}$ ) stets erfïllt sind, wenn die Funktionrn $f_{A}$ in bezug auf die $y_{1}, \ldots, y_{n}$ beschränkte partielle Ableitungen lesitzen.

Spezialisieren wir dieses Resultat auf den Fall einor Differentialgleichung $n$-ter Ordnung

$$
F\left(y, \begin{array}{l}
d y d^{2} y \\
d x
\end{array}, d x^{2}, \ldots, \frac{d^{n} y}{d x^{n}}\right)=0
$$

aus der wir uns wieder die n-te Ableitung in drer Form

$$
\begin{aligned}
& d^{n} y \\
& d x^{n}
\end{aligned}=f\left(x, y, d y, \ldots, \frac{d^{n-1} y}{d x^{n-1}}\right)
$$

ausgerechnet denken wollen, so ist diese Differentialgleichung dem Systeme

$$
\left\{\begin{array}{c}
d y=y_{1}, \quad \begin{array}{l}
d y_{1} \\
d x
\end{array}=y_{2}, \ldots ., \quad \begin{array}{c}
d y_{n}{ }^{2} \\
d x
\end{array}=y_{n-1}, \\
\frac{d y_{n-1}}{d x}=f\left(x, y, y_{1}, y_{2}, \ldots . y_{n-1}\right)
\end{array}\right.
$$

von $n$ Differentialgleichungen äquivalent (vg!. Nr. 2, S. 4). Nach dem oben für ein solches System ausgesprochenen Satze gibt es also ein System von Funktionen

$$
y, y_{1}, y_{2}, \ldots, y_{n-1},
$$

das für $x=x_{0}$ die Werte

$$
y=y_{0}, \quad y_{1}=y_{11}^{\prime}, y_{2}=y_{1}^{(2)}, \ldots, y_{n-1}=y_{i}^{n-1)}
$$

annimmt, in der Nähe von $x_{0}$ stetig ist, den Systeme von Differentialgleichungen (15a) Genüge leistet und durch diese Eigenschaften eindeutig bestimmt ist, vorausgeseizt, daß die sonst willkürlich zu wählenden reellen Anfangswerte

$$
x_{0}, y_{0}, y_{0}, \ldots, y_{0}^{(n-1)}
$$

1) Ein Bereich $B$ heißt konvex, weml er mit zwei Punkten auch alte Punkte ihrer geradlinigen Verbindungsstrecke enthält. 


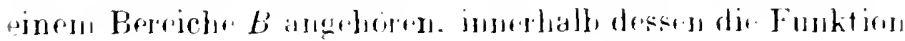

$$
\left(x, y, y_{1} \ldots, t_{n}, 1\right)
$$

tetig und besehräht ist und der l. ipsehit zschen Bedingung ganugt.

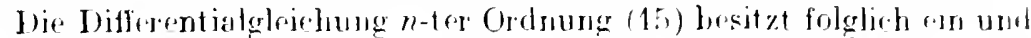
nur oin eindeutiges und stetjers Integral. das liir $a=x_{10}$ den Wort $y_{0}$ ant-

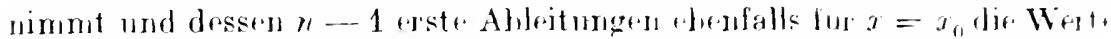

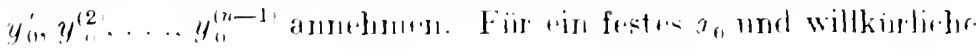

$$
y_{0} \cdot y_{11} \ldots \ldots y_{11}^{n-1}
$$

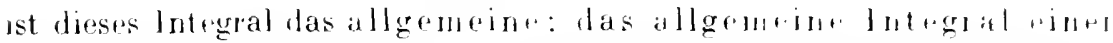
Differentialgleichung n-tor Ordnung langt demateh von" willkürlichen konstanten at).

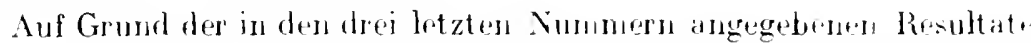
könnè wir jetzt die in der Throrir dre Diffrentialgleichungen zu losend. Aufgabe schärfer fassen. An Stelle der allgemein gehaltuen Aufgabe. dif sämtlichen Funktionssysteme zu findru. die ein grgebenes Systen von Differentialgleichungen erster Orrluung befriedigers. wetzen wir die. folgendr:

Es ist dasjenige Funktionssystem zu hestimmen. das enem gegebenen systeme von Differentialgheichungen G.. nüge leistet und gewissevorgeschriebenrAnfangsholingungen erfiillt, und zu erforseben. Wir dieses funktionseystem vou

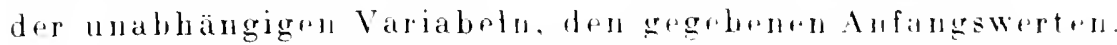
und vonden in den Koeffizinten "ventull neell anftetenden Parametern abhängt.

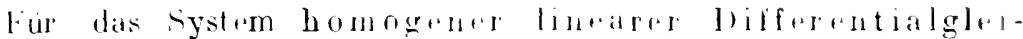

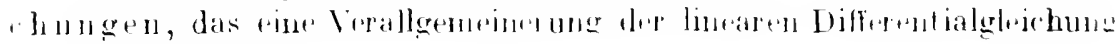
(a), $\therefore$. Li. diarstidl

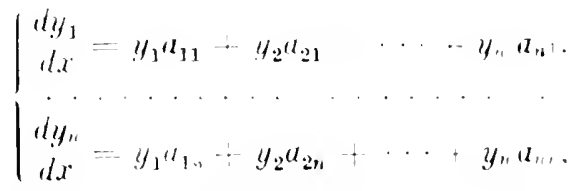

wo die $a_{2 h}(i, k=1,2, \ldots n)$ Funktionen von simb. Haben war \%ur bestimmung des lösungssystems. das für $x=x_{0}$ die Infangswert. $y_{1}=y_{1}^{(1)} \ldots . y_{n}=y_{n}^{(1)}$ annimmt. das Systeni von Differenemgloichungen

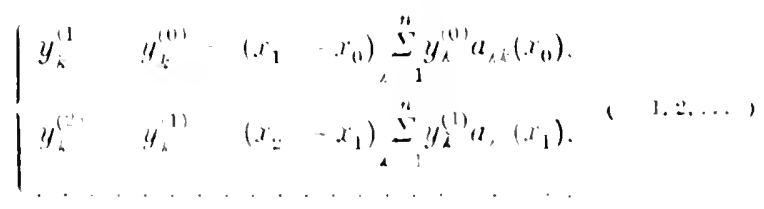




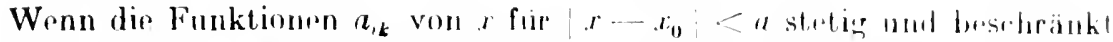
sind, $a_{i k}<M$, so sind die Lipsehitzschen Berdingungrn (1i) wieder

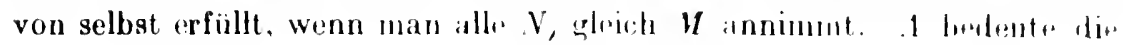
kleiner der beiden Größen a und

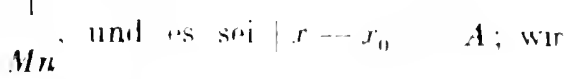
wählen feruer $x_{0}<x_{1} \cdot x_{2}<\cdot \cdot$ Es ist diillı

$$
\begin{aligned}
& \sum_{k=1}^{n} y_{k}^{(1+}-1+n M\left(r_{1}-r_{n}\right): \sum_{k=1}^{n} y^{\prime \prime} \\
& \sum_{k=1}^{n} y_{k}^{2+}: 1+n M\left(x_{2}-r_{1}\right): \sum_{k=1}^{n} y_{k}^{(1)}
\end{aligned}
$$

also

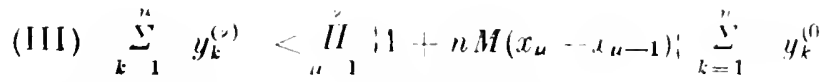
und mit Rücksicht auf $1+a<\rho^{x}$ für $a>0$.

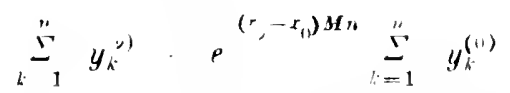

Die $y_{1}^{(\nu)} \ldots, y_{n}^{(\nu)}$ bleiben also unterhalbeiner angebbaren Srhranke, solange die $a_{\mathrm{sk}}$ selbst beschränkt sind.

Wir definieren nuu $n$ Funktioneu $\eta_{:=}=p_{i}(r)$ so. dals zwisehen $x$ und $x_{\nu+1}$

$$
\begin{aligned}
& \text { (*) } \\
& \eta_{k}-y_{k}^{(*)}=\left(x-x_{,}\right) \underset{i}{\stackrel{n}{*}} y_{i}^{(\jmath)} u_{\lambda, k}\left(x_{\nu}\right) \\
& \text { (4) } 1,2, \ldots, n)
\end{aligned}
$$

ist. Dann befriedigen die $\eta_{k}$ das Differentialsystem

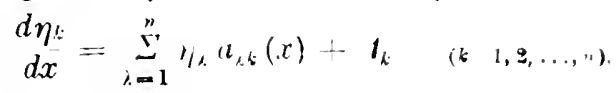

wo in dem Intervall $\left(x, \ldots x_{y+1}\right)$

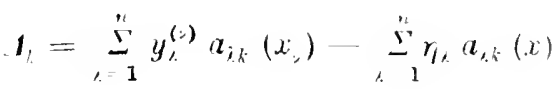

ist. Nun ist aber

$$
\boldsymbol{f}_{k} \leqq \sum_{i=1}^{n}\left|y_{i}^{(\nu)}-\eta_{i} \quad a_{\lambda, k}\left(x_{,}\right)+a_{\lambda, k}(x,)-a_{\lambda, k}(x) \quad \eta,\right| \cdot
$$

also mit Rücksicht auf $(*)$ unı auf $\left|a_{\lambda k}(r)\right|<M$

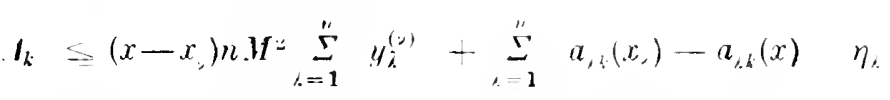

Aus den Ungleichungen (III) und aus der Stetigkeit der $a_{i, k}(x)$ folgt jetzt. $\mathrm{daB}$ die $\boldsymbol{1}_{k}<\varepsilon$ gemacht werden können. inden man $x_{*+1}-x_{z}$ hinreichend klein wählt. Indem man also die $x_{1}, x_{2}, \ldots$ hinreichend eng aneinander rückt, kann man bewirken. daß die Funktinnen $\eta_{i}=\varphi_{i}(x)$ 
das System (I*) mit $\left|\boldsymbol{I}_{k}\right|<\varepsilon$ befriedigen; d. h. diese Funktion m genügen dem System (I) bis auf einen Fehler, der heiner ist als $\varepsilon$.

Hat man nun zwei Funktionssysteme $\eta_{k}$, $\eta_{k}^{\prime}$ nit densellon Anfangswerten für $a=x_{0}$. die das System (I) bis auf einan Fehlor, der kleiner als $\varepsilon$ ist. befriedigen, $d$. h. ist

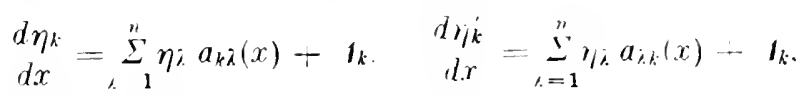

so folgt durch Integration, die ja trotz der Unstetigkeit der $\frac{d \eta_{k}}{d x}{ }_{d, / k}$ an den Stellen $x_{1}, x_{2} \ldots$ erlaubt ist.

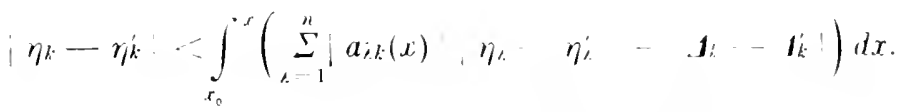

Bedeutet dann $\mu$ die obere Schranke der Differenzen, $\eta_{k} \ldots \eta_{k}$ in dent Intervall $\left(x_{0} \ldots x_{0}+A\right)$, so haben wir

$$
\mu<i_{i_{0}}^{x_{n}+A}(\mu n \mu+2 \varepsilon) d x,
$$

also da $A<n y$ sein sollte.

$$
\mu<\underset{1-n . H_{A}}{2 \varepsilon A}, \quad \stackrel{\mu}{\varepsilon}<\frac{2 A}{1-n .1 / A} .
$$

d. h. die Unterschiede $\eta_{k}^{\prime}-\eta_{k}$ werden mit $\varepsilon$ beliebig klein und zwar so, daß für ein gegen Nıll abnehmendes $\varepsilon$ die Grenzwertı $\eta_{\varepsilon} y_{k}$ interhalb riner angebbaren Grölie bleiben.

Daraus folgt nun, dals sich die $\eta_{k}=\varphi_{k}(x)$ mit Verkleinerung der Abstände zwischen den Punkten $x_{0}, x_{1}, \ldots$ hestimmten, stetigen Grenzfunktionen

$$
y_{i:}=\lim _{x \rightarrow 11} \varphi_{k}(x)
$$

gleichmäßig annähern, von denen man (wie oben in den Nrn. 3 und '́) leicht zeigt, daß sie dem System (I) genügen, für $x=x_{0}$ die vorgesehriebenen Anfangswerte annehmen und durch diese eindeutig bestimmt sind. Die Fortsetzung über das ganze Intervall $\mid x-x_{0}<a_{\text {a }}$ bereitet jetzt keine Schwierigkeiten und wir haben also den Satz:

Innerhalb eines Intervalls der $x$-Achse, wo die $a_{\lambda k}(. l)$ stetig und beschränkt sind, hat das durch das Cauchy-Lipsehitzsche Verfahren 


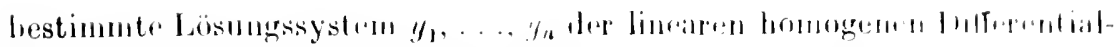
gleichungeren (l) kirinn singulïr. Stelle.

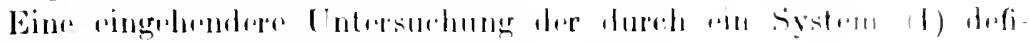

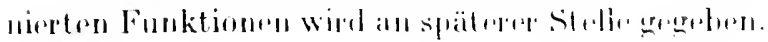

\section{(i. Boispiel ans der analytische'il Ilechanik",}

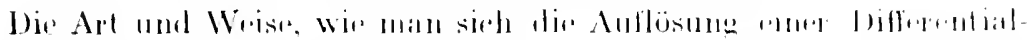

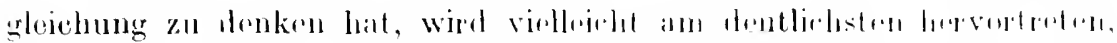

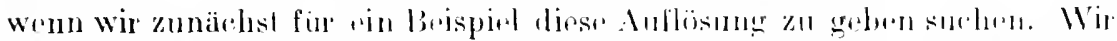

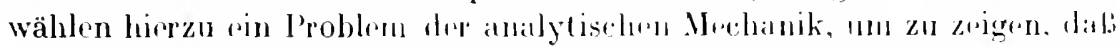

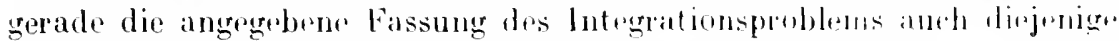

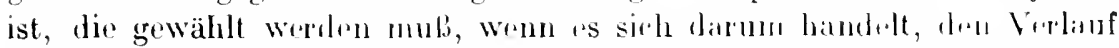

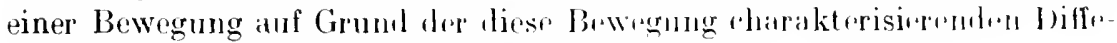
rentialgleichungen zu beschroiben.

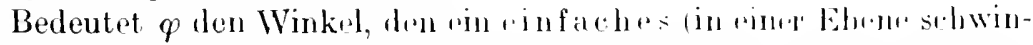
gendes) mathomatisches l'endel von dor Länge l \%m /, it l mit der

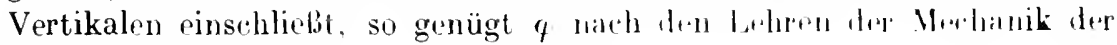
Differentialgleichung

$$
d t^{2}+-\frac{t^{2}}{1} \sin \varphi
$$

wo g die konstante der Gravitation badrented. Birse l)itferentialghleichung

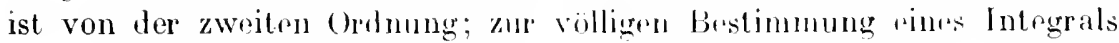

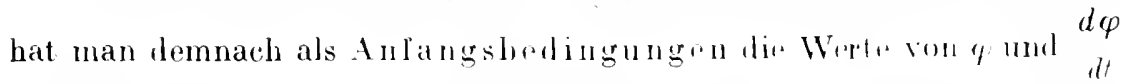
zu einer bestimmten Zeit anzmoeden. Man mula also. un die burrogung dus Pendels völlig zu bestimmen, dir Lage des Pendels und die VVinkrelgrschwindigkeit des pendelndon Punktes in einen Zeitmonente kennen.

Das Pendel möge sich zur Zuit $t=0$ gerade in der Vertikalen betinden, und in diesem Momente sei die Winkelgesehwindigkrit gleich "u. dann haben wir also die Anfangshodingungen

$$
q=0, \quad \frac{d t}{d t}=v_{0}, \text { fïr } t=0 .
$$

Multipliziert man beide Seiten ler Gleirhung (16) mit 2, d/ . s." erhält man

$$
d t\left|\left(\frac{d \psi}{d t}\right)^{2}\right|=\begin{array}{cc}
2 g d \cos \psi \\
l & d t
\end{array}
$$

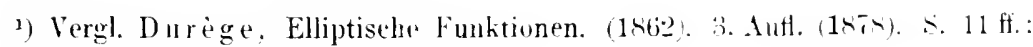
Kirchhoff. Mechanik, (1876). S. $18 \mathrm{ff}$. 
und inden man anf lesiden Sriten nach $t$ integriert.

$$
\left(\begin{array}{c}
d p \\
d t
\end{array}\right)^{2}=\frac{2 g}{l}(\operatorname{ros} \varphi-\cos r) \text {. }
$$

wo $a$ die Integrationskonstante bedrutel. Setzen wir $t$ - 1). so mgibt sich zufolgr dor Anfangsbedingungen (17)

$$
\frac{2 g}{l}(1-\cos (2)
$$

woraus

$$
\cos r_{2}=-1-l_{20}^{2} .
$$

Hierans kennen wir sehon einen Sehlug ziohen, der dir Art. wir der Verlauf der Bewegung von dor Wahl der Anfangsbedingungen abhangt. hervortreten läßı. In der Tat ist:

$$
\begin{aligned}
& \text { fiir } l v_{11}^{\prime \prime}-2 g, \quad \quad l<\frac{.7}{\underline{y}} \\
& \text {. } 2 g<l_{11}^{\prime} \quad \text { ig. } \quad u>\frac{7}{2} \\
& \text { " } \quad l \nu_{0}^{\circ}>4 g . \quad \text { a imaginär }
\end{aligned}
$$

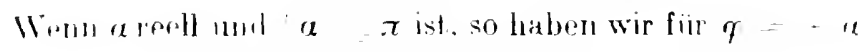

$$
\left.\left.\begin{array}{l}
d p \\
d t
\end{array}-1\right), \quad \operatorname{sgn} \frac{d^{2} p}{d t^{2}}=-\operatorname{sgn}(\cdot a)^{1}\right),
$$

die Funktion $p$ ron $t$ nimut also fur $q=\ldots$ a timm "xtremen Wert (Maximum oder Minimum) an. D. h. wenn a reell ist, oder, wenn $l 0_{0}^{\circ}$. die sogenannte Zontrifugalkrafl zur Zeit les Durchgangs durch dir Vertikale, $t=0$, nicht grölier ist als dir virfarh. Shwere. sehwingt das Pendel zwischen zwei Extremlagen hin und her, nud zwar blibt es unterhall der durch den Aufhängepunkt grhenden Horizontaten. wenn dir Zentrifugalkraft in Momente $t=0 \mathrm{kl}$ iner ist als die doppelte Sehwere. während es sich in entgregengesetzten Falle iibre diese Hurizontale erhebt. Wenn a imaginär ist, d. h. Wenn die Zentrifugalkraft zur Zeit des Durchgangs dureh die Vertikalr größer ist als die vierfache Schwere, sehwingt

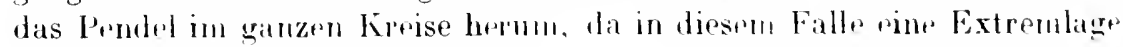
nicht existiegt.

Lassen wir den letztolen Fall heiseite, bechränken uns alou anf atas

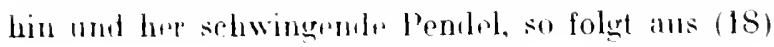

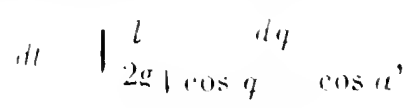

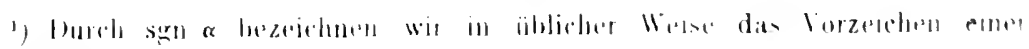
ierllen tiriblice a 


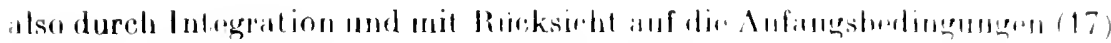

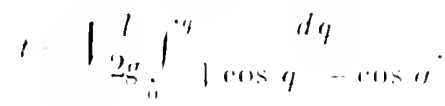

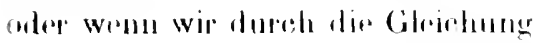

$$
\sin \frac{1}{2} \% \quad x \sin \frac{1}{2} \ell
$$

sine none Integrationsvariahlo $r$ einfiihren nud iituredies

$$
\sin ! a-k
$$

setzen,

$$
\left.1\right|_{l} ^{a} \int_{i}^{1} \quad \begin{array}{ll}
d x & \left.-i^{2}\right)\left(1-k x^{2}\right)
\end{array}
$$

Das hifr anftretende hutegral ist ent olliptischos; wir worlon an späterer Stello (Nr. 29) zoigen, dab, wenn in der Crloichung,

$$
\| \int_{i}^{\cdot r} 1\left(1-x^{2}\right)\left(1-k^{2} x^{2}\right)
$$

$x$ als Funktion von $u$ atufgefalst wird, dies. Funktion einefur jedrn Wert von $u$ eindeutige w. Man bezeichnet dies" Funktion mach Jacolials

$$
x=\operatorname{sinam} u
$$

und sagt, sie gehöre zunı Modul $k$, was man auch in der linzoiehnung hervortreten lassen kann, indem man

$$
r=\sin a m(u ; \bmod k)
$$

sahreibt. Wir finten also

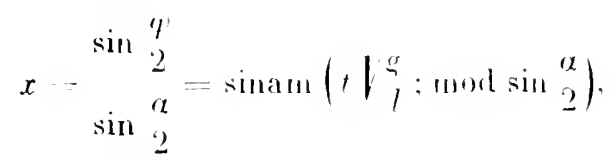

und hieraus fïr $p$ den expliziten Ausrlark

$$
p=2 \arcsin \left|\sin _{2}^{\alpha} \cdot \sin \operatorname{anu}\left(t \mid \frac{g}{l} ; \bmod \sin _{2}^{\alpha}\right)\right|,
$$

der uns $p$ als Funktion der unabhängigen Variabeln $t$, des Infangswertes $v_{0}$ (mit dem $\alpha$ durch die Gleichung (19) verknüpft ist) und nndlich des in den Koeffizienten der Differentialgleichung auftretenden Parameters $l$ (der Pendellänge) charakterisiert.

Wir können aus diesem Beispiele abm noch einf Einsicht gewinnen. die für die ganze Richtung unserer weiteren Studien von entscheidendeur Einflusse sein wird. Die Geschichte der Wissenschaft lehrt, dab eine vollständige Erkenntnis der Eigensehaften der Funktion sinaın $u$ nur dadurch erlangt werden konnte, daß man diese Funktion für komplexe Wert der Veränderlichen " studierte. In der Tat besitzt disse Funktion, wi 
$A$ belund $J$ acobi gezeigt haben, für reclle Werte von a stets eine reylle umil eine inaginäre Periode, und nur auf Grund dieser Eigenschaft (rler sogrnannten doppelten Periodizität) gelang es Jacohi, cine Darstellung j.M Funktion in der Form des Quotienten zweicr beständig konverentu Reihen zu finden, die für die Wertberechnung in hervorragendw Wri: geeignet ist. Was nun für die einfache Differentialgleichung (16) gilt. wird auch für kompliziertere Jifferentialgleichungen gültig bloiben: Inan wird eine tiefere Einsicht in die Natur der Integralfunktioni nur dann gewinnen und für die Wertberechnung lorauchlare Darstellungren dim:as Funktion nur dann geben köınen, wenn man dir Verändrrlichen nirh auf rrelle Werte beschränkt. Wir werden darum in folgend، n lie durth Differentialgleichungen verknüpften Veränderlichen als komplex.. V ..r. änderliche auffassen und von diesem Standpunktr. aus zunächst in rin systematische Entwicklung der Eigenschaften der durch rine Differentialgleichung erster Ordnung vor der Form (10) Nr. 3 (S. 6) definiertrn Funktionen eintreten. 


\section{\%w?itms kipilm. \\ Allgemeine Untersuchnng \\ der liosungen von Diflerentialgleidhungen \\ erster Ordinnng.}

7. Differentialgleichungen erster, Ordnung fïr komplexp Werte der Verinderlichen.

Aufstellung einer Potenzeihe, lie der Differentialgleichung formal genïgt.

In der Differentialgleichung

$$
d y=f(x, y)
$$

werde $x$ als komplexe Variable aufgefalit. Dann genügt es nurht, wir in dem Falle eines reellen $x$, die Funktion $f(x, y)$ als stetige und beschränkte Funktion von $x$ und $y$ aufzufassen, wir werden viehehr annehmen miissen, daß $f(x, y)$ eine monogene (analytische) Funktion der beiden komplexen Veränderlichen $x, y$ sei.

Es möge $\left(x_{0}, y_{0}\right)$ ein Wertepaar der komplexen Variabehn $x, y$ bedeuten, in dessen Ungebung $f(x, y)$ eindeutig, endlich und stetig, oder wie wir (dem Sprachgebrauche der französischen Analysten folgend) sagen wollen, holomorph ist; dann ist $f(x, y)$ in dirser Umgebung, d. h. für

$$
\left|x-x_{0}\right| \leq a,\left|y-y_{0}\right|=b,
$$

nach positiven ganzen Potenzen von $x-x_{0} . y-y_{0}$ entwickelbar:

$$
\begin{gathered}
f(x, y)=A_{00}+A_{10}\left(x-x_{0}\right)+A_{01}\left(y-y_{0}\right)+A_{20}\left(x-x_{0}\right)^{2} \\
+A_{11}\left(x-x_{0}\right)\left(y-y_{0}\right)+A_{02}\left(y-y_{0}\right)^{2}+\cdots
\end{gathered}
$$

und die Koeffizienten dieser Reihe sind nach dem Taylorschen satze durch die Gleichungen

$$
A_{00}=f\left(x_{0}, y_{0}\right), A_{10}=\left(\begin{array}{l}
\partial f \\
\partial x
\end{array}\right)_{\alpha_{1}, y .}, A_{01}=\left(\begin{array}{l}
\partial f \\
\partial y
\end{array}\right)_{x_{1}, y,}, A_{20}=\frac{1}{2}\left(\begin{array}{l}
\partial^{2} f \\
\partial x^{2}
\end{array}\right)_{x_{1}, y_{1}} \ldots
$$
bestimmt.

Es entsteht nun die Frage, ob es eine Lösung der Differentialgleichung (1) gibt, die für $x=x_{0}$ den Wert $y=y_{0}$ annimmt, und die in der Ungebung 
von $x=x_{0}$ holomorph. d. h. nach positiven ganzer Potenzen von $x-x_{0}$ entwickelbar ist. Wir setzen

$$
y-y_{0}+c_{1}\left(x-x_{0}\right)+c_{2}\left(x-x_{0}\right)^{2}-\cdots
$$

in die Differentialgleichurng ein und versuchen zunächst, ob such die Koeffzienten dieser Rrihe so bestimmen lassen. daß sie der Differentialgleichung fornal Genüge leistet.

Dor Einfachheit wegen setzen wir

daun wird

$$
r-x_{0}=\xi, y-y_{0}=\eta_{1}
$$

$$
\begin{gathered}
d y=\begin{array}{c}
d \eta \\
d x
\end{array}= \\
f(x, y)=f\left(\xi+r_{0} \cdot \eta+y_{0}\right)=p(\xi, \eta) .
\end{gathered}
$$

und $p(\xi, \eta)$ ist in der durch die Ungleichungen

$$
|\Sigma|-a,|\eta|-h
$$

definierten Ungebung der Stelle $\xi=0, \eta=0$ in der Forn

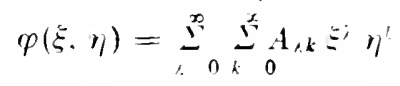

darstellbar. Die Differentialgleichung (1) verwandelt sich in

$$
d_{\eta \underline{\xi}}^{\xi}=\varphi(\Sigma . \eta)
$$

und wir haben die Koeffizienten der Reihe

$$
\eta=c_{1} \xi+c_{2} \xi 2+c_{3}=3+\cdots
$$

so zu bestimmen, daß sie die Differentialgleichung (2) formal befriedigt

Wir bilden. inden wir gliedweise differentiieren.

$$
d \eta=r_{1}+2 c_{2} \xi+i r_{3}=2 \ldots .
$$

dann mub

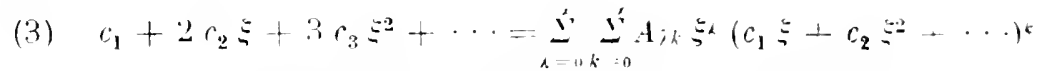
sein. Denken wir uns die auf der rechten Seite stehende Roihe nach Potenzen von $\xi$ geordnet:

$$
\varphi(\xi, \eta)=q_{0}+p_{1} \Sigma+\varphi_{2} \xi^{2}+\cdots
$$

so sind die Koeffizienten $\%$, aus den $A_{\imath k}$ und den $c_{k}$ allein lurch die Uperatonen der Addition und Multiplikation zusammengesetzt. und zwar ist offenbar $\psi_{0}$ von den $c_{k}$ ganz unabhängig, während $q_{\mathfrak{l}}$ nur von $c_{1}$. $\psi_{2}$ nur von $c_{1}, c_{2}$, allgemein $q$, nur von den

$$
c_{1}, c_{2}, \ldots .
$$

ithliangt. Wir setzen darum der Dentlichkeit wegen

$$
q=-q \cdot\left(r_{1} \cdot r_{2} \ldots r^{\prime}\right) \text {. }
$$


Aus (ij) folgt ditum

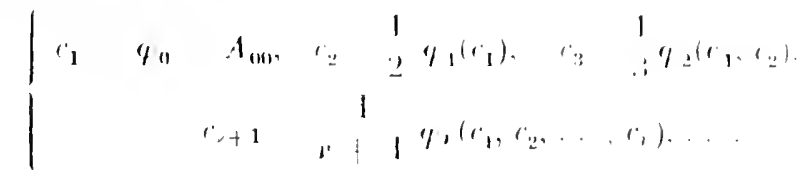

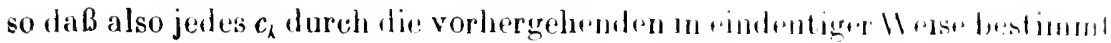
ist. Die mit dirsen co gehildete Reihe

$$
S_{1}^{S} c \%
$$

befriedigt formal die Differentialghichung (2): "s handreft sieh mun noch um die Frage ihrer Konvergenz.

Das hier eingrschlagene Verfahren, die koeffizienten riner Reilur so zu bestimmen, flaf diese Reihe einer vorgelegten Differentialgleichung genügt, wurde sehon von den Analysten des achl zehnt"n Jahrhunderts vielfach angewandt und als die Mothodr der unbestimnten Koeffizienten bezeichnet. Nur hielten jene Analysten dadurch das l'roblen der Integration für erledigt, indem sie nicht bezweifelten, dal.' rin wollbestimmter analytischer Ausdruck auch stets rinen Sinn habe.

Nun werden wir in den nächsten Nummern lirwisen, dali dir formal hergestellte Reihe (5) stets in einer gewissen Ungebung von $\xi=0$ kon-

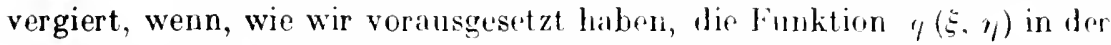
Ungebung von $\xi=0, \eta=0$ holomorph ist. Es ist aber keineswegs überflüssig, die Frage der Konvergenz zu erörtern, woll es vorkommen kann. daß eine Differentialgleichung formal durch eino nach positiven ganzen Potenzen der unabhängigen Veränderlichen fortschreitende Reihe befriedigt wird, und daß diese Reile trotzden fïr keinen vou Null virschiedenen Wert dieser Veränderlichen konvergiert. Wir zeigen dies an dem auch bistorisch bemerkenswerten Brispiel der l)ifferentialgleichung

$$
x^{2} d y+y-i=0 .
$$

Hier ist $f(x, y)=\frac{1}{x^{2}}(x-y)$ in der Ungeloung von $x=0, y=0$ nicht holomorph; es läßt sich aber trotzdem rine Reihe von der Form

$$
y=c_{1} x+c_{2} x^{2}+\cdots
$$

formal so bestimmen, das sie die Differentialgleichung (I) befriedigt. In der Tat findet man nach der Methode der unbestimmten Koeffizienten ohne weiteres

also

$$
c_{1}=1, c_{1}+c_{2}=0,2 r_{2}+c_{3}=0,3 c_{3}+c_{4}=0 \ldots
$$

$$
c_{1}=1, c_{2}=-1, c_{3}=21, c_{4}=-3 ! \ldots
$$


und somit fïr $y$ dir Rrihe

$$
y=x-x^{2}+2 ! x^{3}-3 ! x^{4}+\cdots
$$

die offenbar für jeden von Null verschiedenen Wert von $x$ divergiert $\left.{ }^{1}\right)$.

Die Frage, wolche Bedeutung man solchen divergenten Reihen beilegen kann, wird uns später beschäftigen, vorerst wenden wir uns zur Untersuchung der Konvergenz unserer Reihe (5).

\section{$\therefore$. Konvergenz der antigestellten Reihe. Calcul des limites.}

Die Untrosuchung der Konvergenz der Rouhe (5) scheint auf den ersten Augenblick ziemlich komplizirrt, la ihre kineffizienten einem nicht leicht zu iibersehenden Gesetze gehorchen. Es ist aber Cauchy, der dies. Art der Fragestellung systematisch ringrefuhrt hat, gelungen, diese Untersuchung in äuberst einfacher Weise zu ertedigen, indem or sich einer Methode bedient, die or als caleul des limites (Rechnung mit Grenzen) bezeichnet, md die seitdem in allen Zweigen der Analysis. wo es sich um Konvergenzboweise für Austrüke, die nach der Methode der unbestimmten Koeffizienten hergestellt sind. handelt, mit groben Erfolge angewandt wird.

Das Wesen der Caluchyschen Methode besteht in folgenden:

Wir denken uns an die Stelle der die Funktion $q(5, \eta)$ darstellenden Reihe

eine andere Reihe

$$
\sum_{l=1}^{\infty} \sum_{k=1}^{x} A_{i k}=\eta^{k}
$$

$$
\sum_{x=0}^{x} \sum_{k=0}^{x} B_{\lambda k}=\eta^{k}=y^{k}(\xi, \eta)
$$

gesetzt, dir ebenso wie die ursprïngliche für

$$
i \xi|\leq a,| \eta \mid=b
$$

konvergiert, und deren Koeflizienten $B_{\lambda k}$ positive, reelle Größen von der Beschaffenheit sind, dal.

$$
B_{\lambda k} \geq\left|A_{\lambda k}\right| \text {. }
$$$$
(i, k \quad 0,1,9 \ldots)
$$

Nach Poincaré bezeichnen wir diese Beziehung zwischen den beiden Reihen, oder zwischen den durch diese Reihen dargestellten Funktionszweigen $\eta(\xi, \eta)$ und $\psi(\xi, \eta)$ dureh das symbol

$$
\varphi(\xi, \eta) \ll \psi(\xi, \eta)
$$

1) Siehe zu diesem Beispiel Euler. De seriehus divergentibus, Xovi Comm.

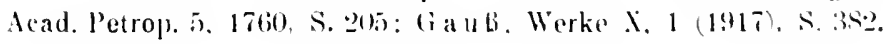


Wir betrachten mun di. Jiffrentialgleichung

$$
d \xi=\psi(\xi, 1))
$$

und setzen darin für ") diu Roilur riu:

$$
\text { 1) }=\gamma_{1} 5+\gamma_{2}=-\gamma_{3}=3, \cdots
$$

Wir bestimmen die $\gamma_{v}$ so, dab diese Roihr der Differentialgleichung (b) formal Genüge leistet; dann setzen sich dir $\gamma$, aus den $B_{\text {,k }}$ offenhar in derselben Woisezusammen, wie die $c_{\nu}$ aus den $A_{i k}$, und da die $B_{\lambda k}$ reell und

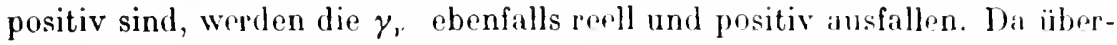
dies die $B_{\lambda k}$ nicht kleiner sind als die entsprechenden $\left|A_{\lambda k}\right|$, so folgt ferner, dab auch

$$
y,\left|c_{\nu}\right| \text {. } \quad(1,1,2,3, \ldots
$$

Es ist also, wenn wir die l'oinearésche Bezeichnung auch auf den Fall von Reihen, deren Konvergenz noch nicht feststeht, iibertragen.

$$
\sum_{\nu=1}^{r} c_{\nu} \Sigma_{\nu} \ll \sum_{\nu=1}^{\infty} \gamma_{\nu} \xi_{\nu}
$$

Wenn nun bekannt wäre, daß die für y aufgestellte Reihe in einer gewissen Umgebung von $\xi=0$ konvergiert. so folgtr hieraus, daf. auch die Reihe

$$
\sum_{x=1}^{x} c_{r} \xi
$$

in derselben Umgebung vou $\xi=0$ konvergent sei; nud dies ist d... Grundgedanke des calcul des limitrs.

Die Wahl der Funktion $\psi(x, y)$. der sogenannten Majorante. kann nun auf mannigfache Art erfolgen. Wir geben zuvörderst das klassische Verfahren von Cauch ${ }^{1}$ ).

\section{Konvergenzbeweis nach Cauchy.}

Es sei allgemein

$$
\varphi(\xi, \eta)=\sum_{\lambda=0}^{\infty} \sum_{k=0}^{\infty} A_{\lambda k} \xi^{\lambda} \eta^{i}
$$

eine im Gebiete $|\xi| \leqq a,|\eta| \leqq b$ unbedingt konvergente Reihe; daun ist $q(\xi, \eta)$ in diesem Gebiete eine holomorphe Funktion der komplexen Veränderlichen $\xi, \eta$. Bedevtet $\|$ die obere Schranke der Werte, die

1) A. L. Cau c hy, Oeuvres. I. Serie. T. VII. Vergl. Briot et Bouquet. Journal de l’École Polytechnique, Cah. 36. S. 136ff. ferner Pic ard, Traité d Analỵse. T. II, (1905), S. 258, 346: H. Poin caré, Mécanique céleste, T. 1. S. 48. u. a. m.: vorl. die Literaturangaben, Enzyklopädie der mathem. Wissenschaften. Bd. Il. A 4 a. S. $201 \mathrm{ff}$. 
$|\eta(\xi, \eta)|$ für $|\xi|=a,|\eta|=l$, anzunehmen vermag. se ist nach einm elementaren Satz der Funktion'utheorie ${ }^{1}$ )

$$
\begin{aligned}
& \operatorname{lin} 1<a^{\prime} b^{h} \\
& (1, k \quad u, 1,2, \ldots)
\end{aligned}
$$

Wemlen wir diesen Satz auf die in den vorigen Nummesn betrachtet. funktion $y(\xi, \eta)$ an, so r rgibt sirh also

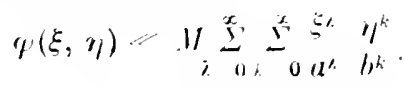

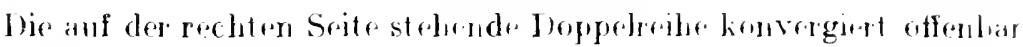
für

$$
|3|<a \cdot y !-b
$$

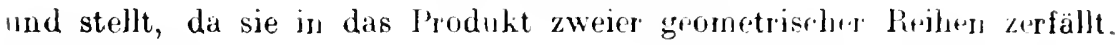
die Funktion

$$
\psi(\xi .11)=\left(\begin{array}{c}
n \\
\left.1-\frac{3}{n}\right)(1-l
\end{array}\right)
$$

dar. Dies ist die Cauch y sche Majorante.

Wir babern nun die Jifferentialgleirhung 2.11 bethathlon:

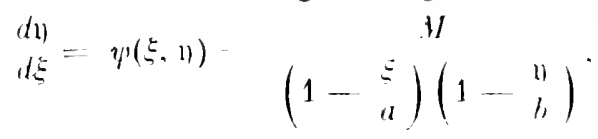

die wir in der form

$$
\left.\left(1-\frac{11}{b}\right) d 1\right)=\begin{aligned}
& 11 \cdot d E \\
& 1 \quad 5
\end{aligned}
$$

sehreibes. In dieser Form sind. we nan zu sign pilegt, die Verander lichen getrennt; wir können also auf beiden Sejten der Geleichung intrsrieres, und finden so

$$
1-2 b=-1 / a \log \left(\begin{array}{r}
1-5 \\
a
\end{array}\right)+\text { const. }
$$

als allesemeenes Integral. Dasjenige partikulise lutegral. das fiis $\Sigma=0 \mathrm{rer}$ schwindet, wrgibt sich in der expliziten Form

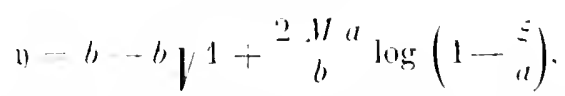

wo die (Mnadralwurzel mit pesition Vorzeichen zu nehmen ist, und dieses Integral ist offenhar in der Umgebung von $\Sigma=0$ holomerph. also durch eine lieihe von der form

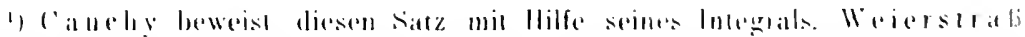

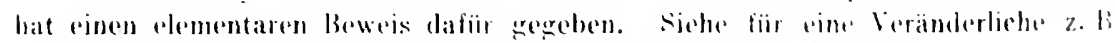

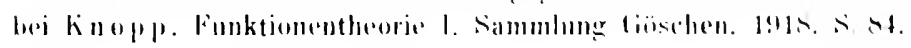




$$
\text { 1) } \quad \gamma_{1}=\gamma_{2}: 2
$$

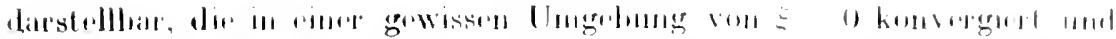

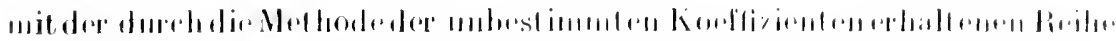

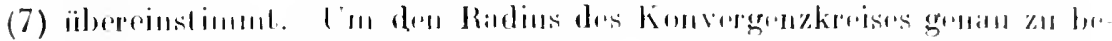

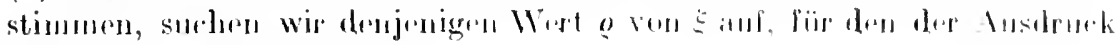
untor dout Murzelzedeles

$$
1+2 M a \log \left(1-\frac{E}{a}\right)=0
$$

ist; wir finden

$$
\text { e } \quad a\left(1+e^{-2.11 \prime \prime)}\right. \text {. }
$$

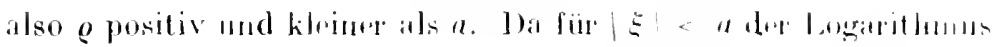

$$
\log (1 \ldots-5)
$$

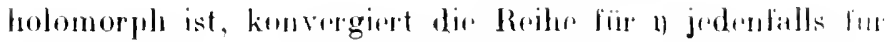

$$
\text { El } 2 .
$$

Nach dem lrinzipe des ralent des limites (No. S. S. : I) konvergint

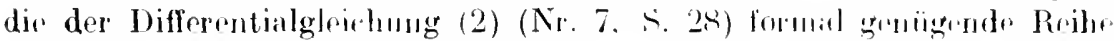

$$
\sum_{1}^{x} r_{k}=h
$$

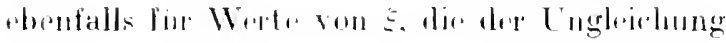

$$
\sum<0
$$

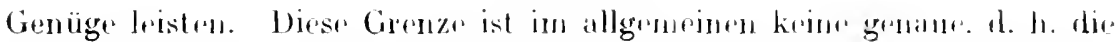
Reihe kann auch noch fïr Werte von E konvergieren. deren absoluter Betrag gröler ist als $g$; uns grenügt es aber, nacligrwiesen zu haben, dals die anfgestellto Reihe allemal innerhalb eines Kreises konvorgent ist. dessen Radius eine stets positive nur von $a, b$ und $\|$ abhängige Crölie ist.

Für die dor ursprünglichon Diffrerentialgleichung (1)

$$
d y=f(x, y)
$$

genügende Reihe

$$
y=y_{0}+c_{1}\left(x-x_{0}\right)+r_{2}\left(x-x_{0}\right)^{2}+\cdots
$$

haben wir demnaclı die Konvergenzbedingung

$$
x-x_{0} \mid<Q
$$

wobei noch hervorzuheben wäre, dafl der Ausdruck $\varrho$ und ebenso der wahre Radius des Konvergenzkreises im allgemeinen auch von den Anfangswerten $x_{0}, y_{0}$ abhängt, da ja $1, a, b$ sich mit $x_{0}, y_{0}$ ändern.

Die für $y$ aufgestelltr Reihe ist der Art ihrer Herleitung nach als solche eindeutig bestimmt, sie stellt innerhalb iłres Konvergenzbereichs ein den vorgeschriebenen Anfangsbedingungen genügendes, holomorphes: 
Integral der Differntialgluichung dar, und res kann auch krin von diesent verschiedenes in der lougebung von $x=x_{0}$ holomorphes Intugral der Differentialgleichung gohen, das für $x=x_{0}$ gleich $y_{0}$ wird. da rin solehes jodenfalls nach positiven ganzen Potenzen von $x-x_{0}$ entwickellyar sein muißte, und demnach die Koeffizionten dirser Entwirklune mit den $c_{1} . r_{3} \ldots$ iibereinstimmen müliten.

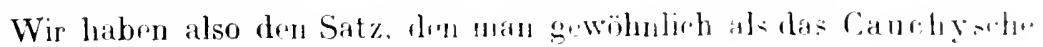
Existenztheorem zu bezejchurn pflect:

Für ein beliebig rewälltes Systrm von Anfangswertuh $x_{0}, y_{0}$, in dessen Ungebung der Differentialquotiont von y nach $x$ holomorph ist. gibt restets rine und nur wine in lur Umgebung von $r=x_{0}$ holomorphe Integralfunktion $y$. di* I ïr $x=x_{0}$ gleich $y_{0}$ wird.

Einr Abänderung der Cauclı yschen Majorante ergibt sich ${ }^{1}$ ), inden Inan an Stelle von $M$ die obere Schrankr $C_{i}$ der $\mid A_{j k} a^{*} b^{k}$ nimmt. Wir. Existenz einer solchen oberen Schranke folgt numittelhar ans dor unbe-

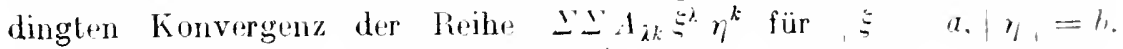

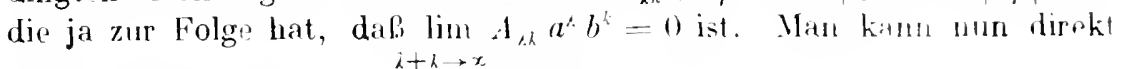
an Stelle des lia uch yehen Ausdrucks (9)

$$
\psi_{1}(\xi \eta \eta)=\frac{i}{\left(1-\frac{\xi}{b}\right)\left(1-\frac{\eta}{b}\right)}
$$

als Majorante wahlen. Man kimn aber aneh statt mit $\psi_{1}(5$, y) mit

$$
\psi_{2}(\xi, \eta)=\left(\begin{array}{c}
\vdots \\
\left(1-\frac{\xi}{a}\right)^{2}\left(1-\begin{array}{l}
\eta \\
b
\end{array}\right)
\end{array}\right.
$$

arbeiten, was den Vorteil mit sich bringt, dafs din entstehende Hilfsdiffrrentialgleichung

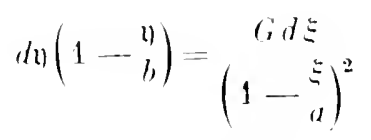

die der Anfangsbedingung $y=0$ fïr $E=0$ ginögndo algobratisehe lüsung

$$
\eta=b-\left(\begin{array}{rrrr}
b^{2} & -2(; b & \Xi & \Xi \\
& 1 & - \\
& & & \vdots a
\end{array}\right)
$$

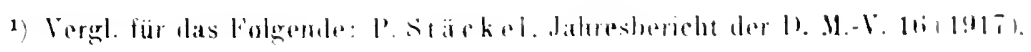
ล. 219 


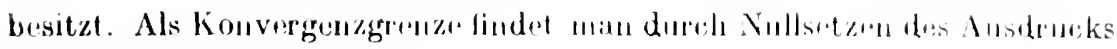
unter dem Wurmelzidelien

$$
|\Xi|<b+2, a b \cdot,
$$

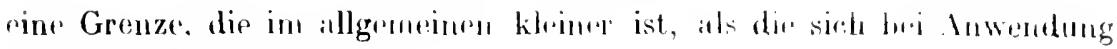
vorl $\psi_{1}(\xi, \eta)$ rerguberude

$$
\left.15 !<a\left(1-e^{\because \prime(a,)}\right)_{1}\right) \text {. }
$$

Die Majorante $y_{2}(\xi, y)$ lieter aber andererseits den Vortril, dab man bei ihrer Anwendung der Throrie dur Funktionen einer komplexen Verindrerlichen ganz entraten kann, so dal. der mit Hilfe von $y_{2}(\xi, 1 /)$ grefïhrl, Konvergenzbeweis auch fiir analytische Funktionm $q(\xi . \eta)$ der regllun Voränderlichrn E. I anwendhar ist.

\section{Ningulire Anfangswerte. Die Ableitung wird so mentlich. daß ihr reziproker Wert holomorph bleibt ${ }^{2}$ ).}

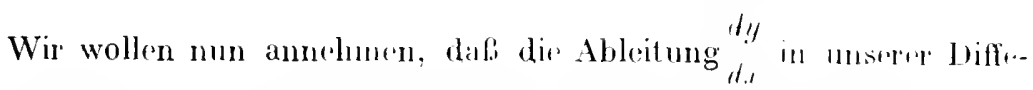
rentialgloichung (1) in der Ungrolung der Anfangswerte $x-x_{1}, y=y_{1}$ nicht mehr holomorph ist.

Es sei zunächst $f(x, y)$ lïr $x=x_{i}, y=y_{1}$ unendlich, aber sobeschafien, daß der reziproke Wert $f(x, y)$ in der Unugebung dieser Stelle holomorph. also in der Form

$$
f(x, y)=\sum_{\lambda=1 k=11}^{x} \underset{\lambda}{\stackrel{x}{x}} B_{\lambda k}\left(x-x_{1}\right)^{\lambda}\left(y-y_{1}\right)^{\lambda}
$$

entwickelbar ist. Wir setzen dann

$$
\begin{gathered}
1 \\
f(x, y)
\end{gathered}=A_{0}(x)+A_{1}(x) \cdot\left(y-y_{1}\right)+A_{2}(x) \cdot\left(y-y_{1}\right)^{2}+\cdots \cdot
$$

wo also $A_{0}(x), A_{1}(x), A_{2}(x), \ldots$ nach positiven ganzen Potenzen von $x-x_{1}$ fortschreitende Reihen bedenten, und jedenfalls

$$
\therefore x_{0}\left(x_{1}\right)=0
$$

1) Da $G \leqq \boldsymbol{M}$ ist, ist diese Konvergenzgrenze unter Unständen gröber ats das durch die Cauchysche Majorante gelieferte $\rho$.

2) Vergl. für diese und die folgende Nummer Briot et Bouquet a. a. O. S. 146; Picard, Traité II (1905). S. $36 \tau \mathrm{ff}$. : Painlevo. Leçons sur la théorie anal. des équat. différ. (Paris 1897), S. 19-20): u. a. m. 


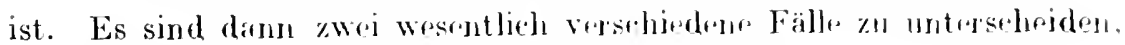
je nachdem nämlich in dor Rejhe der Koefferenten

$$
A_{1}(r) . A_{2}(r) . A_{3}(r) \ldots
$$

einer zu linden ist, der fïr $\theta=\lambda_{1}$ rinen von Null verschiodrnen Wert hat. oder nicht. In lotzteren Falle, wo alle Kueffizionten $h_{k}(x)$ eino Potruz von $x-r_{1}$ als Faktor enthalten, hat also $f(x, y)$ dir Eigenschaft. liir

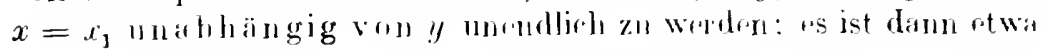

$$
f(r . y)-(y(r, y)
$$

wo der reziprokr Wert von $q(x, y)$ in der Lingehneg von $r=y_{1} . y=y_{1}$ holomorph ist. Jor l'unkt $x=r_{1}$ wird dam für jodes Intrgral der Diflerentialgleichung (1) als singulärre l'unkt fungieren; wir lassen diesen Fall vorläufig beiseite.

Es sei also allgenein

$$
\begin{gathered}
\left.\left.A_{1}\left(x_{1}\right)=0 . A_{2}\left(x_{1}\right) .1\right) \ldots . .1, y_{1}\right)-0, \\
\left.A_{2}\left(x_{1}\right) ! 1\right)
\end{gathered}
$$

oder, da ja

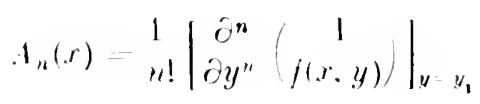

ist, für $x=x_{1}, y=y_{1}$

$$
\partial\left(\begin{array}{l}
1 \\
j
\end{array}\right) \cdots \cdots \quad \begin{array}{ccc:c}
1 & \partial y^{\prime} & 1 & j
\end{array}
$$

gloich Null, aber

$$
\partial y^{\prime}\left(\begin{array}{l}
1 \\
j
\end{array}\right)
$$

von Nill verschioden.

Wir betrachten mun dir Differentialgleideloung

$$
\begin{gathered}
d x=c \\
d y=f(x, y) \\
=A_{0}(x)+A_{1}(x) \cdot\left(y-y_{1}\right)+A_{2}(x) \cdot\left(y-y_{1}\right)^{2}+\cdots
\end{gathered}
$$

dann besilzt diese zufolgo des kxistenztheorems ein in der L'mgehung von $y=y_{1}$ holomorphes Integral, das für $y=y_{1}$ din Mert $.1=x_{1}$ an. nimmt, und dessen crste Ableitung

$$
\begin{aligned}
& d x \\
& d y
\end{aligned}
$$

für $y=y_{1}$ jedenfalls verselowindet. lla fermer 


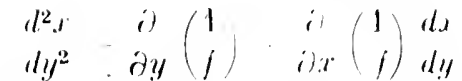

$$
\begin{aligned}
& \begin{array}{l}
d^{3} x \\
d y^{3}=\partial y^{2}
\end{array}\left(\begin{array}{l}
1 \\
f
\end{array}\right)+2 \underset{d}{2} \cdot \partial^{2}\left(\begin{array}{l}
1 \\
f
\end{array}\right) d u
\end{aligned}
$$

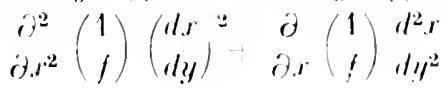

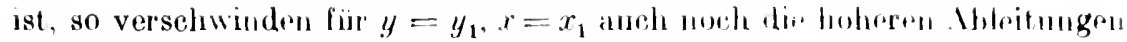
vou $x$ nach $y$ bis zur $\lambda$-tru eimschlieblich, wälrond

$$
\begin{aligned}
& d i+1 x \\
& d y^{\lambda+1}
\end{aligned}
$$

fïr $y=y_{1}$ einen von Null verschiedenen Wert $c_{1}$ brsitzt. Nach den T'a yor schen Satze lautet also die Eutwirklung des definierten Intrograls in dor Umgehung von $y=y_{1}$ :

$$
x=x_{1}+c_{1}\left(y-y_{1}\right)^{2+1}+r_{2}\left(y-y_{1}\right)^{x+\cdots}+\cdots, \quad r_{1} 10 .
$$

Hieraus folgt:

$$
x-x_{1}=\left(y-y_{1}\right)^{\prime+l}\left(c_{1}+c_{2}\left(y-y_{1}\right)+\cdots\right)
$$

und, inden wir bejdreseits die $(\lambda+1)$-te Wurzel auszionen,

$$
\left.\left(x-x_{1}\right)^{\lambda+1}=\left(y \quad y_{1}\right)\left(c_{1}+c_{2}\left(y-y_{1}\right)+\cdots\right)^{\prime}\right)^{1}
$$

Nuu ist der Ansdruct:

$$
\left(c_{1}+r_{2}\left(y-y_{1}\right)+\cdots\right)^{1+1}
$$

in der Umgebung von $y=y_{1}$ holomorph, da ja $c_{1} \mid 0$ ist. Inol folglich in der Form

$$
\gamma_{1}+\gamma_{2}\left(y-y_{1}\right)+\cdots, \quad \gamma_{1}=r_{1}^{1+1} \mid 0 .
$$

darstellbar; wir finden also

$$
\left(x-x_{1}\right)^{\lambda+1}=\gamma_{1}\left(y-y_{1}\right)+\gamma_{2}\left(y-y_{1}\right)^{2}+\cdots
$$

Nach dem aus den Elementen der Funktionentheorie bekannten Satze über die Umkehrung einer Potenzreihe (Satz über die inverse Funkiion) ${ }^{1}$ ) ergibt sich hieraus, dab $y-y_{1}$ nach positiven ganzen Potenzen der auf der linken Seite stehenden Größo

$$
\left(x-x_{1}\right)^{x+1}
$$

entwickelbar ist, wir haben also

1) Siehe z. B. L. Bieberbich. Lehrbuch der Funktionentheorie I (1921) s. 190 . 
(11) $y-y_{1}=\delta_{1}\left(x-r_{1}\right)^{\prime+1}+r_{2}\left(x-s_{1}\right)^{2+1} \ldots . ., r_{1}=\begin{gathered}1 \\ \gamma_{1}\end{gathered} ; 0$.

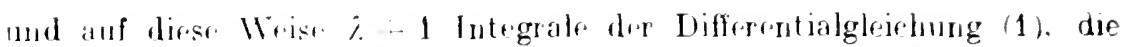
für $x=r_{1}$ dru Wint $y_{1}$ annehmen. Tirdentet in der Formel (11)

$$
\left(x-x_{1}\right)^{1+3}
$$

den Hauptwert dirser $(\lambda-1)$-wertigrn Giralie. d. h.

$$
e^{a+1} \log (x-r)
$$

wo $\log \left(x-x_{1}\right)$ denjenigen Wert des Logarithmus darstellt, der fur reoil positives $x-x_{1}$ reell ist, so gehen die ihbrigen $\gamma$. Integrale aus den eindeutig bestimmten Integralo (11) hervor. inden man an dis Stello von

len Wert

$$
\left(x-x_{1}\right)^{1+1}
$$

$$
\therefore\left(x-x_{1}\right) \wedge+1
$$

satzt, wo \& lin komplex $(\hat{\partial}+1)$-te Einbeitswurzel

$$
\varepsilon=e^{\prime+1}
$$

bedeutet. D. h. mit anderen Wortm, die gulachten $\lambda \div 1$ lntegrale nntstehen aus einem von ihnen. indem man die Variable $x$ i-lnal gesehlossten. Umläufe um den Punkt $x=x_{1}$ vollziehen läbt: dieser Punkt ist also fur jene Integrale ein Verzweigungspunkt. und zwar in der von Rienaul eingeführten Terminologie ein algehraischer Verzwrigungspunt $\lambda$-ter Ordnung.

Im allgemeinen wird es. Wenn $x=x_{1}$ win belieliger Midt der unabhängigen Variabeln $x$ ist, stets Wert $y_{1}$ von $y$ grenen. fir die $f\left(x_{1}\right.$. w) so unendlich wird, daf der reziproke Wert

$$
\begin{gathered}
1 \\
f(x . y)
\end{gathered}
$$

in der Ungebung von $x=x_{1}, y=y_{1}$ holomorple hliht: bedented $z$. B $f(x, y)$ eint rational, Funktion vou $y$, deren Nenner $h(x, y)$ eine ganze rationale Funktion $n$-ten Grades von $y$ ist, so tritt lies firr die $n$ Wurzeln der Gleichung

$$
h\left(x_{1}, y\right) \quad(1
$$

ein, vorausgesetzt. dab sich unter diesen Wurzeln kine befindet. fur die bei $x=x_{1}$ auch der Zähler von $f(x, y)$ verselıwmet. Es wird also in allgemeinen für ein belicbige's $x=x_{1}$ mindestens zwei Lösungen der Differentialgleichung geben, die in $x_{1}$ winen algebraisehen Vorweigungspunt besitzen. 
Dagegen sind dirjenigen $x$-Wurte, fiir dir $/(x, y)$ unathangig von

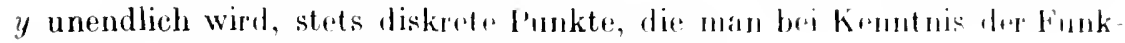
lion $f(x, y)$ von vornherrin anssontern kinu.

\section{Untersuchung des Falles, wo die Integralfunktion selbst mendlich wirl.}

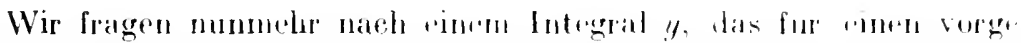

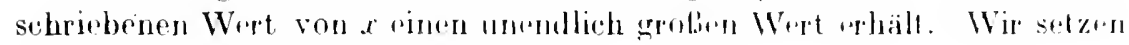
dann in der bifferontialghichung (1)

$$
\frac{d y}{d x}=j(x, y)
$$

an die stelle von!

$$
y=\frac{1}{z}
$$

und ertalten auf diese Weisu fiur z die Mifrerentialgherchung

$$
d z=-z^{2} f\left(x, \frac{1}{z},-f_{1}(x, z)\right.
$$

Es mögr dann $t=i_{1}$ rin Wert sein, von der Besthaffenturl. dab die Funktion $f_{1}(x, z)$ in der Ungebung von $x=x_{1}, z=0$ entwiler selbst holomorph ist, oder dal. sie für llieses Wortepare so unondlich wird, daß ihr reziproker Wert holomorph hleibt. währemel

$$
\left[\begin{array}{c}
\partial^{k} \\
\partial z^{i}
\end{array}\left(\begin{array}{c}
1 \\
f_{1}(x, z)
\end{array}\right)\right]_{\substack{c=x_{1} \\
z=0}} 10
$$

ist. Wenn $f_{1}(x, z)$ für $x=x_{1}, z=0$ selbst holonnorph ist, so gilut es nach den Existenztheoreme ein in ler Ungebung von $x=x_{1}$ holomorphes und für $x=x_{1}$ versehwindendes Integral des Differentialgleichung (12):

$$
z=c_{1}\left(x-x_{1}\right)-c_{2}\left(x-x_{1}\right)^{2}-\cdots,
$$

für das allerdings aueh noch g'wisse erste Kouffizienten

$$
c_{1}, c_{2}, \ldots, c_{k-1}
$$

verschwinden können. während ${ }^{a}$ von Null verschiedren ist. Lamn ist iu der Umgebung von $x=x_{1}$

$$
\begin{aligned}
y=\frac{1}{z} & =c \\
& c_{k}\left(x-x_{1}\right)^{k}+c_{k+1}\left(x-x_{1}\right)^{k+1}-\cdots \\
& =\left(x-x_{1}\right)^{-k}\left[\delta_{0}-\delta_{1}\left(x-x_{1}\right) \cdots \cdots\right.
\end{aligned}
$$

d. h. das für $x=x_{1}$ unendlich werdende Integral drer Differentialgleichung (1) wird mit $\left(x-x_{1}\right)^{k}$ multipliziert in $x=x_{1}$ holomorph. es besitzt also 
in diesem l'unkto einn l'ol (auferwesentlich singuläre Stelle) k-tor Ordnlung.

Wenn $f_{1}(r, z)$ fïr $r \quad r_{1}=0$ so umendlieh wird. dial.;

$$
\begin{gathered}
1 \\
f_{1}(x, z)
\end{gathered}
$$

bolomorph bleibt und $\lambda$ die kleinstr Zahl ist, firr dir. (13) hrosteht. so ist. nach den Ergebnissen der vorigen Nmnmer, in der Ungrobung von $x=x_{1}$.

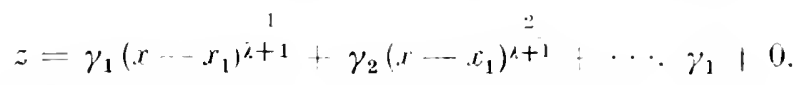

wir haben also lïr das in $x=r_{1}$ unemdlieh wedende hutegrai ron (1) die Entwicklung

$$
\begin{aligned}
& y=1=\begin{array}{c}
1 \\
= \\
\gamma_{1}\left(x-r_{1}\right)^{x+1}+\gamma_{2}\left(r-x_{1}\right)^{z+1}
\end{array}
\end{aligned}
$$

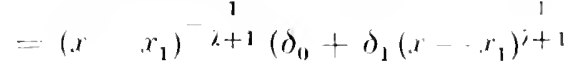

$$
\begin{aligned}
& \left.+\delta_{2}\left(x-x_{1}\right)^{2+1}+\cdots\right) \text {. }
\end{aligned}
$$

d. h. $x=x_{1}$ ist für dieses Intrgral zugleich rin Verzwrigungspunkt t.-ter Ordnung und rine Unendlichkritsstelle; wir sagen in liesen lalle, $y$ besitze in $x=x_{1}$ eine algebraiseh. Unendlichkeitsstolle von der Ord nung 1

In allen bisher betrachteten Fällen ist das lntegral in der Ungehung des betreffenden Wertes der unablä̈ngigen Variabeln nach positiven und negativen ganzen oder gebrochenen Potenzen des lukienents entwickelbar, negative lotenzen können abre stets nur in andlicher Anzahl auftreten. Wir sagen kurz, das Integral habe in diesen Fiallend den Cha a a k ter

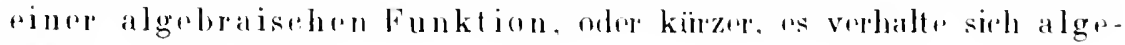
broid ${ }^{1}$ ).

\section{Ahhïngigkeit ron Anfangswerten mol Parametern. Unitait.}

Dir läsung y unserer Differentialgheiehung (1) der Xir. T

$$
d y=f(x, y) \text {. }
$$

die in der Ungebung des Wertes $x_{10}$. wo sie den Wort $y_{0}$ annehunen soll.

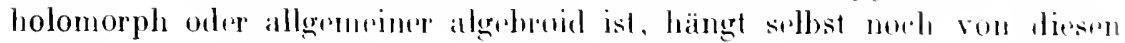

1) Die Rechtfertigung für these bezeichnung ergibt sich darans. dab eine algehraische Funktion stets ain deratigases Virhalten zerigt 


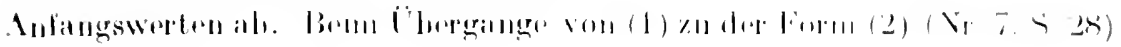
wirl

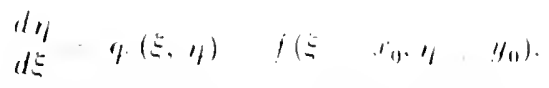

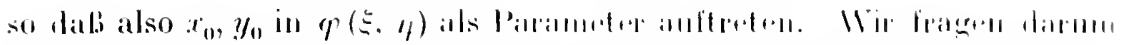

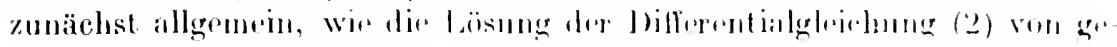

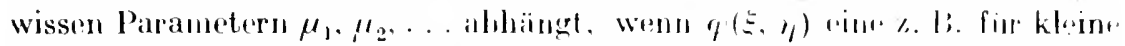

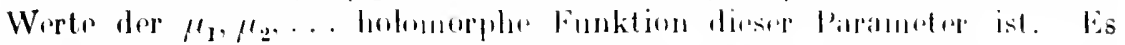

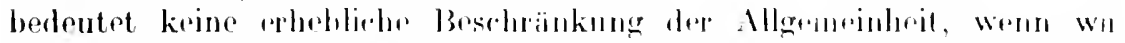

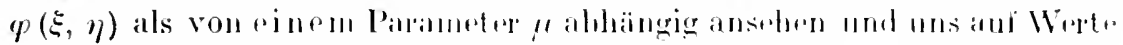

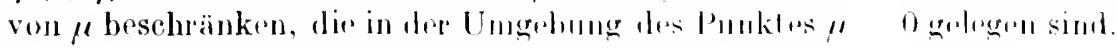
also dir Diffrerentialghinhumer

$$
\text { dI }=\eta(E .11 \cdot 11)
$$

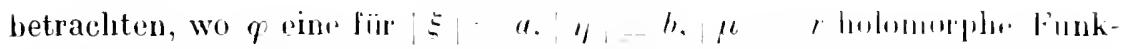

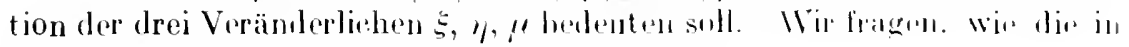
iter Ungebung von $E-0$ holumorphe lïsung

$$
\eta=c_{1} 5-c_{2} 52+\cdots
$$

sich als Funktion von $\mu$ in der Umgehnng von " "verhät. Wir man

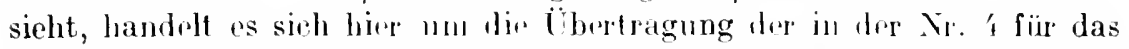
reelle Gebiet angestellin Intersuchmogen anf das Gohiot der komplexen Veränderlichen.

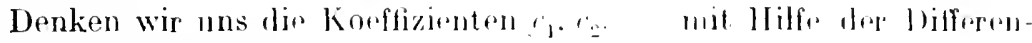
lialgleichung berechmet:

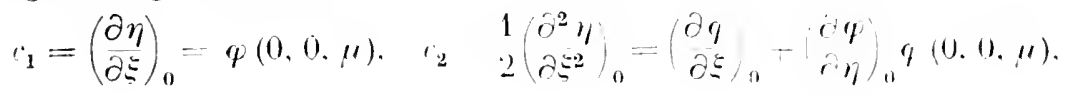

so ergeben sie sich als für $|\mu|<r$ holomorphe Fnnktiomen von $\mu$. Daraus kann man aber noch nicht schliefen. dal. sich die Reih" (5*) in oine nach positiven ganzen Potenzen von $s$ und $\mu$ fortschreitende Reihe mmordnen läßt, die für hinreichend kleine Werte von $\xi$ und $\mu$ unbedingt konvergiert. Es läßt sich dies aber durch eine besondere Untorsuchung ${ }^{1}$ ) feststellen. und zwar am einfachsten durch eine Erweiterung der Majorantenurethode.

Es sei für $|\xi|=a,|\eta|=b,: \prime=r$. 11 die obere Schranke der Werte von $|\varphi(\xi, \eta, \mu)|$ : wir nehmen damn als Majorante dir Funktion

$$
\psi(\xi \cdot \eta \cdot \mu)=\left(\begin{array}{c}
11 \\
\left.1-\frac{\eta}{a}\right)\left(1-\frac{\eta}{b}\right)\left(1-\frac{\mu}{r}\right.
\end{array}\right.
$$

nit der zugehörigen Hilfsdifferentialgleichung

1) Siehe Poinc aré, Méthodes uouvelles de la mécanique céleste $1(1 \$ 96)$. . .4 . 


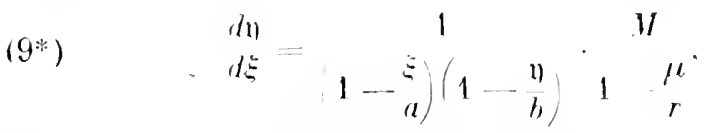

die sich also von der in der $\mathrm{N}_{1}$. 9 betrachteten mur dadurch unterscheidet.

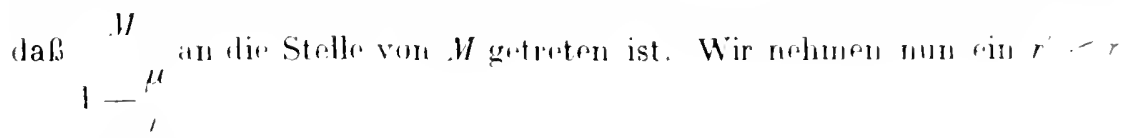
unil sotzon

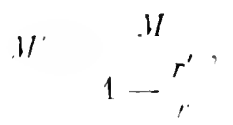

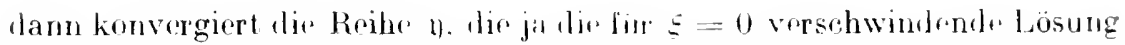
der Hillsdifferentialgloichung (9\%) darstellt, fiir das Gobiet

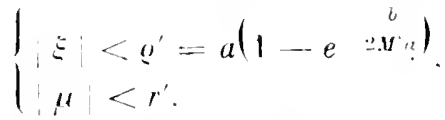

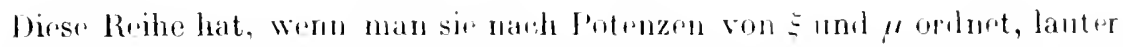
positive koeffzienten und liese simd, wie nan sofort ihersioht, nicht kleiner als dir absoluten Werte der entsperehenden Koeffizionten lue Reihe, die aus (5*) dumb Unorduen nach l'otenzen vou Emad $\mu$ hriror-

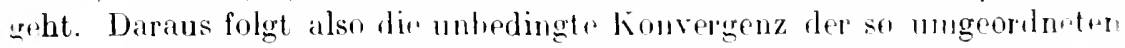
Reihe (5*) fiir las Gebied (*), 1. h. hio fïr $\xi=0$ vesschwindend Lösung $n$ unserer Differenlialgleichung (2*) ist auch als Funktion des l'arameter= " fïr hinreichend kleim. Werte von $\mu$ holomorph. Saturlich gilt der entsprechende Satz auch, wenu $q(E, \eta)$ mehure Paraueter $\mu_{1} \cdot \mu_{2}, \ldots$.nt hält und für himreichend kleine Werte dieser Parameter holosnorph ist

Vir botrachten mun zuvördest dou fall regulär er . Infa meswert".

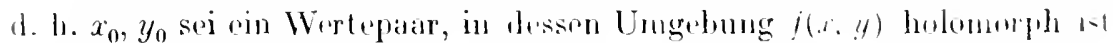

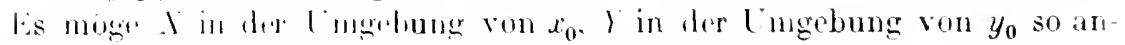

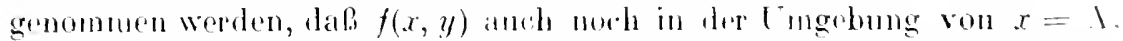
$y$. Y holomorph ist. Das Intergal von (1), das in der Uugebung vin

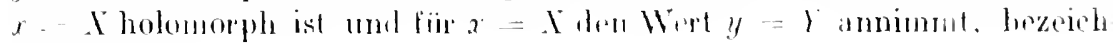
II+ w wir nit

Sitzl mand jutzt

$$
y=l(x ; 1, \gamma)
$$

$$
x \quad \Xi \quad 1, y==, y)
$$

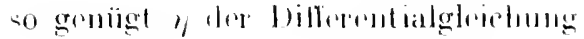

$$
\text { dE= (E+ }
$$

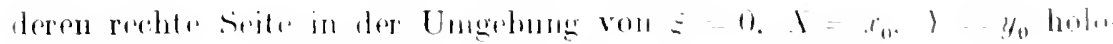

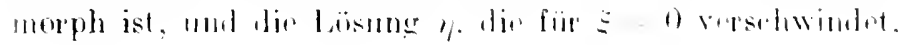




$$
\eta=F(\xi \div-1 ; 1,1)
$$

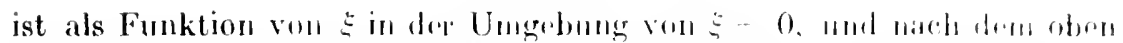

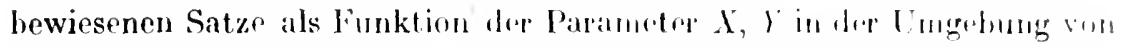
$X=x_{0}, y=y_{0}$ holomorph. $\Lambda$ so ist $y$ math positiven ganzen l'utumen von $x-X, X \quad x_{0}, \quad y_{0}$ "ntwiekelbar und lemmarh $y-I,(x ; X, Y)$

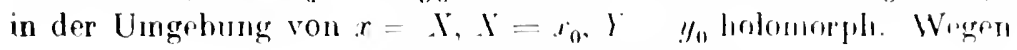

$$
x-X-x-x_{0}-\left(X-x_{0}\right)
$$

ergibt sich. daB das Integral $y=F(x: X, Y)$ als funktion dor drei Veränderlichen $x, X, Y$ holomorpli ist, wonll $x$ and $X$ in der Ungobung von $x_{0}, y$ in der Umgroung von yogelegen ist.

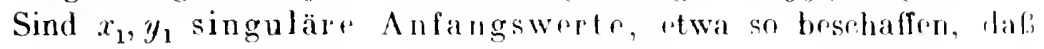
$f\left(x_{1}, y_{1}\right)$ unendlirh wird, aher $f(x, y)$ in der Jumghumg von $x=x_{1}, y=y_{1}$ holomorph bleibt, ohne fiir $x=x_{1}$ unabhängigr von y zu versehwindert (vgl. Nr. 10), dann hat dir Difforentialgleichung

$$
d x=f(x, y)
$$

ein in der Lmgehung vony $y=y_{1}$ holomorphes Integral

$$
x=f ;\left(y ; y_{1}, x_{1}\right)
$$

das für $y=y_{1}$ den Wert $x=x_{1}$ annimment. Liegrt dann X in ther Uumgehmeg von $x_{1}, Y$ in der Ungebung von $y_{1}$, so dal. $f(x, y)$ auch noch in der Ungebung von $x=X, y=Y$ holomorph ist, so wird las Integral

$$
x=G(y ; \gamma, \mathbf{I})
$$

von (I), das für $y=1$ den Wert $t=X$ annimint und iu der liusthumg von $y=Y$ holomorph ist, eine in der Ungebung vou $y=y_{1}, y=y_{1}$, $X=x_{1}$ holomorphe Funktion der drei Veränderlichen $y, X, Y$ sein. Daraus ergibt sich durch Inversion der Gloichung (II), dalis $y$ in der Ungebung von $x=x_{1}, \mathbf{X}=x_{1}, \quad y=y_{1}$ algebroides Verhaltell zeigt. Ein ähnlicher Schluß gilt auch für ein Integral, das in $x=x_{1}$ unendlich wird, iu der Uningebung von $x=x_{1} . \mathrm{I}=x_{1}, \quad,=\infty$.

Wir machen nun Gebranch von diesen Ergehnissen, indru wir die folgende besonders in der Angewandt'll Mathenatik wichtige Frage brhandeln.

Für den Fall regulärer Infangsbedingungen $x_{0}, y_{0}$ haben wir gesehen, daß es ein und nur ein in der Ungehung von $x=x_{0}$ holomorphes Integral gibt, das für $x=x_{0}$ den Wert $y=y_{0}$ annimmt. Wird man etwa durch eine Aufgabe der Angewandten Mathematik auf eine Funktion gefuhrt, die der Differentialgleichung genügen und für $x=x_{0}$ den Wert $y=y_{0}$ annehmen soll, so wird man nicht von vornherein sagen könuen, dab di 
gesuchte Funktion in ler Umeghnng von $x=x_{0}$ geradr holomorph soin inuß. Es könnten also \%weifel entstehen, ob die von uns angegehene Lösung $F\left(x ; x_{0}, y_{0}\right)$ auch wirklich die gesuchlo. Funktion darstollt. Es fragt sich also: gibt us retwa monen dem holomorphen lntegral $F\left(r: x_{0}, y_{0}\right)$ noch

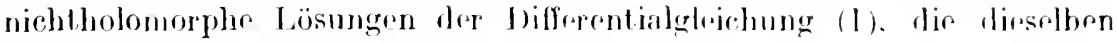
roguläron Anfangsbedingungern ufiillon:

Inn diese Frage zo entschridren. betrachton wir') jetat wioder zwai

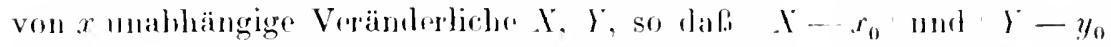

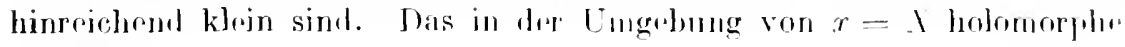
Integlial

$$
y=F(x ; X, Y)=Y+c_{1}(x-X) \div r_{2}(r-X)^{2}+\cdots
$$

las fiur $x=X$ den Wort $Y$ annimmt, ist mach dom Vorhergehemden als Funktion von $x, X, Y$ in dre Ungolung von $r=X, X=r_{0} \cdot Y=y_{0}$

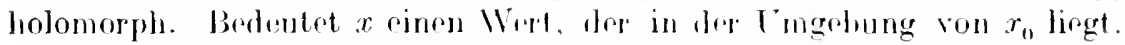
und $y$ den entsprechroblen Wert von $F(x ; I, Y)$, so grihl $\mathrm{es}$ un ein in der Umgebung von $x$ holomorples Intrgral the D)ifforentialgloichung (1).

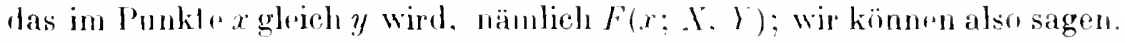
dals das in der Ungebung von $x$ holomorploe lntegral, das im lonkte $x$

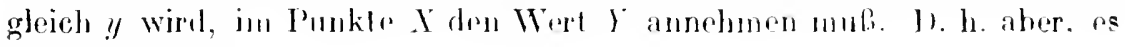
ist jullutisel

$$
I=I \cdot(\mathrm{X} ; x, y) \text {. }
$$

wonn in dieser Gleichung $y=F(x ; X, Y)$ gosetzt wird

Durch die Gleichung

$$
z=I(X ; x, y)
$$

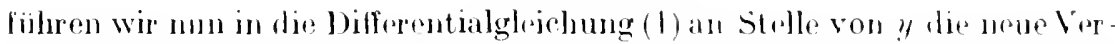
änderliehe z rin. J)a mach dem Vorhorgehenden aus diesor Gilojehung anch worgekelirt

$$
l=l(x ; I, z)
$$

folgrt, so liaberll wir

$$
d y=\frac{\partial f(x ; 1, z)}{\partial x}+\frac{\partial(x: I, z) d z}{\ddot{\partial} z} d r
$$

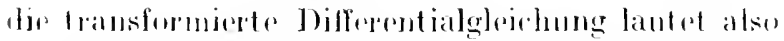

$$
\begin{aligned}
& d z \partial F(x ; X, z) \\
& \text { dr } 0 z \\
& \partial F(x ; \text { I. } z) \\
& +f(x . l(x ; 1 . z)) .
\end{aligned}
$$

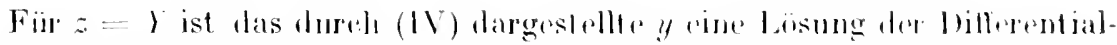

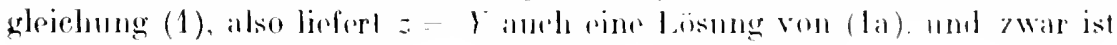

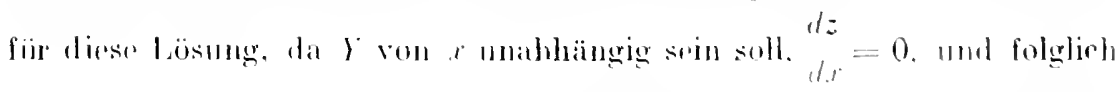

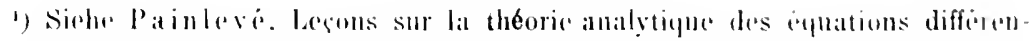

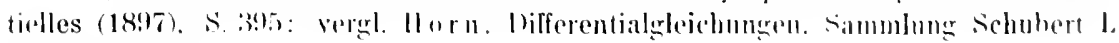
(19)!). S. . 2 ?. 


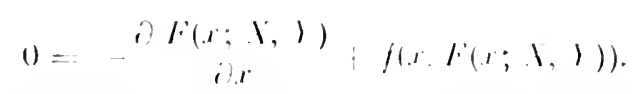

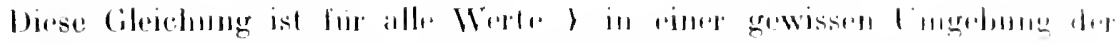

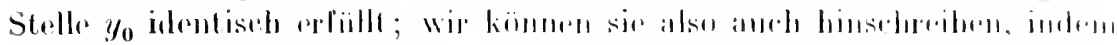

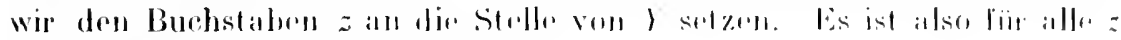
in rimer gewissen (Jugelumer vill y"

$$
0=\frac{\partial(x ; I, z)}{\partial x} \quad f(x, F(x ; I, z))
$$

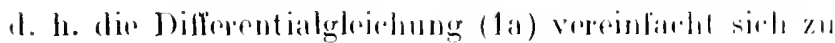

$$
\begin{array}{ll}
\text { dz } \\
\operatorname{ll} r(x ; X, z)
\end{array}
$$

Non ist abou

$$
l=I(X ; 1,1),
$$

also ist

$$
\text { If }(x ; X, Y)
$$

i)

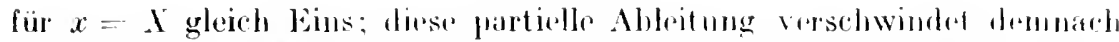

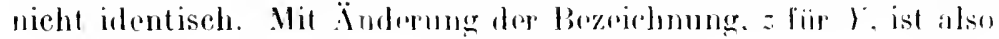

$$
\text { a } F(x ; 1, z)
$$

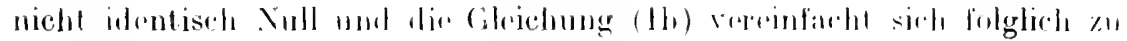

$$
\left.\frac{d z}{d . r}-1\right)
$$

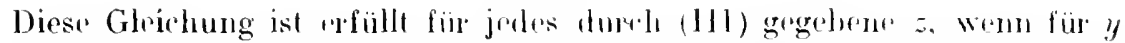
eine Lösung der Differentialghrirhung (1) gesetzt wird, d. h. wrun sich alls ader was dasselbe heißt, aus

$$
z=F(.1 ; x, y) \text {, }
$$

$$
y=F(x: X, z)
$$

$y$ als lösung der Differentialgleichung (1) ergeben soll, so muli z der Cileichung (1c) genügen, also konstant sein. Dabei muissen aber natiurlich $a$ in der Umgebung von $X$ und $y, z$ in rer Ungehung vou $Y^{*}$ liegen. Ist mu $y=p(x)$ irgend eine Lösung ron (1), dir für $x=\mathrm{X}$ den Wert $y=1$ in der Weise annimmt, dab, wemn $x$ in der Ungebung von $X$ liegt, aurh $y$ in der Unigebung von $Y^{\prime}$ rrobleibt, su ist dir entsprechende Lösung

$$
z=F(X ; x, p(x))
$$

von $(1 \mathrm{~b})$ wohldefiniert und koustant. Dil aber für $x=1$

$$
\varphi(\mathrm{X})=Y \text { und } F(\mathrm{Y} ; \mathrm{X}, Y)=\text { r }
$$

ist, so ist dieser konstante Wert von $z$ gleich $Y$. Es ist also

oder

$$
Y=F(X ; x, q(x)) \text {. }
$$

$$
\psi(x)=F(x ; X, \gamma)
$$

d. h. $\varphi(x)$ ist mit dem holomorphen Integral identisch. 
Wenn die Läsung $y=q(x)$ für $x=X$ sich den Wute $y=Y z W a I^{\circ}$ unbegrenzt anuähret, aber nicht dir bedingung erfüllt, dab einem $x$-Werte der Umgrbung von I strets anch ein y-Wurt der Unguhung vou Y entspricht, so kann $q(x)$ von dem holomorphen Integral versehinden sein. wie das

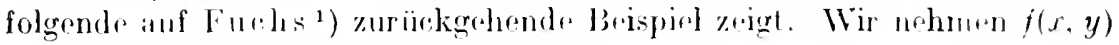
$=-\frac{y^{2}}{x}$. also dir J)ilferential grleichume

$$
d y \ldots . y^{2}
$$

die in der Umgebung von $x=X_{0}$, wh $X_{0} \mid 0$ ist. holomorphe und fur $X_{0}$ verschwindende Jösung ist $y=0$. Schrriluen wir die Differ"nt ialgleichung aber in der Form

$$
\begin{array}{r}
d y \\
y^{2}=
\end{array}
$$

so folgt dureh lntegration

$$
y=\log x+C,
$$

wo $C$ eine willkürliche Konstantr bedeutet. Dir holumorphe Lösung $y=0$ entspricht den Werte $C=\infty$. Es kann aber auch fur eineu endlicben Wert von $C$ der Writ von $y$, der dem $x=\mathrm{X}$ entspricht, beliebig nabe an die Null herangobracht worden, indem man $x$ himpeichend oft den Punkt $x=0$ umkreisen läßt, worhurch ja $|\log x+C|$ grüßer wrmacht werden kanu als jede beliebige positive Gröbe. Diese Benerkung ist für dif Angewandte. Matlomatik nicht ohne Bedeutung, da man ja in lon Anwendungen häufig nicht seharf zu unterschejden imstande ist. ob eine Größe einen gewissen Wert wirklish anniment ode' ihm nur helipbig nahekommt.

\section{1:. Analytische Fortsetzung.}

Wir clenken uns das den reguliaren . Infanerswerten $x=x_{0}, y=y_{0}$ entsprechende, in der Ungebung von $x=x_{0}$ holomorphe Integral

$$
y=F\left(x ; x_{0}, y_{0}\right)=y_{0}-c_{1}\left(x-x_{0}\right)-c_{2}\left(x-x_{0}\right):-\cdots
$$

der Differentialglerehmug (1) hergestellt. Wie dureh dir Ca ue hyshr Majorante grelieferte Konvergenzgrenze (Nr.9, S. 33)

$$
\left|x-x_{0}\right|<0 \quad a\left(1-e^{-2.1 x_{11}}\right)
$$

liefert - wio wis bemerkt haben - micht den genanen lionvergenzkreis

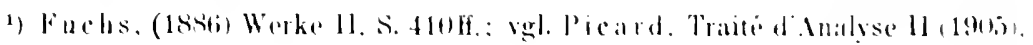
ร. $3(i)$. 


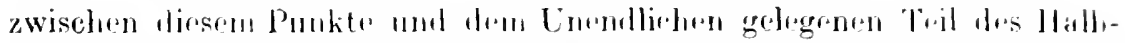

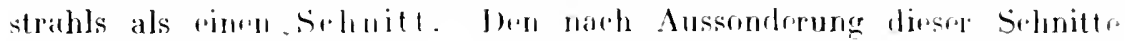

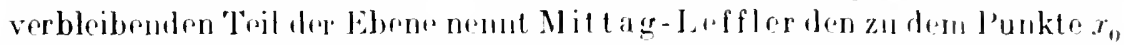
gehörigen Stren. Dieser bildet den Burreich B. innerhalb dessen das Ver-

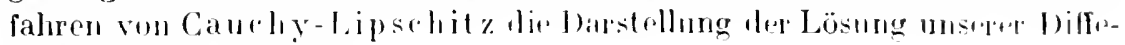
pentialgleirhung liefert.

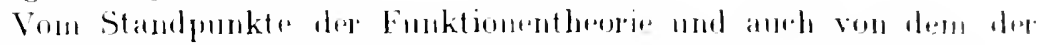

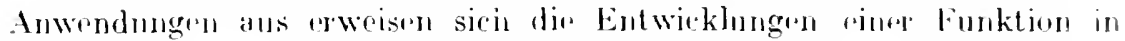
Potenzreihen als dir wichtigsten und brauhhorsten. Diwe Entwick-

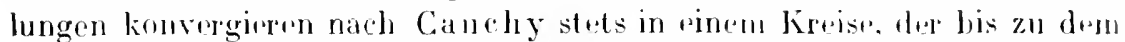
nächstgetegenc'n singulären l'unkte reicht. Das ist anch der tiefere Grund dafïr, dali man in der Theorie der Differentialghriehungen der Einführuner

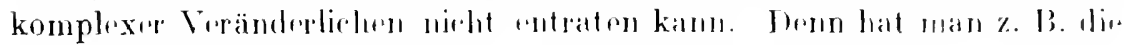

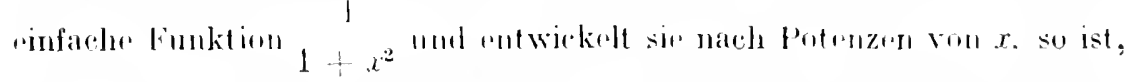

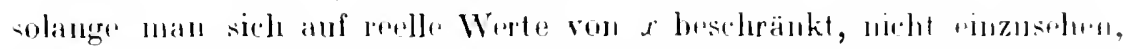

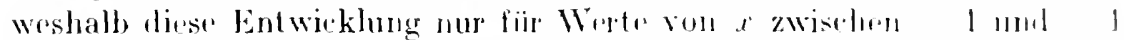

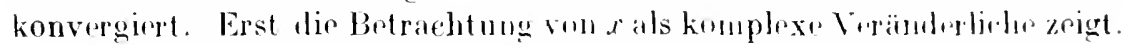

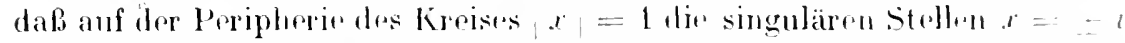

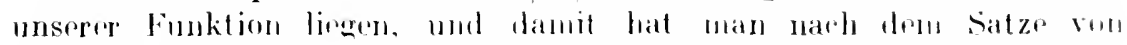
Cauchy den waluren Grund fiir dir Bescluränktheit des Konvergenz-

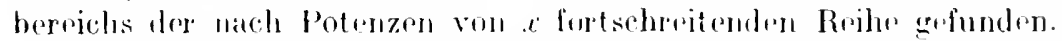

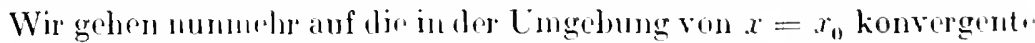
Reihe $F\left(x ; x_{10}, y_{0}\right)$ zur ïre und stellon uns dir Aufgabe. die Natur der durch diese Potenzreihe lefinierten nonogenen Funktion zu erforschen

Zunächst folgt aus dem l'rinzip der analytiseden fortsetzung ${ }^{1}$ ).

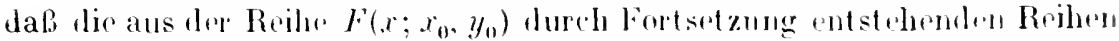
ebenfalls der Jolferntialgleichung (1) genügen. Wenn man sich $f(x, y)$ als Funktion von $x$ anf demselben Wugre fortgesetat denkt. Ist z. B. $f(x, y)$ rine eindeutige funktion von $x$ und $y$, etwa cine rationale Funktion ron $y$. mit in $x$ rindeutigen lioeffzienten, so genögt die gesamter ante

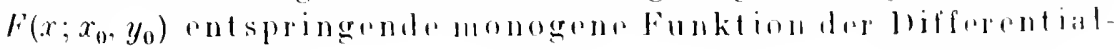
grleichung (1).

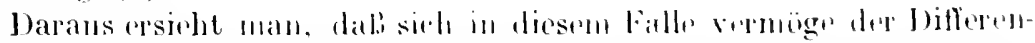
tiakgleichung (1) die analytische fortsetzung der Reiher $F\left(1, x_{0}, y_{0}\right)$ in folgender Weise vollziohen lailit:

Da rine ans $F\left(x ; x_{0}, y_{0}\right)$ dureh Fortsetzunter entstandene Reibe

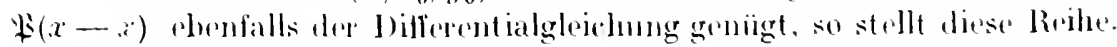

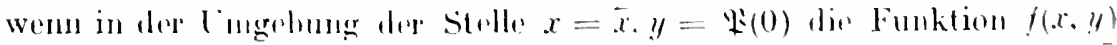

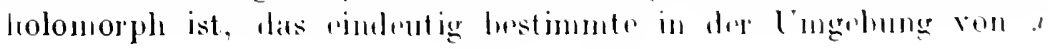

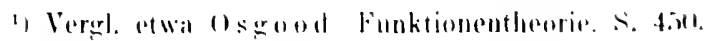




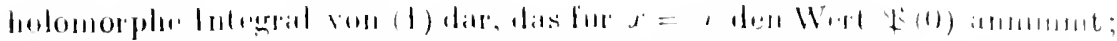

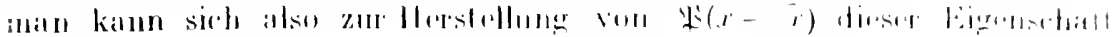

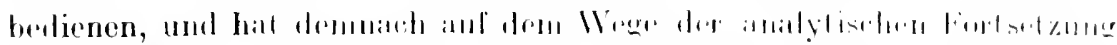

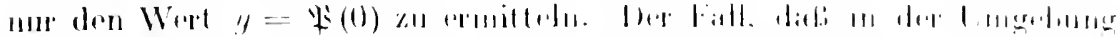

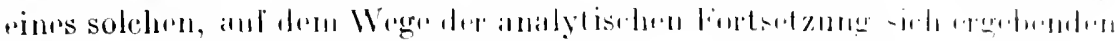

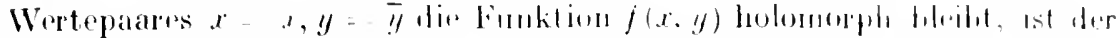

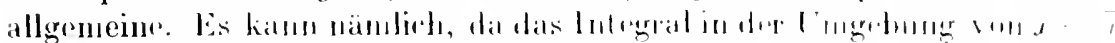

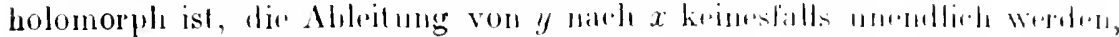

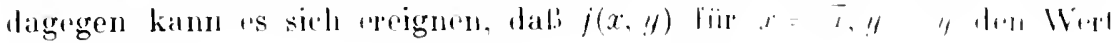

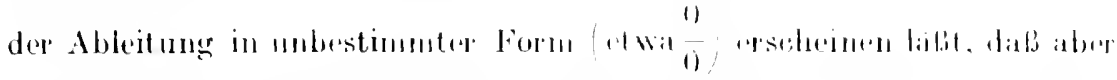

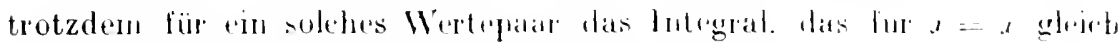
"s wird, in der lingednung von i holomorple bleibt.

so hat z. B. die Dillerentialgrleichume

$$
d y=y-y
$$

die in der Lingrolnum rom a holomorphe Losung

$$
y=\bar{y} \cdot c(x-r) \text {, }
$$

wo c eim. helindige Konstantr bedrutet, und die Lösmag nimme fur $x=x$

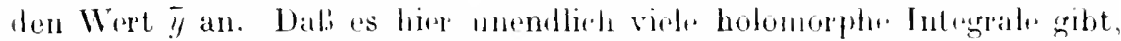

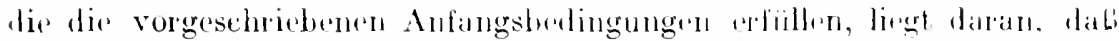
die Anlangswerte $x=\bar{x}, y=$ y krinr reguliarn sind. Lre l'unkt $x=\bar{x}$ wird im Sinne unserer I)efinition (siehe oben S. íi) als singulärer l'nnkt les Integrals y der lofferentialgleichung (*) zu bezeichnen seim, ob woh! or kein singulärer Punkt der Funktion y ist. Nan spricht in liesenn Fallu" von einem auferwesentlich singulären l'unkt des lutugrals (vgl. woiter unten, $\mathrm{Nr}$. . 8 ).

Die Fortsetzung der Reile $F\left(x: x_{0} \cdot y_{0}\right)$ mit Hilfe der Differentialgleichung (1) gibt also, soweit der Ilolonorphinbreich vou $f(x, y)$ reicht, dieselben Ergebnisse, wiי die nach drn ïblichen Methoden ansgelührte analytische Fortsetzung. Wir wollen nun dazu übrrenchen. dit verschiedenen Fälle zu erörtern. die zu Singularitäten der aus dre Reihe $F\left(x ; x_{0}, y_{0}\right)$ entspringenden monogenen Funktion Veranlassung gaben können.

\section{Feste und verschiehbare singuläre Punkte. Der Natz voll Painleré.}

Der einfachste Fall wäre der, wo die Reihe $F\left(x ; x_{0}, y_{01}\right)$ beständig konvergiert, wo also in Endlichen ïberhaupt keine singuläre Stwle vorhanden ist. Lassen wir diesen buiseite, so haben wir für $F\left(x ; i_{0}, y_{0}\right)$ 
und ebenso fur jedr ans dirser Prilur dureh analytische Fortsetzung ent stehende pimen endliclern Konrergenzhalbmesser. Her in allgomeinen

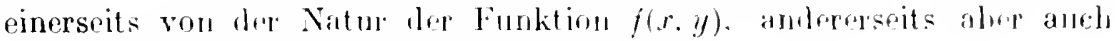
von den Anfangswerten $x_{0}, y_{0}$ abhängen wird.

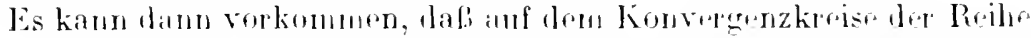
$F\left(x ; x_{0}, y_{0}\right)$ orter eine ans ihr dureh analytische Fortsetzung ant stambenen Roiho rin singulärer Punkt $x$ liegt. für den de Funktion $f(x, y)$ selhst

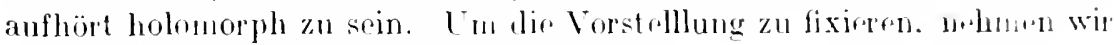
an, dal. $f(x, y)$ cime rational. Funktion

$$
f(x, y)=\frac{g(x, y)}{h(x, y)}
$$

von $y$ sei, wo also $g(x, y), h(x, y)$ ganze Funktionen von y ohne gemeinsamen Teiler hedeuten nögen.

$$
\begin{aligned}
& g(x, y)=\alpha_{10}(x)+\alpha_{1}(x) y+\cdots+a_{, \prime}(x) y^{\prime \prime} . \\
& h(x, y)=\beta_{0}(x)+\beta_{1}(x) y+\cdots+\beta_{n}(x) y^{\prime \prime} ;
\end{aligned}
$$

die Koeffizienten $a_{k}(x), \beta_{k}(x)$ möghn bulingige monogene Funtionen von $x$ sein. Dann sind in Butracht zn ziden:

1. diejenigen $x$-Werte. für die rine bder mehere ler Funktionen $\alpha_{k}(x), \beta_{k}(x)$ rine Singularit ait darbieten ;

2. die $x$-Werte, für die die Xmmerfunktion $h(x, y)$ unahängig von $y$ verschwindet;

3. dir Wertepaare $x, y$, für dir Zähler und Yenner von $f(x, y)$ uleichzeitig rersichwinden,

$$
g(x, y)=0, h(x, y)=0 .
$$

deren $x$ - Werte also der durh Elinination von $y$ zwisehn diesen beiden Gileichungen hervorghenden Resultantengleichung geniigen miissen.

Unanch alie Fälle nit zu unfassin, wodie Funktion y solbst unendlich wird, stellen wir der bifferentialgleithung (1) soglech dip Differentialgleichung (12) ( $\mathrm{Nl}$. 11, S. 39)

$$
d z=-2 j\left(x, \frac{1}{z}\right)-i_{1}(x, z)
$$

an die Seite, die alls (1) dureh dir Transformation $z=\frac{1}{y}$ hrevorgeht: $f_{1}(x, z)$ ist wieder eine rationale Funktion vons 2 Wir haben dann weiter als singuläire Punkto anzusehen:

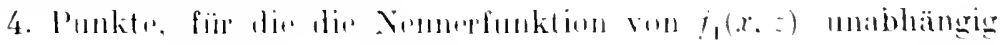
von z versehwintert. mul

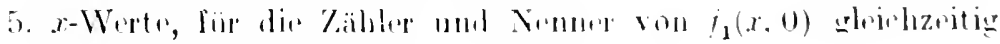
versolhwinden. falls soldele vorhanden sind. 


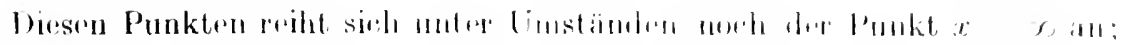

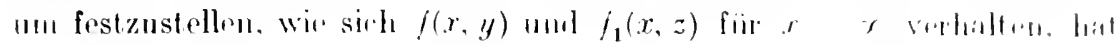

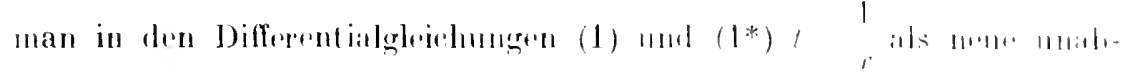

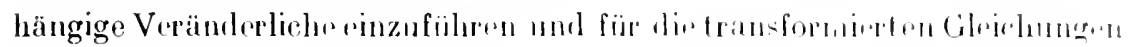
den Punkt $t=0$ zu untersulher.

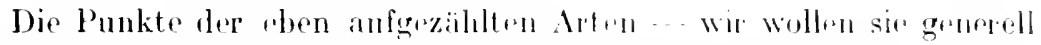

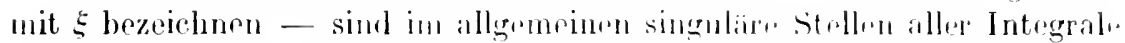

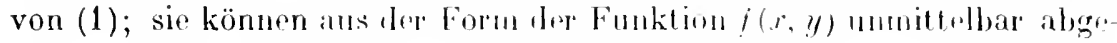
lesen werden und haben mit den Anfangswerten $x_{11}, y_{0}$ des garald be trachteten Integrals nicluts zu tum. Wir nemmen sie dir lestron Singulari täten, oder die singulären Stellen der Differnutialghlıirhung.

Hat man z. B. dir Differentialgleichung

aus der sich

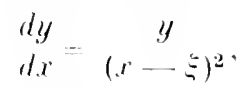

$$
y \text { const. } e^{-{ }^{x}-=}
$$

ergibt, so ist der Punkt $E$ eine solche feste Singularitit dw nlwn muter 2 beschriebenen Art. Das arleiche gilt für $x=0$ in dem Beispirl

dessen Lösıng die Form hat:

$$
d y=\frac{12 y}{x}
$$

$$
y=\text { const. } x^{1}=
$$

Man sieht an diesen beiden Beispielen, daß die Lösung einer Difftrentialgleichung (1) in einem solchen festen singulären Punkte nicht algrebroid zu sein braucht, sondern daselbst eine transzentente Singularität aufweisen kann.

Wir wollen der Einfachheit zuliebe alle verwickelteren Fälle, wie die. daß die festen singulären Punkte Linien oder Fläehrnstïcke der $x$-Ebenr überall dicht erfüllen, ausschliefien und annehmen, daf diese Punkt isoliert liegen. Sie mögen lann durch kleine, sie umgebende Kurven aus der $x$-Ebene ausgeschnitten. und diese Kurven durch Querschnitte an den Punkt $x=\infty$ angeschlossen werden. Die so entstehende einfach zusammenhängende Fläche bezeichnen wir mit $T$. Wir fragen nun nach dem Verhalten der aus der Reihe $F\left(x: x_{0}, y_{0}\right)$ durch analytische Fortsetzung entspringenden Integralfunktion $\Phi\left(x ; x_{0}, y_{0}\right)$ im Innern von $T$.

Wenn wir bei der Fortsetzung unserer Reihe $F\left(x ; x_{0}, y_{0}\right)$ auf einem bestimmten Wege bei einem in der Fläche $T$ gelegenen Wert $x=x_{1}$ anlangen, und es ergibt sich in diesem Punkte $x_{1}$ für die Integralfunktion $\Phi\left(x ; x_{0}, y_{0}\right)$ ein bestimmter endlicher oder unendlich grober Grenzwert 


$$
\lim _{\infty} g\left(r: r_{1} \cdot y_{1}\right)=y_{1}
$$

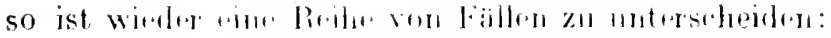

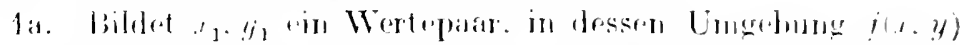

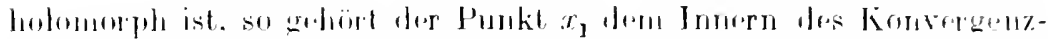
kroises cines Elomonts dre Funktion $\left.\Phi\left(x ; x_{0}, y_{0}\right)^{1}\right)$ and $x_{1}$ ist also

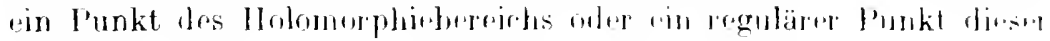
Finulion.

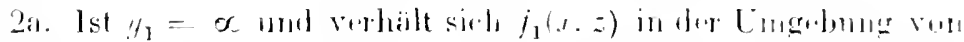

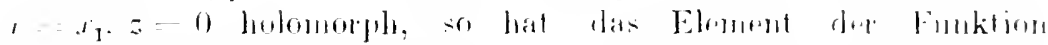

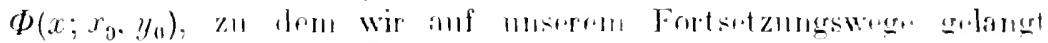

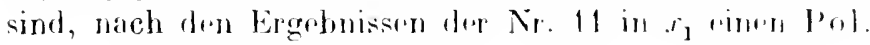

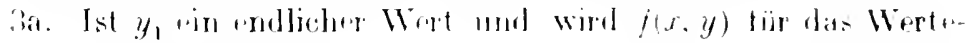

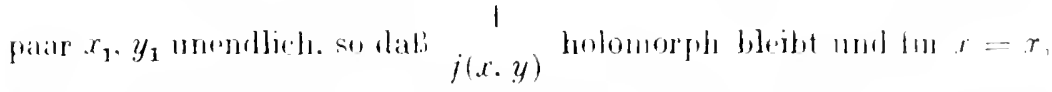
nicht mabhäberier von y versehwindet. dann hat wnser Funktionsarment in $x_{1}$ rinen algehraschen Virzwoignngspunkt.

1a. Ist $y_{1}=x$ und wird $f_{1}(r . z)$ fïr $r=r_{1}=-11411 \cdot 1$ llirh.

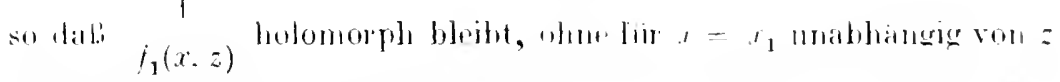
zu verschwinden, dam hat moser Funktionselement in $r=x_{1}$. algehraische Unendlichkeitsstelle.

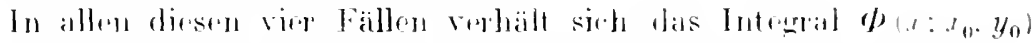

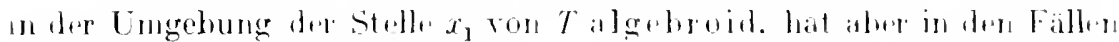

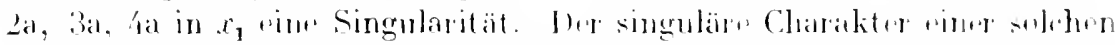

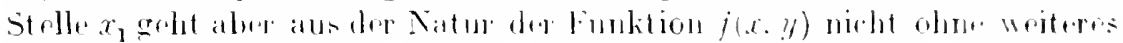

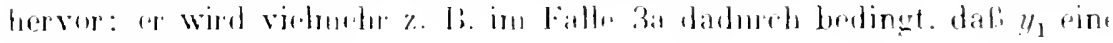
Minzal dow Gloinhme

$$
h\left(x_{1} \cdot y\right)-0
$$

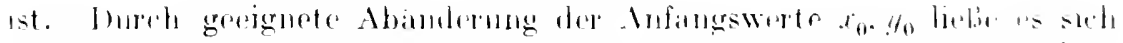

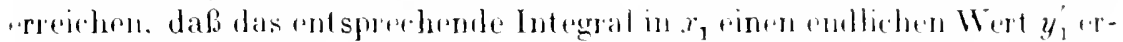

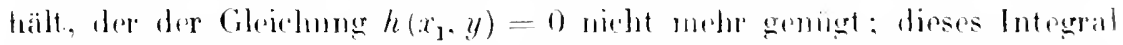

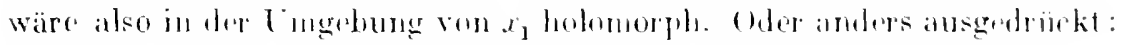

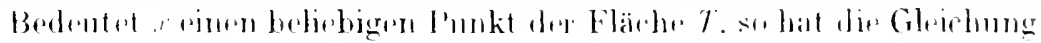

$$
h(\cdot ., y)-0
$$

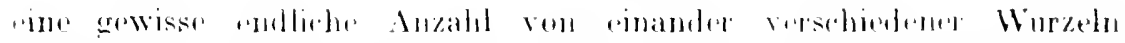

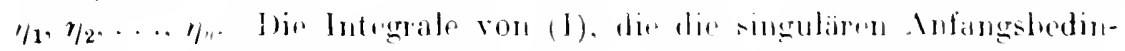

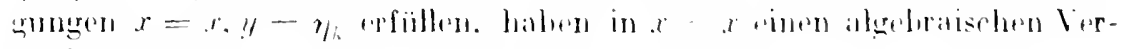

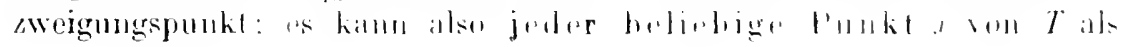

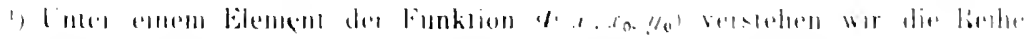

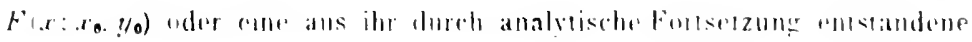




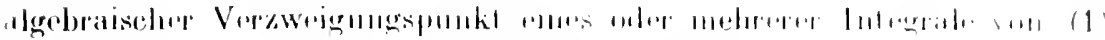

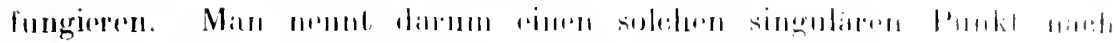

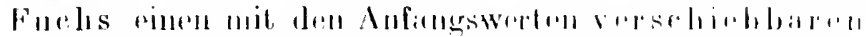

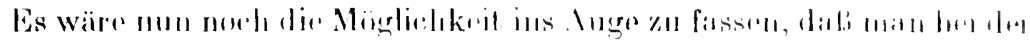

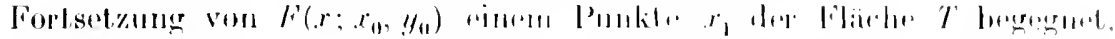

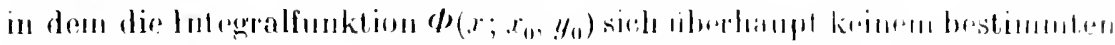

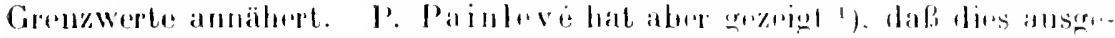

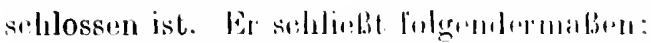

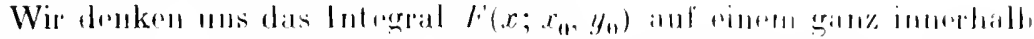

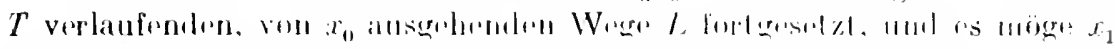

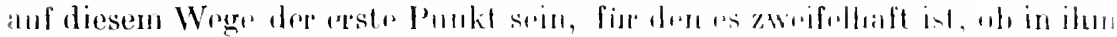

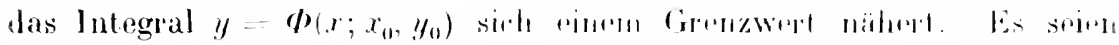

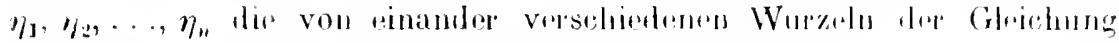
$h\left(x_{1}, y\right)=0$, daun sind zwei Fälle zu muterscheden:

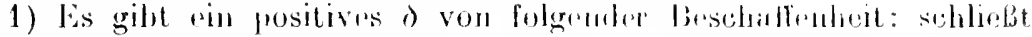

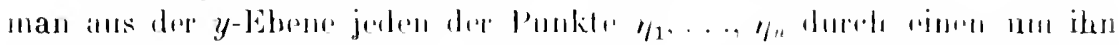

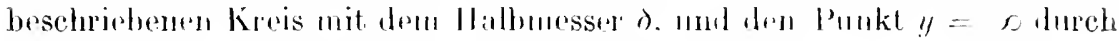

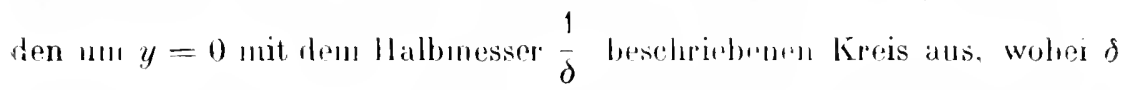

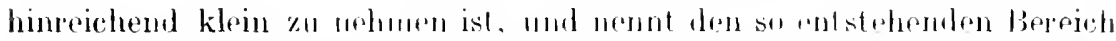
$B_{\delta}$, so mögr das Inlogral y $\boldsymbol{\phi}\left(x: x_{11} \cdot y_{0}\right)$ in holiphiger Nähr von $x=x_{1}$ Wertr annehuren, die in $b_{,}$lingen.

2) Ein solches $\delta$ existiont nieht.

In letzteren Falle lälit sich daher jedem position o nin positives $\varepsilon$ so zoordnen, dab für alle Punkte von $L$, die vor $x_{1}$ liegen nurl fïr di. $\left|x-x_{1}\right|<\varepsilon$ ist, eine ler Ungleichungen

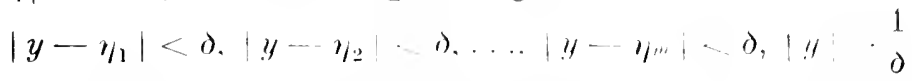

erfiillt ist. Jnd zwar gilt, da dir Ahbilıhng von $L$ anl die $y$-Ehene durch eine stetige Funktion erfolgt, fiir alle in Betracht konmenden Punkte von $L$ nur eine bestimnt "dieser Ungleichungen. Es hätte also in rirsen Falle $y=\Phi\left(x ; x_{0}, y_{0}\right)$ für $x=x_{1}$ entweder pines der Wert. $\eta_{1} \ldots . . \eta_{\text {. }}$. oder $\infty$ zum Grenzwerte.

Im Falle 1) dagegen lälit sich zeigen. daby $y=t\left(x ; x_{0}, y_{0}\right)$ im Punkte $x_{1}$ holomorph ist. Man grenze nämlich un $x=x_{1}$ eine Ungebung $i-x_{1}$ $<\gamma$ so ab, dab, wenn $x$ in diesm. Ungebung liegt, alle Wurzeln dor Gleichung $h(x, y)=0$ den Bereichen

$$
\left|y-1 / 1<\begin{array}{l}
0 \\
2
\end{array}, \quad \cdots\right| y-1 / m\left|\cdots \frac{1}{2} \cdot 1 /\right| \frac{2}{\partial}
$$

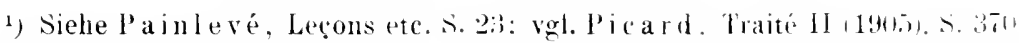
Im folgenden geben wir den Beweis von P'ic ard in einter von stnd. l'lebun berxïhrenden Fassung wieder. 
angehören. I)ann hat $h(x, y)$ in $B_{\text {, }}$ rine positive unture Schranke und folglich $|f(x, y)|$ obenda rine ober. Sclinank", 1 . Schreibt man nun für ein $x$, das anf $L$ liegt, und dessen Abstand von $x$, kleiner ist als $\frac{\gamma}{2}$, als Anfangswert eines Integrals rinen $y$-Wert vor. der dem Bereiche $B_{\lambda}$ a angehört. so wird z. B.der Cauch ysche Konvergenzardius dieses Integrals oberhalb einer angebbaren, positiven Schrankr $\mu$ liegen. Setzt man $\boldsymbol{D}\left(, r_{1}, l_{1,}, y_{n}\right)$ längs $L$ analytiseh fort und wälılt einßn \%wisch"npunkt $x$. dessen Abstand von $x_{1}$ kleiner ist als dir kleinere der Gröben $\frac{1}{2} \gamma$ und $\mu$, und für den der zugehörige Integralwert dem Bereiche $B_{\curvearrowright}$ angehört. so fällt $x_{1}$ in denzu diesen $x$-Werte gehörigen Cauch y selien Konvergenzkreis, das betreffende Integral ist also in $x_{1}$ holomorph. Solche $x$-Punkte existieren aber nach dem Vorhergehenden für unser Integral $\Phi\left(x ; x_{0}, y_{0}\right)$ stets, und darnit ist der Beweis für unsere Behamptung geliefert. Wir haben also don Satz von Paiuleve:

Inncrlatb der Fläche $T$ rerhält sich jedes Integral der Differentialgleichung (1) algebroid; dir verschieblaren singulären Stellen eines solehen Integrals können also nur Pole oder algebraisch. Verzweigungspunktesein.

Den grundsätzlichen Untersehied zwischen festen und mit den Anfangswerten versehiebbaren singulären Punkten hat zuerst (1884) Furhs hervorgehoben ${ }^{1}$ ), nachdem sehon früh M. Hamburger ${ }^{2}$ ) das Aufteten von verschiebbaren. Singularitäten an Beispielen gezeigt hatte. Ein rinfaches Beispiel ist die Gleichung

$$
\left.d y+\frac{x}{y}=1\right) ; g(x \cdot y)=\ldots, h(x \cdot y) \quad y .
$$

deren allgemeines lntegral der Gleirhung

$$
x^{2}+y^{2}=\text { roust. }
$$

genügt. Soll fïr winn ron Null versehiedenen Went $x_{n}$ ron $x . y=0$ sem so wird

$$
y=1, r_{11}^{2}-x^{2}
$$

Da für $y=0$ dir Nennerfunktion $h(x, y)$ verschwindet. hat man es hier mit dem in der $\mathrm{Nr}$. 10 behandelten Falle zu tum, und in der Tat ergiht sich in der Ungebung von $x=x_{0}$ eine Entwiekhng von der Form

$$
y=\left(x-x_{0}\right)^{\prime}:\left(\partial_{0}+\delta_{1}\left(x-x_{0}\right)+\cdots\right) \text {. }
$$

die nach gebrochenen Potenzen von $x \ldots x_{n}$ fortshreitet.

1 Fuchs. Worke H. Sins

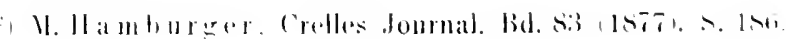




\section{Diflerentialoleichungen mit festen Verzweigungspunkton Riccatische Inillerentialerleichunar.}

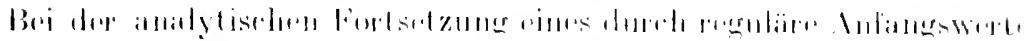

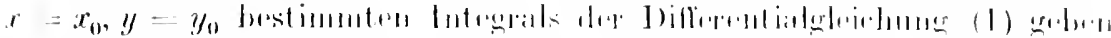

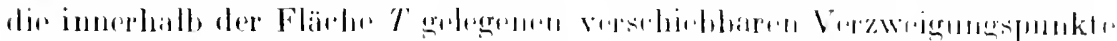

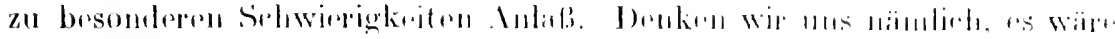

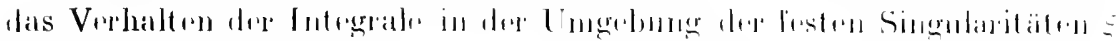

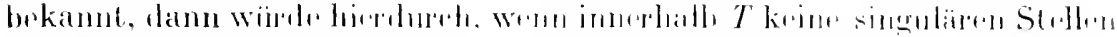

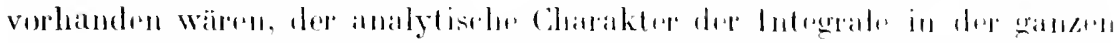

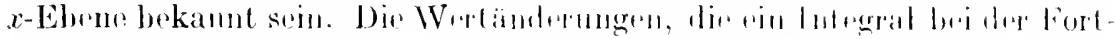

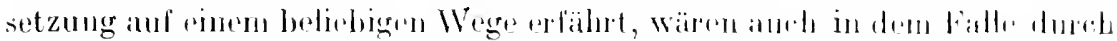

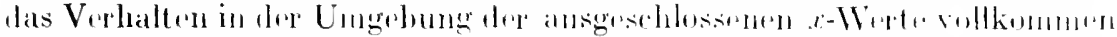

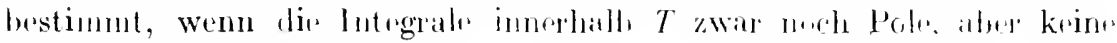

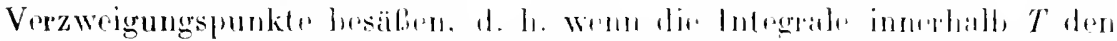

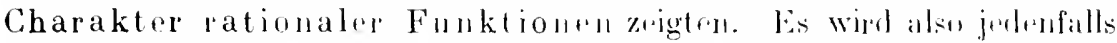
dicjenige Klasse von Different ialgheluhumgen dor Form (1) als diu rinfachste

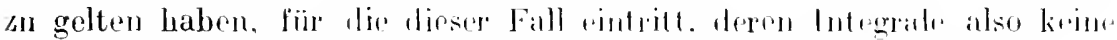

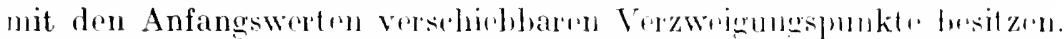

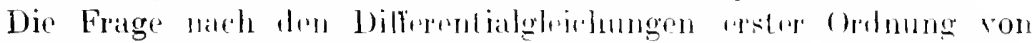

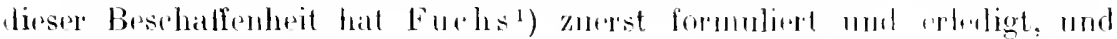

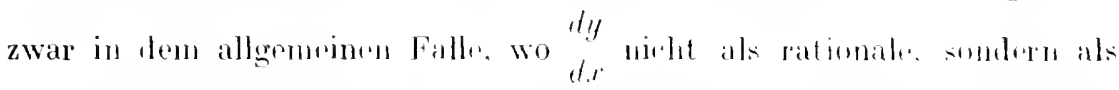

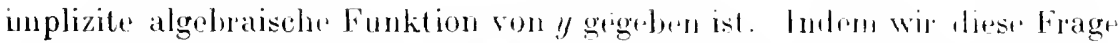
zunächst in dem Falle riner Ditherentialgledehung von fre Form (1), wo $f(x, y)$ eine rationale Funktion von /f bedentun mög", zu brantworten suchen, werden wir zu einer hervoragend wichtigen und interessanten Klasse von Diflerentialgleichungen geführt werlen. Wir butrarhton also wieder die Jifferentialgleichnug (1)

$$
\stackrel{d y}{d y}=f(x, y)=\begin{aligned}
& n(x, y) \\
& h(x, y)
\end{aligned} .
$$

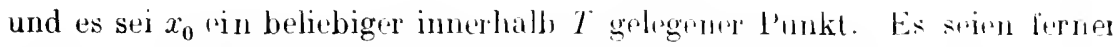
$b_{1}, b_{2}, \ldots, b_{m}$ die Wurzeln der alguhatisehn Glejehung

$$
\left.h\left(x_{0}, y\right)=1\right)
$$

dann werden diejenigen Integrale von (1), die fü $x=l_{0}$, inten de Witt $b_{k}$ annelumen, nach den Ergebnissen der $\mathrm{Nr} .10$ in $t_{0}$ einen Verzweigungspunkt besitzen.

Wenn wir alsu erroben wollen, dab die lntegrate der bifferentialgleichung (1). keine versehiebbaren Verzweigungspunkte besitz'n. so wird

1) Fuchs. 1884. Werke II. S. 350. 


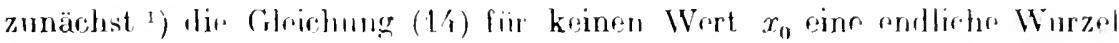
haben dürfen, 1 . h. "s mul. $h(x, y)$ von $y$ umahhängig. also eina Funktion von $x$ allein sein. Jorse denkro wir urs in dir Koelfizienten von g(r.y)

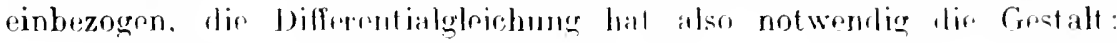

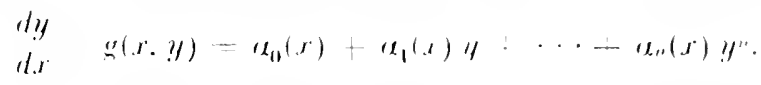

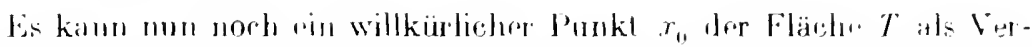
zweigungspunkt rines Integrals fusgiren, wenu lioses Integral fiur $r=r_{0}$ unendlich wird. Sutzen wir also wir in der Nr. 11

$$
1
$$

so wird anch in dere Jofferentialgleichure liur :

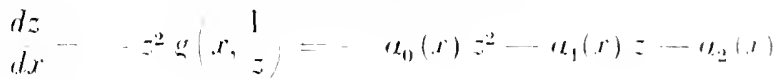

$$
\begin{aligned}
& -\mu_{n}(x)_{z}^{1} \ldots-\ln (x)=1
\end{aligned}
$$

die rechte soite eine ganze rationalu Funktion vol z sin missen. 1. h es muli.

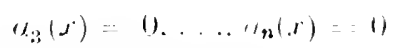

sein. Hir Form der Differentialgleichung (1)

$$
d y=u_{10}(x)+u_{1}(x) ! r+u_{2}(x) ! y^{2}
$$

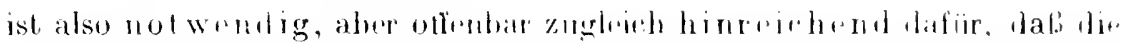
Integrile koine mit den . Infangswerten versehwhbaren Virzwignngs-

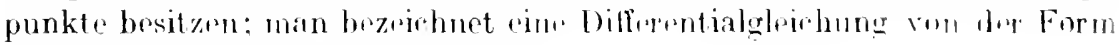
(15) gewöhnlieh als Rivatische ${ }^{2}$ ).

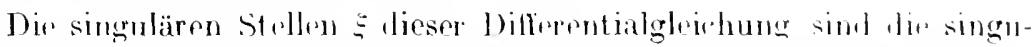

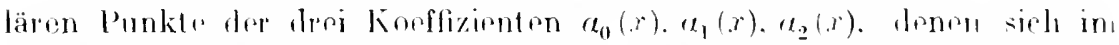

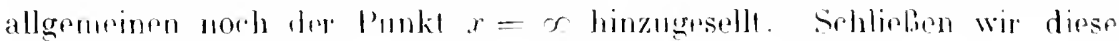

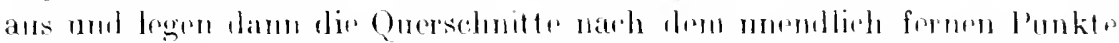

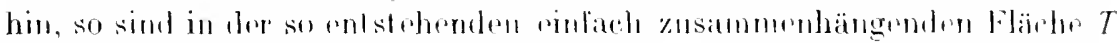

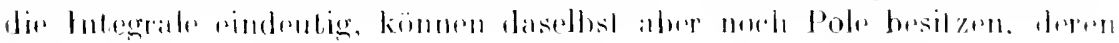

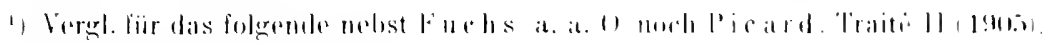

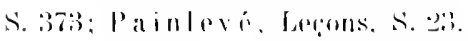

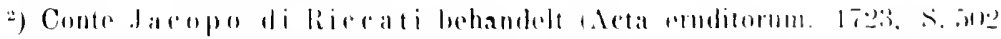

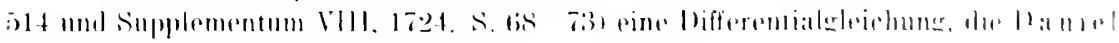

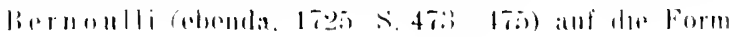

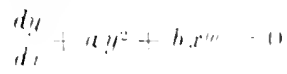

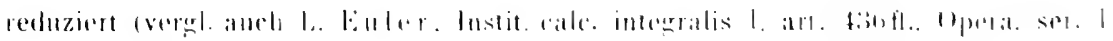

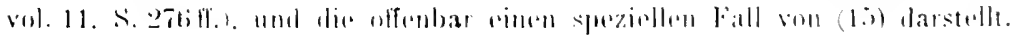




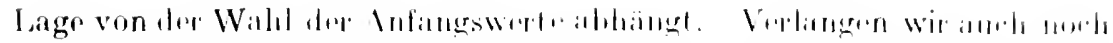

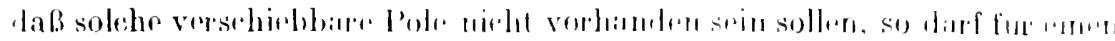

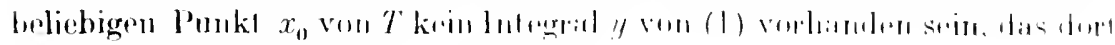

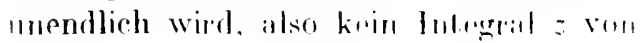

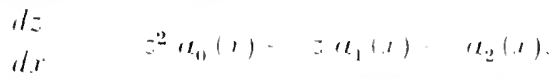

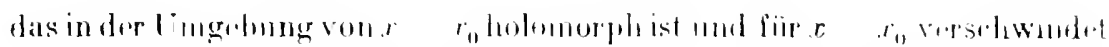

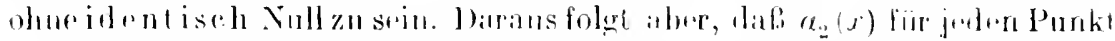

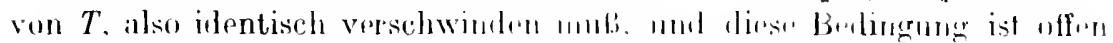

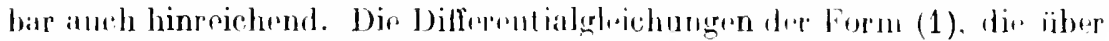

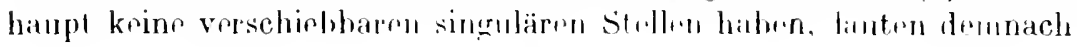

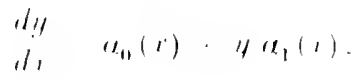

1. h. sie sind li wratr in y und d?

Für die allgemeine Rir.aliseh. Dilferentialgleichung kinn man and der Eigenschaft. daf. ihre Lösmngen immelualh von $T$ krine anderen Singu-

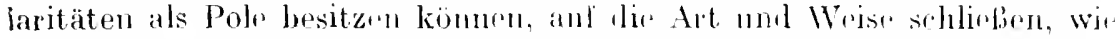

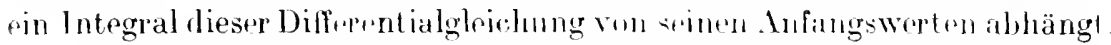

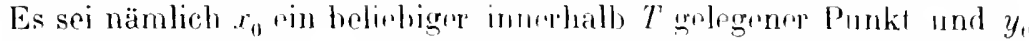

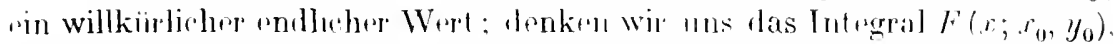

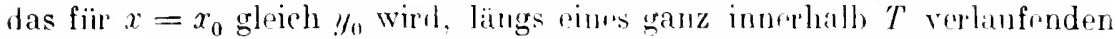
Weges nach einem Punktr $x$ fortgesetzt, sn wijd lep sich fiir las Integral

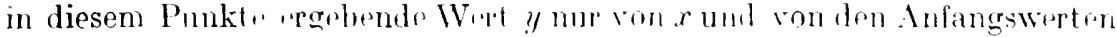

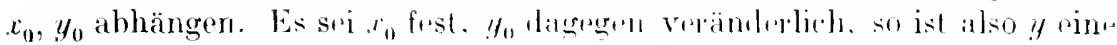

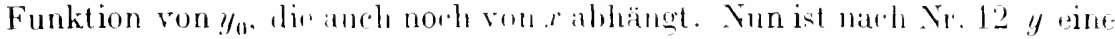

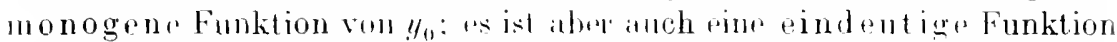
von $y_{0}$. Denn bei festem $x_{0}$ ist dere Wert der Integralfunktion in Punkte $x$, bei Fortsetzung innerhalh $T$. dureh lon Anfangswet $y_{0}$ oindeutig bestimmt, weil ja in $T$ krim Verzweigumgsunktr liogen. Dieser Integralwert ist überdies für jedes $y_{0}$ (am $h_{1}=\infty$ ) rin wolllmstimmter endlicher Wert oder unendlich grob, folglich kinn diese aindentiga Funktion auch keine anderen singulären Stellen als l'ule hrsitzen. sir ist also mach einen bokannten Satze der Funktiommuthmir. ${ }^{1}$ ) eins rationale Funktion 1. h. $y$ ist einerationale Funktion von $y_{0}$. delen Konfizinuten natürlich noch von $r$ abiängull.

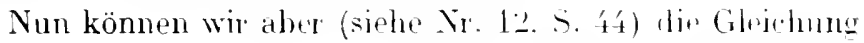

auch in wer Folm

$$
y=F\left(x ; r_{11} \cdot y_{11}\right)
$$

$$
y_{0}=F\left(r_{10} ; l \cdot y\right)
$$

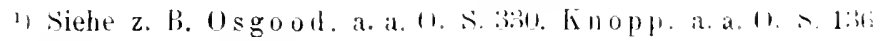


schreiben. Was wir für y als Funktion won yn wschlossen hahen. erilt also genau ebenso fïr $y_{0}$ als Funktion von $y$; d. h. "s ist auch yo wiun rationale Funktion von!y.

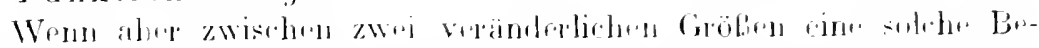

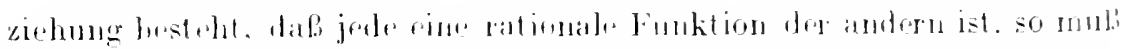

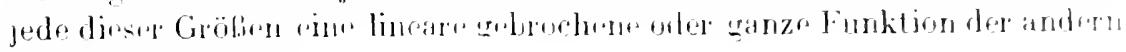
seint as int lululiels

$$
y=\begin{aligned}
& \left(y_{0}-l\right. \\
& \left(y_{0}+l\right)
\end{aligned}
$$

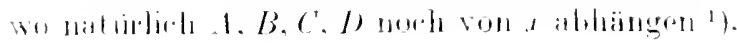

\section{Form des allgemeinen Integrals.}

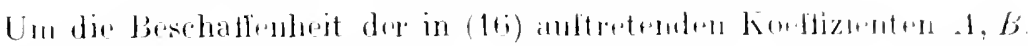

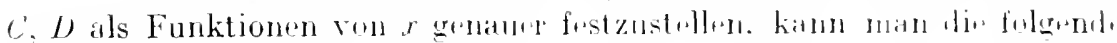
Erwägung anstellen.

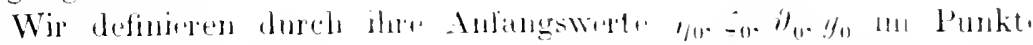

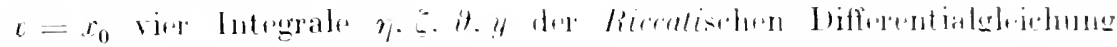
Dalll is

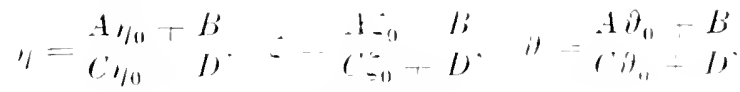

$$
\begin{aligned}
& 1 y_{0}+1 ; \\
& \left(y_{0}+1\right)
\end{aligned}
$$

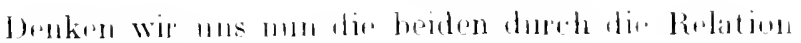

$$
\begin{aligned}
& 11+1) \\
& (2+1)
\end{aligned}
$$

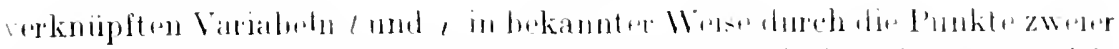

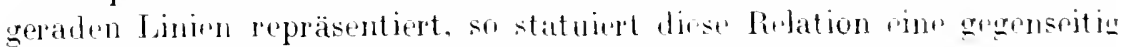

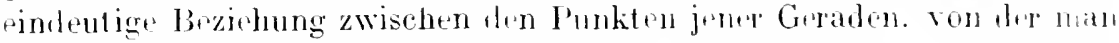
m den Elonenten der analytischen Geometro zojert. dat. sie die allemmonste.

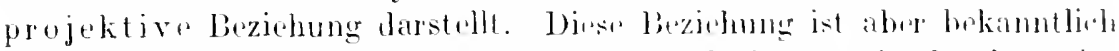

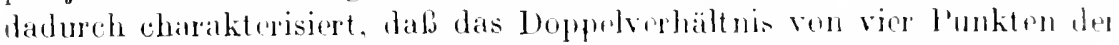

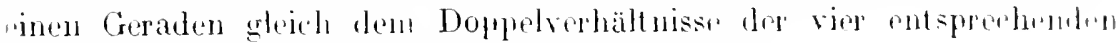
Punkte dew anderen Geraden ist. Den vier Punkten

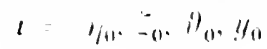

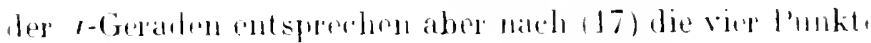

$$
t=1 \%, \therefore, y
$$

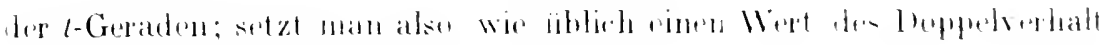

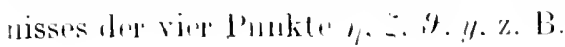

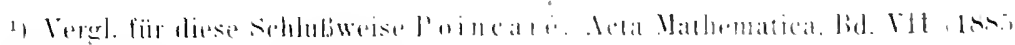

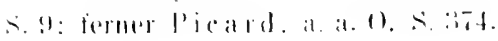




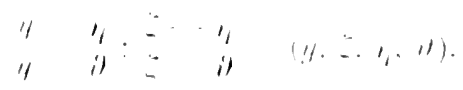

(1) Ist

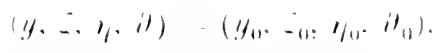

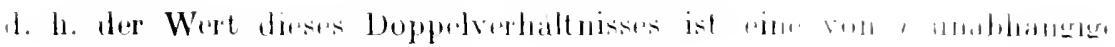
Gröbe $I$. Aus der Glojehumg

$$
(y, \cdots 1, \text { il) }=-l \text {. }
$$

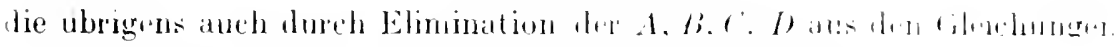

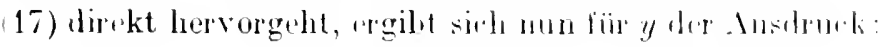

$$
y=\quad y(-i)-r i(-2 y) .
$$

und wonn man hierin $l$ als willkürliche Konstante ansieht. so stellt dieste

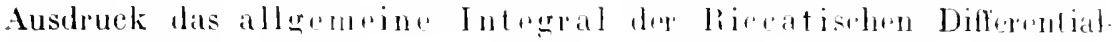
gleichung dar.

Dirses allgemeine Intergal setzt sich also rational audrei partikularen Integralen $y$, it. 20 ammutr ${ }^{1}$ ).

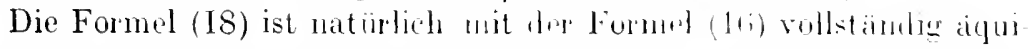
valent, während hier $\Gamma$ als willkürliche Konstante fungiert. sn dort $y_{0}$ :

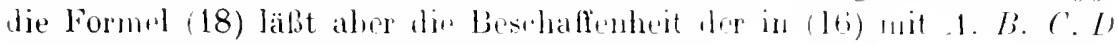
bezeichneten Funktionen von ol heruetreten.

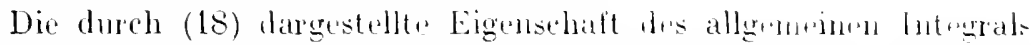

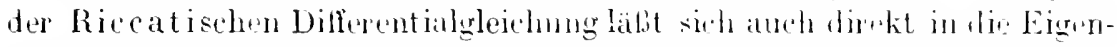
schaft dieser Differentialglelehung mmsetzrn. von der wir ansgegangen

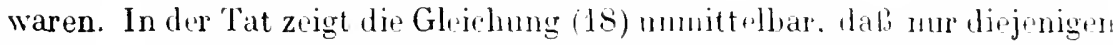

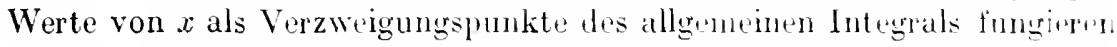
können, für lie eine der Funktionen $\%,-, \theta$ eine Verzweigung triaht. diese sind aber natürlich mit $\Gamma$. onler was dasselb ist, mit dem Anfangswerte $y_{0}$ von $y$ nicht verschibbar. C̈herhampt hängt dit Eigntenschaft einer Differentialgleichung, nur feste Verzweigungspunkt zu besitzen. aufs engste damit zusammen. ob sich las allgeneine Int.ogral als rationale Funktion gewisser partikulärer Integrale darstellen läbt. Ist das allgenein Integral rational durch gewisse partikuläre lntegrale darstellbar, so sint die Verzweigungspunkte nicht verschiebbar; ist es sogar gan z und rational durch eine Anzahl partikulärer Integrale darstellbar, so sim auch die Pole fest ${ }^{2}$ ). Unter den Dilferentialgledelungen von der Form (1) ist also di

1) Dieser Satz rührt ron Eduard Weyr her. Abhandlungen der hïhm Gesellsch. d. Wissensch. (6). \& (185j). S. 30: vergl. anch u. a. Königsberger Lehrbuch der Theorie der Differentialgleichungen (Lejpzig 18s9. S. 322.2.

2) Vyl. hierzu lioenigsberger. a. a. O. S. $96 \mathrm{ft}$. und Acta Mathematica. Bu III (1883). S. $1 \mathrm{ff}$. 


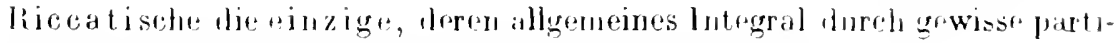
knlare Integrale rational dargrestrellt werden kann')

Fiir die linrare Jiffirentialglrichnong

$$
\begin{array}{lll}
d ! y & & \\
d . r & u_{1} !
\end{array}
$$

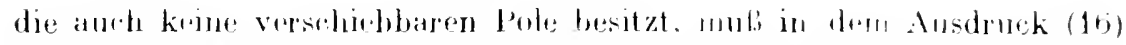

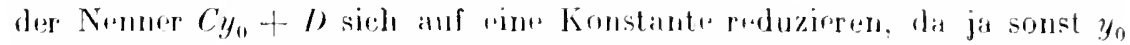

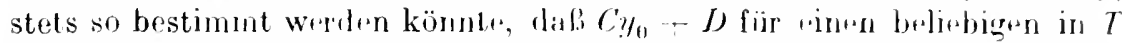
gelegenen $x$-Punkt verschwindot. Das allgemeinf lntregral von (19) ist also rine ganze lineare Funktion der Introgrationskonstantrn yo; das läst sich in diesem einfachen Fallo auch dirokl zoigen. imlem man das allgemeine Intrgral rinre solehen, , linearen Jjiferentialgleichung arster Orlnung" durch ( 2ualraturen darstelle"). Sutzt inan nämlirb

$$
11-116 .
$$

so regibl die Jifferentialgleichmmer:

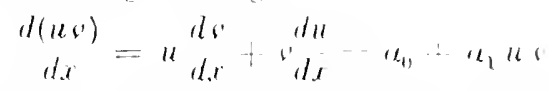

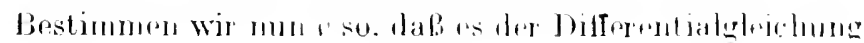

$$
d r=l_{1}{ }^{\prime}
$$

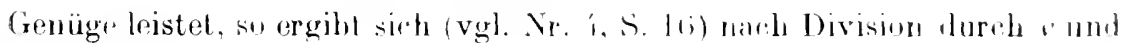
Integration

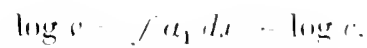

wo c eine willkiurlinh. Konstante bedeutet. also

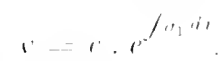

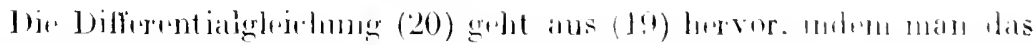

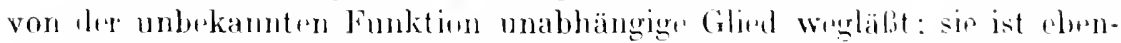

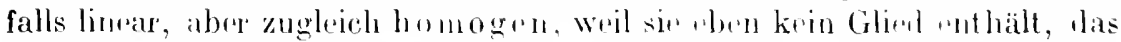

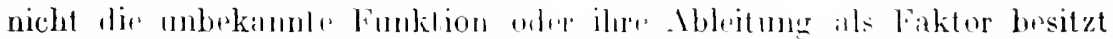

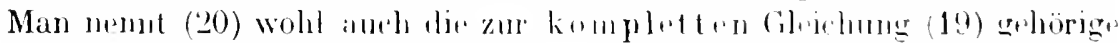

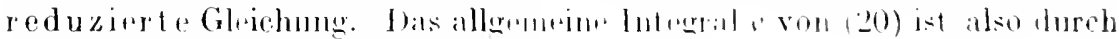
eime Quadratur daresestellt.

Nelumen wir dir kunstant, r.- 1, also

$$
\therefore=r_{1}^{\prime \prime \prime}
$$

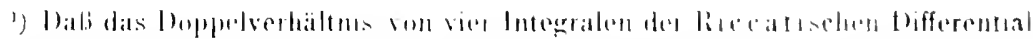

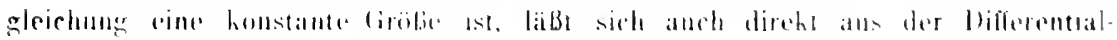

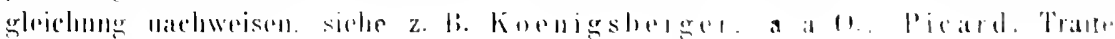
I (1501). S. 1:3ti.

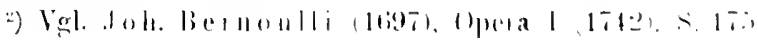




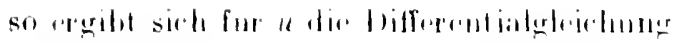

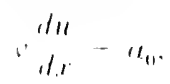

inler

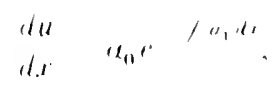

woraus dired Integration

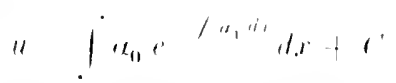

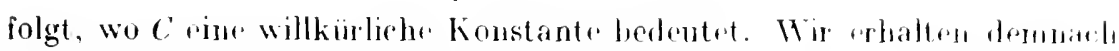
das allgemeino Integral y von (19) in dur Form

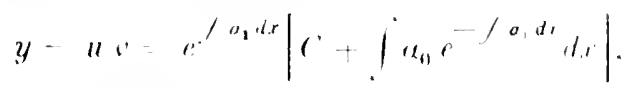

also als ganze lineare Funktion von $C^{\text {. }}$

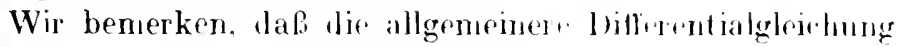

$$
f^{\prime}(y) \frac{d y}{d x}+j(y) q(x)-\chi(x)
$$

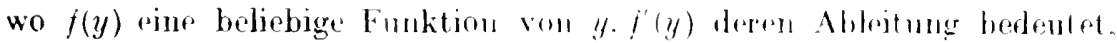
durch dir Substitutiou

auf tie lineare Differentialgleichung

$$
=j(!)
$$

$$
d z+z y(x)-\chi(x)
$$

zur ückgefuhrt werden kanm. Dir sogenannte liernoullıshe lifforentualgleichung (rgl. das Zitat S. (60)

$$
y^{\prime \prime} d y+y(x) y^{\prime \prime+1}=y^{\prime}(x) y^{\prime \prime}
$$

verwandelt sich nach Multiplikation mit

$$
(p-q+1) y=
$$

in einen speziellen Fall dur J)ifferentialglejchung (22). wo nämlich

$$
f(y)=y^{p-q+1} \text {. }
$$

Diese ist also, ebenso wie die Gleichung (22) sulbst. lurch Quadraturen integrierbar.

Wenn es gelungen ist, die Integration viner vorgelegten Differentialgleichung auf Quadraturen zurürkzufiilnen. so ist dadurch zur vollständigen Lösung des Integrationsproblems in dem von uns formulierten Sinme (Nr. 5, S. 20) nur ein erster Schritt getan. Denn wenn es im allgemeinen schon bedeutende Anstrengungen rrfordert, die analytische Beschaffenheit der durch eine einfache Quadratur dargestellten Funktion zu ergr ünden. so ist die Untersuchung eines Ausdruckes, der aus mehreren Quadraturen zusammengesetzt ist, natürlich noch viel komplizierter. In der historischen 
Entwicklung der Wissenschaft spielen solche Differentialgloichungen, deren Integration sich auf Quarlraturen zur ïckfüh"m läßt, insofern eine gowisse Rolle als rinerseits die Quadratur gewissermaßen die einfachste Quelle neuer Funktionsklassen bildet. und andererseits die für die ange-

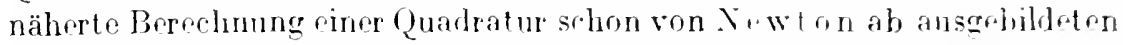
verschiedenartigen Wethorlen für die Wertbestimmung des lntrgrals einer Diffrentialgleichung nutzlar gemarlit werden konnten. wenn es golang, dieses Integral durrh Quadraturen darzustellen. Wir findrn laher bei den äleren Analysten vielfach das Bestreben. eine zur Intugration vorgelegte Differmtialgleichung soleluen Transformationen zu unterwerfen. dab dann die Darstellung les Intragrals durch Quadraturen möglich wird. Nun gelingt es aber nur in den soltensten Fällen, eine solche Transformation ausfindig zu machen; las Problem der Integration wird also durch das Bestreben, derartige Transformationen aufzufinden. nicht nur verschoben, sondern außerordentlich beschränkt. in ähnlicher Weise etwa, wie wenn man sich in der Algrbra die Aufgabe stellte. ein grgebene algebraische Gleichung durch blofir Anwendung von Wurzelzeichen aufzulösen. Su interessant an sich die Aufgabe ist, diejenigen Differentialgleichungen aufzustellen. deren Integration sich auf Quarlraturen zurückführen lälit. so muf. doch dieser Aufgab. als einer ganz speziellen der ihr gebührende Platz in der srstematischen Theorie angewiesen werden. keinesfalls darf sie als das einzige oder erstrebenswerteste Ziel in den Vordergrund der Theorie gerïckt werden. In neuerer Zeit ist es Lie gelungen, durch die von ihn begrindete Theorie der Transformationsgruppen (deren Anwendung in der Theorie der Differentialgleichungen allerdings viel weiter reicht) Methoden zu entwickeln. die zu einer Lüsung der gedachten Aufgabe führen können ${ }^{1}$ ): wir begnügen uns damit, auf diese schöne und wichtige Theorie hingewiesen zu haben, und wollen uns jetzt wieder der Untersuchung der allgemeinen Rice at isehen Differentialgleichung zuwendin.

\section{Znsammenhang der Riccatischen Differentialgleichumg mit linearen homogenen bifferentialgleichmgen.}

Dir allgemeine li icrat ische Differentialgleichung (15)

$$
d y=a_{11}+a_{1} y+a_{2} y^{2} \text {. }
$$

wo $a_{2}, 0$ ist, kann nicht dureh Quadraturen integriert werden *): aher

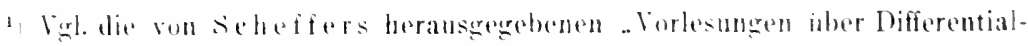
aleichungen" (Lejpzigr 18931 .

2) Ein dirckter beweis dieser behauptung ergith sieh z. B. ans den lenter-

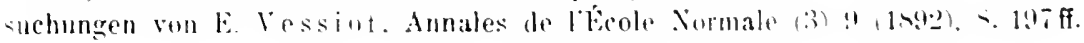




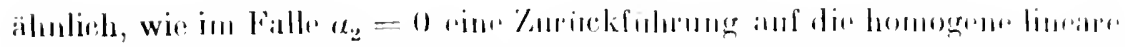

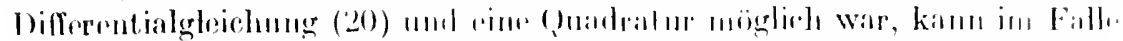

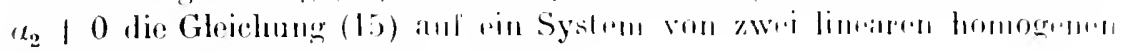
Jiffrentialgleichmmen von der Findu

$$
\begin{aligned}
& \frac{d u_{1}}{d x} \quad\left\|_{1}\right\|_{11}+\|_{2} u_{21} \text {. } \\
& d u_{2}=u_{1} a_{12}+u_{2} a_{22}
\end{aligned}
$$

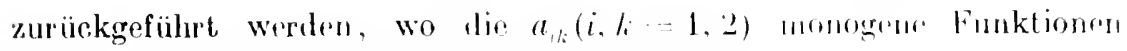
von $x$ bedeuten. Dazu braucht man nur zu sotzen

$$
y=u_{2}, \quad a_{11}=a_{12} \cdot a_{1} \quad\left(a_{11}-u_{222}\right) \cdot a_{2} \quad a_{21},
$$

woraus sich, wem wir the Ableitungen nach $x$ durch Akzentr brzeichnen.

$$
\begin{aligned}
y^{\prime}= & \begin{array}{c}
1 \\
u_{1}^{\prime 2}
\end{array}\left(u_{1} u_{2}^{\prime}-u_{2} u_{1}^{\prime}\right)=u_{11}+u_{1} \frac{u_{2}}{u_{1}}+u_{2} u_{2}^{2}, \\
u_{1} u_{2}^{\prime}-u_{2} u_{1}^{\prime} & =u_{0} u_{1}^{2}+u_{1} u_{1} u_{2}+u_{2} u_{2}^{2} \\
& =-\left(u_{11} u_{1}+u_{21} u_{2}\right) u_{2}+\left(u_{12} u_{1}-u_{22} u_{2}\right) u_{1}
\end{aligned}
$$

ergibt. Die der Substitution $y={ }_{\|_{1}}^{\|_{2}}($ simhe Gl. (2亿)) noch anhaftenul. Willkür gestattet nun, etwa fïr $n_{1}$ dio erste der Gleichungen (23) anzunehmen, worauf dann aus (25) fiir $u_{2}$ die zweit. der Gleivhungen (23) folgt.

Hat man umgekehrt ein beliebig vorgulengtes System vou her Form (23), so befriedigt ler Ausdruck $y=u_{2}$, lin Riceatische Differentialgleichung

$$
y^{\prime}=a_{12}+\left(a_{22}-a_{11}\right) y-a_{21} y^{2} .
$$

Beim Übergang von der Riccalisehen Gleichung (15) zu dem System (23) haben wir noch in der Gleichung

$$
a_{1}=-\left(a_{11}-a_{22}\right)
$$

des Gleichungssystems (2') eine Unbestimmtheit, die wir zur Vereinfachung des Systems (23) benutzon können. Nehmen wir $a_{11}=0$. so ergibt sich

$$
u_{1}^{\prime}=-\alpha_{2} \iota_{2}, \quad u_{2}^{\prime}=\alpha_{0} u_{1}+\alpha_{1} u_{2} .
$$

und indem wir die crste dieser Gleichungen noch einmal differentiieren und für $u_{2}$ und $u_{2}^{\prime}$ ihre Werte einsetzen.

$$
\alpha_{2} u_{1}^{\prime \prime}-\left(\alpha_{2} \alpha_{1}+\alpha_{2}^{\prime}\right) u_{1}^{\prime}+\alpha_{0} \alpha_{2}^{2} u_{1}=0 \text {. }
$$

Es ist dies eine homogene lineare Differentialgleichung zweiter Ordnung für $u_{1}$, und der Zusammenhang zwischen der Riccalischen Gleichung (15) und dieser Gleichung wird durch die Beziehung 


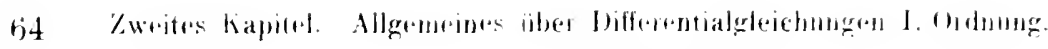

$$
\Rightarrow \quad-\quad 1 / \log u_{1}
$$

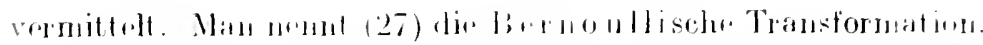

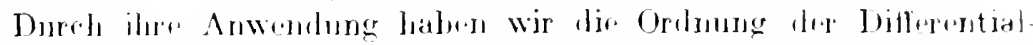

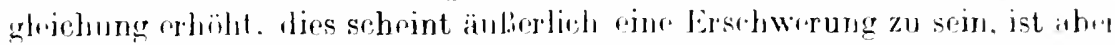
in Wirkliehkrit rine Erloiehterung, da wir durch dies" Transformation

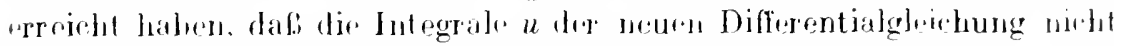

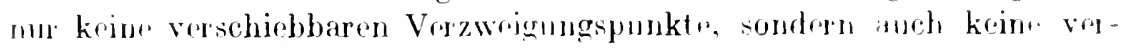
ahiehbalen Pole hesitzom.

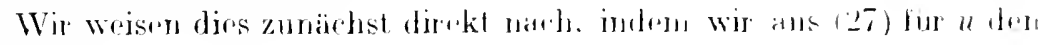
Ausclurek

$$
\text { H. . a }-J u_{2} y d c
$$

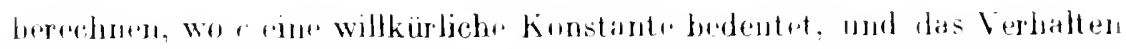
von $u$ untersuchen, wenn $y$ für $x=x_{0}$ pinen versehinharnll lol besitzt.

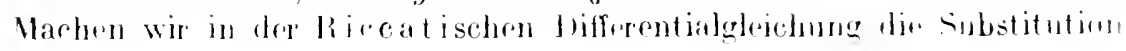

$$
1 \quad 1
$$

wolum firr : die Differentialgloichung

$$
d z-\left(r_{0}=2 \cdot u_{1}=+u_{2}\right)
$$

hervorgent. so ist fiir $x \quad x_{0}$ das betrotfende $=-0$. atso

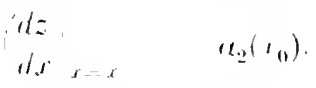

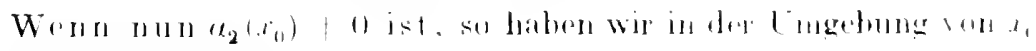
lur : die Entwicklome

woraus sich

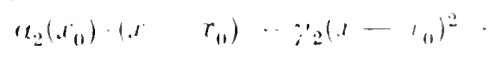

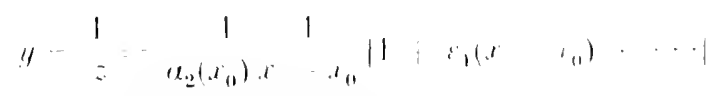

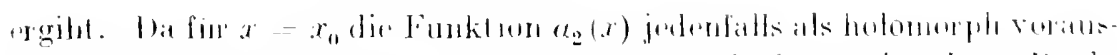

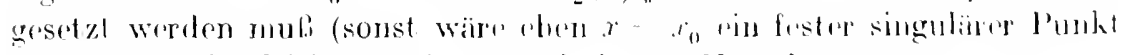

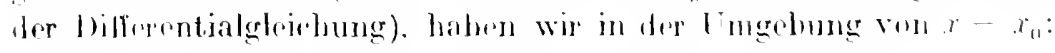

alsu

$$
\left(t_{2}(x) \quad x_{2}\left(x_{0}\right) \cdot \partial_{1}\left(x-x_{0}\right) \quad r_{2}\left(x \quad x_{10}\right)^{2} \ldots \cdots\right.
$$

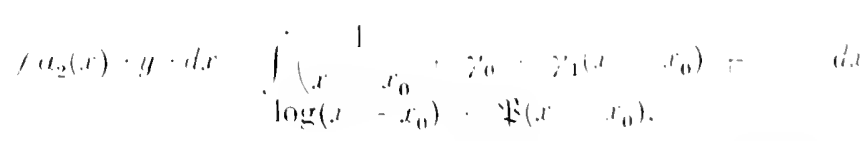

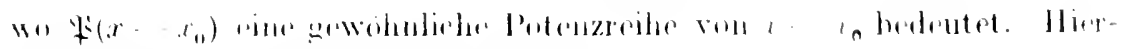

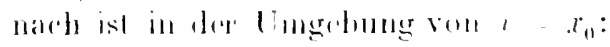




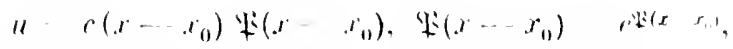

$u$ ist also in der Umgebmur von $r=r_{0}$ holumorph.

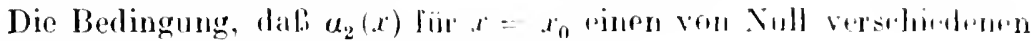
Wert habe, ist für dirses Result at wescullich.

Dividienen wir dic J)ifferentialterleichung (26) doreh $u_{2}$, so rrhält sir die Form

$$
d^{2} u-\left(\begin{array}{l}
\alpha_{2}^{\prime} \\
u_{2}
\end{array}\right.
$$

da $u$ in der Ungebung einer Stellr. wo y salbst holnmorph ist, ollenbar ebenfalls holomorph bleilot, haben wir das wiehtige Resultat:

Reduziert man in are lomogenen linearen Differentialgleichung (26) den Konfizinnten dre hïehstru Ableitung auf Eins, so ist das allermeinr lotegral dieser Groiehung holomorph in der Ungelung jeder stelle $x$, für di, lie Koeffizienten del Differentialgleichung selhst holumorph sind. Die einzigen singulären Punkte dul Integlale sind die singulären Stellen der Koeffizionter, diese sind also säntlich fest; wir nennen sie lir singulären Strllun der Differentialgrleichung.

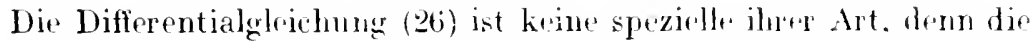
allgemeinste homogene lineare Diffepentialgleichung zweitm Ordnumg

$$
p \frac{d d^{2} u}{d x^{2}}+q_{d x}^{d u}+r u=0
$$

wo $p, q, r$ Funktionen von $r$ bedeuten, lälst sich in dif Form (2tia) boziehungsweise (26) unsetzen. wo

$$
a_{0}=\begin{gathered}
r \\
p^{2}
\end{gathered}, \quad \mu_{1} \quad-\begin{gathered}
q+p^{\prime} \\
p
\end{gathered}, \quad \iota_{22}=l^{\prime}
$$

ist, und wird demnach durch dir Substitution

$$
y=-\begin{gathered}
-1 d \log u \\
p \quad d t
\end{gathered}
$$

in pine $\mathrm{R}$ ic catischo Differentialgleichung für $y$ transformiert. Der ausgesprochene Satz gilt also für jede homogent lineare Differentialgleichung zweiter Ordnung. Er gilt aber auch für ein beliebiges System von der Form (23) und allgemeiner für jedes Systen homogener linearer Differentialgleichungen erster Ordnung

$$
\frac{d y_{k}}{d x}=y_{1} a_{1 k}+\cdots+y_{n} a_{n k} \text {. }
$$$$
(k-1,2 \ldots n)
$$

dessen Koeffizienten $a_{i k}$ monogene Funktionen von $x$ sind. Di, 'Theorie dieser Systeme wird uns später eingehend beschäftigen, wir fügen an dieser Stelle nur noch einige Bemerkungen ïber die Riccat ische Differentialgleichung hinzu.

sehlesinger, Differentialgleichungen. 
Im Innern der Fläch. Thabrn dire Lösungen riner Rireatischen Differentialgleichung keine anderen Singularitänn als Poln. sir verhalten sich in $T$ uresomorph. Die lerage, wie sich diese Lösurgen in dre Ungrbung der festen Singularitäton $\$$ verhalten, ist durch die vorhergelundun Erörterungen aul die analog" Frage für dir linearen Systeme (2.3) oflor die lineare Differentialgleichung zweiter Ordumg (26) zuriurkgrfiihrt. Aun rinfachsten gestaltet sich dirse Frage. wenn die $\alpha_{0}, \alpha_{1}, a_{2}$ iib hatupt k..in. singulären Punkte aufweisen, woler in Entlichen noch in Lnendlichen. d. h. wenn sie koustanten sind. In dirsem Fallr kann in rinem endlichen $x$-Wert iiberhaupt kein Verzweigungspunkt der Integrale liegen. Demn dir Differentialgleichung bleibt offenbar ungeändrert. wenn man $r$ durch $x+$ const. ersetzt; läge also etwa bei $x_{n}$ rin Verzweigungspunkt rines Integrals, so würde das gleiche bei $x_{0}+$ const. zutreffen. man hätte also verschicbhare Verzweigungspunkte. Da somit das Auftreten von Verzweigungspunkten in Endlichen ausgeschlossen ist, kinn aurh $r=x^{\circ}$ kein Verzweigungspunkt sein. d. h. $y$ ist rine rindeutige Funktion von $x$. Wir können diesen Schluf auch lejcht bestätigen. da in dieserm Falle dir Integration der Rirealisehen Differentialgleichurge elementar durchführbar ist.

Schreibt man nämlich bei konstanten $\alpha_{0}, \alpha_{1}, a_{2}$ die Groichung (15) indorseparierten Form

$$
a_{0}+a_{1} y+a_{2} y^{2}-d x
$$

und bezeichnet mit $\varrho_{1}, \varrho_{2}$ die Wurzeln der Gleichung

$$
a_{0}+a_{1} y: a_{2} y^{2}=0 .
$$

so hat man fuir $Q_{1}: \varrho_{2}$

also

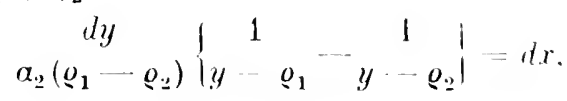

und fïr $\varrho_{1}-\varrho_{2}$

$$
\left.\begin{array}{l}
y-\underline{o}_{1} \\
y-\underline{e}_{2}
\end{array}=\text { const. } e^{a_{2}\left(\varrho_{1}\right.} \underline{o}_{2}\right)
$$

$$
y-e_{1}=a_{2} x+\text { const. }
$$

Es ist also in diesem Falle $y$ in der Tat rine allenthalben eindentige funktion von $x$. 


$$
\text { l)illes Kipiln. }
$$

\section{bifferentialgleichungen erster Ordnung, wo die Al)- leitung als implizite funktion der ahhïmgigen Ver- inderlichen gegeben ist ${ }^{1}$.}

\section{1s. Begriff der Integralfunktion \%.}

Wir haben uns bisher vornehnolich mit dem Falle rine l)ifrementialgleichung erster Ordnung bescläfligt, in der dio Ableitung der nubekannten lunktion $y$ als rationale finktion von $y$ gegeben war. The allgemeinr Fall, wo die Ableitumg rime implizite algebrabehe limbtion ron y ist. wo also $\frac{d y}{d, r}$ einer Gleirhung

$$
F\left(\begin{array}{l}
d y \\
d x
\end{array}, y, x\right)=0
$$

genügt, in der $F$ eine ganze rationale Funktion von dy unl $y$ mit von $x$ abhängigen Koeffizienten bedeutet, erfordert neue Mothoden der Intassuchung, deren Darlegung wir uns jetzt zuwenden wollen.

1) In diesem und dem folgenden Kapitel werden die einfachsten säatze ans der Lehre von den algebraischen Funktionen einer komplexen Veränderlichen als bekannt vorausgesetzt. Man findet diese Sïtze z. B. in den Werken: Appell und Goursat, Théorie des fonctions algébriques (Paris 1905). Landfried, Theorie der algebraischen Funktionen (Sammlung S'chubert XXXI, 1902), II. Stahl. Abriß eine Theorie der algebraischen Funktionen (Leipzig 1911). Eine auf arithmetischer Grundlage fußende Darstellung dieser Theorie gilst das Werk: Hensel und I a n ds berg, Theorie der algebraischen Fu'sktionen (Leipzig 1902).

2) Quellen für diese und die folgenden Nummern sind: Briot und Bouquet. Journal de l'École Polyt. Cah. 36: W. Kaschke, Inaug.-Dissertation, Heidelberg 1883, Acta Mathem. 14 (1890). A. 31: Fuchs, (1884) Werke II, S. 355: Hamburger. Crelles Journal. Bd. 112 (1893). S. $205 \mathrm{ff}$. : Poincaré. Acta Mathematica, Bd. 7 (1885), S. 1 ff.: vgl. hierzu P'ic a rd, Traité III (1908). S. 40. $62 \mathrm{ff}$ : Painlevé. Leçons. S. $47 \mathrm{ff}$.: Forsyth. Theory of differential equations. Vol. Il. (Cambridge 1900), chapter VIII-X. 
Setzen wir $\stackrel{d y}{d x}=s, s 0$ ist durch rie Gleichung (1) $s$ als algebraische Funktion von $y$ definiert. Wir schreiben (1) ausführlicher in der Form

$$
A_{0}(y, x)\left(\begin{array}{l}
d y \\
d x
\end{array}\right)^{m}+A_{1}(y, x)\left(\begin{array}{l}
d y \\
d x
\end{array}\right)^{\prime \prime \prime}+\cdots-A_{m}(y, x)=\mathrm{n} .
$$

worin die $A_{0}, A_{1}, \ldots, A_{m}$ ganze rationale Furktionen von $y$ uit von $x$ abhängigen Koffizienten bedenten, und setzen voraus, daß. die Gleichung

$$
F(s, y, x)=0 \text {. }
$$

aufgefabt als algobraische Gloichung zwischen s, $y$. irreduzibrel sei, daß also $F(s, y, x)$ nicht zerlegbar sei in Faktoren, die setbst ganze rationale Funktionen von $s, y$ mit irgendwelrhen von $x$ abhängigen odrer konstanten Korffizienter sind.

Durch Elimination von s zwischen (2) und der Gleichung

$$
\begin{gathered}
\partial F(s, y, x) \\
\partial s
\end{gathered}=F^{\prime \prime}(s, y, x)=0
$$

bitden wir dir Diskrimimantengleichung

$$
D(y, x)=0 \text {, }
$$

deren linke Seite cine ganze rational. Funktion von $y$ mit von $x$ ah hängigen Koeffizienten ist.

Wir betrachten nun einen Wirt $t=x_{0}$, der die folgenden Bedingungen erfüllt:

1. In der Ungebung von $x=x_{0}$ srien dir. Koeffizienten der ganzen rationalen Funktionen $A_{l}(y, x) \quad(k=0.1 .2 \ldots . m)$ ron $y$ holomorph.

2. Es sei für $x=x_{0}$ dir Funktion $A_{0}(y, x)$ nivht identisel. d. h. unabhängig von $y$, gleich $N u l l$.

3. Es möge für $x=x_{0}$ kein $y$-IVert vorhanden sein, fïr den sämtliche $A_{k i}\left(y, x_{0}\right) \quad\left(l_{i}=0,1,2 \ldots . m\right)$ gleichzritig vershwinden.

Fiir einen solchen Wirt rom i stellt dann dir Gleichung (3) die notwendige und himprichende Bedingung dafür dar, dab dir. Gleichung (2) eine metufacho Wurzol s besitzt, und dic Gleichung

$$
I_{0}(y, x)=0
$$

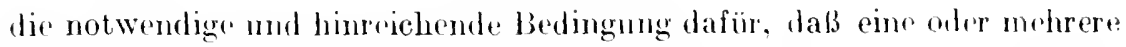
der Wurzeln sivon (2) unendlich grob werden.

Bedeutet also $x=x_{0}, y=y_{0}$ cin Wrepteparr. Lür das weder die Gleichung (3) noch dic Gloiehung (4) orfillt ist. so besitzt die Gleichung

$$
F\left(s, y_{0}, x_{0}\right)=0
$$

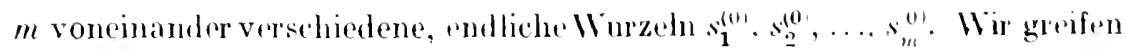
ane bestimmte von ihnen heraus, etwa $s_{k}^{(0)}$. dam lautet die (ileidhung (2) in der Uingebmug vom $x=x_{0}, y=y_{0}, s=s_{k}^{(1)}$ 


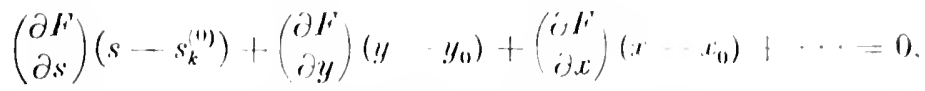

wo die partiellen Ableitungen von $F$ fïr $x=x_{n}, y=y_{0}, s=s_{k}^{(11)}$ zu uchmen sind, was hier (und ebenso im folgermon) durch das Einsehlielien in Klammern ( ) angedentet wirl. ber Koeffiziont $\left(\begin{array}{c}\partial f \\ g s\end{array}\right)$ von $s-s_{h}^{(0)}$ ist vont Null verschieden, da ja $s_{k}^{(1)}$ eine einfache Wurzel ist; wir kömucn also nach dem Satze von der impliziten Funktion ') in eimed hinreichend kleinen Ungebung von $x=x_{0}, y=y_{0}$ die Finnktion $s-s_{k}^{(1)}$ als gewöhnliche Potenzreihe von $x-x_{0}, y-y_{0}$ ohne absolutes Glied

darstellen. Wir setzen

$$
\mathcal{F}_{h}\left(x-x_{0}, y-y_{0}\right)
$$

$$
s_{k}=s_{\hat{k}}^{(0)}+\operatorname{lik}_{k}\left(x-r_{0}, y-y_{0}\right)
$$$$
(k \quad 1,2, \ldots, 14)
$$

und betrachten die $m$ Differentialgleichungen

$$
\frac{d y_{k}}{d x}=s_{k}^{(0)}+H_{k}\left(x-x_{0}, y-y_{0}\right)
$$$$
(k=1,2, \ldots, m) .
$$

Nach dem Existenzsatz der Nr. 9 besitzt jede dieser Differentialgleichungen ein und nur ein in der Ungebung von $x=x_{0}$ holomorphes Integral $y_{k}$, das für $x=x_{0}$ den Anfangswert $y=y_{0}$ annimmt, und das offenbar auch der ursprünglichen Differentialgleicluung Genüge leistet; wir haben also den Satz:

Die Differentialgleiclung (1) besitzt, wenn $x_{0}$ keiner der ausgeschlossenen Werte und $y_{0}$ so beschaffen ist, daB

$$
D\left(y_{0}, x_{0}\right)+0, A_{0}\left(y_{0}, x_{0}\right) \neq 0
$$

sind, $m$ in der Umgebung von $x=x_{0}$ holomorplie Integrale, die für $x=x_{0}$ den Wert $y_{0}$ annehmen und dadurch eindeutig charakterisiert sind, $\mathrm{da} B$ ihre Ableitungen fiir $x=x_{0}$ beziehungsweise gleich einer bestimmten Wurzel der Gleichung werden.

$$
F\left(s, y_{0}, x_{0}\right)=0
$$

Nach dem Unitätssatz der Ni. 12 (S. 45) gibt es außer diesen $m$ holomorphen Integralen kein Integral der Differentialgleichungen (7). das für $x=x_{0}$ den Wert $y_{0}$ annimmt. Inwieweit dies auch für die Differentialgleichung (1) selbst gilt, werden wir alsbald zu erörtern haben; zur vorläufigen Orientierung mögen die folgenden Bemerkungen dienen.

Wenn wir die Differentialgleichung (1) durch ein System von der Form (7) ersetzen wollen, so muß das Wertepaar $x_{0}, y_{0}$ der Bedingung genügen, daß

$$
D\left(y_{0}, x_{0}\right)+0
$$

ist. Nun kann es sich ereigneu, daß die Diskriminantengleichung (3) durch

1) Siehe z. B. Bieberbach. Funktionentheorie I (1921). S. 192. 
ainr Funktion $y=q(e)$ von $r$ idrutisch hofriodigt wird. dir zugleich ein

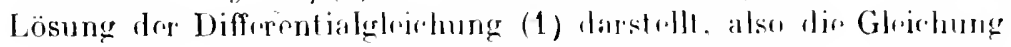

$$
F\left(\begin{array}{c}
d t(x) \\
d x
\end{array}, y(x) \cdot x=0\right.
$$

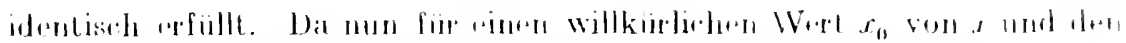

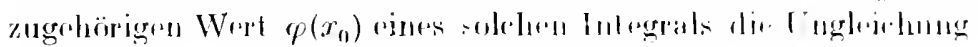

$$
D\left(\varphi\left(x_{0}\right), x_{0}\right) \perp 0
$$

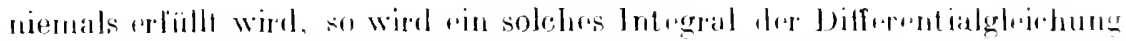

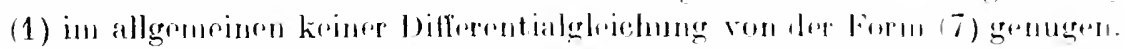

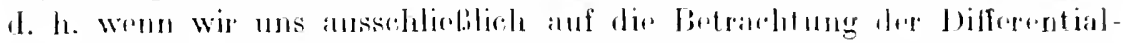
glaichungen von der form (7) besehränkten. so könnten uns dir wio $p(x)$

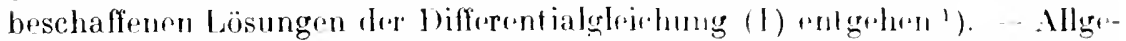
mein gesprochen ist rin Punkt $x_{0}$ für rin lulegral von (1). das in ro rinen

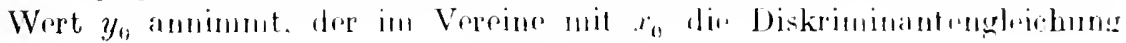

$$
\text { l) } \left.\left(y_{0}, c_{0}\right)=1\right)
$$

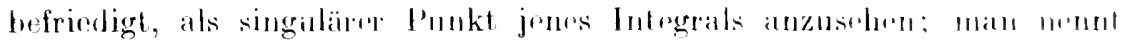

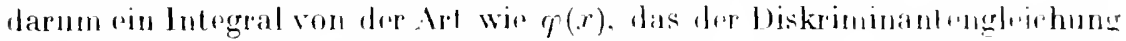

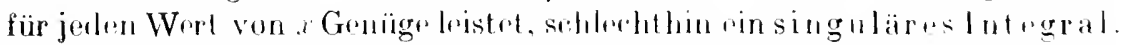

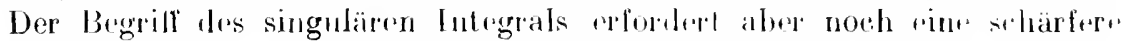
Fassung; wir kommen auf dirse weiter unlen zuriok, nachlenu wir uns

1) Im Riebieto der reellen leränderlichen kalln es anch noch allt andere Weise vorkommen. dab tiosungen von (1) kiner der Differentialgleichungen i

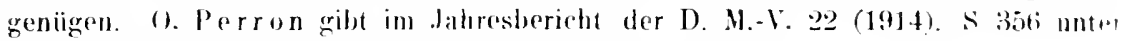
anderen das lolgende lehrreiche beispiel. Wir betrachten

$$
\text { *1 } \quad\left(\begin{array}{l}
d y \\
d . r
\end{array}\right)^{-2}-f(r)^{2}=11 .
$$

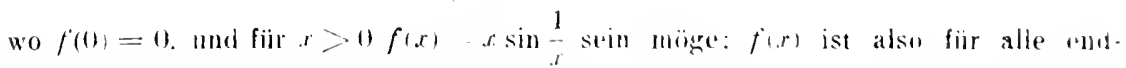
lichen, nichtnegativen Werte von $r$ stetig. Auber den mmitedbar gragebenen Lösungen der hejden Jlifferentialghleichungeren

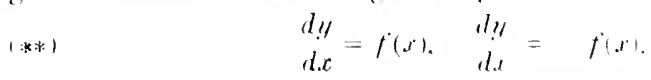

die fiir $d=1$ verschwinden, hat aber die l)ifferentialgleichung (*) now une nitheh viele liir,$d=11$ verschwindende läsungeren. nämlich

wentI

$$
y=1 y_{0}(x)\left|\psi_{1}(x)\right| y_{2}(x)+\ldots
$$

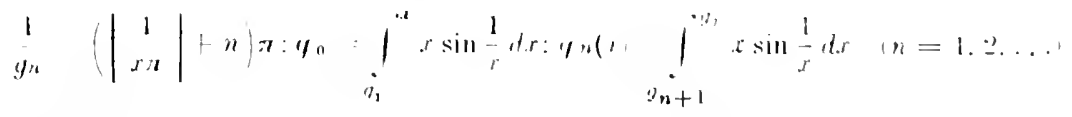

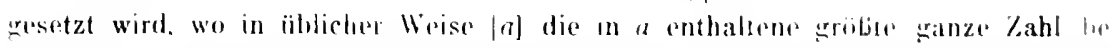
deutet. Wie man sieht. befriedigrt der Ausdruck!" bet boliebigarer IIahl der In zeichen für gewwisse r-Werte die rine, und fïr dis ibbrigen die andere der beiden (ileichungen (**). 


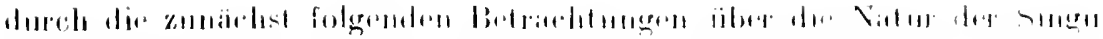

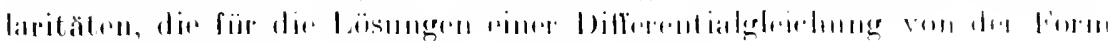

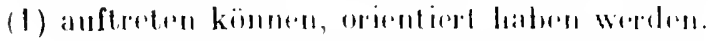

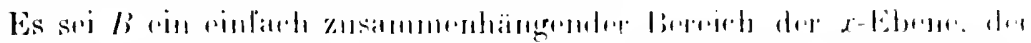

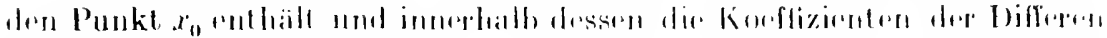

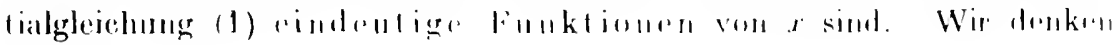
uns dire

$$
y_{1} \cdot y_{2}, \ldots, y_{1 \prime \prime}
$$

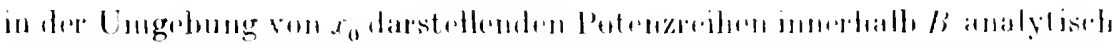

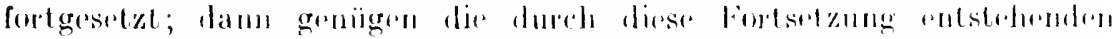

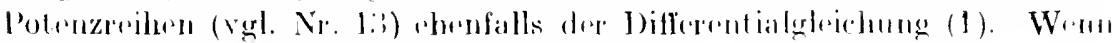

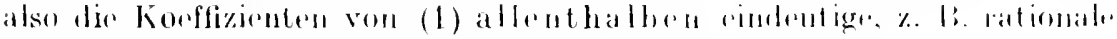

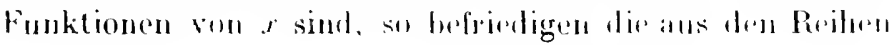

$$
y_{1}, y_{2} \ldots ., y_{m}
$$

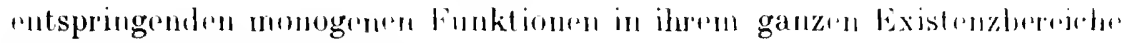
diest Differentialgleichung.

Als singuläre l'unkte dor Integralfunklion sind diejenigen $x$-lVert. anzusehen, Cör dir dir Möglichkeit dor Entwicklung nach positivenganzen

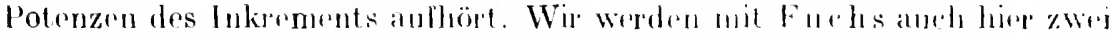

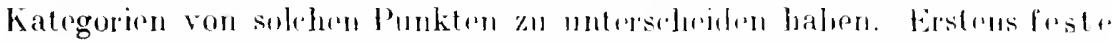

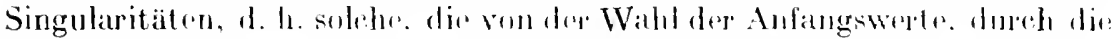

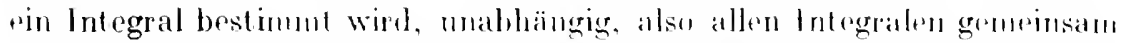

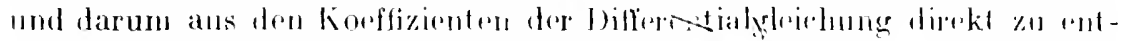
nehmen sind. Zu diesen gehören diejongern $x$-Werter dir wir als dir be

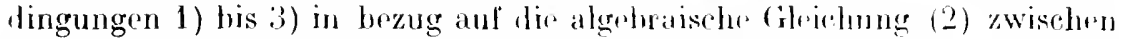
$y$ und s nieht befriedigend von vornherein ausgeschlossen haber, es werden aber noch andere solehe feste singuläre Stellen in havlo dej woiteren Untersuchung hervortreten. Zweitens vorschichbarr Singularitäer, die von der Wahl der Anfangswertı abhängen. Wir kömmen uns auch sofort

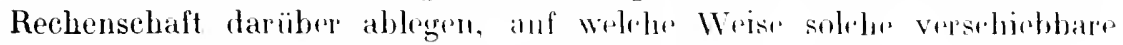
Singularitäten zu Tage treten kömmen.

Wenn sich boi der analytisehen Fortsetzung der Reilun

$$
y_{1} \cdot y_{2}, \ldots y_{m}
$$

für einen Wert $x$ ein Werty der Integlalfumktion orgibt rom der Besehatfortheit, da $B$ das Wertepaar $(\bar{x}, \eta)$ rine der Gleichungen

$$
D(\eta, \bar{x})=0 . \quad A_{0}(\eta, \bar{x}) \quad 0
$$

befriedigt, so ist die Existenz von lntegralfunktionen, die in der Ungeburng von $x=\bar{x}$ holomorph sind und in diesem Punkteden Wert y annehmen, in Frage gestellt. Es wird also in allgemeinen ein solcher Wert $\bar{x}$ für dasjenige Integral oder diejenigen Integrale, die daselbst gleich $\eta$ werden, ein singulärer Punkt sein. Wir werden zunächst das Verhalten der Integral- 
funktion in der Congebung solcher $x$-Worte 70 ergrïnd"u suchen und dabei unser Augenmerk bersonders aul den Fall richter, wo rin derartiger Punkt $x$ ein Verzweigungspunkt rines Intrgrals sein kann. Wenu wir scharf die Bedingungen aufstellen. unter denen rin sokhre Fall eintreten kann. und dann dir Korffizienten der Diffrentialgleichung (1) so rinschränken. dabi diese Bedingungen nirmals orfült simd, so werden wir dirjenge Form der Differentialglrichung (1) anzugromen instande soin, die notwendig und hinreichend dafïr ist. daldi die Integrale keine mit den Anfangswerten verschiebbaren Verzwigungspunkt besitzen. Wir machen die Ermittelung dieser Form der Differentialgleichung zu unserer ersten Aufgabe und werden demgenäb in der folgenden Untrorsuchung. die in allem wesentlichen nach der von Furhs (1884) angegebenen Methode geführt werden soll. immer von Falt zu Fall, gleich den sich rgebenden Beitrag zur Lösung dieser Aufgabe festhalten.

Was dir Untersuchung der Integrale in dre C'mgebung dre festen singulären Stellen atulangt. so hängen dir dabei anzuwendenden Methoden naturgemäßs von der Art und Weise ab. wio dir unahlängign Veränderliele $x$ in dir kiveffizienten

$$
A_{0}(y, x) \ldots \ldots+(y, x)
$$

der Differentialgleichung eingeht.

\section{Untersuchung des Falles, wo der Koeffizient der höchsten Potenz der Ableitumg verschwindet.}

Wir beginmen mit der Untersuchung des Verhaltens der Integralfunktion in der Limgebung einer Steller. wo das hutegral einen Wert $\eta$ anninmot, fïr ten

ist.

$$
1_{0}(1 /, r) \quad 11
$$

1)ir. Gleichung

$$
1_{10}(y, a)=-0
$$

definiert $y$ als Funktion von $x$. Wir betrachten einen Zweig

$$
y=\eta_{i}(r)
$$

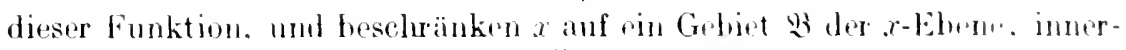
hall dessenl $y(x)$ holomorph ist. C̈berdies setzen wir voraus. dal.s die? Funktion $/(x)$ nicht anch der Diskriminantengleichung Grentur leistet: es soll also firr rin willkïrliches, innerhath

$$
I)(1,(x), x)>0
$$

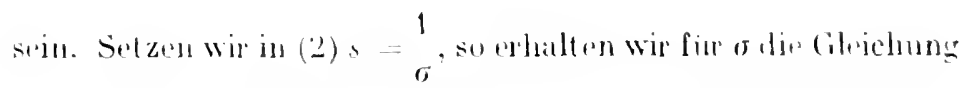

$$
1_{1}(y, x)+._{1}(y, x) \sigma+\cdots+1_{n}(y, x) \sigma^{3}=0 .
$$




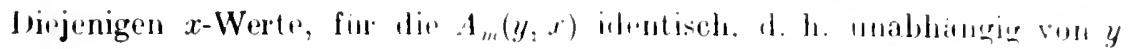

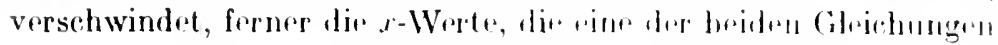

$$
\left.L_{\ldots \prime}(l(x), x)=0, l\right)(t,(x), x)=0
$$

befriedigen, schließen wil ans mud zälıl.n sir zu den festen singularen

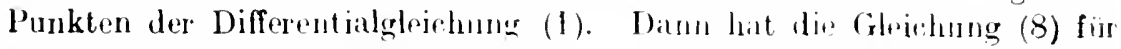
$y=\eta(x)$ die einfache IVurzel $\sigma=0$ und wirl, wenn $y-\eta(x)$ hinreichend klein ist, durch rime Reihe von der Form

$$
\sigma=p_{0} \cdot(y-\eta)+p_{1} \cdot(y-y)^{2}+\cdots
$$

befriedigt, wo die $\varphi_{0}, q_{1}, \ldots$ in der Umgebung allor ni.lit ausgeschlossen.n $x$-Punkte von $\mathfrak{B}$ holonorph sind. Einzelne der $\varphi_{0}, \varphi_{1} \ldots$ können ahor identisch, d. h. fïr jeden Wert von r, versehwimlen; es seien intwa

jedoch

$$
\varphi_{0}=0 . \ldots \varphi_{n-2}=0 \text {. }
$$

$$
4 n+10 \quad(n=1) \text {. }
$$

Dann haben wir also entsprechend der Lösung (9) von (8) eint Lösung $s$ von (2), die für kleine Werte von $|y-\eta|$ in der Form

$$
s=\phi_{n-1}^{1}(y-\eta)^{-n}: 1+\delta_{1}(y-\eta)+\partial_{2}(y-\eta)^{2}+\cdots ;
$$

darstellbar ist, wo anch $\delta_{1}, \delta_{2} \ldots$ in der Umgubung der nicht dusgreschlossenen Punkte von $\mathfrak{B}$ holomorph sind. Wenn aurh $\psi_{n}, 1$ nicht identisch gleich Null ist, so kann diese Funktion gleichwohl für speziellı, innerhalb $\mathfrak{B}$ gelegene $x$-Werte verschwinden; siml solche $x$-VVerte vorhanden, so schließen wir sie aus und zählen sie zu don festen Singularitäten. Es sei $x=c$ ein innerhalb $\mathfrak{B}$ gelegener Punkt. der nicht zu den ausgeschlossenen gehört, so ist also

$$
4 \cdot-1(c)+0
$$

und indem wir $\frac{d y}{d x}$ an die Stelle ron $s$ setzen. hahen wir die Differentialgleichung für $y$

$$
\frac{d y}{d x}=\frac{1}{\varphi_{n-1}^{-}}(y-\eta)^{-n}\left(1+\delta_{1}(y-\eta)+\delta_{2}(y-\eta)^{2}+\cdots ;\right.
$$

Führen wir hierin an Stelle von $y$ die Differenz $y-\eta$ als abhängige Variable ein, so kommt

$$
\begin{gathered}
d(y-\eta)=-\frac{d \eta}{d x} \\
d x \quad=\frac{1}{\varphi_{n-1}}(y-\eta)^{-n}\left\{1+\partial_{1}(y-\eta)+\partial_{2}(y-\eta)^{2}+\cdots ; .\right.
\end{gathered}
$$

oder wenn wir ausmultiplizieren und

setzen:

$$
y-\eta=u
$$

$$
\frac{d u}{d x}=-\frac{d \eta}{d x}+{ }_{\varphi_{n-1}}^{u-n}+\psi_{1} u-+^{-1}+\cdots-\psi_{n}-\chi_{-1} u-\cdots
$$




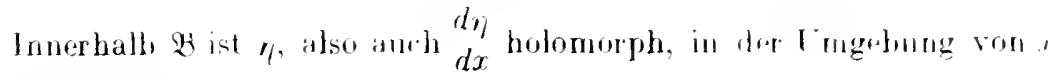
sind aush

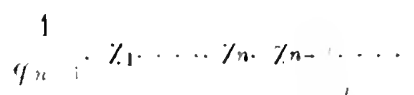

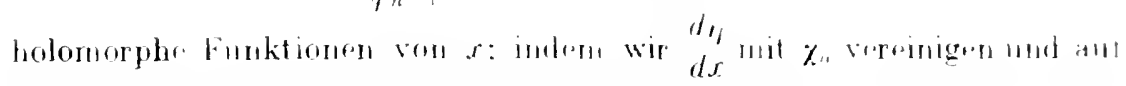

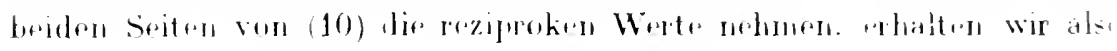

$$
\begin{gathered}
d r \\
d u
\end{gathered}=4 n-1 \|^{\prime \prime} 1 . \varepsilon_{1} u \cdots \varepsilon_{2} u^{2} \ldots \ldots
$$

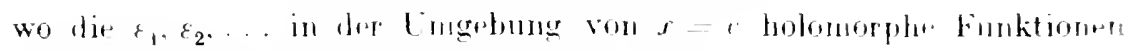
von $x$ bedenten. Wir fimden demanch endlich

$$
d x=u^{n}: i_{0}(x) \quad \cdots i_{1}(x) u \quad-\cdots ; \quad i_{10}(e)-q_{n} i(r): n \text {. }
$$

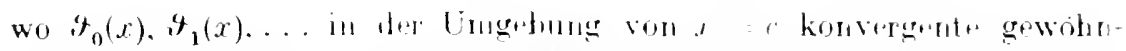

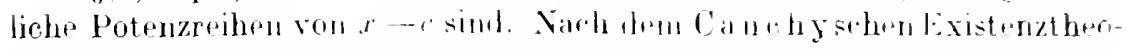
reme der Nr. 9 besitzt difse Diflerentialgledelung ein und mor rin in i.. Urugebung von $u=0$ holomorphes lntegral $x$. das fir $u$ o den 15 in anninumt. Hud fïr das wegend des liaktors $u^{2}$

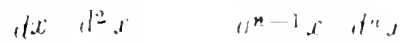

$$
\begin{aligned}
& \| u^{\circ} d / u^{2} \cdots \cdot d u^{n}: d u
\end{aligned}
$$

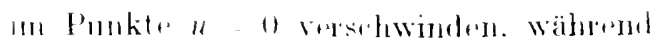

$$
\begin{aligned}
& d n+1 x \\
& d u^{n-1}
\end{aligned}
$$

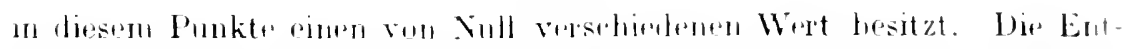

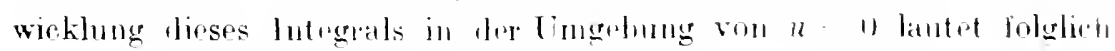

$$
x-r=y_{n+1} u^{n+1} \quad \gamma_{n+2} u^{n+2} \cdots y_{n+1} \quad 0 .
$$

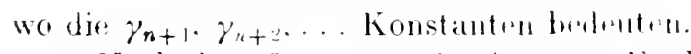

Nach dem Satz von der invesen Fumktion folgt himans

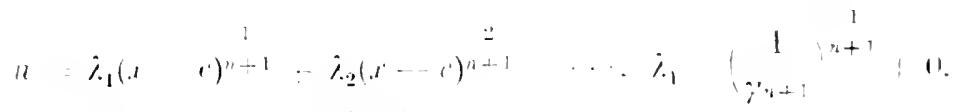

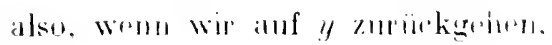

$$
y=y(x)=\lambda_{1}(x-c)^{n+1}+\lambda_{2}(x-c)^{n+1}
$$

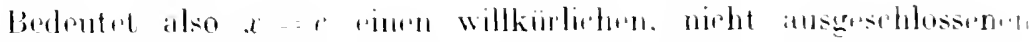

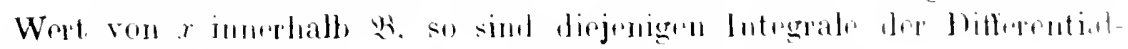

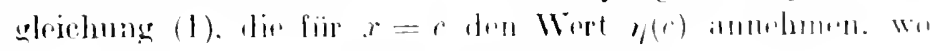

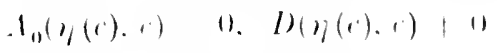

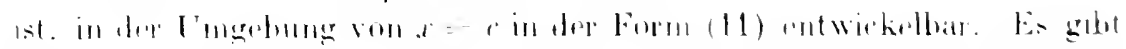

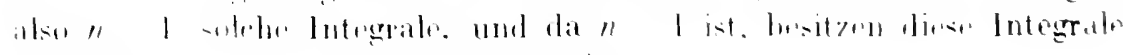

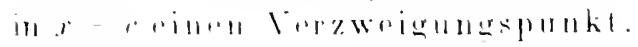




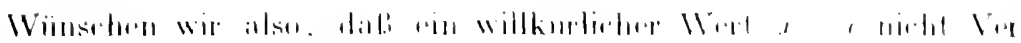

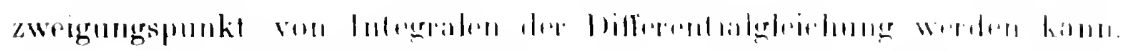

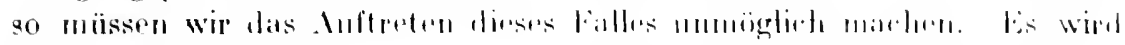

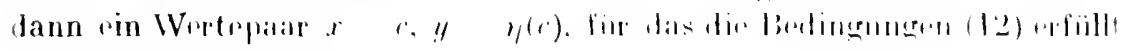
sind, wicht gehwn dirftrll. Al. h.

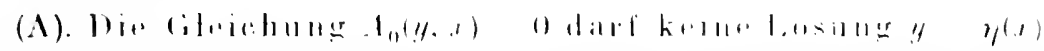

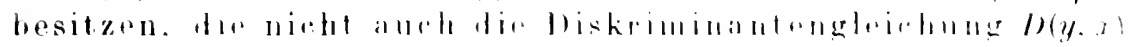
- 0 berrinligt.

\section{2(1. Untersuchnng des Falles, wo die Diskriminante rerschwindet. Vorluereitendes.}

Wir wenteu uns dem Fallo zu, wo fiir rimen Wirt a die Intrgralfunktion rinen Wert $y$ annimmt. der mil $x$ zosammengenommen der Gleichung

genïgt.

$$
10(\pi, x)=0
$$

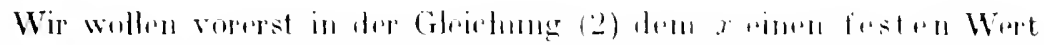

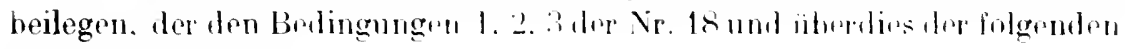
Bedingung geniigt:

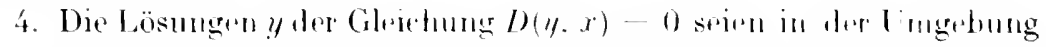

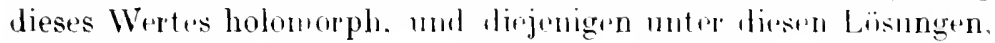
die fïr rin willkürliehes $r$ von riflamler rerselibelen sind. mögen auch für den betrachtoten festen $i$-WVert versehiedene Wirte besitzen.

Die Tatsache, daf a diesen lesten Wirl bedented. hringen war dadurch zum Ausidruck, dab wir bei F. D. A, und " Hell Burbstaben $x$ weglassen.

Wenn wir für $y$ dic Lösung y der Gleichung $D(y)=0$ pinsetzen, so besitzt die Gleichung $F(\therefore, \eta)=0$ mindestens rine mehrfache Wurzel ... Es sei $s=5$ eine solehr. etwa $\lambda$-fach. Wurzel, so dab also fïr $y=\eta_{1}, x=$ auch noch

$$
\begin{aligned}
& F \quad \partial^{2} F \quad \ldots O^{k-1} F
\end{aligned}
$$

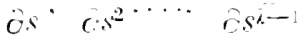

verschwinden. Von den $m$ in allgrmeinen voneinander verschientemen Funktionszweigen $s_{1}, \ldots s_{m}$, div dir Gleichung $F(s, y)$. O hefriedigen. nehmen $\lambda$ für $y=\eta$ den gemeinsamen Wert - all: "s seien dits die Tweige $s_{1}, s_{2}, \ldots . s_{\mu}$. Diese sondern sich in $Z_{y}$ keln ron der Art, dali die zu einem Zyklus gehörigen Zweige sich bei Lmläufen von y um dril Punkt , 
zyklisch permutiercn '). Wenn $s_{1}, s_{2}, \ldots, s_{a}$ einen a-gliedrigen Zyklus bilden $(\alpha \quad \lambda)$, so besteht für dirse a \%weige für hinreichend kleine Werte von $\mid y-\eta$, eine gemeinsame Darstellung von der Form

(13) $s_{v}=\zeta+s_{1}(y-\eta)^{\frac{1}{x}}+g_{2}(y-\eta)^{\frac{2}{x}} \ldots \ldots$

$(1,2, \ldots, n)$

in dem Sinne, dal. für lie verschiedenen $s_{1}, s_{2} \ldots, s_{u}$ die a verschiedenen Werte von $(y-\eta)^{\frac{1}{x}}$, also

$$
e^{2 g \pi i}(y-\eta)^{\frac{1}{a}} \quad(g 0,1,2, \ldots, 1,-1
$$

zu nehmen sind. Der Punkt $y=\eta$ ist, wenn $\alpha>1$ ist, ein Verzweigungs punkt $(\alpha-1)$-ter Ordnung für die den Zyklus bildenden Funktionszweige $s_{1}, \ldots, s^{\alpha}$. Ist die dem $!=\eta$ entsprechende mehrfache Wurzel der Gleichung $F(s, \eta)=0$ unendlich großu, $\zeta=\infty$. so hat man $s=\frac{1}{\sigma}$ zu setzen und erhält entsprechend der Darstellung

$$
\sigma_{1}=g_{1}(y-y)^{\frac{1}{\alpha}}+g_{2}(y-y)^{\ddot{\alpha}}+\cdots
$$

der fiir $y=\eta$ rerschwindenden Zweige der transformierten Creishung

$$
\sigma^{n} F\left(\frac{1}{\sigma}, y\right)=0
$$

eine Darstellung der $s_{\nu}$, die nach positiven und negativen Potenzen von $(y-\eta)^{\frac{1}{a}}$ fortschreitet, negative Potenzen aber nur in endlicher Anzabl enthält. Wenn in (14) z. B. $g_{1}, g_{2}, \ldots, g_{k}$, gleirh Null sinfl, aber $g_{k}$ von Null verschiedon ist, so hat man

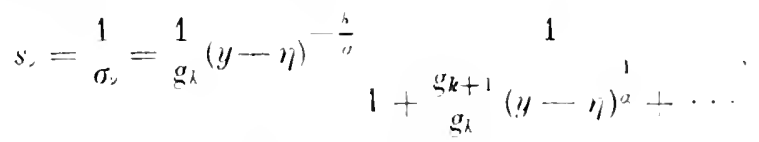

also für hineichend kleine Werte von $y-$-"

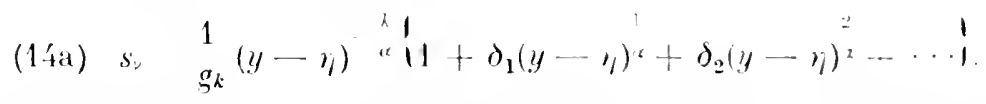

Vir denken uns nun $x$ wieder veränderlich nit der Einschränkung. dafs für alle Werte. die $x$ annehmen kann, jedenfalls die Bodingungen 1. bis 4. erfüllt sind. Die mehrfache Lösung $s$ der Gileiehung

$$
F(s, \eta(x), x)=0
$$

wirl dann auch eine Funktion $-(x)$ von $x$ sein. Wir besehränken nun $\boldsymbol{x}$

1) Die Yerteilung in Zykelu und die Bestimmung der Ordmungszahlen det

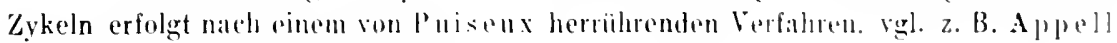
und Goursat. al. al 11. S. listif. 
auf ein Gebint R, innerhalb dessen noben don berdingungen 1. his 4. anch noch die Bedingung crfiillt ist, dal. $\zeta(x)$ loolomorph hl.ihl.

Machen wir daun in (2) dir Substitution

$$
\begin{aligned}
& y-\eta(x)=\boldsymbol{\eta}, \\
& s-\zeta(x)=;,
\end{aligned}
$$

so verwandelt sich die Gleichung (2) in

$$
\tilde{J}(j, n, r)=0
$$

ihre Diskriminante bezcichnen wir mit $D_{1}($ l) $x)$. Solangs $x$ auf den Bereich $\mathfrak{B}$ beschränkt ist, bleibt (zufolge der Bedingung 4.) die Differenz zwischen $\eta(x)$ und einer anderen Lösung der Diskriminantengleichung $D(y, x)=0$ dem absoluten Betrage nach oberhalb einer angebbaren Schranke. Man kanu also in der v-Ebene un $v=0$ einen Kreis $h$ mit, von Null verschiedenem Halbmesser so beschreiben, daß solange $x$ in $\mathfrak{B}$ und $\mathfrak{y}$ in $K$ bleibt, $D_{1}(\mathfrak{y}, x)$ nur im Mittelpunkte von $K$, dort aber für jeden Wert von $x$ verschwindet. Wir haben dann für die dem Zyklus $s_{1}, \ldots, s_{\alpha}$ entsprechenden Lösungen $z_{1}, \ldots . \delta_{\alpha}$ der Gleichung (15) die der Entwicklung (13) entsprechende Entwicklung

$$
\lambda_{\nu}=g_{1} u^{\prime}+g_{2} \eta^{\prime \prime}+\cdots,
$$

wo die $g_{1}, g_{2}, \ldots$ jetzt monogene Funktionen von $x$ sind. Diese Funktionen hahen für jeden Punkt des Bereichs $\mathfrak{B}$ eindeutig bestimmte, endliche Werte, die sich mit $x$ stetig ändrrn; sie sind folglich in der Umgebung jeder Stelle von $\mathfrak{B}$ holomorph. -. Dir gleichen Ergebrisse gelten in sinngemäßer Abäuderung, wenn $\zeta(x)=\infty$ ist.

\section{Der Fall, wo die Diskriminante verschwindet, der Koeffizient der höchsten Potenz der Ableitung aber nicht.}

Wir machen nun ausdrücklich die Annahme, daß der die Diskriminantengleichung $D(y, x)=0$ befriedigende Funktionszweig $y=\eta(x)$ nicht auch die Gleichung $A_{0}(y, x)=0$ befriedigt. Es soll also innerhalb R $A_{0}(\eta(x), x)$ nicht identisch verschwinden; spezielle $x$-IVerte, für die

$$
\mathcal{A}_{0}(\eta(x), x)=0
$$

ist, schließen wir aus und zählen sie zu den festen Singularitäten. Die mehrfache Lösung $s=\zeta(x)$ der Gleichung $F(s, \eta(x), x)=0$ ist dann sicher endlich und innerhalh $\mathfrak{B}$ holomorph. In der entsprechenden Entwicklung

$$
s=\zeta(x)+g_{1}(y-\eta)^{\frac{1}{\alpha}}+g_{2}(y-\eta)^{\frac{2}{u}}+\cdots,
$$

die für hinreichend kleine Werte von $|y-\eta|$ gilt, sind dann die $g_{1}, g_{2} . \ldots$ 
holonorphe Funktimen ron $x$, von denen aber einzelne noch identiseth verschwinden können. Es sei $g_{1}, g_{2}, \ldots, g_{k-1}$ gleich Null, aber $g_{k} \neq 0$ : solite $g_{k}$ für spezielle $x$-lWerte verschwinden. so schlieben wir liese aus und zählen sie zu den festen Singularitäten. Es ist dann

$$
(13 i 1) \quad s=-(x)-g_{k}(y-\eta)^{\frac{k}{a}}+g_{k+1}(y-\eta)^{2-1}-\cdots \text {; }
$$

setzen wir hirrin $d y$ an die Stelle von $s$ so haben wir für $y$ die Differentistgreichung

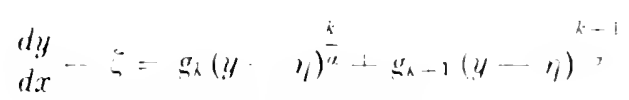

oder fuir $y--\eta$ die Differentialglejchung:

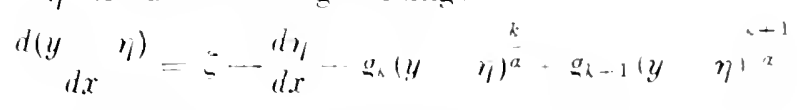

Es ist mu möglieh. dati die Lösung $y=\eta(x)$ der Diskiminantongleichung auch der Differentialgleichming (1)

$$
F\left(\begin{array}{l}
d y, y, x) \quad 0 \\
d x, y, x)
\end{array}\right.
$$

Cienüge leistet. Dann muB also $s=\frac{d \eta}{d x}$ eine Losung der Gleichung

$$
F(s, \eta(x), x)==0
$$

sein. Es könnte sich ferner erejgnen. dab $\frac{d \eta}{d x}$ geradr we mehrfir h. Lösung dieser Gleichung. eventuell sogar die von uns betrachtete meh. fache Lösung $\zeta(x)$ ist. Diesen Fall behandeln wir nachher.

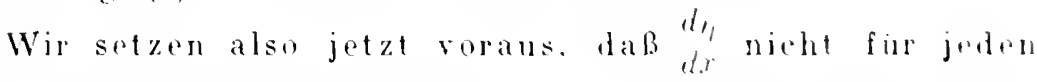
Wert von $x$ mit $:(x)$ iblereinstimnt. Sollte fur speziellr r-Mint

$$
d y-(x)-0
$$

sein, so schliefen wir diese $r$-Worte ans.

Wir setzen

$$
\text { ly } 11 n^{2} \text {, }
$$

dann genügt nach (17) 1 der Differentialgkeichung

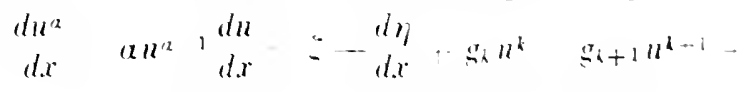

woraus fiir : $x$ als Funktion von $a$ die Mifferent ialglechelung

$$
d x=\zeta \frac{d \eta}{d y+a_{k} u^{k}+g_{k+1} u^{k+1} \ldots .}
$$

folgt. 


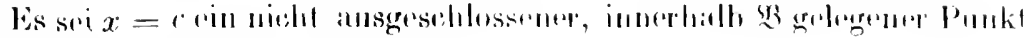
der $x$-Elente, damn isl fuir $x$,

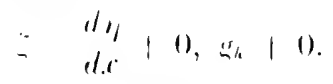

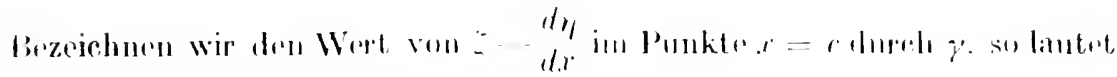

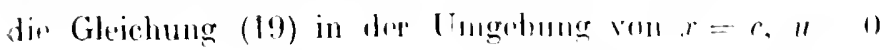

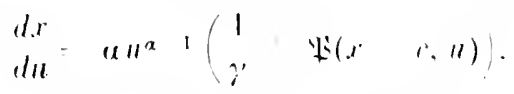

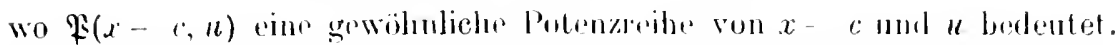
Nach dem Existenzthenreme von "a uc hy (Nr.9) besit zt dirse Differentialglachung ein und nur ein in der Umgrbung von $u=0$ holomorphes Integral $x$, das für $u=0$ den Wert $r=c$ annimmut, und für dieses Integral ist in Punkte $u=0$

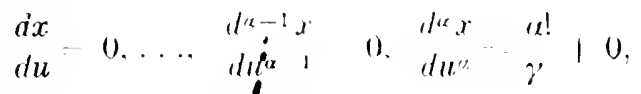

soine Entwicklung in ler Unghthug von $11=0$ lautet folglich

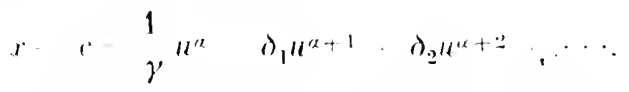

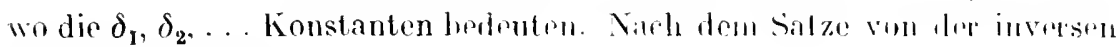
Funktion folgt hicraus

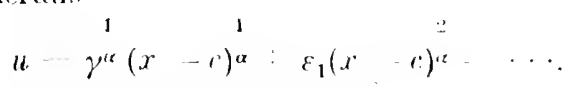

und hiernach ist in der Jmgehung von $r=c$

$$
\begin{aligned}
y-\eta= & \mu^{a}=\gamma(x-c) \quad \mu_{1}(x-c)^{1+\frac{1}{\alpha}} \\
& +\mu_{2}(r-c)^{1+2}+\cdots
\end{aligned}
$$

Bedeutet also $x=c$ einen belirbigen nicht ausgeschlossenen Wort von $x$. so sind diejenigen Integrale der Differentialgleichung (1), die für $x=$ ? den Wert $\eta(c)$ und deren Ableitungen für $x=c$ den Wert $\zeta(c)$ annehmen, $"$.

$$
D(\eta(c), c)=0, A_{0}(\eta(c) . c)+0,\left(\begin{array}{l}
d \eta \\
d x
\end{array}-\zeta(x)\right)_{x \ldots} \mid 0
$$

ist, in der Umgebung von $x=c$ in der Form (20) entwickolbar. Es gibt stets $\alpha$ solche Integrale, nnd wrm $\alpha>1$ ist, haben diese Integrale in $x=$, einen Verzweigungspunkt.

Wenn wir wïnschen, daf der willkürliche Punkt $x=c$ nicht ak Verzweigungspunkt gewisser Integrale von (1) soll fungieren können. so muß dieser Fall unmöglich sein. d. h.

(B) $)_{1}$ Wenn $\eta(x)$ eine Lösung der Diskriminantengteichung ist, die die Gleichung

$$
A_{0}(y, x)=0
$$


nicht befriedigt. wnel einer mehrfachen Wurzol $(x)$ der Gleichung

$$
F(s, \eta(x), x)=0
$$

ein Zweig, der dureh dir Gleichung

$$
F(s, y, x)=0
$$

definierten algebraischen Funktion $s$ von $y$ entspricht. der sich für $y=\eta$ verzweigt $(\alpha>1)$. so mub $\zeta(x)$ mit der Ableitung von $\eta(x)$ übereinstimmen: $\eta(x)$ mul. also jedenfalls ein lntegral der bifferentialgleichung (1) sein.

Nun könnte zu $y=\eta(x)$ noch eine von $\zeta(x)$ verschiedene mehrfache Wurzel $\vartheta(x)$ der Gleichung $(\alpha)$ gehöron, der solch. Zweige der durch $(\beta)$ definierten algebraischen Funktion $s$ von $y$ entsprechen. die sich für $y=\eta(x)$ verzweigen. Dann wäre aber jedenfalls

$$
t(x)+\frac{d y}{d x}
$$

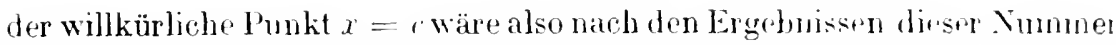
ein Verzweigungspunkt für diejenigen Integrale von (1), dir in $x=c$ den Wert $\eta(c)$ und deren Ableitungen in $r=c$ den Wert $\forall(c)$ annehmen. Um diese Möglichkeit auszuschließen. haben wir, wenn vrschiebbare Vrrzweigungspunkte nicht auftreten sollen, als weiter" Bedingung hinzuzıf ügen :

$(\mathrm{B})_{2}$. Es $\mathrm{muB}$

$$
=d_{1}
$$

die einzige mehrfache lösung der Gleichung (a) sein. der solehr Zweige. der duroh $(\beta)$ definireten algebraisehen Funktions von y entsprechen. die sich fiir $y=$ " verzweigen.

Ehe wir witer gehen, deuten wir den in dieser Xumumer abgehandelten fall noch geometrisch.

Nach (20) ist für die a Integralr. die in $t=c$ den Wirt $r_{j}(c)$ annehmen.

$$
\left|\begin{array}{c}
d(y-y) \\
d x
\end{array}\right|_{x}=\because\left|\begin{array}{l}
d y \\
d x
\end{array}\right|_{x} \quad y-\frac{d y}{d x} .
$$

also nach der Definition ron $\gamma$

$$
\begin{aligned}
& d y \quad-(1) \\
& d . r=1
\end{aligned}
$$

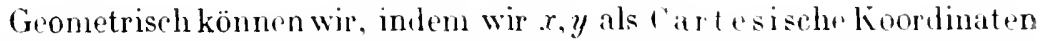
ansehen, jede Integralfunktion durch eine Kurro (Integralkurve) repräsentieren. Die durch (20) in der Ungebung von $x=c$ dargestellten Integrale werden also a kurven geben, die einander in Punkte $x=c, y=\eta(c)$ berühren, da für sie in diesem P'unkto $\frac{d y}{d x}$ den gemeinsamen Wert $\zeta(c)$ 


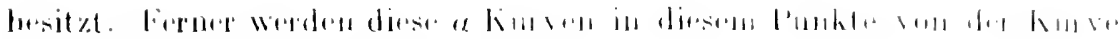

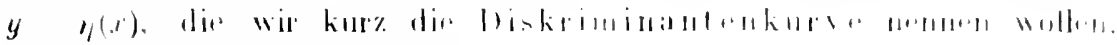

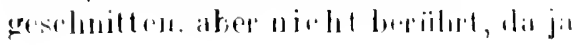

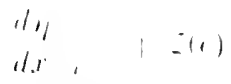

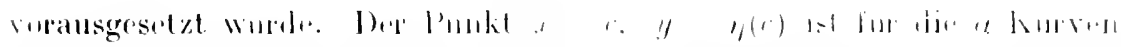

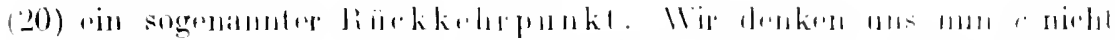

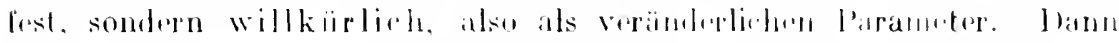

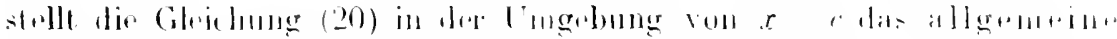

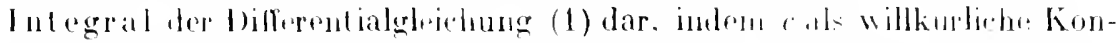

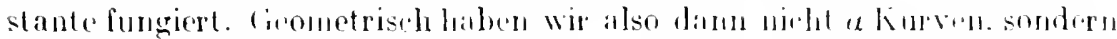

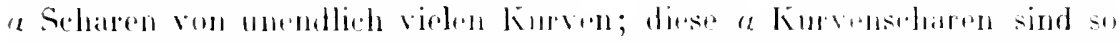

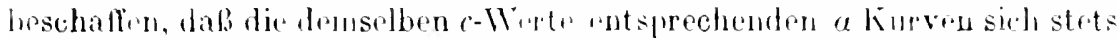

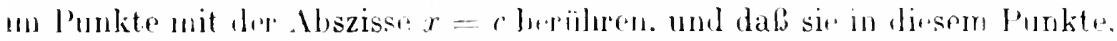

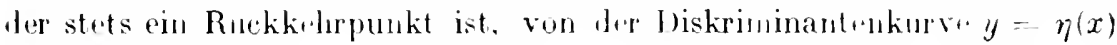
geschnitten werden. Es is also $y=\eta(i)$ der grommlatish. Ore

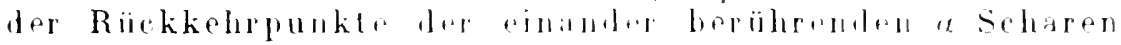
vou lntegralkurin').

\section{2.). Intersuchme der singulairen Integrale.}

Hi. Lösung

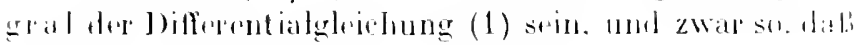

$$
\begin{array}{ll}
d,(x) \\
4 l & -(x)
\end{array}
$$

tine mohrlache Lösung der Cileitehumer

$$
F(s, 1,(x), y)=0
$$

1st. Das Integral $f(x)$ hefriedigt dann die beiden Gleichungen

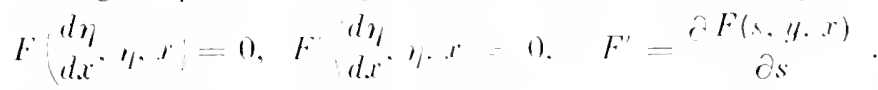

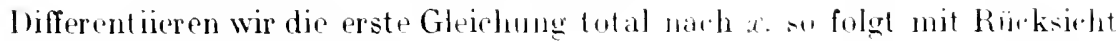
duf die zweite. daf aucls

$$
\begin{array}{ll}
a d, \quad r F \\
\because r d r
\end{array}=0
$$

sein mub. Danit also dic Ditlerentialghleichung (1) ein Integral ron der fiir $\eta(x)$ angegebenen Beschatfrnheit hesitzt. ist notwendig, dab dif drei Gloichungen

(22) $\quad F(s, y, x)=0 . \quad F^{\prime \prime}(s, y, x)=0, \frac{\partial F}{\partial y} s+\frac{\partial}{\partial x}=0$ S. 158.

1) Vergl. (i. Harboux. Bullelin des sciences Mathématiques. T. 411873. 
gleichzeitig befriedigt weruen. indem man fïr $s . y$ gewiss. Funktionen von $x$ sutzt.

Wenn man die Diffrrentialgleichung (1) beliebig wählt und die drei Gleichungen (22) ansetzt, so werden sich als gemeinsame Lösungen dieser Gleichungen iun allgemeinen gewisse diskrete Wertetripel $(s, y, x)$ rrgeben. Eine Differentialgleichung (1) besitzt also ..iu allgemeinen" kuin Integral von der für $\eta(x)$ gefordrorten Beschaffenheit: die Existenz eines solchen bedingt das Bestehen von gewissen Beziehungen zwischen den Koeffizienten der Differentialgleichung.

Unter Voraussetzung des Bestehens der Gleichung (21) lautet (17) (S. 78) wie folgt:

$$
\text { (17a) } \frac{d(y-\eta)}{d x}=g_{k}(y-\eta)^{\frac{h}{k}}+g_{k+1}(y-\eta)^{k+1}-\cdots \cdot
$$

wenn wir also wioder

setzen, so ist

$$
y-y=u^{\alpha}
$$

$$
\begin{aligned}
& a u^{u-1} \frac{d u}{d x}=g_{k} u^{k}+g_{k}+1 u^{k+1}+\cdots \\
& d x \\
& d u=g_{k}+g_{k+1} u+g_{k}+2 u^{2}-\ldots
\end{aligned}
$$

Bedeutet $c$ einen willkürlichen, nicht ausgeschlossenen $t$-Wert innerhalb $\mathfrak{B}$, so ist $g_{k}$ für $x=c$ von Null verschieden. die Differentialgleichung (23) hat also in ler Ungebung von $u=0 . x=r$ div Form

$$
d x=u^{x-1-k}+2(x \cdots c u) \text {. }
$$

wo $\mathfrak{P}(x-c, u)$ eine gewölnnliehe Potenzruhe ron $x-c$ und $u$ brdentet. Wir haben jotzt die beilen Fäll+

$$
\text { 1) } a-1-k=0 \text {. }
$$

zu unterseheiden und gesundert zu behandeln.

Im Falle I) haben wir. da $k$ eine positive ganze Zahl ist. a $-1=k$, also

$$
k<a .
$$

Die rrehte Seite der Diffrentialglechung (2) ist in der Lmgebung von $u=0, \quad x=-c$ holomorph. nach dem Existenztherem gibt is demnach win und nur ein iu der Umerehung von $u=0$ holomorphes lntegral $x$. das für $u=0$ den Wert $c$ annimmt, und für dirses lntogral ist die ( $a-k)$-te Ableitung die erste, die für $u=0$ nicht versehwindet; "s lautet also:

$$
x-i=\gamma_{1} u^{n-i}+\gamma u^{i} i+i+\cdots . \quad(\because 1+0) \text {. }
$$


Nach dem Satze von der inversen Funktion folgt hieraus

$$
\begin{aligned}
& \left.u=\delta_{1}(x-c)^{2}{ }^{1}+\delta_{2}(x-c)^{2 x-k}+\cdots . \quad()_{1}+1\right) \\
& \text { (25) } y-\eta=u^{\alpha-}-\varepsilon_{1}(x-c)^{x-k}+\varepsilon_{2}(x-c)^{\alpha+k}+\cdots \quad\left(\varepsilon_{1} \mid 0\right) \text {. }
\end{aligned}
$$

In der Ungebung von $x=c$ strilt uns diese Rriher $a-k$ Integrale der Differentialgleichung (1) dar, die für $x=c$ den Wort $\eta(c)$ annehmen und daselbst einen Vorzweigungspunkt besitzen, wonn $\alpha-k>1$ ist.

Wenn wir dafür sergen wollen, daf der willkïrliche Punkt $x=$, nicht als Verzweignngspunkt gewisser Integrale soll fungieren können. so haben wir also der Differentialgcleichung die Berlingung aufzuerlegen. daß $\alpha-k=1$ sei, d. h.

$(\mathrm{C})_{1}$. Wenn in der Entwicklung (17) (S. 78 ) die Zahl $a-1-k$ nicht negativ ist, so muß sir gleich Null sein.

Wir wollen nun die Bedeutung der Integrale (25) und ihre Beziehung zu dem Integrale $y=\eta(x)$ erörtern. Es ist im Punktr $x=c$ nicht nur der Wert der $\alpha-k$ Integrale (25) gleich $\eta(c)$, sondern auch der Wert ihrer ersten Ableitungen stinmt für $x=c$ mit den Worte von $\frac{d \eta}{d x}$ überein, da ja nach (25)

$$
\left(\begin{array}{c}
d(y-y) \\
d x
\end{array}\right)_{x=c}=0
$$

ist. Betrachten wir wiederun, ähnlich wie in der vorigen Nummer, $c$ als willkürliche Konstante oder als veränderlichen Parameter, so liefert (wie dort) die Gleichung (25) die Darstellung des allgemeinen Integrals von (1) in der Umgebung von $x=c$. Geometrisch gedeutet, stellt (25) $\alpha-k$ Scharen von Integralkurven dar; die Diskriminantenkurve $y=\eta(x)$, die jetzt selbst auch eine Integralkurve ist, hat die Eigenschaft, daß sie in j ed e m ihrer Punkte $x=c, y=\eta(c)$ ron den $\alpha-k$ zusammengehörigen und einander berührenden Individuen der Scharen (25) berührt wird, sie stellt also die Enveloppe dieser $\alpha-k$ Scharen von Integralkurven dar. Der Unterschied gegenüber dem in der vorigen Nummer behandelten Falle besteht also darin, daß dort jeder Punkt $x=c, y=\eta(c)$ der Diskriminantenkurve $y=\eta(x)$ ein Rückkehrpunkt, also ein singulärer Punkt gewisser Integralkurven war, während hier die Diskriminantenkurve $y=\eta(x)$ selbst eine Integralkurve ist, die in jedem ihrer Punkte $x=c$, $y=\eta(c)$ gewisse $a-k$ Integralkurven berührt. Der Berührungspunkt ist aber im geometrischen Sinne nicht notwendig ein singulärer Punkt jener $\alpha-k$ Integralkurven. In der Tat hat man z. B. für $\boldsymbol{\alpha}=2$, für die Kurven (25) in der Ungebung von $x=c$ die Entwicklung

$$
y=\eta(x)+\varepsilon_{1}(x-c)^{2}+\varepsilon_{2}(x-c)^{3}+\cdots .
$$


Man nonnt das lntegla] $y=\eta(x)$ in diesen Fallo rin siuguläres (vol. Nr. 18, S. 70).

Die Brodrutung ninus singulärn Integrals ist also dit.

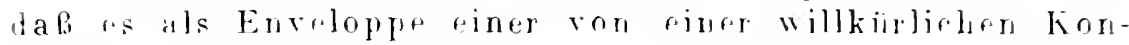
stanter abbängigen Sehar von lntegalkurvon merbeint.

Denkt man sirh diese Sehar vou Integralkurven dureh fin. Glejehungr vou der Form

$$
\phi(y, r . r)=()
$$

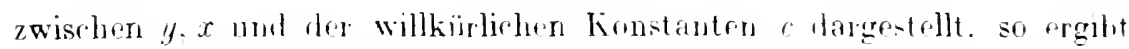
sieh, wi. aus den Elementen der Dufferentialyehnumg bekannt ist. di.

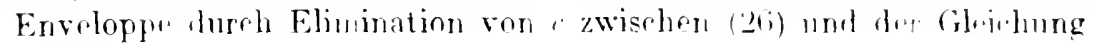

$$
\frac{q}{r \cdot r}=11
$$

Eine Gleichung vou der Forn (26) wrunt man eine allgrment Int.gralgleichnng der Differentialgleirhung (1). Gibt man dem "rinen speziellon konstanten Wert. so liefert die Glejehung (26) rin sogenanntes partikuläres Integral; las singuläre Integral. orler die Enveloppe unterscheidet sich von den partikulären Integralen dadurch. dab es in allgemeinen aus (26) nicht dureh Spezialicierung ler lionstanten c hervorgeht. In gewissen besonderen Fällen kann allerdings auch dir Envoloppe durch die Gleichung (26) fïr rinen speziellen konstanten Wert rou gegeben werden; in einem solchen Fall. sagt man. dafi ${ }_{2}(x)$ zugleich singuläres und partikulärs Integral ron (1) sei. In buzug hirauf wird dio Butrathtung des Falles il) lehreich seiu. der wir uns jotzt zuwendest.

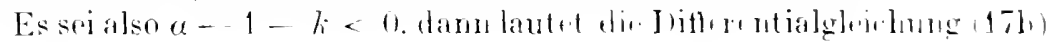

$$
d u=a_{a}^{1} u u^{k} a+1(g-\cdots u+1 u \cdots \cdots) .
$$

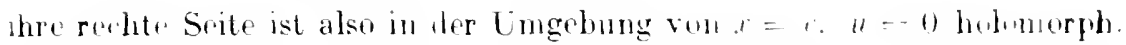
Es gibt folglich ein und nur "in in der Tmgelumg von $x=8$ hulnmorphws Integral $u$. das fiir $x=$ e versohwindel: dieses Integral ist aber offonbar $u=0$ solbst. Wiu Jifferentialghelehung (17a) hesitzl dennach als oinziges Integral $y$, das für $r=c$ den Wert $\eta(c)$ ammimnt und narh irgenelwete her Potenzen von $x-$ entwikelbar ist, uas Integral $y=y(x)$, das also hier nieht als Enveloppe einer Schar vou lutegralkurvell. d. h. nublit als singuläres, sondern als partikuläres Integral auftritt. Fs ist abrer natiorlich nicht ausgeschlossen, dab fïr rinen andern. Henfalls zur mehrfachem Wurzel $;(x)$ der Gleivhung

$$
l(s, y), x)=1)
$$

grehörigen, pi-gliceldigrn \%yklus von \%weigen des algebraischen Funktion $s$ von $y$ die Entweklung in der I nugelung ron $y=\eta$ die Form 


$$
s \quad z(x) \quad h_{0}(y \quad y)^{\prime}+h_{1}(y \quad y)^{x+1}+\cdots \cdot \quad\left(h_{1}, 11\right)
$$

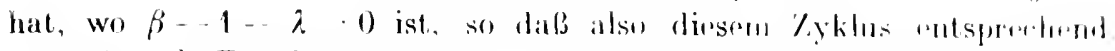

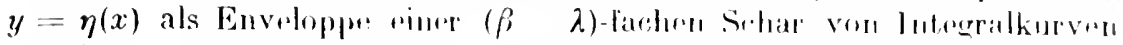

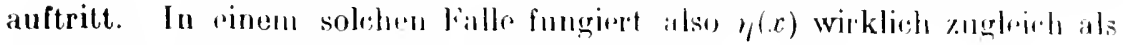
partikuläres und als singruläres Integral (vorl. olun S. 8' $)$.

Da in lem Fall, II) $l=r$ kein Vermwrigungspunkl s.in kitum, ist

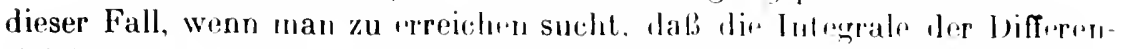
tialgleichung (1) krine vorschichbaren Verzwrignngspunkto besitzen sollon als zulässig zu betrachten; inu Satze (C) ist also hin\%n\%nfügen:

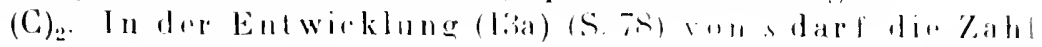
$\boldsymbol{a}-1-k$ nogativ suill,

oler imlem wir (C: $)_{1}$ mit $\left(C_{1}\right)_{2}$ voreinen:

(C). In der Entwicklung (13a) (S. Ts) nuls u - Isrin.

\section{2:3. Untersuchung der Fiille, wo der Koeffizient der höchsten Potenz der Ableitung zugleich mit der Diskriminante verschwindet. und wo das Integral selbst unendlich wird.}

Es sei nun $y=\eta(x)$ eine gemeinsane Lusung dor lwillan Gleichungen

$$
\left.A_{0}(y, x)=0, \quad D(y, x)=1\right) .
$$

dann besitzt die Gleichung

$$
F(s, \eta(x), i)=1)
$$

die mehrfache Wurzel $s=\infty$. Jiesim eutsprexhen gewisso 7ykeln vor Zweigen, der durch die Gleichnng

$$
F(s, y, x)=0
$$

definierten algebraischrn Funktions von $y$, die für $y=\eta(x)$ den gemeinsamen Wert $s=\infty$ anmehmen, also in $y=\eta(x)$ algebraisch unendlich werden. Betrachten wir einen sulchen $\alpha$-gliedrigen 7yklus. so wird dieser in der Umgebung von $y=\eta(x)$ in der Form (14a) (S. Thi), also in der Form

$$
s=\left(y-\eta_{j}\right)^{-\frac{k}{a}\left(g_{0}+g_{1}(y-\eta)^{\alpha}+g_{2}(y-\eta)^{2}+\cdots\right)}
$$

dargestellt. wo $k$ eine positive ganze Zahl bedeutut. die wir uns so gewählt

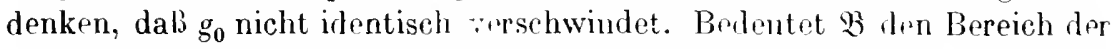
$x$-Ebene, innerhalb dessen die Funktion $\eta(x)$ holomorph ist, so sinul dif $g_{0}, g_{1}, \ldots$ ebenfalls innerhalb \& holomorphe Funktionen von $x$. Diejenigen $x$-Werte, für die gogleich Null wird. denken wir uns ausgeschlossen.

Setzen wir $\frac{d y}{d x}$ an die Stelle vous. so folgt ans $(27)$ die Differentialgleichung: 


$$
\frac{d y}{d x}=(y-1)^{-k}{ }^{k}\left(g_{0}+g_{1}(y-\eta)^{\prime \prime}-\cdots\right) .
$$

und wenn wir wiederum

einführen:

$$
y-\eta=u^{2}
$$

$$
\begin{aligned}
& \alpha u^{u-1} \frac{d u}{d x}=-\frac{d \eta}{d x}+u^{-k}\left(g_{0}+g_{1} n \ldots \cdots\right) . \\
& \begin{array}{l}
d x=\alpha u^{k+1-1}-\frac{1}{d \eta} u^{k}+g_{0}+g_{1} u+\cdots \\
-\frac{d x}{d n}
\end{array}
\end{aligned}
$$

Ist nun $x=c$ ein beliebiger innerhalb $\mathfrak{B}$ gelegener Wert, der nicht zu den ausgeschlossenen gehört, für den also $g_{0}$ nicht verschwindet, so ist in der Ungebung von $x=c, u=0$

$$
d x=u \iota^{k+u-1}+(x-c, u),
$$

wo $\mathfrak{B}(x-c, u)$ eine gewöhnliche Potenzruihe von $x-c$ und $u$ bedeutet Da jedenfalls

$$
k-+a-1>0
$$

ist, so besitzt diese Differentialgleichung nach dem Existenztheorem deI Nr. 9 ein wohlbestimmtes, in der Ungebung ron $u=0$ holomorphes Integral $r$, das für $u=0$ den Wert $c$ annimmt. Da die $(k+u)$-te Ableitung dieses Integrals die erste ist, die für $u=0$ nicht verschwindet. so lautet seine Entwicklung in der Ungehung von $n=0$ :

$$
r-c=\gamma_{1} u^{k+n}+\gamma_{2} u^{k+a+1}+\cdots,
$$

also folgt nach dem Satze von der inversen Funktion

$$
u=\partial_{1}(x-c)^{k+c t-c} \partial_{2}(x-c)^{k+1}+\cdots
$$

woraus sich für $y$ die in der Ungebung von $x=c$ giiltign. Entwicklung

$$
y=\eta+\varepsilon_{1}(x-c)^{k+x}+\varepsilon_{2}(x-c)^{k+n+1}+\cdots
$$

ergibt. Wir halun also $k+a$ Integrale, die für $x=c$ den Wert $y=\eta(c)$ annehnen, mud diese Integrale besit zen, da $k+a>1$ ist. in $x-c$ jedenfalls einen Vorzweigungspunkt. In ïbrigen ist dieser Fall dem in den Nummern 20. 21 belaandelton ganz analog; da nämlich $\eta(x)$ in der Ungebung von $x=c$ holomorph ist. so ist $\frac{d \eta}{d x}$ für $x=$ " ondlieh. also vion $s=\infty$, was hier dem $\zeta(x)$ der Nr. 21 entspricht, versehieden.

Soll das Aufteten von versehiebbaren Verzweigungspunkten ansgeschlossen sein, so larf der hier diskutierte Fall nicht auftreten, d. I.: (D). Die Gleichung $A_{0}(y, x)=0$ darf keine Lösung $y={ }_{1}(x)$ 


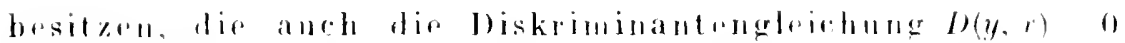
bofriodigl.

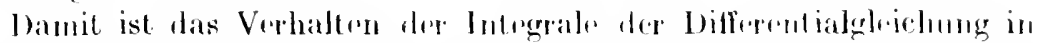

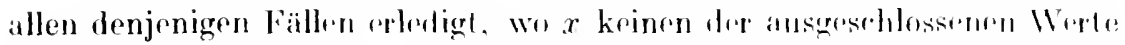

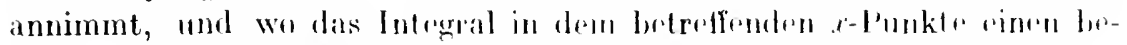

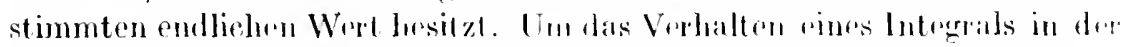

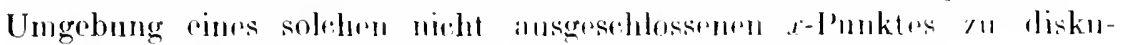

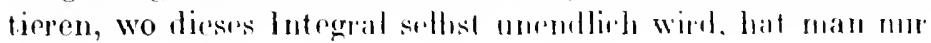

$$
\begin{aligned}
& 1 \\
& y
\end{aligned}
$$

zu sotzen wnd fïr dir. Infforentialgleichung in z dits Verhalten derjenigun Integrate zn untersuchen, dir in dem brotreflonden $x$-Punkteversehwinden. Da nach den Ergebnissen dor luen abgeschlossenen Untersuchung $z$ in der Ungebung eines jeden solchen $x$-Wortes mach ganzen oder gobrochenen positiven Potenzen des Inkrements rntwickelbar ist, so ist ein solcher $x$-Wert für ein dasellost unendlich werdrudes Integral $y$ entweder ein rinfacher Pol oder eine algebraische Uncudlielikeitsstelle, je nachdoun das in diesem Punkte verschwindende Intrgral $z$ in der Ungebung desselhen holomorph ist oder sich verzweigt.

Wenn wir für dir Integrale von (1) das Anftroten verschirbbarer Verzweigungspunkto vermoiden wollen, so werden wir also dafür sorgen müssen, daß ein willkürlichrr l'unkt $x$ (der nicht zu don ausgeschlossenen gehört) kein Verzwrigungspunkt für das in dissem P’unkto vorschwindende Integral z sei. Wir werden also die Differentialgleiehumg für z den Bedingungen (A), (B), (C), (D) zn muterwerfen halen. 1. h.:

(E). Setzt man in der Differrntialghrichung (l) für $y$ den Wert $z^{-1}$ ein, so miissen die Bedingungen (A), (B), (C). (D) auch für die sich so ergebende Differentialgleithung erfüllt sein.

Wenn wir jetzt die Gesamtheit dor Punktr i ins Auge fassen, die wir für die Differentialgleichung (1) als anszuschlielsende bezeichnet haben, und noch diejenigen Punkte hinzufügen, die für die Differentialgleichung in $z$ aus ähnlichen Gründen auszuschließen sind, wenn wir ferner noch leststellen, ob der Punkt $x=-\infty$ auszuschließen ist oder nicht, wis durch die Substitution

$$
x=\begin{aligned}
& 1 \\
& 5
\end{aligned}
$$

und Untersuchung des Punktes $\xi=0$ für die transformierte Differentialgleichung geschehen kann, so erhalten wir eine gewisse Menge von $x$-Werten, die wir als die festen singulären Punkte rler Differentialgleichung (1) bezeichnen werden. Wir wollen auch hier - wie in der Nr. 14 


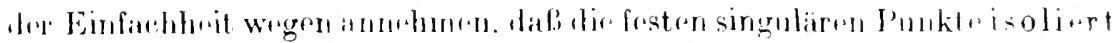

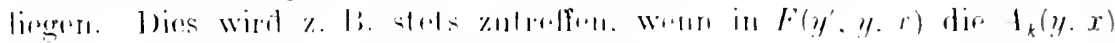

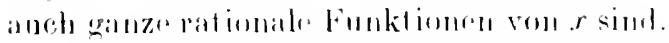

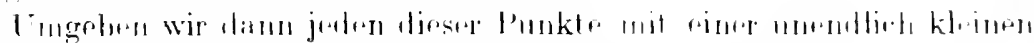

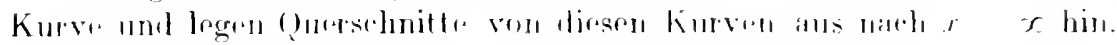

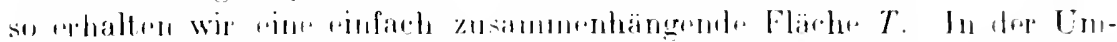

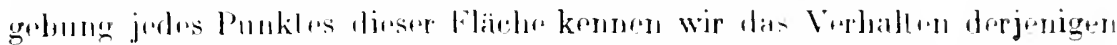

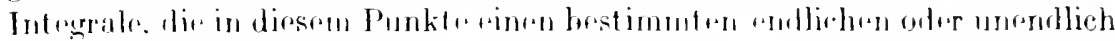

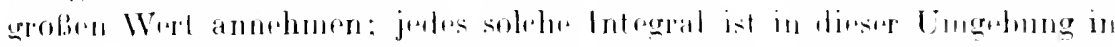

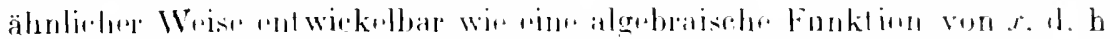

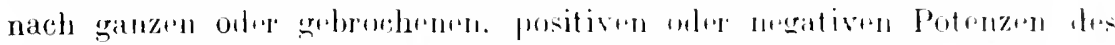

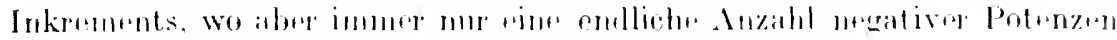

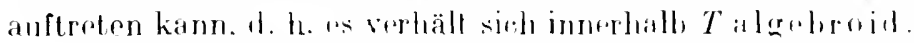

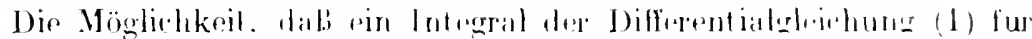

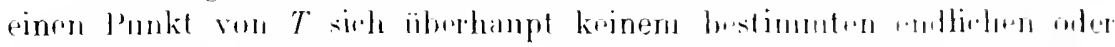

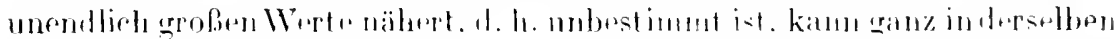

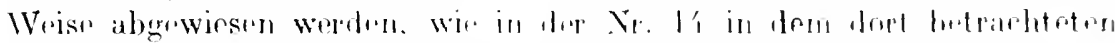

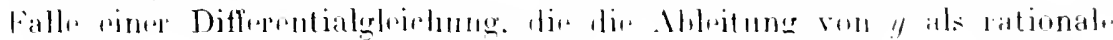
Funklion von y defininel ${ }^{1}$ ). Wau hat

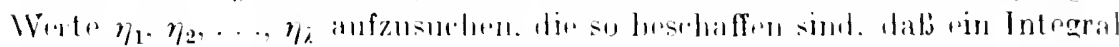

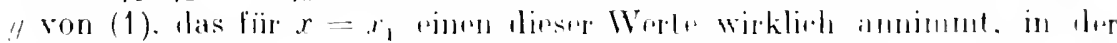

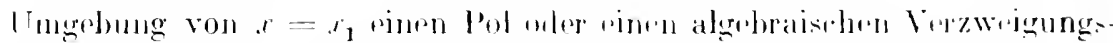

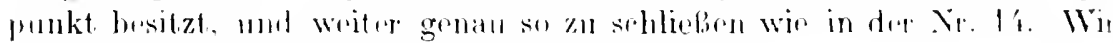
haben demnach den allermorimen siatz:

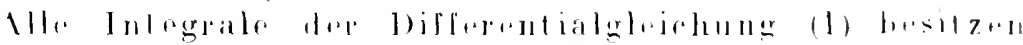

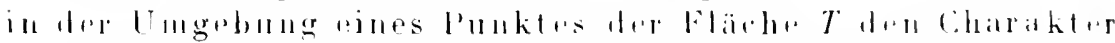

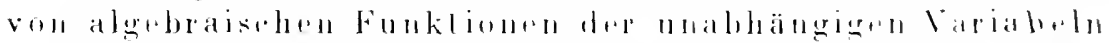

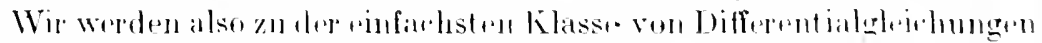

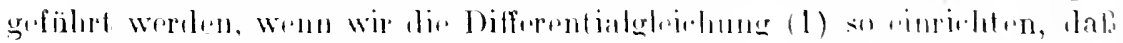

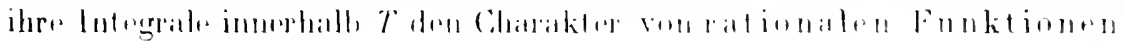

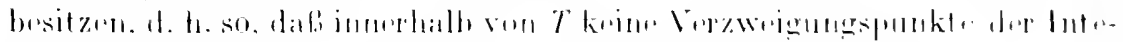

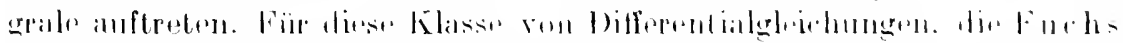

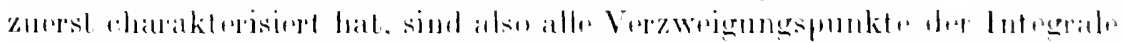

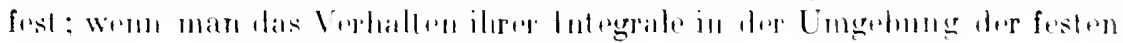

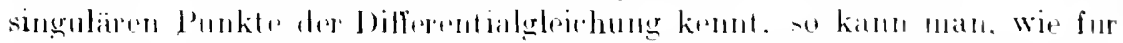

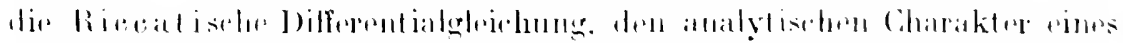

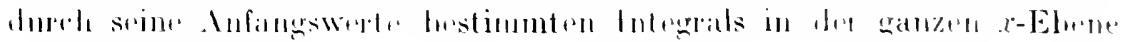

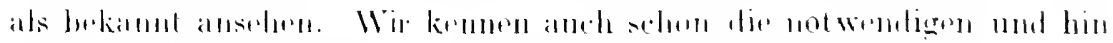

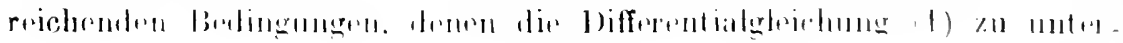

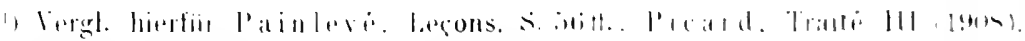

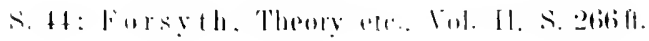




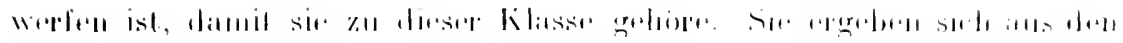

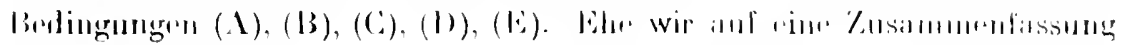

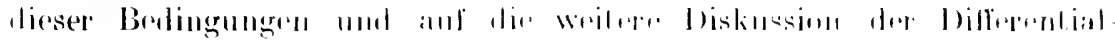

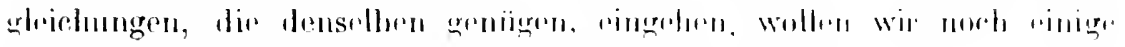

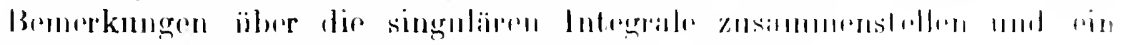

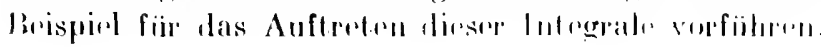

\section{4. Öber die 'Theorie der singulären Integrale.}

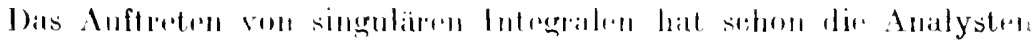

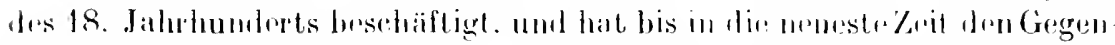

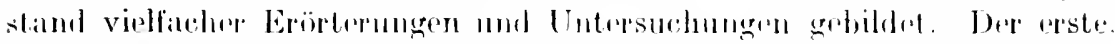
der die Existenz dieser Art von lutegralen wahreremommen late, war Clai-

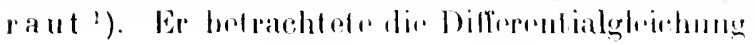

fiir dis

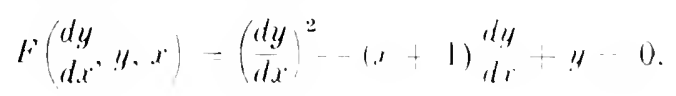

$$
\begin{aligned}
& f(s, y, x)=x^{2}-(x+1) x+y=0, \\
& F^{\prime \prime}(s .11, x)=2 x-(x+1)=0 \text {. } \\
& l)(y, x)=y-\left(\begin{array}{c}
x+1= \\
2,1
\end{array}\right.
\end{aligned}
$$

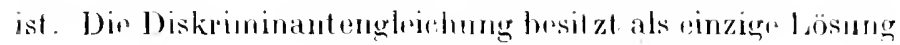

die Gloichung

$$
y \quad y(x)=\left(\begin{array}{c}
x+1 \\
2
\end{array}\right)^{2} \text {. }
$$

$$
F(s, y(x), x)=x^{2}-(x+1)+\begin{gathered}
x+1=2 \\
2
\end{gathered}=0
$$

besitzt als melufache (doppolte) Wurzel

$$
s=-(c)=\begin{array}{r}
r+1 \\
2
\end{array}
$$

es ist also

$$
-(x)=\begin{gathered}
l, f(x) \\
d x
\end{gathered}
$$

Die Entwicklung (17a) (S. 82) rergibt sich ummittelbat in der Form

$$
d(y-\eta)=(\eta-y)^{2 !}
$$

wir haben also $k=1, a=2, a-1-h=0$. 's liegt somit der Fall I)

1) A. C. Clairaut, Histoire de IAcadémie de Paris. 1734. S. $196 \mathrm{At}$. Cber hierher gehörige frühere Bemerkungen Taylors vergl. H. Cantor. Gesehichte der Mathematik. Bd. III (1901). S. 460. 
der Nr. 22 vor, d. h. $\eta(x)$ ist ein singuläres Integral. Aus (29) folgt durch Integration .

$$
x-c=-2(\eta-y)^{2},
$$

wo $c$ die willkürliche Konstante bedeutet: also haben wir (vgl. (25))

$$
y-\eta=-\frac{1}{4}(x-c)^{2}
$$

woraus sich. wenn wir $c_{1}=!(1+r)$ setzen,

$$
y=c_{1}^{2}+(x+1) c_{1}
$$

als allgeneines Integral nit der willkürlichen Konstarten $c_{1}$ ergibt. Jjese Gleichung stellt geometrisch eime Schar von geraden Linien dar. als deren Enveloppe sich in der Tat die durch das singuläre Integral

$$
y=\frac{i+1}{2}
$$

repräsentierte Parabel ergibt.

Wie man bemerkt, erhält man die allgemeine Integralgleichung (30) aus der Differentialgleichung (28). indem inan $d y$ durch die willkürlich" Konstante $c_{1}$ ersetzt.

In ähnlicher Weise wird auch die allgemeine Cileichung

$$
y=x_{d x}^{d y}+f\left(\begin{array}{l}
d y \\
d x
\end{array}\right)
$$

wo $f$ eine beliebige Funktion von $d y$ mit konstanten Kueftizimten bedeutert. integriert. Man bezeichnet diese Gleichung gewöhnlich als Clairaut sch. Differentialgleichung. Man gelangt zu ihrem allgemeinen luterralr. indenu man die Gleichung differentiiert. In der Tat ergibt sich hurh l)illm.mtiation

$$
\frac{d y}{d x}=\frac{d y}{d x}+x \frac{d^{2} y}{d x^{2}}-f^{\prime}(d y) \frac{d^{2} y}{d x^{2}}
$$

wo $f^{\prime}$ die Ableitung ron $f$ bedeuted; und hieraus folgt weiter

Die Gleichung

$$
d x^{2} y\left(x+f^{\prime}\left(\frac{d y}{d x}\right)\right)=0
$$

$$
x+f^{\prime}\left(\begin{array}{l}
d y \\
d x
\end{array}\right)=0
$$

liefert in Falle der Differentialgleichung (2S) das singulat" luteglal Die Gileichungr

$$
d x^{2}=0
$$

gibt awrimal integrient

$$
4=e_{1}+1+2 .
$$


wo $c_{1}, c_{2}$ Integrationskonstanten bedenten; setzt man diesen Wort von y in die Differentialgleichung (31) rin, so kommt

$$
r_{1} r+r_{2}-r_{1} \cdot i\left(c_{1}\right)
$$

1. h. $c_{2}=f\left(c_{1}\right)$, es ist also in der Tat

das allgemeine Integral.

$$
y \quad c_{1} x-\cdots f\left(c_{1}\right)
$$

Wie wir in der $\mathrm{Nr} .22$ benerkt haben, muß ein singuläres Integral $\eta(x)$ der Differentialgleichung (1) die Eigenschaft haben, dafs die drei Gleichungen (22) (S. 81 ) befriedigt werden, wenn man

$$
y=\eta(x), \quad s \quad \begin{array}{cc}
d \eta /(x) \\
d x
\end{array}
$$

setzt. Daraus folgt (vgl. a. a. O.), daß rine Differentialgleichung (1) im allgemeinen kein singuläres Integral besitzt. Andererseits haben wir gesehen, daß sich, wenn die allgemeine Integralgleichung (26)

$$
\Phi(y, x, c)=0
$$

bekannt ist, durch Elimination von $c$ zwischen dieser Gleichung und

$$
\frac{\partial \Phi}{\partial c}=0
$$

das singulär" Integral ergibt. Da nun dirsf Elinination in allgemeinen möglich ist, schloß L a grangel), der dieses Verfahren zur Auffindung des singulären Integrals zuerst angegeben hat, daf eine Differentialgleichung (1) im allgeneinen ein singuläres Integral besitzt. Dieser scheinbare Widerspruch galt lange Zeit hindurch als unlösbares Paradoxon. Hamburger, dem wir in bezug auf die Theorie der singulären Integrale in unseren Auseinandersetzungen gefolgt sind, hat aber gezeigt ${ }^{2}$ ), daß ein genaues Studium der allgemeinen Int egralgleichung (26) zu ebendenselben Bedingungen für die Existenz eines singulären Integrals führt, wie das Studiun der Differentialgleichung selbst. Diese zuerst von H amburger aufgestellten Bedingungen lauten nach den Ergebnissen der Nummern 21, 22 zusammengefaßt wie folgt:

Venn $y=\eta(x)$ eine Lösung der Diskriminantengleichung ist, so sind drei Fälle möglich:

1) $y=\eta(x)$ ist keine Lösung der Differentialgleichung (1); dann sind diejenigen partikulären Integrale $y$ von (1). die in dem willkürlichen Punkte $x=c$, in dessen Umgebung $\eta(x)$ holomorph ist, den Wert $\eta(c)$ annehmen, in der Umgebung von $x=c$ in der Forn

$$
y-\eta=\mathbb{P}(x-c)
$$

entwickelbar, wo $\mathfrak{P}(x-c)$ eine nach positiven ganzen oder gebrochenen

1) 1774. J. L. Lagrange, Veurres. Bd. IT, S. $1 \mathrm{ff}$ : vergl. ebenda S. $585 \mathrm{ff}$.

2) M. Hamburger. Crelles Journal, Bd. 112 (1893). S. 205 ff. 
Potenzen vou $x$-r fortschreitende. Peibe befented, in der der Exponent des Anfangsgliodes nicht größer ist als Eins.

2) $y=\eta(x)$ ist ain siuguläros Integral, d. h. Firroloppe riner Schar von Integralkurven; dann habes diese Integralkurven in der Vmgebung von $x=c$ wine Entwicklung von der Form $(\gamma)$. in dre der Exporient des Anfangsgliedes (vgl. (25)) grölser ist als Eins.

3) y $\eta(x)$ ist rin partikulïres, prenturll zugleich win singuläres Integral; dann ist fur wine Gruppe von Integralun. die in $r=r$ den Wert $\eta(c)$ annelmen, $y-\eta(x)=0$. nnd wom für diw ibrigen dieser Integrale dè Anfangsexponent der Entwicklung $(\gamma)$ nicht gröber als Eins ist, so ist $\eta(x)$ nur "in partikuläres, wonn dagegen für cinige dieser Integrale der Anfangsexponent gröber als Eins ist. so ist $\eta(x)$ zugleich partikuläres und singuläres Integral.

Auf eine Wiedergabe der an dit allgemein lntegralgleichung anschließenden Untersuchunge'n von Il a mb urger könmen wir hier nicht eingehen, da das genaue Studium dieser Integralglunchung flilfsmittel aus der Theorie der partiolten Differentialgleichungen rfordert ").

1) Vargl. datïr If orn. Differentialgleichungen. Sammlung schuber L 194:s. ᄂ. $348 \mathrm{ff}$ 
Virites \apitm.

\section{Differentialgleichungen mit festen Verzweigungs- punkten.}

\section{2.:. Zusammenfassung der Bedingungen für das Nirhtanftreten verschiebbarer Verzweigungspunkte. \\ Briot- und Bouquetsche Differentialgleichungen.}

Wie schon am Schlusse der Nr. 23 bemerkt wurde. enthalten bereits die im vorigen Kapitel unter (A) bis (E) formulierton Bedingungen die notwendigen und hinreicluenden Einschränkungen, denen dir Koeffizienten der Differentialgleichung

$$
F\left(\frac{d y}{d x} \cdot y \cdot x=A_{0}(y \cdot x) \quad \frac{d y}{d x}+\cdots+A_{m}(y \cdot x)=0\right.
$$

zu unterwerfen sind, damit die lntegralu inmerhalh der in dur. Mr. 23 nit 7 bezeichneten Fläche den Charakter rationaler Funktionen hahen, oder. wie wir mit Fuchs kurz sagen wollen, danit dir Differentialgleichung (1) nur feste Verzweigungspunkte besitze.

Zunächst ergibt die Zusammenfassung der Bedingungen (A) (S. 75) und (D) (S. S6), daß die Gleichung $A_{0}(y, x)=0$ üherhaupt keine (endliche) Lösung $y$ besitzen darf. d. h. die ganze Funktion $A_{0}(y . x)$ null. von $y$ unabhängig, also eine bloße Funktion von $x$ sein. Danı kann man aber mit dieser Funktion von $x$ durchdividieren und erhält so als Koeffizienten der höchsten Potenz der Ableitung in (1). die Eins.

Setzen wir in (1). wo also jetzt $A_{0}(y \cdot x)=1$ vorausgesetzt wird.

$$
y=1
$$

so folgt für z die Differentialgleichung

$$
\begin{gathered}
\left.-\begin{array}{c}
1 \\
z^{2}
\end{array}\right)^{\prime \prime}\left(\begin{array}{l}
d z \\
d x
\end{array}\right)^{\prime \prime}+A_{1} z^{1} \cdot z^{-1} z^{\prime \prime-1}(d z)^{\prime \prime-1} d x \\
-\cdots+A_{m}\left(\begin{array}{l}
1 \\
z
\end{array}, x\right)=0 .
\end{gathered}
$$

Wenn wir hierin die Nenner durch Multiplikation mit einer geeigneten Potenz ron z entfernen, d. h. die Differentialgleichung so umformen, daß 
94 Viertes Kapitel. Differentialgleichungen mit feston Verzweigungspunkten.

ihre Koeffizienten ganze rationale Funktionen von $z$ sind, so müssen nach (E) (S. 87) auch für diese Differentialgleichung dir Brodingungen (A) und (D) erfüllt, d. h. der Koeffizient der $m$-ten Potenz von $\frac{d z}{d x}$ muß von $z$ unabhängig sein. Hieraus folgt aber, dale der Korffizient $A_{k}(y, x)$ in (1), für $k=1,2, \ldots, m$, in $y$ höelıstens vom $2 k$-ten Grade ist.

Was die Bedingungen (B) (S. 79,80) und (C) (S. 85) anlangt, so ist zu bemerken, daß jeder von Null verschiedenen Lösung $y=\eta(x)$ der Diskriminantengleichung $D(y, x)=0$ von (1) tine Lösung $z=\begin{gathered}1 \\ y_{y}(x)\end{gathered}$ der Diskriminantengleichung $D_{1}(z, x)=0$ der Differentialgleichung (2) in $z$ entspricht. Soweit also solehe Lösungen von $D_{1}(z, x)=0$ in Frage kommen, sind die Bedingungen (B) und (C) für die Differentialgleichung (2) von selbst erfüllt, wenn sie für (1) gelten. Es kann aber vorkommen. daß die Diskriminante $D_{1}(z, x)$ von $(2)$ noch eine Potenz von $z$ als Faktor enthält; in diesem Falle müssen die Bedingungen (B) und (C) für die Lösung $z=0$ ausdrüicklich hinzugefügt werden ').

Wir erhalten so den folgenden. im wesentlichen von Fuchs herrührenden Satz $\left.^{2}\right)$ :

Die notwendigon und hinreichenden Bedingungen dafur. daB die Integralu der Difforentialgleichung (1) nur feste Verzweigungspunkte besitzen, sind:

1) Die Differentialgleichung lat die Form

$$
F\left(\begin{array}{l}
d y \\
d x
\end{array}, y, x\right)=\left(\begin{array}{l}
d y \\
d x
\end{array}\right)^{m}+A_{1}(y, x)\left(\begin{array}{l}
d y \\
d x
\end{array}\right)^{\prime \prime}+\cdots-1_{\mu \prime \prime}(y, x)=0 .
$$

wo $A_{1}(y, x), \ldots, A_{m}(y, x)$ ganze rationale funktionen von " mit von $x$ abhängigen Koeffizienten bedeuten und $A_{\boldsymbol{k}}(y, x)$ höchstens vom Grade $2 k$ in $y$ ist, für $k=1,2 \ldots \ldots m$.

2) Ist $y=\eta(x)$ eine Lösung der Diskriminantengleichung $D(y, x)=0$ und $s==(x)$ eine mehrfache Wurzel der Greichung

$$
F(s, \eta(x), x)=0 .
$$

der solele Zwoigr der dureh

$$
F(s, y, x)=0
$$

definierten algebraisehen Funktions ron y entsprechen,

1) Dies ist z. li. der Fall für die Differentialdskededunng

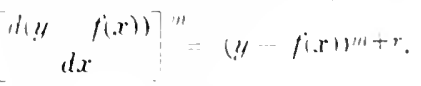

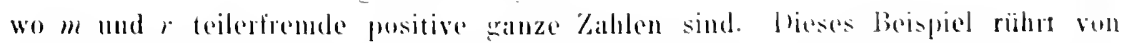
Hill und licrey her (l'roe of the London Math. Soe 12). 9. 1910. S. 2:31). die auf diese zuletzt genannte Bedingrung ansdrïcklich hingewiesen haten.

2) L. Fuchs. Berliner sitzungsberiehte 1sist. S. int: Werke II. S. 3tit 
die sich füry $\eta(x)$ verzwrigrn, so mul $\zeta(x)$ mit $\frac{d \eta}{d x}$ äbereinstimmen, also $\eta(x)$ jedenfalls eime lösung der Differentialgleichung sein.

3) In der Entwiekelung lieser Zweige nach Potrnzm von $y-\eta(x)$

$$
\left.s-\frac{d y}{d x}=g_{k}(y--1) \alpha+2 k+11 y-1\right)^{h+1}
$$

mu $B k>a-1 \operatorname{sein}$.

4) Die Bedingungen 2) und 3) müsson allch erfüllt sein fur die Differentialgleichung, die aus dor gegebenen durch die Transformation $:=\frac{1}{y}$ hervorgeht.

Die durch diese Bedingungen charakterisierte Klasse von Differentialgleichungen der Form (1) spielt also hier dieselbe Rolle, wie die Rice a tische unter den Differentialgleichungen, in denen $\frac{d y}{d x}$ als rationale Funktion von $y$ gegeben wird.

Ein interessanter und wichtiger Spezialfall von Different ialgleichungen der Form (1), die in diese Klasse gehören, d. h. keine verschiebbaren Verzweigungspunkte besitzen, ergibt sich, wenn wir die Kocffizienten der Differentialgleichung. d. h. also die

$$
\Lambda_{1}(y, x) \ldots, \Lambda_{m}(y, x)
$$

als von $x$ unabhängige, ganze rationale Funktionen ron $y$ (uit konst anten Koeffizienten) voraussetzen. In diesesu Falle enthält also $F$ die unabhängige Veränderliche überhaupt nicht explizite, wir können die Differentialgleichung demnach in der Form

$$
F\left(\begin{array}{l}
d y \\
d x
\end{array}, y\right)=0
$$

schreiben.

Im Falle der Riceatischen Differentialgleichung (Nr. 17, S. 66) lief sich der analoge Fall durch elementare Integration erledigen, das ist hier nicht nöglich, wir können aber. ähnlich wie in der $\mathrm{Nr} .17$. folgendermaßen schließen:

Offenbar bleibt die Differentialgleichung (3) ungeändert, wenn wir $x+c$ an die Stelle von $x$ setzen wo $c$ eine willkürliche Konstante bedeutet; ist also

$$
y=f(x)
$$

eine Lösung von (3), so ist auch $f(x+c)$ eine Lösung, und zwar. la sie eine willkürliche Konstante enthält, die allgemeine Lösung.

Wäre nun z. B. der im Endlichen gelegene Punkt $x=x_{0}$ ein Verzweigungspunkt der Integrale von (3), so würde auch $x_{0}+c$ für gewisse 
Integrale als Vorzwojgungepunkt fungieren: dies ist aber nirht möglich. dit die Verzwoignngspinkto nicht verschiebhar sein sollten. Wäre ein soleher

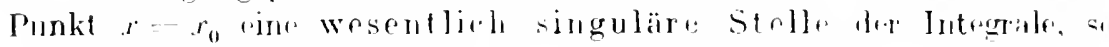

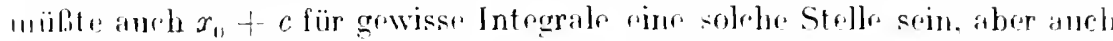
dies ist nirht möglich. da wir wisson. dal. sich jedes Integral innerhalh

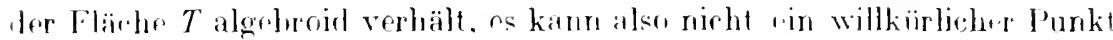

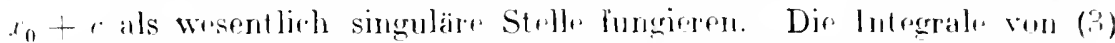
verhalten sirle demach fiir allo rendlichen Werte von $x$ wir rationale Funk-

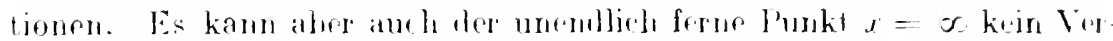

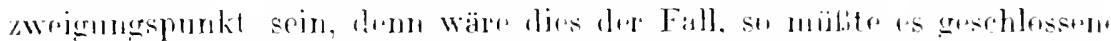
Wrge in der $x$-kbene geben. anf denen fortgesetzt rie Integrale von (3) rine Wertänderumg crfahren: da aher die Integrale in der Ungebung jedes Punktes, der innerhall dus rom riner ereschlossenen kurre begrenzten

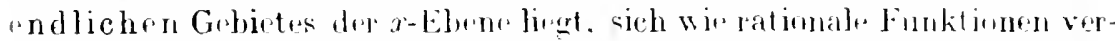

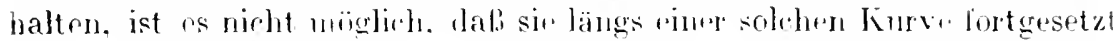

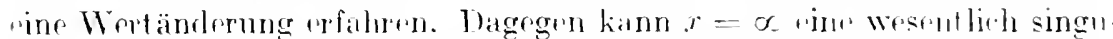

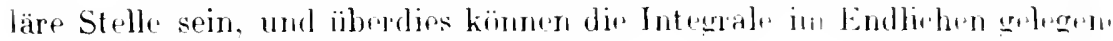
lole haben. Wir haben alsu don Sat\%:

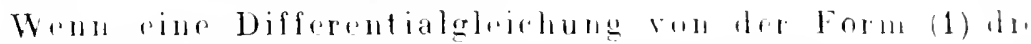

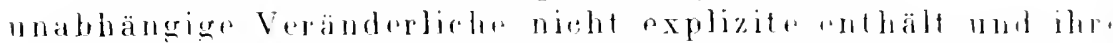
Integrale keine nut den Infangswerten versehieblare Vue aweigungspunkt besitzen. oder mit andepen Worten. Wons

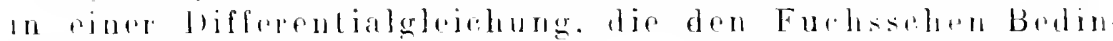

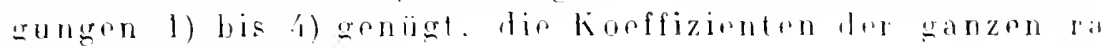
tionalen Funktionfin

$$
1,(!), \ldots) \ldots A,(\eta, r)
$$

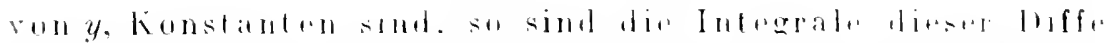
rentialgreichung rindrutier Funktionon ven $x$, li, unr im

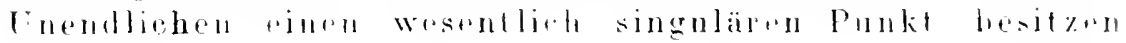
k̈̈нnกn.

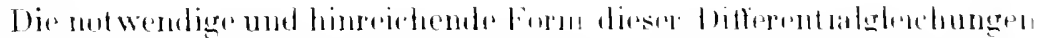

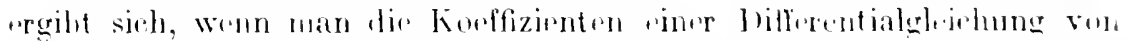

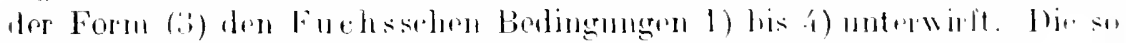

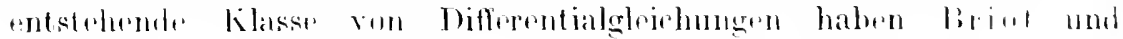

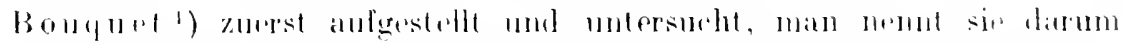

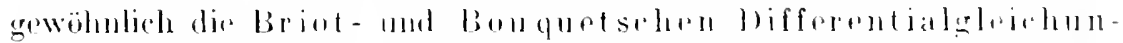

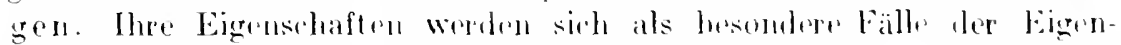

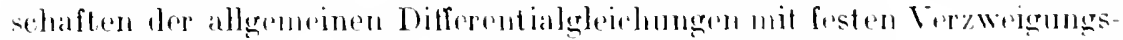

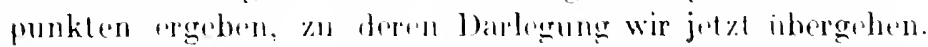

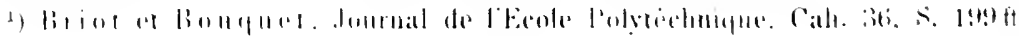




\section{Rang eince algebraischen Gleichung. Rung Null, Eins mul Kwei.}

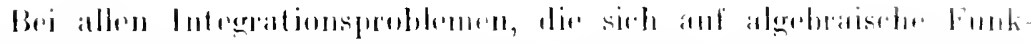

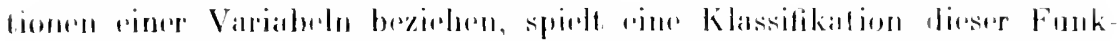

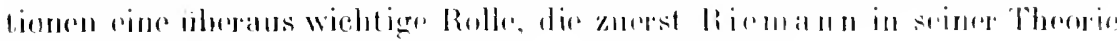

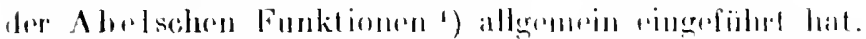

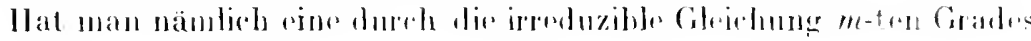
in s:

$$
F(x, y)=0
$$

Irfiniorte algehraisehe Funktion s von y. umb handelt as seb darum. das Integral

$$
\text { Is dy }
$$

auszurechnen, so zeigt sirh, dal. die Schwierigheit dieses Problems nicht von dem Grade $m$ der Glcichung (4), sondern von riner anderen Zahl ab-

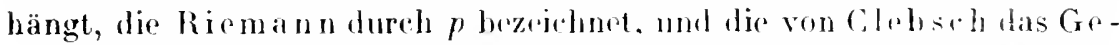
sehlecht der durch (4) dofinierton algebraischere Kurve, ron Wrierstra $B$ der Rang der Gleichung (4) ode der algehraischen Funktions von $y$ genannt worden ist. Dieso positive ganzo Zall hat die merkwirrdige Eigensehaft, ungeändert zu bloihren, wenn man von der Glairhung (4) durch die Substitution

$$
\left\{\begin{array}{l}
s=\eta(\sigma, y), \\
y=y(\sigma, \eta),
\end{array}\right.
$$

wo $\varphi, \psi$ rationale Funktionen der besiden neuen Variabeln $\sigma, y$ bedenten. zu rine Gleichung zwisthen or und $\eta$

$$
\Phi(\sigma, \eta)=0
$$

ibbergeht, vorausgesetzt, dab sich aus den Gleichungen (4), (5) auch ungekehrt $\sigma, \eta$ als rationale Funktionen, dre dureh dic Gloichung ('s) miteinander verknïpften Variabeln $s, y$

$$
\left\{\begin{array}{l}
\sigma=j(s, y), \\
\eta=g(s, y)
\end{array}\right.
$$

ausslrücken lassen. Man nemnt eine solche Transformation (5) eine eindeutig umkehrbars rationate oder auch eine birationale Transformation.

Wenn z. B. in (4) der Grad $m=2$ ist, so darl man ohne wesentliche Beschränkung der Allgemrinhuit roraussetzen, dab diese Cileichung die Forin

$$
s^{2}=R(y)
$$

1) 1857. B. Riemann. Werke. 1892. S. s8ff.; vergl. z. B. Appell und Goursat, a. a. 0. S. $222 \mathrm{ff}$; Picard, Traité II 11905), S. $417 \mathrm{ff}$. 
besitzt, wo R(y) rime ganze rationale Funktion von y berdeuter. dir in latuter vomeinander verschiedene linwarn Faktoren zerlegt werdon hann: ws s.i

$$
R(y)=\left(y-a_{1}\right)\left(y-a_{2}\right) \ldots\left(y-a_{n}\right) .
$$

wo also $a_{1}, a_{2}, \ldots, a_{n}$ sämtheh voneinamber versehiended sind Man nennt

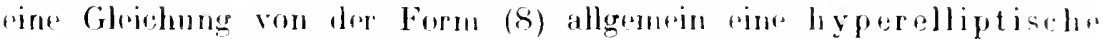

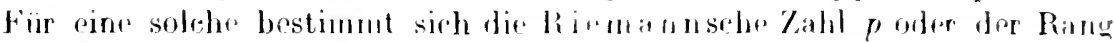
so, dab fior ein ungerades $n$.

dagegen fiur ain gerades $n$

$$
p=n-1
$$

$$
p=\frac{n-2}{2}
$$

ist. Die Glevehung ist also fïr $n=2 p+1$ mm $n=2 \mu+2$ rom Rang. i. d. h

$$
\begin{aligned}
& \text { fiir } n=1,2 \text { vou Riunge } p=0 \text {. } \\
& ., n=.3 .4 \quad . . \quad, . \quad p=1 \text {. } \\
& \text {, } n=5,6, . \quad . . \quad \mu=2.11 \mathrm{sw}
\end{aligned}
$$

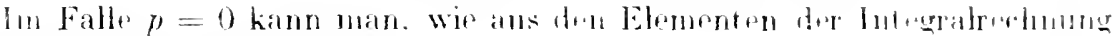
briannt ist. das Intrgral

$$
\int P(s, y) d y .
$$

wo $P$ eine rationale Funktion der dureh die Gleichung (S) rerkumplom Variabeln $s$, y bedentet, durch clementare Funtionen (algebraisehe Funhtionen, Logarithmus, Areustangens) in expliziter form bermhnem, Es

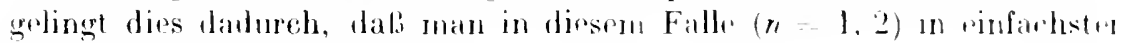
Wrise rine rationale Funktion

$$
t=y(s, y)
$$

von sumd y anfinden kann, dureh die sich s und y rational sn darstollen lassen.

$$
\begin{aligned}
& s=p(t), \\
& y=\psi^{\prime}(t),
\end{aligned}
$$

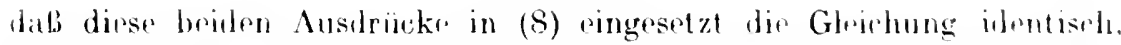
d. h. für jeden Wert von $t$ befriedigen. Führt man dann in (10) $t$ als 11 . In. Variable "in, so orhält man

$$
f P(s, y) d y=f P\left(\downarrow(t), \psi^{\prime}(t)\right) \psi^{\prime}(t) d t .
$$

d. h. das Integral riner rationalen Funktion von 1 .

In Falle $p=1$, wo also $R(y)$ eine ganze Finktion dritten oder vierten Grades ist, gelingl die explizite Darstellung nims Integrals vou der Form

$$
f(s, y) d y
$$

dureh elementare Funktionen in allgemeinen nicht mehr; dats Intrgral ist 


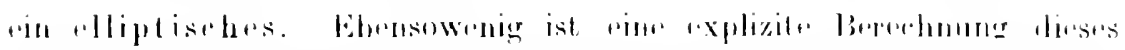

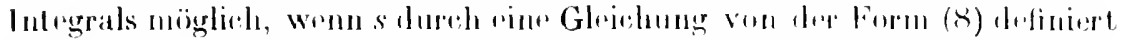

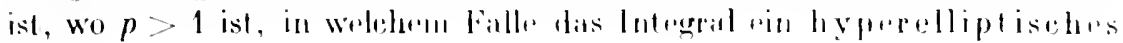
lexibt.

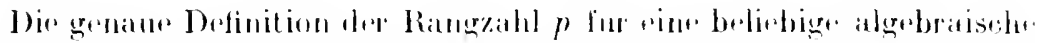

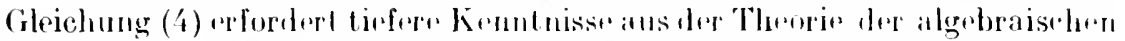

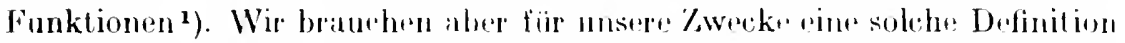

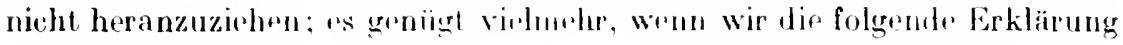
zu Grunde legen.

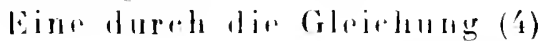

$$
l(s, y)=0
$$

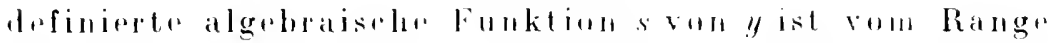

$$
p=0,1,2 \text {, }
$$

wenn die Gleichung (4) dureh biralional. Transformation

$$
\begin{array}{ll}
s=\phi(\sigma, \eta) & \sigma=f(s, y) \\
y=\psi(\sigma, \eta) & \eta=g(s, y)
\end{array}
$$

ill risn hyperelliptis.he Gluichung

$$
\sigma^{2}=R(\eta)
$$

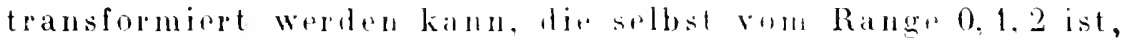
wo also im Falle $p=0$ ler Gral der ganzen Funktion $R(\eta)$

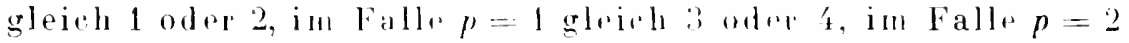
gloieh 50 der $t$ ist. In jubem anderen Falle ist der Rang der Gleichung ('́)gröbor als 2. Die Koeffizinnlen der ratiunalen Funktionen

$$
p, q, f, g, h
$$

bestimmen sich auf algebraische Woise aus den horffizirnten der Gleichung (4).

Im Falle $p=0$ sind $\sigma$ and $\eta$ wurch rinen Parametrr $t$, der selbst rational in $\sigma$ und $\eta$ ist, rational darstellbar, in diesen Falle kann also dite obige Definition auch dureh die folgende ersetzt werden:

Die Gleichung (4) ist vom Rang" Null. W"nn sich ein. rationale Funktion $t$ von $s$ und $y$

$$
t=h(s, y)
$$

so angeben läßt, daßs,y als rationale Funktionen von $t$

$$
s=\Phi(t) . y=\boldsymbol{\Phi}(t)
$$

so darstellbar sind, dab diese Ausdrüke in (4) eingesetzt diese Gleichung identisch bofriedigen.

1) Wir verweisen nebst der Abhandlung Riemanns namentlich auf die bereits $S .67$ genannten Werke von Appell und Goursat. Hensel und Landsberg. Landfried. 


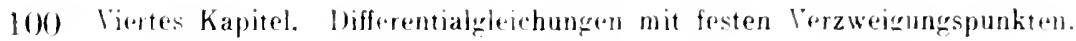

In diesem ralle ist also in lonterat

$$
f(s, y) d y \text {. }
$$

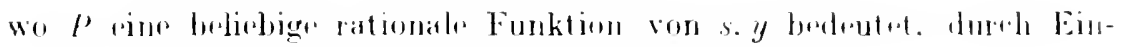
fiilurnng ron / als nemer Variaboln anf dir lourun

$$
\int P\left(\Phi(t), \quad(j(t)) i j^{\prime}(1) d t .\right.
$$

1. h. anf das Intrgral riner rationalen Funktion von t moduzierlar. Man

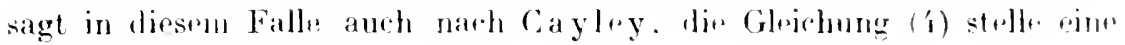
Unikursalknrou dar.

\section{Gleichungen rom Range Null.}

Dir Bedeutung des Ranges p. der dureh dir Glwichung

$$
F(s, y, x)=0
$$

definiorten algebraischen Funktions von y fïr has Jroblum der Integration der Differentialgleichung (1)

$$
F\left(\begin{array}{l}
d y \\
d x
\end{array}, y, x\right)=0
$$

hat in dem Falle der Briot- und Bonquet sehen Differentialerlefehung. wo also dir Korffizienten von (1) von $x$ unabhängig sind. zurest Hemit: $\left.{ }^{1}\right)$ zur Geltung gebracht. In seinen Untersurhungun uber di* Differentialgheichungen von der Form (1) mit festen Virzwrigmngspunkten hat Fuchs gleichfalls die Klassifikation dieser Gloichungen navh dem Range der Glejchnng (11) (in der. wie auch bisher immer. $r$ die Rollw rines Parameters spielt) in Angriff genommen und die Fäle $p=0.1$

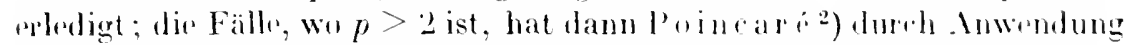

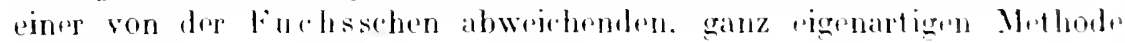
untersucht.

Nach dem Vorgange von Furhs behandeln wir zunähst den Fall $p=0$.

Wenn dio dureh (11) definierte algehraisehe Funktion s ron $y$ rom Rangr Null ist, so kann man rine rationale Funktion

$$
t=h(s, y)
$$

rons sumd $y$ finden. durch dies $s$ und $y$ rational:

$$
s=\Phi(l), \quad y=\boldsymbol{\Psi}(l)
$$

darstrlbar sind. Die Karlizienten der rationalen finghtion h. $\phi$, is hängru in allgtmoinon noch voll $x$ ah, uml zwar sind sie algebraisch alus den Kooffizienten der Globhume (II) zusammengestetzt.

1) Ch. Hermite. Cours (lithographii) de lEcole l'olytechnipue 15i3: rysl. hierzu noch furbs. Comptes Rendus 1sis. S. 116i3. Werke 11. S. 2sis und W. Rasehke. 1883. Acta Hathematica. Bd. 14 (1s:?0). S. 31.

$\Rightarrow$ II. Poincare, Aeta Mathematica. Bd T (18si). S. $1 \mathrm{ff}$. 


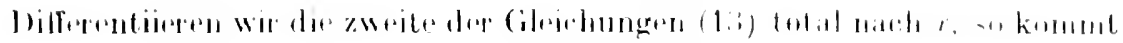

IIII) M siud offenbar

$$
\begin{aligned}
& \text { dy idi dipill } \\
& \text { ar a.t i.t } 1 / r^{\circ}
\end{aligned}
$$

$$
\begin{aligned}
& \text { r.j क्य } \\
& \therefore \text { i }
\end{aligned}
$$

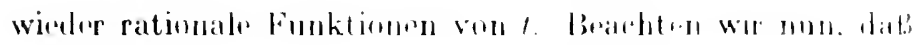

$$
\therefore \quad d y \quad-d(1)
$$

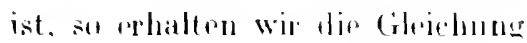

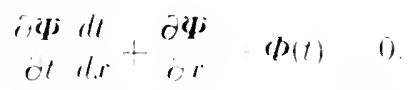

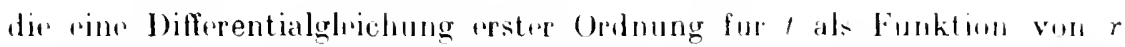
darstellt, 11. z. eine solelue. lie de als rationale funklion voll thestimmt.

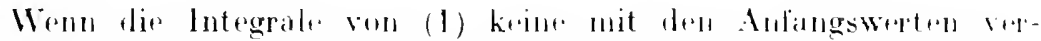
sehithbaren Verzweigungspunkte besitzen. so hat aud dire rationale Funk-

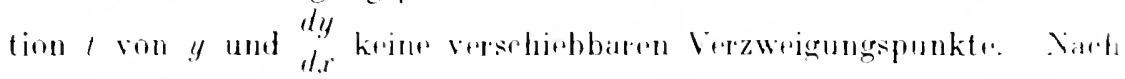

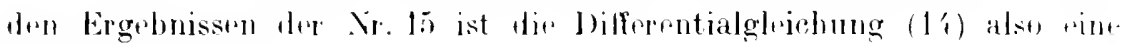
liveratisphe, il. h.

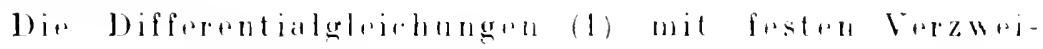
gungspunkten, in denen dir zwischen dy nudy bestehende

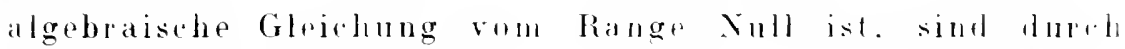
rine rationalo Substitution anf Rirratisuthe Joiffrentialghrichungen reduzierbar.

In dem besonderen Falleder liriot-umb bumpuetsehen l)ifferent ialshrichung, wo die Koeffizienten von (1) und (11) von $x$ unahlängig sind. wroden anch die Koeffizienten der rationalen Funktionen $h(s, y), \Phi(t)$. $\Psi(t)$ Konstanten sein, da sir sich ja aus den Kireftizienten der Gleirhung (11) algebraisch zusammensetzen. In diesem Falle sind alsu ameh di." lireffizienten der Riceat ischen bifferentialgleichung (14) ron r mablıängig. diest Gleichung hat alsu di“ Form

$$
d t=t_{11}-1_{1} t \cdots 1_{2} t^{2} .
$$

wo $A_{0}, A_{1}$. A, Konstantru herdeuten.

Wenn dir Gleichung

$$
A_{0}+1_{1} t \rightarrow 1_{2} t^{2}=0
$$

die voneinander versehiedenten Wurzeln $Q_{1}$. $Q_{2}$ hat. so folgt ans der in der 


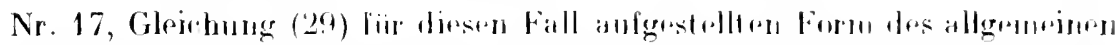

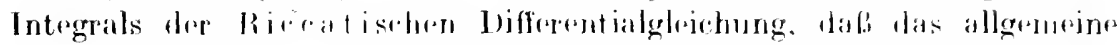

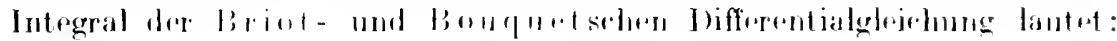

$$
\left.y=y^{\prime}(l)=\bar{\psi}\left(\rho \cdot 1_{2}^{\prime} e_{1}-\underline{o}_{2}\right) x\right) \text {, }
$$

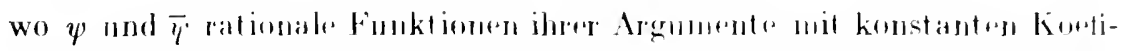

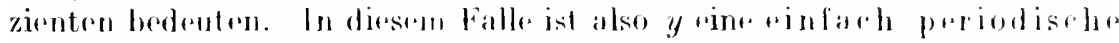

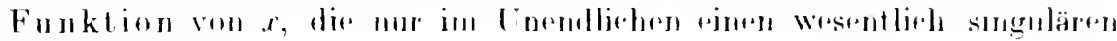
Punkt hesitot.

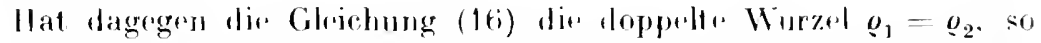

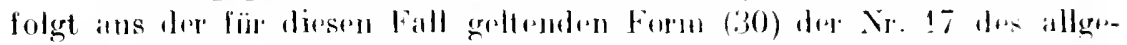
meinen Integrals der Ric.ealisehen Jifferentialgleichung. dals die all-

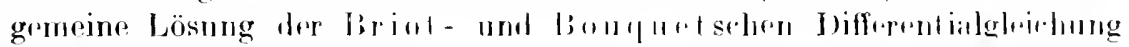
aine rationale Funktion

$$
y=\psi(l)=\bar{\psi}(x)
$$

von $t$ und folglich alleh ron $x$ ist.

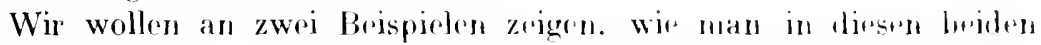
Fällen die Integration einer solehen Br. tialgleichung zu vollziehen hat.

Es seit

$$
F\left(\begin{array}{l}
d y \\
d x
\end{array}, y\right)=\left(\begin{array}{l}
d y \\
d x
\end{array}\right)^{2}+\frac{d y}{d x}\left(y^{2}-1\right)+4-y^{2}=0:
$$

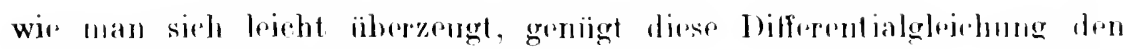
Furbeschen Brelingungern. Wir haben fornes

$$
f^{\prime}(s, y)=s^{2}+s\left(y^{2}-4\right)+4-y^{2}=0:
$$

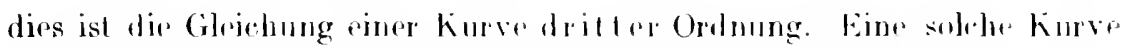
ist vom Geschlcelete Null, wem sie rinen Hoppelpunkt besitzt. Dirs ist in der Tat der Fall, denn das Wertepatar

borfrienligh dir Gileirhumgen

$$
s=2 . \quad y=0
$$

$$
F(s, y)=0, \begin{aligned}
& \partial F \\
& \partial s
\end{aligned}=0, \begin{aligned}
& \partial F \\
& \partial y
\end{aligned}=0
$$

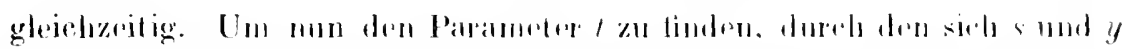

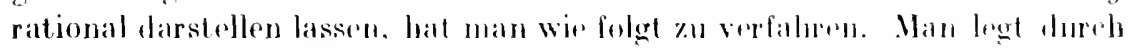
den Joppelpunkt s $2, y=0$ pin strahllitsolhel

$$
x-2=1 \mathrm{y},
$$

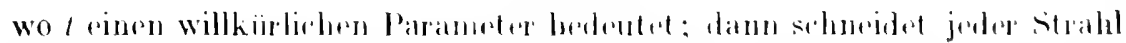

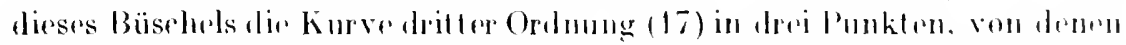

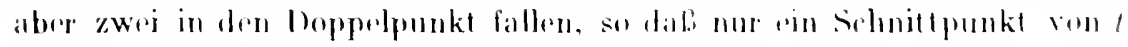

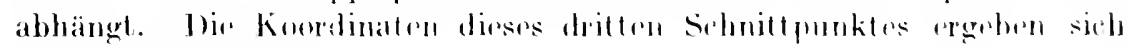
leichl in der form 


$$
19+1,1,12,
$$

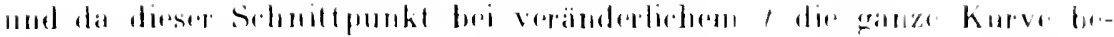

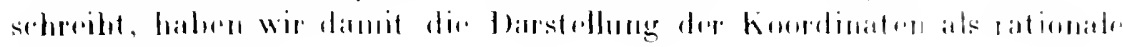
fintiotion vorn

$$
1+2-2
$$

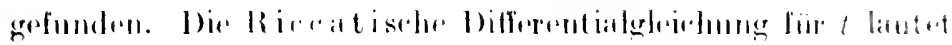

$$
d t=1^{2}
$$

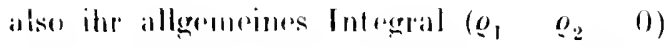

wir haben folglich

$$
y=-t^{2}+1=\frac{1}{t}=c^{x+x-c,}
$$

d. h. $y$ ist in der Tat rime rationalo Funktion.

Wir betrachten ferner die Gleichung

$$
F\left(\begin{array}{l}
d y \\
d x
\end{array}, y\right)=\left(\begin{array}{l}
d y \\
d x
\end{array}\right)^{3}-3\left(\begin{array}{l}
d y \\
d x
\end{array}\right)^{2}-9 y^{4}-12 y^{2}=0,
$$

die ebenfalls den Fuchssehen Bodingungen genügt. Wir hitben

$$
F(s, y)=s^{3}-3 s^{2} \quad 9 y^{4}-12 y^{2}=0 \text {; }
$$

setzon wir hirein

so stellt dir Glpichung

$$
y^{2}=z
$$

$$
s^{3}-3 s^{2}-9 z^{2}-12 z=0
$$

wieder eine Kurve dritter Ordnung vom Geschlechte Null dar, denu

$$
s=2, \quad z=\frac{2}{3}
$$

ist ein Doppelpunkt. Durrh dirsen legen wir das Strahlbuschel

$$
3 z+2=1(s-2) \text {, }
$$

dann ergeben sich die Koordinaten des mit 1 veränderlirhan Sohnittpunkes in des Form

$$
\begin{aligned}
3 & =t^{2}-1, \\
3 z & =t^{3}-3 t-2,
\end{aligned}
$$

wir finden also zwische'n $y$ und $t$ dir Gleichung

$$
3 y^{2}=t^{3}-3 t-2,
$$

die, wenn man $y, t$ als Koordinaten auffaßt, wieder eint Kurve druttr Ordnung mit dem Doppelpunktr $y=0, t=-1$ darstellt. Legen wir durch diesen Doppelpunkt das Strahlbüschel 
104 Viertes Kapitol. Differoutialgleichungen mit festen Verzweigungspunkten.

$$
y=(1+1) 1
$$

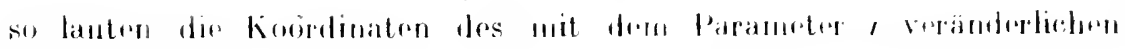
Sihnittpunktos

$$
1=\lambda^{2}+2, \quad ! \quad \therefore\left(\iota^{2}+1\right) \text {. }
$$

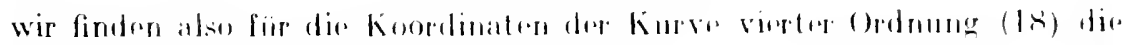

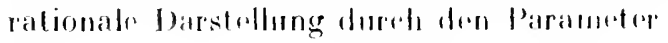

$$
\begin{array}{ccccc}
y & y & y s & 21 \\
1+1 & 3 z+2 & 2 & : 3 y^{2} & \text { s }
\end{array}
$$

in del Forni

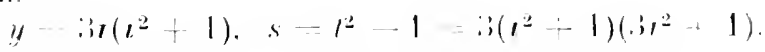

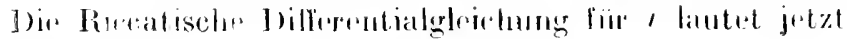

$$
d t=1+t^{2} \text {. }
$$

ihr allowereines Integral ist also

$$
1=\ln (x+r) \text {. }
$$

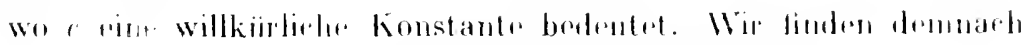

$$
y=810^{3}(x+c)+3 \operatorname{tg}(x+r) \text {, }
$$

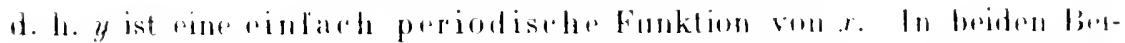

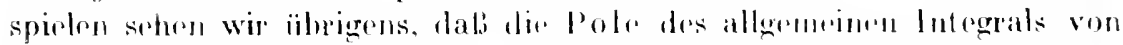
der willkurlichen Kunstanten r abhängers, also mit dert Anfangswerten versehinbluar sind').

\section{로. Gleichungen rom Range Eins.}

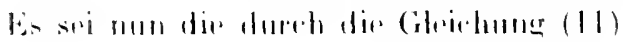

$$
F(x, y, r) \quad 0
$$

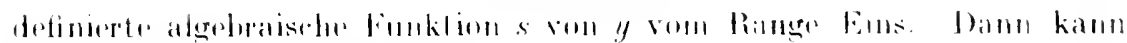
man zwei homeh die Gibirhmer

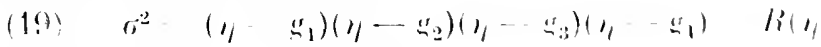
verknopfte rationale finmkliomm

$$
\sigma=f(s, y) \text {. }
$$

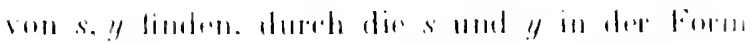

$$
\text { (21) } \quad x=q(6.1,9, y \quad 4(6.3)
$$

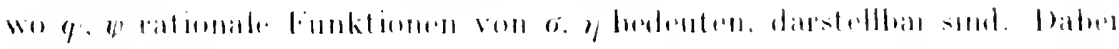
hälgखण

$$
\because 1.82 .
$$

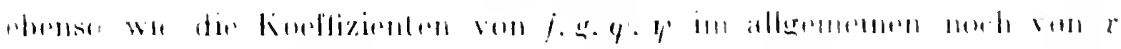

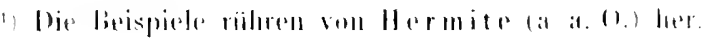




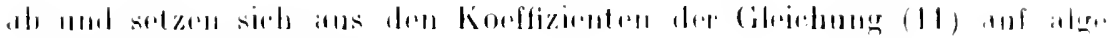

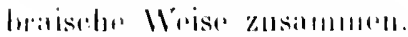

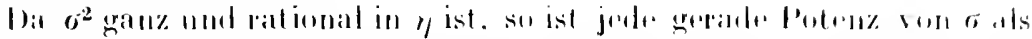

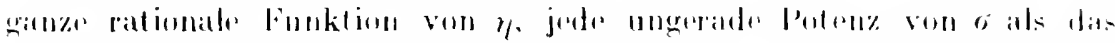

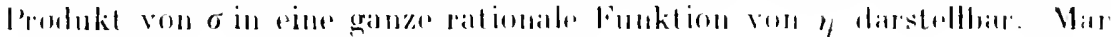

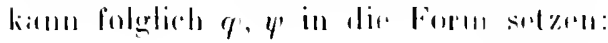

$$
\begin{aligned}
& \therefore \psi(\sigma, y) \quad \eta(\eta)+\psi_{1}(\eta) \mid R(\eta) \\
& y=\quad y(\sigma . y) \quad y_{2}(y) \cdot \frac{y_{2}(y) \mid l(y) .}{y_{11}(y)} .
\end{aligned}
$$

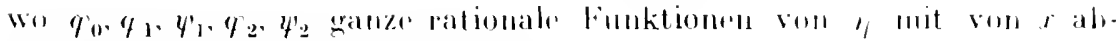
liägrigen kooffizienten bedouton.

Bilden wir nun

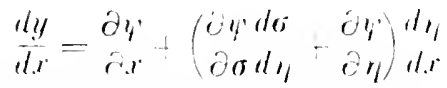

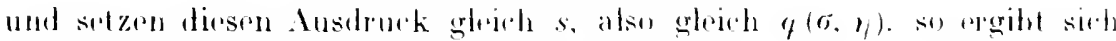
fiir y dir Jifferent ialgheichung erster Ordumng

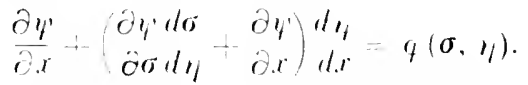

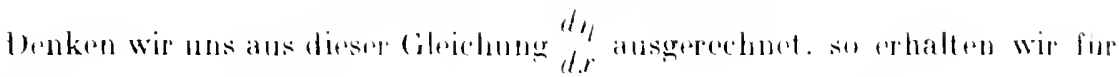
dy aine rationale Funktion von o und $\%$, dir wir in der Form

$$
\begin{array}{ll}
d_{1}-1 & =b \mid R(1)
\end{array}
$$

scluriben kömen, wo A. B. C' ganze rationale Funktionen vou y mit von $x$ abhängigen Koeffizienten bedenten. Hierans Colgt. dali y der Jifferenlialghichung roster Orhumng

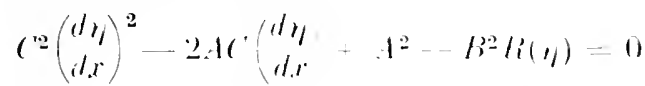

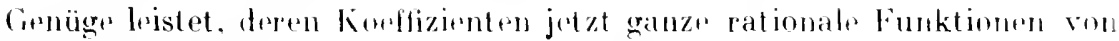
$y$ sind.

Wenn nun y keine mit den Infangswerten velsehiebbare Viazwaigungspunkte besitzt. so gilt das gleiclut vous mol folgheh nach (20) auch

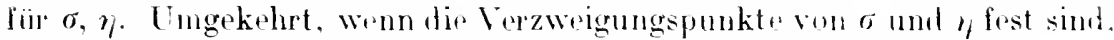
so sint zufolge von (2l) auch dir Virzweigungspunkte von y fest.

Es muf also erstens dir J)ifferentialgleichung (23) den Fuchssehen Berlingmingen genügen. und zwritrus düren die Verzweigungspunkt von $\sigma$ nicht von der wilkzülichen komstanten des allgemeinen Integrals

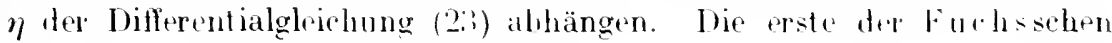


106 Viertes Kapitel. Differentialgleichungen mit festen Verzweigungspunkten.

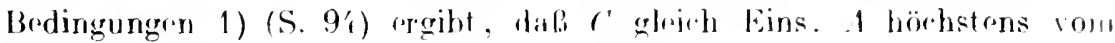
zweiten und

$$
A^{2}-B^{2} l i(\eta)
$$

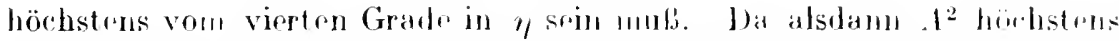
vom vierten Grade und $R(\eta)$ genan vom vierten Grarde in $\eta_{f}$ ist. so mon $B$ von $\eta$ mabhängig. also eine blolise Funktions von s

$$
B=\hat{h}(r)
$$

srin; is sid

$$
A:=A_{11}+A_{1} \eta+A_{2} \eta^{2},
$$

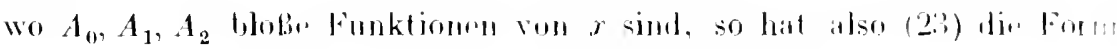

$$
\left.d x=A_{0}+A_{11} 1+A_{2} \eta^{2}+\lambda(x)\right) h(\eta) \text {. }
$$

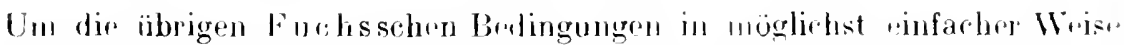
befriedigen zu können, wenden wir auf y rine Transformation an. dir von l'oilleiri') angegeben worden ist.

Wir setzen nämlirh

$$
\eta=\begin{aligned}
& a t+\beta \\
& \gamma t+\delta
\end{aligned} .
$$

wo $a, \beta, \gamma, \delta$ noch zu bestimmende Funktionen von $x$ berdenten. dip des Bedingung

$$
\alpha \partial-\beta \gamma=1
$$

Genüge leisten, und bestimmen diese finktionen derart. dab

$$
\text { liirl } \eta=g_{k}, \quad l=a_{k} \text { sei, } \quad(k=1,2,3) \text {. }
$$

wo die $a_{1}, a_{2}, a_{3}$ von $x$ unabhängig, also Konstanten sind. Wir hitbent dann die Gleichungen

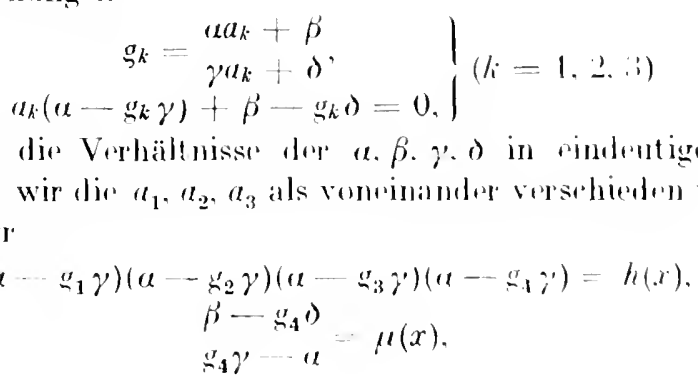

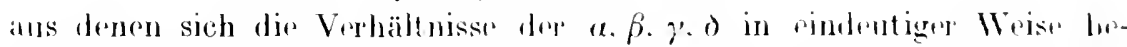
stimmen, wemn wir dir $a_{1}, a_{2}, a_{3}$ als vominamder versehiedn voranssetzen.

Setzen wil

so nimunt $\sigma^{2}$ die formo an:

Finner wird

$$
r^{2}=h(\eta)=\frac{h(x)}{(\gamma t+\delta)^{4}}\left(t-a_{1}\right)\left(t \quad a_{2}\right)\left(t \quad a_{3}\right)(t-\mu(l)) .
$$

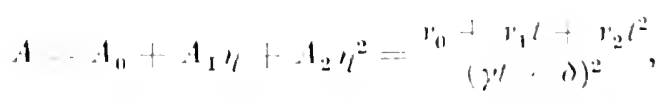

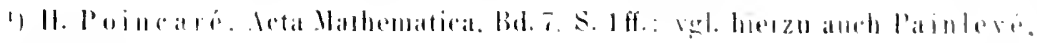
lacolts. Si. (i) ff. 


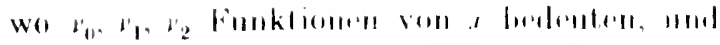

$$
\begin{aligned}
& \begin{array}{lc}
d y & 1 \\
d x & (\gamma t-t))^{2} d x
\end{array}
\end{aligned}
$$

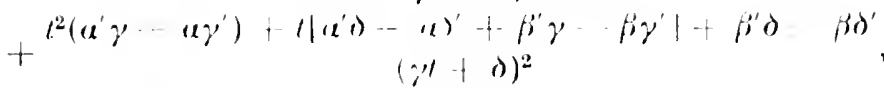

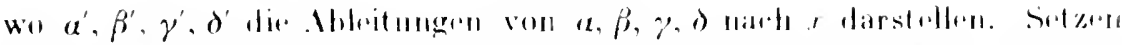

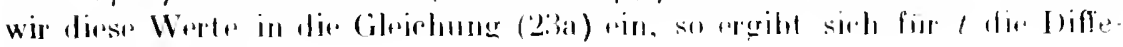
rentialghoirlhung

$$
\begin{gathered}
d l=\lambda_{0}+\lambda_{1} t+\lambda_{2,} l^{2} \\
+l^{\prime}(x) \mid\left(t \cdots a_{1}\right)\left(t \cdots a_{2}\right)\left(t-a_{3}\right)(t-\mu(x)),
\end{gathered}
$$

wo $\lambda_{0}, \lambda_{1}, \lambda_{2}, P(x)$ wohlbestimmtr Funktimen ron $r$ bodenten.

Es sind dann 1 , und

$$
u=\mid\left(t--a_{1}\right)\left(t-a_{2}\right)\left(t-a_{3}\right)(t-\mu(x))
$$

rational dureh s und $y$ darstellhar, mol umgokehrt ist annl

$$
s=\frac{\Phi_{1}+\Phi_{1} \cdot u}{\Phi_{0}}, y=\frac{\Phi_{2}+\Phi_{2} \cdot "}{\Phi_{0}},
$$

wo $\Phi_{0}, \Phi_{1}, \psi_{1}, \Phi_{2}$, ifi, ganze lationale funktionen van $/$ bedeuten deren Koeffizienten noch von $x$ abhängen und sich rbenso wie $\mu(x)$ aus den koeffizienten der Gloiehung (11) aul algobraisehe Weise zusammensetzen. Es mun num dir. Dilforentialgleichung (24) festr Verzweigungspunkte besitzen, und dir Verzweigungspunktr von $\|$ müssen von der willkürlichen Konstanten des allgemoinen lntegrals $t$ von (24) unabhängig sein.

Für die Differentialgloichung (24) ist dif erste der Furhsschen Bedingungen erfüllt, da sie breits für (23a) erfüllt war. $I_{n}$ dic übrigen Bedingungen [2), 3), 4), S. 9', 95] formulieren zu können, beachten wir, dab in unserem Falle die Diskriminantengleichung nichts anderes ist als

$$
u^{2}=\left(t-a_{1}\right)\left(t-a_{2}\right)\left(t-a_{3}\right)(t-\mu(x))=0 \text {. }
$$

Dio gedachten Bedingungen reduzieren sich alse rinfach darauf, dat.

$$
a_{1}, a_{2}, a_{3}, \mu(x)
$$

Integral'von (24) sein mïssen. Da $a_{1}, a_{2}$. $a_{3}$ Konstanten sind, mula also zunächst

$$
\lambda_{0}+\lambda_{1} a_{k}+\lambda_{2} a_{k}^{2}=0
$$

sein. Da aber $a_{1}, a_{2}, a_{3}$ vonteinander verschieden sind. so fulgt hieraus

$$
\lambda_{i}=\lambda_{1}=\lambda_{2}=0 \text {. }
$$

Setzen wir nun in (24) $\mu(x)$ an dir Stelle von 1 so folgt

$$
\frac{d \mu(x)}{d x}=0
$$

d. h. $\mu(x)$ ist ron $x$ unahhängig. also eine Konstante: wir hezeichnen dieste durch $a_{4}$, 


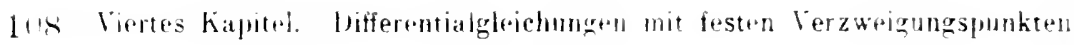

$$
\|(r)=a_{1} .
$$

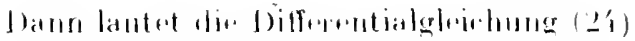

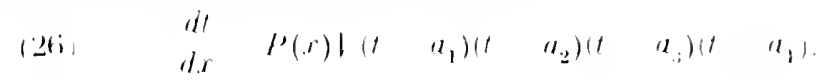

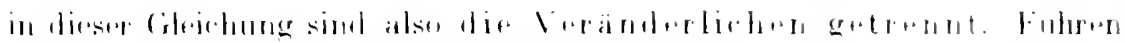

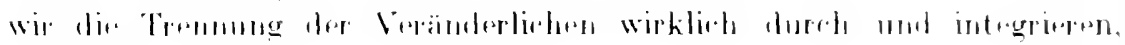
so kemmont

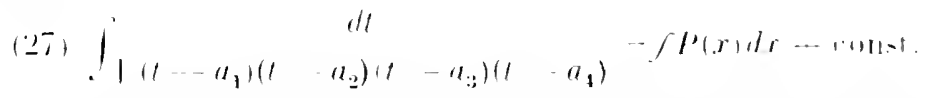

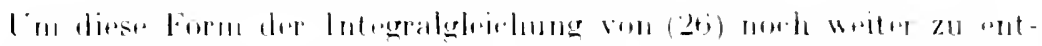

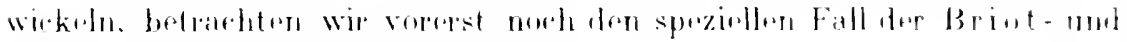

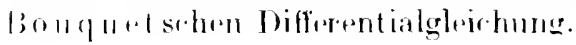

\section{2!! Briot - and Bonquetsche (ileichung vom Range Eins.}

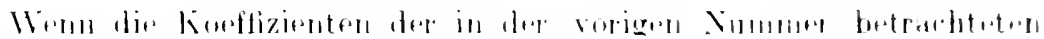

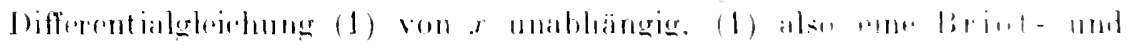

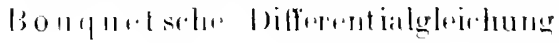

$$
F^{\prime} \cdot \operatorname{li} \cdot y=0
$$

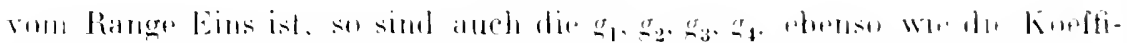

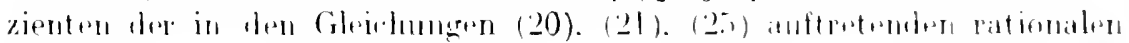

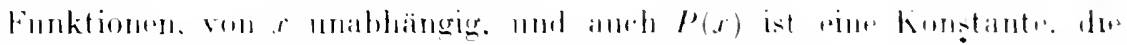

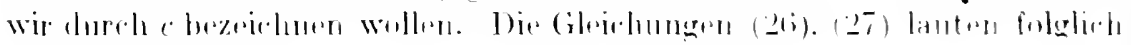
in lixing Fallo:

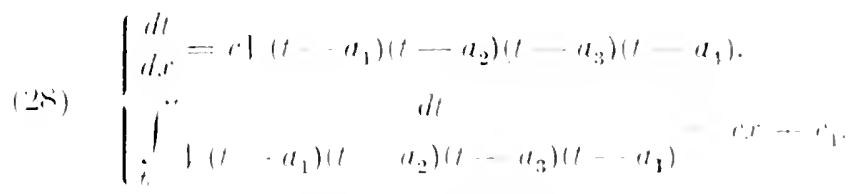

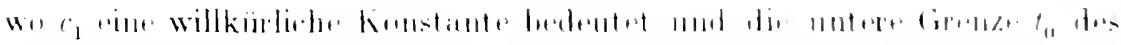

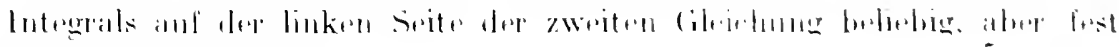

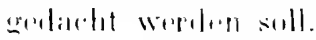

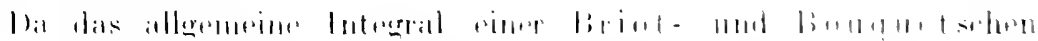

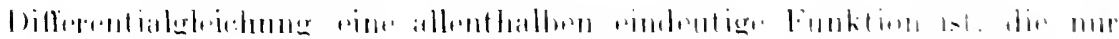

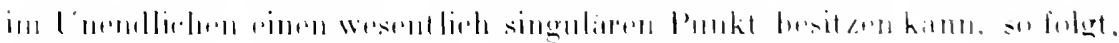

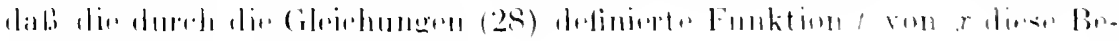

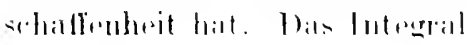

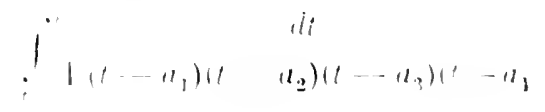




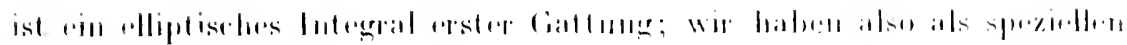

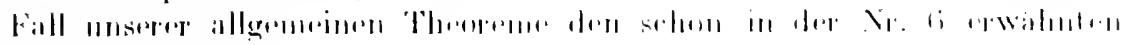
wirlitigen Siat $\%$ :

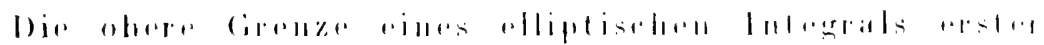

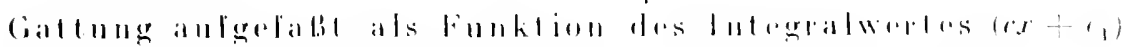

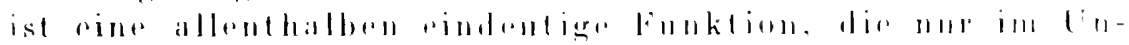

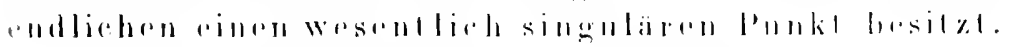

Man mement diese Funktion aime olliptisthr.

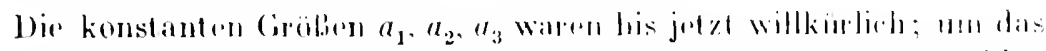

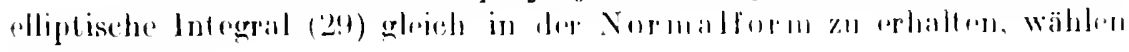
wir speziell

$$
a_{1}=0, \quad a_{2}=1, \quad a_{3} \ldots x_{2}
$$

Wir haben dann zur Bestimmming der Koeffizienten a. o. $\beta \hat{\gamma}, \hat{\gamma}$, der in der $\mathrm{Nr} .28$ (S. 106) angegebenen linearrn Funktion

dir Gloichungen

$$
\eta=\begin{aligned}
& \theta t+\beta \\
& \gamma t+\delta
\end{aligned}, \quad(\nu)-\beta \gamma=1,
$$

Ind wenn wir noch

$$
\begin{gathered}
\beta-g_{1} \delta=0, \\
\left.a-g_{2} \gamma+\beta-g_{2}\right\rangle=0, \\
\alpha-g_{3} \gamma=0 .
\end{gathered}
$$

$$
u_{4}=\begin{gathered}
1 \\
k^{2}
\end{gathered}
$$

setzen, so.konnm wir diu Gleichung (26) allgemein in der form

(26a)

$$
d t=P(x) \mid \text { t } t(1-t)\left(1-k^{2} t\right) .
$$

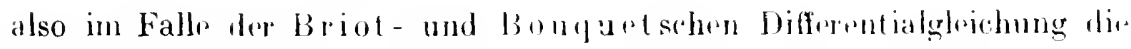
Gleichungen (28) in der Form

(28a)

$$
\left\{\begin{array}{l}
d t=c \mid 4 t(1-t)\left(1-k^{2} t\right) . \\
\int_{0}^{t} \mid \frac{d t}{4 t(1-t)\left(1-k^{2} t\right)}=r t+c_{1}
\end{array}\right.
$$

schreiben, wo wir noch $t_{0}=0$ gewählt haben.

Setzen wir im Falle der Briot-und Bonquetsohen Differentialgleichung

$$
c x+i=11,
$$

und führen in dem elliptischen Integrale erster Gattung dureh die Gleichung

$$
t=z^{-2}
$$

eine neue Integrationsvariable ein, so ergibt sich 
110 Viertes Kapitel. Differentialgleichungen mit festen Vorzweigungspunkten.

$$
u=\int_{i}^{n} \quad 1\left(1-z^{2}\right)\left(1-k^{2} z^{2}\right)
$$

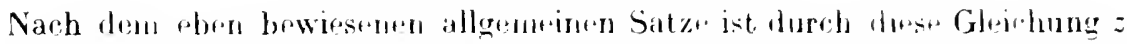

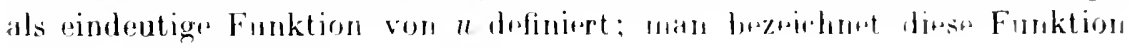
nach Jacobi dureh

(vgl. Nr. f. S. 25) nud s+tzt

$$
z=\sin : 111 \|
$$

$$
\begin{aligned}
& 11-z^{2}=\cos a \| n u \\
& \mid 1-k^{2} z^{2}=\Delta \text { aाl } u .
\end{aligned}
$$

Wir haben also in diesen Zeicher

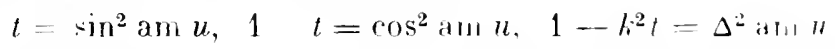

J)as allgemeine Integral $y$ der liriot- und Bunquet seh.n Differentualgleichung vorn Range Eins. das nach (25) in der Form

$$
y=\frac{\Phi_{2}+\psi_{2} \mid t_{4} t(1-t)\left(1-k^{2} t\right)}{\Phi_{0}}
$$

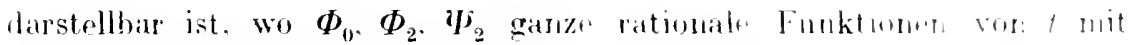
konstanter lioffizienten berlusten, lautet also

$$
y=K\left(\sin a m\left(c x+c_{1}\right) \text {, cos am }\left(c x-c_{1}\right) . \Delta a m\left(r x-r_{1}\right)\right) \text {. }
$$

wo $R$ eine rationale Funktion andeutet.

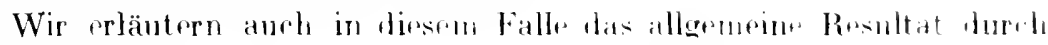
pin Beispiel. Es soi

$$
F\left(\begin{array}{l}
d y \\
d x
\end{array}, y\right)=\left(\begin{array}{l}
d y \\
d x
\end{array}\right)^{3}+3\left(\begin{array}{l}
d y \\
d x
\end{array}\right)^{2}+y^{\mathrm{b}}-4=0
$$

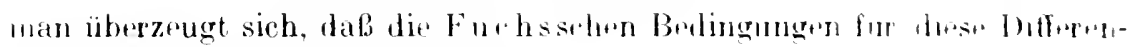
lialg]richung erfiillt sind. Es ist

$$
F(s, y)=s^{3}+3 s^{2}+y^{6}-4=0
$$

sitzen wir $y^{3}=\zeta$, so haben wir die Gleichung

$$
s^{3}+3 s^{2}+-2-1=0,
$$

die eine Kurve dritter Ordnung in den rechtwinkligen koordunaten s. darstellt. Der Pinkt

$$
s=-2 . \quad \div=0
$$

ist rin Joppolpuntet. Legen wir durch ihn das Strahlbuseluel

$$
\Sigma=(s+2) \boldsymbol{c} \text {. }
$$

so simb die koordinaten des mit $t$ veranderlichen sihntepunts

$$
s=1-r^{2}, \quad c=3 i-r^{3},
$$

fiir s, y rergibt sich also die loarstellung:

$$
s=1-r^{2}, y^{3}=3 i-r^{3} .
$$

Die Gleithung zwisehen y und stellt in den rexhtwinkligen Keorinaten 


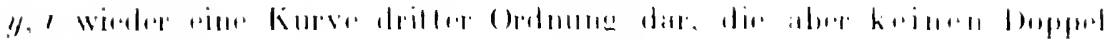

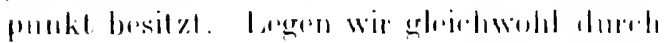

$$
\text { !l }(), \quad, 0
$$

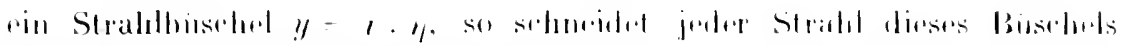

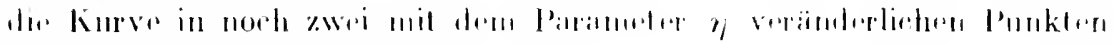

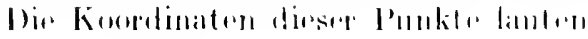

$$
\begin{aligned}
& 1: 1: 3 \\
& 11+y^{3}, y
\end{aligned}
$$

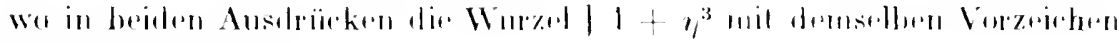
all mehmont ist.

Setzon wir also

$$
\sigma-11+r^{3} \text {. }
$$

so haben wir fiir s.y dir: 1)arstellunger

$$
s=1-1: y^{3} \cdot y=\frac{1,1:}{\sigma} .
$$

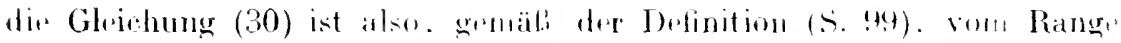

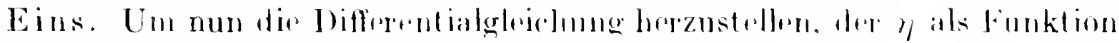
von $x$ geniigt, hildum wir

$$
d y=\frac{1: 32-r^{3}}{d y} d^{3} d y
$$

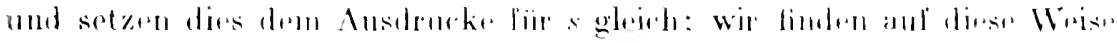

$$
d_{r}=-\frac{2}{1.3} \sigma=-\frac{2}{13} 11+r^{3}
$$

woraus sich

$$
-\overline{2}^{2} x+c_{1}=\int \frac{d y}{11+\eta^{3}}
$$

rrgibt. Es handelt sich nunmehr noch um den Übergang zur Normalform.

Bezeichnen wir eine der komplexen Wurzeln der Gleichung

dureh $u, z$. B.

$$
1+r^{3}=0
$$

$$
a=\frac{1}{2}+\frac{1}{2} 1-\overline{3}
$$

so ist

die Substitution

$$
1+\eta^{3}=(\eta+1)(\eta-\alpha)\left(\eta+\alpha^{2}\right)
$$

$$
\eta+1=\left(1-a^{2}\right) z^{2}
$$

verwandelt dann das elliptischr lntegral (31) direkt in

$$
\int \frac{d \eta}{1+\eta^{3}}={ }_{11}^{2} \dot{j} \quad \frac{d z}{+\left(1-z^{2}\right)\left(1-k^{2} z^{2}\right)}, \quad\left(k^{2}=1-\alpha\right)
$$


112 Vitrtes hapital. Differentialgleichungen mit festen Verzweinngspunktril

Wir finden somit

$$
\begin{array}{ll}
11+k+c^{\prime}=\int_{j}^{z} \mid\left(1-z^{2}\right)\left(1-k^{2} z^{2}\right) \\
13
\end{array}
$$

wo C: "rine willkürlichr Konstant,

$$
r=r_{1}^{2} \mid 1+\mu
$$

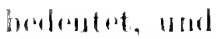

$$
y=\frac{\eta 1: 3}{\sigma}=\begin{gathered}
|i|\left(1-u^{2}\right) z^{2} \quad 1 \mid \\
k^{2}(1+u): 1=2\left(1-z^{2}\right)\left(1-k^{2} z^{2}\right)
\end{gathered}
$$

also schlioblich, woun wir

sitzon,

$$
\begin{array}{r}
11+{ }^{2} x+r=5 \\
13
\end{array}
$$

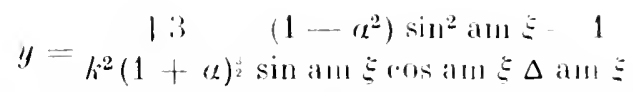

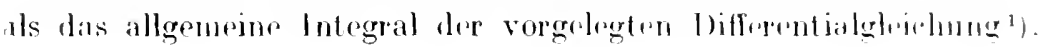

\section{Integration der Differentialgleichung rom Range Eins mit festen Verzweigungspunkten.}

Wir kehren nun zu der allgemeinen Dilferentialgleichung mit frsten Verzweigungspunkten zuriek, fïr die dir durch die Gleirbung (11)

$$
F(s, y, r)=0
$$

definierte algebraisehe Funktion $s$ von $y$ vom Ramere Eins ist. und wollum uns auch hier die konstanten Gröben $a_{1}, a_{2}, a_{3}$ so gewählt deruken. dals

$$
a_{1}=0, \quad a_{2}=1, \quad a_{3}=\propto
$$

ist. Die vierte Wurzel der Gleichnog $u^{2}=0$ ist damn, win in der Nr. 2e gerorgt wurde, rbenfalls vou $x$ mabhängig; wir setzon. Wie in Falle der Briot - und Bouguetschen Differentialghichung. diese vinte Wurkel

$$
t_{1}=\begin{gathered}
1 \\
k^{2}
\end{gathered}
$$

Es ist dann nach (25) s, $y$ in the Form

$$
\begin{aligned}
& \int s=\Phi_{1}+i_{1} \mid i_{1}(1-t)\left(1 \cdots i^{2} t\right) .
\end{aligned}
$$

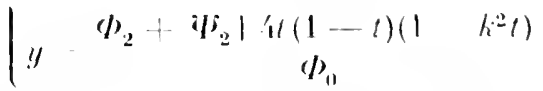

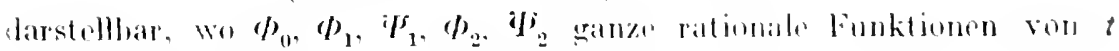

1) Dieses Berispiel stammt von biriot und bolly lat. a. al. ". 


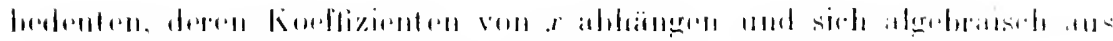

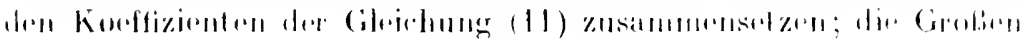

$$
\text { 1. } 1 \text { 1 } 1(1-1)\left(1-1 i^{2} t\right)
$$

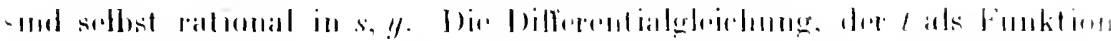

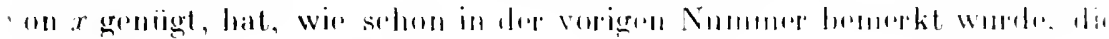
Finm (26ia)

$$
\text { dl } l(x) \mid \operatorname{ll}(1-l)\left(1 \ldots k^{2} l\right) \text {; }
$$

es ergiht sich atso, wents wir

$$
f(x) d x=d u
$$

setzon:

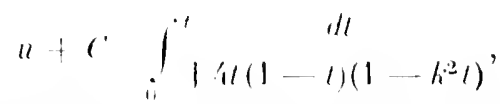

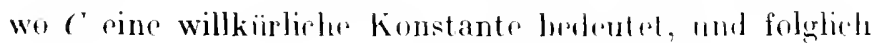

$$
t=\sin ^{2} a n+\left(u+c^{\prime}\right),
$$

$14 t(1-l)\left(1-k^{2} l\right)=2 \sin a m\left(u+l^{\prime}\right) \cos \operatorname{am}\left(u+c^{\prime}\right) \Delta a \operatorname{ar}\left(u+c^{\prime}\right)$, worin $u$ gleich einem bestimmten Werte dos Integrats

$$
f(x) d x
$$

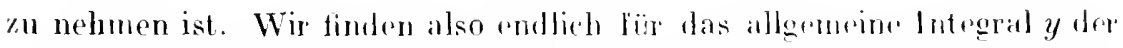
Differentialgleichung mit lesten Vurzwigungspunkten lwa Austruek

$$
\begin{gathered}
y=R \Gamma \sin a m\left(f P(x) d x+(), \cos \operatorname{am}\left(f P(x) d x+C^{\prime}\right),\right. \\
\Delta a u\left(f P(x) d x+C^{\prime}\right) \mid,
\end{gathered}
$$

wo $R$ den Algorithmus riner rationalon Funktion brentert, depen lioeffzienten sich algebraiseh aus den Koeffizienten der Differentialgleichung zusammensetzen. Diese Form des allgemeinen Integrals setzt aurh dir Eigenschaft desselben, keine versehicbbaren Verzweigungspunkte zu besitzen, in Evidenz; in der Tat kann die Konstanto ' zwar die Lage dre Pole, nicht aber die der Verzweigungspunkte von $y$ beeinflussen.

Von besonderem Interesse ist noch die folgende Bemerkung. Dir Größe $k^{2}$, der sogenannte Modul dos elliptischen Integrals (32). und dra aus der Umkehrung dieses Integrals entspringenden elliptischen Funktion sin am, ist eine Konstante. Dies ist nicht von vornherein evident: denn wie man leicht einsicht, ist $k^{2}$ nichts anderes als der Wert:

$$
\frac{g_{1}-g_{3}}{g_{4}-g_{1}}: \frac{g_{2}-g_{3}}{g_{2}-g_{1}}
$$

des Doppelverhälthisses (vgl. Nr. 16, S. 59) der vier Glöben $g_{1}, g_{2}, \varepsilon_{3}$, $g_{4}$. die, wie aus ihrer Definition ( $\mathrm{Nr}$. 2S, S. 104) herrorgeht, in allgemeinen Funktionenvon $x$ sind. Dic Eigrnschaft der Differentialgleichung (1), feste Verzweigungsunkte zu besitzen, bewirkt ps, dali dieses Doppelverhälthis einen von $x$ unabhängigen Wort hat.

Sehlesinger. Differentialgleichungen. 


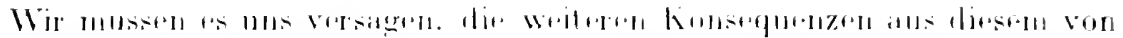

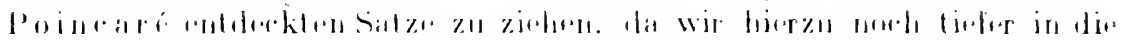

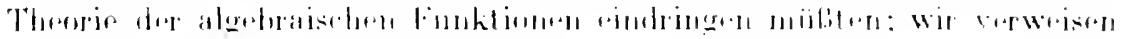

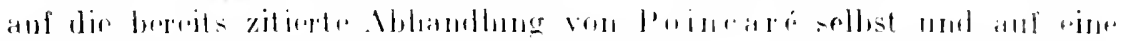

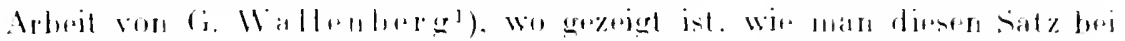

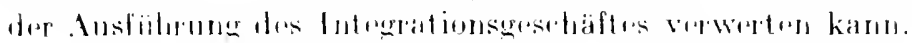

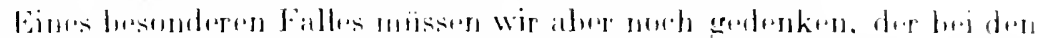

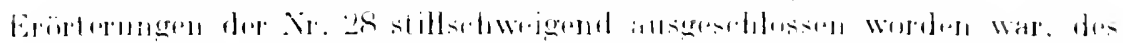

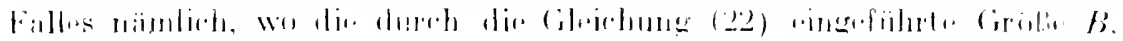

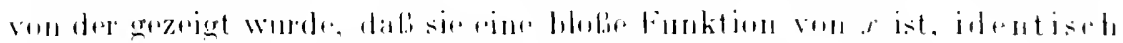

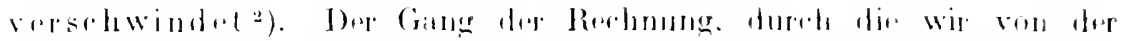

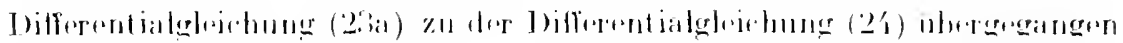

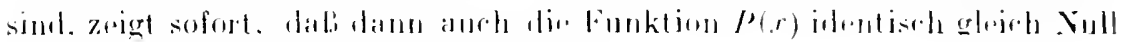

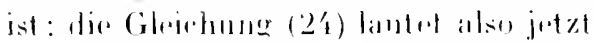

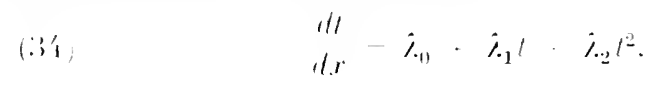

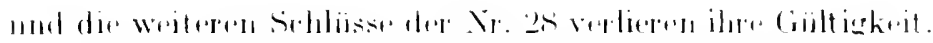

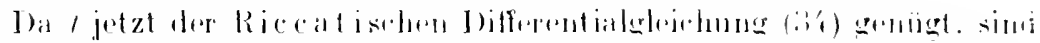

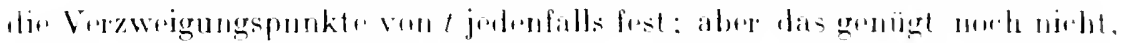

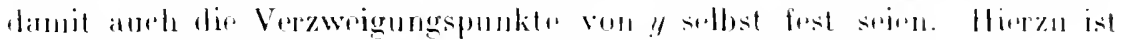

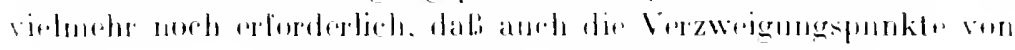

$$
\left.u=1\left(l-a_{1}\right)\left(t-a_{2}\right)\left(t-a_{3}\right)(t-, 1,1, r)\right)
$$

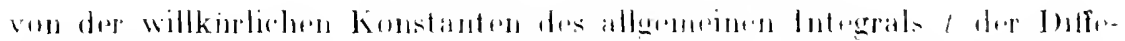

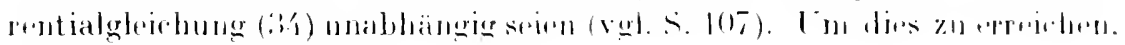
verfahrent wir wie folgt.

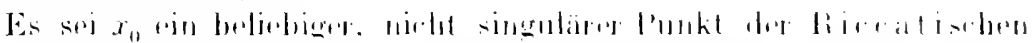

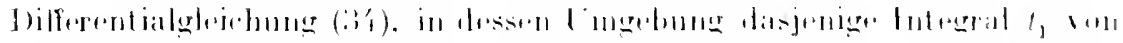

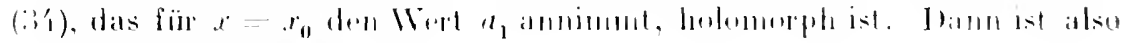
in dieser Jomerounger

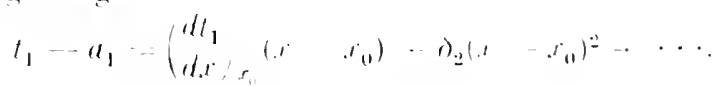

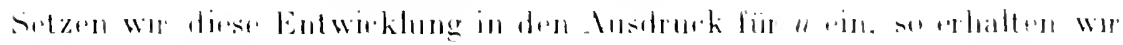

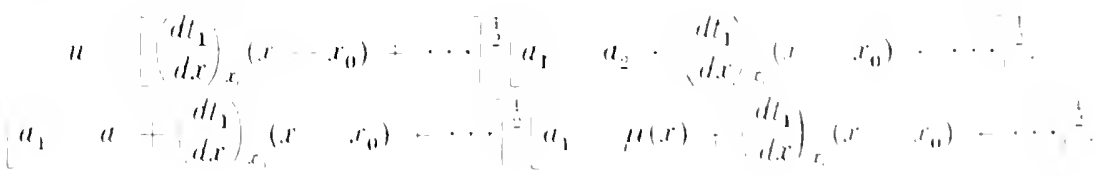

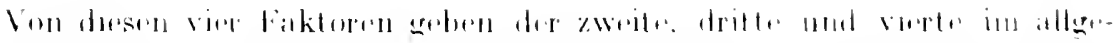

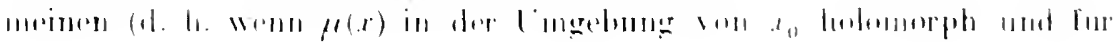

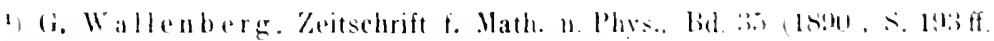

2. Verol. hierfür l'ainlevi. Lerons, S. bitfl. 


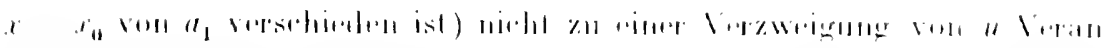

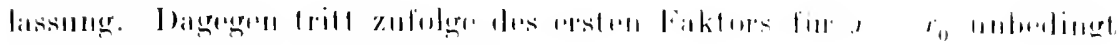

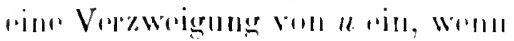

$$
\left.\left(\begin{array}{l}
d l \\
d r
\end{array}\right), \quad, \quad 1\right)
$$

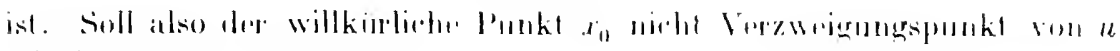
sin kïnnen, so mul.i

$$
\left\{\begin{array}{l}
d 1 \\
(1,)_{\substack{r=r_{1} \\
t=t_{1}}}=0
\end{array}\right.
$$

d. h. also, da $e_{0}$ rin willkirlichere $r$ Mirt ist.

$$
\lambda_{11}+i_{1} a_{1}+i_{22} a^{\prime \prime}-i b
$$

stin, umel ebroso folgt, dab atreh

$$
\begin{aligned}
& \lambda_{a_{1}}+\lambda_{1} a_{2}+\lambda_{2} a_{2}-0 \\
& \left.\lambda_{n_{1}}+\lambda_{1} a_{3}+\lambda_{n_{2}} a_{3}-1\right)
\end{aligned}
$$

spin mïssen. Hirrerarh ist also notwendie

$$
i_{11}=\lambda_{1}-\lambda_{.2}-i i_{1}
$$

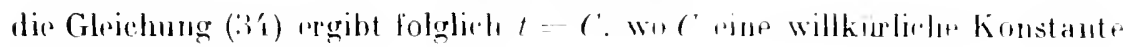
berderitet.

Damn hat alome ulie fiorm

$$
u-1\left(c^{\prime}-a_{1}\right)\left(c^{\circ}-a_{2}\right)\left(c^{\prime}-a_{3}\right)\left(c^{\prime}-\mu(r)\right) \text { : }
$$

jecle Wurzel des Gleichung

$$
C-\mu(x) \div 0
$$

gibt im allgemeinen zu rinem Verzweigungspunkte von u Veranlassung, und da dirse Wurzeln vou $C^{*}$ abhängell. so hätten wir also fiir u versohirbbare Vorzweigungspunktr. Wrun nirlit $/ 1(x)$ koustant ist. Vungokehrt ist dir Brelingung

$$
\mu(r)=a_{1}=1 \cdot 0 \text { onst }
$$

offenbar auch hinprichemt. D)as allgemeine Integral y von (1) lantet dant $\operatorname{nach}(25)$ :

$$
y=\frac{\Phi_{2}\left(C^{\prime}\right)+\Psi_{2}\left(C^{\prime}\right) !\left(C^{v}-a_{1}\right)\left(C^{*}-a_{2}\right)\left(C-a_{3}\right)\left(C^{\prime}-a_{4}\right)}{\Phi_{0}\left(C^{\prime}\right)}
$$

wo die korffizienten der ganzen rationalen Funktionen $\Phi_{2}, \Psi_{2}, \Phi_{0}$, im allgemeinen von $x$ abhängen, sich aber in algebraischer Weist ans der Koeffizienten der Differentialgleichung (i) zusammensetzen.

In diesem Falle ist also $y$ durrle algebraisehr Operationen aus den Koeffizientru der Differentialgleichung zusammengesetzt; wenn diese Koeffizientru z. B. selbst algebraische Funktionen ron $x$ sind, so ist $y$ ebenfalls eime algebraische Funktion ron $x$. 


\section{Adbitionstheorem der elliptischen Funktionen.}

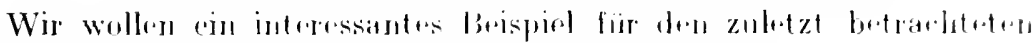

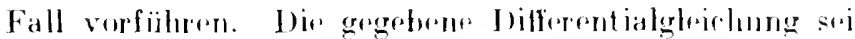

$$
\begin{aligned}
& d y-1, y y(1-y)\left(1-k^{2} y\right) \\
& 14 x(1-x)\left(1-k^{2} x\right)
\end{aligned}=0,
$$

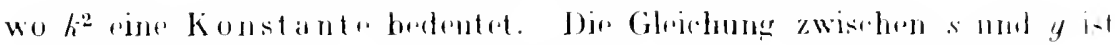
offenbar vom Range Eins: fernes argibt sirh sofort durch lntegration

$$
\int_{0}^{y} 14 y(1-y)\left(1-k^{2} y\right)=\int_{0}^{x} 14 x(1-t)\left(1-k^{2} x\right) \text {. ondet. }
$$

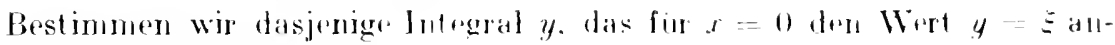
nimmot, s0 molls

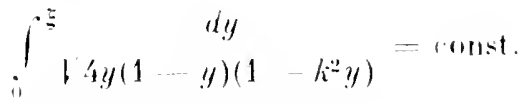

sem, wir haben also fïr das betreffendr. Introgral y

$$
\begin{aligned}
& \int_{0}^{y} 1 y(1-y)\left(1-k^{2} y\right) \\
& =\int_{0}^{x} 14 x(1-x)\left(1-k^{2} x\right) \int_{i}^{d} 14 y(1-y)\left(1-k^{2} y\right) .
\end{aligned}
$$

wo jetzt $\xi$ auch als willkürliche konstante gelten kanm. Setzen wir

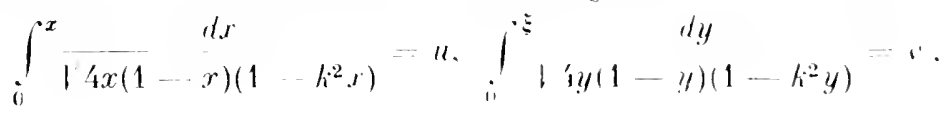

so ist

und wir baben

$$
\int_{i}^{y} 1 y(1-y)\left(1-h^{2} y\right)=u \cdots
$$

(36a)

$$
\left\{\begin{array}{l}
\left.y=\sin ^{2} a+11(u+)^{\prime}\right) . \\
x=\sin ^{2} a 111 u . E-\sin ^{2} a t 11
\end{array}\right.
$$

llieralls folgt ohne weiteres dab die Verzwrigungspunte der Fonktion y von E unabhängig sind, was ïhrigens durch Amwendung der Futheschen

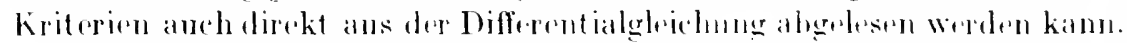

Eigentlieh sedurint hier die Einführung von $t$ in die bifferential-

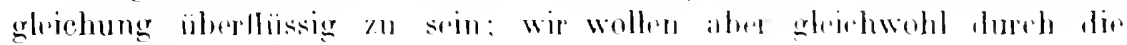
Gileichung

$$
\begin{aligned}
& 1 y \quad 1 x !(1-1)\left(1-\frac{k-2}{12}\right)+111(1-x)\left(1-k^{2} x\right) \\
& 1-k^{2} x !
\end{aligned}
$$

alls der sich leieht 


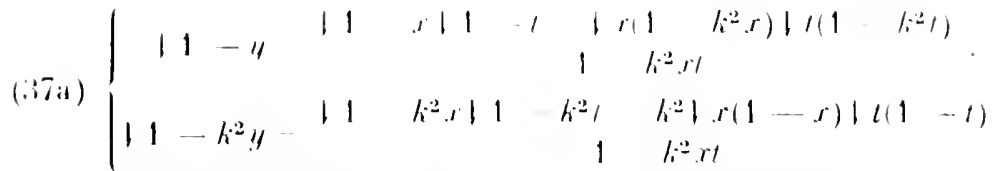

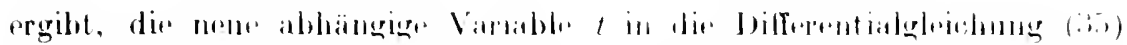

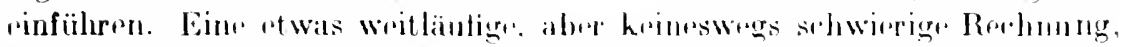

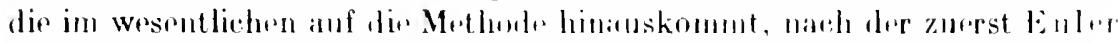

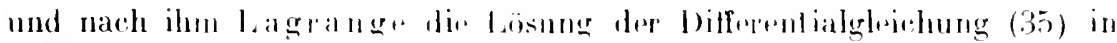

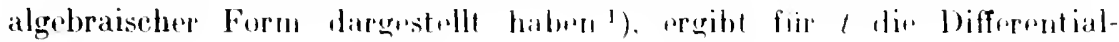
gleichung

$$
d x \quad 11
$$

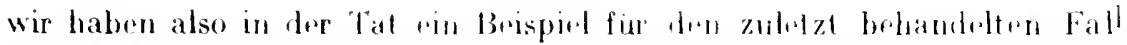
Da die Koeffizienten von (3i) algebraisele finktionen von r sind. ist das allgemeine Integral y solbst algobraisoh: wir rehalten os, inderu wir in (37) für $t$ die willkürliche honstantr.

rinsertzen in der Form

$$
t=5
$$

$$
\text { (38) } 1 y=|x|(1-5)\left(1-k^{2} \xi\right)+1 \Xi 1(1-r)\left(1-k^{2} \cdot x^{2}\right) .
$$

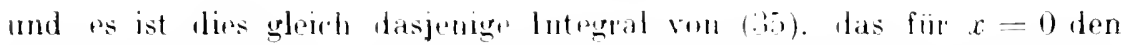
Wert $y=\$$ annimmt. Da dimses Integral aher durch seille Anfangsbeelingungen eindentig bestimmt ist, folgt hieraus, da dis die Glojeloungen (36) und (38) vällig äquivalent silld.

Die Tatsache, dab das allomemin. [ntwgra] der Differentialgleichung (35) algebraisch ist. hat Eul.r (at. a. 1.) entdeckt. Dir fundanentale Wichtigkeit dieses Satzes wird am deutlichsten hrrortroten. Wenm wir in (38) für $y, x$, s ihre Ausdrïck als olliptische Funktionen. wir sie durch die Formeln (36a) gegeben simd, einführen. Dir Glrirhung (3S) lautet dann:

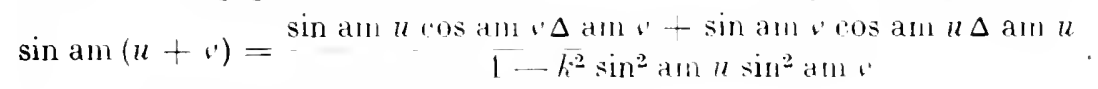

und analog ergeben dir Globlumgern (.97a)

$$
\begin{aligned}
& \cos \operatorname{am}(u+v)=\begin{array}{c}
\cos \operatorname{am} u \cos a m u-\sin \text { am } u \Delta \operatorname{ann} u \sin a m v \Delta \text { dul } v, \\
1-k^{2} \sin ^{2} \operatorname{ann} u \sin ^{2} \operatorname{am} v
\end{array}
\end{aligned}
$$

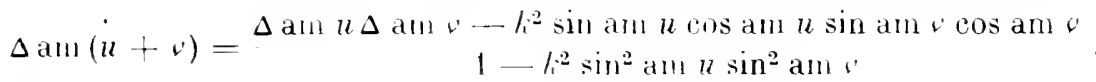

1) Euler 1761. siehe Inst. calc. integralis 1 (1768). Sect. 2. Lap. 6. Opera. Ser. I. vol. 11. S. $391 \mathrm{ff}: \mathrm{Lag}$ a a ge $176 \mathrm{~s}$. siehe Théorie des fonctions analytiques. nouv. éd. 1si3, chapitre XI: vgl. z. B. H. Durège. Theorie der elliptischen Funktionen. 5. Antl. 1908. S. 44. 


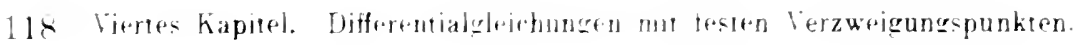

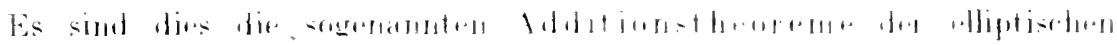
Funtitinnell

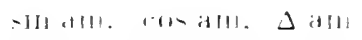

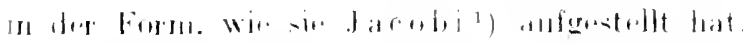

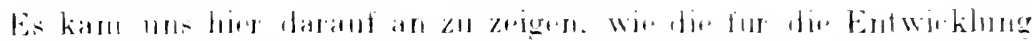

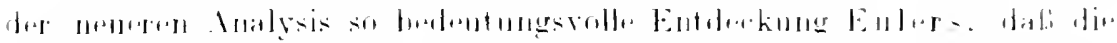

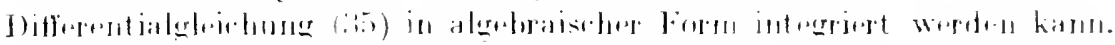

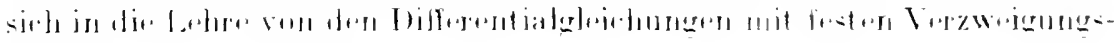

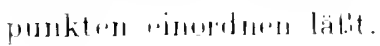

\section{i:. Gleichungen rom Range Zwei. Zusammentassung der Resultate.}

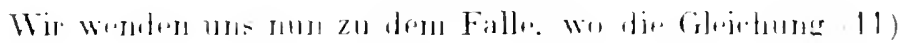

$$
F(x . y \cdot r)=0
$$

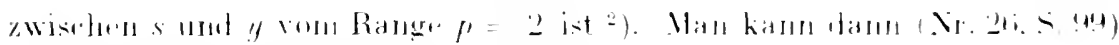

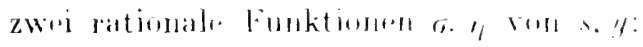

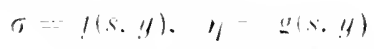

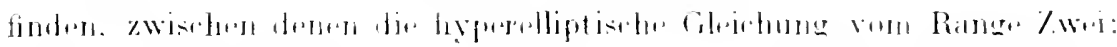

$$
(40) \sigma^{2}=l_{1}(1)=\left(1,-g_{1}\right)\left(1,-g_{2}\right)\left(1,-g_{3}\right)\left(1,-g_{4}\right)\left(1,-g_{5}\right)\left(1,-g_{6}\right)
$$

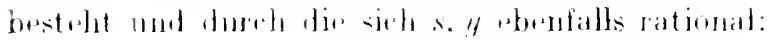

$$
s=q(\sigma ., 1) . \quad y=y\left(\sigma . \eta_{1}\right)
$$

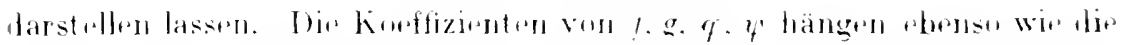

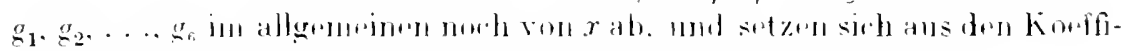

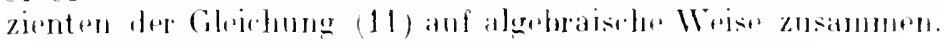

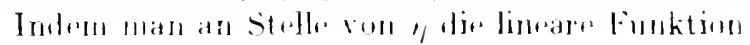

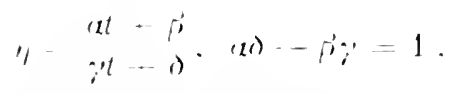

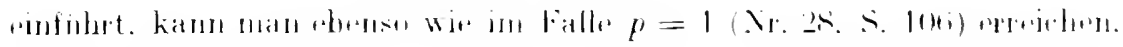

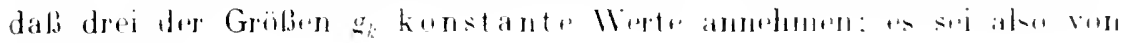
vormbrem

$$
\because_{1}=t_{1} \cdot \quad u_{2} \quad u_{2} \cdot u_{3}=u_{3}
$$

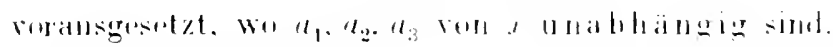

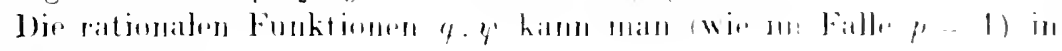
die Fin'll set\%en:

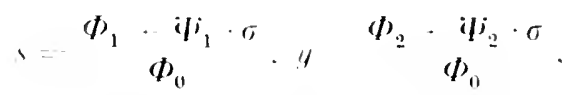

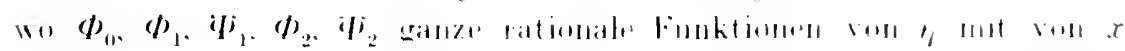

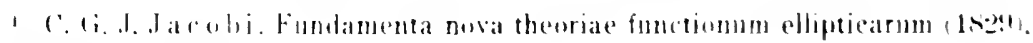
art. 1h. llerke I. St. Sis.

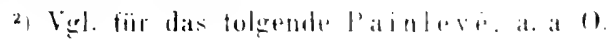




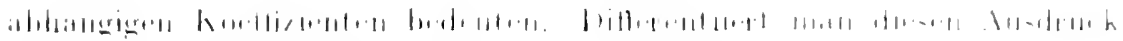

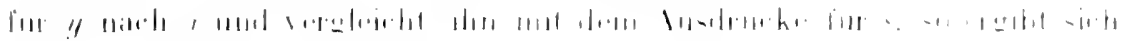

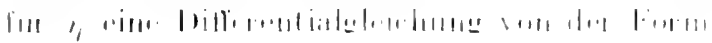

$$
\begin{array}{lll}
11 & 1
\end{array}
$$

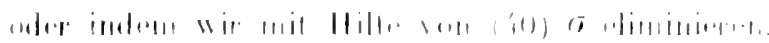

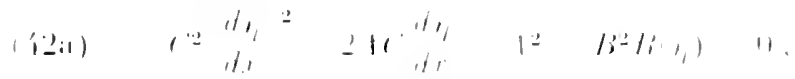

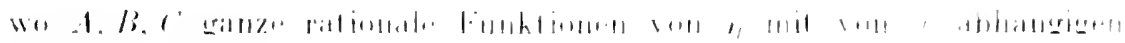

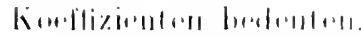

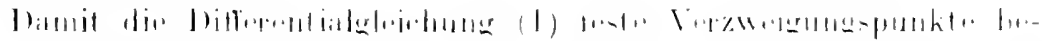

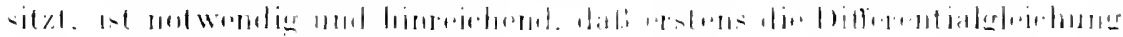

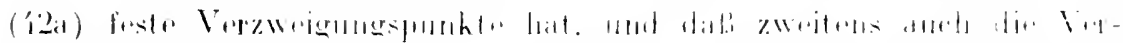

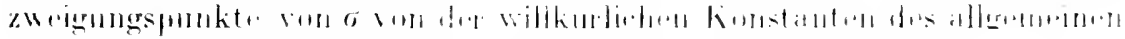

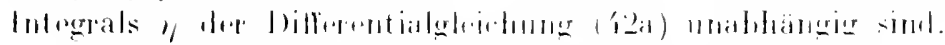

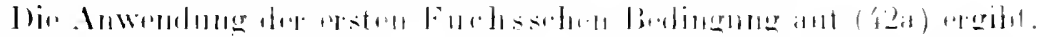

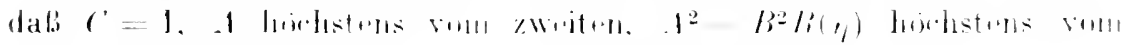

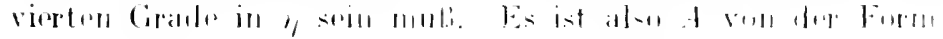

$$
1 \quad 1, \cdot 1,1, \cdot 12,2 \text {. }
$$

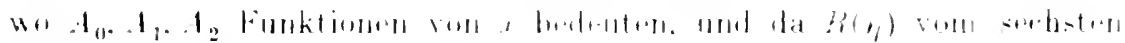

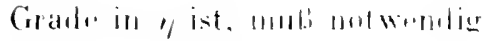

$$
\text { is } 11
$$

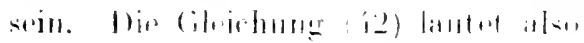

$$
t_{1}-1_{11}+1_{11}-1_{21} 1^{2}=
$$

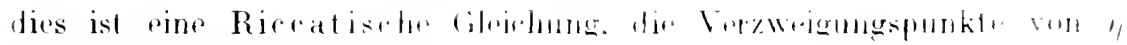
sind also bejeits festgelingt.

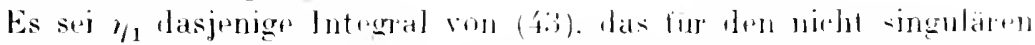

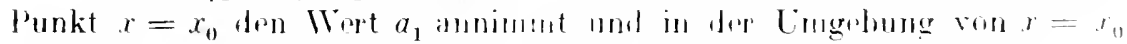

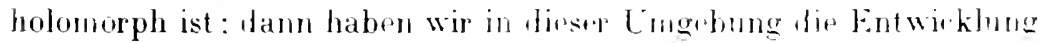

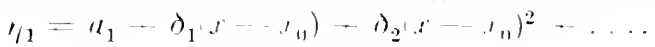

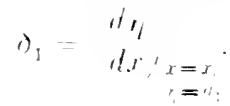

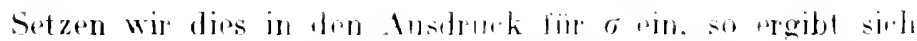

$$
\begin{aligned}
& \sigma=\left\{r_{1}\left(r-r_{11}\right)-r_{2} x-r_{11}\right)^{2}-\cdots \mid \text {. } \\
& \prod_{k=2}^{i}\left[\left(l_{1}-g_{l}+r_{1}\left(t-r_{11}\right)-r_{2}\left(r-r_{11}\right)^{2} \ldots \ldots .\right.\right.
\end{aligned}
$$

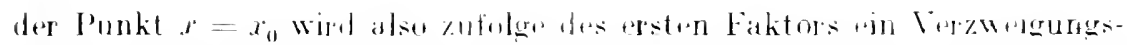

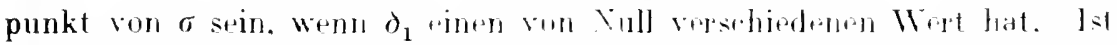

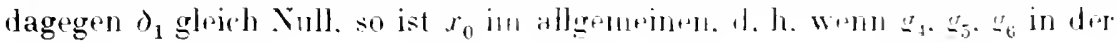




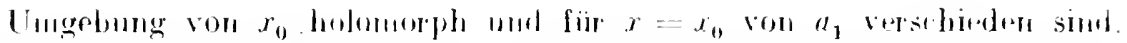
kein Verzweigungspunkt von o. Sull als, der willkïrliche Punkt $r_{0}$, kein

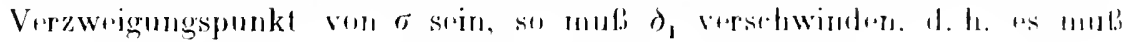

$$
A_{11}+A_{1} a_{1}+A_{2} a_{1}^{2}=0
$$

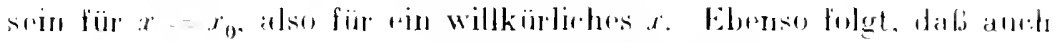

$$
\begin{aligned}
& A_{11}+A_{1} a_{2}+A_{2} a_{2}^{2}=0 \\
& A_{1}+A_{1} a_{3}+A_{2} a^{2}=0
\end{aligned}
$$

sion misseren: wir habme longliels

also nach (

$$
\left.A_{11}=0.1_{1}=0.1_{2} \quad 1\right) .
$$

$$
d x=0
$$

d. h. $\eta$ ist rime willkürliche Konstante $\eta=C^{\prime}$, uml

$$
\sigma=1\left(C-a_{1}\right)\left(C-a_{2}\right)\left(C-a_{3}\right)\left(C-a_{1}\right)\left(C-g_{5}\right)\left(C-g_{6}\right) .
$$

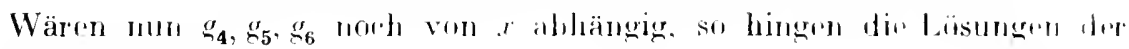
Gleichungen

$$
C-g_{4}=0 . \quad\left(\quad-g_{5}=0 . \quad\left(-g_{6}=0\right.\right.
$$

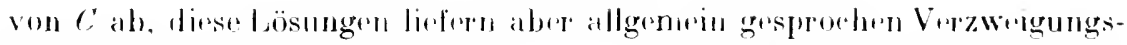

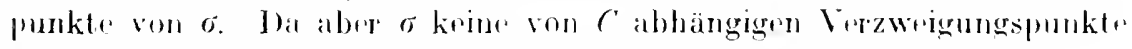

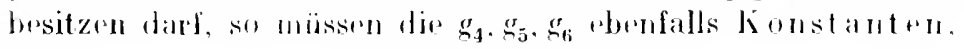

sein.

$$
g_{4}=a_{1}, g_{5}=a_{5}, a_{6}=a_{6}
$$

Wern also die Jifferentialgleirhung (1) feste Verzweigungspunkle.

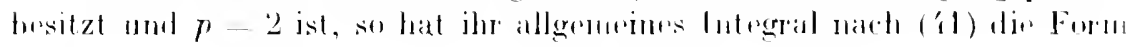

$$
\left.y=\Phi_{2}\left(r^{\prime}\right)+\Phi_{2}\left(c^{\prime}\right) \mid 1 c^{\prime}-a_{1}\right)\left(c^{\prime}-a_{2}\right) \ldots\left(c^{\prime}-a_{6}\right):
$$

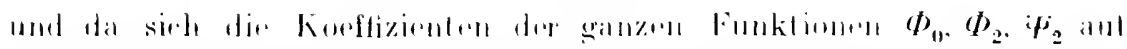

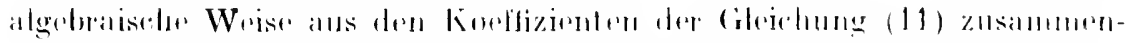

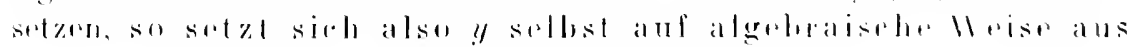

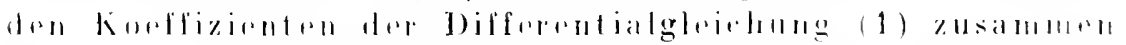
sind dir kerplizienten von (1) inshesondere alentratische

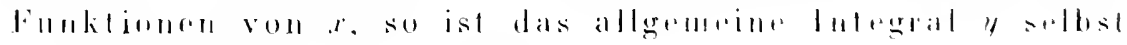

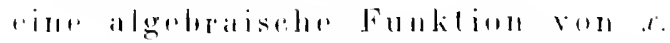

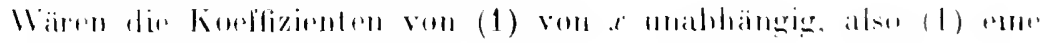

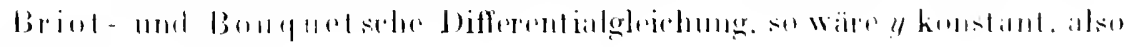

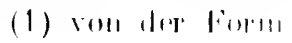

$$
\text { dis } 1)
$$

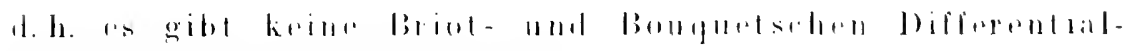
whichungen wour Range lowi. 


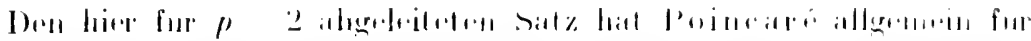

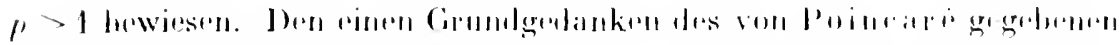

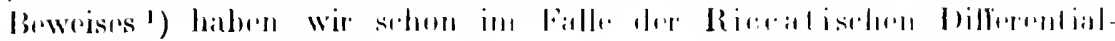

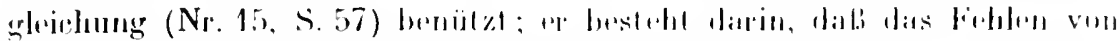

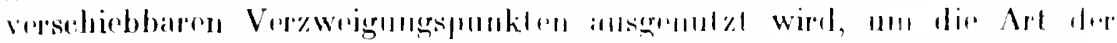

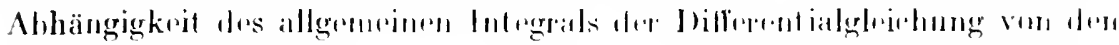

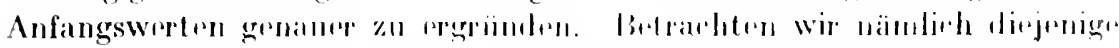

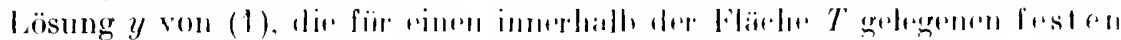

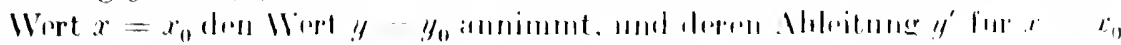

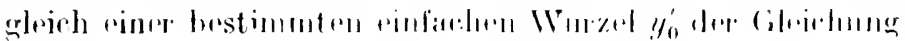

$$
\left.l\left(x, y_{1}, x_{11}\right) \quad 1\right)
$$

wird $\left.{ }^{2}\right)$, so dalli alsil
$\left(1_{10}\right)$
$F\left(y_{0}^{\prime}, y_{10}, x_{0}\right)$
1)

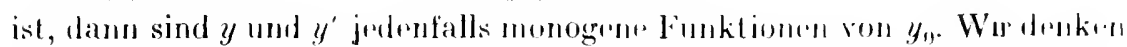
uns num das Integral $y$ von $x_{n}$ aus auf rinem ganz innerhall $T$ verlaufonden Woge nach einem lunktי $x$ hin lortgeselzt, damn sind, da immerhall, $T$ keine Verzweigungspunkte der Funktion y liegest. die Werte won y und y' in $x$ dureh dir in $x_{0}$ rorgeseleriebenen Anfangswerte $y_{0}$, yo rindentign

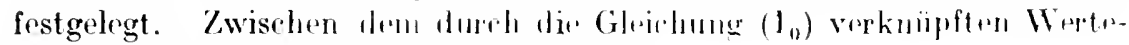

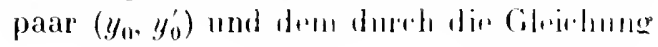

$$
\left.F\left(y^{\prime}, y-x^{\prime}\right)-1\right)
$$

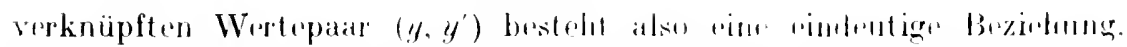

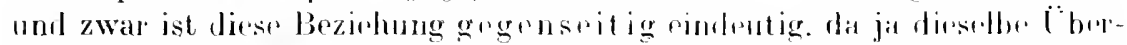

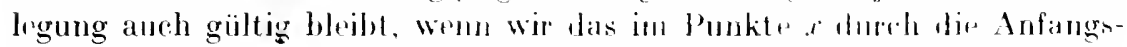

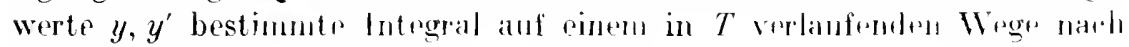
dem Punkte $x_{0}$ hin fortset\%+n. Es ist also

$$
\begin{array}{cl}
y=\Phi\left(y_{1}^{\prime}, y_{0}\right) & y_{0}=\Phi_{0}\left(y^{\prime} \cdot y\right) \\
y^{\prime}=i\left(y_{0}^{\prime} \cdot y_{11}\right) & \left.y_{0}^{\prime}=\psi_{01} \cdot y^{\prime} \cdot y\right) .
\end{array}
$$

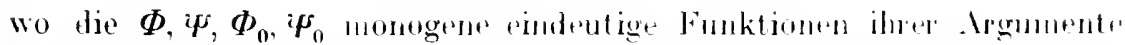
bedeuten, die natiorlich noch $i_{0}$ und $r$ als Parameter enthalten, wud durnt

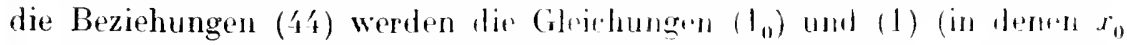
und $x$ als Parameter anzusphen sind) imrinander tramsarmiert. Ein genaueres Eingehen auf den analytischen Charaktw dew zwisehen (y'. y) und $\left(y_{0}^{\prime}, y_{0}\right)$ bestehenden Ahhängrigkeit führt. ähnhwh wi* in der Nr. 15 . zu den Schlusse, dals die eindeutigen Funktionen $\Phi . \Psi^{*} . \Phi_{0} . \Psi_{0}$ rationale Funktionen von $y_{0}^{\prime}, y_{0}$ bzw: $y^{\prime} . y$ sinul: man kam aber hier dasselbe ans einem Satze ron Picarl ${ }^{3}$ ) ersehliefien, der besagt, lalij eine grgenseitiog

1) Poincaré. a. a ().. val. fïr das folgende Pric ard. Traiti d Analyse III (1905), S. $82 \mathrm{ft}$. : P a inlevi, a. a. (1. S. 70) ft.

2) Werte $y_{0}$. fïr die alle Wurzeln dieser Gleichung mehrfache sind. gubt es nur in endlicher Anzahl: diese ïben auf die folgende C̈berlegung keinen Einflub aus.

$\left.{ }^{3}\right)$ Siehe Picard. Traité d.Inalyse III (1908). S. 6i3. 


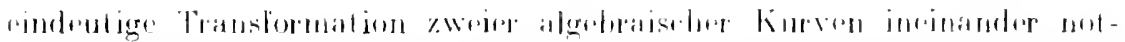

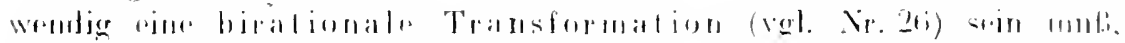

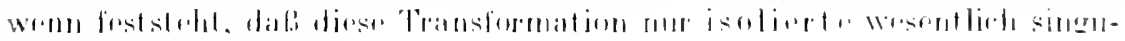

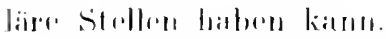

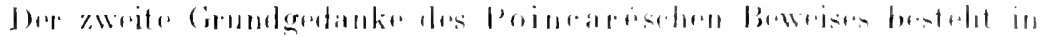

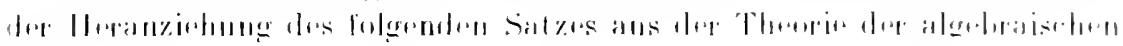
Finnklimorn' $\left.{ }^{\prime}\right)$ :

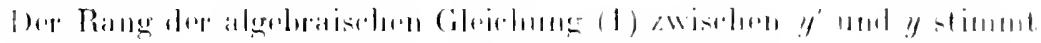

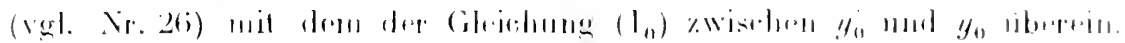

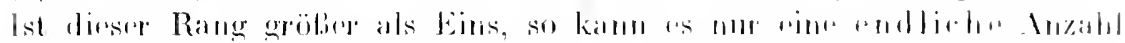

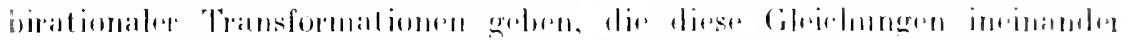

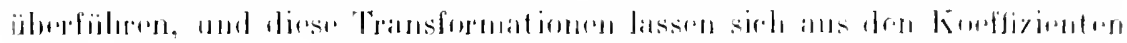

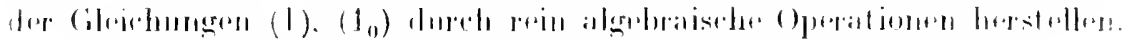

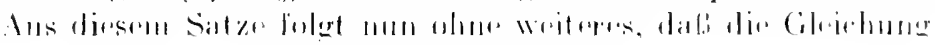

$$
y-p\left(y_{10}^{\prime}, y_{10}\right) \text {. }
$$

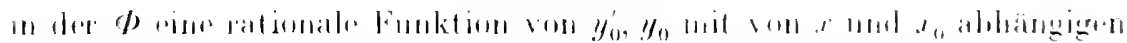

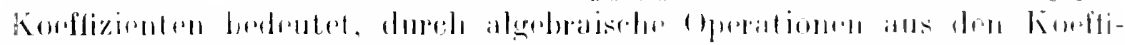

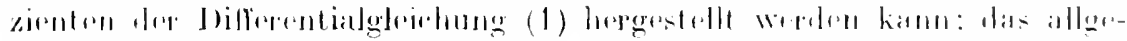

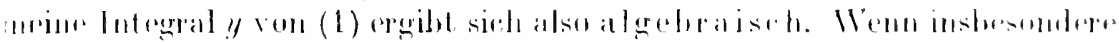

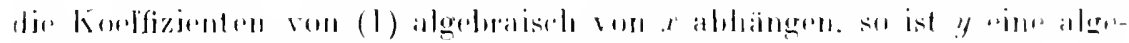

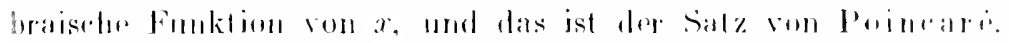

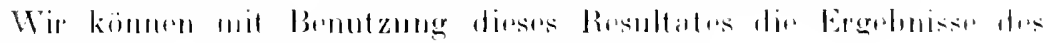

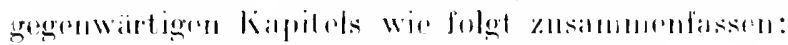

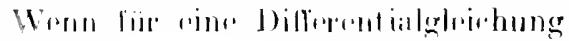

$$
F\left(\begin{array}{l}
d y, y, y \\
d, y
\end{array}\right)-0 \text {. }
$$

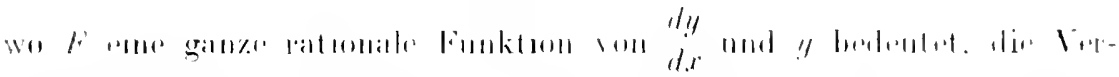

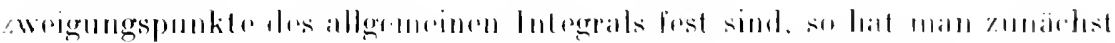

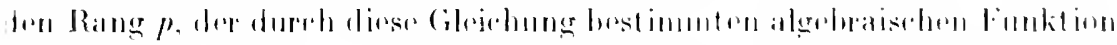

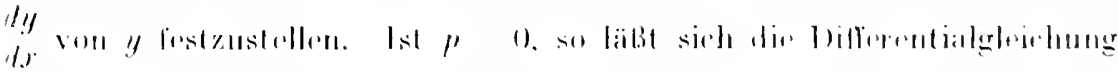

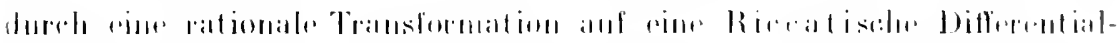

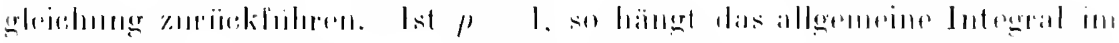

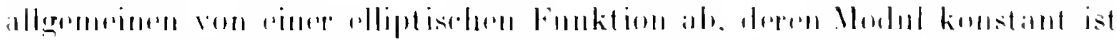

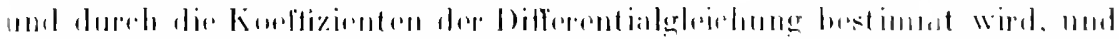

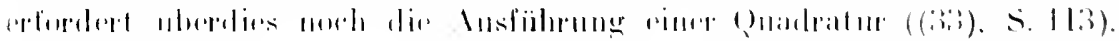

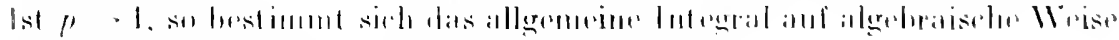

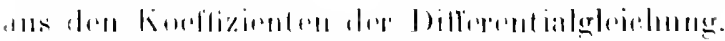

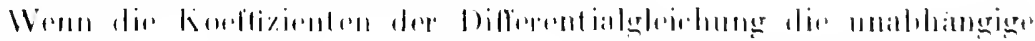

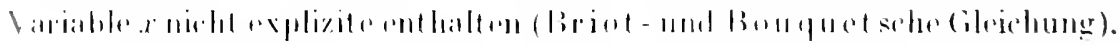

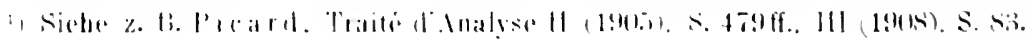




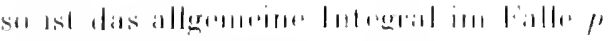

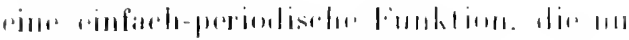

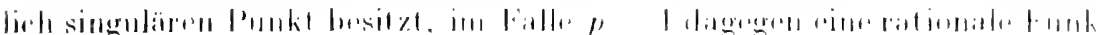
lioll

$$
\text { sill il1111, 1.11s } 111111, \Delta: 111111 .
$$

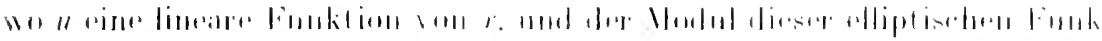

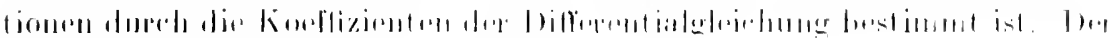

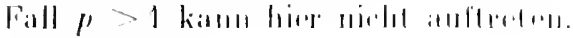

\section{Geschichtliches. Weitere Fragestellungen';}

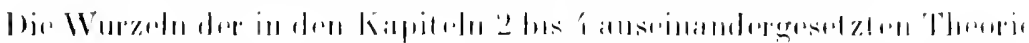

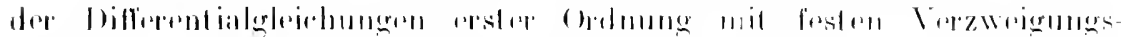

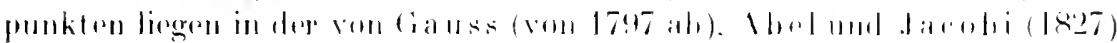

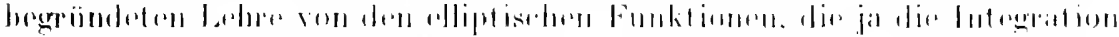

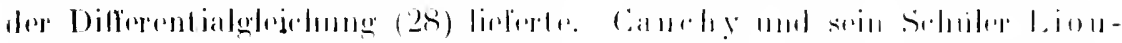

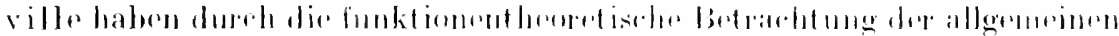

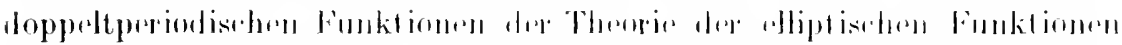

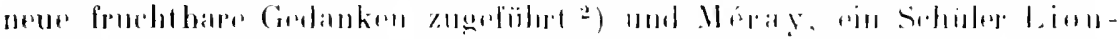

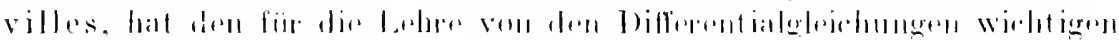

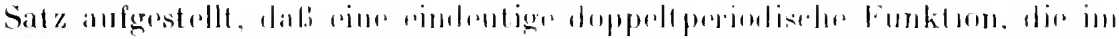

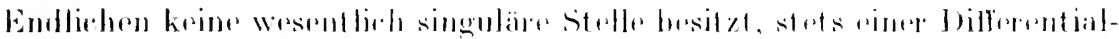

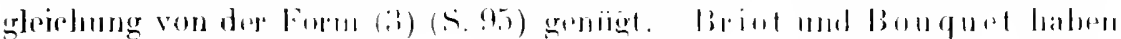

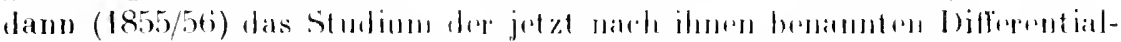

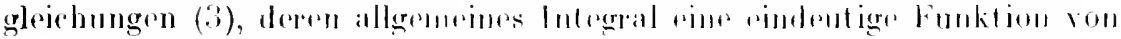

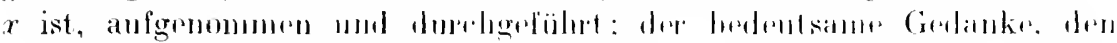

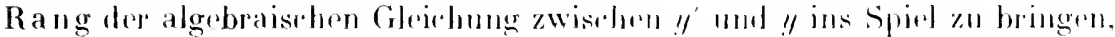

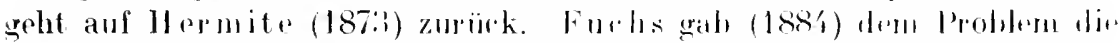

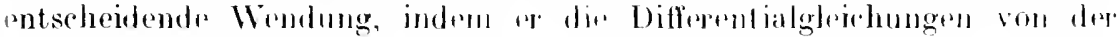

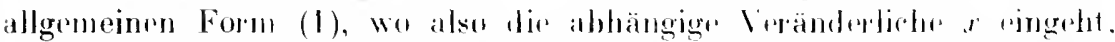
betrachtote und in den Diflerentialgleiwhmgen, dio keine versehiebbaren

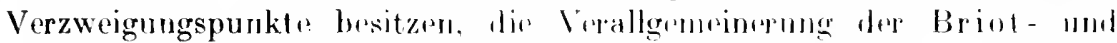
Bouquetschen Gleichnngen mkanmle. Es ist zu bementern, dab dir Me-

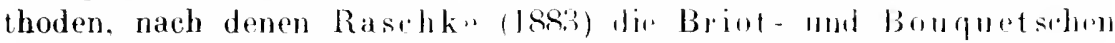
Differentialgleichungen behandelte. rime wertvolle Vorarbeit für alin Untersuchungen von Fuchs bildeten. boindare gab mmittelbar narh dem

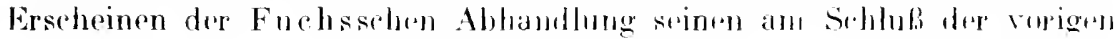

") Vgl. hierzu den Artikel II B (i von E. Hill, in der Enzyklopädie deI Math. Wiss.. Bd. 112 (1921), S. 563 ff.

2) Siehe z. B. Liouville, Lecons sur les fonctions doublement périodiques faites en 1847. Crelles Journal s8 (1880). S. $27 \overline{7}$. 


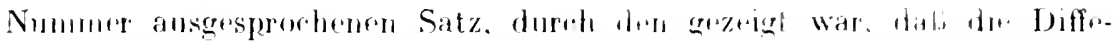

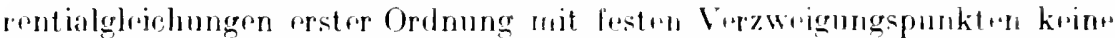
bis rahin unbekannten Transzendenten. iushesondere krino neman rindentigen Funktionen defineren. - Painl.ró eab (1887) dur.h sein.

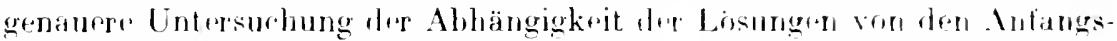

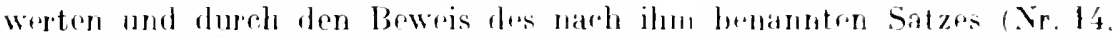

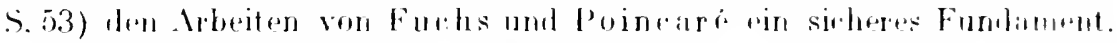

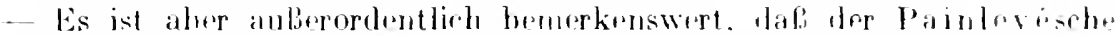

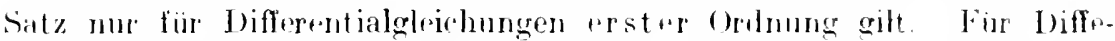
rentialglejehungen höherer Ordnung orler fïr systeme ron Jiffermitialgleichungen erster Ordnumg kann es sehr wohl vorkommen. dab sieh an len mit den Anfangswerten verschiobbarrn singulären Punkten die Intrerale nicht mehr algebroid verhalten'). So hat die Thiferentialgleingung zweitur ordumng

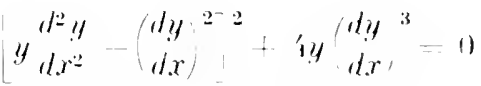

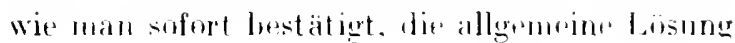

$$
y=C_{1} e^{r} c^{r} \text {. }
$$

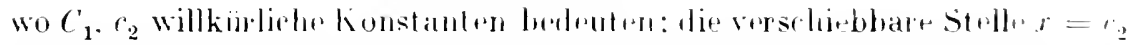

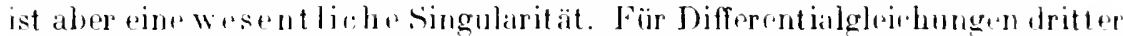

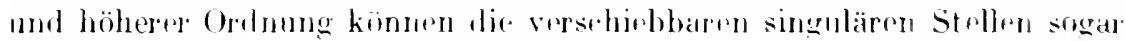
linien refïlen, was - wir Painlevi erezeigt hat - für Differential-

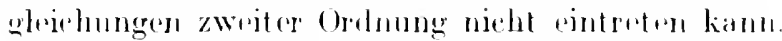

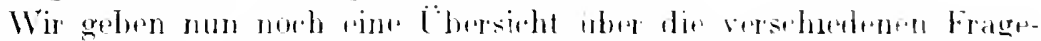

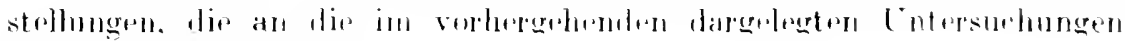
angeknupft worden sind.

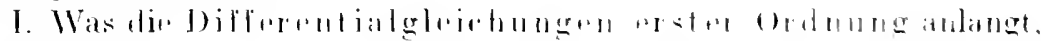
st haben wir

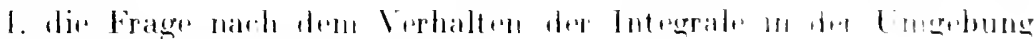

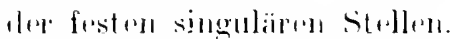

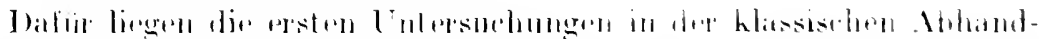

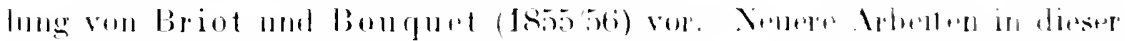

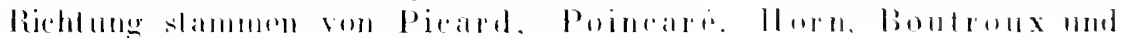

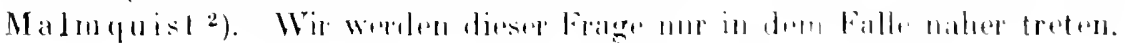

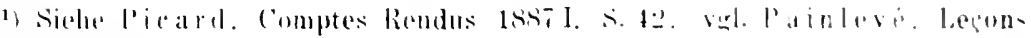
atte. S. 394. $41: 3$.

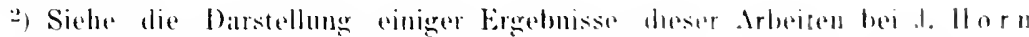

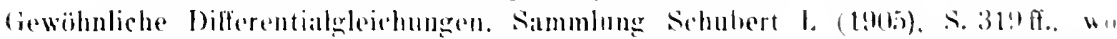

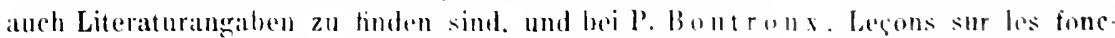
tions ditinies par les inquations differentielles du premier ordre. Paris lons.

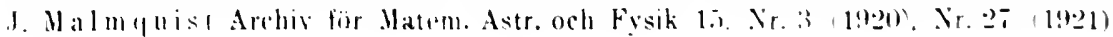




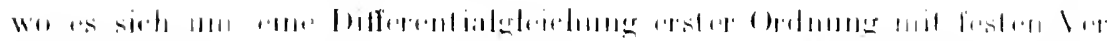

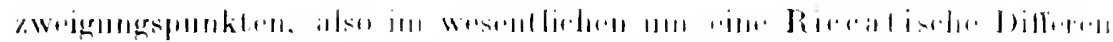

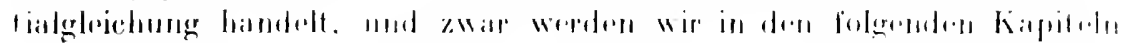

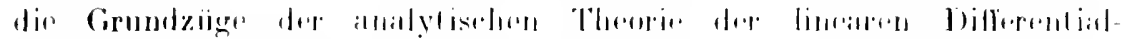

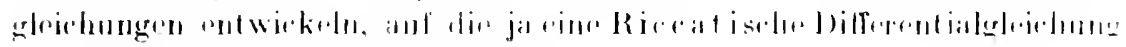

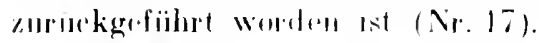

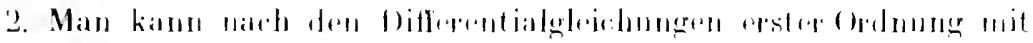

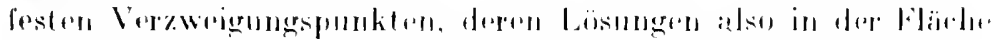

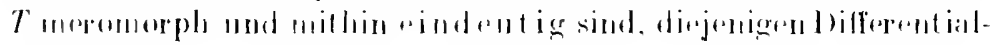

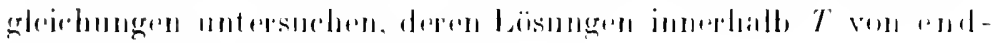
licher Vinlalentierkil sibl.

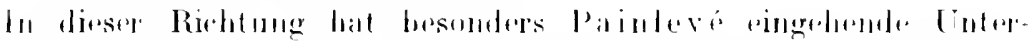

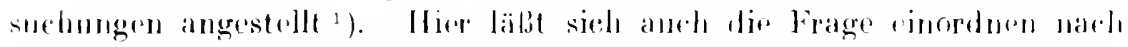

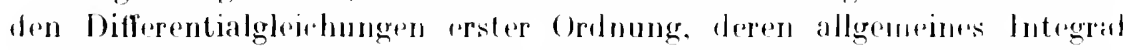
eine algebraische Funktion ler mabhängigen Vorändolichen ist. Sir.

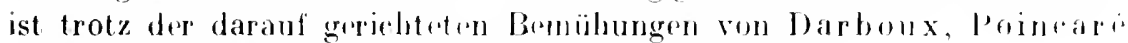
und Autonno 2 ) ron ine liosung noch weit entlernt.

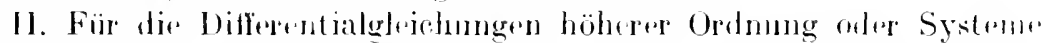
von Differentialgleichungen erster Ordnumg kommen in Frage:

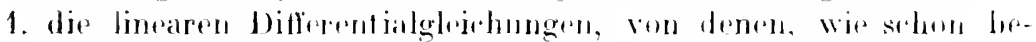
merkt, in den lotgenden Kapitoln die Rode soin wird;

2. die Frage nach den Difterentialgheluhugen zwoiter mod hïherel Ordmung, deren Lösungen krine anderen versehieblearen Singularitäten als Pole hesitzen.

Painlevé hat (von 1900 an) nawh einer ihm eigentiunlichen Mr-

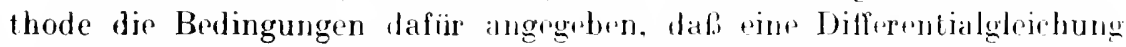
zweiter Ordnung die genannte Eigensehaft besitzt, und hat darlurch u. a. einen Typus von Differentialgheichungen ent dockt, deren allgemeinr Lösung eine neue eindentige Transzendente ist ${ }^{3}$ ). Später (1905) hat Schlosinger gefunden, daß die Theoric der linearen Differentialgleichungen zu eincr Klasse von Differentialgleichungen bezw. von Systemm ron Diflerentialgleichungen beliebig hohrr Ordnung fiihrt, leren Integrale nur verschiebbare Pole haben. Wir weden über diess. Untersuchungen in neunten Kapitel berichten und dort auch die nouere Literatur ibbes. diesen Gegenstand zusamminstellen.

1) Siehe Painlevé. Leçons etc. S. 92 ff.. und Note zu dem in der Fubnote $\left.{ }^{2}\right)$ S. 124 genannten Buche von Boutroux; vergl. ferner J. I a Imquist. Acta Mathematica 36 (1910), S. 297. 42 (1920). S. 317.

2) Siehe Literaturangaben iu dem oben genannten Enzyklopädieartikel von Hilh.

3) Eine Behandlung dieser Painlevéscheı Transzendenten gibt Horn. a. a. 0. S. 379, wo auch Literaturangitben zu tinden sind. 
fionfte kapitul.

\section{singuliare stellen linearer Diflerentialgleichungen.}

\section{Die lineare homogene Differentialsleichung erster Ordnung.}

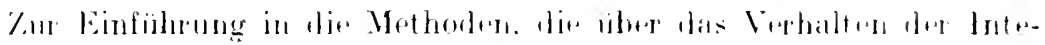

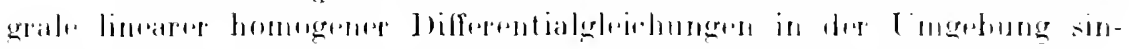

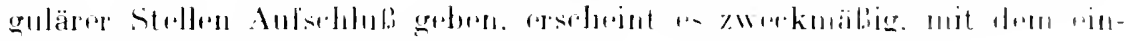

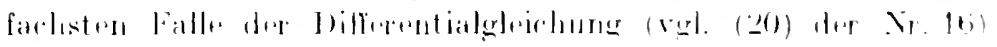

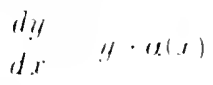

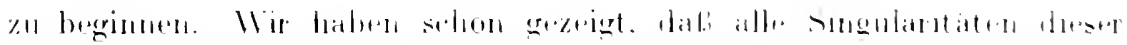

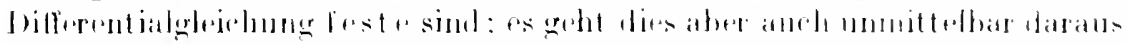

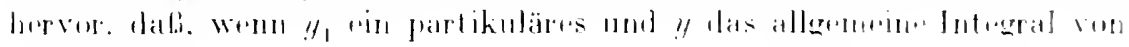
(1) berdintet.

alar,

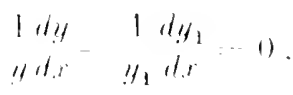

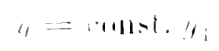

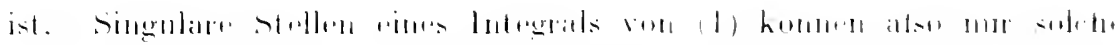

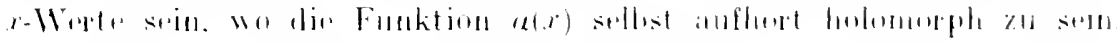

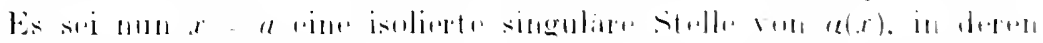

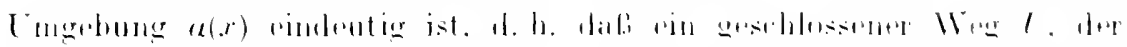

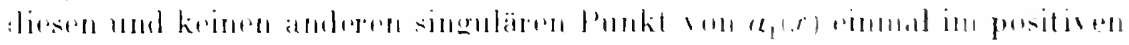

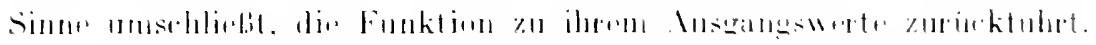

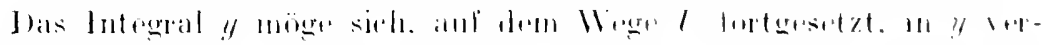

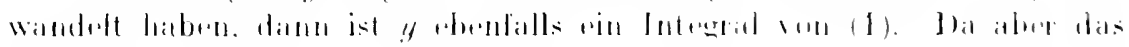

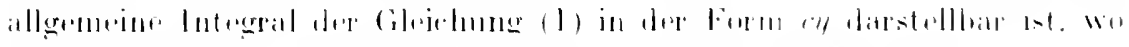

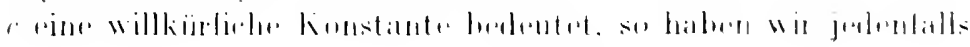

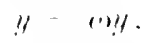

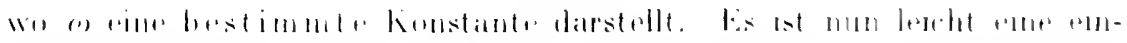

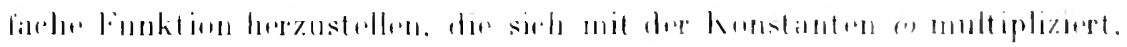

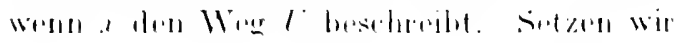




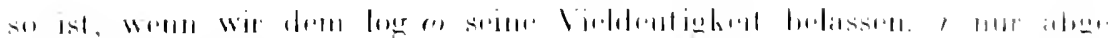

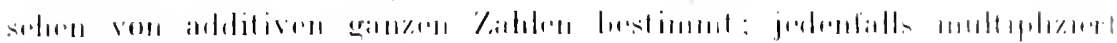

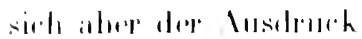

$$
\text { 1) }(1, \quad 11)^{\prime}
$$

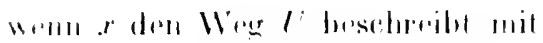

line ()uotiont

$$
\text { i2 (") }
$$

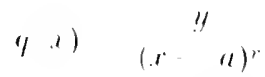

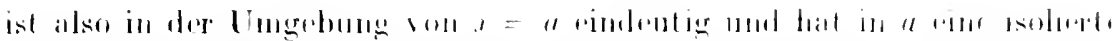

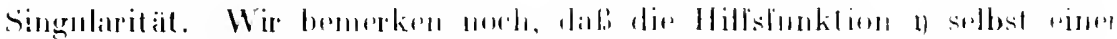

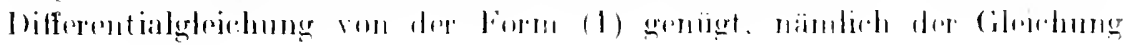

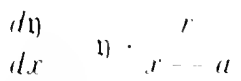

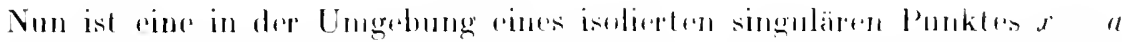

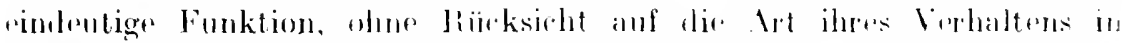

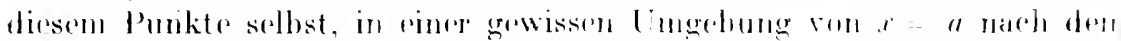

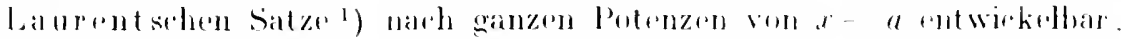

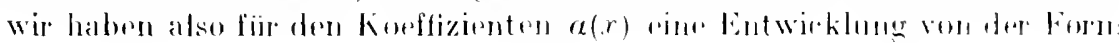

$$
r(, r) \sum_{k=\pi}^{+\infty} r(x \quad a)^{k}
$$

und lim $q(x)$ rine ähntiche Entwicklume

$$
y(x)=\underset{l}{\stackrel{x}{x} \gamma(x)} u)^{k}
$$

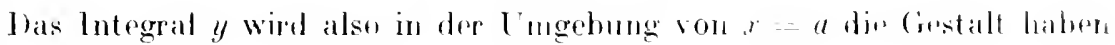

$$
y=(l i-a)^{r} \sum_{k=-\infty}^{+\infty} \gamma_{k}(r-a)^{t}:
$$

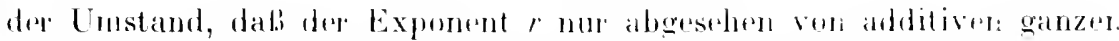

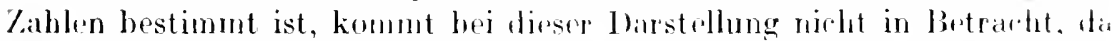

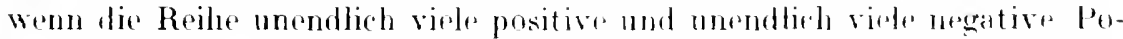
tenzen von $(x-a)$ enthäl, die Äuderung von $r$ mon rine ganze Zahl nur rine Verschiobung der Glieder inmerhalb dor Roihe nath sich zieht.

Dadurch ist das Verhalten dur liosung $y$ in der lingebung der singuären stelle $x=a$ qualitativ bestimmt. Um num noch die Darstellung der Lösung in dieser Umgebung quantitativ angeben zu kömnen. mul: man suchen, $r$ und dip Koeffizienten $\gamma_{k}$ wirklich zu berwhnen, wenn dir Korffizienten $c_{k}$ der Entwicklung von $a(x)$ bekannt sind. J)iese Aufgabr ist. wio man sich dureh Einsetzen der Reihen in die Differentialgleichung solort ïberzengt. dureh Anwendunes der Methode der unbestimmten Koef-

1) Siehe z. B. Knopp. Funktionentheorie (1918), S. 11!) 


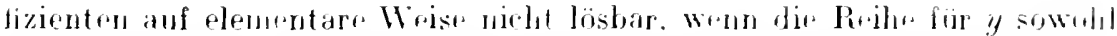

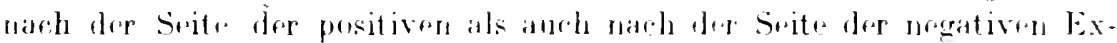

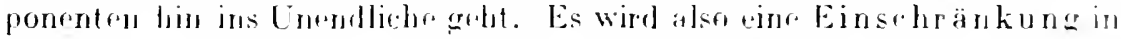

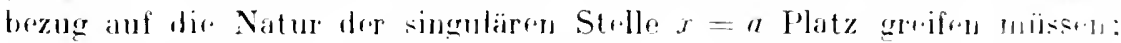

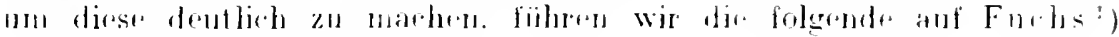
zourürkgehende. Bezerichnung rist.

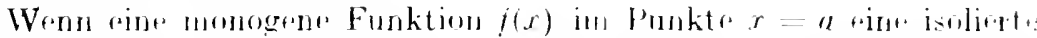

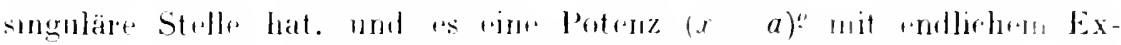

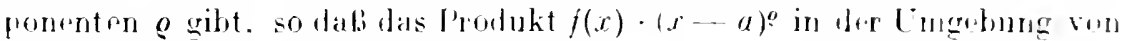

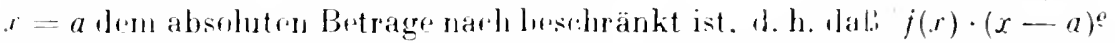

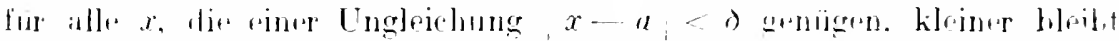

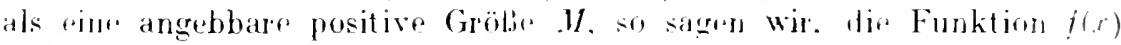

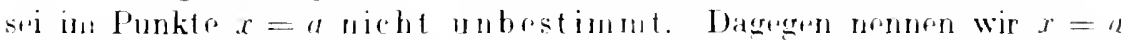
wiren Punkt der Lnbustimmtheit für we Funktion $f(x)$. Wenn fine solehr Potenz $(x-a)^{o}$ nirht "xistiert. Eine ni "ht isclierte singulär" Stelle soll stets als Punkt der Conbestimmiturit angesthen werden. In

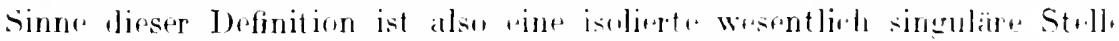
riner eindeutigen Funktion an l'unkt deg lnhestimmtheit: disgegen wird ». B. wine Funktion $q(x)$. dir in $x=a$ ainen Pol ron indlirher Ordnune besitzt. und phenso das Proulukt airer solchun Funktion mit einer Potriz

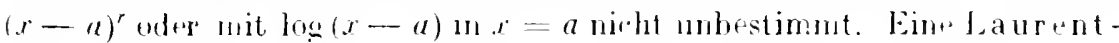
whe Reihe $\sum_{k=-\infty}^{+\infty} \gamma_{k}(x-a)^{k}$ lat die stalle $x=a$ zum Punkte der Cubre stimmtheit dann umd mur dann. wenn die Anzahl der l'otenzen mit nogatiwn Exponenten unendlich erob ist. Wie dirse bezeichnung auf das Verhalten einer Fonktion firr $x=x$ anzuwenden ist. bedarf wohl keiner besonderma brörterung.

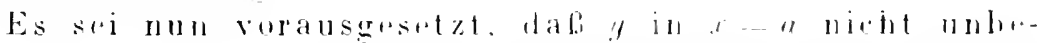

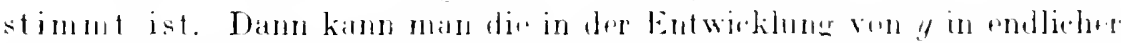

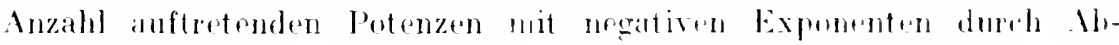

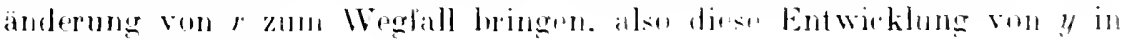
dir Form setzen:
(IV:I)
$y=1.1$
a) $\left(\gamma_{1}+\gamma_{1}(x-a)\right.$
$\cdots+$

in

\section{$\because 0+0$}

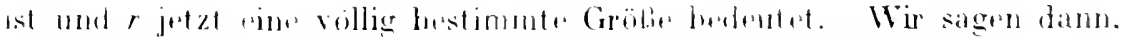

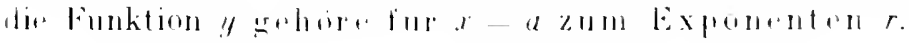

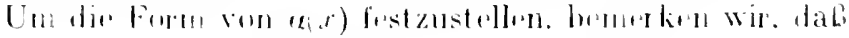

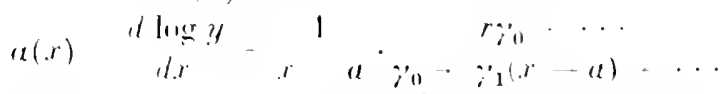

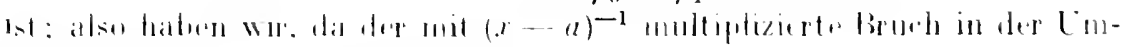
gebung ven $x=a$ holemerph ist,

1) I. Fuchs. 1siti. Werke 11. $\therefore$. 394. 


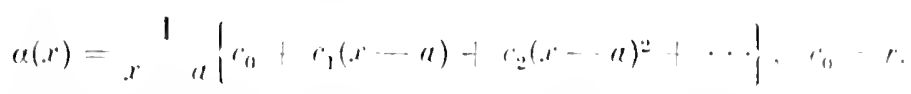

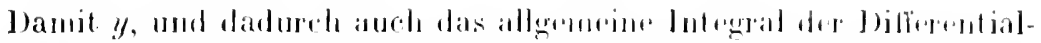

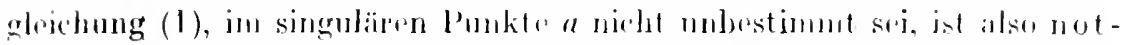

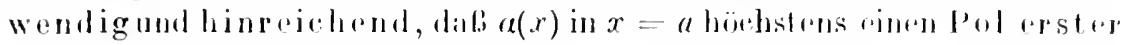

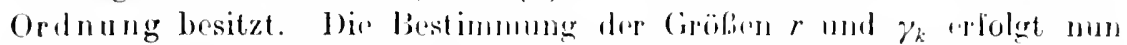
dureh Einsetzen in die Differentialghlegelung nach der Methorle der unbestimmten liocffizienten. Man rehält

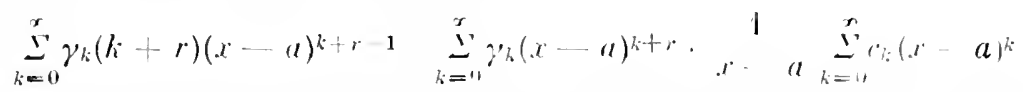

also, inden man beidersojts mit $x$ - a multipliziort und dir Koeffizienten gleich hoher Potunzen von $x-a$ rilimder glojehsetzt:

(2) $\gamma_{\nu}(\nu+r)=c_{0} \gamma_{\nu}+c_{1} \gamma_{\nu-1}+\cdots+c_{\nu} \gamma_{0}$

$(, \quad 0.1,2, \ldots)$, was eine Rekursionsformel für die $\gamma_{0}, \gamma_{1}, \ldots$ liefert. Insbesondere ergibt sich für $z^{\prime}=0$

$$
\gamma_{0}^{\prime} r=r_{0} \dot{\gamma}_{1} \text {. }
$$

es bleibt also das von Null versehieden rorausgesetzte $\gamma_{n}$ willkürlich, und der Exponent $r$ bestimmt sich zu $c_{v}$, d. h. er ist gloich dem Koeffizienten von $(x-a)^{-1}$ in der Entwicklung von $a(x)$ oder dem Cauchyschon sehen Residuun von $\alpha(x)$,

$$
r=\operatorname{Ros}_{x=a} \alpha(x) .
$$

Wir laben also in diesen Falle die quantitative Bestimmung dar Entwicklung des Integrals geleistet.

Wir gehen nun einen Seluritt weiter, indem wir statt uns wie bisher auf die Umgebung einer einzelnen singulären Stelle zu beschränken, das Verhalten in der ganzen Ebene studieren. Wir wollen zuvöderst $a(x)$ als allenthalben eindeutige Funktion von $x$ voraussetzen und dann die Forderung aufstellen, daß $y$ für keinen Punkt der $x$-Ebene unbestimmt sein möge. Dann darf $a(x)$ in der ganzen $x$-Ebene (den Punkt $x=\propto$ eingeschlossen) keine anderen Singularitäten als einfache Pole besitzen, und hieraus folgt '), daß $\alpha(x)$ eine rationale Funktion sein muB.

Es seien die in Endlichen gelegenen einfachen Pole von $\alpha(x)$

$$
a_{3}, a_{2}, \ldots, a_{\sigma}
$$

so hat also $a(x)$ die Gestalt

$$
a(x)=\frac{h(x)}{\left(x-a_{1}\right)\left(x-a_{2}\right) \ldots\left(x-a_{\sigma}\right)},
$$

wo $h(x)$ eine ganze rationale Funktion ron $x$ bedeutet. Um noch dir Bedingung dafür zu finden, daß auch $x=\infty$ kieine Unbestimmtheitsstelle von $y$ ist, führen wir in die Differentialgleichung (1)

1) Siehe z. B. Knopp. a. a. O., S. 136. 
Fünftes Kapitel. Singuläre Stellen linearer Differentialgleichungen.

$$
d ! y=\left(a-n a_{1}\right) \ldots(x)
$$

durch diu Prinsformation

$$
r=\begin{aligned}
& 1 \\
& \xi
\end{aligned}
$$

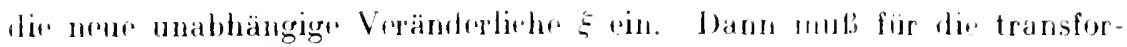

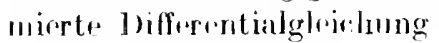

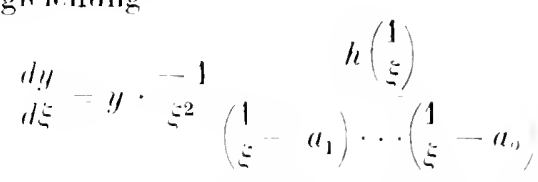

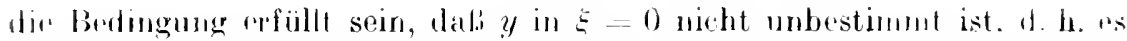

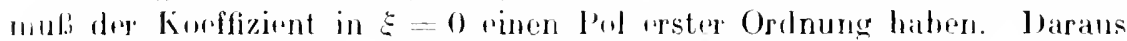

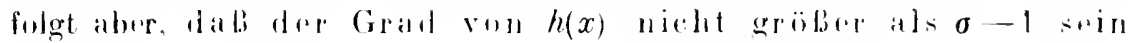
(1) If f.

Donkt man sirh damn $u(r)$ in Partialbriolur zerlegl:

$$
r(x)=\sum_{k=1}^{0} r_{k} a_{k},
$$

so regibt sieh das allermenum hutegral von (1) in der Form

$$
\text { !l= ronst. } \prod_{h}^{0}\left(x-a_{k}\right)^{r}
$$

in Übereinstimmung nit Anu oben fii] die Exponenten $r$ gofumdenen Ergebnis.

Dagegen wïrde, wenn ler firad $m$ von $h(x)$ gröber als o- I ware.

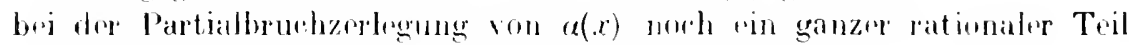
$g(x)$ vom Gratr m- $\sigma$ alltrotent.

$$
r(x)=m(x)+\stackrel{\ddot{n}_{1}}{y_{k}} r_{k} .
$$

und has allgumuine lotigral vou (1)

$$
y=e^{f} y(x) d x \prod_{1}^{\prime \prime}\left(x-u_{k}\right)^{r} k
$$

häte m diesem Falle in $x=\infty$ rimen l'unkt der l nbestmunthrit.

J)ir. I)ifferout ialgleichmng

$$
d y=y \cdot \sum_{k=1}^{n} x-r_{k}
$$

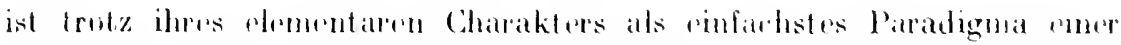

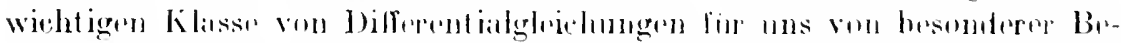

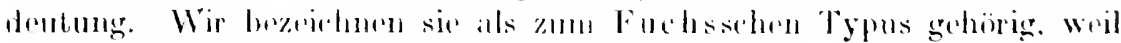

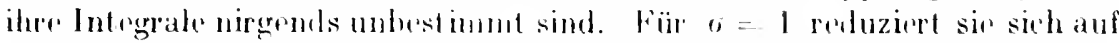

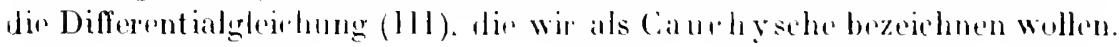




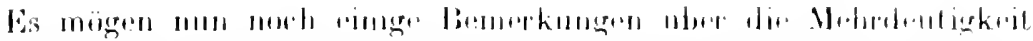

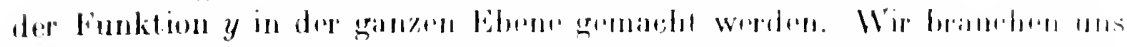

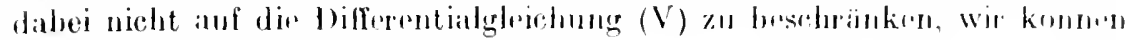

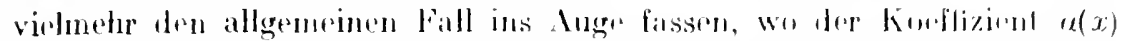

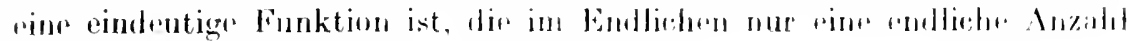
singularer l'unkte $a_{1}, a_{2}, \ldots, a_{0}$ besitzt.

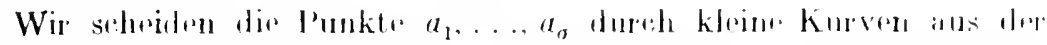
Ebene aus und verbinden diese dureh bohnitte $l_{1}, l_{2}, \ldots, l_{a}$ mit dem IJ-

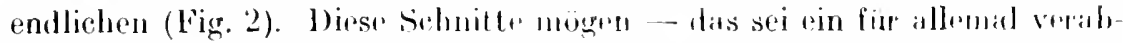

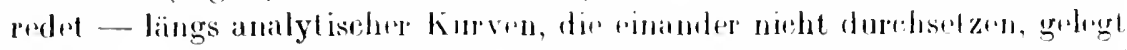

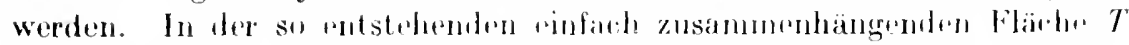
ist das lutegral $y$, tas fiir $x=x_{0}$ dun Antiangswert $y=y_{0}$ annimul, allenthalben holomorph, also rimbentign bestimmt. Es fragt sich nun, was mil y geschicht, wenn wir nicht innorhalh $T$ verbleiben, sontern auf rimm Wugr $u$ fortsetzen, der gewisse der Quersibutto l. überschreitet. Wenn dann y in y iibergegangen ist, so genügt auch $y$ dor. J)ilf.rentialgleichung (1), es ist also $y=c_{n} y$. wo $c_{u}$ rine für den 1 reg $u$ charaktr-

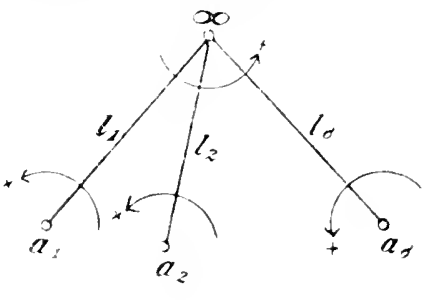

Fis. 2. ristische Konstante bedrutet. Fiir dir Wirkung des Wioges 11 anf diu Wertänderung der Funktion ist aber non maligebend, in welcher Firihrnfolge und in welelenes Sinne diestr Wogg dir anzelnen sehnitte $l_{1} \ldots l_{\text {, }}$ durehquert. Wir menten rinen tinfachen Selhleifenweg s, pinen solehen, der nur einen Schnitt l, ïberschroitert, und sagen, dir lंberschreitung erfolge im positiven Sinne. Wrnn der I'bergang in derjenigen Richtung erfolgt, die der Brwegung rines im Punkte $a_{\text {, }}$ befestigt gridachten Uhrzeigers entgegengesetz ist. Analytisch kam diestr Sinn also dadurch definiert werden, dal. das Argument von $x$ - $a_{s}$, in diesom Sinne wächst, oder dal.) siete $\log \left(x-a_{y}\right)$ bei Übersehreitung von $l_{3}$ in

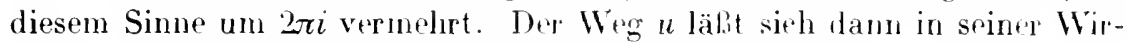
kung auf $y$ durch die Aufeinanderfolge soleher teils in positiven, teils itn negativen Sinne durchlaufenten Schleifenwege s, arsotzen. Multipliziert sich $y$ bei der Fortsetzung längs des im positiven Sinne durchlaufenen Schleifenweges. $s$, d. h. also nach rinmaliger Umkreisung dos

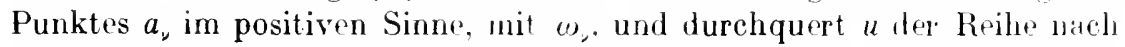
$g_{\alpha}$-mal den Schnitt $l_{\alpha}$, dann $g_{j}$-mal den Schnitt $l_{j}, \ldots$. wo $g_{\mu}, g_{j} \ldots \ldots$ positive oder negative ganze Zahten bedeuten, je nachden die Ubreschreitung im positiven oder negativen Sinne $\left|g_{g}\right|$-mal erfolgt, so ist

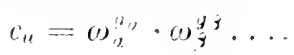


Man sieht, dal. anf dirse Weise die Mchrdeutigkeit der Funktion y vollkommen beherrscht wird, wenn man die zn den einzelnen Sehleifenwegen $s_{2}$ gehöricen Multiplikatoren os, kennt.

Dir quantitation Bestimmung dieser $\omega$, ist in Falle der Differentialgleichung von Fuchsschen Typus sofort gegeben, inden nämlich

$$
(i)_{\nu}=e^{2 \pi i r_{\nu}}
$$

$(2=1,2, \ldots, a)$

ist. Ferner kommt es hier, d. h. für die fine Differentialgleichung (1). nur darauf an, wie oft der Weg $u$ im ganzen die Schnitte $l_{1} \ldots \ldots l_{n}$ überschritten hat, wobei Überschreitungen desselben Schnitts in positiven und negativen Sinne sich in ihrer Wirkung aufhrben. weil ja in dem Ausilruck (VI) der Wert des Produktes von der Reibenfolge der Faktoren unablıängig ist. Diese hier trivial erscheinende Bemerkung wird später Bedeutung gewinnen, wenn wir die analogen Betrachtungen für lineare Differentialgleichungen von höherer als der ersten Ordnung oder für Systeme von Differentialgleichungen erster Ordnung durchführen werden.

\section{Systeme vou zwei linearen Differentialgleichungen. Matrizenkalkül.}

Die in der vorigen Nummer gegebenen Entwicklungen beziehen sich auf den besonderen Fall der Riceatischen Differentialgleichung (15) der Nr. 15, wo $a_{0}=0, a_{2}=0$ ist. Im allgemeinen Falle hatten wir ein System von zwei linearen Differentialgleiehungen erster Ordnung oder eine lineare Differentialgleichung zweiter Ordnung als der Riceatischen Differentialgleichung gleichwertig gefunden. Wir wollen nunmehr die Theorio der Systeme von der Form

$$
\left\{\begin{array}{l}
d y_{1}=y_{1} a_{11}+y_{2} a_{21} . \\
d x \\
d y_{22}=y_{1} a_{12}+y_{2} a_{22} \\
d x
\end{array}\right.
$$

entwiekeln und werden dabei sehrittwrise die Analogie zu verfolgen suchen zu den in der $\mathrm{Nr}_{\mathrm{r}} 34$ für die eine Differentialsleichung (1) gefundenen Ergebnissen. Die einzelum Siduste sind durh die mit den römischen Ziffern (I) bis (VI) bezeichneten Gleidhungen angedeutet.

lin Existenzbeweis fïr die Integrale des Systems ist bisher nur fär reelle Wrete der Veränderlichen $x$ erbratht am den Beweis für das komplexe Grbiet bequem auseinandersetzen zu können, sollen cinige formale Entwicklungen vorausgesehicht werden. bei denen man vorerst. wenn von lutegralen die Redr ist. an die inn redlen Gebiete definierten denken mag. 
Es seien zwoi partikulär Jiosungssysteme voll (A) $y_{11}$. $y_{12}$ und $y_{21}, y_{22}$ doreh ilore Anfangswerte fiir $r=x_{0}$ eregebon:

$$
\begin{aligned}
& y_{11}-y_{11}^{(0)}, y_{12}-y_{12}^{(0)}, \\
& y_{21}=y_{21}^{(1)}, y_{22}-y_{22}^{(1)} ;
\end{aligned}
$$

dann bestehen also dio vire Girichungen

$$
\text { dyik } y_{11} u_{1 k}+y_{i 2} u_{2 k} \text {. }
$$$$
(1,5=1,2)
$$

Brdenten nun $y_{1}$, y. das allgemoine lösungssystem, so sotzen wir

$$
y_{1}=r_{1} y_{11}+c_{2} y_{21}, y_{2}=c_{1} y_{12}+c_{2} y_{22}
$$

und stelken uns die Aufgabe, die Natur dur Größen $r_{1}, c_{2}$ zu erforschen.

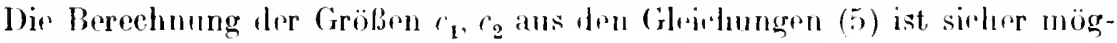
lich, wenn die Determinante

$$
\boldsymbol{t}=\begin{aligned}
& y_{11} y_{12} \\
& y_{21} y_{22}
\end{aligned}
$$

nicht identisel verschwindet. Wir nohmen darum an, dal.s dir Anfangswerte (4) so gewählt seien, daß

$$
t_{0}=\begin{aligned}
& y_{11}^{(0)} y_{12}^{(1)} \\
& y_{21}^{(0)} y_{2:}^{(1)}
\end{aligned}, 0
$$

sei. Dann ergeben sich die $r_{1} . c_{2}$ aus (5) als differentiierbare Funktionen von $x$. Wir wollen nun die Gleichmugen $(5)$ differentiieren und für die Ableitungen $y_{i k}^{\prime}$ ilore Werte aus $\left(A^{\prime}\right)$, fiir die $y_{k}^{\prime}$ ilıre Werte aus $(A)$ einsetzen; dann kommt

$$
y_{1} a_{1 k}+y_{2} a_{2 k}=c_{1}\left(y_{11} a_{1 k}+y_{12} a_{2 k}\right)+r_{2}\left(y_{21} a_{1 k}+y_{22} a_{2 k}\right)+c_{1} y_{1 k}+c_{2}^{\prime} y_{2 k}
$$

und mit Berücksichtigung der Gleichungen (i)

$$
c_{1}^{\prime} y_{1 k}+c_{2}^{\prime} y_{2} k=0 \text {. }
$$

Da aber die Determinante I nicht identisch verschwindet, so folgt aus den beiden Gleichungen (6), $c_{1}^{\prime}=0, c_{2}^{\prime}=0, \mathrm{~d} . \mathrm{h}$. dit $c_{1}, c_{2}$ sind Konstanten. Das ist in gewissen Sime das Analogon der Gleichung (I).

Diese Analogie kann abor noch sinnfälliger gemacht werden. wenn man neben den $y_{i k}$ ein zwoites Paar von partikulären Lösungen $u_{11}, u_{12}$ und $u_{21}, u_{22}$ betrachtet, für das auch dip Determinante

$$
E=\begin{array}{ll}
u_{11} & u_{12} \\
u_{21} & u_{22}
\end{array} \mid
$$

nicht identisch verschwindet. Han hat dann entsprechend den Creichungen (5) die Beziehungen

$$
u_{i k}=c_{i 1} y_{1 k}+c_{i \Omega} y_{2 k}
$$

mit konstanten $c_{1 k}$. und da nach dem Multiplikationssatz der Determinanten 
134 Fiinftes Kapitr. Singulare stellen linearer Differentialgleichungen.

$$
\begin{aligned}
& u_{11} \|_{12} \ldots c_{11} c_{12}, y_{11} y_{12} \\
& u_{21} u_{22} \quad r_{21} c_{22} \quad y_{21} y_{22}
\end{aligned}
$$

ist, so bat dir Joetrominomto

$$
r=r_{11} r_{12}
$$

rimon von Null versehiedemen Wert. Man kann mun narh einem in don verschirdensten Teifon dor Mathematik iblichren Verfahren die vier Gleichungen (7) in aine symboliseh" Form zusammenfassen, indem man

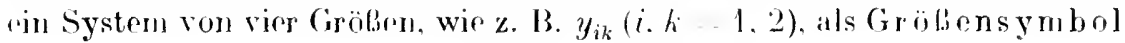

$$
\left(\begin{array}{ll}
y_{11} & y_{12} \\
y_{21} & y_{22}
\end{array}\right)=\gamma
$$

als eine sogenannte Matrix ${ }^{1}$ ) ansioht und für diese Matrizen Rowhnungsregeln aufstellt. J)ir wichtigsto unter diesen Rogeln ist dir humposition oder Multiplikation, die so repklärt wird, daf man

$$
\left(\begin{array}{ll}
u_{11} & u_{12} \\
u_{21} & u_{22}
\end{array}\right)=\left(\begin{array}{ll}
c_{11} & c_{12} \\
c_{21} & c_{22}
\end{array}\right)\left(\begin{array}{lll}
y_{11} & y_{12} \\
y_{21} & y_{22}
\end{array}\right)
$$

setzt, wenn dis $u_{i k}$ durch dir Glejehongen (7) gegeben sind. Man sagt dann, $U$ sei die aus (' mul $V$ (in dieser Reihenfolge!) komponiorte odor zusammongeselzto Matrix, oler $U$ entstehe aus $Y$ durch linkskomposition mit $C$ oder aus $C$ durch Reehtskomposition mit $Y$. Im allgemeinen ist C' y von IC verschieden; die Komposition ist im allgemeinen nicht kommutativ, es gilt aber allemal (Gl. (8)):

Wenn insbesondres

$$
\left|C^{\prime}\right\rangle|=| \gamma C^{\prime}|=| C|\cdot| \gamma \mid \cdot
$$

$$
\text { C') } \mathrm{YC}
$$

ist, su heibren (' und Y vertauschbare Matrizen.

Jefiniert man dip Adlition zweice Matrizen dureh diw Glowhnng

$$
\left(\begin{array}{ll}
a_{11}+b_{11} & a_{12}+b_{12} \\
a_{21}+b_{21} & a_{22}+b_{22}
\end{array}\right)=\left(\begin{array}{l}
a_{11} a_{12} \\
a_{21} a_{22}
\end{array}\right)+\left(\begin{array}{l}
b_{11} b_{12} \\
b_{21} b_{22}
\end{array} .\right.
$$

so ist diese Opration resichtlich kommutatil: fermer gilt das distri. hilivf Gosetz

$$
C(A+B)-(: 1+C B
$$

und firr Mdation mol Komposition das assoziative fiesetz

$$
\begin{gathered}
(A+B)+C \\
(A B) C=A\left(B C^{\prime}\right) \quad 1 B C .
\end{gathered}
$$

1) Die Matrix. deren bitemente durch keine Buchstaben mit loppelindex dargestellt sind. bezoichnen wir im folgrenden stets durch den entsprechenden groben Buchstahen. Dieser grobe Buchstahe zwischen vertikalen strichen soll den Wert der Determinante der Matrix bedenten, also $2 . B, \quad r=t$. 


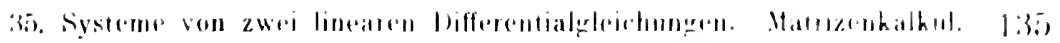

Hit Matrix

$$
\begin{aligned}
& 111 \\
& 111
\end{aligned}
$$

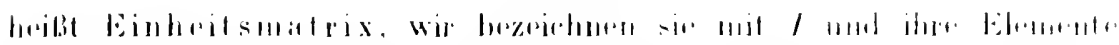

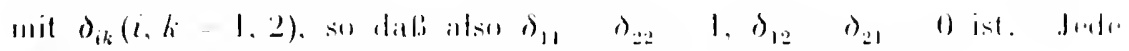

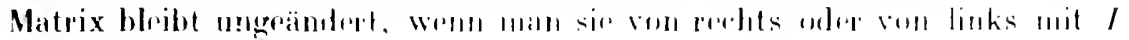
knomponiert.

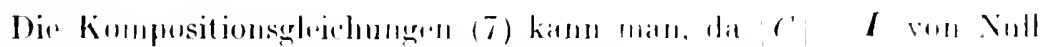
versehirden ist. mach den $y_{1 k}$ anflösen mud fimdel

$$
\text { ly } \quad r_{1} u_{1 k}+r_{2} \|_{2 k} \text {, }
$$$$
(1, k \quad 1, \because)
$$

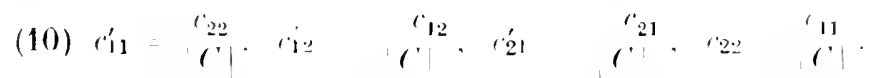

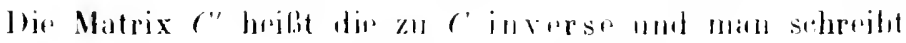

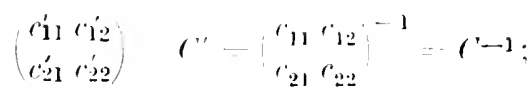

offenbar ist damu anch (' dir inverse Matrix von (" mml man hat

$$
\begin{gathered}
C^{\prime \prime}=\sigma^{\prime \prime}=I \\
\left.c^{\prime}\right) c^{\prime \prime}=1 .
\end{gathered}
$$

Ans

folgt

(9a)

$$
\left.l^{\prime}-r^{\prime}\right)
$$

$$
1=1-1 ;-1
$$

denn es ist

$$
\text { U } Y^{\circ}-1 C^{\circ}-1=C \zeta \gamma^{\circ}-1 C^{\circ}-1-C^{\prime} / C^{\circ}-1=C^{\circ}-1=1 .
$$

Sind die Elenente $y_{i k}$ der Maldix $y$ difurentiierbare Funktionen von $r$. so schreiben wir

$$
\left(\begin{array}{cc}
d y_{11} & d y_{12} \\
d x & d x \\
d y_{21} & d y_{22} \\
d x & d r
\end{array}\right)=\begin{aligned}
& d r \\
& d r
\end{aligned}
$$

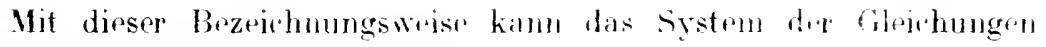
$\left(A^{\prime}\right)$ in die symbolische Ciloichung

$$
\text { d) } d x=1
$$

zusammengefabt werdell. Wit $\mid Y !+0$ ist. können wir statt dessen auch scluruibri

$$
\frac{d y}{d x}=-1:
$$

wir sehen, dads atiu Korffizienten des Differentialsystems (A) in eintach.r Weise durch die Elemente der Matrix $Y$ dargestellt Werdun können. so 
dat. also durre die Matrix $Y$ der gesante Inhalt des Differentialsystems (A) erschöpft wird. Wir nennen l' rine Integralmatrix des Differentialsystems (A) oder $\left(\Lambda^{\prime}\right)$.

Es treten hier zwoi wohl zu unterscheidende formen des bifferentialsystems (A) auf. Einmal dir ursprüngliche Form (A), für die die allage meine Lösung $y_{1}, y_{2}$ in der Form (5) durch die Elemente $y_{d k}$ der Integralnatrix $Y$ dargestellt wird. Dann aber die Form (A'). wo dir Elemente rinep Intogralmatrix selbst vorkommen. J)e Cbengang von der lutegralmatrix $Y$ zo der lntegralmatrix $l^{*}$ wird durch dir Gleichung (9) oder

$$
U=C r
$$

wrmittelt. wo $C$ ano konstante Matrix uit nicht versehwindender Jeterminante bedeutet. Ungokehrt stellt

$$
Z=I Y
$$

stets eine Integramatrix von (A) dar. Wemn $I$ irgendeino konstante Matrix nit nicht verschwindender Determinante bedeutet. In der Tat. ist nach den Koupositionsgleichungen (7)

also

$$
z_{i n}=\gamma_{i 1} y_{1}+\gamma_{12} y_{2} \text {. }
$$

folglich nach ( 12 )

$$
d x=r \cdot d Y^{\circ}
$$

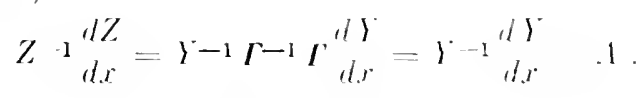

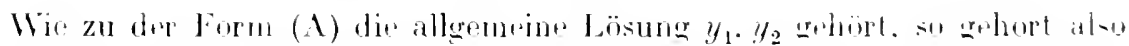

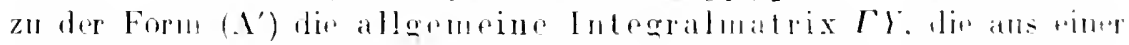
partikulären lntegraluatrix I' durrh linkskompositinn nit ain+r willkürlichen konstanten Matrix $r$ ron nicht vershwindender beter-

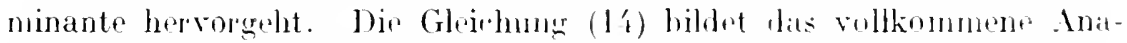

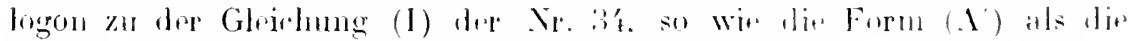

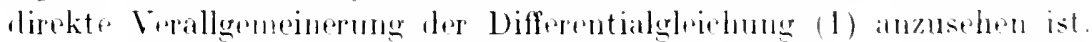

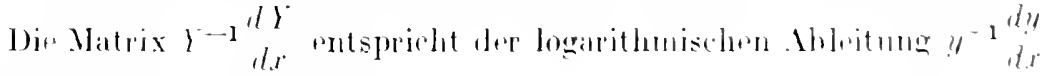
rintringtion. wir sehreiben

$$
\sum_{-1} d Y^{2} \quad D
$$

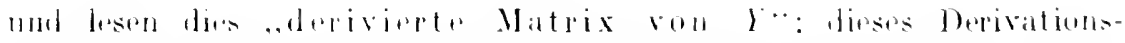

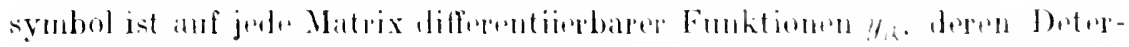
minatute nicht versehwindet. anwendbar. Wir kinnen das System (A) jotzt in der Form
(A)
$I_{x} Y \quad \ldots .1$ 


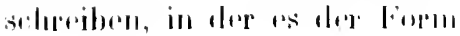

$$
\frac{\log m !}{d i}
$$

der bilferentialghteinlumer (1) mulsprieht.

\section{Existenzbeweise im roellen unl komplexen Gebiet.}

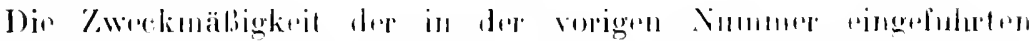

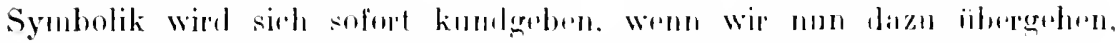

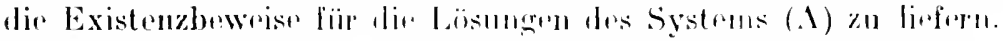

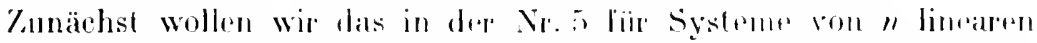
Differentialgleichmogen trster Ordmung dargelegte Cauchy-lipsehilzsehe Verfahren der Matrizenaufassung anzupassen suchen. Fïr das Differentialsystem (A), wo jetzt dir $a_{i k}$ als besehränktr und stetige Funktionen vorausgesetzt werden, shreriben wir in Punkte $x=x_{0}$ zwri systeme von Anfangswerten, also eine Anfangsmatrix

$$
\left.\left(\begin{array}{ll}
y_{11}^{(0)} & y_{12}^{(0)} \\
y_{21}^{(0)} & y_{2: 2}^{(1)}
\end{array}\right)=y^{0} 0\right)
$$

mit nicht versehwindender beterminante vor. Entsperehend den cileichungen (11) der Mr. s brkommen wir dann

$$
\begin{aligned}
& y_{i k}^{(1)}-y_{i k}^{(1)}=\left(x_{1}-x_{i n}\right) \sum_{h=1,2} y_{i k}^{(1)} a_{\lambda k}\left(x_{i n}\right) . \\
& y_{i k}^{(2)}-y_{i k}^{(1)}=\left(x_{2}-x_{1}\right) \underset{\lambda=1 . \nu}{\underset{\nu}{\Sigma}} y_{i \lambda}^{(1)} a_{\lambda k}\left(x_{1}\right) \text {. }
\end{aligned}
$$

oder

$$
\begin{aligned}
& y_{i k}^{(1)}=\sum_{\lambda=1}^{N} y_{i \lambda}^{(0)}\left(a_{\lambda i}\left(x_{0}\right)\left(x_{1}-x_{i 1}\right)+\delta_{\lambda k}\right), \\
& y_{i k}^{(2)}=\sum_{i=1} y_{i \lambda}^{(1)}\left(a_{\lambda k}\left(x_{1}\right)\left(x_{2}-x_{1}\right)+\delta_{k k}\right),
\end{aligned}
$$

oder endlich in Matrizenform

$$
\begin{aligned}
& Y^{(2)}=Y^{(0)}\left(.1\left(x_{0}\right)\left(x_{1}-x_{0}\right)+I\right) \\
& Y^{(2)}=Y^{(1)}\left(1\left(x_{1}\right)\left(x_{2}-x_{1}\right)+I\right) \\
& \left.. . . . . . . . .{ }^{1}\right)
\end{aligned}
$$

1) Multipliziert man alle Elemente $a_{i k}$ der Matrix $A$ mit einer Gribie $r$. so kommt das auf die Komposition von $A$ mit der Ylatrix

$$
\left(\begin{array}{cccc}
x & 1 & \ldots & 0 \\
1 & x & \ldots & 0 \\
0 & \ldots & . & 0 \\
0 & 1 & \ldots & x
\end{array}\right)
$$


In dieser Form tritt die Analogie mit den Gleir:hungen (b), die in der Nr. \& fïr dir rinf Differentialgloichnng (a) anfgestollt wurden, klarer hervor als in den Gloighumgen (11) drer Nr.5.

Man kann mu mit den Gloichungen (17) ganz ähnlirh verfahren. wir in der Nr. '́ mit den Gleirhungen (b), wenn man an die Stalle der dort angewamen Multiplikation dir Komposition der Matrizen treten läßt. Teilt man das Intrerall $\left(x_{0} \ldots r\right)$ dureh dir $m-1$ Teilpunkte $x_{0}<x_{3}<x_{2}<\cdots<x_{m}=x$, so dalj jedes Teilintervall kloiner als $\varepsilon$ ist, so folgt ans den Giloichungen (17)

$$
Y(k)=Y^{(k-1)}\left(A\left(x_{k-1}\right)\left(x_{k}-x_{k} 1\right)+I\right)
$$

$k \quad 1.2, \ldots, n$,

durch Komposition

$$
Y(m)=\operatorname{lil}_{k=1,2, \ldots, m}^{(I /}\left(A\left(x_{k-1}\right)\left(x_{k}-r_{k} 1\right)-I\right) .
$$

wo das Zeichen II dir Komposition der Matrizen und zwar in der am FuBe angedeuteten lieihenfolge anzeigt. In der Nr.5 wurdr narligewiesen, daß die $y_{i k}^{(m)}$ sirh den zu den Anfangswerten $y_{i k}^{\prime \prime}$ grhörigen lösungen $y_{c k}$ des Differentialsystems (A) als Grenzwertun nähern. wenn $\varepsilon \rightarrow 0$ goht. Es ist also

$$
\left(\begin{array}{ll}
y_{11} & y_{12} \\
y_{21} & y_{22}
\end{array}\right)=\gamma=Y(0) \lim _{\varepsilon \rightarrow 0} \prod_{k=1, \ldots, n}\left(A\left(x_{k} 1\right)\left(x_{k}-x_{k-1}\right)+I\right) .
$$

und wenn wir, wie in der $\mathrm{Nr}$. 4, wieder das Produktintegralzeichon

$$
\text { (18) } \int_{x_{k}}^{x}(A d x+I)=\lim _{\varepsilon \rightarrow 0 k=1,2, \ldots m} I_{k \rightarrow 1}\left(.1\left(x_{k-1}\right)\left(x_{k}-x_{k} 1\right)+I\right)
$$

cinführen, so können wil in der form

$$
y=\gamma(0) \int_{i_{i}}^{x}(.1 / x-I)
$$

die Integralmatrix des Systems (A) odor (A) darstollen. die fiur $r=x_{0}$ die Anfangswerto $Y^{\prime(0)}$ annimmt. Hio Integralmatrix

$$
\int_{x_{0}}^{x}(1 d x+1)
$$

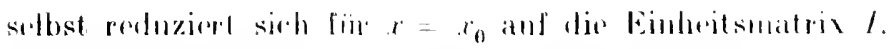

herans. die offenbar mit jeder Matrix vertauschhar iss. Man dentet diese Multiplikation deshalt durch die einfache Schreibweise 1.4 cider $A$. $r$ an und nennt $r$ im Gegensatz zu der Matrix a eine skalare Gröbe. In desem simne ist im Texte die Schreibweise $f\left(x_{0}\right)\left(x_{1}-x_{0}\right)$ zu verstehen. wo $A_{1} t_{0}$ die Matrix der $a_{i k}\left(x_{0}\right)$ brientet. 


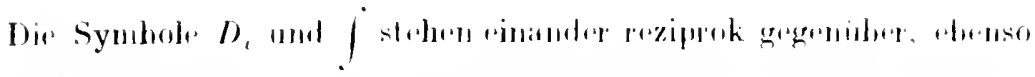

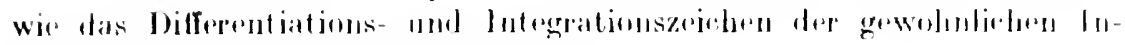

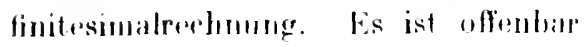

$$
\begin{aligned}
& \left.D_{x}\right|_{i_{1}} ^{x}(1,1+1)=.1 \\
& \left.\int_{x_{n}}^{x_{x}}(L)_{x}\right)(x+I)=1 .
\end{aligned}
$$

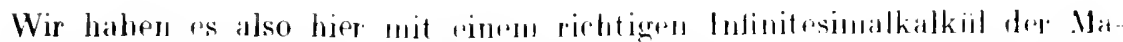

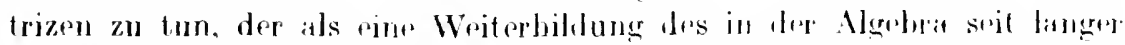
Zeit iblichen algehraisehen Matrizenkalkiils anzusehen ist. I"hrigens ist

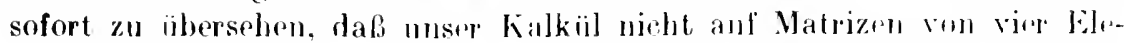
menten beschränkt ist, sondern ohem weiteres anf Matrizen von $n^{2}$ Elrmenten $a_{i k}(i, k=1,2, \cdots, n)$ ïbertragon werden kam. Wir besthränken

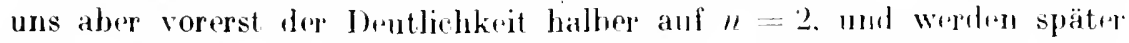
mit Wrengen Worten die Verallgenneinermug auf ain belirbiges " vornehmen. Wir folgen darin dem Brogriander dirses [nfinitesinalkalküls dur Matrizen, Vito Volterra ${ }^{1}$ ). der freilich rinen rtwas anderen Ausgangspunkt genommen und anch in einzelnen andres Methoden angewandt hat. ${ }^{2}$ )

Wir wenden uns nummehr zmon komplex.fu Gibirte mul setzen demgemä $B$ voraus, da die $a_{,}$in einer gewissen Ungebung von $r=x_{0}$ holomorph, also in der Form

$$
a_{i k}=a_{i k}^{(1)}+a_{i k}^{(1)}\left(x-x_{0}\right)+\cdots
$$

darstellbar sejen. Nehnurn wir fiir die Elemente yik der hureh die Anfangswerte $\gamma^{r(0)}$ zu bestimmonden Integraluatrix $\gamma^{\circ}$ lir Eutwirklungen an

$$
y_{i k}=y_{i k}^{(0)}+y_{i k}^{(1)}\left(x-x_{0}\right)+y_{i k}^{(2)}\left(x-r_{0}\right)^{2}+\cdots .
$$

so ergeben sich die Koeffizienten dieser Entwiklungen zunärhst formal

1) V. Volter ra, Memoria della Societi Italiana delle Scienze detta dei XL, t. 11 (1887) und XII (1899).

2) Für die hier gegebene Darstellung rgl. s'lılesiuger. Crelles Journai 128 (1906), S. 263 und Vorlesungen ïber lineare Differentialgleiclungen. Leipzig 1908. Die Definition des Produktintegrals kaun auch erfolgen. wenn man die Funktionen $a_{i k}$ nicht als stetig. sondern nur als integrierbar (im Sinne von Riemann) voraussetzt. Natürlich befriedigen die so definierten Funktionen $y_{i k}$ das Differential. system (A) nur an den Stellen $x$, wo alle $a_{i z}$ stetig sind. 
durch Einsetzen in die Differentialgleichungen $\left(A^{\prime}\right)$. In Matrizenform haben wir

also eingesetzt:

$$
\begin{gathered}
d Y=r A, \\
d r=A^{0}+A^{(1)}\left(x-r_{0}\right)+\cdots \\
Y=r^{(0)}+r^{(1)}\left(x-r_{0}\right)+\cdots,
\end{gathered}
$$

$$
\sum_{\nu=0}^{\infty}(r+1) Y^{(\nu+1)}\left(x-x_{0}\right)^{\prime}=\left(\sum_{\nu=0}^{\infty} \gamma^{(\nu)}\left(x-x_{0}\right)^{\nu}\right)\left(\sum_{,=0}^{\infty} A^{(\nu)}\left(r-x_{0}\right)^{\prime}\right) ;
$$

durch Vorgleichen der Koeffizienten gleichhoher Potenzen von $\left(x-x_{0}\right)$ auf beiden Seiten finden wir so die Rekursionsformel

(22) $(v+1) Y^{(\nu+1)}=Y^{(())} A^{(\nu)}+Y^{(1)} A^{(\nu-1)}+\cdots+Y^{(\nu)} A^{(0)} . \quad(\nu=0,1,2, \ldots)$

Uu nach den calcul des liuites abschätzen zu können, inerken wir den folgenden einfarhen Satz ïber Matrizen von vier Elementen an: Sind

$$
\left(\begin{array}{ll}
\alpha_{11} & \alpha_{12} \\
\alpha_{21} & \alpha_{22}
\end{array}\right)=A, \quad\left(\begin{array}{ll}
\beta_{11} & \beta_{12} \\
\beta_{21} & \beta_{22}
\end{array}\right)=B
$$

zwei Matrizen und hat man die Ungleichungen

$$
\left|\alpha_{i k}\right| \leqq M, \quad\left|\beta_{i k}\right| \leqq Y \text {. }
$$

so gilt nach der Kompositionsformel

$$
A B=\left(\begin{array}{ll}
\alpha_{11} \beta_{11}+\alpha_{12} \beta_{21} & a_{11} \beta_{12}+\alpha_{12} \beta_{22} \\
a_{21} \beta_{11}+\alpha_{22} \beta_{21} & \alpha_{21} \beta_{12}+\alpha_{22} \beta_{22}
\end{array}\right)
$$

fïr jedes Element der komponierten Matrix die Ungleichung

$$
\left|\alpha_{i 1} \beta_{1 k}+\alpha_{i 2} \beta_{2 k}\right|<2 . M N \text {. }
$$

Ist also $a^{(\nu)}$ eine positive Größe, die gleirh lem Doppelten des gröbten unter den absoluten Boträgen der $a_{i k}^{\prime \prime}$, und $y^{0)}$ eine positiwe Größre, die gleich dem gröbten unter den absoluten Buträgrn der $y_{i k}^{(u)}$ ist, so ist natch $(*)$

$$
y^{(j)}=y^{(0} a^{(1)}
$$

keineslials kleiner als der größte unter dent absoluten Beträgen der Elemente der Matrix

und woll wir allgemein

$$
Y(1)=\gamma^{\prime(1)} \mathrm{All} .
$$

(2i) $(v+1) y^{(v+1)}=y^{(0)} a^{(\nu)}+y^{(1)} a^{\nu-1}$

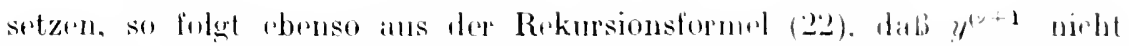
kleiner ist, als der gröbte unter den absoluten beträgent der Elentente der Mattrix $y^{\prime \prime 1)}$, d. h.

$$
y^{(x+1)} \quad y_{i k}{ }^{+1)}
$$

Nun folgt aus den rinfarhstrn Sätzen ibler Potenzreihen, dab die Reihr 


$$
a^{(6)}+a^{(1)}\left(x-r_{0}\right)+\cdots
$$

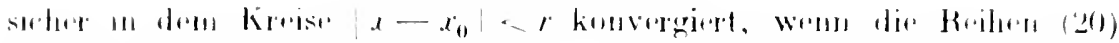

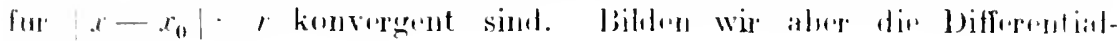
strichung

$$
d x=n\left(a^{(1)}+a^{1)}\left(x \quad r_{0}\right)+\cdots\right),
$$

so wird diese olfenhar durch die lieihe

$$
\begin{aligned}
& f^{x}\left|a^{101}+a^{1}\left(x-x_{11}\right)+\cdots\right| d x \\
& \text { y) }=\mathfrak{y}^{(1)} e^{r_{0}} \quad y^{(0)}+y^{1)}\left(x-x_{n 1}\right)+y^{(2)}\left(x-x_{01}\right)^{2}+\cdots
\end{aligned}
$$

befriedigt; diese Reshe konvergiest folghich för $\left|r-r_{0}\right|<r$. Xareh dem Prinzip des calcul des linites gilt also zufolge der Ungleichungen (2') dasselbe für die Reihen (21), wotheh dor Beweis lür die Integratexistenz des Differentialsystems $(A)$ erbracht ist. Zugleich ist gezeigt, daß der Konvergenzbereich der lntegralreilien (21) sicher bis zu den $x_{0}$ am nächsten gelegenen singulären Punkte der Korffizienten $a_{i k}$ reicht, d. h. daß die Lösungen des Infferentialsystems (A) keine anderen singulären Stellen haben können als die Kooffizienten $a_{i}$.

Nach der in der $\mathrm{Nr}$. 13 angegebenen Methode kann nun weiter geschlossen werden, dabis das zu Beginn dieser Nummer im reellen Gebiete auseinandergesetzte Cauchy-Lipschitzsche Verfaliren anwendbar bleibt, wenn man es auf einem von $x_{0}$ ausgehenden nar'h $x$ hinführenden geraden oder krummlinigen Wege zur Anwendung bringt, vorausgesetzt, daß dieser Weg keine der singulären SteHlen der $a_{i k}$ passiert. Auf́ diese Weise hat man die Möglichkeit. das Produktintegral

$$
\int_{x_{0}}^{P_{x}}(A d x+1)
$$

erstreckt aul einem beliebigen solchen Wege zu definieren, und kann nun mit diesem genạ so operieren wie mit dem gewöhnlichen Integral in komplexen Gebiet.

Legt man von den singulären Punkten der $a_{i k}$ aus Schnitte $l$ nach dem Unendlichen, so erhält man eine Fläche $T$, innerhalb deren das Produktintegral einen vom Wege unabhängigen, eindeutig bestimmten Wert besitzt. Auf die Wertänderungen, die es erfährt, wenn der Integrationsweg die Schnitte $l$ überschreitet, kommen wir gleich nachher zurück.

Wir fügen hier noch eine Abschätzungsformel für die Elemente der durch das Produktintegral gegebenen Integralmatrix hinzu, die als das Analogon des Mittelwertsatzes der gewöhnlichen Integralrechnung gelten kann. Aus der Fläche $T$ sondern wir einen abge- 


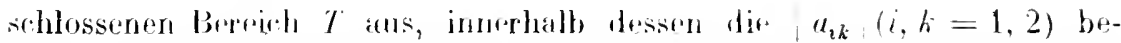
schränkt sind. Es möge dann $g(x)$ die Funktion won $r$ berdeuten, dife an jeder Stulle von $T$ gleich dern gröbten der vier Wertre $\left|a_{a k}(x)\right|$ ist. Dieso Funktion ist natiorlirh nicht monogen, aber sir ist. wir die $\left|a_{i k}(x)\right|$ selbst, längs jerter stetigen Kurre von endlicher Bogenlänge, dir ganz innerhalf, $T$ verläuft. stetig mod infolgerlessen integrimbar. Aus ifen Differentialgleirhungen $\left(A^{\prime}\right)$ folgt dann

$$
\begin{aligned}
& d y_{i k} \\
& d d^{\prime}
\end{aligned}
$$$$
\text { , } 1,21
$$

Nun ist liir eine monegene Funktion $/(.$, ) der komplexn Veranderlichen $x$ offenbar stets

$$
\left|f(x)-f\left(x^{\prime}\right)\right| \geq\left\|f(x)|-| f\left(x^{\prime}\right)\right\| .
$$

man hat also

dabei ist

$$
d f(x) \geq \begin{gathered}
d|f(x)| \\
d x
\end{gathered}
$$

$$
\begin{aligned}
& d|f(x)| \\
& d x
\end{aligned}=\lim _{x^{\prime} \rightarrow x}|f(x)|-! f\left(x^{\prime}\right)
$$

zu nehumen. wo die Annäherung von $x^{\prime}$ an $t$ in einer bestimmten. von ir ausgehenteri Richtung erfolgt.

Aus (26i) folgt durch Addition

$$
d y_{i 1}+\frac{d y_{12}}{d x} \geq 2 g(x):\left|y_{11}\right|+\mid y_{x 2}: i,
$$

also mit Rürksicht anf (27)

$$
d d^{\prime}\left|y_{i 1}\right|+\left|y_{i 2}\right|:=2 g(r):\left|y_{11}+\right| y_{i 2}:
$$

Lutegriert man hice auf beiden Seiten anf pinem innerbalb $T$ zwisehen $x_{0}$ and $x$ verdanfenden Wege $1 f$ mit stetiger Tangente. so ergibt sir $h_{1}$

$$
\left|y_{i 1}\right|+\left|y_{12}\right|=e^{2 i^{2} f^{x} g(x) d r}\left(y_{i 1}^{(i) 1}-y_{i 2}^{(1)}\right) .
$$

wenn $y_{i k}^{(1)}$ dir Anfangswerte der $y_{i k}$ in $x=x_{0}$ bezedelunen. Labit man also insbesendere $y_{i k}$ die Elemente des auf dem Wegr II ersterekten Produktintegrals (25) bedrutent, so ist $y_{i k}^{(0)}=\partial_{\imath k}$. also

Ind a potiori

$$
\left|y_{11}\right|+\left|y_{i-2}\right|=\left.e^{y^{x}}\right|^{x} y(x) d r
$$

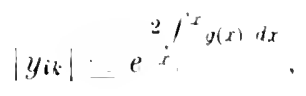




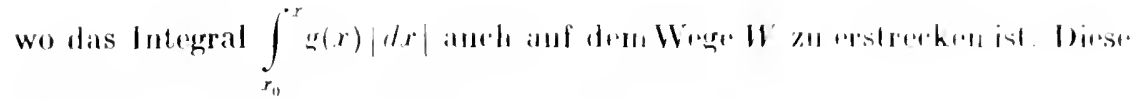

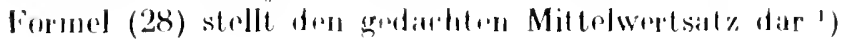

\section{Methode der sukzessiven Approximationen. Mehrdeutigkeit.}

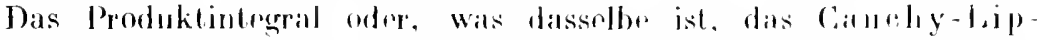
schitzseler Verfahren gibt uns zwar cine Darstellung der Intregralmatrix

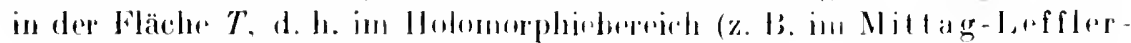

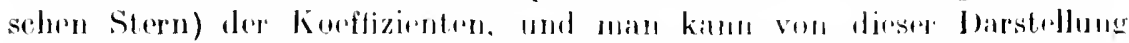

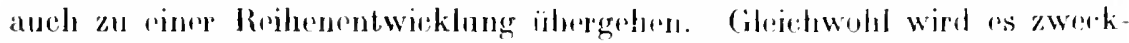
mälig sein, eine andere. äuberst wiehtign" Reihenentwirklung anzugeben dir im ganzen Holomorphirbepoch giiltig ist, mod dir naeh der sogr-

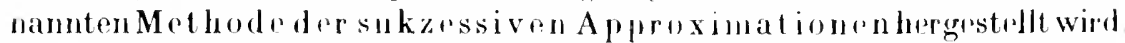

Wir bezeichnen wieder mit $Y$ die durch das leoduktintegral gergebene Integralmatrix von (A) unl betrachten din Matrix ilner Anfangswerte $I$ selbst als erst. Annäherung von $\zeta^{\circ}$ Man bildr mun die Folge weiterer Näherungsmatrizen $C^{1)}, l^{\gamma^{2}}, \ldots$ in folgender Wrise:

$$
\begin{aligned}
& \frac{d}{d x}=I .1=.1 \\
& \frac{d U^{(2)}}{d x}=U(1) A, \\
& d C^{(3)}=I^{(2)} A \text {. }
\end{aligned}
$$

Wenn wir dann die aus den Elementen $\int_{x_{n}}^{x} a_{t} d x$ gebildetes Malrix kurz dum h $\int_{\dot{x}_{0}}^{x} A d x$ bezeichnen, so ergibt sich aus der Folge der Grichungen (29) durch Integration längs eines in $T$ verlaufenden Weges II

(29a)

$$
\begin{aligned}
& U(\mathbf{1})=\int_{\dot{x}_{0}}^{x} A d x, \\
& U(2)=\int_{i_{1}}^{x} U^{(1)} A d x, \\
& U(3)=\int_{x_{0}}^{x} U^{(2)} A d x .
\end{aligned}
$$

1) Die Formel rührt von H. F uhr her: vgl. Jahresbericht der U. M.-V. 2. 3 (1919), S. 168, wo sie für das reelle Gebiet entwickelt ist. 
Wir zeigen nun. daß dir Reihe

$$
I+U^{(1)}+L^{(2)}+\cdots \text { in inf. }
$$

in dem ganzon Bereich $T$ (siehe die vorige $\mathrm{Xr}_{\text {. }}$ ) unbedingt und gleinhmälig konvergiert und daselbst die Integrahnatrix ' darstellt.

Es sei innerhalb $T$

$$
\left|a_{i k}\right| \leqq M .
$$

Hat unser Integrationsweg 11 die endlichr Länge ss so ist zunächst

$$
u_{k}^{(1)} \leqq u s .
$$

Durch Anwendung der Forme! (*) (S. 140) auf dir komponierto Matrix $I^{11} A$ ergibt sich dann aus der zweiten dro (ileichungen (29a)

$$
u_{i k}^{(2)} \leqq \int_{i}^{x} 2.4 s .11 d r^{\prime} \leqq 2 . H^{2} \frac{s^{2}}{2} .
$$

dann weiter aus der dritten

und allgemein

$$
u_{d k}^{(3)} \leqq \int_{x_{u}}^{x} 4 y^{2}{ }_{2}^{s^{2}} \cdot y d x=2^{2} \cdot y^{3} \frac{s^{3}}{3 !}
$$

$$
u_{i k}^{(\nu)} \leqq 2^{\nu-1} u^{\prime \prime}{ }^{s} \cdot
$$

Die Reihen $\sum_{\nu=0}^{\infty} u_{i k}^{(\nu)}$. wo $u_{c k}^{\left(u_{k}\right)}=\delta_{c k}$ ist. konvergieren also wir eine Exponentialreihe. Verbindet man $x_{0}$ mit den Randpunkten des Bereichs $T$ auf den kürzesten in $T$ verlaufenden lligen, so bleiben die Längen dieser Wege unterhalb einer endlichen Schranke $\sigma$. Ersetzt man in (3I) $s$ durch dieses $\sigma$, so gelten diese Ungleichungen a potiori für jeden in Innern und auf den Rande von $\bar{T}$ gelegenen Punkt $x$. Die Reihn

$$
\delta_{i k}+u_{i k}^{(1)}+u_{i k}^{(2)}+\cdots \text { in inf. }
$$

konvergieren also in der Tat unbedingt und gleichmäfig innerhalh des Bereiches $\bar{T}$; sie konvergieren aber auch. wenn der Integrationsweg über die Begrenzung von $T$ hinausgeht, rorausgesetzt. dalis ex eine endliche länge hat. und dab die $a_{i k}$ auf ihm beschränlit bleibern.

Wir zeigen nun, daf die Reihen (32) das Diffrentialsysten (A) befriedigen. Dazu ist vor allem nötig nachzuweisen. daf. sie gliedweise differentiintan sind. Es ist

$$
d U^{(n)}=I^{(v-1)} A
$$

ałš zumächst rein formal

$$
\sum_{v=1}^{x} d t^{(\nu)}=\sum_{\nu=1}^{\infty} l^{(\nu-1)} A .
$$




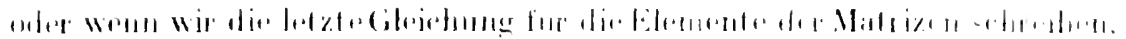

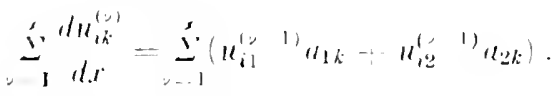

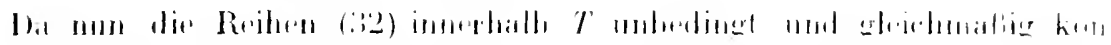

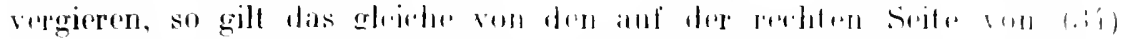

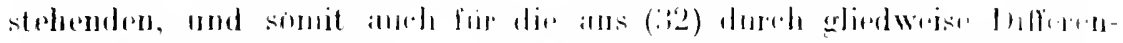

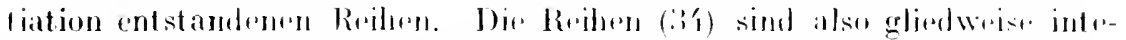

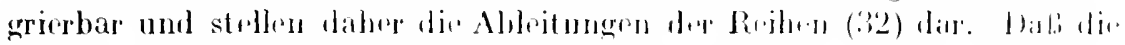

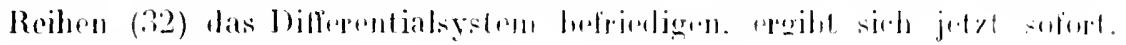

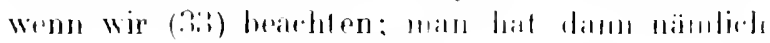

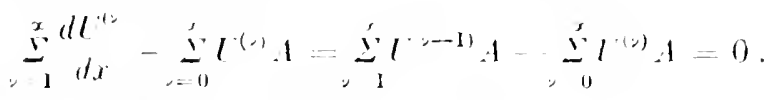

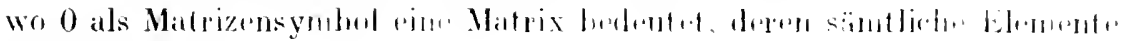
verschwindern.

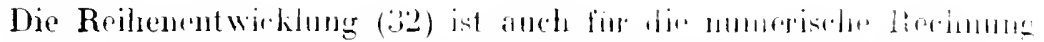

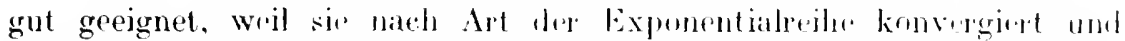

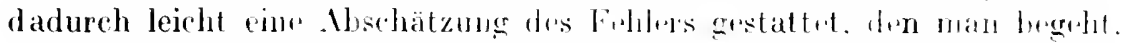

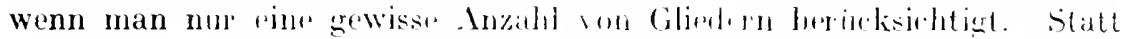

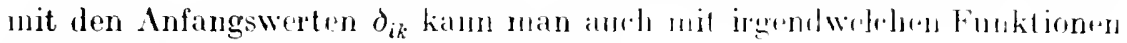
als , "rsten Nähermosworten" hesimnen.

Besonders wichlige ist eine Folgerung, dir sirh aus der Entwirklung

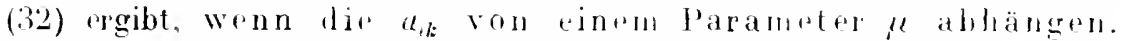
Wenn z. B. die $a_{i k}$ ganze rationalu Funktionen des Parameters !l sind. so gilt das gluiche auch fiir all' $u_{i k}^{(v)}$. Dir : $a_{i k}$ bleiben, wron $\mu$ beschränkt ist, unterhalb einer angrobaren endlichen Schranke 11, also konvergieren die Reihrn $\Sigma u_{i k}^{(x)}$, solange $\mu$ heschränkt isl. unberlingt und gleichmäfig und können daher nach Potenzen ron $\mu$ geordnet werlen. Dir durrh diese Reihen dargestellten Lösungen des Difforentialsystems (A) sind also ganze transzendente Funktiomen ron "l. Djesre Satz stammt von Poincaré ${ }^{1}$ ).

Die Anwendung der Methode dep sukzessiven Approximationen aul lineare Differentialgleichungen geht aul Cauchy zurïch ${ }^{2}$ ). Spätc haben Caqué (1864), Fuchs (1870), Peano (1888), der letztere mit Anwendung des Matrizenkalküls, die Hethode weiter nntwickelt. Für heliebign nichtlineare Differentialsystente hat sich brsunders Pitard ${ }^{3}$ ) s.h. "ingehend mit dieser Methorle boschäftigt.

1) Geuvres II. S. 310: vol. P. Gïn the r. Crelles Journal 107 (1889). S. 312.

2) Siehe A. Cauchy, Leçons etc. rest. par lAbbé Moigno. II (1844). S. 702. wo die Methode für lineare Ibifferentialgleichungen zweiter Hrdnung auseinandergesetzt wird.

3) Vgl. E. Picard, Traite I Analye II (19015). ‥ 340. 351. 


\section{Wertänderungen der Integralmatrix hei Umläufen. Kanonische Integralmatrix.}

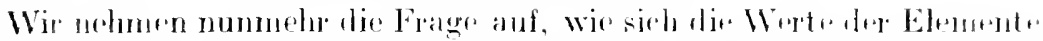

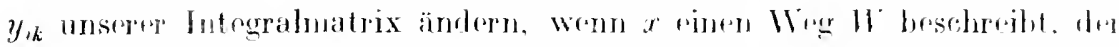

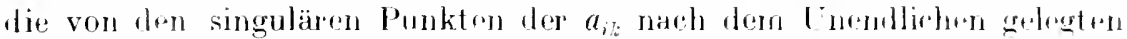

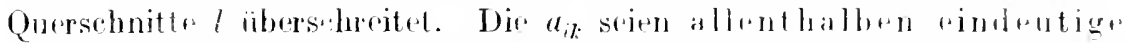
Funktionen, dann genïgen auch die aus don $y_{2 k}$ durch Fortsetzung

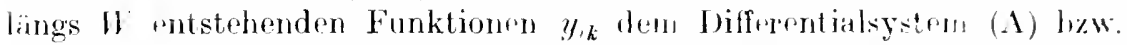
$\left(A^{\prime}\right)$. Man hat lolglieh, wrenn der Migr $\|^{\circ}$ in gusehlosserer ist. narh Cil. (7) der Nr. 35

$$
\bar{y}_{i k}=c_{i 1} y_{1 k}+c_{12} y: k,
$$

wo die $c_{i k}$ Konstanten bedeuten, oder in Matrizuntorn

$$
Y=C Y \text {. }
$$

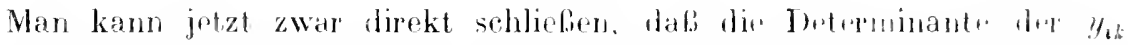

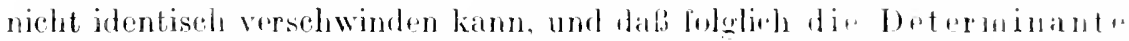
der cik von Null versehieden suin mali. Es ist aber nuiblieh

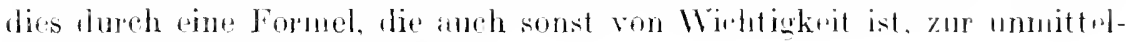
baren Anschaumb zol bringen.

Brtrachten wir nämbich die Delerminante / l | dre lutegralnatrix

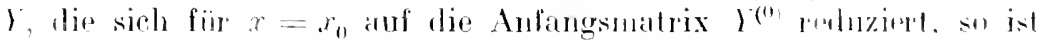

$$
\begin{aligned}
& d|l|= \\
& d x
\end{aligned} \quad \begin{aligned}
& y_{11}^{\prime} y_{12} \\
& y_{21}^{\prime} y_{22}
\end{aligned}+\begin{aligned}
& y_{11} y_{12}^{\prime} \\
& y_{21} y_{2: 2}^{\prime}
\end{aligned} .
$$

also mit Rücksieht auf dir Differentialgleichungen (.1)

$$
\left.\begin{array}{l}
d|\gamma| \\
d . r
\end{array}=\mid \begin{array}{ll}
y_{11} a_{11}+y_{12} a_{21} & y_{12} \\
y_{21} a_{11}+y_{22} a_{21} & y_{22}
\end{array}+\begin{array}{l}
y_{11} y_{11} a_{12}+y_{12} a_{22} \\
y_{21} y_{21} a_{12}+y_{22} a_{22}
\end{array}=\left(a_{11}+a_{222}\right)\right) .
$$

woraus sich

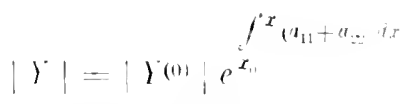

regibt, wo das Integral von $x_{0}$ bis $x$ and rimen immerhalb der Flarder $T$ verlaufromen Wege za astreken ist. Wir sebro ans dieser ven datebi

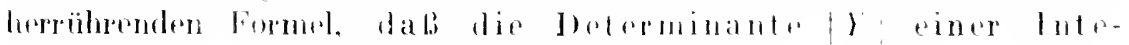

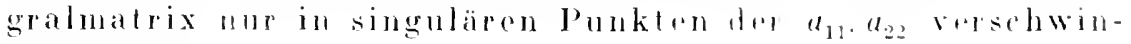
dril kanll.

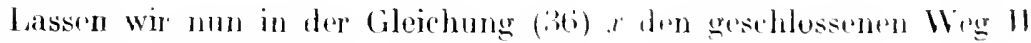
beschreiben, so rhalten wir

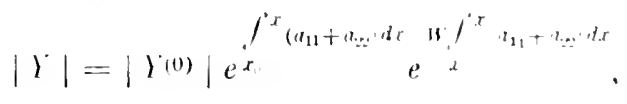




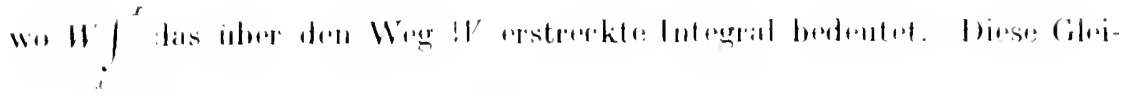

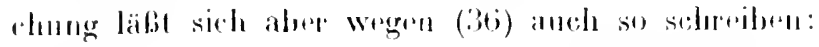

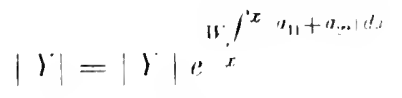

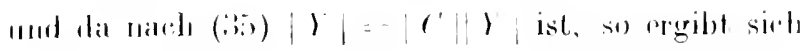

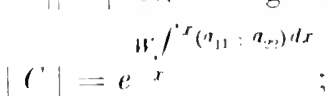

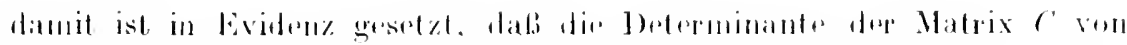

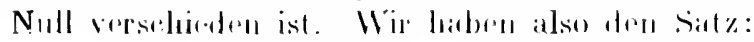

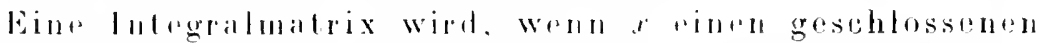
lleg bestereihl, von links her mit rimer konstanten Matrix nieht verselewindender Joterminanle komponielet.

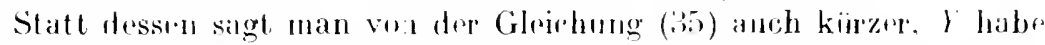

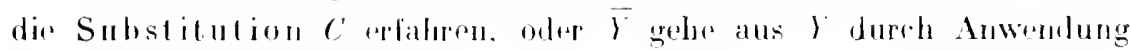
der Substitution (' hervor.

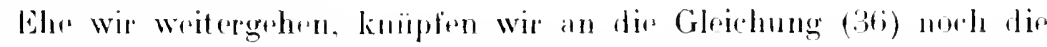

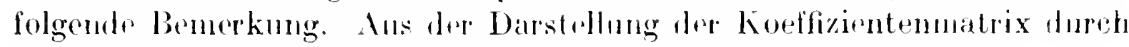

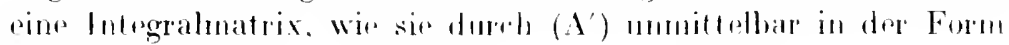

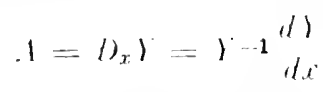

gegeben wird, folgt, dali liir diejenighn Plnkte, wo dit $y_{i k}$ holomorph sind und die Determinante $/ \gamma \mid$ nicht versehwindet, auch die Koeftizienten $a_{i k}$ holomorph sein mussen. l)agregen wird ein Punkt $x=\alpha$. in dessen Ungebung liw $y_{i k}$ holomorph sind, in dem aber $|\gamma|=0$ ist, notwendig ein singulärre Punkt der Koeffizienten $a_{i k}$ soin. In der Ungebung einer solehen Stelle ist $|\gamma|$ jelenfalls holomorph, alsu ron der Form

$$
|Y|=\partial \cdot(x-\alpha)^{2}+\partial_{y+1}(x-a)^{\nu+1}+\cdots, \quad(x) \neq \omega
$$

wo $v \geqq 1$ ist. Dir Darstellung (40) zeigt dann, daß dir $a_{i k}$ in $x=a$ einen Pol haben; wir haben es hier, nach der in der $\mathrm{Nr} .13$ (S. 49) eingeführten Bezeichnung, nit einem auberwesentlich singulären Punkte der Differentialgleichung (A) zu tun.

Wir kehren nunmehr zı der Gleichung (35) zurück, die die Viertänderung einer Integralmatrix kennen lehrt, die dem geschlossenen Weg oder Umlauf $W$ entspricht. Für eine lineare Differentialgleichung erster Ordnung multipliziert sich jedes Integral $y$ bei einem solchen Unlauf mit einer und derselben bestimmten lionstanten, die also für diesen Unlauf charakteristisch ist. Hier tritt an dit Stelle der Multiplikation mit einer Konstanten die Linkskomposition mit einer konstanten Matrix; 
wir fragen: Wie ändert sinh diese komatante Natrix. Wenn wir statt $I$

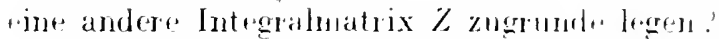

Es bestrht dir Bezirhung (Gl. (9) $\mathrm{N}_{r}$.35)

$$
Z=I Y, \quad ;=I-1 Z \text {. }
$$

wo $I$ eine konstante Matrix mil nicht rorshwimdender Detorminante bedeutet. Wenn also $Z$ durch den I mlauf 11 in $Z$ übergeht. so ist

$$
Z=\Gamma Y=I C Y=I C I-1 \%:
$$

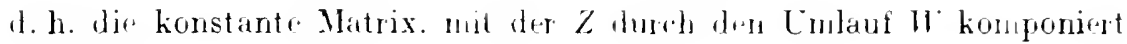
wird, ist

$$
l \prime=i(l) .
$$

Wir sagen von dieser Matrix. sif sfi mit $C$ ähnlich und sie gehe aus $C$ durch Transformation mit $r$ hervor. Wir haben also hier nicht dine für den Unlauf $t 1$ charaktuistische Matrix. sondern di. Gesamtheit aller zu $C$ ähnlichen Matrizen (42), wo $I$ rine willkürliche, konstante Matrix mit nicht ressehwindender Jeterminante bedeutet, ist hier das fü ${ }^{\circ}$ Charakteristische. Tabui ist klar, daß die Gesamtheit (42) in keiner Wrise von der zufälligen Wah] der Integralmatrix $Y$ und damit der Matrix $C$ abhängt. daf viehnehr. wenn $C^{\prime}$ irgendeine Matrix von der Form (42) bedeutet, diese Gramtheit auch in der Form $\boldsymbol{\Gamma} C^{\prime} \boldsymbol{I}^{-2}$ geschrieben werden kaun. wo $\boldsymbol{\Gamma}$ wieder eine willkürliche, konstante Matrix mit nicht verschwindender Determinante ist. Es entsteht so dje Frage nach den, was für die Zugehörigkeit ein $r$ Matrix zu der Gesantheit ('2) rharakteristisch ist. d. h. nach den charakteristischen Invarianten eines Systens ähnliher Matrizen. Diese Invarianten werden für don Weg II selhst charabteristisch sein. während die einzelne Matrix von dre zufälligen Wahl her lusgangsmatrix $Y$ abhängt.

Die hier anfgeworfene Frage erwoist sieh fü unsere ganze Theorie von fundanentaler Bedeutung. Wir werden zu ihrer Beantwortung kommen. wenn wir uns bemulhen. aus der Gesantheit der ähnlichon Vatrizen (42) die in gewissen Sinne pinfachst auszuwählen: d. h. nit anderen Worten, wir wollen eine lntegrahnatrix answahlen. deren Wortanderung bei desn Cinlauf it sich in möglieh-t ninfacher Form darstellt.

Da liegt es nahe zu versuchen. al es nieht lösungen des lofferentialsystems (A) giht. die sich bei dem bindanf 11 a verbalten. we die

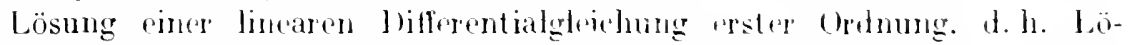
sungen, die sich bei den Lndauf 11 mit kon-tanton Fateren multiph-

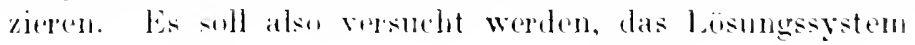

$$
\begin{aligned}
& t_{11}=p_{1} y_{11}+p_{2} y_{21} . \\
& y_{12}=p_{1} y_{12}+p_{2} y_{22},
\end{aligned}
$$




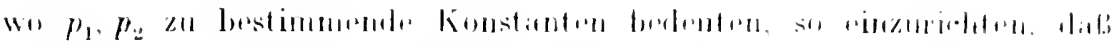

$$
\begin{aligned}
& y_{1}=p_{1} y_{11}+p_{2} y_{21}=(1) m_{1} \\
& y_{2}=p_{1} y_{12}+p_{2} y_{22}=(1) \eta_{2}
\end{aligned}
$$

wirk, wo a rine Konstante ist.

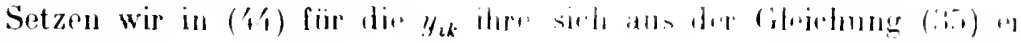
gebenten Wertr.

$$
y_{i k}=c_{11} y_{1 k}+r_{i 2} y_{2 k}
$$

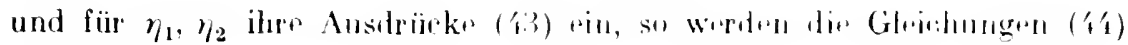
erfiillt, werm

$$
\begin{aligned}
& p_{1} c_{11}+p_{2} c_{21}=(1) p_{1} \\
& p_{1} c_{12}+p_{2} c_{22}=(1) p_{2}
\end{aligned}
$$

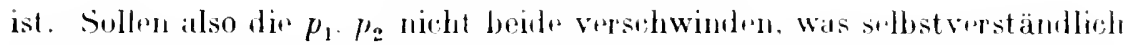
gefordert werden muli, so muls in den beilen linearen lomoginen Griachungen (45) dir Determinante der Koeffizienten

$$
\begin{array}{cc}
c_{11}-c_{12} & c_{21} \\
c_{12} & c_{22}-c_{1}
\end{array} \mid=0
$$

sein. Diese Gleichung zwoiten Grades fiir "o hribt dir charakteristische Gleichung oder Fundamentalghirhung der Matrix $C$. Sir hat jedenfalls von Null verschiedene Wirzeln, da $r^{r}+0$ ist. Ist $\omega_{1}$ ein

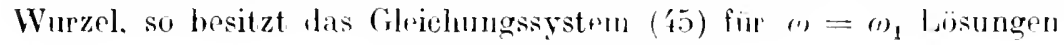

$$
p_{1}=p_{11} \cdot p_{2}=p_{12},
$$

dir nicht beide vessehwinden, und dir nur abgesehrn ron finem gemeinsamen Proportionalitätsfaktor bestimnt sind. Dirse lösungen. in (43) eingesetzt. geben ein Lösungssystem

$$
\begin{aligned}
& y_{11}=p_{11} y_{11}+p_{12} y_{21} \\
& y_{12}=p_{11} y_{12}+p_{12} y_{22}
\end{aligned}
$$

von (A), das sich brim Umlauf $W$ tatsächlich nur mit den konstanten Faktor $\omega_{1}$ multipliziert. - Hat die Fundamentalgleichung (46) zwei verschiedene Wirzeln $\omega_{1}, \omega_{2}$, so finden wir der anderen Wirzel ()$_{2}$ entsprechend ein zweites Lösungssystem

$$
\begin{aligned}
& \eta_{21}=p_{21} y_{11}-p_{22} y_{21} . \\
& \eta_{22}=p_{21} y_{12}+p_{22} y_{22}
\end{aligned}
$$

von (A), das sich bei dem Umlauf $1 t^{\prime}$ nit $\omega_{2}$ multipliziert. Die $r_{i k}$ bilden eine Integralmatrix, denn "sist

$$
\begin{aligned}
& \eta_{11} \eta_{12}=p_{11} l_{12} \quad y_{11} y_{12} \\
& \eta_{21} y_{2 \cdot 2}=p_{21} p_{2 \cdot 2} \quad y_{21} y_{22}
\end{aligned}
$$

und dir Determinante $|P|$ ist von Null verschieden, da die Verhälnisse $p_{11}: p_{12}$ und $p_{21}: p_{22}$ nicht einander gleirh sein können, wenn $\omega_{1}+\omega_{2}$ ist. Ehe wir den Fall erörtern. wo die Fundamentalgleichung (46) zwei gleiche Wurzeln hat, zeigen wir, dal. diese Gleichung insofern nieht von 
der Wahl der Matrix $C^{\prime}$ abhängt. als sire für alle ähnlichen Vatrizen (42) dieselbe ist.

In der Tat ist die linke Seite on (46) die I)eterminante der Matrix $C-I(\omega)$. Bilden wir nun aus $D$ die analoge Matrix $D-I(1)$, so ist nach (42)

$$
\text { l) }-I(1)=I C I^{\prime} 1-I(1)=I^{\prime} C I^{-1}-I^{\prime}(I(1)) I^{\prime-1}=I^{\prime}(C-I(1)) I^{-1} \text {; }
$$

wir haben also

$$
|D-I(1)|=\left|I^{\prime}\right| C-I(0)|| C^{-1} \mid=C^{\prime}-I_{(\prime)} .
$$

d. h. für ähnliche Matrizen stimmen nicht nur dio Fumdamentalgleichungen, sonfern sogar dir linken Seiten dieser Gleichungen überein. Damit haben wir also in der Tat Invarianten für das System aller ähnlichen Matrizen gefunden, nänlich die Koeffizienten der Fundamentalgleichung. Es fragt sich nun noch, ob lirse auch charakteristisch sind, d. h. ob zwej Matrizes. fiir dir diese Invarianten übereinstimmen, auch stets ähnlich sind.

In dem Falle. wo $\omega_{1}+\omega_{2}$ ist. hat die Intrgralnatrix

die Eigenschaft, da?

$$
\left(\begin{array}{l}
\eta_{111} \eta_{12} \\
\eta_{121} \eta_{22}
\end{array}\right)=\boldsymbol{H}=P Y
$$

$$
\boldsymbol{H}=\left(\begin{array}{cc}
\omega_{1} & 0 \\
0 & \omega_{2}
\end{array}\right) \boldsymbol{H}
$$

ist. Wir haben also in diesem Falle (vgl. (41) und (42))

oder

$$
\left(\begin{array}{ll}
\omega_{1} & 0 \\
0 & (1)_{2}
\end{array}\right)=P C P^{-1}
$$

$$
C=P-1\left(\begin{array}{cc}
\omega_{1} & 0 \\
0 & \left(\omega_{2}\right.
\end{array}\right) P \text {. }
$$

Gehen wir statt yon $Y$ von der Integrahnatrix $Z$ aus, so ist

also

$$
\boldsymbol{H}=P Y^{\circ}=P I^{-1} Z \text {. }
$$

$$
D=I^{\prime-1}\left(\begin{array}{cc}
(1) & 0 \\
0 & \omega_{2}
\end{array}\right) P^{\prime-1} .
$$

Es lassen sich also alle mit einander ähnlichen Matrizen (42) dureh Transformation in die Form

$$
\left(\begin{array}{cc}
(1)_{1} & 0 \\
0 & (1)_{2}
\end{array}\right)
$$

überführen; wir bezeichnen für den Fall verschiedener Wurzeln der Fundamentalgleichung (49) als die kanonisehe Form dirses Systems ahnlicher Matrizen.

Weik man ungekehrt von einer Matrix D. daß sie durch Transformation in die kanonische Form (49) ïbergefïhrt werden kaun. d. h. daß eine Matrix (l existiert, so dab 


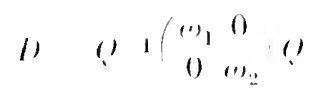

ist, sol lial minll

$$
\text { (1)- () } 12(1,-1()
$$

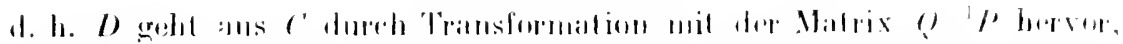

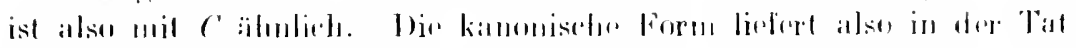

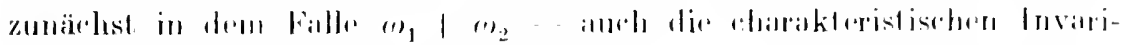
amlon des Systems ïhulicher Matrizent.

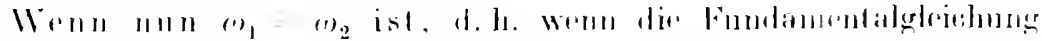

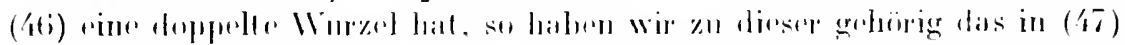

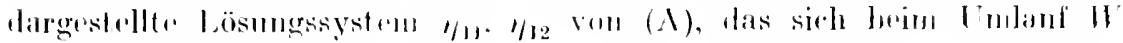

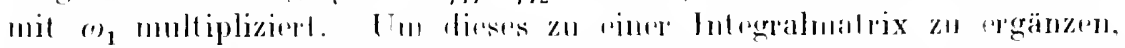

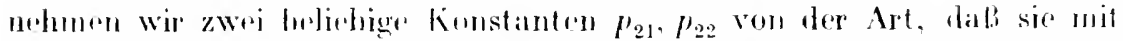

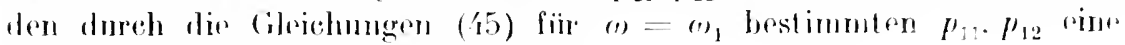
von Null versohiofen, Drterminante

bilden. Sitzen wir damm

$$
p_{11} p_{22}-p_{12} p_{21} \mid 0
$$

so wird dir lntegralmatrix

$$
\eta_{2 k}=p_{21} y_{1 k}+p_{22} y_{2 k} .
$$

$(k=1,2$,

$$
\left(\begin{array}{l}
\eta / 11 / 2 \\
\eta_{22} \eta_{2.2}
\end{array}\right)=H=P \gamma
$$

hrim Undauf H mit riner konstanten Matrix von der Form

$$
\left(\begin{array}{ll}
(1) & 0 \\
\gamma & 0
\end{array}\right)
$$

komponiert, deren Fundamentalgleichung

$$
\left(a_{1}-(\prime)\right)(\delta-(\prime))=0
$$

mit (4ti) ïbereinstinuncen, also die doppelte Wurzel ("), haben mus. Es ist folglich $\delta=()_{1}$. Wälerend $\gamma$ noch nnbestimmt bleibt. Die Matrix $\boldsymbol{H}$ verwantelt sich also dureh dere Tublanf $\mathrm{IV}^{\circ}$ in

$$
H=\left(\begin{array}{cc}
\omega_{1} & 0 \\
\gamma & \omega_{1}
\end{array}\right) \boldsymbol{H}
$$

und wir haben noch zwei Fällo zu unterschrilen, je nachdem

a) $\gamma-10$, b) $\gamma=0$ ist.

In ersten Falle $\gamma \mid 0$ kann diese Gröbe $\gamma$ vermöge der bei der Auswahl von $p_{21}, p_{22}$ noch rorhandenen Willkür jeden beliebigen ron Null verschiedenen Wert erhalten, wir können es z. B. stets so einricht+n. dati $\gamma=1$ wird. Wir bezeichnen dann

$$
\left(\begin{array}{cc}
0 & 0 \\
1 & (1)_{1}
\end{array}\right)
$$

als die kanonische Form des Systens ähnlicher Matrizen (42). 
In zweitrn Fall $\gamma=0$ hat anch das tösurgssystrm $\eta_{21}$. $\eta_{22}$ die Eigensehaft, sich beim Umlauf $I V$ mit dre konstanton $\omega_{1}$ zu rultiplizieren, die $p_{21}, p_{22}$ inüssen also denselben Gleirhungen (45)

$$
\left(s_{1}\right) \quad \begin{aligned}
& p_{1} c_{11}+p_{2} r_{21}=w_{1} p_{1} \\
& p_{1} r_{12}+p_{2} c_{22}-m_{1} p_{2}
\end{aligned}
$$

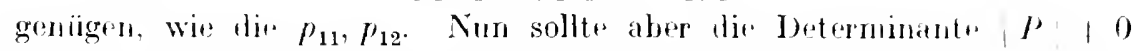
soin; dir Verhälnisse $p_{11}: p_{12}$ und $p_{21}: p_{22}$ diirfen also nirlit ïherein-

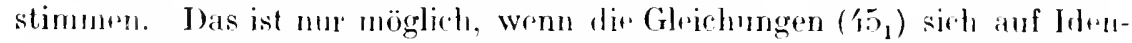
titäen roduzieren, d. h. wenn alle iher Koeffizienton gloich vill simul. In diesem Falle ist also

$$
r_{11}=\prime_{1}, r_{22}=\prime_{1}, r_{12}=r_{21}=0 \text {. }
$$

so dal.s sich also jode Integralnatrix Y beim! Vmatul II mit Mre Matrix

komponiert

$$
\left(\begin{array}{cc}
\omega_{1} & 0 \\
0 & \omega_{1}
\end{array}\right)
$$

Zusammenfassend können wir also sagen: ] )ir Matrix C kann durm Transformation stets in eine dre droi kanonisrlon Formen (49), (50). (51) iibergefiilnt werden. Die erste Fornt "ntspricht dem Falle. wo die Fundamentalgleichmng $|C-I \omega|=0$ zwei vessehiedene Wurzeln hat, dic zweite dem, wo diese Cileichung eine doppelte Wurzel $\omega_{1}$ hat. ohne daB alke vier Elemente der Determinante $\left|C-I \omega_{1}\right|$ rerschwimene, die dritt" den, wo das letztere eintritt. Alle ännlichen Matrizen haben dirselbe kanoniselıe Form und offenbar sind - wie in Falle $\omega_{1} \mid \omega_{2}$, $s$ ) anch allgemein - zwei Matrizen, die dieselhe kanonisehe Forul haben. auch stots ähnlich.

In dieser rein algebraischen Form bildet der ansegsprorher satz. we wir sohen verden, das algehraiseho Greppen der gamzen analytiseh+n Theorie tes Differentialsystems (A). Anf unser Ausgangsproblem, das

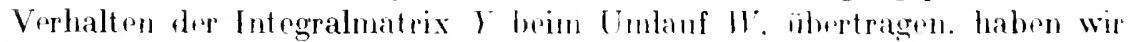
las Ergebnis:

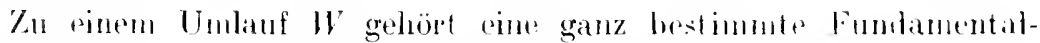
gleiehumg (46) und rine Integralmatrix $H$. depen Wretanderung hei

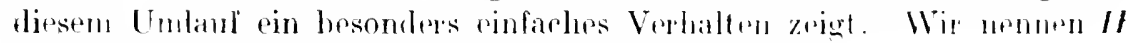

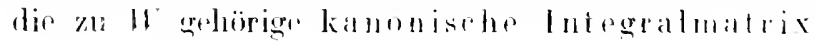

\section{Darstellung der Integralmatrix in der Ungebmng einer} isolierten singulizen Stelle. ('all chysche Differentialsysteme.

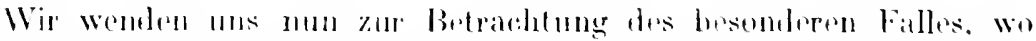

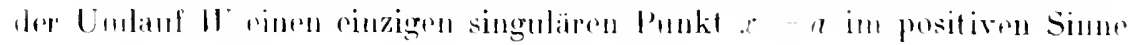




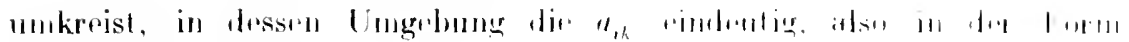
l,anroutsehro Rrihur

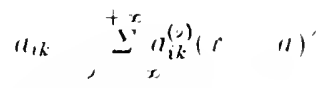

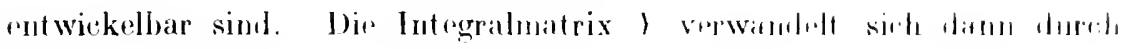
don Unlanf in

$$
\gamma=r i
$$

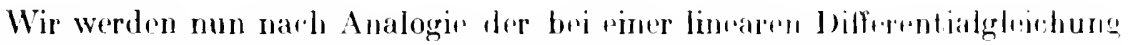
erster Ordnung in der Nr. 34 angestrilten C̈berlogungen fragerl, ob wil rino Matrix ? leicht angobbarer Funktionen $\mathfrak{y}_{i k}$ finden können, dir bu drm Undaul II in

$$
\text { ?) }
$$

ïbergeht? Wir wollen mit einer in der Agebra der limearen Transturmationen üblichen Bezoichnung von zwei Matrizen, die, wir hior Y and !), bei einem Unlanf $W \mathrm{~V}$ mit derselben konstanten Matrix konnonient werden, sagen, sie seien in brzug aul den Unlauf $W^{\prime}$ kogredient.

In dor Nr. 34 lioforte dio Cauchyschr Differentialglejehong (III) die gesuchte Funktion n. Wir versuchen dementsprechend anch hior ein Cauchysches Differentialsystem zu bilden. d. h. oin Tifferentialsystem

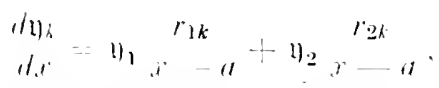$$
\left(\begin{array}{lll}
k & 1 & 2
\end{array}\right.
$$

wo die $r_{k k}$ Konstanten bedeuten, und fragen, ob sich diese $r_{i k}$ sh brstimmen lassen, dal.s eine Integralmatrix ?! von (B) mit unserer Intrgrahmatrix $Y$ in bezug auf $I V$ kogreilient sei:

Was zunächst die Integration des Systrms (B) anlangt, st brmerken wir, dali durch Einfïhrung vor

$$
\text { t. }=\log (x-a)
$$

als neuer unabhängiger Veränderlicher, (B) in das tystem mit konstanten Koeffizienten

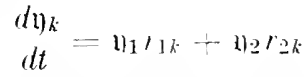

übergeht. Dip pine Infferentialgleiehung mit konstanten Kopfizienten

$$
d \mathfrak{l t}=\mathfrak{u} \cdot r
$$

wird durch $\mathfrak{n}=$ const. $e^{\text {th }}$ gelöst. Man kann nun ganz analog zu der allgemeinen Lösung des Systems (C.) gelangen, wenn man in naheliegender Verallgemeinerung eine Exponentialfunktion der Matrix $R$ bildet.

Was man unter riner positiven ganzzahligen Potenz einer Matrix $P$ zu verstehen hat. liegt anf der. Hand; es ist 


$$
k^{2}=k \cdot R, K^{3}=k^{2} R=R K^{2} \text {. usw. }
$$

Danarh kann man ganze rationale Funktionen riner Natrix mit skalaren. d. h. Zahlkoeffizienten herstell+n. z. B.

$$
a_{0}+a_{1} k+a_{2} k^{2}
$$

wo dir $a_{0}, a_{1}, a_{2}$ reelle oder kumplexe Gröben bedruten. Nan kán abrer anch woitergehen und unenulliche Potenzreihen riner Matrix untersurden ${ }^{1}$ ).

Hat man eine Potenzreih".

$$
\alpha_{0}+\alpha_{1} t-\alpha_{2} t^{2}-\cdots,
$$

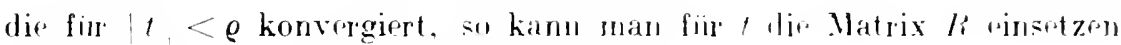
und fragen. wann die Elemente der Matrix

$$
a_{0}-a_{1} l i-a_{2} l^{2}-\cdots
$$

konvergent sein werden? Dunt man sich $K$ in dit kanonisch. Form transformiert :

$$
l=P=\begin{array}{lll}
r_{1} & 0 \\
\gamma_{2} & r_{2}
\end{array}
$$

wo $\gamma$ nur dann von Null verschieden scin kann. wrnn $r_{2}=r_{2}$ ist. so ist offenbar für jedes positive ganzzahligo $\gamma$

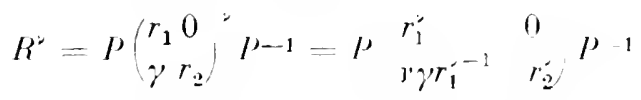

und man kann folglich die Matrix (5:i) in der Forul

$$
\left.p\left(\alpha_{0}+\alpha_{1} \begin{array}{l}
r_{1} 0 \\
\gamma r_{2}
\end{array}\right)+\alpha_{2} \begin{array}{ll}
r_{1}^{2} & 1) \\
2 \gamma r_{1} & r_{2}^{2}
\end{array}-\alpha_{3} \begin{array}{c}
r_{1}^{3} \\
3 \gamma r_{1}^{2}
\end{array} r_{2}^{3}\right)-\cdots p^{\prime-1}
$$

schreiben. Da die derivierte Reihe $a_{1}-2 \alpha_{2} t+3 a_{3} t^{2}-\cdots$ ron (52) ohenfalls für $|t|<\varrho$ konvergiert. so ist also für die Konvergenz der Matrix (53) notwendig and hinreichend, dals die Wurzeln $r_{1}$. $r_{2}$ der zu $R$ gehörigen Fundamentalglei"hung lem Konvergenzkreise der Reihe (52) angehören.

Nimmt man fïr (52) die beständig kenvergente Exponentialdeihe. so erhält man lie Exponentialfunktion der Matrix $R$

$$
e^{R}=I+\frac{l 1}{1 !}+\frac{l^{2}}{2 !}+\cdots
$$

die demnach für jede Matrix $h$ komvergiert. Bedenten $h$. s zwei vertauschbare Matrizen. so dali also $R S=s h$ ist. dann ist auch jete Potenz von $R$ mit jedor fotonz von is und bolglieh atueh en mit $e^{s}$ vertauschbar: man fimlet also dureb komposition:

1) Es kommt das anf dasselbe hinans, wie wenn man P'utenzreihen sun höheren komplexen Gröben, die ans mehreren llanpteinheiten gebildet sind. betrachtet. In dieser form hat kd. Weyr im Bulletin des Sciences Mathem. (2) $X I$ (1887). S. 20: das l'rollem behandelt und das in folgenden wiedergegebene Konvergenzkriteriom aufgestellt. 


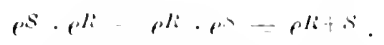

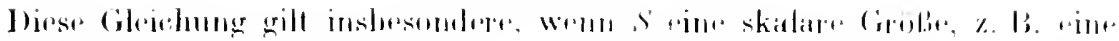

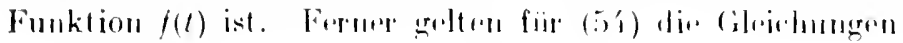

$$
\begin{aligned}
& \text { e li }\left(e^{k}\right)^{-1} \text {, }
\end{aligned}
$$

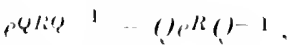

derent Broweris and der lland lioner.

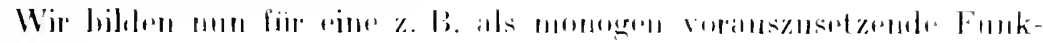
lioll $f(l)$

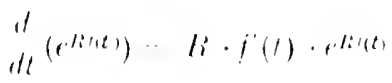

und witer

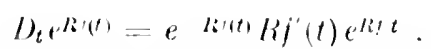

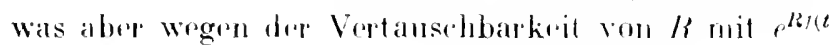

$$
H_{t} e^{(t / t)}=l i f^{\prime}(t)
$$

ergibt. Also ist $p^{k f(t)}$ ein" Integralmatrix les loffrerentialsystems

$$
d l_{1 k}=\left(n_{1} r_{1 k}+\eta_{2} r_{2 k}\right) l^{\prime}(l) \text {. }
$$

Für $f(t)=t$ erhalten wir oine Intrgrahuatrix

$$
\because=e^{n t}
$$

des Systems (C), und zwar resluzient siclo diose Intogralnatrix für $t=0$ auf die Einheitsmatrix $l$. J)ir allgemeinste Integralmatrix von (C) hat also die Form

$$
\text { Pe } e^{k t}
$$

wo $P$ eine willkürliche konstante Matrix mit nieht versehwindender Determinante bedeutet.

Für rlas Caurliysche Systrm (B) haben wir dengenäl die Intrgralmatrix

(58)

$$
\stackrel{\eta}{l}=\rho R \log (x-a) \text {. }
$$

die wir nach Analogie der ,.skalaren" Formel $(r-a)^{r}=e^{r \log (r-a)}$ auch in der bequemeren Gestalt

(58a)

$$
y)=(x-a)^{R}
$$

schreiben können. Wenn $x$ den geschlossenen Umlauf 11 vollzieht, der den Punkt $a$ im positiven Sinn" umkreist, so repwandelt sich ? in

$$
\text { y) } \left.=e^{R(\log (x-a)+2>i}=e^{R 2>i}\right)
$$

Wir fragen nunmehr, ob sirh dir Matrix $R$ stets so bestimmen lärt, daß $\mathfrak{Y}$ mit der Integralmatrix $I$ von (A) in bezug auf den Umlauf ${ }^{\prime}$ kogredient sei, d. h., dal.

$$
\rho R \cdot 2 \pi l=C
$$

wird? Es handelt sich hier also um dir Inversion der Matrizenfunktion $e^{R \cdot 2.7 i}$, d. h. gewissermaßen un das Analogon des Logarithmus einer 


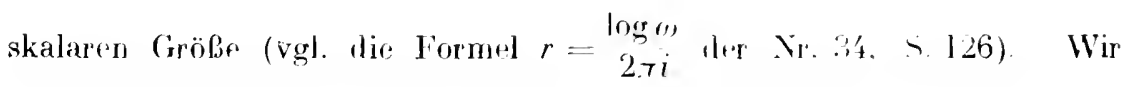
können diese Inversion hier in folgender Wrist vernehmen. Es sei

$$
C=P^{-1} \Omega P
$$

wo Q dir kanonisehe Form von $C$ bedeutrt, alsi) wine dur drei Formen

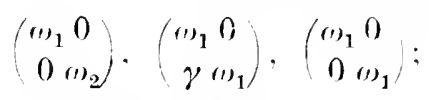

wir nehmen $\gamma^{\prime}=2 \pi i \omega_{1}$. Wir bilden dam

$$
r_{1}=\frac{\log \omega_{1}}{2 \pi i}, r_{2} \quad \stackrel{\log \omega_{2} \omega_{2}}{2 \pi i} .
$$

wo den Logarithmen ihre volle Mehrdeutigkeit belassen werdr. on dafir also die $r_{1}, r_{2}$ nur abgesehen von additiven ganzen Zahlen bestinnt sind; nur in Falle der zweiten Form (61), wo ()$_{1}=\left(m_{2}\right.$ ist. n+hmen wir $r_{1}=r_{2}$, dagegen können sich im Falle der dritten Form $r_{1}$ und $r_{2}$ um eine ganze Zalhl $g$ unterscheiden. Entsprechend derjenigen der drui Formen (61), die $\Omega$ besitzt, bezeirhnen wir dann mit $\sum$ dir entspreshende der drei Matrizen

und setzen

$$
\left(\begin{array}{ll}
r_{1} & 0 \\
0 & r_{2}
\end{array}\right),\left(\begin{array}{cc}
r_{1} & 0 \\
1 & r_{1}
\end{array}\right) \cdot\left(\begin{array}{ll}
r_{1} & 0 \\
0 & r_{1}+g
\end{array}\right.
$$

$$
R=P^{P-1} \Sigma P^{\prime}
$$

dann ist

$$
\rho^{k \cdot 2 n}=C^{\prime} \text {. }
$$

und man sieht auch leicht, daß die mit der angegebenen Willkurlirhkeit in den $r_{1}, r_{2}$ behaftete Matrix $R$ die allgemeinste ist, dir diest Gleidhung befriedigt. Die mit diesen $l i$ gebildete Matrix $:)=(, r-a)^{R}$ ist nuit $l$ in bezugr aul $\mid V$ kogreslient.

Die Analogie mil den Betrachtungen der Nr. 34 fuhrt nun ohne weiteres zur qualitativen Darstellung der $y_{k}$ in der lingehung von $r=a$. Da

ist, so ist

$$
1-(Y, y=(?) !
$$

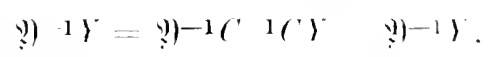

die Elentente der Matrix

$$
(1)=?-1\rangle
$$

sind folglich in der fomgehung von $x=a$ eimlentigr. Sir haben former.

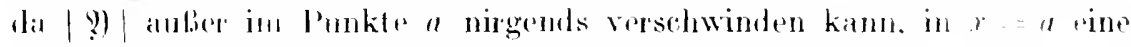

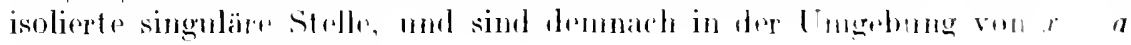
in Landentseler Reihern

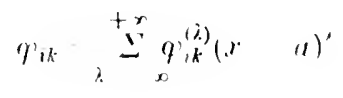




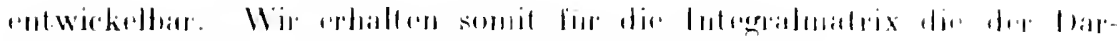

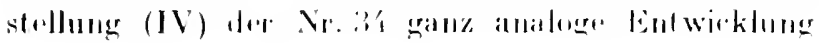

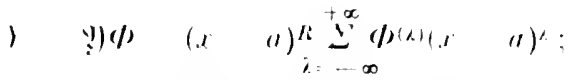

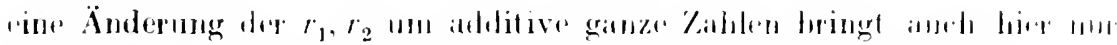

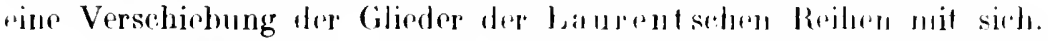

Wir geben neben diment symbolischen formeln, deren lindentumer wesentlich anf der suggestiven Kraft dor Analogie mit dem Falle der

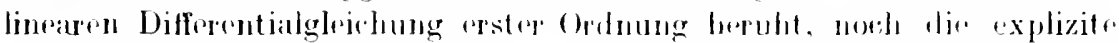

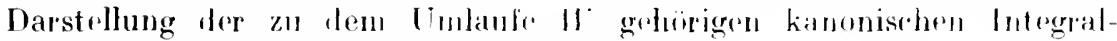
matrix H. Für diese hatten wir ( $\mathrm{Xr}$.: is) !

$$
\boldsymbol{H}=\underline{I H}
$$

es wird also an die Stolle von ?l) jetzt pinfach $(x \text { a })^{2}$ tretern. Ju nachdem welche der drei Formen (6.3) nun I hat, rhalton wir fir $(x-a)$ 亡 die drei Typen

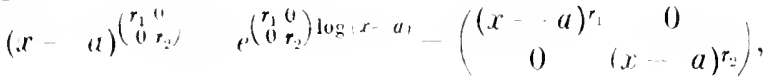

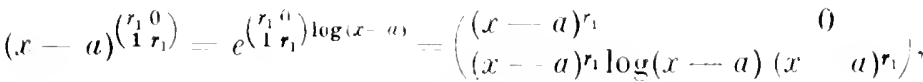

$$
\begin{aligned}
& \left(\begin{array}{ll}
x \quad a
\end{array}\right)^{\left(\begin{array}{cc}
r_{1} & 0 \\
1 & r_{1}+g
\end{array}\right)}=e^{\left.\left(\begin{array}{cc}
r_{1} & 0 \\
0 & r_{1}+g
\end{array}\right) \log x-a\right)}=\left(\begin{array}{cc}
(x \cdots a)^{r_{1}} & 0 \\
0 & (x-a)^{r_{1}+g}
\end{array}\right)
\end{aligned}
$$

und damit fijr dir $\eta_{i k}$ lie Darstellungen:

$$
\begin{aligned}
& r_{11}=(x-a)^{r_{1}} / q_{11} . \quad t_{12}=(x-a)^{r} / t_{12} . \\
& \eta_{21}=(x-a)^{r}=4_{21} . \quad r_{122}=(x-a)^{r}+\psi_{22}: \\
& r_{111}=(x-a)^{r_{1}} q_{11} . \quad 1_{12}=(x-a)^{r_{1}} q_{12}, \\
& \gamma_{/ 21}=(x-a)^{r_{1}}\left(q_{21}+q_{11} \log (x-a)\right) \text {. } \\
& r_{22}=(x-a)^{r_{1}}\left(q_{22}+q_{12} \log (x-a)\right) \text {; } \\
& r_{111}=(x-a)^{r_{1}} q_{11}, \quad r_{12}=(x-a)^{r_{1}} q_{12}, \\
& r_{/ 21}=(x-a)^{r_{1}+g} q_{21} . \quad r_{i 22}=(x-a)^{r_{1}+y} q_{22.2} \text {. }
\end{aligned}
$$

Zur quantitativen Bestimnung der $r_{k k}$ und der Koeffizienten $\varphi_{, k}^{(i)}$ in den Reihen $\varphi_{i k}$ hat man die in (65) dargestelltr Lösung $\gamma$ in das Differentialsystenı ( $A^{\prime}$ ) einzusetzen und die Koeffizienten gleich hoher Potenzen ron $x-a$ zu rergleichen. Un diese Rechnung einfach ausfïhren zu können, bemerken wir, daß für zwei Matrizen differentiierbarer Funktionen $Y$ und $P$, deren Determinanten nicht identisch verschwinden, dir Derivationsformel gilt:

$$
D_{x}(Y P)=I^{3-1} D_{x} Y \cdot P+D_{x} P,
$$


deren Beweis unmittelbar ans dor I) finition des Symbols $D_{s}$ (..r. 35) folgt. Wir haben also für $Y=? \mid \Phi$ :

$$
D_{r} \gamma=\left(D-1 D_{s}(y) \cdot \Phi+D_{x} \Phi=A .\right.
$$

und da g) rine Lösung des Cauchyschen Systems (B). d. h.

$$
\left.l_{r}: l\right)=r
$$

ist. so erhalten wir ans (bit), indem wir mit $\phi$ won links her komponterem und nit $x-a$ durchmultiplizierens.

$$
R \Phi+(r-a) \frac{d}{d r} \Phi=\Phi .1 \cdot(r-a):
$$

darin ist

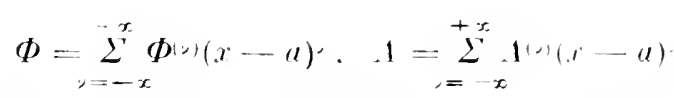

zu setzen. Durch Koeffizientenvergleichung ergibt sich ditnu

$$
\left(R+r^{\prime}\right) \Phi\left(=\sum_{1+\mu=0-1} \Phi^{(\lambda)} \mathcal{A}^{(n)} .\right.
$$

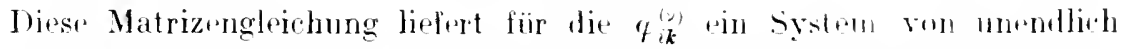
vielen linearen Gleichungen mit unendlich vielen Inbekannten. das int allgenteinsten Falle nur durch transzendente Methonden gellost wriden kann. Eine besonders degante Lösung hat Helge ron Kuch mit Hilfe von Determinanten unendlich hoher Ordmung gegeben ${ }^{1}$ ). Wir wollen uns aber auf die Betrachtung der Fälle heschränken. wo dir lobsung jones Systems linearer Gleichungen durch elementar" algebrais whe Methoden erfolgen kanu, es ist das der Fall. wo in den laurentsehen buihern $\Phi$ nur eine endliche Anzahl negativer Exponenten vorkonmut. also dep Fall. wo die Lösungen I in dem Punkter $r=a$ nirht unhestimnt werden. Wir bezeichnendirsen Fall kiirzer als den der Bestimmtheit. $E s$ ist das bleibende Verdienst von Furhs, dipere klass. von singularitäten hervorgehoben und sie eingehend untersurht zu haben 2). Durch diese Beschränkung erst. ist dir Entwiekhng der andytisehen Therie der linearen Dilferentialgleichungen ermöglicht worden.

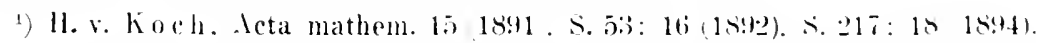
S. 337: vel. die Darstellung bei II orn. at. a. (). S. 2leff und lui f. Riesz. less systimes d'équations linéaires a une infinite d’inconnues. Paris 1913. S. 15ti. An diesen Stellen werden vorwiegend statt der Systeme lintare I) ifferentialgleichungen bïherer Ordnung behandelt.

2) L. Fuchs, 1 s65. Werke I. s. $111 \mathrm{ft}$. 
Sirrlistis Kilpit.l.

\section{Untersuchung der singuliiren stellen, wo die Integrale nicht mbestimmt werden.}

\section{Limeare Differentialgleichung zweiter Ordunng. Hestalt dor Koeftizienten in der Umgehung einer singulären Stelle der Bestimmtheit.}

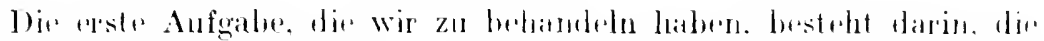
Form der Koeffizienten $a_{i k}$ in der Congebung eine singulären Stelle anzugeben, an der die Lisumgen unseres Differentialsystens nieht unbestimmt werden. Es zeigl sich. daf rine rinfarhe notwemlige und hin.

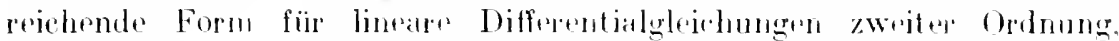

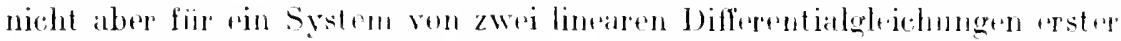

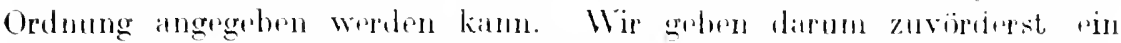
Verfahren, das die Lösung des Systens (1) auf die oiner Differentialgleichung zweiter Ordnumg zuriekzufïhren lehrt.

Differentiert man aie Gleichungen les Systeme (1)

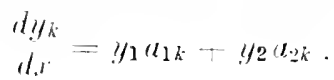

so kornmt

$$
y_{k}^{\prime \prime}=y_{1}^{\prime} a_{1 k}+y_{2} a_{2 k}+y_{1} a_{1 k}^{\prime}+y_{2} a_{2 k}^{\prime} .
$$

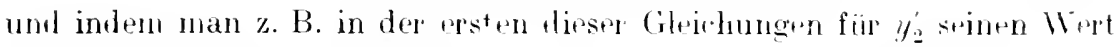
aus (A) einsetzt, erhält mau

$$
y_{1}^{\prime \prime}=y_{1}^{\prime} a_{11}+y_{1}\left(a_{12} a_{21}+a_{11}^{\prime}\right)-y_{2}\left(a_{22} a_{21}+a_{21}^{\prime}\right) \text {. }
$$

Wenn nun $a_{21} \neq 0$ ist, so gibt dir erstr der Gleichungen (A)

$$
y_{2}=a_{a_{21}}^{1}\left(y_{1}-y_{1} a_{11}\right) \text {; }
$$

wir finden also

(2) $y_{1}^{\prime \prime}=y_{1}^{\prime}\left(a_{11}+a_{22}+\begin{array}{c}a_{21}^{\prime} \\ a_{21}\end{array}\right)+y_{1}\left(a_{12} a_{21}-a_{11} a_{22}+a_{11}^{\prime}-\begin{array}{c}a_{11} a_{21} \\ a_{21}\end{array}\right)$.

d. h. eine lineare homogene Differentialgleichung zweiter Ordnung für $y_{1}$. Wäre $a_{21}=0$, aber $a_{12} \neq 0$, so könnte in derselben Weise eine ebensolche Differentialgleichung für $y_{2}$ hergestull werden. Sollten beide hoeffi- 


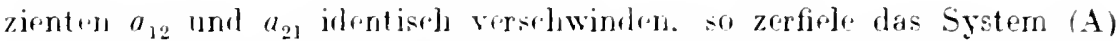
in zwei Differentialgle.dmugen erstre Ordnung fïr $y_{1}$ und $y_{2}$ allein; diesen Fall können wir also leiscitr lassen, d. h. wir können annehmen. daß wenigstens riner der beiden Korffizienten $a_{12}$. $a_{21}$ nicht identisch, verschwindet. Es sei dirs für $a_{21}$ der Fall, dann gilt also für $y_{1}$ die Differentialgleichung zweiter Ordmug (2). und wrin diese gelost ist. so wird $y_{2}$ durch (1) unmittelbar gegeberl.

Wir sohen. dali dir Koeffizienten der Differentialgleiehung (2) und "henso dir der Relation (1) sich rational zusammensetzen aus den $a_{k}$. und ihren Ableitungen. sie gehören. wie man sagt. demselben Rationalitätshereiche an. wir die koeffizenten des Systems (A): wenn z. B. die $a_{i k}$ rationale Funktionen von $x$ sind. so sind rs ateh die Korffizient'n in (2) und (1).

Hat man umgekehrt rine lineare Differmtialgleirhung von der Form (2)

$$
u^{\prime \prime}+p u^{\prime}+q u=0 .
$$

so kinn man durch dir substitution

snforl zn dent System

$$
u=z_{1} \cdot u^{\prime}=z_{n}
$$

$$
\begin{aligned}
& d z_{1} \\
& d x \\
& d z_{2} \\
& d x
\end{aligned}
$$

ubergehen. Sind $z_{i k}$ die Elemente einer Integralmatrix von tit). also

$$
z_{1}-c_{1} z_{11}+c_{2} z_{21} \cdot z_{2}=c_{1} \overline{1}_{12}-c_{2}=2
$$

mit willkïlichen Konstanten $c_{1}$. C2 das allgemene Lösungssystem diess systrms. so stellen

$$
z_{11}=u_{1} \cdot z_{21} \cdot u_{2}
$$

«wei partikuläre Lösungen von (3) dar. für dir nach der esten der Gloi(lougen (4)

ist. Firner liefort

$$
i_{12} \quad u_{1} \cdot i_{22}=u_{2}
$$

$$
u+c_{1} u_{1}-c_{2} u_{2}
$$

dir allgeneene Lösung ron (3). Man sieht. dal. in ditrem falte die be-

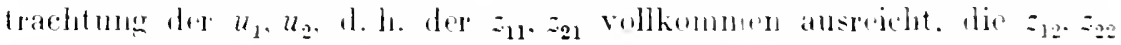

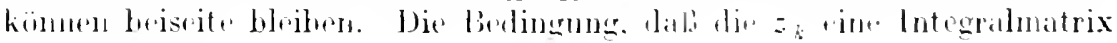
bilden. wair

in muserem Falle ist also

$$
\text { 7. } 10 \text { : }
$$

$$
\left.u_{1} u_{2}-u_{2} u_{1} \quad u_{1}^{2} \begin{array}{cc}
d & u_{2} \\
d_{2} & u_{1}
\end{array}\right)+0,
$$




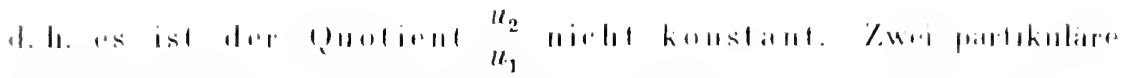

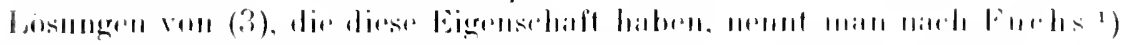

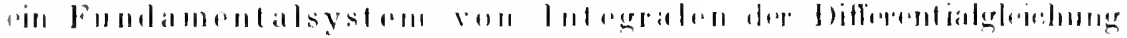

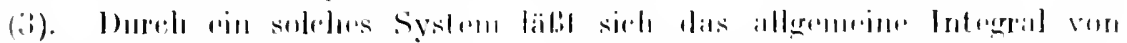

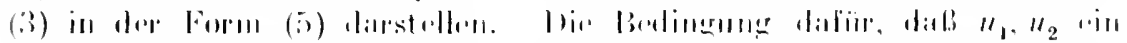

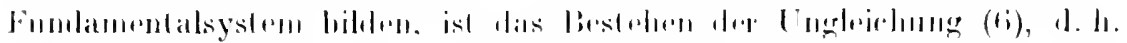

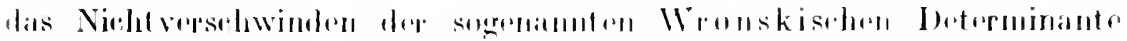

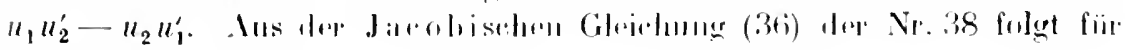
IIISIPII Fill. WO

(7) $\quad a_{11}-0, a_{21} \quad 1, a_{12} \quad \eta, a_{22} \quad p$

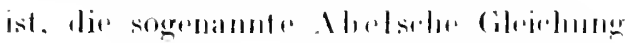

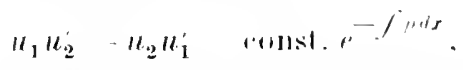

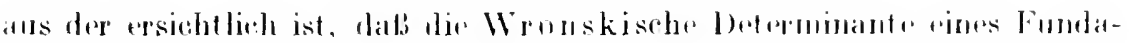
mentalsystrms nur in dro simendären Punkten des Kowfizionten p versithwinden kann.

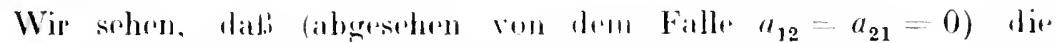

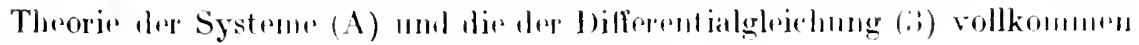
atufrinanter zurïckgefiihrt werelen kännon.

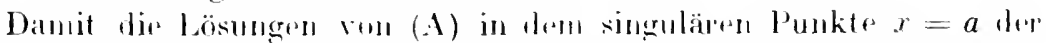
Konffizionten nieht mobestimmt werden, gronigt as, dab diese Eigen-

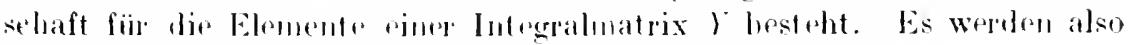

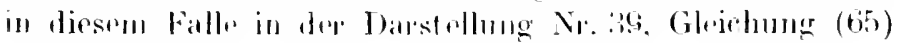

$$
\gamma=\text { ? }
$$

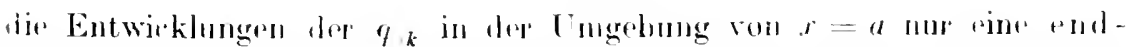
lirhe Anzahl negativer Potenzen inthalten. Damn enthät auch die Entwieklung der Jetopminante $|\Phi|$ nur rint emllirhe Anzahl negaliver Potenzen, mud la

$$
|1|=|? !||\phi|
$$

ist, so wirl auch jedes Element lew Matrix

$$
11-\Phi 1 ? 1
$$

in $x=a$ nicht mubestimml sin. l)a

$$
\left.A=D_{x}\right)-1-1 \frac{d 1}{d x}
$$

ist. so sind auch dir $a_{i k}$ im Punktr $x=a$ nicht unbestimut, und da sie in ller Ungebung von $x=a$ rindeutig srin sollten, so haben sie daselbst ainen Pol. Das gleiche gilt dann auch von den Koeffizienten der Differentialgleichung (2) und überträgt sich danit ohne weiteros anf dir beliebige lineare Differentialgleichung zwoiter Ordnung (3).

1) L. Fuchs 1in6s. Werke 1. S. 117.

schierifiger. Differntialgiticlinn: 
Wï untersuchen nun zuvörlerst dir Jifferentialgleichung (3). Wenn dir Lösungen ron (3) in $t=a$ nicht nubustimut simd, so hat das Fumdamontalsystru, das der 7.1 $r=a$ gehörigen kanonischer Intrgralmatrix des Systrms (4) entspricht. mol las wir als das kanonisch. Fundamentalsystem bropichuer wollen. in der Timgubung ron - div Form

$$
\begin{aligned}
& r_{1}=(1-a)^{r} / 11 . \\
& r_{2}=(x-a)^{r}:\left[1 / 21-k i q_{11} \log (1-a)\right] .
\end{aligned}
$$

wo dir $\psi_{11}, q_{21}$ nur eine wulliche Anzahl negativer lenterzon erothalten und $k$ glejeh 0 orlor 1. aber allemal glrieh Null ist. wonn $r_{\text {, von }} r_{2}$ vepschiecten ist.

Wir zirhen daun dir höchste negative lotenz $(r-a)^{-a}$ ans $f_{11}$ heraus, vereinigen sie mit $(x-a)^{r_{1}}$ mod "rhalten anf liese Wrise für $v_{1}$ die Darstolluner

$$
\therefore \quad(i-a)^{r_{1}-J_{1}}(x-i)
$$

wo jetzt $r_{1}-g$ vollkommen bestimnt mol $\gamma_{1}(x-a)$ in der Lomgehung von $x=a$ holomorph. und fïr $r=\|$ von Null versthieden ist. Damn ziehen wir aus $p_{21}$ und $k q_{11}$ die höchste nogative Potenz $(r-a)^{-h}$ herans. so dalli r.g lie Form rhält:

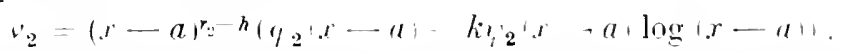

wo $f_{2}(x-a)$. k $y_{2}(x-a)$ in dor Lugrebung ron $x=a$ holomorph. und in $x=a$ nieht beide gleichzeitig gleich Null sind. Dadureh ist anch $r_{2}-h$ vollkommen frstgelegt. Wir lwegchmen mun dirst rindoutig festgelegten Exponenten $r_{1}-g, r_{2}-h$ wiedor mit $r_{1}, r_{2}$ und sohroibun also das kanonische Fumdamentalisstem in der Form

J)i" londinkt"

$$
\begin{aligned}
& \therefore \quad i r-a r^{r} t_{1} \text {. }
\end{aligned}
$$

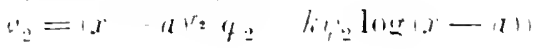

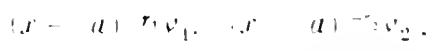

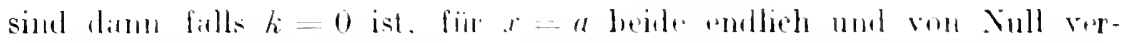
sehiedell. dagegen wird. Wrme $k \mid 0$ ist. das zweite fiir $r$ a so mondlich, wio dre Ausdruck

$$
8=101+k \cdot 2010 \log 2-11
$$

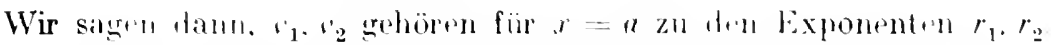

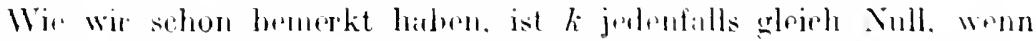

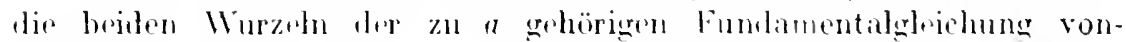

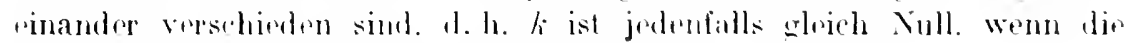
bifferenz der Exponenten $r_{1}, r_{2}$ krine gan\% \%all ist.

Auch dide folerende bermerkmug ist oft von Nutzen.

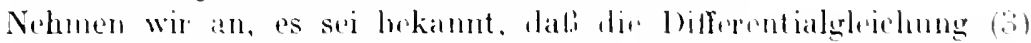

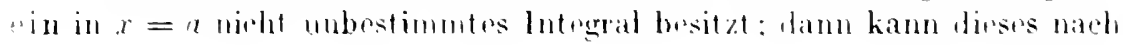


den allgemeinen Ergohnissen in der limgubung von $t=a$ jedmofalls in der Form

$$
\left.\left.v_{2}=-x-a\right)^{r}: q_{2}(x-a) \cdot k \cdot 1_{2}(x-a) \log (x-a)\right) \text {. }
$$

largestellt werden, wo $\varphi_{2}, \psi_{2}$ gewöhnliche Potrnzreihen bedenten. Iassen wir dann $x$ un den Punkt a don positiven Imanf / beschruben. so inultipliziert sich

$$
(x-a)^{r_{1}} \text { mit perir. }
$$

und $\log (x-a)$ vermolnt $\operatorname{sich}$ un $2 \pi i$; alsu repwandelt sich $\varphi_{2}$ in

$$
v_{2}=e^{2 \pi i r_{2}{ }_{2}}+e^{2} \operatorname{vr}(x-a)^{r_{2}} \cdot{ }_{2}(l-a) \cdot 2 \pi i \cdot k .
$$

und $\omega_{2}$ ist ebenfalls rin Intrgeal. Dann ist aber auch

$$
e_{2 \pi i}^{2 \pi i r_{2}}\left[o_{2}-e^{\left.2 \pi i r_{2} \nu_{2}\right]}=k \cdot(x-a)^{r_{2}}: i_{2}\left(t^{\prime}-a\right)\right.
$$

ein Integral, d. h. wenn ein Integral $\nu_{2}$ vorhanden ist, das in $x=a$ nicht unbestimmt wird, so ist der mit dem $\log (x-a)$ multiplizierte Ausdruck selbst ein Integral, es gibt also dann stets auch ein in Reihenform darstellbares Integral, das in Punkte a nicht unbestimmt ist.

Wir suchen nun das Verhalten der Koeffizienten der Differentialgleichung (3) in der Ungebung von $x$ a festzustellen, unter dor Voraussetzung, daß die Integrale in diesem Punkte nicht unbestimmt werden, daß also das kanonische Fundamentalsystem die Form (9) besitzt.

Setzen wir in der Differentialgleichung

$$
u=v_{1} \text { ivd.r. }
$$

woraus

$$
\begin{aligned}
& u^{\prime}=v_{1} v^{\prime}+v_{1}^{\prime} / v_{d} r . \\
& u^{\prime \prime}=v_{1} v^{\prime}+2 v_{1}^{\prime} v+v_{1}^{\prime \prime} l v d x .
\end{aligned}
$$

so ergibt sich mit Rücksicht darauf, dal. $v_{1}$ der Differentialgleichung (3) genügt, für $v$ die Gleichung erster Ordnung:

$$
d v+v\left(\begin{array}{c}
2 v_{1}^{\prime} \\
v_{1}
\end{array} d p\right)=0 .
$$

Setzen wir das allgemeine Integral dieser Gleichung

in den Ausdruck

$$
r=e^{-i\left(\frac{2 v_{i}}{n}+p\right) d r}
$$

$$
u=v_{1} i v d x
$$

für $v$ ein, so genügt $u$ der Differentialgleichung (3). und umgekehrt bofriedigt

$$
f=\stackrel{d}{d x}\left(\begin{array}{l}
u \\
v_{1}
\end{array}\right)
$$

der Differentialgleichung (10). wenn $u$ irgendeine Lösung von (3) bedeutet. Also genügt auch 


$$
d \quad \frac{d}{d x}\left(\begin{array}{l}
b_{2} \\
r_{1}
\end{array}\right)
$$

der Jifferentialgleichung (10). In der Umgebung von $x=a$ ist

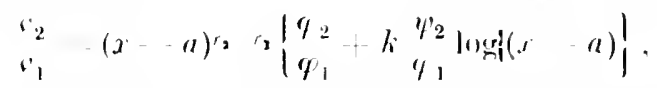

also ist dirser Quotient im Punkto $r=a$ keinesfalls mubrestimul mud

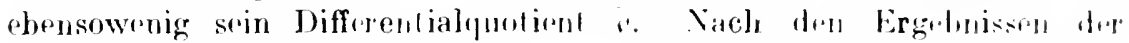
$\mathrm{Nr}$ 34 (S. 129) folet aluer daraus, dal.i das Introgral von (10) im Punkte a richt mobstimut ist, lal. Mre Korffoirnt dieser Differentialgleichung in $x \Rightarrow a$ einen Pol erster Orrmmer besitzt: wir haben a!so in der Ungrobung von $x=a$

$$
\begin{array}{cccc}
2 r & & W(x-a) \\
r & r & x-a
\end{array}
$$

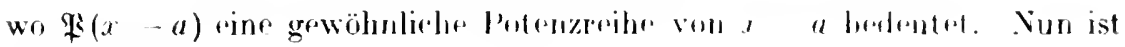
aber forner

$$
2 r_{1}^{\prime}=2 \frac{r_{1}}{x-a}+2 \frac{q_{1}(x-a)}{r_{1}(x-a)}-2, r_{1}+\mathrm{L}(x-a) .
$$

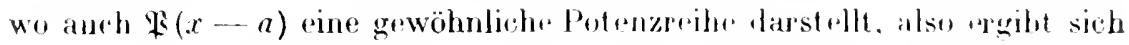

$$
p=\begin{aligned}
& \mathfrak{P}(x-a) \\
& x-a
\end{aligned} 2^{r_{1}}, \mathfrak{F}(x-a) \begin{gathered}
\mathfrak{F}_{1}(x-a) \\
x-a
\end{gathered} .
$$

wo $\mathfrak{F}_{\mathbf{1}}(x-a)$ rine grwöhuliahe Potrnorihe von $x-a$ bedentet, al. h. $p$ verhält sich in der Ungebung voun $x=a$ wie rine rationale Funktion und hat in diesen Punkte einen l'ol rester Ordnung.

Aus aler ideutischen Gleichung

brrechurn wir

$$
\because+p \cdot \because+q \cdot 1=0
$$

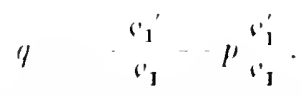

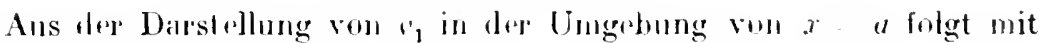

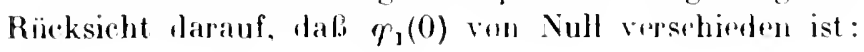

$$
\begin{array}{ll}
r 1 & 1 \\
r & x^{\prime}-a
\end{array}
$$

wo $\mathbb{F}_{3}(x-a)$, 疋 $1(x-a)$ gewöhnliche Potenzreihen von $x$ - a bedenten; also haben wir

$$
\begin{aligned}
& \text { q } \quad(x-a)^{2}: W_{1}(x-a)+W_{1}(x \quad a) \cdot W_{3}(x \quad a) ! \\
& \mathbb{H}_{2}(x)
\end{aligned}
$$

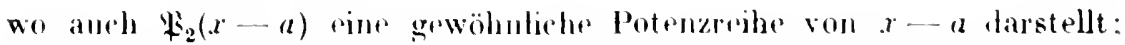
d. h. q verhäl sich in der Ungehung voll $x=$ a chenfalls wir eine rationale Fonktion und hesitzt dasellest rimen Pol zweiter Grdnung. 


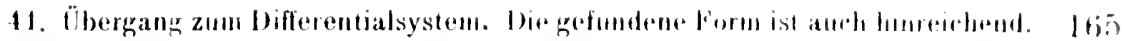

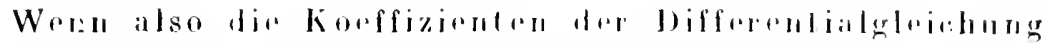

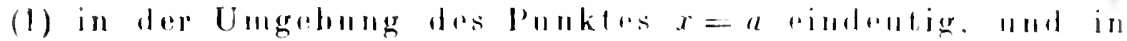
diesen Punkte die Integrale nicht mubestimmt simd, so bit

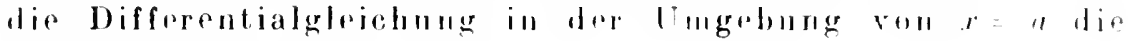
for III:

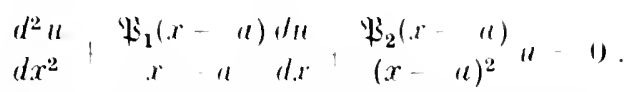

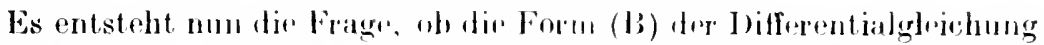

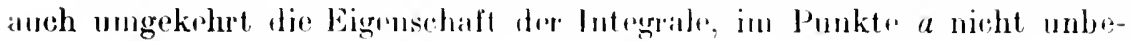
stimmt zu sein, nach sief zieht. l)ie Antwort auf diese Frage wird bejahend ausfallnu, bedarl aher otwas woitgehendor Untersuchungrn, denen wir uns jetzt zuzuweminn habron.

\section{1. Übergang zum Differentialsystem. Die gefundene Form ist auch hinreichend.}

Geht man von drun Differentialsystem (A) aus. dessen Koeffzienten $a_{i \boldsymbol{k}}$ in $x=a$ höclistens einen Pol habrn. mol wo $a_{21} \mid 0$ ist, so befriedigt $y_{1}$ die Differentialglrichung zwriter Ordnung (2). Wir identifizierrn nun diese Differentialgleichung mit (3), d. h. wir sutzen

$$
y_{1}=\|,-p=a_{11}+a_{22}+\begin{aligned}
& a_{12}^{\prime} \\
& a_{21}
\end{aligned} .-\cdots=a_{12} a_{21}-a_{11}{ }_{22}+a_{11}^{\prime} \cdot a_{11} a_{21}^{\prime}
$$

und nehmen an, dak $p$ und $q$ von der in dor vorigen Nummer gefundenen Form sind. Von (3) gehen wir dann durch dio Transformation

$$
z_{1}=u, \quad z_{2}=(x-a) u^{\prime}
$$

zu einem linearen Differentialsystem für $z_{1}, z_{2}$ über. Es ist

$$
\begin{aligned}
& u^{\prime}=z_{1}^{\prime}=x_{2}^{z_{2}} u^{.} \\
& z_{2}^{\prime}=u^{\prime \prime}(x-u)+u^{\prime},
\end{aligned}
$$

also folgt aus (13), wonn wir für $u^{\prime \prime}$ seinen aus (B) berechneten Wert pinsetzen,

$$
z_{2}^{\prime}=\frac{1-\mathfrak{B}_{1}(x-a)}{x-a} z_{2}-\mathfrak{B}_{2}(x-a) z_{1}
$$

Die Gleichungen (12) und (14) zeigen, dal $z_{1}, z_{2}$ pinem linearen Differentialsystem genügen. dessen Koeffizienten im Punkte $x=a$ einen Pol erster Ordnung liaben.

Zwischen dem Differentialsystem (A) und dem gefundenen Differentialsystem für $z_{1}, z_{2}$ besteht nun die folgende Beziehung. Es war $y_{1}=u$. also nach (1) 


$$
y_{2}=-a_{a_{21}}^{1}\left(u a_{11}-u^{\prime}\right) .
$$

d. h. wir finden

$$
\begin{aligned}
& y_{1}=z_{1}, \\
& y_{2}=-\frac{1}{a_{21}}\left(z_{1} a_{11}-\frac{z_{2}}{x-a,}\right.
\end{aligned}
$$

Die Gleichungen (15) haben die Form:

$$
\begin{aligned}
& y_{1}=z_{1} s_{11}+z_{2} s_{21}, \\
& y_{2}=z_{1} s_{12}+z_{2} s_{22} .
\end{aligned}
$$

wo die $s_{i k}$ sich in $r=a$ wir rationale Funktion'n verhalten, d. h. dort höchstens einen lol haben. Ind ' $S$ | 0 ist: wir könnf also das bisher erzielte Ergebnis auch so aussprerhen:

Damit die Lösungen des Differentialsystems (A). dessru Koeffzienten in der Umgebung der isolierten singulären Stelle $x=a$ eindeutig sind, in diesem Punkt nicht unhestimmt werden. mule es möglich sein. durch eine Transformation von le Form (16) zn einem ebrensolehen Differentialsysten übrzugehen. Ifssen Korffzienten im Punkte $x=a$ höchstens rinen Pol erster Ordmung habern.

Man kann die Jifferentialsystemo fïr $z_{1}, z_{2}$ und $y_{1}, y_{2}$. dir durch eine Transformation von der Form (16) anseinander hervorgchen, in bezug anf einen Umbaf $W$, der den singulären Punkt a umschliebt, kogredient nenmen. Bedeutet nämlieh $Z$ eine Integralmatrix des Differentialsystems fiir $z_{1}$. $z_{2}$. so ist

$$
Y=Z \cdot S
$$

offenbar eine Intograluatrix des Systems för $y_{3} \cdot y_{2}$ uml $y$. $Z$ sind 111 Sinne der Definition der Nr. 39 (\$. 153) in bezug auf len I Mulauf

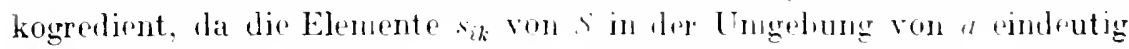
sind. Aber hier haben die sik noch dir hesonder. Eigensehaft. sieh im Punkte $r$ "wic rational' Funktionen zu verlalten: wir bringen dies

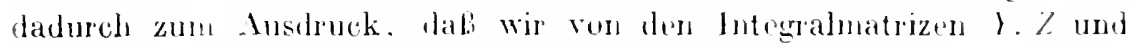
ebenso von den zugehörigen Differentialsystemen sagen, sir seien in

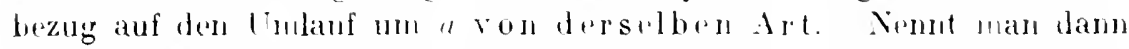
noch ein Differentialsystem. dessin korffizienten in dent l'unkte $x$ - a hüchstens cinen Pol crster Ordmung haben. cin fïr dicsen Punkt kanonisehes. so kann man moser Fregchnis kury se anseprechen:

Wenn die Lösungen eines Differentialsystems (A) mit in der Ln-

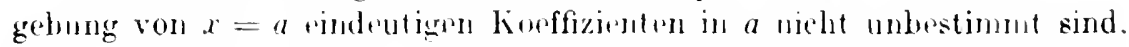
so muld (A) mit ainom für den l'unkt " kanonisehen Differentialsystem

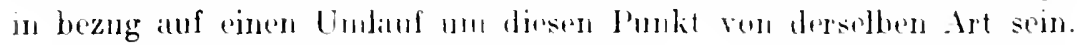

Wir legen jetzt rin beliebiges frir $x$ a kimoniseless lifferentialsystrmu zugrumble, also 


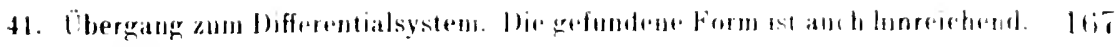

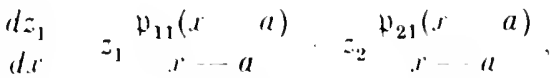

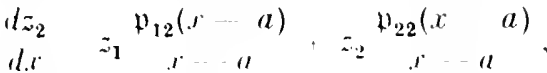

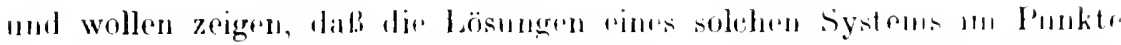

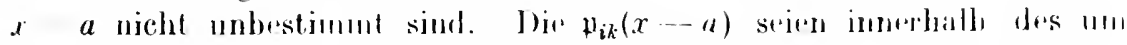
$x$ a beschriebenen Korises $K$ holomorph mon as soi im lumen vor $K$

$$
\left|p_{i k}(x-a)\right|-M \text {. }
$$

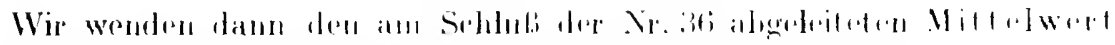

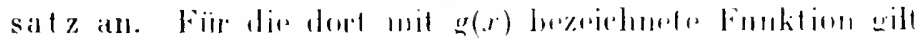

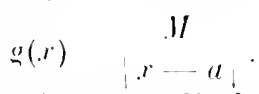

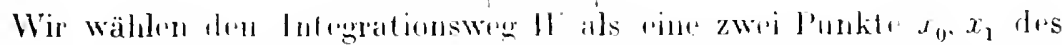

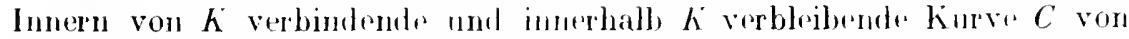
'ndlicher Bogenlängr. von der wir nowh folgendes voranssetzen wollen. Wrinn in Polarkoordinater

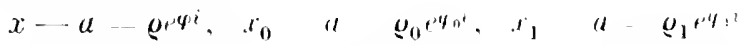

gesetzt wird, mol $x$ sieh anf $C$ vom $x_{0}$ nach $x_{1}$ hin hewegt, so sullen gund o niemals vom Wachsen zum Almehmen orler mmgekehrt iihergehen (monoton bleiben). nnd die Gesamtändernug ron of soll kleinor als $2 \pi$ sein. Es seien dann z, dir Elommon dex durch das Promblintugral

$$
\int_{i .}^{x_{1}}(x-a)^{d x-1}
$$

definierten lntegralnatrix von (C), wo dir Intrgration längs $C$ zu arstrecken ist. Dann ist

und wir haben folglich

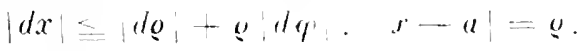

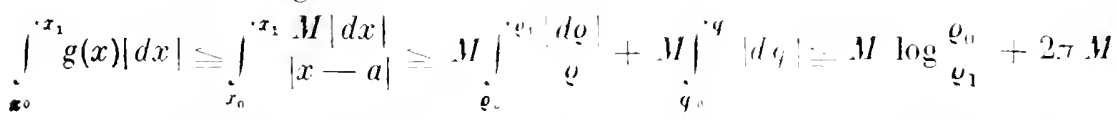

Der gedachte Mittelwertsatz liefort also für $\varrho_{1}<\varphi_{n}$ die Lngleichung

$$
|z, k| \leqq\left(\begin{array}{l}
\varrho_{0}, 2 M \\
\varrho_{1}
\end{array}\right) \quad e^{+\tau} M
$$

d.h. aber, daß $z_{i k}(x-a)^{2 M}$ besehräukt ist: nach der in der Nr. 34 (S. 128) gegebenen Definition heißt das aber. dal. $z_{i k}$ in $x=a$ nicht unbestinmt ist ${ }^{1}$ ). Wir haben also den von Sauvage ${ }^{2}$ ) gefuntenen Satz:

1) Der vorstehende Beweis ist aine von H. Fuhr herrührende Vereinfachnng des von Schlesinger, Crelles Journal 13:2 (1907) S. 247 gegebenen Beweises: vergl. auch G. D. Birkhoff. Transactions of Am. Nath. Soc. 11 (1910). S. 194.

2) L. Sauvalge. Annales de J'Ecole Norm. (3) IIl (18kti): verl. l'oincaré 1884. lenves II. ‥ 312. 313. 


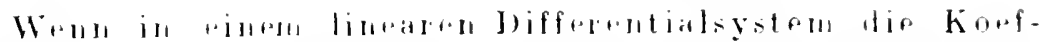

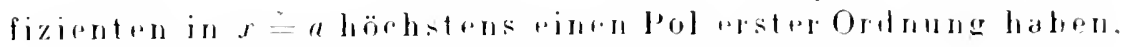
so sind dir Lösungen in liesem Punktr nirlat unbestimut.

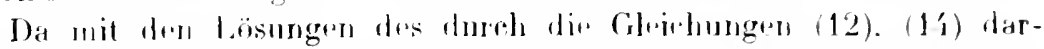
gestelltun Differentialsystems fïr $z_{1}$. $z_{2}$ andh die lösungen dor Differen-

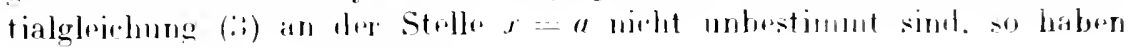
wir den siat\% ron Fur his $\left.{ }^{1}\right)$.

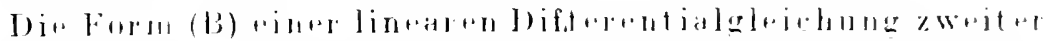

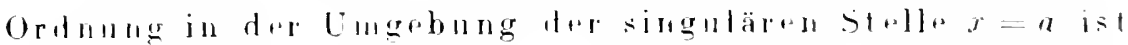

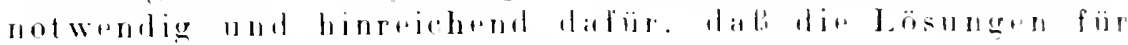
$x=a$ nirlit nubestimmt warlin.

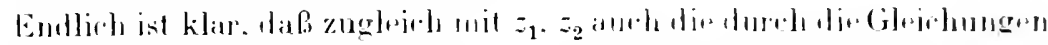

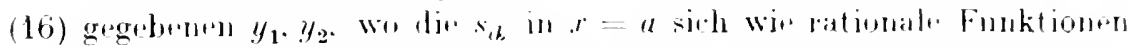

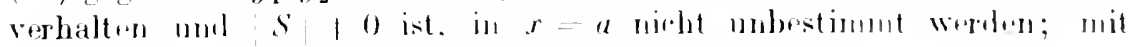
Rücksinht anf das oben ausgesprombur Ergebnis haben wir also don Satz:

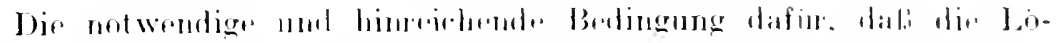
sungen rines limearen lilferentialsystems (A). dessent Konflizinnten in

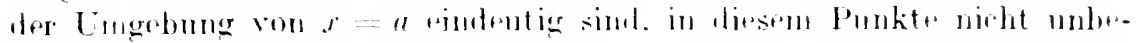

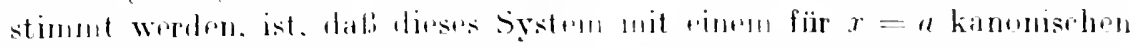

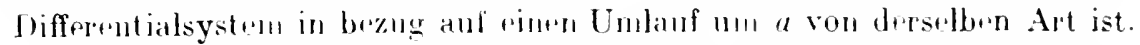

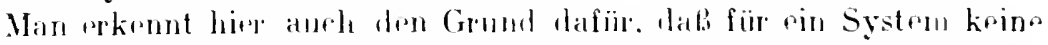
notwemdige Form der Konffizienten von gleicher Einfachhest rorhander

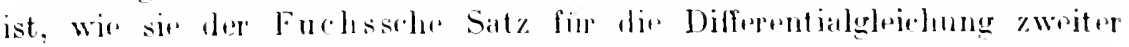

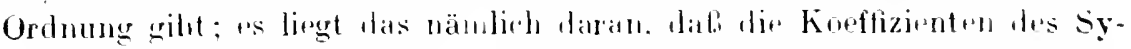

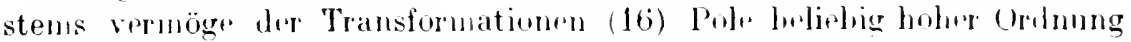

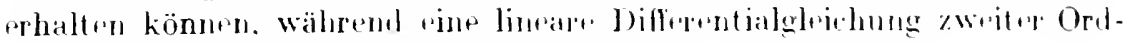

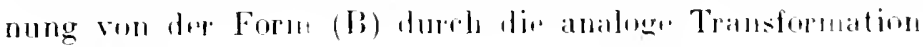

$$
\text { " } s_{1} u+s_{2} \| \prime \text {. }
$$

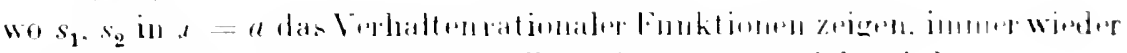

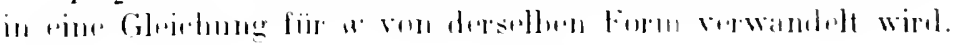

\section{Beredinung der Integrale in der Imgehmug einer Stelle der Bestimmtheit fïr Differentialgleithungen zweiter Mrinmug.}

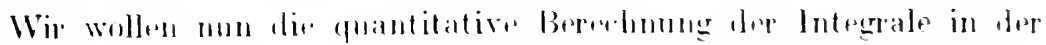

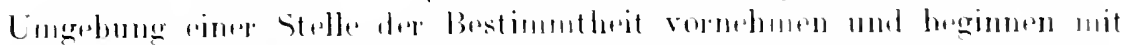

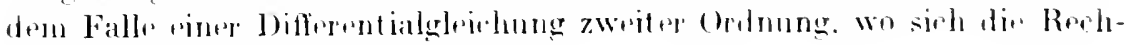

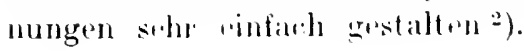

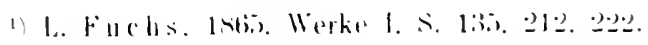

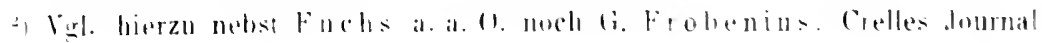




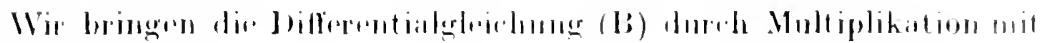
$(x-u)^{2}$ anf div Form

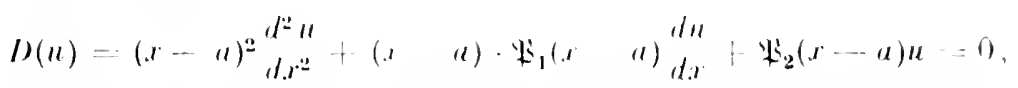

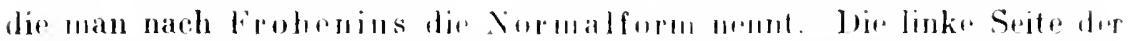

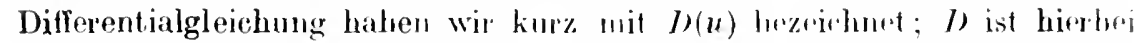

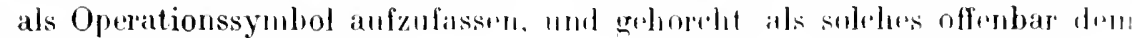
distributivon Grsetze

$$
\left.l\left(u_{1}+u_{2}\right) \quad l\right)\left(u_{1}\right)+l\left(u_{2}\right):
$$

ebenso ist fïr ein komstantes ;

$$
\omega(r u)=r l(u) \text {. }
$$

Wenn dir Intrgate von (17) in $x=$ a niuht unbestimnit simb. so gibt es nach der in der $\mathrm{Nr} .40$ (S. 16i2. 16.3) gemachten Bemerknng jertrnfalls auch ein Integral. las in der Ungubung ron $r=11$ in der Form

$$
=(r \cdot a)^{r} \sum_{i=11}^{x} r_{k}(x+a)^{k}
$$

darstellbar ist. Jie Konvergenz dieser Reihe in derjenigen Ungebung von $x=a$, für die die Reihen $\mathbb{B}_{1}(x-a)$. $\mathfrak{P}_{2}(x-a)$ kowvergent sind, ist nach dem Vorhergehemblu als grsichret anzusehen.

Setzen wir die Reihe (18) in die linke Srite Hor Differentialgleiehung (17) ein, so ergibt sich:

$$
D(u)=D\left(\sum_{k}^{\infty} r_{k}(r-a)^{r+k}\right)=\sum_{k}^{\infty} c_{k} D\left((x-a)^{r+k}\right) .
$$

Wir werden also veranlalit, den Ausilruck

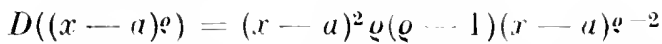

$$
\begin{aligned}
& +(x-a) \cdot \mathfrak{P}_{1}(x-a) \cdot \varrho(x-a)^{0}-1+\mathfrak{P}_{2}(x-a) \cdot(x-a) ?
\end{aligned}
$$

zu untersuchen, wo $g$ eine beliebige konstante Gröbe bedeutet: mannennt diesen Ausdruck nach Frobenius dir charakteristisehe Funktion der Differentialgleichung.

Wir setzen diese in die Form

wo also

$$
\left.D((x-a) !)=(x-a)^{e}\right)(x \cdot 0) \text {. }
$$

$$
f(x, g)=\varrho(g-1)+\mathbb{H}_{1}(x-a) \cdot g+\mathbb{H}_{2}(x-a)
$$

eine in der Ungebung vor $x=a$ holomorphe Funktion brdentet. Es sei nach Potenzen von $x-a$ entwickilt:

$$
f(x, g)=\sum_{i=1}^{\infty} f_{i}(g)(x-a)^{\wedge} .
$$

Bd. 76. (1873). S. $214 f f .$, Bd. 89). (1875). s. 317ff. und 1. Hefftel. Einleitung in die Theorie der lin. Differentialgleichungen (1893): siehe auch L. Schlesingel, Handbuch des Theorie der lin. Differentialgleichungen Bd. 1, 1895. S. $154 \mathrm{ff}$. 
dann ist mach dem Taylorschen Satz:

$$
\begin{aligned}
& i_{0}(0) \quad 0(0-1)+\varphi \Phi_{1}(0) \cdot W_{2}(0) \text {. } \\
& i_{1}(0) \quad 0 \mathbb{P}_{1}(0) \cdot \mathbb{L}_{0}^{\prime}(0) \text {. }
\end{aligned}
$$

Wir fimders hiernarh

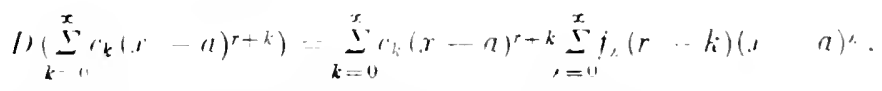

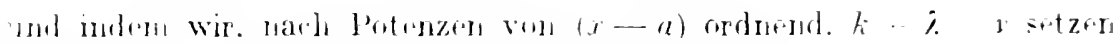
und den ganzen Ausdrum mit Null vergleichnon.

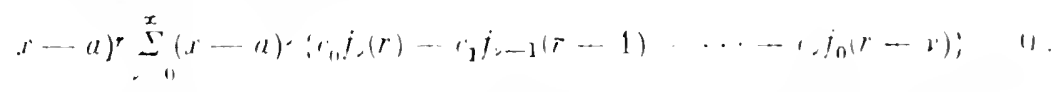

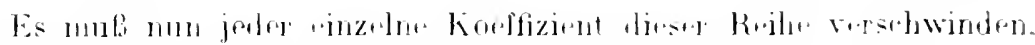

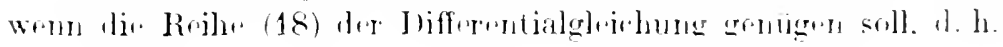

(21) $c_{0} j_{2}(r)+c_{1} i_{1}(r-1) \ldots \ldots i_{1}(r-r)=0$.

Firr $r-0$ ergiht sirh

$$
\left(n i_{0}(r)-1\right):
$$

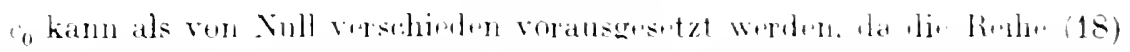
sorst nicht zum Expromenten r. sondern zu eilum höheren Expententen gehören wïrde: wir finden also zuvörderst. da li dor Expontent $r$ pine Wurzel lor Glojeluma

$$
(22) \quad i_{0}(0)=0\left(0 \quad \text { 1) } \quad 0 L_{1}(0) \quad L_{2}(0)-0\right.
$$

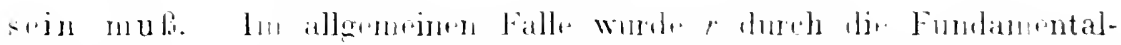

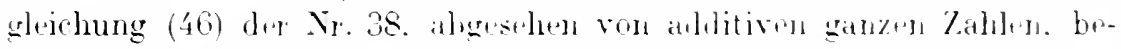
stimmt: hier bestimmt sich $r$ vollkommen als Wurzel der Gleichung

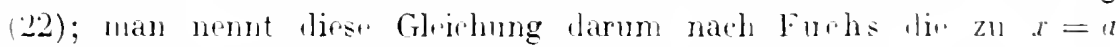
goürige determinierende Fumbanentalgleichung. Es ist be -

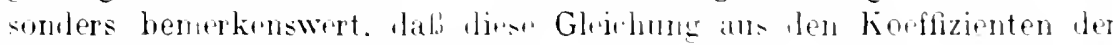

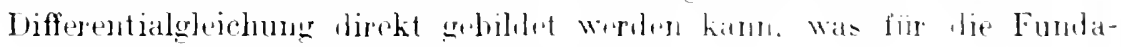
mentalgleichung nisht dir Fall war.

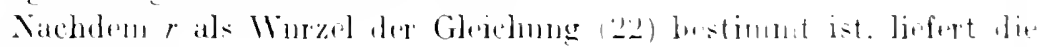
Gloichung (21) fiur $r=1$

$$
{ }_{0} j_{1}(r)-i_{10} j_{0}(r+1)
$$

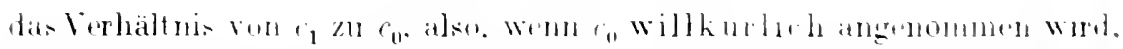

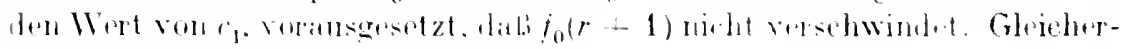

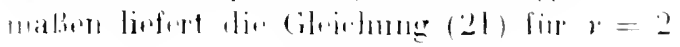

$$
\left(n l_{2}(r)-r_{1} !_{1}(r-1) \cdots c_{2} r_{0}(r-2) \quad 1\right)
$$

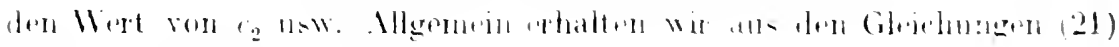

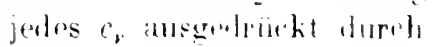

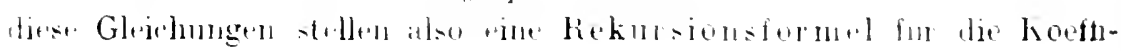




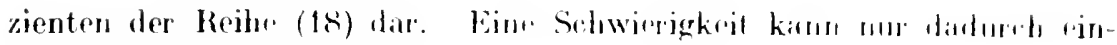
treten, dal. für cin gewisses $y$

$$
f_{0}(r+v)=0
$$

wird. Unu dieser vonläufig ans dem Wrene zu gelen, wählen wir $r$ als diejenige Wurzel r dre determinierenden Fundamentalgleichung, derren roeller Teil nicht kleiner ist, als der der anderen Wurzel; dann ist offenthar fiur jedes positive $v$

$$
f_{0}\left(r_{1}+v\right) \mid 0 \text {. }
$$

die sukzessive Berechnumg der co alse strets möglich. Wir habron sonnit das zum Exponenten r gehörige Integral

$$
u_{1}=(x-a)^{r_{1}} \sum_{k=0}^{x} c k(x-a)^{*}
$$

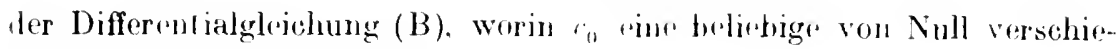
dene Konstante bedeutel.

Für die andere Wurzel $r_{2}$ der Gleichung (22) wiirdr alles unverändert bestehen hleihren, wenn drer Ausdruck

$$
f_{0}\left(r_{2}+v\right)
$$

für kein positives ganzzahliges $r$ verschwindet. Nun kann aber

nur dann bestehru. weun

$$
f_{0}\left(r_{2}+g\right)=0
$$

$$
r_{2}+g=r_{1} \text {. }
$$

die Schwierigkeit, dab $f_{0}\left(r_{2}+v^{\prime}\right)$ verselwwindut. wird also dann und nur dann eintreten. wenn die Differenz $r_{1}-r_{2}$ der Wurzeln der determinierenden Fundamentalgleichung piur positive ganze $\mathrm{Zahl}$ ist.

Diesen Fall, sowie auch denjenigen, wo die beiden Wurzeln der determinierenden Fundamentalgleichung einander gleich sind, lassen wir vorläufig beiseite; dann finden wir also entsprechend der Wahl $r=r_{2}$ das zum Exponenten $r_{2}$ gehörigo Integral

$$
u_{2}=(x-a)^{r}: \sum_{k=0}^{\infty} \bar{c}_{k}(x-a)^{k} .
$$

wo $\bar{c}_{0}$ willkürlich, aber von Null verschieden ist und die übrigen $c_{k}$ durch die Rekursionsfornel (21) für $r=r_{2}$ geliefert werden. Da $r_{2}-r_{1}$ weder eine ganze Zahl noch Null ist, kann der Quotient.

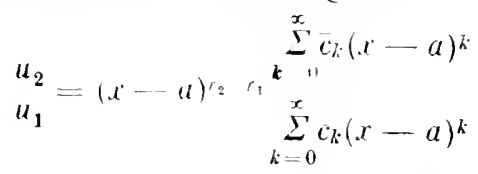

nicht koustant sein; $u_{1}, u_{2}$ bilden also ein Fundamentalsystem.

Die Wurzeln der determinierenden Fundanentalgleichung stehen hiernach. wenn ihre Differenz waler Null noch eine ganze Zahl ist, mit 


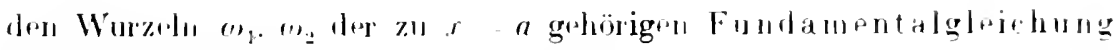
in Aer Broiphung

$$
\begin{array}{cccc}
r_{1} & \log (1)_{1} \\
2 \pi i
\end{array} \quad r_{2.2} \quad \begin{aligned}
& \log (1)_{2} \\
& 2 \pi i
\end{aligned} .
$$

und wir sehen zugleinh den Grund. weshall der Fall. wo die Differenz der Wirzeln $r_{1}, r_{2}$ eine ganze Zahl orler Null ist. Sohwerigkeiten bereitet: in diessul Falle wärr nämlìh

$$
\left(m_{1} \quad()_{2}\right. \text {. }
$$

so dalis es allgemein zo redern, "in Fumbanentalsystem von der Form $u_{1}, u_{2}$ gar nicht gibt, indron das einr der hauonischen Integrale tinen Logarithmus enthalten kann. Wir wonden wus jetzt zur Erledigung dirses bisher ausgeschlossenen Falles.

Wir schicken die Behandlung aines einfarhen mul inturessantm Spezialfalles voraus $\left.{ }^{1}\right)$.

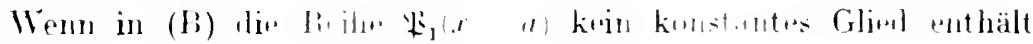
und $\mathfrak{R}_{2}(r$ a) nit $(r$,

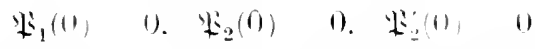

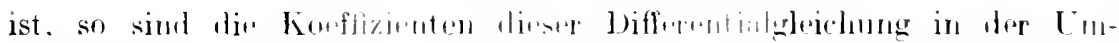
gebung von $r=a$ hutumorph. Also ist anh has allgmmeime Integral holomorph; wir wollon mun zusehern. in wh.lur Form sich die Darstellung des allgemeinen lntegrals in dar Lingulung von $x=a$ ergibt.

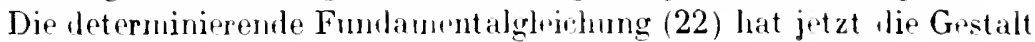

$$
\text { o(e } \quad 1)=0 \text {. }
$$

die Differenz ihrer Wurzent 0.1 ist also rine ganze Zahl. A. h. wir habert unseren Ausnahnoffall. Gleichwohl tritt hier keime Sehwierigknit auf, denn

$$
j_{1}(0)-Q \mathbb{L}_{1}^{\prime}(0)-\mathrm{PL}_{2}^{\prime}(0)
$$

reduzient sich für $y=0$ auf Null so lafe lin Rekursionsformed (21) für $r=0$ sowohl bei $r \quad 0$ als aneh bei $r \quad 1$ kime bistimnung der $c_{0} \cdot c_{1}$

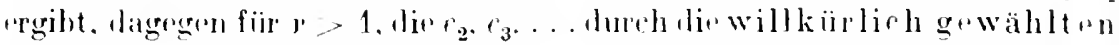

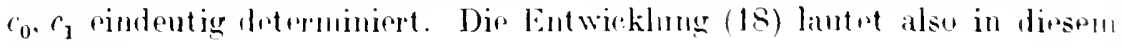
Falle

$$
\| r_{11} c_{1}(x-a) \cdots c_{2}(x \quad a)^{2}, \ldots \text { in inf.. }
$$

wo din con willkïrliche Konstanten hedeuten. sir stellt also das allgemrine lntrgral in der Ungebung der Stolle $x=a$ dar. Um rin partikuliires lntegral zu bestimmen. hat man uh+r $c_{0} c_{1}$ z.l verfügen. d. h. man hat für $x=a$ die Wirte

$$
\lim _{x \rightarrow \infty} u r_{1} \lim _{1, \ldots} d x
$$

vorzuschriben. Fïr eine Stalle. in deren Lmgehung die kouffizienten dor Diffrentialgloichung (3) (S. 160 ) bolomorpb

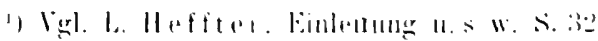


sind, wirl also rin partikulares lufegral hestimmt, indem

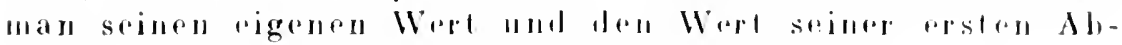
leitung in diesem l'unkte willkijplich festsetzt.

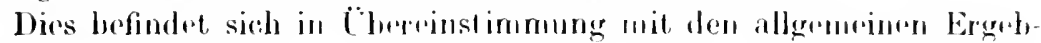
nissen der $\mathrm{Nr} .36$.

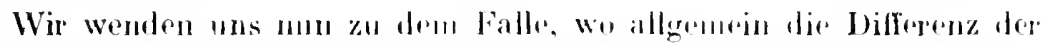

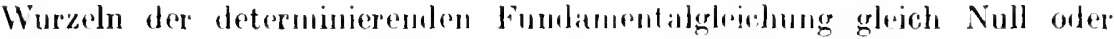
riner ganzen Zahl ist. Womm $r$, die obrn (S. 171) fosterelegte Bedeutung behält, so möge also

$$
r_{1} \quad r_{2} \quad s
$$

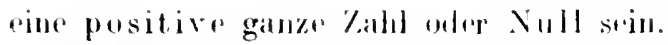

Machen wir mit dem zum Exponenten $r_{3}$ gohorigen lutrgrale

$$
u_{1} \quad(x-a)^{r_{3}} \sum_{k}^{\infty} r_{k}(x-a)^{k}
$$

in der Differentialgleichung (B) dic Substitution

$$
u-u_{1} f(x) x \text {. }
$$

so genügt $v$ der linearen l)iffrentialgleichung erster Ordmung (vgl. Nr. 40. S. $163, \mathrm{Gl} .(10))$

$$
d x+\left(2 u_{1}^{u_{1}}+\begin{array}{c}
\mathbb{X}_{1}(x-a) \\
u_{1}-a
\end{array}\right)=0 .
$$

die wir, da in der Ungubnug von $x=a$

ist, in der Form

$$
\begin{aligned}
& u_{1}^{\prime} \\
& u_{1}=r-a
\end{aligned}=r_{1}+\partial_{1}(x-a)+\cdots
$$

$$
d x+v\left\{\begin{array}{c}
x_{1}(0)+2 r_{1}+\varepsilon_{0}+\varepsilon_{1}(x-a)+\cdots !=0, \\
x-a
\end{array}\right.
$$

oder endlich, da nach $(22)$

ist, in der Form

$$
r_{1}+r_{2}=-\left(\mathbb{L}_{1}(0)-1\right)
$$

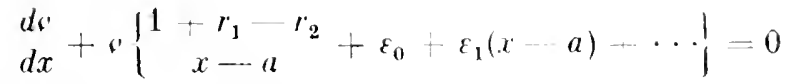

schreiben können. Aus den Ergebnissen der Nr. 34 folgt, daß das Integral dieser Differentialgleichung für $x=a$ nicht unhestimmt ist und zum Exponenten

$$
-\left(1+r_{1}-r_{2}\right)
$$

gehört; in der Tat finden wir auch mmittelbar, inden wir di" Gleichung (24) integrieren,

$$
v=(x-a)^{-\left(1-\gamma_{1} r_{2}\right)}\left(\gamma_{0}+\gamma_{1}(x-a)+\cdots\right),
$$

und wenn wir jetzt dies in (23) einsetzen, so ergibt sich ein zweites Integral von (B) in der Form:

$$
u_{2}=u_{1} /(x-a)^{-1+r_{1}-r_{2}}\left(\gamma_{0}+\gamma_{1}(x-a)+\cdots\right) d x .
$$


Beachten wir nun, dals $r_{1}-r_{2}$ glosich der nichtuegativen ganzen Zahl $g$ sein sollte, so folgt hei Ausführung dres mit $u_{1}$ multiplizierten Integrals:

$$
\begin{aligned}
& n_{2}=u_{1}\left\{_{1-g}^{\gamma_{0}}(x-a) a+\cdots \gamma_{1}^{1}(x-a)\right. \\
& +\gamma_{g} \log (x-a)+\boldsymbol{x}_{0}+\boldsymbol{\iota}_{1}(x-a)-\cdots ! \mathrm{j} .
\end{aligned}
$$

and wenn wir für $u_{1}$ seine Entwicklung einsetzen und beachtert, daf

$$
r_{1}-g=r_{2}
$$

ist, so finden wir fïr $u_{2}$ rine Darstrllung vou der Form

$$
u_{2}=(x-a)^{r_{2}}: \varphi_{2}(x-a)+\gamma_{9} y_{2}^{\prime}(x-a) \cdot \log (x-a) ; .
$$

wo $p_{2}(x-a), \psi_{2}(x-a)$ gewöhnliche Potenzreihen von $x-a$ beclutent. und die Konstante $\gamma_{g}$ jedenfalls von Null verschieden ist, wenn $g=0$ ist; dagegen kanr! $\gamma_{g}$ für ein wesentlich positives verschwinden. Der Ansdruck

$$
\gamma_{g}(x-a)^{r_{2}} y_{2}(x-a) .
$$

der den Faktor von $\log (x-a)$ bildet, unterscheidet sich von $u_{1}$ mur durch einen konstanten Faktor. Wir sehen, daf. aurh in dron Falle eines ganzzahligen Wertes von $r_{1}-r_{2}$ die Größen

$$
e^{2 \pi \pi r_{1}} . \quad e^{\check{2} \pi \cdot r_{*}}
$$

die Wurzeln der zu $x=a$ gehörigen Fundanentalgleichung sind. Wenn $\gamma_{g}=0$ ist, wird auch das zweite Integral $n_{2}$ logarithmenfrei sein. Ein Beispiel dafïr bildet der oben hehandelte Fall, wo dir Koeffizienten von (B) in ler Ungebung vou $x=a$ holomorph sind; in diesem Falle ist $r_{1}=1, r_{2}=0$. Ein writerer interessanter Sombreall tritt oin. wenn $r_{1}, r_{2}$ ïberhaupt voneinander verschindene ganzzahlige Wiete haben und $\gamma_{g}$ verschwindet. Ist dam wonigstens rines her $r_{1}, r_{2}$ argativ, so hat das allgemeine lntegral in $x=a$ rinen Pol. simd $r_{1}, r_{2}$ nichtnegativ. so ist das allgemeine Intrgral in liw Ungehung voul $r=a$ holomorph. Ist alier nicht $r_{1}=1, r_{2}=0$, so lat wenigstens riner der Kooffizienten der Differentialgleichnng $(B)$ in $x=a$ wirklieh ninen Pol nud wir hatwn es mil einem auloerwesentlich singulärett Punkte zu tun (rgl. Nr. 13, S. 49). Für einen solchen Punkt mul. llann nach Nir. 38 (S. 1í7)

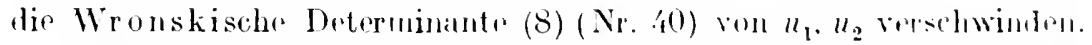

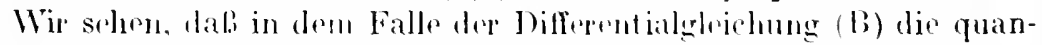
titative Berechung des kanonischen Funtamentalsystems dureh algebraische Operationen möglich ist. Wir hoben nech besonders hervor. dab dasjenige Integral, das zur der Wurzel der doterminierenden Fumbanentalgleichung gehört, Heren preller Tril nicht kleiner ist. als der der anderen.

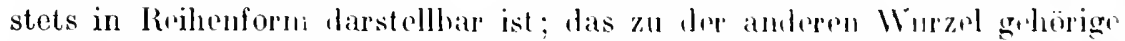
Integral kann, wenn die Differenz der Wurzeln der determinieremden 
Fundanentalgleichung einn gatn\% Zarhl ist, rinen Logarithmus whthalten, es enthält diesen Logarithmus unberdingt, weun die beiden Wurzetn dor determinierenden Fundamentalgleichung zusammenfall'n.

Da die Herstellung der Rrihenentwisklungen für die Integrale in den Anwendungen von besonderer Wichtigkeit ist, geben wir in dor folgenden Nummer einige den Anwendungen intnomurene Brispiele.

\section{tö. Beispiele. Verhalten der Integrale im Unendlichen.}

1. Dir Cauchysche Differentialgleichung ${ }^{1}$ ) lautet

$$
\left.d x^{2}+x-a d x^{-+} \quad b-a\right)^{2} u=0,
$$

wo $A, B$ Konstanten sind; sie kann durch dir Substitution (4) in ein Cauchysches Differentialsystem verwandelt werden; wir können dit Integration aber auch leicht direkt vollziehen. Dif determinierende Fundamentalgleichung für $x=a$ lautet

$$
\underline{Q}(\underline{\omega}-1)+A \underline{\underline{\omega}}+B=0 .
$$

Hat diese zwei verschiedne Wur\%ln $r_{1}, r_{2}$. so rrgoben sich die Lösungen

$$
u_{1}=(r-a)^{r_{1}} \cdot u_{2}=(r-a)^{r_{2}} .
$$

während für $r_{1}=r_{2}$

(28) $\quad u_{1}=(x-a)^{r_{1}}, \quad u_{2}=(x-a)^{r_{1}} \log (x-a)$

ist, in Übereinstimmung mit den für das Canchy sche Differentialsysten gefundenen Lösungen. Führt man in (25) durch dip Substitution

$$
t=\log (x-a) ; x-a=e^{t}
$$

$t$ als unabhängige Veränderliche ein, so verwandelt sich (25) in dir Diff s. rentialgleichung mit konstanten koeffizienten

$$
\frac{d^{2} u}{d t^{2}}+(A-1) \frac{d u}{d t}+B u=0 \text {; }
$$

die Gleichung (26) oder

$$
\text { (26a) } \quad \varrho^{2}+(A-1) \varrho+B=0
$$

heißt hier die charakteristische Gleichung. Entspreehend (27) und (28) haben wir das Fundamentalsystem

$$
\begin{array}{ll}
u_{1}=e^{r_{1} t}, & u_{2}=e^{r t} . \\
u_{1}=e^{r_{1} t}, \quad u_{2}=t e^{r_{1} t} .
\end{array}
$$$$
\left(r_{1}+r_{2}\right.
$$$$
\left(r_{1}=r_{1}\right.
$$

Studiert man z. B. die Bewegung des mathematischen Pendels fü unendlich kleine Amplituden, so kann man in der Differentialgleichung (16) der Nr. $6 \varphi$ an die Stelle von $\sin \varphi$ setzen und erhält

1) A. L. Cauchy, Exercices d'Analyse ttc. I (1840)). S. 262. 


$$
d t^{2}+\frac{g}{l} \tau=0 .
$$

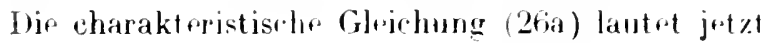

$$
e^{2}-\frac{g}{l}=0 \text {; }
$$

sis hat die beiden vomeinander versehiederm Wurzuln

$$
r_{1}=i\left|i \cdot r_{2}=-i\right|_{i}^{l g} \text {. }
$$

also bildrn

$$
\left.e^{*}\right|_{i} ^{y} \cdot e^{+\left.\right|^{a}}{ }^{4}
$$

(in Fumlanmentalsystrm. Statt dessun kamm matl ameh das in rombler Form, rrscheinemde Fumlamentalsystem

$$
\sin t \mid \frac{g}{1} \cdot \cos t / \frac{g}{l}
$$

nehmen. Man hat also liss allgemmin. Integral

$$
q=r_{1} \sin t\left|\frac{\pi}{l}+r_{2} \cos t\right| \frac{g}{i} \text {. }
$$

und das partikulärr lntegral. das fïr $t=0$ rersebwinder und lessen Ableitung für $t=0$ gloich ${ }^{\prime}{ }_{1}$ ist. lautort:

$$
q=\left.\left.v_{a}^{\prime}\right|_{g} ^{l} \sin t\right|^{\frac{g}{l}} \text {. }
$$

Die Form der Differentialghenhung (30) tritt übrhanpt bei allen Bewegungsvorgängen auf, die in kleinen Schwingungen pint's Punktes von der Mass" 1 um rine Ruluelage bestehen. Erfolgt dir Buwegung auf der $u$-Achse. und hat der Punkt zur Zeit $t$ die Ahszisse $u$, wirkt auf ihn

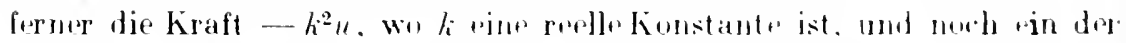
Gesthwindigkeit $\frac{d u}{d t}$ propontionaler dämpfender Widtrstand $-\varepsilon_{d t}^{d u}$. wo $\varepsilon>0$ ist. so lauted die Differentialgleiclung der bewegung ${ }^{1}$ )

$$
t^{2} u+\varepsilon \frac{d u}{d t^{2}+k^{2} u=0} d t
$$

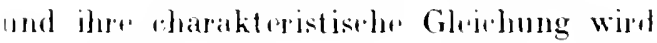

$$
\underline{Q}^{2}: \varepsilon \underline{\varphi}+k^{2}-0 \text {. }
$$

Setzt man

$$
\mu^{2}=k^{2}-\varepsilon^{2} .
$$

so sind drei Fälle 2.11 mulersederiden:

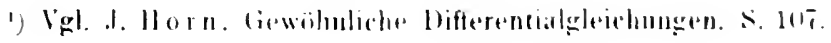




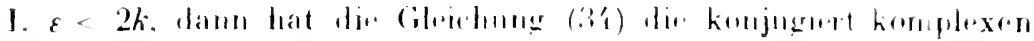
IInrzeln

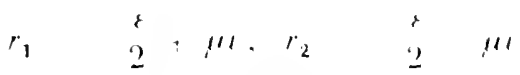

und die allgemenere Lösung laule nach (27a)

$$
\text { u- } r_{1} e^{\left(\frac{\varepsilon}{2}+m\right) t}+c_{2} e^{( \pm \mu)^{t}} \text {. }
$$

wo $c_{1}, c_{2}$ willkürliche Konstanten hedenten. oder Wonn man

setzt,

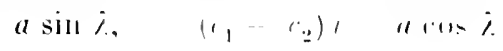

$$
\left.\| \quad \text { ut } \frac{t^{2}}{2} \sin (11) \cdot i\right)
$$

Für $\varepsilon=0$, d. h. fïr lmh Fall ungedänpfter S.thwingungers, hat Iman, wif beim l'endel, eine einfach freiodisehe Bewegung mit der Schwingungsdauer $T=\frac{2 \pi}{k}$. Ist dagegen $0 . \varepsilon \cdot 2 k$. so hat man

$$
d u+a^{\prime}, \stackrel{\varepsilon}{\prime} \cdot \sin (\mu t+\lambda) \text {. }
$$

WO

$$
\begin{aligned}
& a \mu \cos \lambda-\frac{\varepsilon}{2} \sin \lambda=a \sin \lambda . \\
& a \mu \sin \lambda+\frac{\varepsilon}{2} \cos \lambda \quad \| \cos \lambda
\end{aligned}
$$

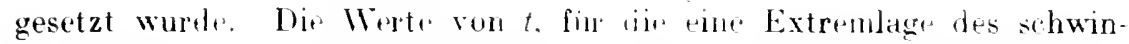
genden Punktes rintritt, also $\underset{d t}{d u}=0$ ist, bilden wine arithnetische Reihe mit der Differenz ${ }_{\mu}^{\pi}$ : Her Zeitunterschied zwischen den Eintreten zweier aufeinander folgender Maxima oder Minima ist konstant gleich 2.7 ." kann auch wieder als Schwingungsianer lezelchmet werden. Die Abstände der Extremlagen des schwingenden Punktes rom Punkte $u=0$ bilden eine geometrische Ruihe nit dem Quotirnten e " : man nennt «T das logarithmische Dekrement.

"II. $\varepsilon=2 k$; dann ist $r_{1}=r_{2}$ und (siehe $(28 \mathrm{a})$ )

die allgemeine Lösung.

$$
u=e^{-s t}\left(c_{1}+c_{2} t\right)
$$
lautet

III. $\varepsilon>2 k$, dann sind $r_{1}, r_{2}$ reell negativ und die allgemeine Lösung

$$
u=c_{1} e^{t_{1} t}+c_{2} e^{r^{t}}
$$




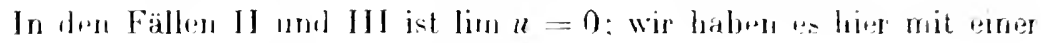
aperiodisehon Browgung zut tun.

2. Jir Aufgabe, den Spanmugs- und Joformationszustand riner dünneu. aus rinem homogenen und isotropen Stoff bestrhenden Ringflächensehale konstarter Wandstärke nutw dre Voranssetzung dre Griil-

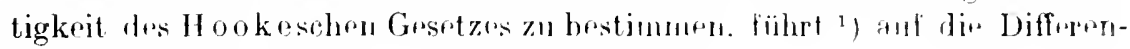
tialgleichung

$$
\begin{gathered}
(\lambda-x)^{2}\left(1-x^{2}\right) u^{\prime \prime}+(\hat{x}+t)\left(1-i s-2 x^{2}\right) u^{\prime} \\
-\left[1-i k \lambda x+(i k-1) x^{2}\right] u=0
\end{gathered}
$$

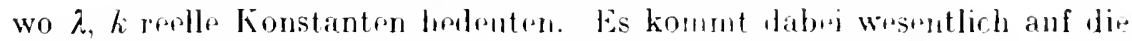
Entwicklung d+r Lösungen in der Uungebung der singulären Stellen $x=+1, x=-1$ an. Man sieht, dal. nach Jivision mit den Koeffi. zienten von $u^{\prime \prime}$. dir. Differentialgheichung in der Ungulung dirser heiden

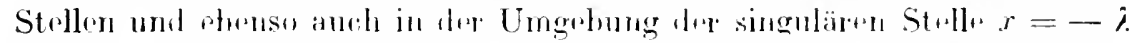
die Form (13) hat. Dir hösungen verhalton sirh also an dirsen Stellen bestimmt. Fïr $x=1$ z. li. källl man

$$
(x-1)^{2} \psi_{4}(x-1) u^{\prime \prime}+(x-1) \psi_{1}(x-1) u^{\prime}+\psi_{2}(r-1) u=D(u)
$$

als dit Normalform der linken Seite von (is) answhen. wn

$$
\begin{aligned}
& \mathbb{F}_{0}(x-1)=(1+x)(\lambda+x)^{2} . \\
& \mathfrak{H}_{1}(x-1)=-(\lambda+x)\left(1-\lambda_{x}-2 x^{2}\right) . \\
& \mathfrak{H}_{2}(x-1)=(x-1)\left[1-i k \lambda+(i k-1) x^{2}\right]
\end{aligned}
$$

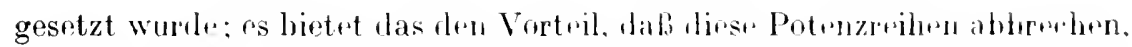
worlureh sich die weitere Rerhnung erhehlich vereinfarht. Di, rharakteristischr Funktion ( $\mathrm{Nr}_{\mathrm{r}} 42$. Gl. (19)) lautet damn

$$
\begin{aligned}
f(x, \varrho) & =\mathscr{F}_{0}(x-1) \varrho(\varrho-1)+\mathcal{F}_{1}(x-1) \varrho-\mathcal{H}_{2}(x-1) \\
& =f_{0}(\varrho)+f_{1}(\varrho)(x-1)+f_{2}(\varrho)(x-1)^{2}+f_{3}(\varrho)(x-1)^{3} .
\end{aligned}
$$

und dir determinierende Gleichung ist

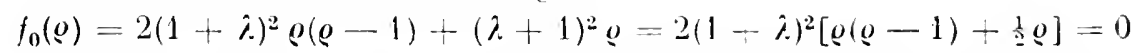
mit den Wurzeln $r_{1}=0, r_{2}=1_{2}$. Dir expliziten . Ausdrüeke für $\dot{f}_{1}(\varrho)$. $f_{2}(\varrho), f_{3}(\varrho)$ sind auch loicht horzustellon. Wir hiben also oin in der Umgebung vou $r=1$ holomorphes Integral

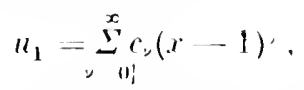

in tent in wilkürlich bleibt, Während dir äbrigen korffizinten $c$ sich durth dir Rekursionsformel

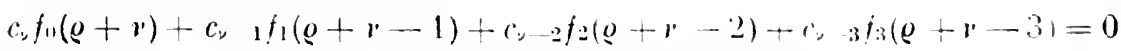
bestimnen. Dir Berehohung des zweiten hutegrats

1) II. Wissler. Festigkeitsberechnung ron Ringtlitchenschalen. Disiertation Techn. Hochischute Zürich, 1!nti. 


$$
u_{2} \quad(x-1) \sum_{y=11}^{s} c_{\nu}^{\prime}(x-1)
$$

gestaltet sich am rinfachsten. Wenn man dir Difforntialghoichung (35; durch die Substitution

$$
11 \quad \mid 1-r^{2} r
$$

in eine Differentialgloichnng fur "transforminl. Mir lam für $x=1$ als Wurzeln der doterminierenden Gleirhung $r_{1}^{\prime}=0 . r_{2}^{\prime}=-1$ hesitzt. Das der Wurzel $r_{1}^{\prime}=0$ entsprechende Integral $r_{1}$ ist in we Ungebung von $x=1$ wieder holomorph und rerwindelt sich durch Multiplikation mit $11-x^{2}$ in $u_{2}$, was aueh wiedor mit ninem willkïrlichen konstanten Faktor (dem Anfangskoeffizienten dor Potenzerihe $v_{1}$ ) lohaftet orseheint. Das allgemeine Integral von (35) hat also die Form

$$
u=u_{1}+11-x^{2} u_{1} \text {. }
$$

Für reelle Werte von $x$ hat man dann als Konvergenzberoich.

wenn $\lambda \geq 1$ ist, $-1<x<3$.

wenn $\lambda<1$ ist, $-\lambda<x<\lambda_{0}+2$.

Behandelt man noch den Punkt $x=-1$ in ähnlicher Woise. so hat man für alle in der Praxis rorkommenden Fälo bramehbar Entwicklungen des allgemeinen Integrals.

3. Die Aufgabe, alle analytischen Verbiegungen der Kugel vour Halbmesser $1 \mathrm{zu}$ finden, führt $\left.{ }^{1}\right)$ auf die Differentialgleichung

(36) $x^{2}\left(1-x^{4}\right) u^{\prime \prime}+x\left(1-8 x^{2}-x^{4}\right) u^{\prime}-k^{2}\left(1-x^{4}\right) u=0$.

in der $k$ eine positive ganze 7ahl bedeutet. Darin ist $x=\operatorname{tg} \%$ ' wo $p$ die geographische Breite auf der Kugel bedeutet. In Endlichen hat die Differentialgleichung die singulärea Punktr 0. 1. - $1, i$. - $i$; an jedem dieser Punkte zeigen die Integrale bestimmtes Verhalten. Für $x=0$ lautet die determinierende Fundamentalgleichung

$$
\varrho(\underline{o}-1)+g-k^{2}=0 \text {. }
$$

$\mathrm{Zu}$ ihren Wurzeln $+k,-k$ gehören Integrale von der Form

$$
u_{1}=x^{k}\left(c_{0}+c_{1} x+\cdots\right), \quad u_{2}=x^{-k}\left(c_{0}^{\prime}+c_{1}^{\prime} x+\cdots\right) \text {. }
$$

Für $x=1$ und $x=-1$ hat die determinierende Fundamentalgleichung

$$
g(o-1)-2 g=0
$$

die Wurzeln 0 und 3. Zu der Wurzel 3. als der größeren, gehört sicher ein in Reihenform darstellbares Integral, aber auch der Ansatz

$$
u=c_{0}+c_{1}(x \pm 1)+c_{2}(x \pm 1)^{2}+\cdots
$$

führt zu einer Bestimmung der Koeffizienten $c_{0}, c_{1}, c_{2}, \ldots$ durch Rekursionsformeln; es gehört also auch zu der Wurzel 0 ein in Reihenforı:

1) H. Liebmann. Bedingte Flächenrerbiegungen usw.. Sitzungsberichte des Bayr. Akademie d. Wiss. 1920. S. 21. insbesondere S. 37. 
darstellbares Integral. 1. h. das allgem.ine Integrat von (36) ist in der Ungebung von $x=1, x=-1$ holomorph. Wir hahen as hipr mit dußerwesentlich singulären Punkten zu tun.

Firr $x=$ - $i$ hat die determinieremde Fundaumentalgherehune

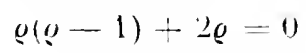

dir Wurzeln 0 und - 1; zu 0 als der gröberen gohort ein holonorphes Integral. aber der Reihenansatz zeigt, dal. auch bei dru zu deu Exponenten - 1 gehörigen Integral kein Logarithmus auftritt. Dir allgemeine Lösung von (36) hat also in $x=i$ und $x=\ldots i \mathrm{j}$ " win n Pol erster Ordnumg. Danit ist das Verhalteu der lutrgrale in Endlichen vollkommen geklärt, die allgemeine Lösung ist in der ganzen Ebene neromorph. Wenn $k$ keine ganze Zahl wäre. so wäre $x=0$ ein Verzweigungspunkt. ahwr die Produkte $x^{k} u_{1}, x^{k} n_{2}$ wären in der ganzen Ebror meronorph. Lir einzigen Pole dieser Produkte sind $x=z i$. dies können dureh Multiplikation nit $\left(x^{2}+1\right)$ beseitigt werden. Also sind $x^{k}\left(x^{2}+1\right) u_{1}, x^{k}\left(x^{2}+1\right) u_{2}$ in der ganzen Ebene holomorph, und wir wollm nun noch prüfen, wie sich diese Funktionen für $x=\propto$ verhalten. Das bietut keinerhei Schwierigkeit. Wenn wir in der Differmitialoledehung (:36)

$$
E=1
$$

als neue unabhängige. Veränderlich» rinführen und dann di. Cintersuchung der Lösungen für $\xi=0$ vornehment.

Hat man allgenein dir Differentialgleichung (3)

$$
u^{\prime \prime}+p u^{\prime}+q u=0 \text {. }
$$

wa $p$ und $q$ in der Ungebung von $t=\alpha$ eindentig sind, und fuhrt $\Sigma=\frac{1}{r}$ als nene unahhängige Veränderdiche on, so ist

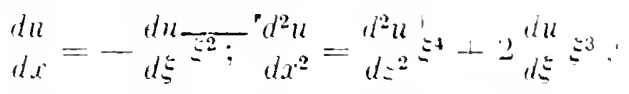

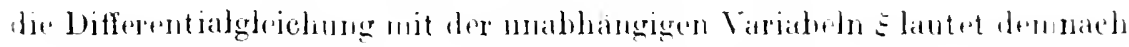

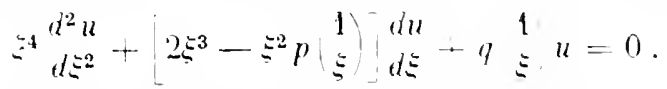

und as sind mun die Koeffizienten dieser Differentialgledehung in der L'mgebung von $\xi=0$ rindeutig. Damit die Integrale in $\xi=0$ nicht unhestimmt werden. ist nach den Ergebnissen der Nummern 40. 41 notwendig und lisureichend. dati in der Umerebung ron $\Sigma=0$

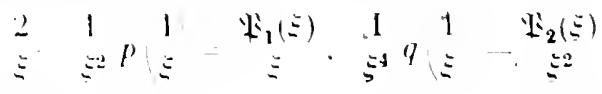

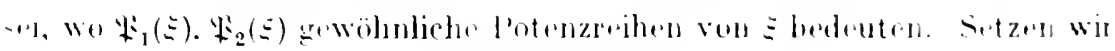

$$
2-W_{1}(\xi)-
$$


" lautet also dir Form der Konffizienten p $(x)$. g(x) in dw limgundung von $x=\infty$

$$
p(x)={ }_{r}^{1} \&_{1}\left(\begin{array}{l}
1 \\
x
\end{array}\right), \eta(x) \quad \begin{gathered}
1 \\
r^{2}
\end{gathered} \psi_{2}\left(\begin{array}{l}
1 \\
x
\end{array}\right)
$$

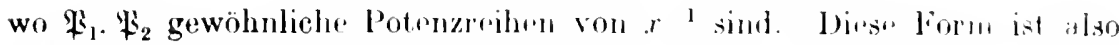
notwendig und hinreichend dafiur, dial. die Integralr von (3) iun Purkte $x=\infty$ nicht unbestiunt. wrorden.

Die determinierende Fumbanmtalgleichung der Ibifferentialgleichung (37) fuir $:-0$ lautet

odes

$$
o(0-1) \cdot \varphi\left[2--W_{1}(0)\right]-W_{2}(0) \quad 0 \text {. }
$$

$$
\varrho(\varphi-1) \quad \mathbb{F}_{1}(0) \varrho \cdot \mathfrak{P}_{2}(0): 0,
$$

sic gilt auch als determinierende Fundamentalgleichnng von (3) fiur $x=\infty$; ihre Wurzeln geben dif Exponenten (von $x^{1}$ ), zu denen dif Elemente des kanonischen Fundamentalsystems für $x=\infty$ gehören.

Für unsere Gleichnng (36) ${ }^{1}$ ) ergibt sich, daß die Lösungen in $\xi=0$ nicht unbestimmit werden. und daf. dip determinierende Fundammntalgleichung

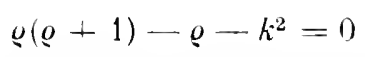

die Wurzeln $\pm k$ besitzt. Wir schließen daraus, daß dip Produkt.

$$
x^{k}\left(x^{2}+1\right) u_{1}, \quad x^{k}\left(x^{2}+1\right) u_{2}
$$

in $x=\infty$ je einen Pol zwoiter Ordnung besitzen. also ganze rationale Funktionen zweiten Grades sind. Man findet aus den Rekursionsformeln die Koeffizienten dieser ganzen Funktionen und danit für $u_{1}, u_{2}$ die expliziten Darstellungen

$$
u_{1}=x^{k} \begin{gathered}
1+k+(1-k) x^{2} \\
1+x^{2}
\end{gathered}, \quad u_{2}=x^{-k} \begin{gathered}
1-k+(1+k) x^{2} \\
1+x^{2}
\end{gathered} .
$$

\section{Direkte Behandhung der Differentialsysteme.}

Aus theoretischen Gründen wollen wir die für Differentialgleichungen zweiter Ordnung dargelegten Methoden auf Systeme zu übertragen suchen. Wenn für das System (A)

$$
\frac{d y_{k}}{d x}=y_{1} a_{1 k}-y_{2} a_{2 k}
$$

die Elemente einer Integralmatrix $Y$ und folglich die allgemeinen Lösungen in dem singulären Punkte a nicht unbestimunt sind. und es bedeutet $\mathfrak{Y}$ die zu $\zeta$ für einen Unlauf um a kogrediente Cauchysche Matrix. so treten in den Laurentschen Reihen, die die Elemente der Matrix.

1) die ïbrigens 'durch dit Transtormation $\xi=\frac{1}{x}$ in sich selbst ïhergeht 


$$
\Phi=? ! 1 \%
$$

bilden. negative Potenzon nur in endlieher Anzahl auf. Die Matrizen ) und 2$)$ sind also hier in lerzug auf einen Umlauf um "von derselben Art. Für die kanonische Integralnatrix

$$
I I=(r-a)^{2} \Phi,
$$

wo also $\Sigma$ eine der drei Formen (63) der Nr. 39 (S. 156) hat. könmen wir dann die in den $r_{1}, r_{2}$ noch willkürlichen ganzen Zahlen so einrichten, daß

1. die Elemente vou $\Phi$ in der Ungetung von $x=a$ holomorph $\operatorname{sind}$,

2. diese Elemente nicht alle für $l=a$ verschwinden.

Es seien $r_{1}, r_{2}$ schon von vornherein so groühlt: dann sagen wir. dal. dic kanonische Matrix $H$ zu der Exponentenmatrix $\Sigma$ gehöre. und nennen $\Sigma$ die zu $x=a$ grehörige doterminiorends Matrix. du Gleichung

$$
\because-I r \mid=0
$$

die zu $x=a$ gehörige determinierende Fundamentalglejehung Nach den Jacobischen Satz (Nr. 38) ist

$$
\{\boldsymbol{H}\rangle=\text { const. } e^{\int(1, \cdots \cdots) \cdot d x} \text {. }
$$

Da die $a_{k}$ in $x=a$ nur einen Pol haben können, ist in der Ungebung von $x=a$

also

$$
a_{11}+a_{22}=\frac{a_{k}}{(x=a)^{k}}+\cdots+\frac{a_{1}}{(x-a)}+\beta_{0}+\beta_{1}(x-a)+\cdots .
$$

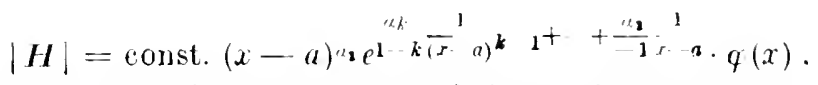

wo $q(x)$ in der Ungebung von $x=a$ holomorph und $q(a)=0$ ist. Da $x=a$ für $|\boldsymbol{H}|$ keine Unbestimutheitsstcll, sein kann, so muB $l \leqq 1$ sein. d.h. $a_{11}+u_{22}$ hat in $x=a$ höchstens oinen Pol erster Ord. nung. Wir finden also

wo

$$
\mid \boldsymbol{H}\}=\text { const. }(x-a)^{a_{2}} q(x), q(a)+0 .
$$

$$
a_{1}=\operatorname{Res}_{a}\left(a_{11}+a_{22}\right)
$$

ist. Für die Cauchysche Matrix $(x-a)^{2}$ fergilt der Jacohisebe Satz endlich hahen wir

$$
i(x-a)^{2}=\text { const. }(x-a)^{r_{1} \cdot r_{2}} \text {, }
$$

$$
\boldsymbol{\phi}=(x-a)^{r} \psi^{\prime}(x) \text {. }
$$

wo $r \geqq 0$ und $\psi^{\prime}(x)$ in der Unigebung von $x=a$ holomorph und $\psi^{\prime}(a)+0$ ist. Da aher

ist, so finden wir

$$
H_{1}=\left|(x-a)^{2}\right|, \phi \mid
$$

$$
r=\operatorname{lies}_{12}\left(a_{11}+a_{22}\right)-\left(r_{1}-r_{2}\right) \quad 0 .
$$




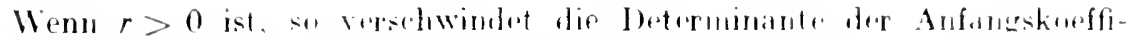

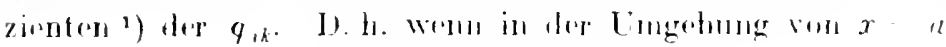

grandzt winl, ist

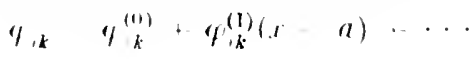

$$
\begin{aligned}
& f_{11}^{(11)} q^{(1) !} \\
& q_{2 !}^{(1)} q^{(i)} \\
& 2 ! 2
\end{aligned}
$$

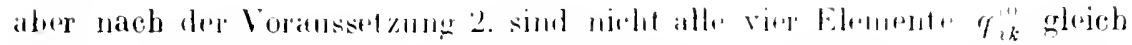

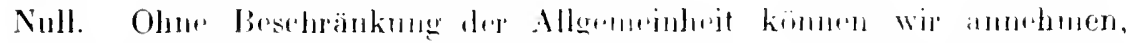

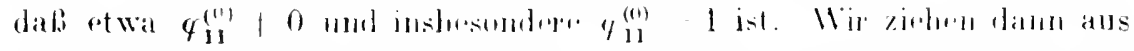

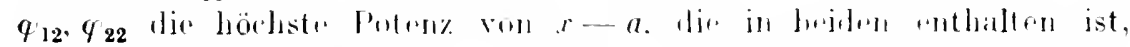

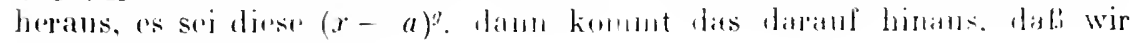
$\Phi$ in dir Form sitmill:

$$
\Phi=\Phi \cdot\left[\begin{array}{ll}
1 & \prime \prime \\
0 & (x-a)^{a}
\end{array},\right.
$$

unl in der Matrix $\Phi$ sind dir Elemente der prsten Spalte dirselhen wie in $\Phi$, während in der zweitren Spalte sicher niclat dir Anfangsglieder beider Elmonte rerselowindon. Ist $\psi_{12}(a)=0$. su hat die Deteminante

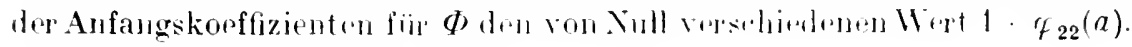
Ist $\varphi_{12}(a) \mid 0$, so hilifen wir

$$
\Phi\left(\begin{array}{cc}
1 & -\eta_{12}(a) \\
0 & 1
\end{array}-\Phi:\right.
$$

dadurch wird die reste Spalte nicht geandert und in do zwoitru Spalte ist $\varphi_{12}(a)=0$. Ist $\mathscr{f}_{22}(a)+0$. so hat dir Duterminante der Anfangskoeffizienten in $\Phi$ einen von Null versehiedemen Mert. ist ${ }_{22}(a)=0$, so kann wie bei $\Phi$ die höcliste in $f_{12}$ und /22 auftretende l'otenz ron $x$ - a herausgezogen werden. Setzen wir dirses Verfahren fort. so hann der Fall, dali die Elenente der zwpiten Spaltr gleichzeitig verschwinden, nur eine endliche Anzahl von Malen auftreten. da ja in $\Phi$ / keine höhere Potenz von $x$ - $a$ als lie r-te steekt. Wir kommen also schließlich zu einer Darstellung von der Form

$$
\Phi=i \mu \cdot(; .
$$

wo die Determinante der Anfangskoeffizientrn von 4 i nicht verschwindet. und $G$ aus Matrizen von der Form

$$
\left(\begin{array}{lc}
1 & \text { const. } \\
0 & 1
\end{array}\right),\left(\begin{array}{l}
1 \\
1(x-a)^{g}
\end{array}\right.
$$

zusammengesetzt ist. Die Determinante $\left.\right|_{i} ;$ ist eine Potenz ron $x-a$. Gehen wir dann vou (A) durch die Transformation

$$
\begin{aligned}
& y_{1}=z_{1} g_{11}+z_{2} g_{21}, \\
& y_{2}=z_{1} g_{12}+z_{2} g_{22}
\end{aligned}
$$

$\therefore$ d. h. der von $x-a$ freien (ilieder. 
zu den Differmtialsystem von derselben Art

( $\left(X^{\prime}\right)$

$$
d z_{k} \quad z_{1} b_{1 k} \quad z_{2} b_{2 k}
$$

über, so hat dieses die kanonische Integralmatrix

$$
\text { 7. } H^{-1} \quad(x \quad a)=i s,
$$

und in $\psi^{j}$ ist die Determinant" der Anfangskomfizienten von Vull ropschieden.

Wir könmen also. wenn $r>0$ ist, durch eine Transformation von der Form (42) stets zu einem Differentialsystem (A') von derselben Art übergehen, für das dir dem $r$ rntspreehende Zahl gleich Null ist.

Bilden wir die derivierte Matrix von \% so ist narh ler Derivation.. formol (D) der Nr. 39

$$
D_{r} Z \quad B=i \mu \quad 1 \stackrel{\Sigma}{\perp} a j i-D_{r} i j ;
$$

die Elemente von is sind holomorph, und da $|\psi j|$ in Punkte a von Null verschieden ist, sind auch die Elentente von $\psi^{-1}$, und folglich die vou $D_{x} \Psi$ holonorph. Wir sehen also, daß die Koeffizienten $b_{\text {th }}$ des Differentialsystems ( $\left.\mathrm{A}^{\prime}\right)$ in $x=a$ höchstens rinen Pol erster Ordnung haben, also ist $\left(\mathrm{A}^{\prime}\right)$ ein für $x=a$ kanonisches System.

Es sei in dre Ungebung ron $x=a$

$$
\begin{aligned}
& b_{i k}=x-a+b_{i k}^{(11)}+b_{i l i}^{(1)}(x-a) \cdots \cdots \\
& l_{i k}=y_{i k}^{(1)}+\psi_{i k}^{(1)}(x-a) \cdot \cdots .
\end{aligned}
$$

dann liefert die Gleichung (43) orter

$$
i p h(x-a)=\mathcal{L}^{\prime} i \beta+(x-a) \frac{d \psi}{d x} .
$$

inden wir beiderseits die Koeffizienten gleich hoher Potmzen von $r \ldots a$ vergleichen. dureh dis absoluten Glieder

$$
\Psi^{(11} S \quad \Sigma \boldsymbol{J}^{\mathrm{w}}
$$

und dureh die Korffizienten von $(x-a)^{x+1}$

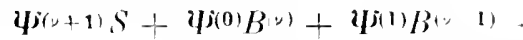

$$
\begin{aligned}
& =\Sigma y(v+1)+(v+1)(v i+1) .
\end{aligned}
$$$$
\varphi^{(\nu)} B^{\prime \prime}
$$

Aus (4/) regilot sich

$$
S=i \beta^{\prime(1)} 1 \cdot \Sigma \cdot \boldsymbol{i}^{(0)} \text {. }
$$

d. h. die deforminierende Matrix $\Sigma$ ist mit der Residuenmatrix S Her Koeffizienton $b_{, k}$ ähnlich und zwar ist $\Sigma$ einfach die kanonisehe Form dir Matrix $S$. Insbesondere ist $|S-I r|=0$ die determinierende Fundamentalgleichung. Hat man $\Sigma$ auf diese Weise bestimut, so liefert (45) eine Rekursionsformel zur Berechnung der Koeffizientemuatrx

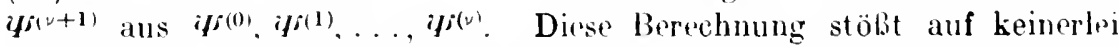
Sehwierigkeit, wenn dir Determinante der Koeffizienten $\psi_{i k}^{(\nu+1)}$ von Null 


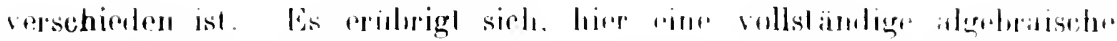

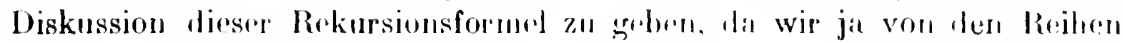
phk ansgegangen sind; man findet rime soldhe - in nowh allgemeineren

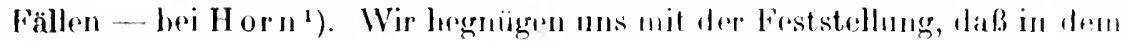

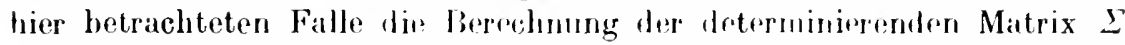
und der Koeffizienten der Ruihenenl wicklungen $\psi_{i k}$ ans den lint wisklungskoeffizienten der $b_{1 k}$ auf algetmaische Wise möglieh ist.

Nicht fïr jedes in Punkte $x=a$ kanonische Systron ist die lit-dingung erfiillt, dab die Determinaute der Anfangsedieder in der Matrix is von Null versehieden ist. Aber man kann dies dureh rine Transformation von der Form (42) stets erreich"n. Wir sagen, wenn dies der liall ist, das System $\left(\Lambda^{\prime}\right)$ habe in $x=a$ die Normalform. In diesem Fille: lautet also die Entwicklung cinee breliebigen lntegralunatrix in der. Unugebung von $x=\|$

$$
r(x-a)^{s}(y(\theta)+y+(r-a)+\cdots),
$$

wo $r$ eine beliebige konstante Matrix, $S$ die Residuenmatrix der $b_{i k}$ und $\Psi^{(i)}$ eine Matrix mit nicht verschwindender Determinante bedeutot. Wir solum hier das vollständige Analogon zu der Ent wicklung (IVa) der Nr.34 (S. 128)

Wir bemerken, dals die Erörterungen dieser Nummer vorwiegend theoretische Bedeutung haben, indem sie von der Entwicklung der Lösungen ausgehend dir Transformation anf die Normalform zeigen. Wenn ein vorgelegtes Differentialsystem daranlhin untersucht werden soll, ob seine Lösungen sich in einem singulären Punkte $x=a$ bestimnt verhalten, so ist es an bequemsten, dir: Transformation auf eine lineare Differentialgleichung zweiter Ordnung auszuführen. Ergibt sich für diese die Form (B), so stellt man nach deil Methoren der Nr. 42 das kanonisehe Fundanentalsystem her; dieses bildet dann mit seinen Ableitungen eine Integralmatrix, auf die man die Methode lieser Nummer anwenden und auf diese Weise zu einem System von dur Normalform gelangen kann.

\section{Riccatische Differentialgleichung und beliebige Iifferential- gleichung erster Ordnung ").}

Die in den Nrı. 40, 42 fiir lineare Differentialgleichungen zweiter Ordnung abgeleiteten Resultate sollen nunmelır zunächst auf dir Rircatische Differentialgleichung übertragen worden.

1) J. Horn. Mathematische Annaten 39 (1891). s. $391 \mathrm{ft}$.

2) Vgl. für diese Nummer: F uchs. Berliner Sitzungsberichte 1685. S. 279ff. Werke II. S. 391: Briot et Butuquet. Jomnal de l'École Polyt. Cah. 3it. 185ti

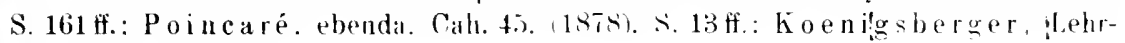
buch etc., S. $373 \mathrm{ff}$. 
Marihen wir in der Differentialgleirhung (B) (S. 165) dir Substitution

$$
y=(x-a) \stackrel{d \log u}{d x}, \quad u=e^{i,{ }^{y}}{ }^{d r},
$$

sis ergibt sich

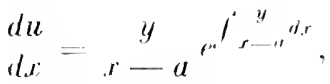

$$
\begin{aligned}
& \mathbb{R}^{2} u=\left\{\begin{array}{c}
y^{2} \\
d x^{2}
\end{array} \mid(x-a)^{2}+x-a d y-(x-a)^{2}\right\} e^{e^{i}=a^{d x}} .
\end{aligned}
$$

nnd somit für $y$ die Rircatische Differentialgleirhung

$$
d y=-y^{2}+\left[\mathfrak{F}_{1}(x-a)-1\right] y+\mathfrak{F}_{2}(r-a) .
$$

Bedeuten $u_{1}, u_{2}$ irgendein Findammalsystem von $(B) . \gamma_{1} \cdot \gamma_{2}$ willkürliche Konstantron, so ist

$$
y=(x-a)^{d \log \left(\gamma_{1} u_{1}+\gamma_{2} u_{2}\right)}=(x-a) \begin{aligned}
& \gamma_{1} u_{1}^{\prime}-\gamma_{2} u_{2}^{\prime} \\
& \gamma_{1} u_{1}+\gamma_{2} u_{2}
\end{aligned}
$$

das allgeneine Integral von (48). Der l'unkt $x=a$ ist also anct für dir Integrale der Riccatisthen Glerhung (48) kein Punkt der Unbestimmtheit.

Die Form (48) orduet sich den Fallu unter, wo in einw Differentialgleichung

$$
d y=j(x, y)
$$

die Funktion $f(x, y)$ nnahhängig von $y$ mendlich wirk, während ihn reziproker Wert in allgeneinen holonorph blejht, ein Fall, der in der Sr. 10 ausgeschlossen worden war, weil or zu viner festru Singularität führt. Wir können nummelır die Beschaffenheit der Integrale von (48) in der Ungebung von $x=a$ vollkommers angebers.

Es seien $u_{1}, u_{2}$ dic beiden Elemente des z.n $x=a$ gelörigen kanonischen Fundanentalsystems, wir sie in der . Yr. 42 aufgestellt worden sind. Dann ist, falls beide lntegrale in Roihenform darstellbar sind, das allgeneme Integral $y$ von $(48)$ in der Ungebung ron $x=a$ in der Form

$$
y=(x-a) \begin{gathered}
(x-a)^{r_{2}-1} \psi_{2}+r \cdot(x-a)^{r_{1}}{ }^{1} y_{1} \\
(x-a)^{2} q_{2}+c \cdot(x-a)^{r_{1}} q_{1}
\end{gathered}=\begin{aligned}
& y_{2}+c(x-a)^{r_{1}} \cdot \psi_{1} \\
& y_{2}+c(x-a)^{r_{1}} \cdot q_{1}
\end{aligned}
$$

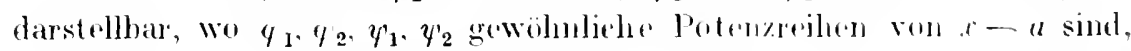
dir für $x=$ " nicht verschwinden, und $r=\gamma_{1}$, lie willkürliche lionstante bedeutet. Das allgemeine Integral ist also ju der Lingehung von $x=a$ nach positiven ganzen Potrizen von $x$ - a mul

$$
1(x-a) \div,
$$

entwickelbar. Besonders bemerkenswert sind die beiden lntegrale 


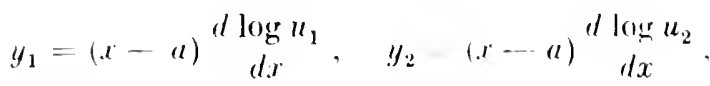

die in der Ungebung vorn $r=a$ dic firm:

$$
\begin{aligned}
& y_{1}=r_{1}+\delta_{1}(x-a)+\delta_{2}(x-a)^{2}+\cdots, \\
& y_{2}=r_{2}+\varepsilon_{1}(x-a)+\varepsilon_{2}(x-a)^{2}+\cdots
\end{aligned}
$$

haben, also daselbst holomor th sind und in $x=$ a lurichungsweise die Werte $r_{1}, r_{2}$ annehmen.

Fiir dir Wertepaare $x=a, y=r_{1}$ und $x=a, y=-r_{2}$ fiillt in der Entwicklung des Zählers der rochten Srite der Gleichung (/A) mach Potenzen von

$$
x-a, y-r_{1} \text { beziehumgswerise } x-a, y-r_{2}
$$

das konstantr. Glied weg, da jal $r_{1}, r_{2}$ die Wurzeln dre Gleichung (22)

$$
\varrho(\omega-1)+t_{1}(0) \varrho+i_{2}(0)=0
$$

sind; der Differentialquotient $\frac{d y}{d x}$ erscheint also für diest Wertepare in der unbestimmten Form $\frac{0}{0}$, rin Fall. der bei den allgemoinen Untersuchungen des zweiten Kapitels elrenfalls ausgeschlossen war. Wir sehen bier zugleich wieder ein Beispial für die in der Nr. 13 (S. 49) rrwähnte Möglichkeit, daß ein gewisses Integral, das für $x=x$ den Wert $y=y$ annimmt, in der Ungebung von $x=x$ lıolomorph bleiben kanm, obwohl für dieses Wertesystenı der Differentialquotiont $\frac{d y}{d x}$ in unbestimuntor Form erscheint.

Wenn die Entwicklung des Integrals $u_{2}$ in der Unigebung von $x=a$ einen Logarithmus enthält, so ist $r_{1}-r_{2}=g$ Null odler eine positive ganze Zahl; in diesem Falle lautet die Darstellung des allgen rinen Integrals in der Umgebung von $x=a$ :

$$
\begin{gathered}
y=(x-a)^{c(x-a)^{r_{1}-1} \psi_{1}+(x-a)^{r_{1}-y-1}\left[\chi_{1}+\chi_{2} \cdot(x-a) \log (x-a)\right]} \\
c(x-a)^{r_{2}} \varphi_{1}+(x-a)^{r_{1}-g}\left[\varphi_{2}+\psi_{2} \log (x-a)\right] \\
=\frac{\chi_{1}+\chi_{2} \cdot(x-a) \log (x-a)+c(x-a)^{g} \psi_{1}}{\varphi_{2}+\psi_{2} \log (x-a)+c(x-a)^{g} \varphi_{1}}
\end{gathered}
$$

wo $\varphi_{1}, \psi_{1}, \varphi_{2}, \psi_{2}, \chi_{1}, \chi_{2}$ gewöhnliche Potenzreihen von $(x-a)$ bedeuten, von denen $\varphi_{1}, \psi_{1}$ für $x=a$ von Null verschiedene Werte haben, während $\chi_{1}, \chi_{2}$ und $\varphi_{2}, \psi_{2}$ für $x=a$ nicht gleichzeitig verschwinden. In diesem Falle ist also $y$ nach positiven ganzen Potenzen von $x-a$ und $\log (x-a)$ entwickelbar. Dem Integrale $u_{1}$ entsprechend, gibt es ein in der Umgebung von $x=a$ holomorphrs Integral

$$
y=(x-a)^{\frac{d}{\log u_{1}}} \frac{{ }^{2}}{d x}=r_{1}+\partial_{1}(x-a)+\partial_{2}(x-a)^{2}+\cdots,
$$

das für $x=a$ den Wert $r_{1}$ anninmut. 
Wir wollen den Fall. wo $r_{1} \quad r_{2}$ weder eine ganzo Zahl noch Nill! 1st. als den allgemeinon otwas näher ins Auge fassen

Setzt man

$$
x-a=\xi . y-r_{j} \ldots \eta \text {. }
$$

wo $\lambda$ pine der Zahlen 1. 2 hedeutet. so ist in der Unuebuna won $三=0$. $\eta=0$

woselbst

$$
y^{2}+\left[L_{1}(x-a)-1\right] y+\mathscr{L}_{2}(x-a)=-\alpha \xi-\beta \eta-[\xi, y]_{2}
$$

$$
\begin{aligned}
& a=\mathscr{W}_{1}^{\prime}(0) r_{i}-\mathscr{W}_{2}^{\prime}(0) . \\
& \beta=2 r_{1}+\mathfrak{W}_{1}(0)-1 \quad 2 r_{i} \quad\left(r_{1}-1,1\right.
\end{aligned}
$$

ist und $[\xi . \eta]_{2}$ die Gesamtheit der Glieder zweiter und höherer Dimension in $5 . \eta$ bedeutet. Dir Differentialgleichung

$$
\xi \frac{d \eta}{d \xi}=\alpha \xi+\beta \eta+[\xi, \eta]_{-}
$$

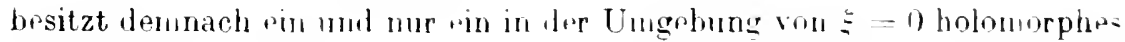
Integral

$$
\eta_{1}=\delta_{1} \xi+\delta_{2} \xi 2+\cdots .
$$

das für $5=0$ verschwindet. Die Vuraussetzung. dal. $r_{1}-r_{2}$ werder gleich Null noch gleich einer ganzen Zahl sei. bedingt für $\beta$. laß dies" Größr keine ganze Zahl sein kann. Wirn $\beta$ eine negative ganze Zahl oder Null ist. so gibt 's, wie wir gesehen haben. noch immer ein in der Ungebung von $x=a$ holomorphes Integral der Differentialgleichung (48). das für $x=a$ den Wert $r_{\lambda}$ annimmt, dem tann ist $r_{\lambda}$ eben diejenige Wurzel der determinierenton Fundamentalgleichung (22), deren reeller Tril nicht kleiner ist als der der anderen Wurzel. Ist $\beta$ keine positive ganze Zahl und der reelle Teil von $\beta$ positiv. so ist das allgemeine Integral von (50) nach positiven ganzen Potenzen von $\Xi$ und $\leqq$ entwickelbar. Wir haben also den Satz:

Die Differentialgleichung (50), die aus (48) durch dir Substitution (49) herrorgegangen ist. besitzt. Wenn $\beta$ kein positive ganze Zahl ist. ein und nur ein mit \$ zugleich vor. sehwindendes und in der Ungehung ron $\xi=0$ holomorphes Integral.

Der besondere Charakter der Differentialgleichung (50) als Riccatischer Gleichung gibt sich darin kund, dab das Aggregat [\$. $\eta]_{z}$ nur die erste und zweite Potenz von $y$ enthalten kann. Der chen ansgesproehene Satz gilt aber unahhängig von diesen Unstande. I. h.:

Wenn in der Differentialgleichung (50) die rechte seite eine beliebige in der Ungehung ron $\xi=0 . \eta=0$ konvergente gewöhnliehe Potenzreihe ohne konstantes Glied bedeutet. worin der koeffizient $\beta$ der ersten Potenz ron $r$ keine posi- 


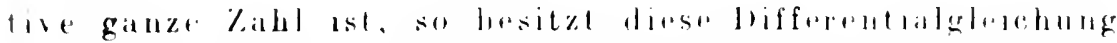
mu nut nur rin mit $\xi$ geleichzeitig verschwimbendes nurl in ler Ungobung von E O holomorphes Intregal.

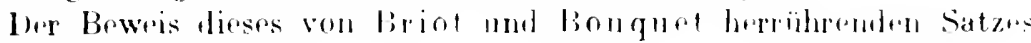
lst äuberst riufach.

Setzen wir nämlirh

$$
\eta_{1} \quad \partial_{1}=-\partial_{2}=2 \text {. }
$$

In die Differentialgleiehung $(50)$ rin, so prgilu sieh

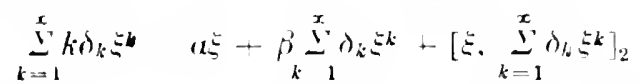

The Vergleichung les lowfizinten der gitrichhohen Potenzen von E auf beiden Seiten dieser Gleichung liefort fïr $k=1$ :

$$
\delta_{1}=a-\beta \delta_{1} \cdot \delta_{1}=\stackrel{u}{\iota-\beta}
$$

und allgenoin

$$
k \delta_{k}=\beta \delta_{k}+q_{k}\left(\partial_{1} . \delta_{2} \ldots . \partial_{k} 1\right) .
$$

wo $\varphi_{k}$ eine ganze rationale Funtion we $\delta_{1}, \delta_{2} \ldots . \delta_{k}$, hedentet. deren Koeffizienten sich aus $\alpha, \beta$ und den Koeflizimten von \&, 1/, ganz un! rational zusammensetzen.

Dif Rekursionsforturl

$$
r_{k}=\frac{q_{k}\left(\partial_{1}, \partial_{2}, \ldots \delta_{k} 1\right)}{k-\beta}
$$$$
k=12 ., \quad S=01
$$

liefert stets endliche bestimnte. Werte für die $\partial_{k}$, da zufolge unserer Voraussetzung $\beta$ keine positive ganze Zahl. also

$$
k-\beta+0
$$

ist. Um die Konvergenz der so formal hergestellten und die Differentialgleichung (50) befriedigenden Reihe zu erweisen. vergleichen wir die $\dot{b}_{k}$ mit den durch die Rekursionsformel

$$
n \gamma_{k}=\beta_{;} z_{k}-q_{k}\left(\gamma_{1} \cdot \gamma_{2} \ldots \ldots \gamma_{k-1}\right)
$$$$
\dot{i}=1.2 \ldots \gamma=0 \text { ) }
$$

definierten Größen $\gamma_{k}$.

Diese $\gamma_{k}$ sind, wie nan sofort duersieht, nichts anderes als die Koeffizienten der nach positiven ganzen Potenzen von $\$$ fortschreitenden Entwicklung derjenigen Lösung der Gleichung

$$
n z=\alpha s-p z+[\xi, z]_{2} \text {. }
$$

die für $\xi=0$ verschwindet. Sach den Satze von der impliziten Funktion besitzt nämlich eine Gleichung von der Form

$$
\text { 为 }(\xi . \Sigma)=0 \text {, }
$$

wo $\mathfrak{P}(\xi, z)$ eint in der Ungebung von $\xi=0, z=0$ konvergente gewöhnliche Potenzreihe bedeutet, die kein konstantes Glied enthält, stets eine und nur eine Lösung $z$, die in der Unigebung von $\xi=0$ holomorph ist 
und für $\xi=0$ versehwindet. vorausgesetzt, daß der Köffizient der erston Potenz von $z$ in $\mathfrak{B}(\xi, z)$, 1. h.

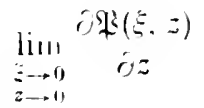

einen von Null verschiedenen Wert bat. Also konvrorgiart dis Pritue

$$
\sum_{k=1}^{x} \gamma_{k}=k
$$

in der Ungebung von $\xi=0$ jedenfalls. werm

$$
n-\beta+0
$$

ist.

Wenn nun der reolle Teil won $\beta$ negativ ist, so nehuen wir $n=1$ : ist dagegen der reelle Teil von $\beta$ positiv, so wählen wir $n$ als diejenige bwstimute positive ganze Zahl, fïr die der reelle Teil von $n-\beta$ dern absolut $n$ Betrage nach kleiner als $\frac{1}{2}$ ist. Es sei $\beta$ in seinen reellent und intaginären Bestandteil zerlegt:

$$
\beta=\beta_{1}+\beta_{2} i
$$

dann ist für ein positives ganzzahliges $\lambda$

also, da

$$
|n+\lambda-\beta|^{2}=\left(n-\beta_{1}\right)^{2}=2 \lambda\left(n-\beta_{1}\right)-\lambda^{2}+\beta_{2}^{2},
$$

ist,

und folglich

$$
\lambda \leq 1 .\left|n-p_{1}\right|<!
$$

$$
\lambda^{2}=2 \lambda\left(n-\beta_{1}\right)>0 \text {, }
$$

$$
|n+\lambda-\beta|>|n-\beta| \text {. }
$$

Wir haben also für $k \leqslant n$

$$
\left.\left|\partial_{k}\right\rangle=q_{k}\left(\partial_{1},\right)_{2} \ldots, \partial_{k} 1\right)
$$

d. h. $\left|\partial_{k}\right|<\left|\gamma_{k}\right|$, und somit ist die Konvergenz der Reihe

$$
\eta_{1}=\sum_{k=1}^{x} \delta_{k}=k
$$

erwiesen. Es eriibrigt noch, den Beweis der Unitat zu liefern

Nehmen wir an, die Differentialghleirhung (50) besäbe noch pin zweites für $\xi=0$ verschwindendes und in der Imgebung ron $\xi=0$ holomorphes Integral

$$
-=\sum_{k=1}^{x} \varepsilon_{k}
$$

dann folgte für die Differenz

$$
i_{1}-\div=:
$$


die Differentialgleichung

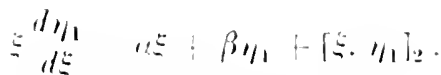

$$
\begin{aligned}
& =\begin{array}{ll}
d \\
\text { dE }
\end{array}
\end{aligned}
$$

$$
\varepsilon \frac{d z}{d \xi}=\beta z+z\left(r_{1}=+r_{2}=2 \ldots \cdots\right) .
$$

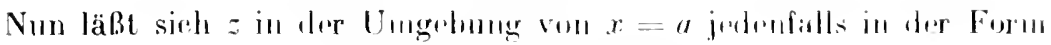

$$
z-c_{m} \xi^{m}+c_{m+1} \xi^{m+1}+\ldots
$$

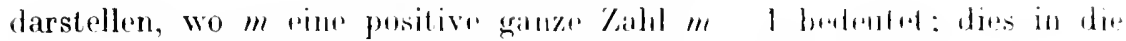

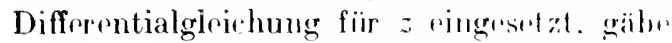

$$
m c_{m} \xi^{m}+(m+1) c_{m+1} \xi^{m+1}+\ldots=\beta c_{m}=m: \ldots .
$$

es inüBts folglieh

$$
\beta=m
$$

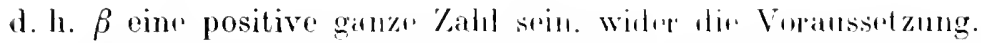

Wie Poincaré gozeigh hat $\left.{ }^{2}\right)$, gilt für dir allgemeine Differentialgleichung (50) auch dre Satz, dals ihr allgempines futrgeal in der Uutgebung von $\xi=0$ mach positiven ganzen Potruzen rou E mud s's entwickelt werden kann, wenn $\beta$ krime positive gawn \%ahl und dop reelle Teil von $\beta$ positiv ist: wir gelone den Bewers dirses Satzes hier nicht wieder.

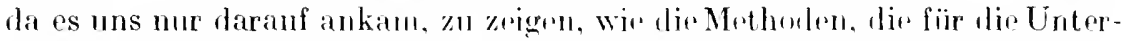
suchung des Verhaltens der Intrgeale ler Rircatisebun Ilifferentialgleichnng in ter Ungelnumg der simgulären Stellen entwiekell worden sind. auf die Untersuchung der Integrale belinhiger Differentialghoichungen

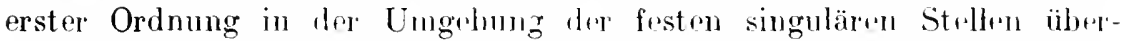
tragen werden können. Währeml abor, wie sich aus dem Folgemlen ergeben wird, für die Riccatische Gleichmng aus lem Vrrhalten der Integrale in der Ungebung der (festen) singulären Stellen, auf deren Verlauf in der ganzen Ebene der unabhängigen Variabeln geschlossen werden kann, ist dies für die allgemeine Differentialgleichung erster Ordnung mit beweglichen Verzwrigungspunkten richt in derselhen Wrise möglich.

\section{Der Fuchssche Typus.}

Wir wollen num, ähntich wir in der $\mathrm{Nr}$. 34 fïr rine Differentialgleichung erster Ordnung, den Fall betracht'n. wo die Lösungen des Systenıs (A) bez. einer linearen Differentialgheichung zweiter Ordnung in ter ganzen Ebene, den unendlich fernen Junkt mit eingeschlossen. krinen Punkt der Unbestimmtheit besitzen. und beginnen mit der Untresschnng der Differentialgleichung zweiter Ordnung (3) (S. 160).

1) H. Poincaré. Journal de l'École polyt.. Cah. 45. s. 13. 


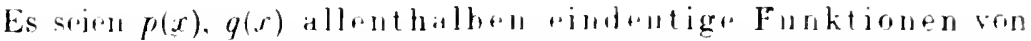
$x$. Dann miissen $p(x), q(x)$ in dror Ungebung rines jeden im endlichen gelegenen singulären Prntetes a rlic Form

$$
p(x)=\begin{gathered}
W_{1}(x-a) \\
r-a
\end{gathered}, \quad \eta(x)=\begin{gathered}
\mathbb{W}_{2}(x-a) \\
(x-a)^{2}
\end{gathered},
$$

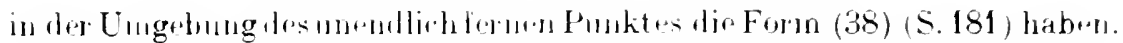

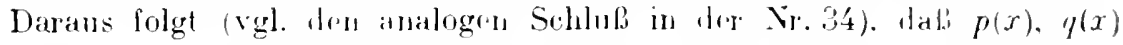
rationale Funktionen von $t$ sem miissen. und laf die in endlichen ge-

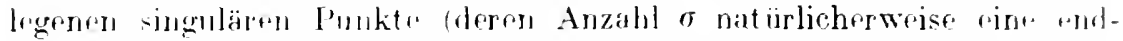

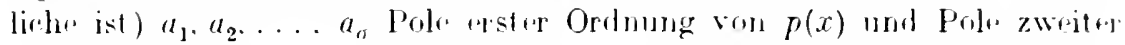
Ordmong von $q(x)$ sind. Aus der für $x=x$ geltunden Form (38) ergibt sich ferner. dafi dor Zähler von $p(x)$ höehstens vom Gréde $\sigma-1$. dow

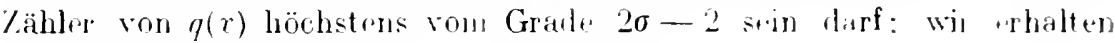
also fïr die Koeffizionter von (3) dir Form

$$
p(x)=\prod_{k=1}^{n(x)} \cdot q(x)=\prod_{k=1}^{n} h(x)
$$

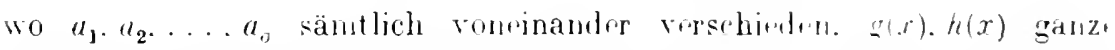
rationale Funktionen. die erste höchstrus vom $(\sigma-1) t+1$. dir zweito

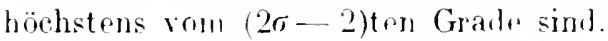

Von piner Differentialelerdung (3). Iren Koeffizienten rational. Funktionen von der Form (51) simb, oder was dasselbo heibt. Heren Integrale keinem Punkt der Unbestimmitheit besitzon. sagen wir, dalis sie zun Fuchsschen Ty yus gohör. Diuser Typus linearer Differntialghiohngen wurde nämlich zuerst von Fuchs in semer im 6r. Bande des Coplluselen

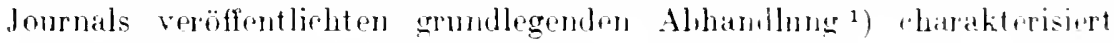
mud hat soitden den Grogenstand der Lntersuchungon von Furhs solhst und virles andorer Mathenatiker gohidet. Die dureb dims Untersu-

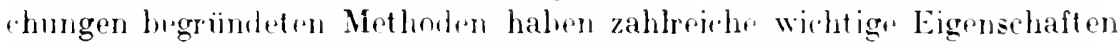

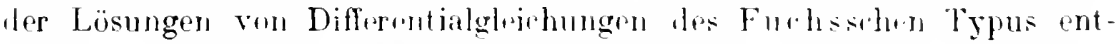

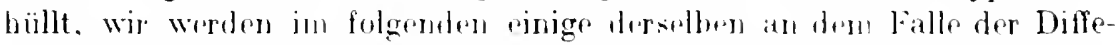

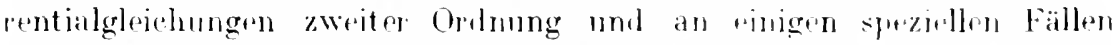

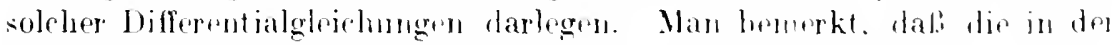

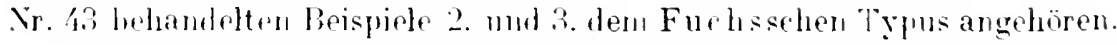

Wir beginnen not der Aufstelhng eine lieziehunge die zwischen arn Wurzeln hor zu allon singulären Punkten der Differntialgleichung

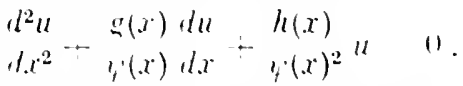

$$
\begin{aligned}
& y^{\prime}(x)=\left(x-a_{1}\right)\left(x-a_{2}\right) \ldots\left(x-a_{0}\right) .
\end{aligned}
$$

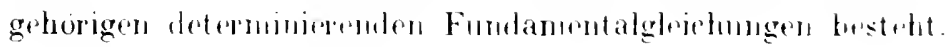

1) I. Fuchs, Intio. Wintir 1. \& lisuff. 


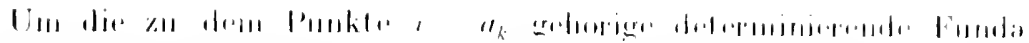

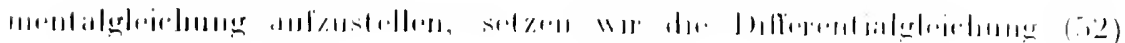

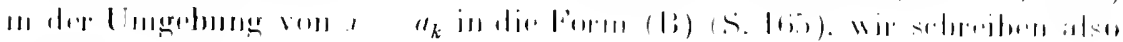

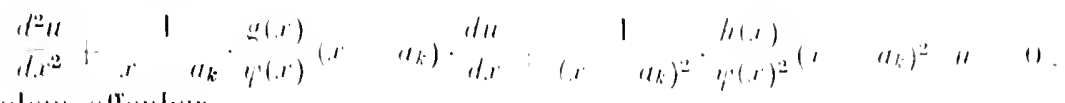
IIIInII ollumbal

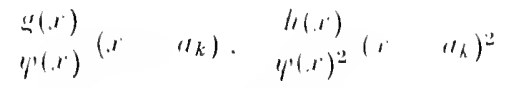

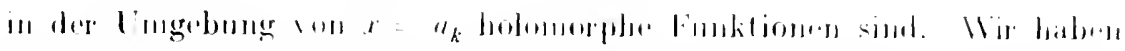

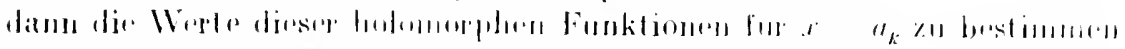

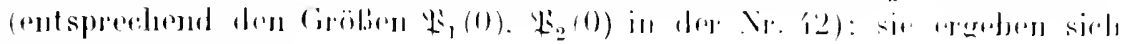
sofort in ine finrull

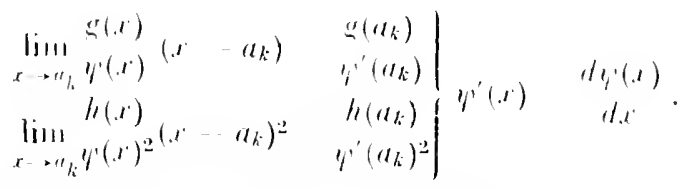

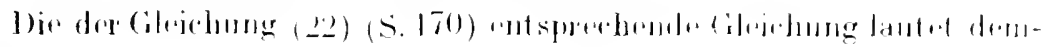
แaเ')

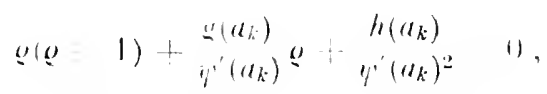

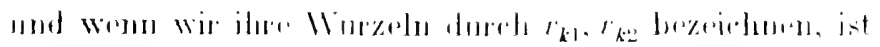

$$
r_{k 1}+r_{k 2}-\cdots \frac{u\left(a_{k}\right)}{y^{\prime}\left(a_{k}\right)}-1 \text {. }
$$

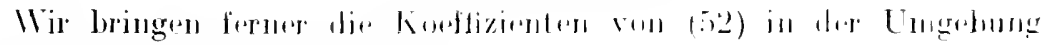
von $x=$ or auf die Furns (sis)

und selzen

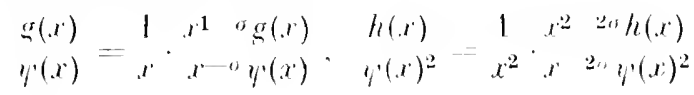

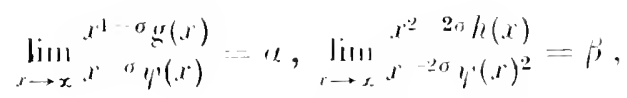

damn lautet die $z u x=$ s gehörige determinierende Fundamentalgledehung

$$
\underline{g}(\underline{0} \div \mathbf{1})-\alpha \underline{\alpha}+\beta=0 \text {. }
$$

Bozeichneu wir ihre Murzeln dureh $r_{x_{1}}, r_{x_{2}}$, so ist also"

$$
r_{x 1}+r_{x: 2}=12-1 \text {. }
$$

Nun ist in Partialbriclse zorlegt:

\section{und folglich}

$$
\begin{aligned}
& g(x) \\
& y(x)=\sum_{k=1}^{g} y^{\prime}\left(a_{k}\right) \cdot x-a_{k},
\end{aligned}
$$




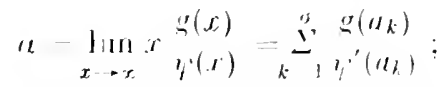

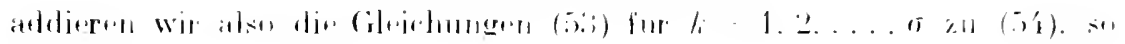
kommit

$$
\sum_{k=1}^{n}\left(r_{k} \cdot r_{k 2}\right) \quad r_{x}+r_{x} \quad 0-1 .
$$

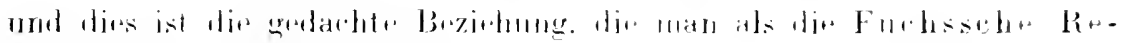

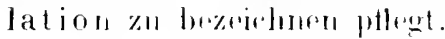

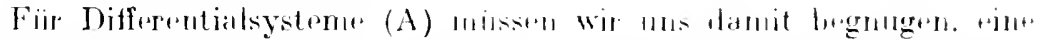

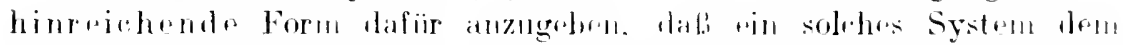

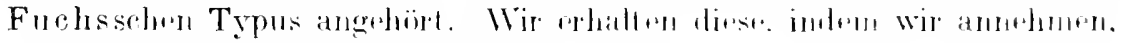

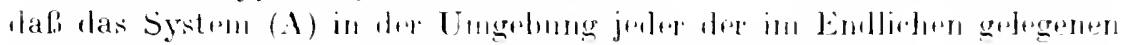

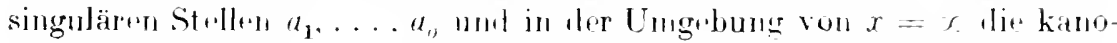

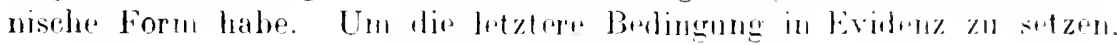

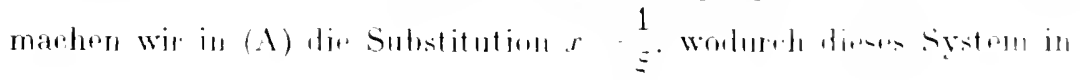

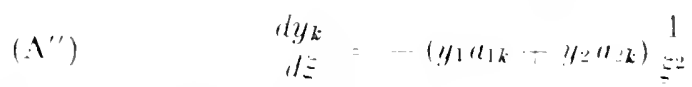

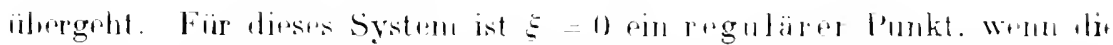

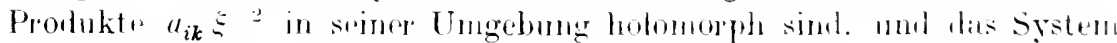

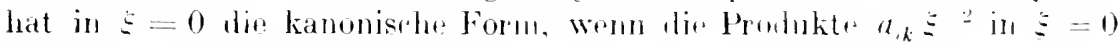
einen Pol erster Ordmung haben. Auf $r$ x iibutragen fimben wir also:

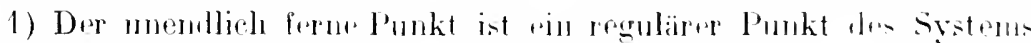

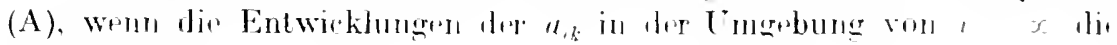
Form halinil

$$
a_{k}=a_{i k}^{(1)}+r_{i k}^{a^{(2)}}+\ldots \text { in } \mathrm{mi} \text {. }
$$

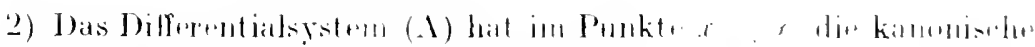

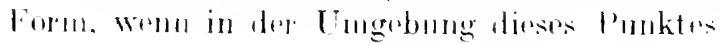

$$
a_{, k} \quad r_{i k}^{(a+1)}+\frac{a_{i k}^{(1)}}{r^{2}}+\frac{a_{i k}^{(2)}}{x^{3}}+\cdots \text { lll inf. }
$$

ist.

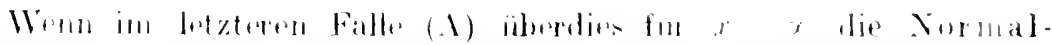

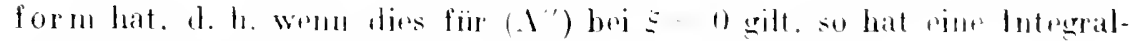

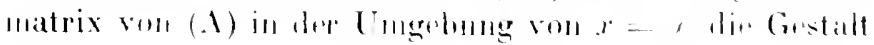

$$
\left(\begin{array}{l}
1 \\
n^{(n+1)}()_{0+1}
\end{array}\right.
$$

wo dio Elementw : on of

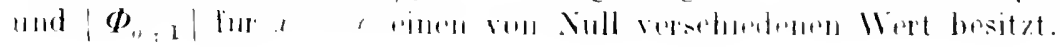




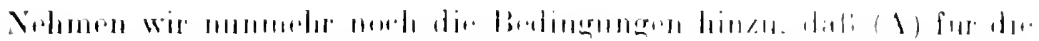

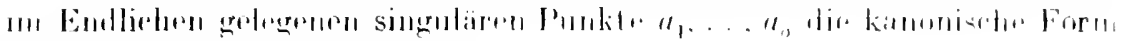
hatben soll, so lanterl also (.1)

$$
\begin{array}{lll}
d y_{k} & y_{1}-w_{k}(r) \\
d x & y_{1}(r)
\end{array} \cdots y_{2} \begin{gathered}
y_{2}(r) \\
y(r)
\end{gathered} .
$$

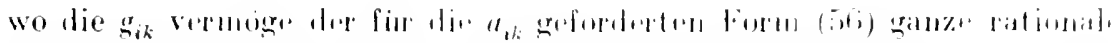

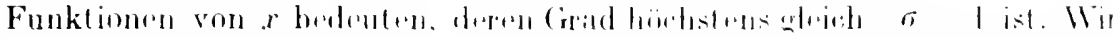

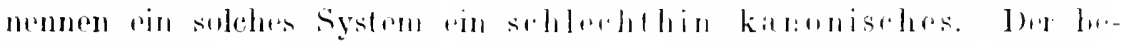

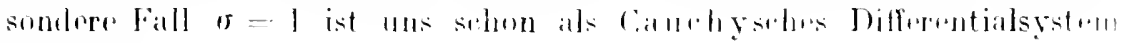
hegrenget.

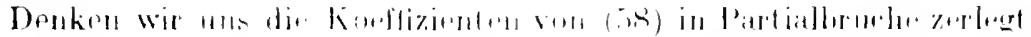

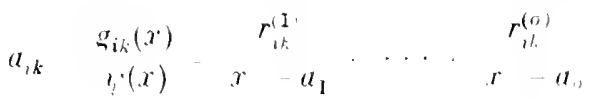

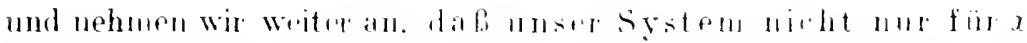
sondern auele fïr jollon der im Eudlirhongelegenen singulären Punktr dio Normafform habr. lann sind (siohe Nr. H) die zu den Punkten $r-a$, gohiorigon Residunmatrizen $f^{(2)}$ mit dol.

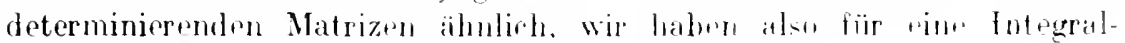
matrix in der Umgehmug von $x=a$, lie Darstallung

$$
\left.\left(x-a_{i}\right)^{l i(\infty)} \phi\right)
$$

wo die Elemente von $\Phi$, in Her Ungelung ron $r=a$, holomorph sim und $\left|\Phi_{\text {, }}\right|$ fïr $x=a$, nicht rersehwindet.

Bedeuten nun $r_{1}^{(*)} \ldots r_{n}^{\prime \prime}$ dis Wurzeln der determiniermatu Fundamentalgleichung

$$
\text { ili Ir Ir - o }
$$

fïr $x=a_{\text {, }}$. wo wir dor Einhritlichkrit zuliehe den nnendlich formen Punkt mit $a_{\sigma+\mathrm{I}}$ bezeichnen. so ist

$$
r_{1}^{(*)} \cdots r_{2}^{(\nu)}=r_{11}^{(\nu)}-r_{22}^{(*)} \text {. }
$$

und da sich durch Vergleichung von (56) mit $(59$

$$
\sum_{i=1}^{\sigma+1}\left(r_{11}^{(\nu)}+r_{22}^{(\nu)}\right)=0
$$

ergibt, so haben wir für die Wurz.ln aller determiniegenden Fumbamentalgleichungen die Relation

$$
\sum_{\nu=1}^{\sigma+I}\left(r_{I}^{(\alpha)}-r_{2}^{(j)}\right)=0 \text {. }
$$

die als Ubertragung der Fuchsschen Relation auf System anzusehen ist. 
Das sehluhthin kanmisehe Systran

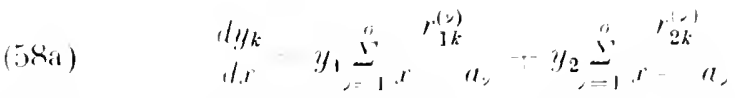

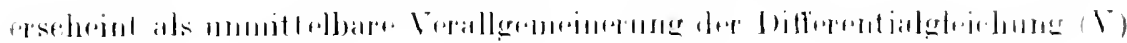
lar Nr. : ín.

\section{Das Integrationsproblem. Fundamentalsubstitutionen.}

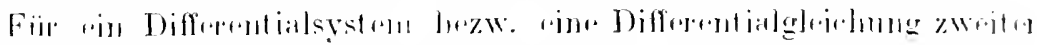

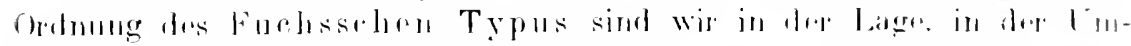

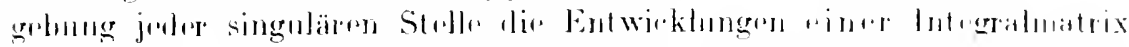

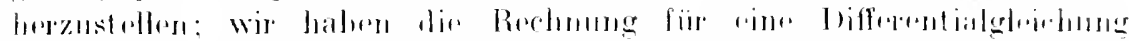

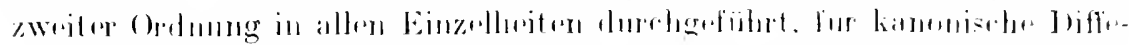

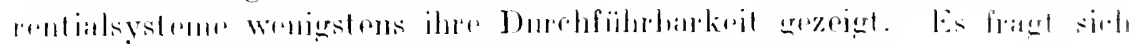

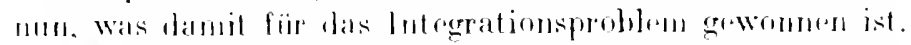

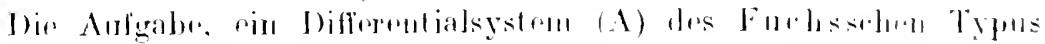

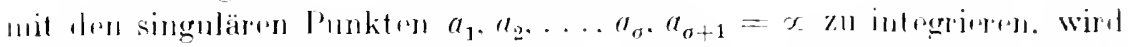

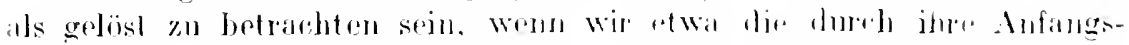

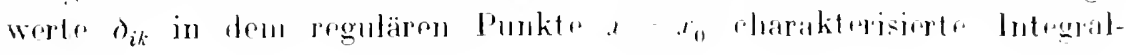
matrix

$$
y=\int_{x_{1}}^{r r}\left(11, x^{\prime}+l\right)
$$

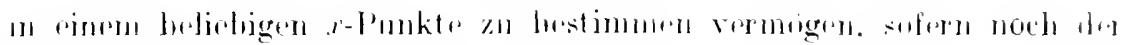

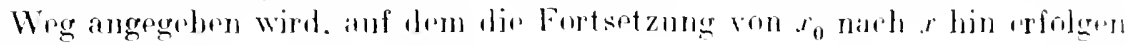
soll.

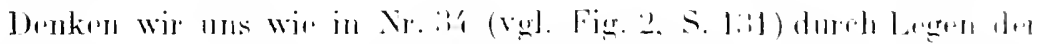

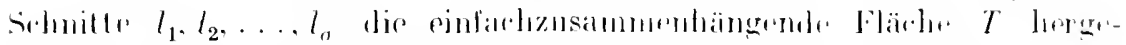

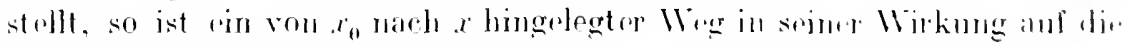

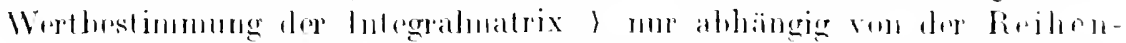

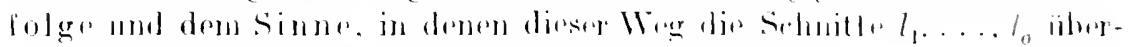

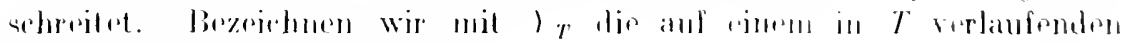

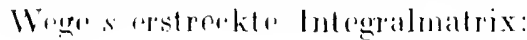

$$
\left.r_{T}(s) \int_{x_{0}}^{-s}(1 d x+l)^{1}\right)
$$

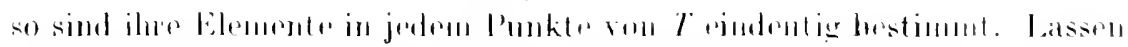

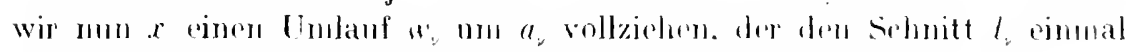

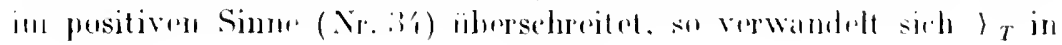

$$
\text { 【.Y.T. }
$$

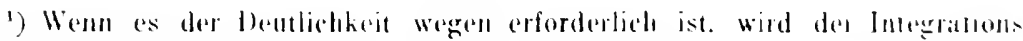
woy desu l'roduknintegralzedehen in tilammern vorgesertzt. 


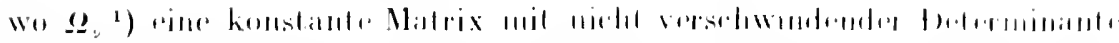

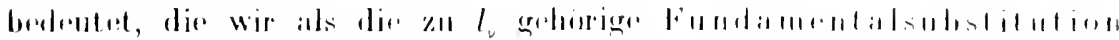

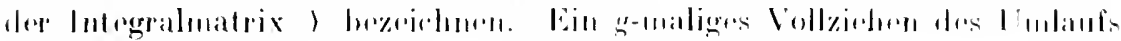

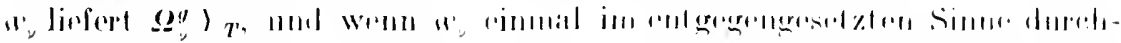

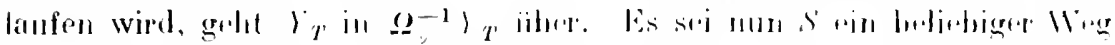

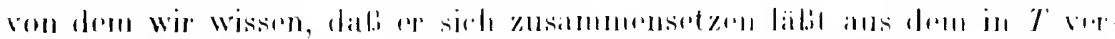

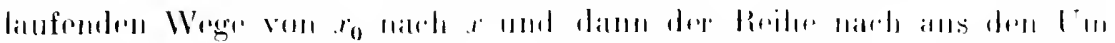

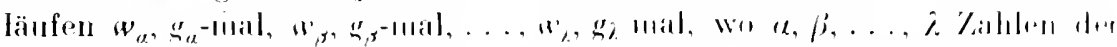

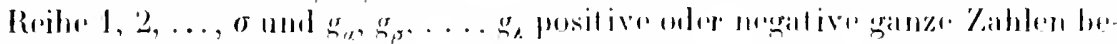

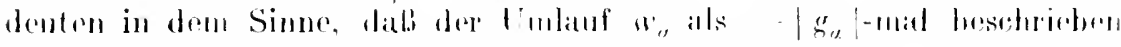

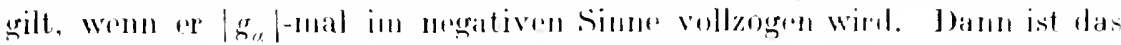

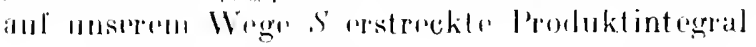

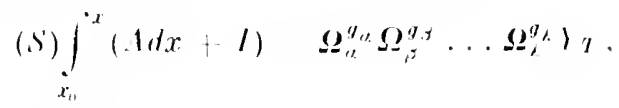

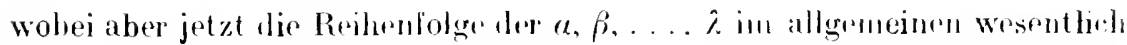
ist, so dafi also in dor hahlonfolgn $\alpha, \beta, \ldots . \lambda$ sehe wohl zwri nicht un-

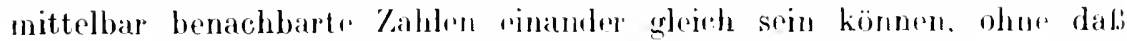
es gestattet wäre, dir bozïglichril Potenzen des browffemolen $\varrho_{k}$ mitcinander zu vereinigen. Nur in dern ganz speripllon fialle, wn die $\Omega$. miteinander vertanselhhar sind, ist die Roihenfolge ghrohgïltig, wie das

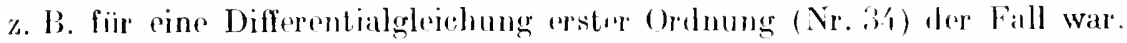

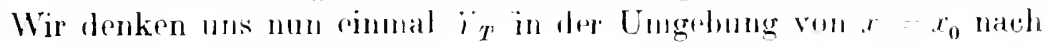
Potenzen von $x-x_{0}$ entwirkolt:

$(62) \quad Y_{T}=\gamma^{\prime}(0)+\gamma^{(1)}\left(x-x_{0}\right)+\gamma^{(2)}\left(x-x_{0}\right)^{2}+\cdots-\phi ;$

diese Entwicklung, deren Harstellung vermögt der Rekursionsformel sedr einfach ist, konvergiert gewil, bis zu dem $x_{11}$ an näclusten gelegenen singulären Punkte, es sei dieser $a_{k}$. Wir stellen uns dann die in der lingebung von $a_{k}$ gültige Entwicklnng einer lntegralmatrix $H_{k}$ nach dem in der Nr. 42 für Differentialgleichungen zweiter Orolnung, in ler Nr. 4́㇒ für Systeme in der Normalform gelehrten Verfahen her, wolei zu bemerken ist, daß wir für Differentialgleichungen zweiter Ordnung als Integralmatrix die Elemente rines Fundamentalsystoms $u_{1}, u_{2}$ und ihr Ahleitungen $u_{1}^{\prime}, u_{2}^{\prime}$, also

$$
\left(\begin{array}{ll}
u_{1} & u_{1}^{\prime} \\
u_{2} & u_{2}^{\prime}
\end{array}\right)
$$

aulzufassen haben. Als $\boldsymbol{H}_{k}$ hätten wir also in dieseu Falle das zu $\boldsymbol{u}_{k} g_{\mathrm{g}}-$ hörige kanonische Fundamentalsysteu und seine Ableitungon zu Wählen Die gedachte Entwicklung hat dann die Form

1) Wir sehreiben I., statt $\Omega^{(*)}$ : die Elemente dieser Matrix sollen mit $\omega_{i k}^{(*)}$ bezeichnet werden. 
(tii) $\quad H_{k} \quad\left(l-a_{k}\right)^{R_{k}} \phi_{k}$,

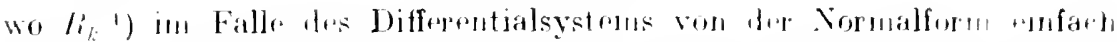

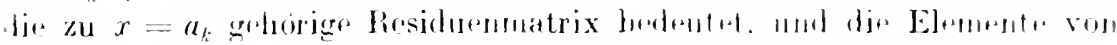

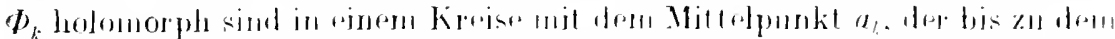

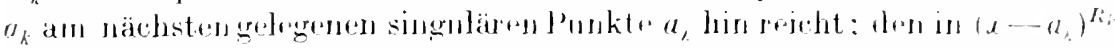

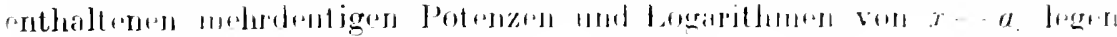

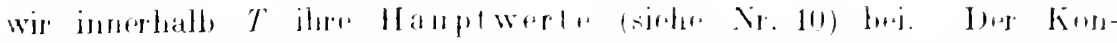

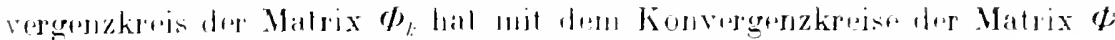

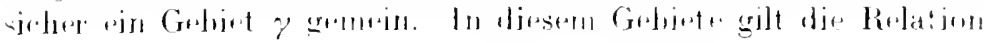

(1) $\quad b_{k}\left(l \quad a_{k}\right)^{l}: t_{i}$.

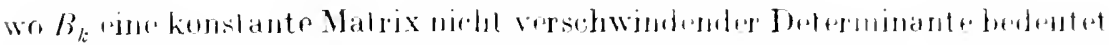

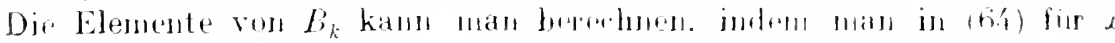

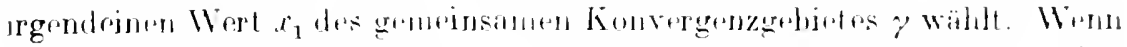

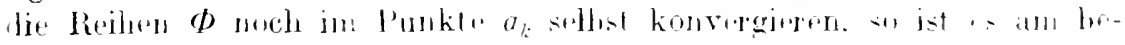

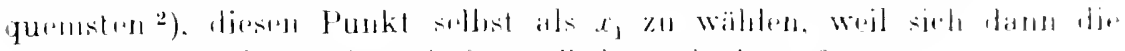

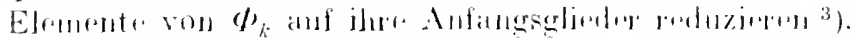

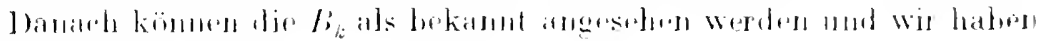

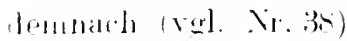

$$
\Omega_{k}-B_{k} e^{2+k_{k}} B_{i}{ }^{\prime}
$$

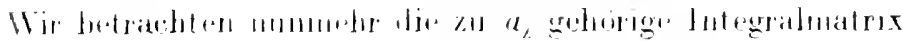

$$
H_{l} \quad\left(.1-\left(l_{1}\right)^{l_{i} ;}, p_{1}:\right.
$$

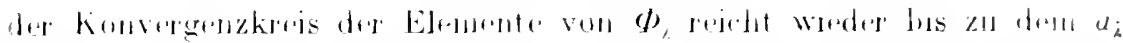

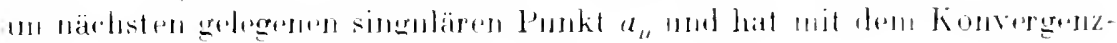

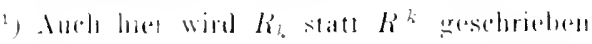

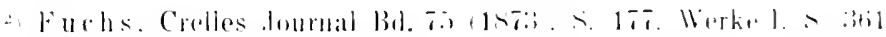

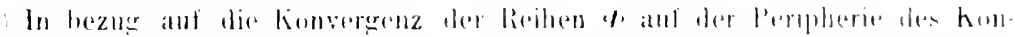

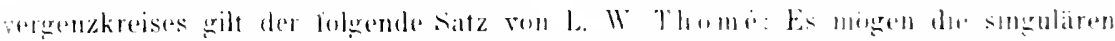

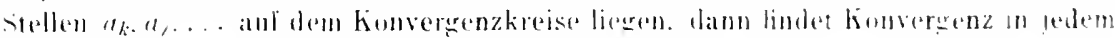

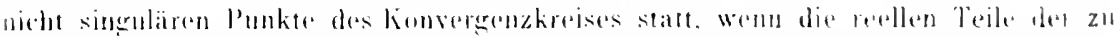

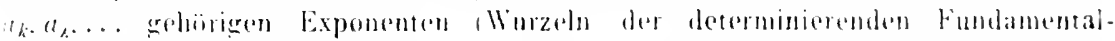

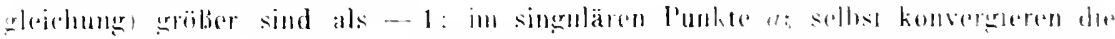

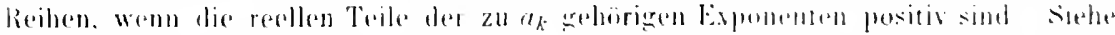

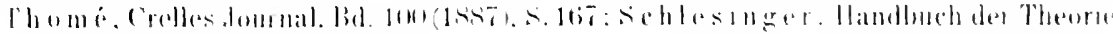

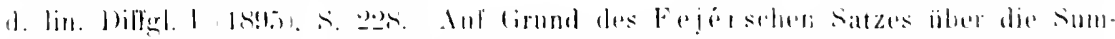

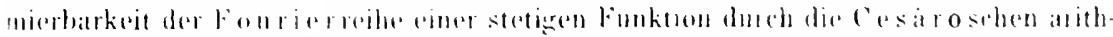

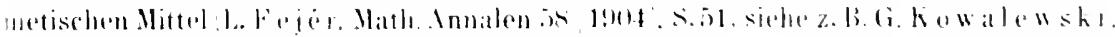

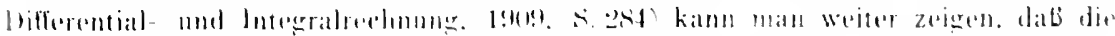

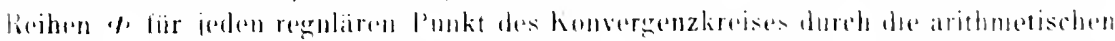

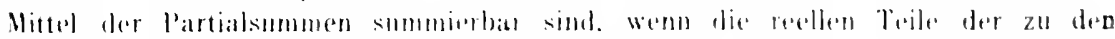

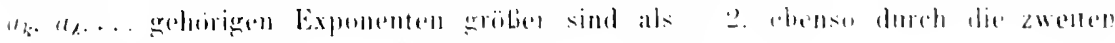

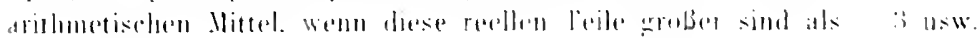




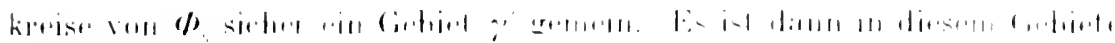

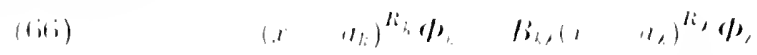

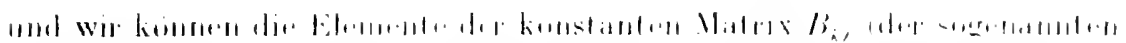

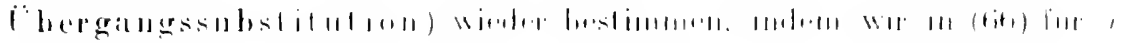

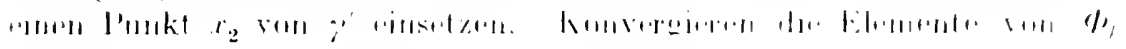

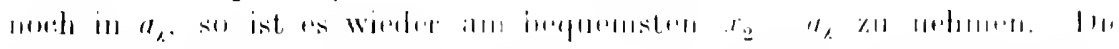

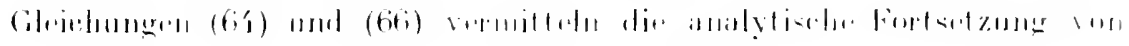

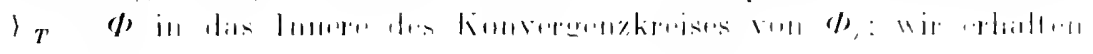

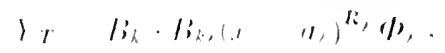

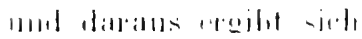

$$
\therefore \quad l i k, i_{i}, \therefore+l i, l i
$$

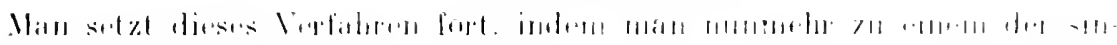

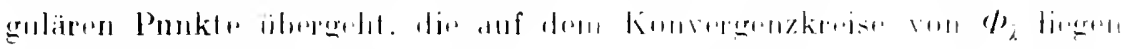

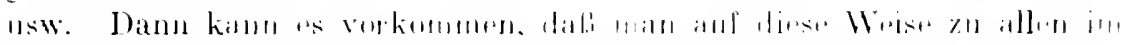

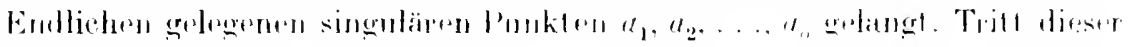

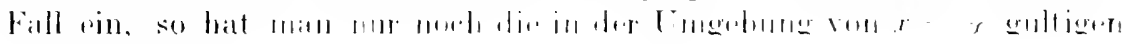
Enlwioklungers

$$
1 \quad l \quad l n+1
$$

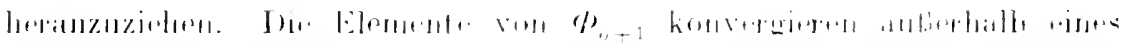

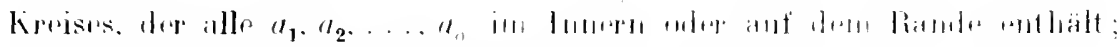

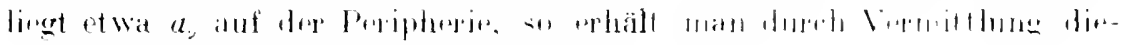

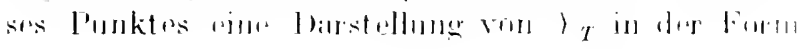

$$
l_{1} \quad l_{n+1} i_{n+1} R_{n+1}
$$

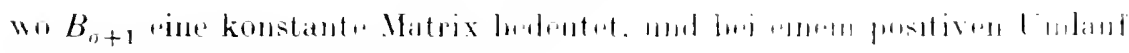

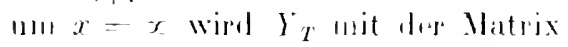

$$
2_{n+1}-B_{n-1}, n^{2}+R_{n+1} B_{n+1}^{-1}
$$

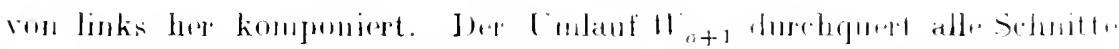
l.....l, und zwar in nugativen Sinne; denken wir mos diese Quer-

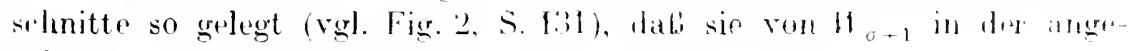
grthenen Reihenfolge grotroffen wertum. dann ist also

$$
\Omega_{0+1}=\Omega_{1}^{1} 2_{2}^{1} \ldots 2_{0}^{1} \text {. }
$$

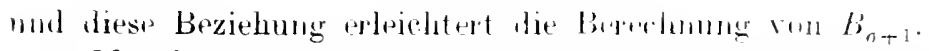

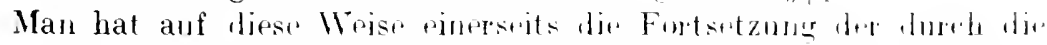

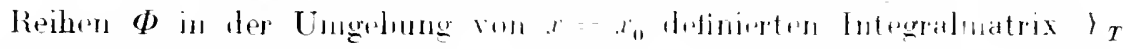

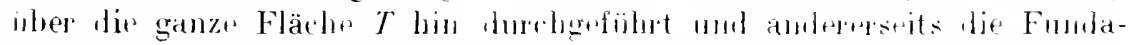
mentalsubstitutionen $\varrho_{1}, \ldots . \Omega_{6}$, heregestellt. so dal.' las Introgationsproblem als gelöst angesplen welen kinn. 


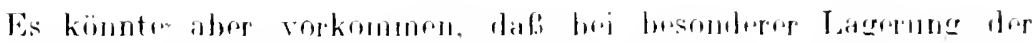

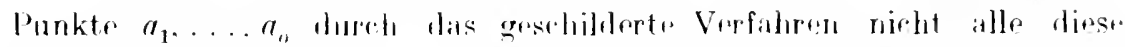
Punkte rerricht werden. Joann mul. der Zusammonhang zwisehen den

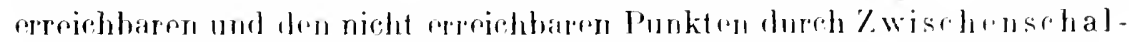

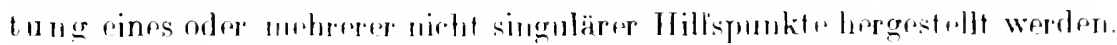

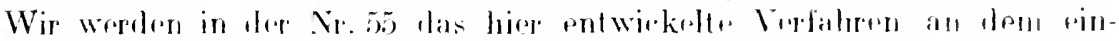

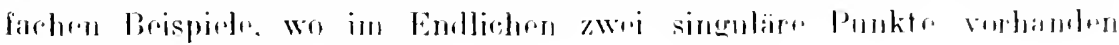
sind, wirkliels derrhfiihrert.

\section{4r. Monodromiegrupe. Der Artbegriff. Fundamentallemma.}

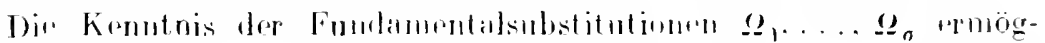

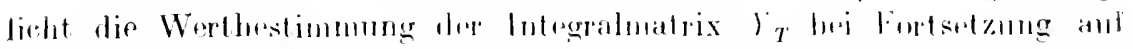

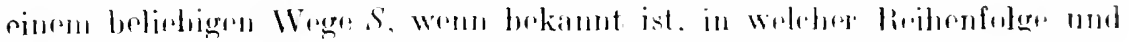

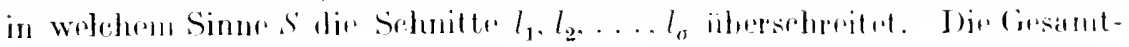

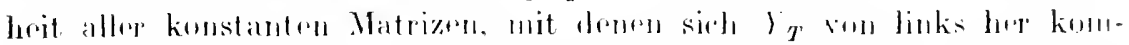

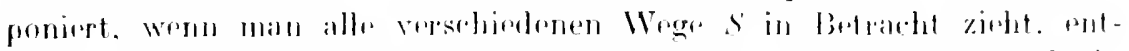

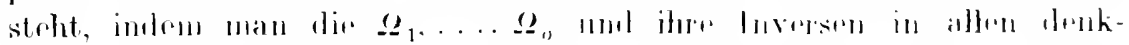

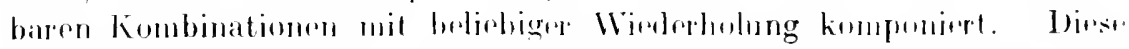

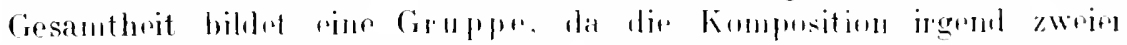

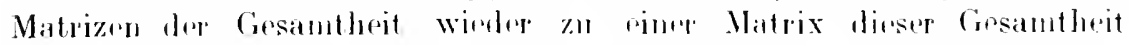

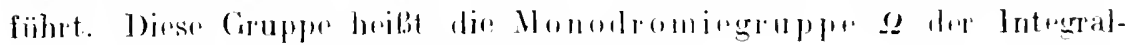
matrix $I_{T}$, man sigt, sic soi aus dru $\varrho_{1} \ldots \ldots \varrho_{0}$ als Fumbanental-

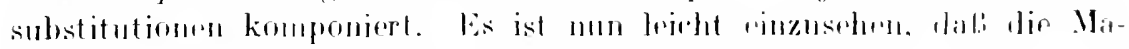

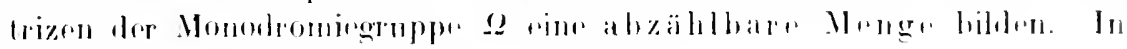
dre Tat ist jule Matrix rou 2 in der Form

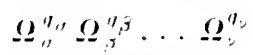

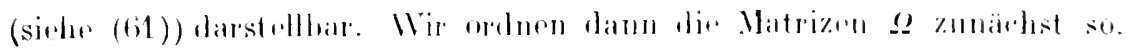

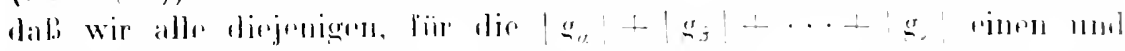
denselben Wert hat, in rine Klasse zosammenfassen. Iu julor Klassu

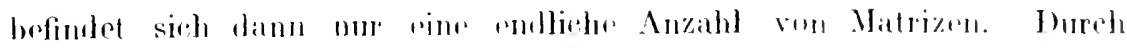

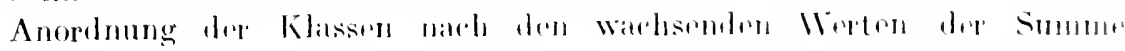

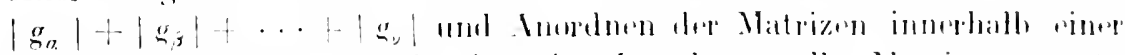

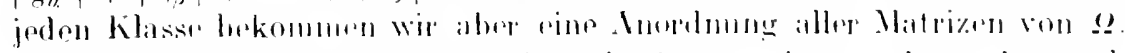

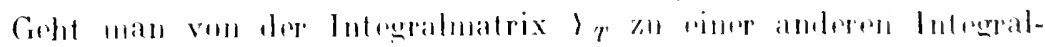

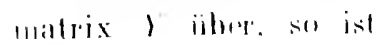

$$
1 \quad \gamma^{\circ} \cdot \gamma_{T}
$$

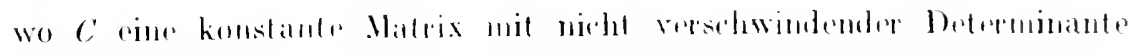

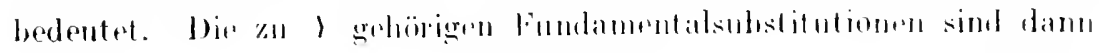




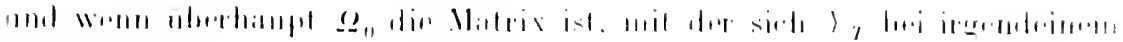

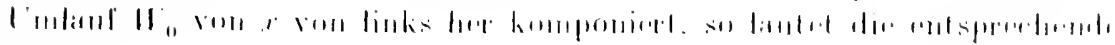
Matrix firr l:

$$
\varrho_{11} \quad\left(\Omega_{11}{ }^{\prime-1}\right.
$$

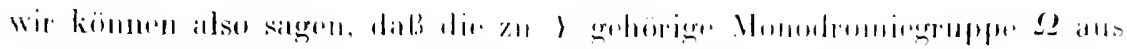

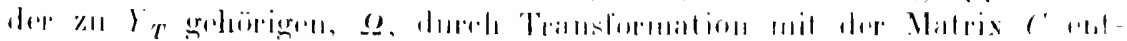

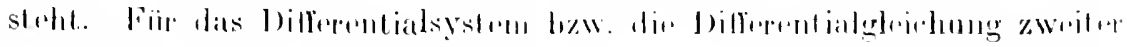

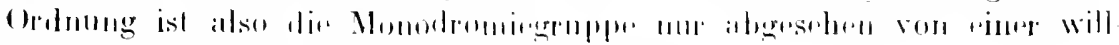

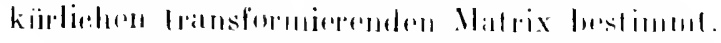

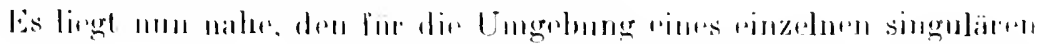

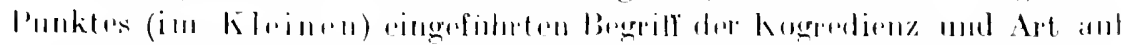

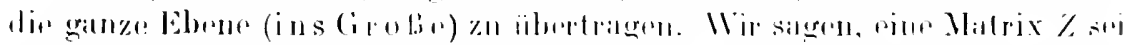

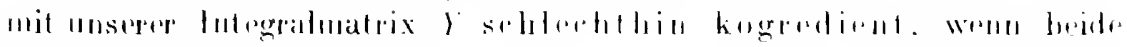

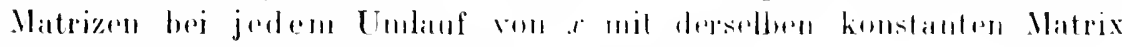
von links lore komponimet werelon. Silnt man

$$
1 \%=1 ; \text {. }
$$

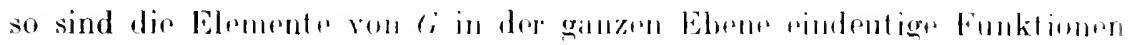

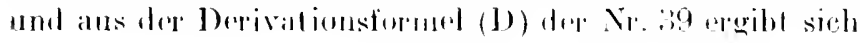

$$
\left.I_{x} \%=(;-1 /)_{r}\right)(;)_{x}(; \text {. }
$$

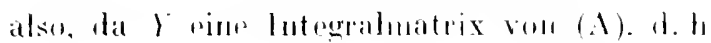

$$
\left.1)_{x}\right\rangle \ldots .1
$$

ist. haben wir

(69) H. li.

wo $B$ dureh die Gleichung

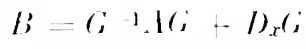

bestimmt ist. Wir sagen auch ron den Jifferentialsystemen (A) mor

$$
\begin{aligned}
& d z_{k} \\
& d x
\end{aligned}=z_{1} b_{1 k}+z_{2} b_{2 k}
$$

dab sie kogredient seien. Wemn num inshesomber anch die Elemente der Matrix $Z$ keine Unbestimnthrilsstello habers, so haben and dit eindeutigen Funktionen $g_{i k}$ dirse Eignsehaft mol sind folgheh rationale Funktionen von .x. Das Diffinutialsystem (li). dem die $z_{i k}$ genügen, gehört dann amch zum Fuchsschen Typus, wir sagen, ps si mit (A) schlechthin von derselben $A r t^{2}$ ) und übertragen diese Ausdrucksweise auch auf die Matrizen $Z$ und $r$ selbst. Wir erhalten umgekehel ancl stets eine mit $Y$ zu derselben Art gehörige Matrix, wenn wir $/ r^{\prime}$ bihlen, wo $G$ irgendeine Matrix rationale Funktionen bedentet. Im folgenden wollen wir uns ahep stots die Vordussetzung himzudenken.

1) Dieser Artbegriff (espece) geht auf Poinc aré zuriek. siehe Acta math. i, 1884, S. 209. Oenvres II. S. 41.2. insbesondere s. 411.;. 


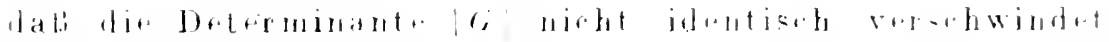

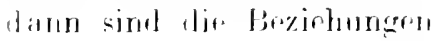

$$
\%=\gamma 1 ; \%, \quad \% ;
$$

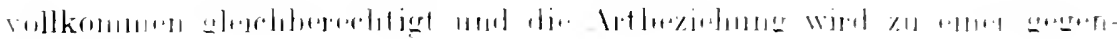
$\therefore$ itients.

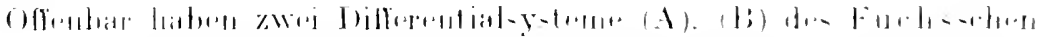

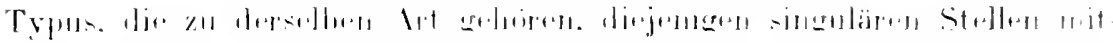

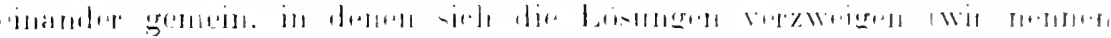

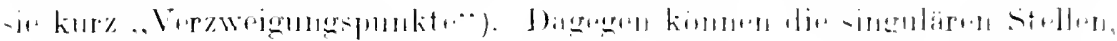

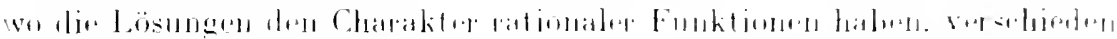

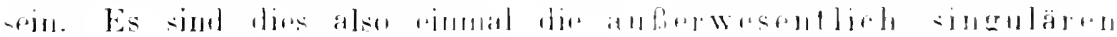

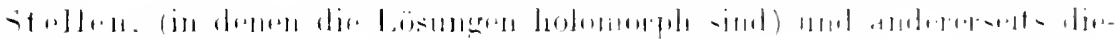

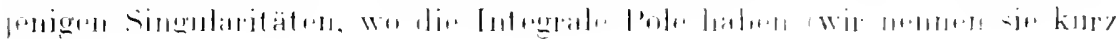
... Folori)

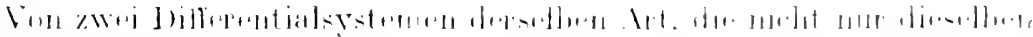

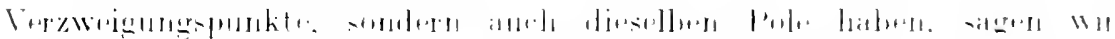

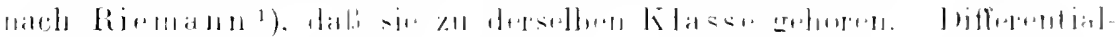

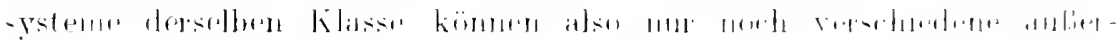

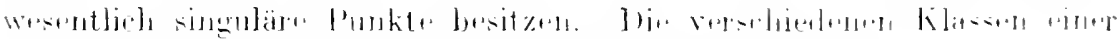

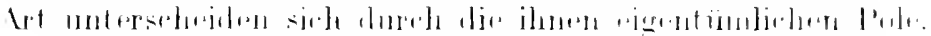

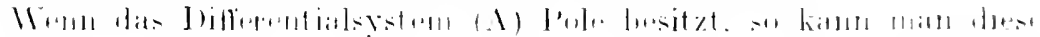

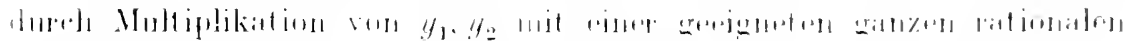

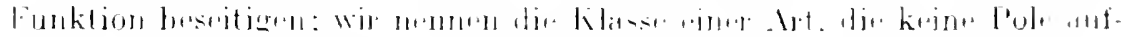

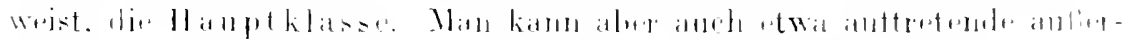

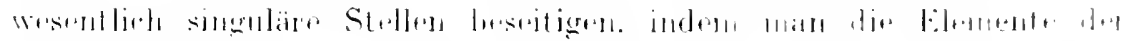

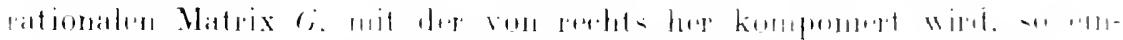

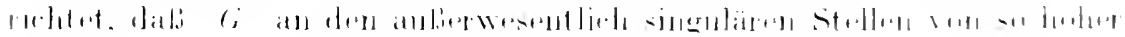

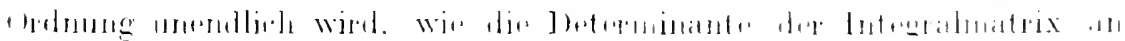

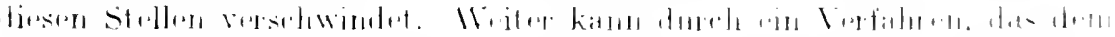

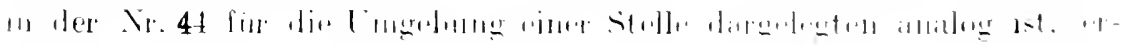

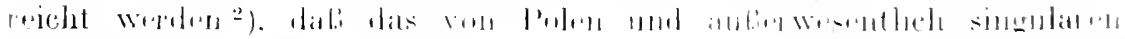

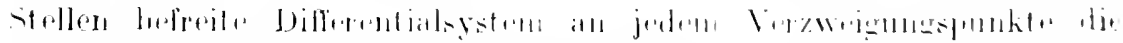

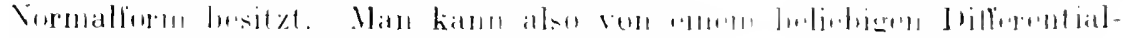

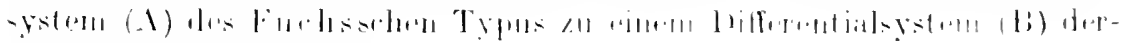

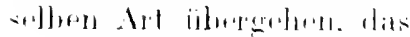

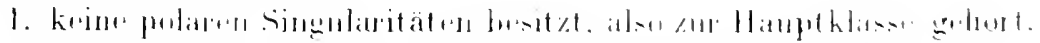

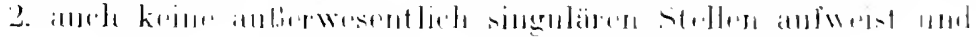

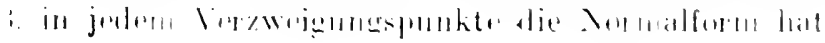

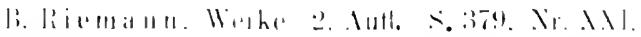

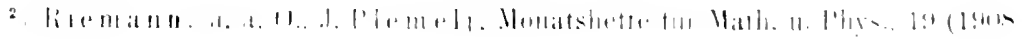
$\therefore .11$. 


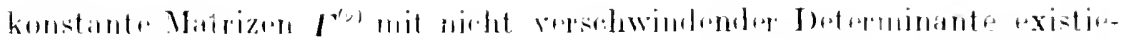
rrn, liï dir

$$
\text { (i) } \Gamma(1) \text { (U) }
$$

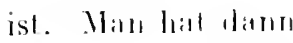

$$
\left.\Gamma \quad B_{2}(x-a,)^{R^{(2)}} \Phi, . \quad \Gamma=B,(r-a,)^{R^{1 / 2}} d\right) .
$$

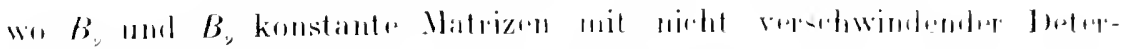

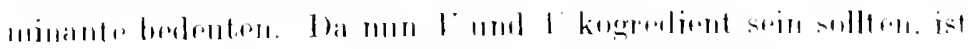

$$
B, p^{2}, i R^{(2)} B, 1 \quad B, p^{2}{ }^{1}, R^{(-)} B, \mathrm{I} \text {. }
$$

also nach (7I)

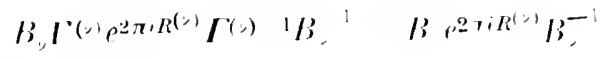

Es regiht sich also

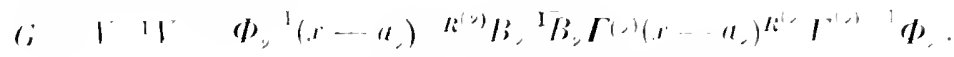

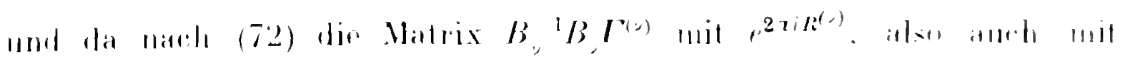
$\left(\right.$. $-(a)^{R^{(-)}}$vertansehbar ist. so haben wir

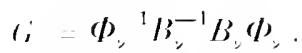

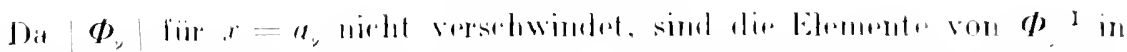

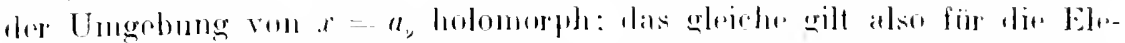

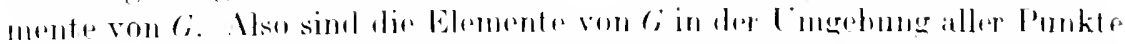

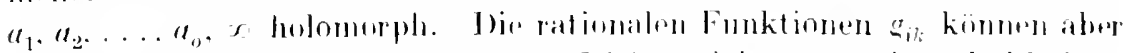

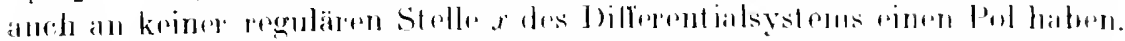

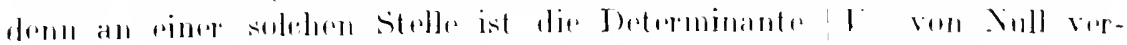

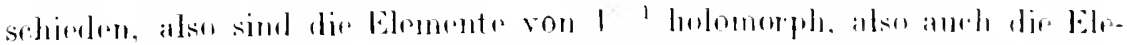

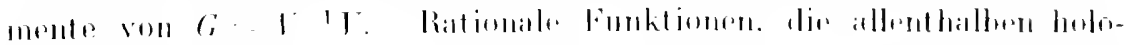

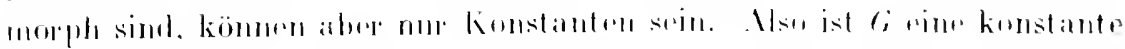

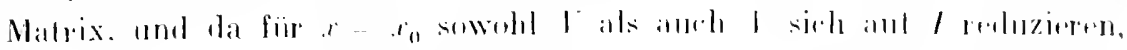

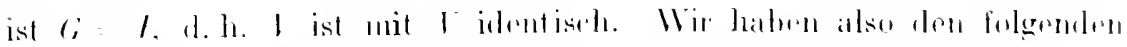

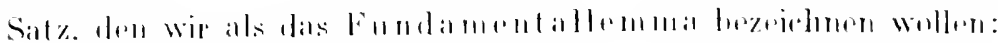

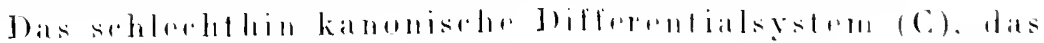

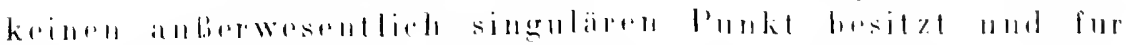

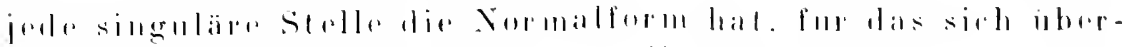

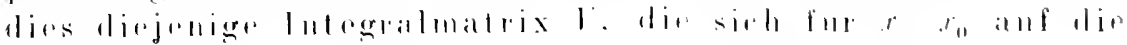

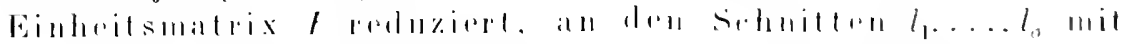

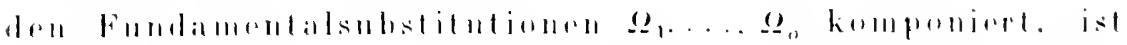

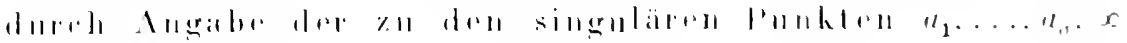

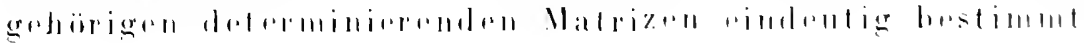




\section{Konstantenzilulungen. Riemamusder Hilferentialsysteme.}

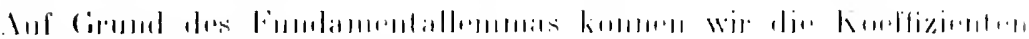

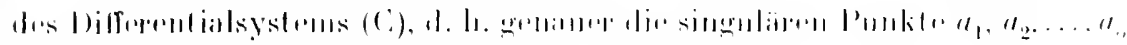

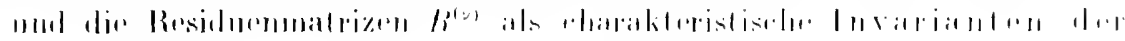

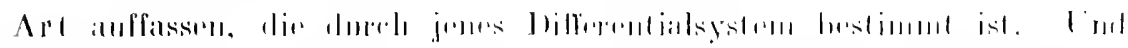

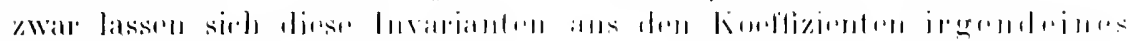

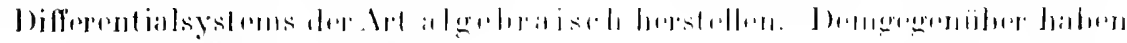

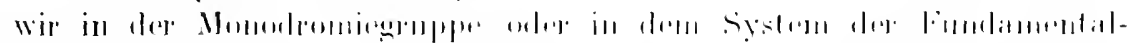

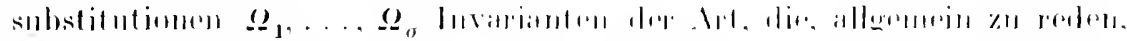

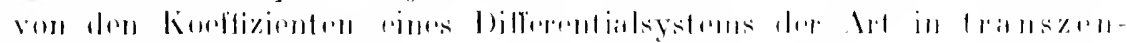

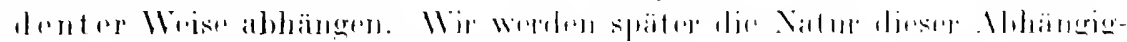

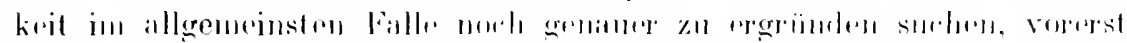

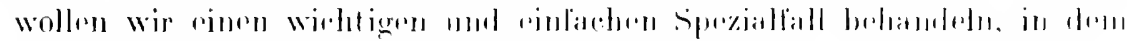

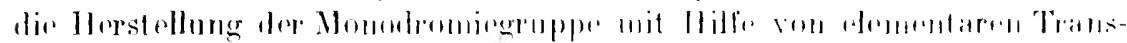
zomelentem gelingt.

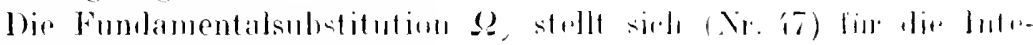
gralmatrix 1 in der Form dar:

$$
\text { I. }=B, 2 \pi R^{(-)} R^{-1} \text {. }
$$

wenn in lew lingolmug vin $x$ a

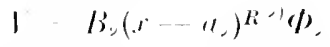$$
(1,-1.2 \ldots, 1)
$$

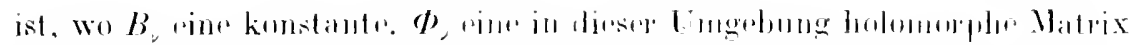

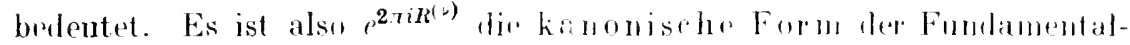

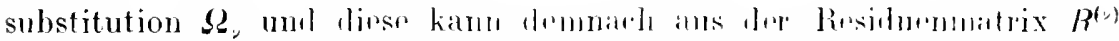

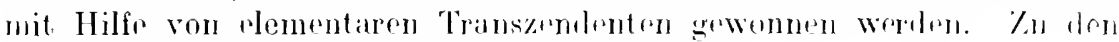
Formeln (7is) tritt now für $x=$,

$$
\Omega_{s+1}=B_{\sigma+1} p^{2} \boldsymbol{z} \cdot R^{(1)+1)} B_{j+1}^{-1},
$$

wo zwischen den $\Omega_{1} \ldots . . \Omega_{\sigma} . \Omega_{o+1}$ dir Buziehmug (6i ) (. Ni. ti) unu zwischen den $R^{(1)} \ldots . K^{(0)}, R^{(0+1)}$ div Beziehung

$$
-R^{(n+1)}=R^{(1)}+\cdots+R^{(0)}
$$

bestcht. Nun enthalten die $\Omega_{1} \ldots . \Omega_{\sigma}, \Omega_{\sigma+1}$ im ganzen $i(\sigma+1)$

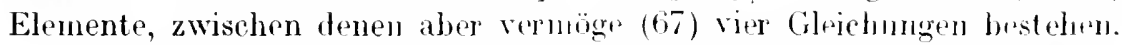

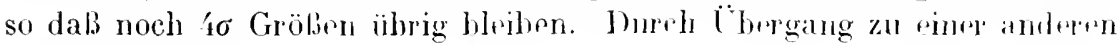
Integralmatrix

$$
\mathrm{r}=\mathrm{C}
$$

verwandelt sieh (siehe Nr. 48) jedes $\Omega$, in $\left(\Omega, C^{-1}\right.$; man kamm lie konstante Matrix $C$ nun dazu benutzen, um über lroi von jenen 40 Grölien nach Belieben zu verfïgen. Es bleiben also to-3 wesentliche Konstanten in den $\Omega_{1}, \ldots, \Omega_{0}$. Die kanonische Form einer Matrix I, nnt- 


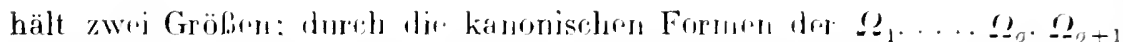
kennen wir afse $2(\sigma+1)$ Gröben. zwischen denen aber vermögr (67) orler (74) noch rime Pration bestrht. so dal. $2 \pi-1$ brkinnt" Gröfen

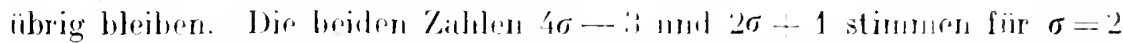

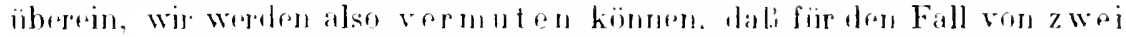
inn Endlichen gelegenen singulären Punklon $a_{1}$. $a_{2}$ dir. Brestimnnug der Fundanentalsubstitutionen dureh dir kanenisehen Formen von $\Omega_{1}$. $\Omega_{2}$.

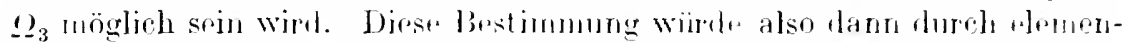

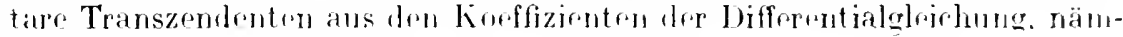

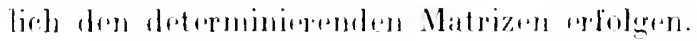

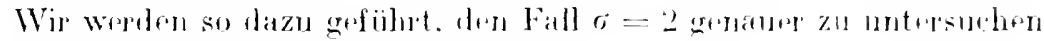
und betralleten also das J)ifferentialspestem
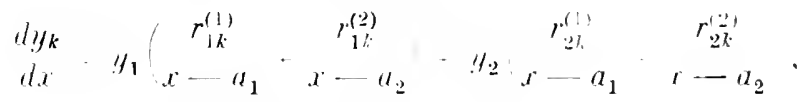

$i=1.2)$

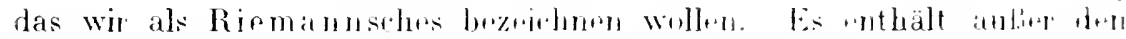
$a_{1}$. $a_{2}$. die wir als fest ansthen wollen, merh die acht konstanten l'ara-

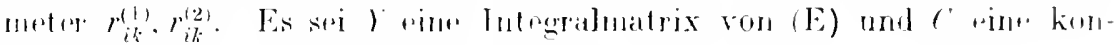
stante Matrix mit nichtrerselowindender Duterminante. Sotzen wit

$$
Z=Y C^{\prime} \text {. }
$$

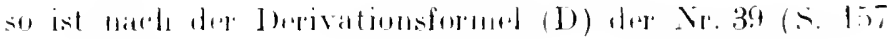

$$
\left.D_{x} \%=C^{\prime} \cdot D_{x}\right\rangle \cdot(\cdot)
$$

atso befriediegt $\%$ wenu

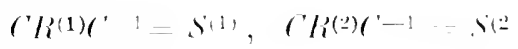

gersetzt wird. das Jifferentialsysteme

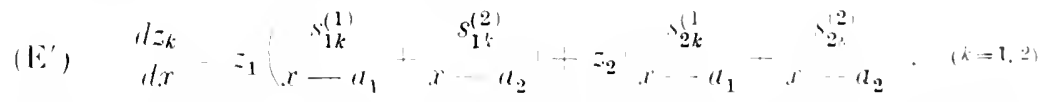

Ans (76) folgt. dali die determinierenden Vatrizen bei den singuären

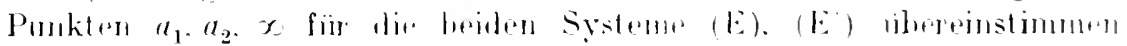

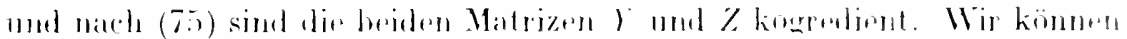

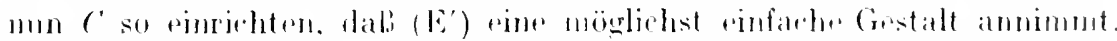

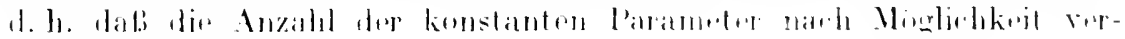
ringert wird. lis ist

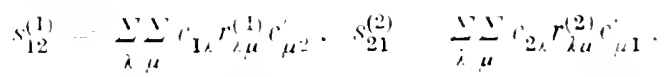

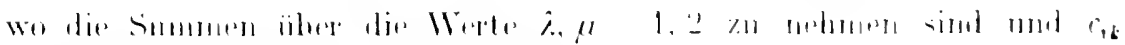
die Elementer von r-1 leedentent. also

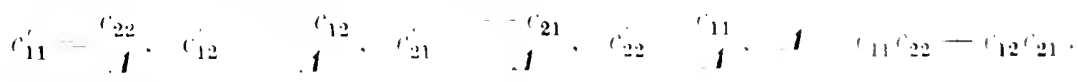

Wir bestimneren mun die rik so. dal.i

$$
(1)-0 . \quad s_{2}^{(2)}=0
$$




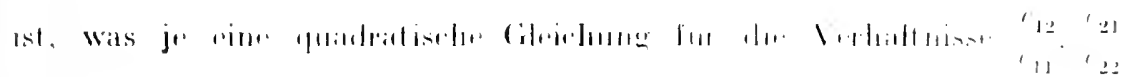
liifiut. llit

$$
\begin{array}{llll}
11 & 12 & 11 & 11 \\
121 & 1 & 11 & i_{22}
\end{array}\left(\begin{array}{cc}
1 & 12 \\
& 11 \\
i_{21} & 1 \\
i_{22} & 1
\end{array}\right)
$$

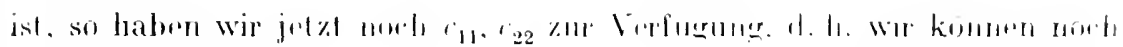

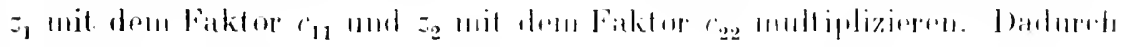

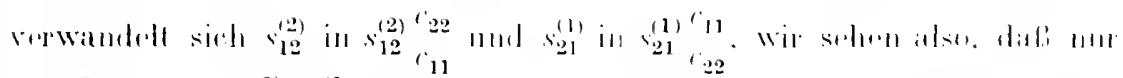

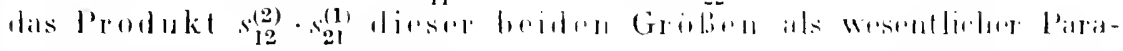

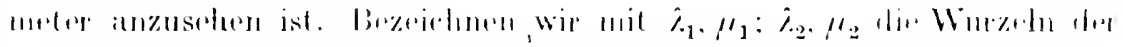

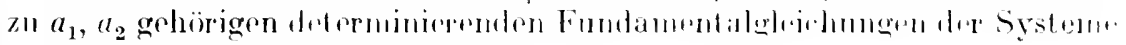
(E) und (li'), so ist wenom (77)

$$
s_{11}^{(1)}=\hat{\gamma}_{1}, \quad s_{22}^{(1)}=\mu_{1}, \quad s_{11}^{(2)}=\hat{\varkappa}_{2}, \quad s_{22}^{(2)}=\mu_{2} .
$$

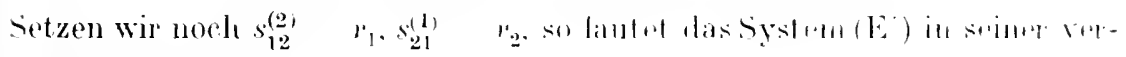
(infachten Form:

$\left.(1)^{\prime}\right)$

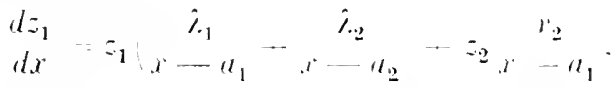

$$
\begin{aligned}
& d z_{2}=\Sigma_{1} v_{1}-u_{2} z_{2} \mu_{1} u_{1} \mu_{2} u_{2} .
\end{aligned}
$$

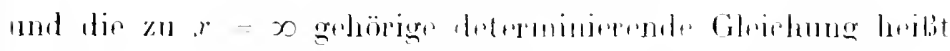

$$
\left(\lambda_{1}+\lambda_{2}+r\right)\left(\mu_{1}+\mu_{2}-r\right)-r_{1} r_{2}=0 \text {. }
$$

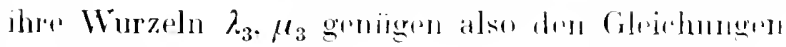

$$
\begin{aligned}
& \lambda_{1}+\lambda_{2}+\mu_{1}+\mu_{2}-\lambda_{3}-\mu_{3}=0 . \\
& \left(\lambda_{1}+\lambda_{2}\right)\left(\mu_{1}-\mu_{2}\right)-l_{1} \mu_{2}=\lambda_{3} \mu_{3} .
\end{aligned}
$$

deren erste dir Fuchssehe Retation ist. Durch die aweite Gleiehung bestimmt sich $\jmath_{1} \cdot r_{2}$; da, wie oben bemerkt wurde, nur dirses Produkt als wesentliche Konstante zu grelten hat. su enthält (E') nur noch fïnt Parameter, und wir können sagen. daß die Koeffizienten des Differential-

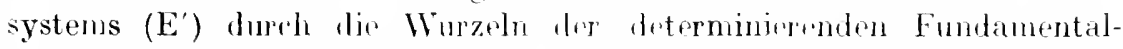
gleichungen $\lambda_{1}, \mu_{1} ; \lambda_{2}, \mu_{2} ; \lambda_{3}, \mu_{3}$. zwishlen denen noeh dir Fuehssch. Relation (78) besteht, vollkommen festgelegt sind. Jedes Riemannsehe Differentialsystem (E), dessen determinierende Fundamentalgleichungen die Wurzehn $\lambda_{1}, \mu_{1}: \lambda_{2} \cdot \mu_{2}: \hat{\lambda}_{3}, \mu_{3}$ haben. whe dureh wint Transformation ron der For ${ }^{\prime}=Z C^{\prime}$. also

$$
\begin{aligned}
& y_{1}=z_{1} c_{11}-z_{2} c_{21} \\
& y_{2}=z_{1} c_{12}-z_{2} c_{22}
\end{aligned}
$$

aus (E') hervor. Ein Riemannsches system ist also, abgu- 


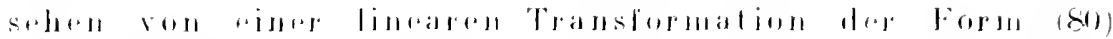

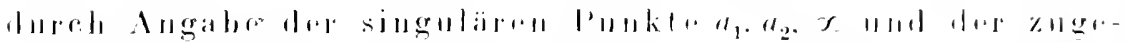

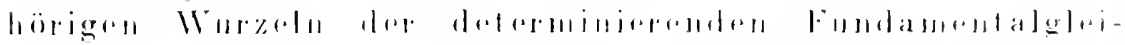

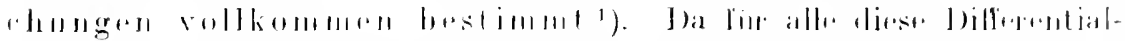

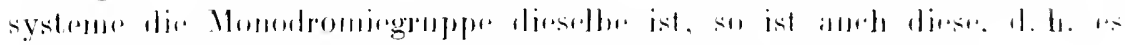

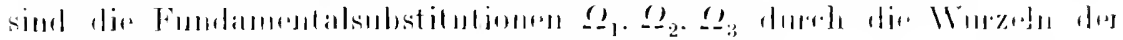

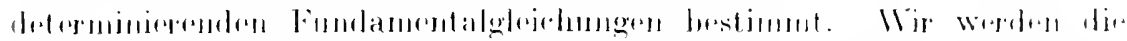

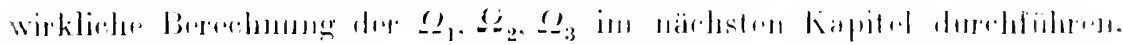

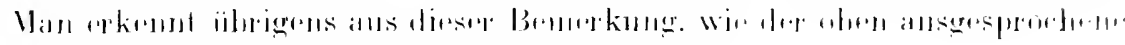

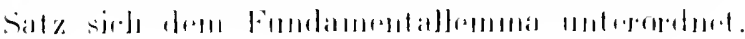

\section{io. Vereinfachung des Riemannschen Differentialsystems. Gauksche Differentialgrleichung.}

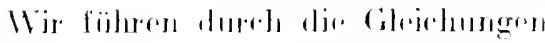

$$
\begin{aligned}
& z_{1} \quad H_{1}\left(x-a_{1}\right) u_{2}\left(.1-u_{2}\right)^{i_{2}} \\
& \therefore \quad l_{2}\left(l \quad a_{1}\right)^{m+1}\left(i \quad a_{2}\right)^{\prime 2}
\end{aligned}
$$

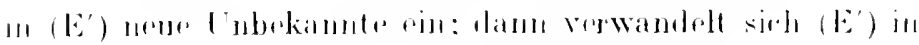

$\left(E^{\prime \prime}\right)$

$$
\begin{aligned}
& \int\left(r-u_{1}\right) \frac{d u_{1}}{d d^{2}}=-0_{1} u_{1} \cdot r_{2} u_{2} . \\
& \mid\left(x-u_{22}\right)_{a}^{d u_{2}} \quad v_{1} u_{1}+u_{2} u_{2} .
\end{aligned}
$$

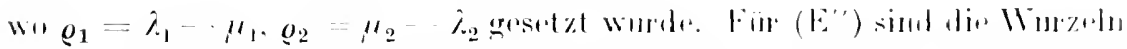

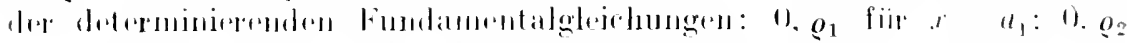

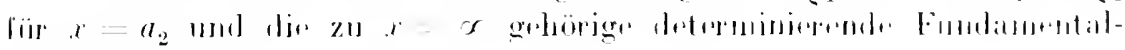
glojehumg limblot

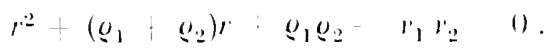

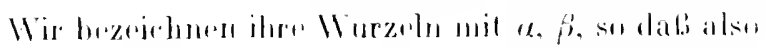

$$
\begin{aligned}
& l Q_{1}+\theta_{2}+u \cdots p l|l| \\
& l_{1} \theta_{2} \quad b_{1} r_{2} \quad a_{j} ;
\end{aligned}
$$

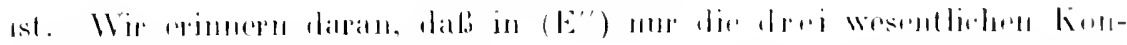

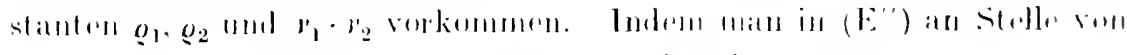

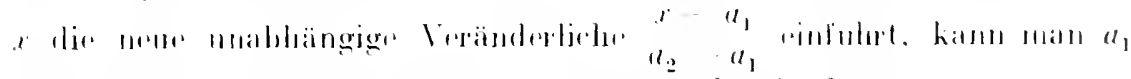

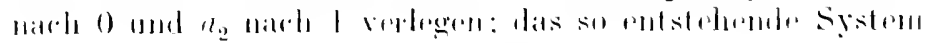

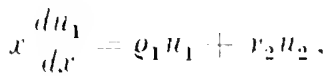

$$
\begin{aligned}
& \left(\begin{array}{lll}
1 & 1
\end{array}\right) \frac{d u_{2}}{d x^{2}} \quad n_{1} u_{1} \quad g_{2} u_{2}
\end{aligned}
$$

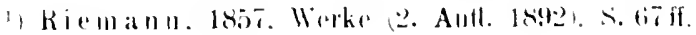




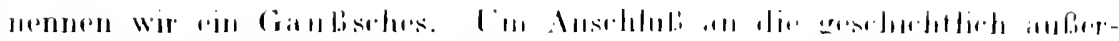

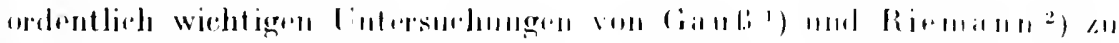

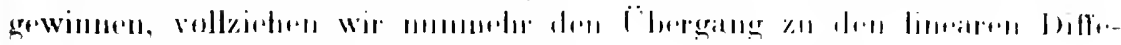

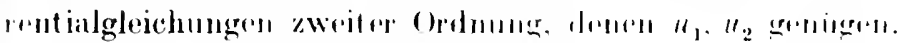

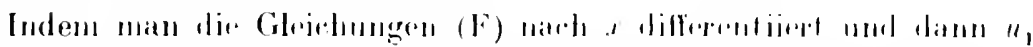

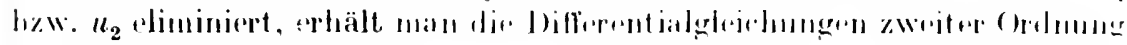
(8i3) $r(x-1) u_{1}^{\prime \prime} \quad\left|\left(\varrho_{1} u_{2} \quad 1\right) r \quad \varrho_{1}+1\right| u_{1}^{\prime}+\left(r_{1} l_{2}-\varrho_{1} \varphi_{2}\right) u_{1}$.

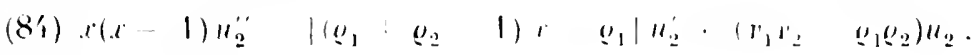

dir heide von der frorm

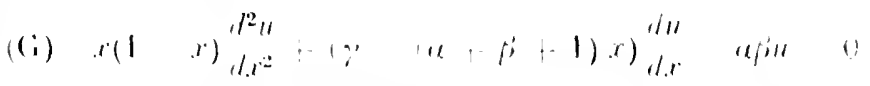

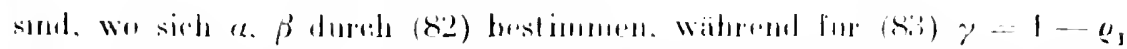

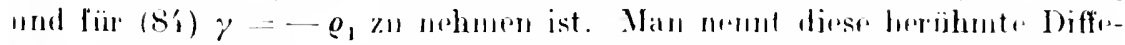

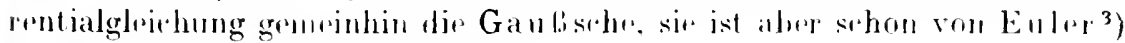
anfgestellt und untersuchl werriterl.

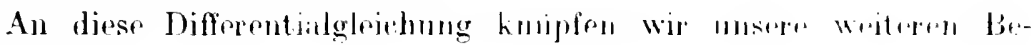
trachtungen.

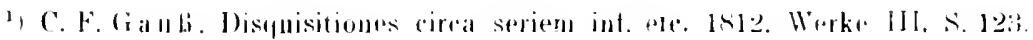

$\Rightarrow$ a. a. (I.

3) L. Euler. Spreimen transformationis singularis seriermu Mi. Tlu des Eneströmschen Index). Nova Arta l'etropolitand Xll 1801), datiert vom :3. 9. 17in: rogl. auch Inst. calc. integralis II. Sect. 1, cap. YlJl. prothl. 123. Opera. Series I.

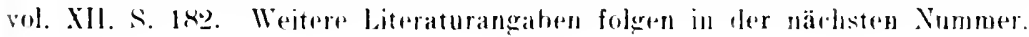




\section{Siebentes Kapitel. \\ Die Gaubche Differentialgleichung.}

\section{Aufstellung des kanonischen Fundamentalsystems fiir den Nullpunkt.}

Wir stellen uns die Anfgabe. die Gaubsehe Differentialgleichung (G) im Sinne der Nr. 47 zu integrieren. mnd beginnen danit, daß wir zunächst das zu $x=0$ gehörige kanonische Fundanentalsystem aufst ellen.

Un die Differentialgleichung auf die Xornalform zu bringen. hätten wir dip linke Seite nuit $x$ zu multiplizieren und durch $1-x$ zu dividieren: das letztere wollen wir aber. ähnlich wie wir es schon in den Beispiel 2. der Nr. 43 getan haben, unterlassen, da sich dadureh dir Rechnung homplizieren wiirde; wir setzen also

(1) $D(u)=x^{2}(1-x) \frac{d^{2} u}{d x^{2}}+x[\gamma-(\alpha+\beta-1) x] d x-u \beta x u=0$. dann lautet die charakteristische Funktion

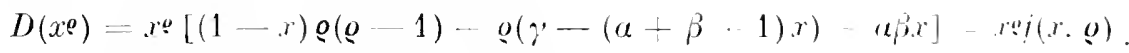

Ordnen wir $f(x, g)$ nach Potenzen von $r$.

so ist

$$
\begin{gathered}
f(x, \varrho)=\sum_{i=11}^{\infty} f_{\nu}(\varrho) x^{\prime} \\
=\varrho(\varrho-1)-\varrho \gamma-x\left[\quad \varrho(\varrho-1)-\varrho(u-\beta-1)-u \rho^{\prime}\right] .
\end{gathered}
$$

$$
\begin{aligned}
& f_{0}(\varrho) \quad \varrho(g-1)+\gamma \varrho . \\
& f_{1}(\varrho)=-\varrho(\varrho-1)-\varrho(\alpha+\beta-1)-\alpha \beta . \\
& f_{\nu}(\varrho)-0 .
\end{aligned}
$$$$
(,=2 ;, \ldots
$$

Die determinierende Fundimentalgleichung lautet

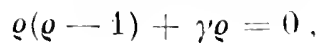

ihre beiden Wurzhn sind also

$$
0,1-\gamma
$$

Es sei $r$ eine dieser Wurzeln, dann lautet die Rekursionsformen für die Koeffizienten der der I)ifferentialgleichung genügrenden Reihe 


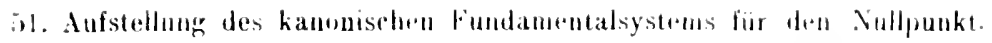

$$
\left.\sum_{0=11}^{r} x^{r+}, \quad r_{0} \mid 1\right)
$$

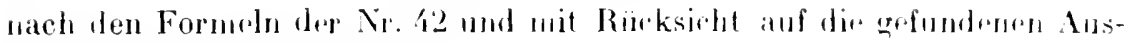
lroucke für die $f_{2}(g)$ rinfach

$$
c_{y-1} l_{1}(r+1-1)+c f_{0}(r+1) \quad 0 .
$$

$(,-: 2, \quad)$

Ilieraus folgt

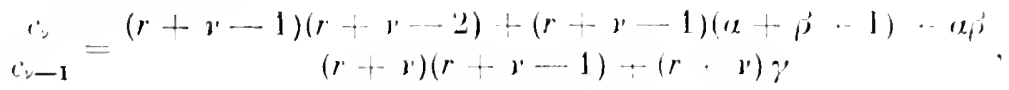

$$
\begin{aligned}
& =\frac{(r+r-1+a)(r \quad b-1+\beta)}{(r-v)(r+v-1+\gamma)} .
\end{aligned}
$$

also ergibt sich, da

$$
c_{y}=c_{,} c_{,} \quad c_{\nu-1} c_{\nu-2} \ldots c_{1}
$$

(2) $c_{\nu}=\frac{(r+\alpha+v-1)(r+\alpha-\gamma \quad 2) \cdots(r+\alpha)(r+\beta+1 \quad 1)(r+j-\gamma-2) \cdots(r-\beta)}{(r+v)(r+v-1) \cdots(r+1)(r+\gamma+v-1)(r+\gamma+\gamma \quad 2) \cdots(r-\gamma)} c_{\eta} \cdot$ wo $c_{0}$ eine noch willhïliche. aher von Null verschiedene Konstante hwdentet.

Wir setzen vorläufig voraus, dab dir loilferenz der Wurzein dex determinierenden Fundamentalgleichung, also $1-\gamma$, weder Null noch eine ganze Zahl sei.

Nehmen wir dann zuvörderst $r=0$ und wählen $c_{0}=1$. so ist

(2a) $\quad c_{\nu}=\frac{\alpha(\alpha+1) \cdots(\alpha+1-1) \beta(\beta-1) \cdots(\beta+r-1)}{\gamma(\gamma-1) \cdots(\gamma+1-1) \cdot 1 \cdot 2 \cdots v}$,

das zum Exponenten 0 gehörig' Integral lautet also in der Ungebung von $x=0$ :

$$
u_{01}=1+\begin{gathered}
a \cdot \beta \\
1 \cdot \gamma^{2}
\end{gathered} x+\begin{gathered}
\alpha(\alpha+1) \beta(\beta+1) \\
1 \cdot 2 \cdot \gamma(\gamma+1)
\end{gathered} x^{2} \cdots \cdots
$$

Man bezeichnet nach Gaub diese Reihe durch

$$
F(\alpha, \beta, \gamma, x)
$$

und nennt sie die GauBsche oder hypergeometrische Reile. Sie findet sich schon bei Euler ${ }^{1}$ ) und Joh. Friedr. Pfaff ${ }^{2}$ ) u. z. als Lösung der Differentialgleichung (G): Gaul hat sie in einer 1812 veröffentlichten Abhandlung ${ }^{3}$ ) losgelöst von der Differentialgleichung untersucht. namentlich ihre Konvergenz geprïft und auch die Art ihrer Abhängigkeit von den $\alpha, \beta, \gamma$, die er das erste, zweite, dritte Element der Reihe nennt ${ }^{4}$ ), in den Kreis seiner Betrachtungen gezogen. Nach Gaulj hat sich

i) a. a. 0 .

2) J. Fr. Pfaff. Disquisitiones analyticae. Helmstadii 1797.

$\left.{ }^{3}\right)$ C. F. GauB, Werke Bd. III (1866). S. $123 \mathrm{ff}$.

4) $x$ wird als das vierte Element bezeichnet. 
K ummer ${ }^{3}$ ) mit rer gedachten Rrihe besmäftigt. inden ar dir Differentialgleichung (G) zum Ausgangspunkte nahru. Auf die Differentialgleichung ist GauB in eines erst aus srinen Nathlasse hurausgegebrenen Fortsetzung ${ }^{2}$ ) seiner erwähnten Abhandlung ringrgangen. Von neufren Bearbeitungen der Gaubsehen Differentialgleichung sim aulier der bereits grnannten Abhandlung Riemanns und der Fuchsschen Abhandlung im 66. Bandr von Crelles Journal, wo die daselbst rutwickelten Prinzipien der allgemeinen Theorir der linearen Differentialgleichungen anf die Difforentialgleichnng (G) als Beispiel angwwandt werden. zu nennen pine ausfïhrliche Monographie von (roursat ${ }^{3}$ ) unt dir. I)arstelhungen bei Camille Jordan ${ }^{4}$ ), Picard "). Kornigstrerger "). forner sei aluf dir einschlägigen $A$ bschnitte des .. Handbuchs der Theorif der lineden Differentialgleichungen" verwiesen. wo auch dit iibrige Literatur angegeber ist.

Un das zwrite Element les zu $x=0$ gehörigen kanonischen Fundamentalsystems anfzustellen, nehmen wir $r=1-\gamma$ umd wienler $c_{0}=1$ Es ergiht sich dann

$$
\begin{gathered}
(\alpha \quad \gamma+1)(\alpha-\gamma+2) \cdots(\alpha \quad \gamma+\nu)(\beta-\gamma-1)(\beta-\gamma-2) \cdots(\beta-\gamma-1) \\
(2 \gamma)(3-\gamma) \cdots(v+1-\gamma) 1 \cdot 2 \cdots v
\end{gathered}
$$

wir sehen. lab dieser Austruck aus dem vordin fiir $r=0$ gefumdenen dadurch hervorgeht, dabi an Stelle von

$$
\begin{gathered}
\alpha, \beta \\
a-\gamma+1, \beta-\gamma+1,2-\gamma
\end{gathered}
$$

gesetzt werden. Das gesuchte zweile Element hat also in der L'mgebmer von $x=0$ die Form

$$
u_{02}=x^{1-\gamma F} F(\alpha-\gamma+1, \beta-\gamma+1,2-\gamma, x) .
$$

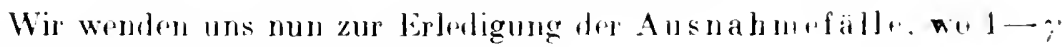
gleich Null culer einer ganzen lathl ist.

\section{Frlediguma der Ausnahmefälle.}

Es søj zunächst $1-\gamma$ eine negatiw ganze Zahl:

$$
1-\gamma \quad-g, g>0 \text {. }
$$

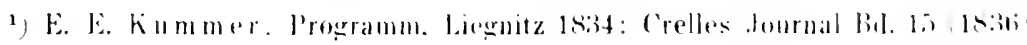
S. $39 \mathrm{lf}$.

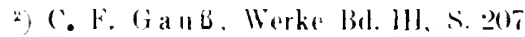

3) E. Coursat. Pariser These (1882). Annales de lkeole Nomale. Seraell. [id. 10. Suppl.

4) C. Jordan. Comrs d Analyse (2). ed.) 11] (1896). S. 2031).

$\therefore$ E. Pirard. Traiti d Analyse IIl (1896). S. 291.

") L. Kornigsterger. Lehrinch rete. s. tio. 


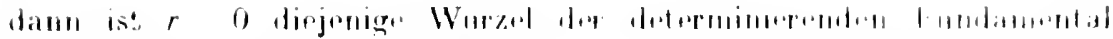

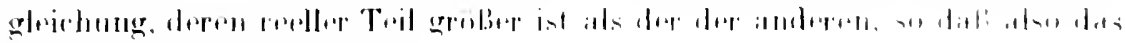
zol $r=0$ gahorigen lutugral

$$
u_{01} \quad l(r e ., i, j, t)
$$

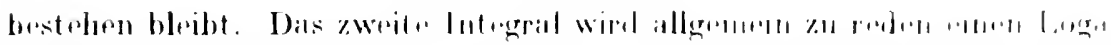

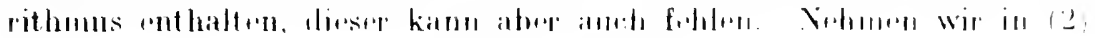

$$
1-\gamma \quad-g \text {. }
$$

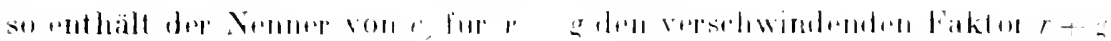

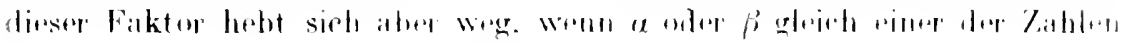

$$
1,2, \ldots, g
$$

s. Dats lutengal $u_{02}$ lwhilt also fiur?

$$
1 \gamma \quad \text { \&. } \alpha, \beta \quad 2, \ldots
$$

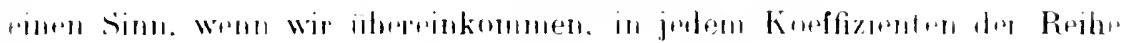

$$
F(\alpha \quad \gamma=1, \beta-\gamma-1,2 \gamma, x)
$$

im Zähler und Nenner diu verseluwindenden Termer wrgzulasson. Damit

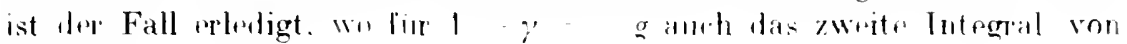
l.ogarithuen frei ist.

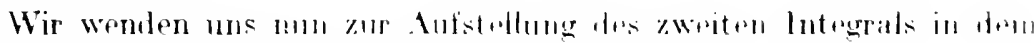
Falle. wo g eine positive ganz. Zahl uder Null ist mol keine der Gröben

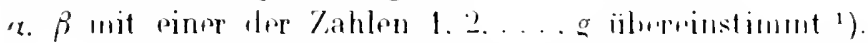

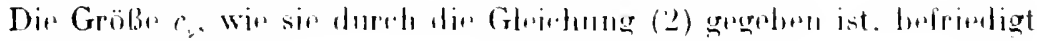
lit Rokursionsformel

$$
(2,1 / 1, r+r-1)-r f_{0}(r \quad v)-0:
$$

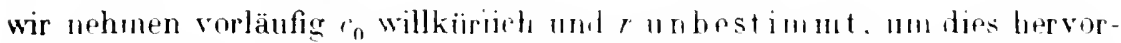
treton zu lassen. bezeichnen wir dun. Auselpuck (2) statt durch r, dureh $r,(r)$

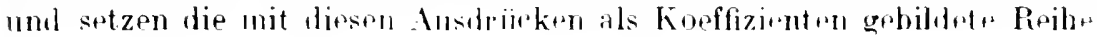

$$
\sum_{\nu=0}^{x} c_{\nu}(r) x^{r+\nu}=u(x, r)
$$

ganz formal in die linkr Spitu dre Dilfrentialglei,humg rin:

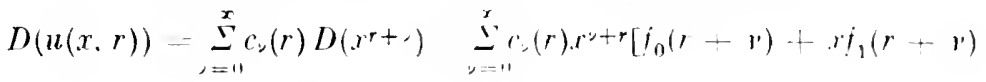

$$
\begin{aligned}
& =c_{0}(r) x^{r} f_{0}(r)+\sum_{\nu=1}^{x} x^{y+r}\left[c_{2}(r) f_{0}(r-r)+c_{,}{ }_{1}(r) f_{1}(r-r \ldots-1)\right] .
\end{aligned}
$$

also ist, da zufolg" der Glrichung (i) die Sumur auf der prohten seite verschwindet:

$$
D(u(x . r))=c_{\mathbf{0}}(r) x^{r} f_{0}(r) .
$$

Wir wenden nun auf diese litentität ein Verfahren an, Jas auf 1) Alembert zurückgeht. der es für die lineare Differentialgleichung mit

1) Vigl. G. Frobenius. Crelles Journal Bd. ib (1873). S. 2eltff.: I. Heffter. Einleitung etc.. S. 227ff.: E. Goursat. a. a. O. 
konstanten Korffizienten angeguben hat ${ }^{1}$ ), indem wir gleich benterken. dab die in folgenden rein formal auszuführenden Differentiations- und substitutionsprozesse zil Ausdrücken fïhren werden, deren Konvergenz dis: unseren allgemeinen Sätzen a posturiori folgt.

Wir wählen das noch willkürlirh" $c_{0}(r)$ in frolgender Weis":

$$
\begin{aligned}
& (r-1)(r-2) \cdots(r-2 g) \\
& r_{0}(r)=(r+a) \cdots(r+a+g-1)(r+\beta) \cdots(r-\beta-g-1)
\end{aligned}
$$

und setzen

$c_{v}(r)=\begin{gathered}(r+a) \cdots(r+a+r-1)(r-\beta) \cdots(r-\beta-r-1) \\ (r+1) \cdots(r+1)(r+g+1) \cdots(r-g-r)\end{gathered} .(1=: 2 ;$ $c_{0}(r)=1$.

dann ist offenbar

und folglich

$$
c_{g+,}(r)=c_{g+,}(r) c_{0}(r)=c_{*}(r+g)
$$$$
1,=1: 2 \ldots y
$$

Ninn ist

$$
\begin{aligned}
& u(x . r)=c_{0}(r) x^{r} \sum_{\nu=0}^{?} c_{\nu}(r) x^{\nu}-\sum_{\nu=g+1}^{x} c_{\nu}(r) x^{r+} \\
& =r_{0}(r) x^{r} \sum_{i=0}^{g} c_{c}(r) x^{r}-\sum_{i=1}^{x} c_{\nu}(r+g) x^{r}+v+g .
\end{aligned}
$$

$$
f_{1}(r)=r(r-1)+\gamma r=r(r-g) \text {. }
$$

die rechte Seite dor Identität (4) enthält dennach, wenn wir für $c_{0}(r)$ den angegebenen Ausdruck einsetzen, den Faktor $(r+g)^{2}$ : ps rerschwindrt folglich für $r=-g$ nicht nur diese rechte Seite selbst. sondern aulh ihre partielle Ableitung nach $r$. A. h. es ist rein formal

$$
D\left[\begin{array}{c}
\partial u(x, r) \\
\partial r
\end{array}\right]_{r=-g}=0 .
$$

Nun ergibt sich durch gliedweise Differentiation nawh ,

$$
\begin{aligned}
& \begin{array}{c}
\partial u(x, r) \\
\partial r
\end{array}=u(x, r) \log x-x^{r} c_{0}(r) \stackrel{D}{p}=0_{c_{i}}(r) x^{\prime} \\
& +x^{r} c_{\nu}^{\prime}(r) \underset{\nu=0}{\stackrel{g}{S}} c_{\nu}(r) x^{\nu}+x^{r} \underbrace{s}_{\nu=1} c_{\nu}^{\prime}(r-g) x^{\nu+\theta} .
\end{aligned}
$$

wo die Akzente Ableitungen nach $r$ bedeuten. Für $r=-g$, wofur zufolge der getroffenen Dispositionen alles endlich bleibt, ist aber

setzen wir fermet

$$
u(x,-g)=\underbrace{x}_{=11} c_{\nu}(-g) x^{-g+}=\sum_{==1}^{\infty} c_{\nu}(0) x^{\nu}=F(u, p, \gamma, \alpha) ;
$$

$$
\begin{aligned}
& c_{0}^{\prime}(-g) x^{1} ;+c_{1}(-g) x^{2-;} \quad \cdots-c_{2} \quad 2(-g) x^{-1} \quad c_{;} \quad 1(-g):
\end{aligned}
$$

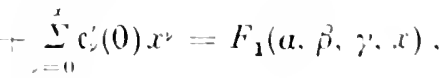

1) J. d Alembert, Mémoires de lacadémie de Berlin. 1its. S. 2833t 
so folgt

$$
\left[\begin{array}{c}
\partial u(x, r) \\
\partial r
\end{array}\right], F_{1}(\alpha, \beta, \gamma, x) \quad F(\alpha, \beta, \gamma, x) \log x,
$$

und dies ist die explizit. Form des zwoiton Integrals $u_{62}$. Dir Konvergenz

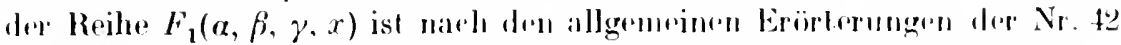

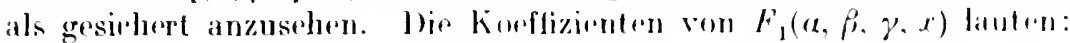

$$
\begin{aligned}
& r_{0}^{\prime}(-g) \lim _{r, q}(r-g)^{-1} c_{0}(r) \\
& =(-1) r_{(\alpha-1) \cdots(\alpha-\gamma+1)(\beta-1) \cdots(\beta \quad \gamma+1)} \cdot{ }^{(\gamma-1) !(\gamma-2) !} .
\end{aligned}
$$

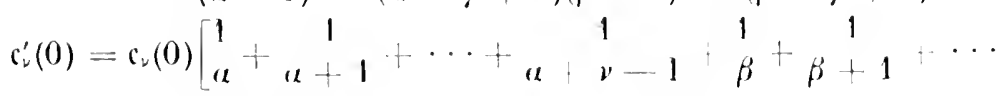

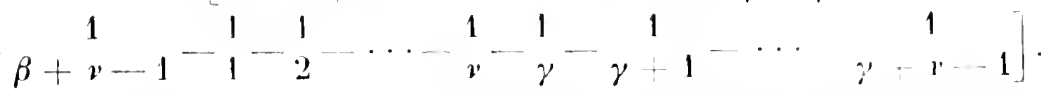

Für $g=0$ ist $F_{1}(\alpha, \beta, \gamma, x)$ eine nach positiven gatuzen Potenzen von $x$ fortsehreitende Reihe.

Es bleibt nunmelır noch der Fall. wo $1-\gamma$ eime positive ganze Zahl:

$$
1-\gamma=h
$$

ist. Dieser Fall lälist sich auf Iron ohoun behandolten zur'ickführen, indem man in dip Differentialgleichung

$$
u=x^{1-r}
$$

einsetzt. "genügt dann einer ( raubschen Differentialgleichung, worin an die Stelle von

$$
\alpha+1-\gamma, \beta+1-\gamma, 2 \stackrel{\gamma}{2},
$$

getreten sind. Die Differenz der Wurzeln der zu $x=0$ gehörigen determinierenden Fundamentalgleichung ist jetzt $\gamma-1 \rightarrow h$, also eine negative ganze Zahl. Wenden wir die vorhin erlangten Resultate auf die Differentialgleichung für $v$ an und ïbertragen sie dann auf $u$, so ergibt sich:

Wenn $a+1-\gamma$ oder $\beta+1-\gamma$ mit einer der Zahlen 1. $2, \ldots, h$, also $\alpha$ oder $\beta$ mit einer der 'Lahlen

$$
1-h, 2-h, \ldots, 0
$$

übereinstimmt, so bleiben die beiden Integrale

$$
F(\alpha, \beta, \gamma, x), \quad x^{1-\gamma} F(\alpha+1-\gamma, \beta+1-\gamma \cdot 2-\gamma, x)
$$

bestehen, falls man übereinkonmt, im Zähler und Nenner jedes Kouffizienten von $F(\alpha, \beta, \gamma, x)$ die versehwindenden Terme wegzulassen. In jedem anderen Falle bleibt das Integral

$$
u_{02}=x^{1-\gamma F}(\alpha+1-\gamma, \beta+1-\gamma \cdot 2-\gamma, x)
$$




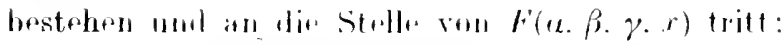

$$
\begin{aligned}
& u_{01}, \gamma^{2} \gamma\left[F_{1}(\alpha+1-\gamma, \beta+1-\gamma \cdot 2 \gamma \gamma)\right. \\
& +F(\alpha+1-\gamma \cdot \beta+1-\gamma \cdot 2-\gamma, c) \log x] .
\end{aligned}
$$

Damit ist fiir $x=0$ das kanonische Fumbancritalsystom in joden Falle aufgestellt: wir fassien dir Resultate in lem folgenden Srhemit 7.11samnen:

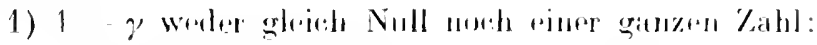

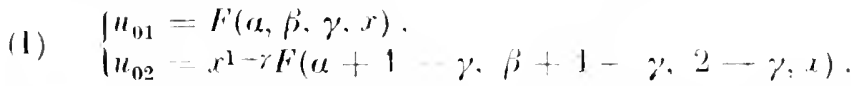

2) $1-\gamma-g . g$ gleirh Null orler riner positiven ganzen Zahl:

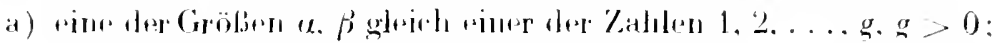
die Reihnen (1) behalten ihren Sinn, wernn man die versehwiu-

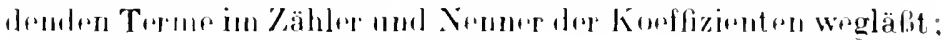

b) $a, \beta>1,2 \ldots, g, g=0$ :

$$
\begin{aligned}
& u_{01}=F(\alpha, \beta, \gamma, x) . \\
& u_{02}=F_{1}(\alpha, \beta, \gamma, x)+F(\alpha, \beta, \gamma, \gamma) \log x
\end{aligned}
$$

i) $1-\gamma=h$, h gloich oiner positiven garnen Zaht:

a) eine Are Gröben u. $\beta$ gleich riner der Zahhlnn $1 \quad h .2-h$ .... 0); dir Reihen (I) behalten ihren Sinn unter der für zas) getroffenen Fistsetzung .

b) $u, \beta+1-h, 2-h \ldots, 0$ :

$$
\begin{aligned}
& n_{01} \quad r^{1}\left[f_{1}(\alpha+1-\gamma, p+1-\gamma 2-\gamma x)\right. \\
& +f(\alpha+1-\gamma \beta \beta+1 \quad \gamma \cdot 2 \quad \gamma, x) \log x] \text {. } \\
& n_{02} \quad x^{1-\gamma f}(u+1-\gamma \beta+1-\gamma 2-\gamma(r) \text {. }
\end{aligned}
$$

\section{Kanonische Fundamentalsysteme fïir a 1 , r $r$}

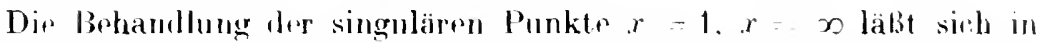
einfacher Wrise anf die berents erledigte des Punktes $r=1$ ) zuriickfiihren

Setzen wir zonäight bei $r=1$

$$
5=1-r
$$

und führen in $(G)$ E als urur unahhängige Variahhe rin. so rerwamlelt

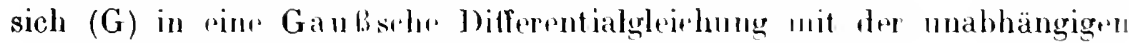
Veränterlichen E. wo an Stelle voul

$$
\text { u. } \beta, \quad \beta, \quad \alpha+\beta \gamma
$$

treten. Wir erhalton folglich firr $\xi 0$ onder $x \quad 1$ das kimonisehe Fun-

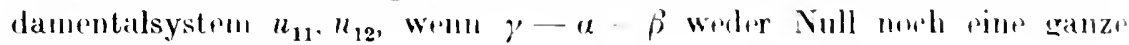
Zahl ist, in der Form: 


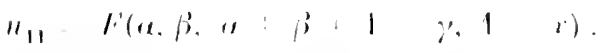

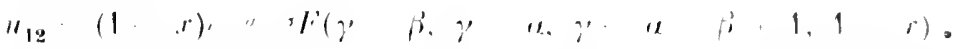

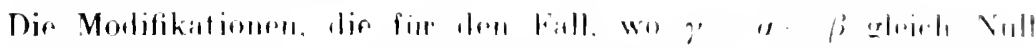

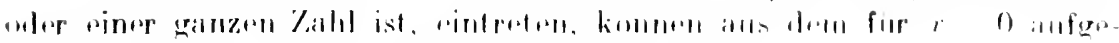

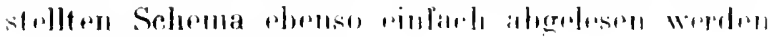

Bei $r=$ sotmon wir

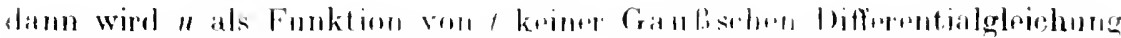

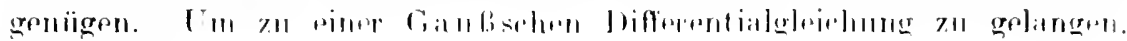
selzen wil

"1 " 11 .

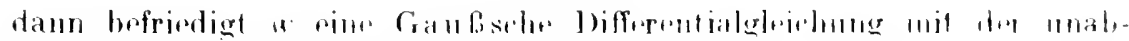
hängigen Verändopliehen /. Worin at Stolle voll

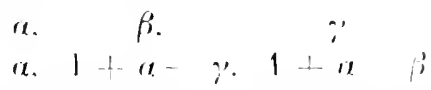

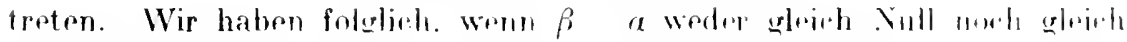
einer ganzen 7ahl ist. fur 1 O las Fumbamentalsystem:

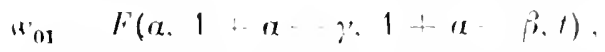

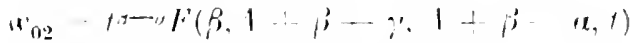

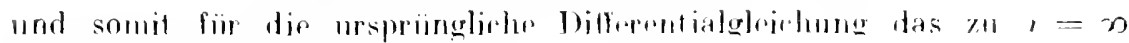
grehörige Fundamentalsystem:

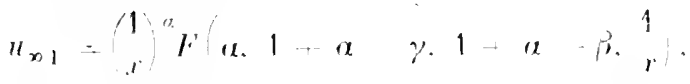

$$
\begin{aligned}
& u_{x_{2}}=\left(\begin{array}{l}
1 \\
x
\end{array}\right)^{\prime} r(\beta .1 . \beta \quad \gamma \cdot 1-\beta \quad \beta \quad 1
\end{aligned}
$$

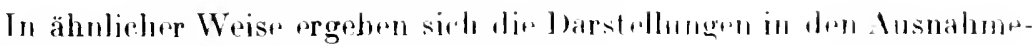
fällen. wo $\beta$ - re eine ganze 7.ahl orler Xull ist.

\section{4. lionvergenzbereiche der anfigestellten Reihenentwicklungen.}

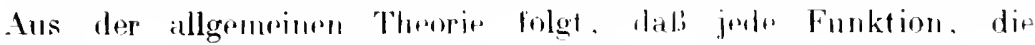

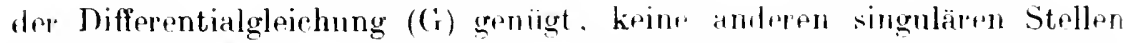
besitzt als $0,1, \infty$. Das Integral $u_{01}$ ist ïberdies in dre Umgramug von $x=0$ holomorph (vorausgesetzl. dalli l. $\gamma$ krinr positive ganze 7ahl ist), die für dieses Integral gefumlene Roihrnentwirklung

$$
F(\alpha, \beta, \gamma, x)_{i}^{\prime}
$$

konvergiert folglich inmerhalb eines um $x=0$ als Mittelpunkt besehrimbenen Kreises, der sich bis zum näehsten singulären Punkte, 1. h. bis zı $x=1$ hin erstreckt. 
Dir Gaufsche Rrihe F( $(\alpha, \beta, \gamma, x)$ ist also fïr Worte von r. deren absoluter Betrag kleiner ist als Eins. konvergent.

Das läbt sich anch dirokt ans der expliziten Form drer Koeffizientm rschließen. Naeh Gloichung (2a) (S. 211) ist nämlich der Ouotiont des

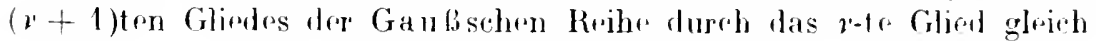

$$
\begin{aligned}
& (u+v)(\beta+v) \\
& (v+1)(\gamma+v)
\end{aligned} x .
$$

Dieser Quotient nähert sich für ins Unendliche warhsendes r hei willkürlichen Werten der $\alpha, \beta, \gamma$ den Grenzwerte $x$; nach dom d'Alembertschen Konvergenzkriterium ist die Reihe daher konvergent. wenn $x<1$. divergent, wemn $|r|>1$.

Die Frage der Konvergenz der Gauls schen Roihr auf dor Peripherie des Konvergenzkreises hat Gaulin der ersten (ron ihm sullst veröffentlichten) Abhandlung über diese Roihe für renlle Wirte Aer $\alpha$. $\beta$. $\gamma$ untersucht. Weierstrab. ${ }^{1}$ ) hat die Gaulisehe Mothode anf len Fall komplexer Werte der $a, \beta, \gamma$ ausgerlehnt. Es argibt sich in Cbrominstimmmng mit dem in der Nr. 47 angefïhrten Satze rom Thome, dal.i

$$
F(\alpha, \beta, \gamma, x)
$$

für Werte von $x$, Aleren absoluter Betrag gleich Eins ist. ansgenommen den Wert $x=1$, konvergent ist, falls der reolle Teil von $;-\alpha-\beta$ gröBer ist als - 1; wenn der reelle Tril von $\gamma-u-\beta$ positiv ist, so komvergier die Reihe auch noch für $x=1$.

Da für unbestimmte $\alpha, \beta, \gamma$ (wo also keiner der ...Ausuahmefäle. (untritt) die för $u_{12}, u_{11}, u_{12}, u_{x_{1}}, u_{\infty 2}$ anfgestellten Reihenentwicklungen sämtlich durch den Algorithnus der Gaubschen Rrihe gegeben werden. folgen nun unmittelbar die Konvergenzbezirke dieser Roihon. Will man auch die Ausnahmefällo mit umfassen, so bedient man sich am rinfachsten des Satzes, dals der Konvergenzkreis einer Potrnzreile bis zu dem den Mittelpunkte am nächsten gelegomen singulären Punkte reicht. Man rekennt unmitelbar. dal. dir im folgenden zusammengestallten lionvergenzberible vorhanden sind:

Da diese Konvergenzhereir:he zusammengenommen die ganze $x$ Ebeme, zum Toil ameh mehrfach, ïherdeckrn, simd wir für die Gaulschr Differentialgluichung in aler glïcklichen Lage, fïr jed.n $x$-Wert wenigstens ein Fundamentalsystem dureh msere Roihenentwicklungen dargestedlt zu haben. Um die Integration in dem in der Nr. to prazisierten simme als

1) K. Weirestrab. Crelles lournal. Bd. il Isobi. S. 1: Wurke l. 1sit. S. 17\%. 


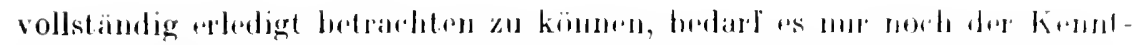

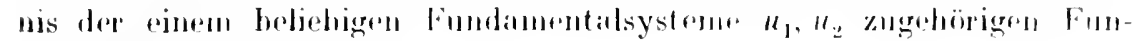
damentalsuhstitutiomen.

\section{Bestimmung der Fundamentalsubstitutionen.}

Wir setzen, mu dir Vorstellumer za fixirren, vorans, dab das Fundamentalsystem $u_{1}, u_{2}$ durch seine Anfangswerte in einnon Punkto der $x$-Ehene gegeben sei, der dem Konvergrnzbreriche der Rrilnen $u_{x_{1}}, u_{x_{2}}$ angehört, also a berhalb des mon den Nullpunkt mit dron Radius Eins beschriebenen Kreises liegt. Jann sind also die Ausdrückr drer $\left(u_{1}, u_{2}\right)$ durch $\left(u_{\infty 1}, u_{\propto_{2}}\right)$ als bekinnt anzusehen und es handelt sich un die Aufstellung der Fundamentalsubstitutionon für dieses letztere Fundamentalsystem. Dabei ist zu bearhten, dab fïr die lineare Diffrentialgleichung zwoiter Orduung die Matrix

$$
\left(\begin{array}{ll}
u_{x 1} & u_{x_{1}}^{\prime} \\
u_{x 2} & u_{\infty 2}^{\prime}
\end{array}\right)
$$

als Integralmatrix zu gelten hat. Wird diese Matrix von links her mit der konstanten Matrix $C$ komponiert, so verwandeln sich $u_{x_{1}}$. $\|_{x_{2}}$ in

$$
c_{11} u_{x_{1}}+c_{12} u_{x_{2}}, c_{21} u_{\times 1}+c_{22} u_{x_{2}} \text {; }
$$

wir sagen dann kurz, dali $u_{\infty 1}, u_{\infty 2}$ die lineare Substitution $C$ refahrer baben. Der Einfachheit wegen beschränken wir uns anf den Fall unbestimmter $\alpha, \beta, \gamma$, schlioßen also das Aliferen der Ausnahmefälle aus.

Wir denken uns, wie allgemein vorgesehen, die Punkte $x=0,1, \propto$ durch kleine Kurven ausgeschlossen und 0,1 mit $\infty$ durch die beiden Querschnitte $l_{0}, l_{1}$ verbunden, die wir $z$. B. so legen können, daB $l_{0}$ von 0 nach $-\infty$ längs der negativen reellen Achse, $l_{1}$ von 1 nach $; \infty$ längs der positiven reellen Achse verläuft. In der so entstehenden einfach zusammenhängenden Fläche $T$ sind die durclı analytische Fortsetzung der

$$
u_{01}, u_{02}, u_{11}, u_{12}, u_{\propto 1}, u_{\infty 2}
$$

innerhalb $T$ entstehenden Funktionszweige eindeutig und endlich, wir bezeichnen sie mit denselben Buchstaben wie die Reihen, aus denen sie entsprungen sind, und wollen ebenso unter $F(\alpha, \beta, \gamma, x)$ nicht allein die für $|x|<1$ konvergierende Reihe, sondern auch den aus dieser Reihe durch analytische Fortsetzung innerhalb $T$ entspringenden und daselbst eindeutigen Funktionszweig verstehen. Die mehrdeutigen Faktoren, die in der Darstellung der sechs kanonischen Integrale auftreten, fixieren wir durch die Festsetzung, daß bej Fortsetzung innerhalb $T$

sei.

$$
\lim _{x \rightarrow 1} x^{1-\gamma}=\lim _{x \rightarrow 0}(1-x)^{\gamma-\alpha-\beta}=\lim _{x \rightarrow 1} x^{-\alpha}=\lim _{x \rightarrow 1} x^{-\beta}=1
$$


Es handelt siels num an dir Herstellung der Substitutionen. die $\left(n_{\infty 1}, n_{x_{2}}\right)$ arfährt, weun $x$ dir Q (uersehnitte $l_{0} . l_{1}$ inn positiven sinne überschreitet; wir bezeichnen diese mit

$$
\Omega_{0}:\left(\begin{array}{ll}
\beta_{11}^{(1)} & \beta_{12}^{(1)} \\
\beta_{21}^{(0)} & \beta_{22}^{(1)}
\end{array}\right), \Omega_{1}=\left(\begin{array}{ll}
\beta_{11}^{(1)} & \beta_{12}^{(1)} \\
\beta_{21}^{(2)} & \beta_{22}^{(1)}
\end{array}\right) .
$$

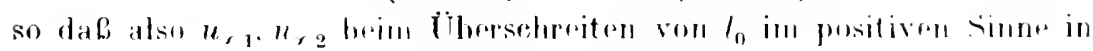

$$
\begin{aligned}
& \beta_{11}^{(1)} u_{\infty 1}-\beta_{12}^{(1)} u_{x_{2}} \\
& \beta_{31}^{(1)} u_{\infty 1}-\beta_{32}^{((1)} n_{\infty 2}
\end{aligned}
$$

und beim Cherseherenten ven $l_{1}$ im positiven Simme in

$$
\begin{aligned}
& \beta_{11}^{(1)} u_{n_{1}}+\beta_{12}^{(1)} u_{x_{2}} . \\
& \beta_{21}^{(1)} u_{\infty 1}+\beta_{2 !}^{(1)} u_{s}
\end{aligned}
$$

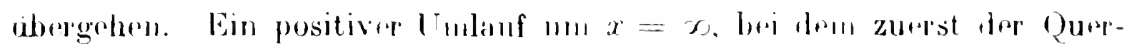

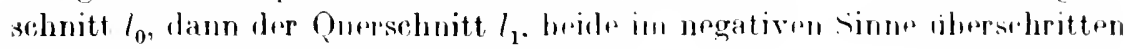
werden, verwandelt $n_{n, 1} n_{\infty 2}$ in

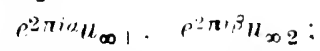

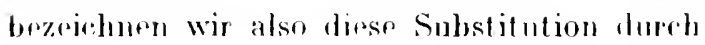

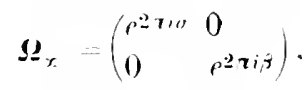

so ist (vol. Xir. 47. Cilichoung (67). S. 199)

$$
\Omega_{\infty}=\Omega_{11}^{-1} \Omega_{1}^{1} \text {. }
$$

Die inverse Matrix von $\varrho_{\infty}$ läBt sich offenbar so zusatumensetzen:

$$
\Omega_{x}^{-1}=\Omega_{1} \Omega_{0} \text { : }
$$

komponieren wir hier briderseits mit $\varrho_{-1}{ }^{1}$ von remts her, so ergibt sich

$$
\Omega_{1}-\Omega_{x}^{1} \Omega_{0}{ }^{1} \text {; }
$$

von dieser Darstellung von $\Omega_{1}$ wrerden wir soglfirh Colbratuh zu machen haben.

Wir schreiben die zwisehen on und 0 rimerseits, o mol 1 andererseits vermittemone Übergangssubstitutionen wie folgt: ${ }^{1}$ )

$$
\left\{\begin{array}{llll}
u_{x 1}=a_{0} u_{111} & b_{11} u_{02} . & u_{x 1} & a_{1} u_{11} \cdot b_{1} u_{12} . \\
u_{x 2}-c_{0} u_{01} & d_{01} u_{02} . & u_{22} & u_{1} u_{11}-a_{1} u_{12} .
\end{array}\right.
$$

Wenn dann $x$ den Quersehnitt $l_{1}$ einmal in positiven Sinne überschreitet, so verwimbln sieh $n_{11}, n_{12}$ in

also $u, 1, u_{n 2}$ in

$$
n_{11}, e^{2} \eta(i-12-3) u_{13} \text {. }
$$

$$
\begin{aligned}
& \mid a_{1} n_{11}+b_{1} \rho^{2 x_{i} ;-n "} " n_{12} \text {. } \\
& \text { le } u_{11}+\partial_{1} e^{2 v(y-\alpha} \text { r) } \|_{12} \text {. }
\end{aligned}
$$

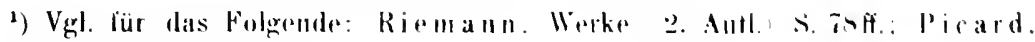
Traite III (190) s. s. $315 \mathrm{ff}$. 


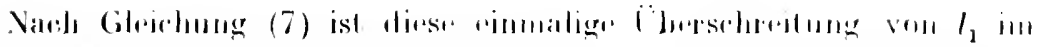

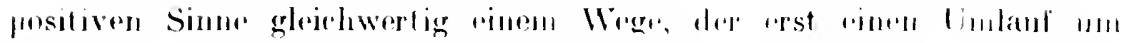

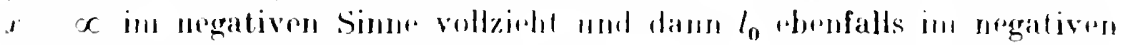

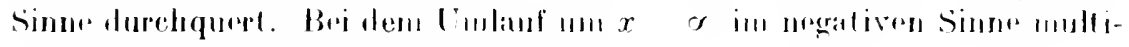
plizieren sich $u_{\alpha_{1}}, u_{, 2}$ bezirhungswoise mit

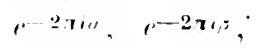

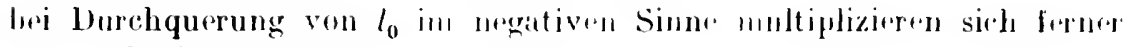
$u_{01}, u_{02}$ heziehungsweise mit

$$
\text { 1. e }-2 \pi(1 \text {; }) \quad e^{2 \pi i \gamma},
$$

durch Anwondung der esten dor Chergangssubstitutionen (8) rrhalten wir folglich dir Worte. in dir $u_{s_{1}}, u_{x_{2}}$ anf dem gesolithlerten Wege übergefiihrt werdon, in der form

$$
\mid \begin{aligned}
& e^{2 \pi i \alpha}\left(a_{0} u_{01}+b_{01} e^{2 \imath \pi} u_{02}\right), \\
& e^{2 \pi j}\left(c_{0} u_{01}+r_{0} e^{2 \pi i \gamma} u_{02}\right) .
\end{aligned}
$$

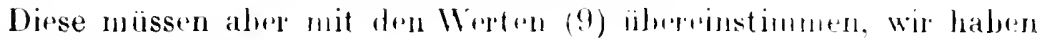
also dii Gleichungen

(11) $a_{1} u_{11}+b_{1} e^{2 \cdot a l ;}$ a g) $u_{12} \quad e^{-2 \pi u}\left(u_{0} u_{01}-b_{0} e^{2 \pi i_{\gamma}} u_{02}\right)$,

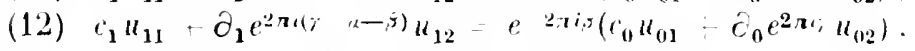

denen wir noch die aus (8) folgrmulon cileichungen

$$
\begin{aligned}
& u_{1} u_{11}+b_{1} u_{12}-u_{0} u_{01}-b_{0} u_{02}, \\
& c_{1} u_{11}+\partial_{1} u_{12}=c_{0} u_{01}+\partial_{0} u_{02}
\end{aligned}
$$

an die Seite strllen. Eliminimen wir zwischen den Glorehnngen (11), (13) einerseits und (12), (14) anderestils arst $u_{11}$. dann $\|_{12}$ und ver-

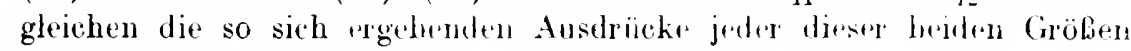
miteinander, so erhalten wir

$$
\begin{aligned}
& 0=u_{01}\left[a_{0} \partial_{1}\left(e^{-2 \pi i u}-1\right)-c_{0} b_{1}\left(e^{-2 \pi i \rho}-1\right)\right] \\
& +u_{02}\left[b_{0} \partial_{1}\left(e^{2 \pi_{1}(;-\cdots)}-1\right)-\partial_{0} b_{1}\left(e^{2 \cdots(;-;)} \cdots 1\right)\right] \text {, } \\
& 0=u_{01}\left\lfloor a_{0} c_{1}\left(e^{\left.-2 n a_{1} \gamma-j\right)}-1\right)-c_{0} a_{1}\left(e^{-2 \pi u(\gamma-u}-1\right)\right\rfloor \\
& +u_{02}\left[b_{0} c_{1}\left(e^{2 \pi}-1\right)-\partial_{0} a_{1}\left(e^{2 \pi u}-1\right)\right] .
\end{aligned}
$$

Dies sind homogene linear" Relationen mit konstanten hoeffizienten zwischen $u_{01}, u_{02}$, da aher $u_{01}, u_{02}$ ein Fundanentalsystem bilden, sind solche Relationen nur möglieh, wenn die einzelnen Koeffizienten verschwinden. Wir rhalten also vier Gleishungen, dir wir mit Rücksieht larauf, daB

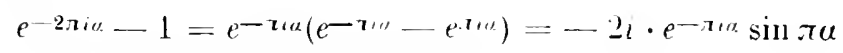

usw. ist, in ler Form schreiben könnem:

$$
\begin{aligned}
& \begin{array}{l}
a_{0}=\begin{array}{l}
b_{1} e^{-\pi} \sin \pi \beta \\
i_{0}
\end{array} \partial_{1} e^{-\pi i \alpha} \sin \pi u
\end{array}=\begin{array}{l}
a_{1} e^{-\pi i \gamma} \sin (\gamma-\alpha) \pi \\
c_{1} e^{-\pi i \omega} \sin (\gamma-\beta) \pi
\end{array},
\end{aligned}
$$

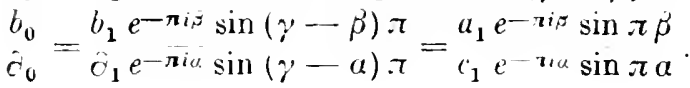


Nun ist (vgl. Nr. 47. Gleichung (65) . S. 19র')

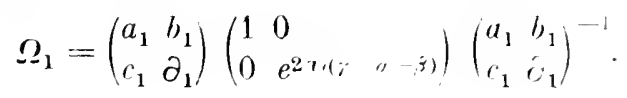

und da, wenn wir

setzen,

$$
a_{1} \partial_{1}-b_{1} c_{1}=\delta
$$

ist, so ergibt sich

$$
\left(\begin{array}{ll}
a_{1} & b_{1} \\
r_{1} & \partial_{1}
\end{array}\right)^{-i}=\left|\begin{array}{cc}
\partial_{1} & b_{1} \\
o & \delta \\
-c_{1} & a_{1} \\
\delta & \delta
\end{array}\right|
$$

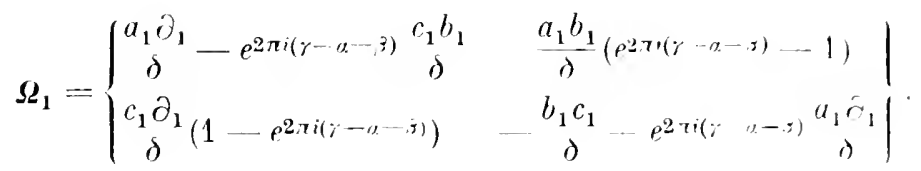

Setzen wir nun

so ist

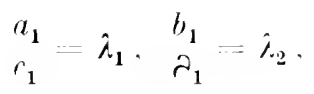

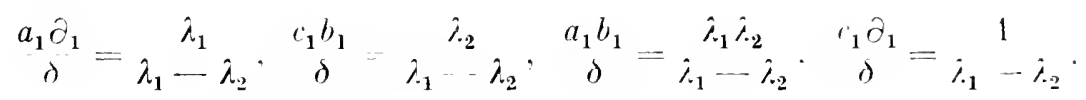

d. h. die Koeffizienten der Substitution $\varrho_{1}$ hängen mur von $\lambda_{1}$. $\lambda_{.2}$ and bekannten Gröfien ab. Nun ist aber z. B. vermöge der Gleichung (15)

$$
\lambda_{2} \lambda_{1} \frac{\sin (\gamma-\alpha) \pi \cdot \sin \pi \alpha}{\sin (\gamma-\beta) \pi \cdot \sin \pi \beta},
$$

es handelt sich also nur noch um die Bestimmung del Gröbe $\lambda_{1}$.

Zu diesem Ende setzen wir in der zweiten der Chergangssubstitutionen (8) für $x$ den Wert 1 ein. Dann ist

$$
\lim _{x \rightarrow 1} u_{11}=1 \text {. }
$$

und wenn wil voraussetzen, daf. der reelle Tril des Exponenten $:-a-\beta$ positiv ist,

ferner ist

$$
\lim _{x \rightarrow 1} u_{12}=0:
$$

$$
\begin{aligned}
& \lim _{x \rightarrow 1} n_{x_{1}}=F(u, \alpha-\gamma+1, \alpha-\beta+1,1) . \\
& \lim _{x \rightarrow 1} u_{x_{2}}=F(\beta, \beta-\gamma+1, \beta-\ell+1,1) .
\end{aligned}
$$

bezeichnen wir also den Wert, den der durch die Gaubsehe Reihe 


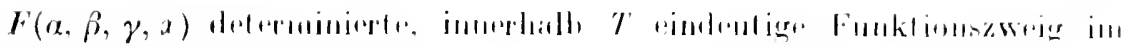
Punkte $x=1$ ammimumt '). mit

$$
f(\mu, \beta, \gamma, 1) \quad f(\mu, \gamma, \gamma) \text {. }
$$

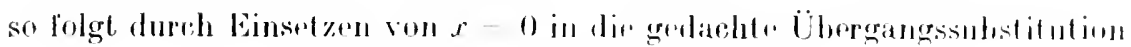

$$
\begin{array}{lllllll}
a_{1} & i(\alpha, \beta & \ddots & -1, \alpha & \beta & 1), \\
c_{1} & i(\beta . \beta & \ddots & 1, \beta & \ell & 1)
\end{array}
$$

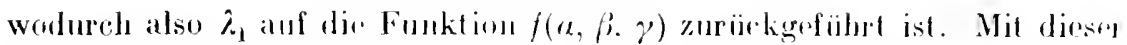

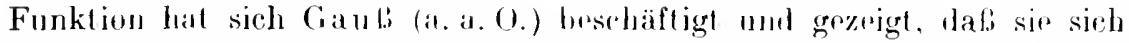
durch die sogenannten Eulersehere lntegrale ansibürken Jälit; wir kommen auf riese Darstellung machher zuriick. Sihen wir jem. Funktion vorläufig als bekinut an, so ist also $\hat{\lambda}_{1}$ hrekinnut, mud somit auch die Fundamentalsubstitution $\Omega_{1}$ lrkannt.

Durch $\Omega_{1}$ und $\Omega_{2}$ läbt sich aber dir Fundanentalsubstitution $\Omega_{0}$ sofort darstellen, os folght nämlich atss (6)

$$
\Omega_{0}-\Omega_{1}^{-1} \Omega_{s}^{-1} \text {. }
$$

Damit ist das Intrgationsprobleme fiir die Gatulische Differentialgleichung in Fallo monestimmter $\alpha, \beta, \gamma$ als golöst anzusehen ${ }^{2}$ ). (irnauer gesprochen ist es anf die Bestimmung von $f(\alpha, \beta . \gamma)$ zur irekgefiihrt.

\section{Multiplikator. Adjungierte IDifferentialgleichung. Identitüt ron $L$ a grauge.}

Die Bestimmung vou $f(\alpha, \beta . \gamma)$ grelingt an pinfaclsten, Wemn man sich einer Darstellung der Lösungen der Gau los:hpn Differentialgleichung durch ein bestimmtes Integral bedient, die zuerst von Euler angegeber worden ist. Um zu dieser Darstellung zu gelangen, müssen wir einigr Betrachtungen rorausschicken, die sich auf beliebige lineare Differentialgleichungen beziehen, und die aneh liir andere Fragen von llichtigkeit sind.

Wir nehmen die Differentialgleichung in der Form

$$
P_{x}(u)=p \frac{d^{2} u}{d x^{2}}-q_{d x}^{d u} \div r u=0 \text { : }
$$

der Charakteristik, die die linke Seite dieser Gleichung bezeichnet, haben wir den Index $x$ angehängt, un auch die unabhängige Variable der Differentialgleichung hervortreten zu lassen.

1) Vgl. z. B. L. W. Thomé. Crelles Journal, Bd. 87 1879), S. 22.2.

$\Rightarrow$ In bezug auf andere Formeh und für die einschlägige Literatur werde auf Bd. I des Handbuches von L. Schlesinger verwiesen. 


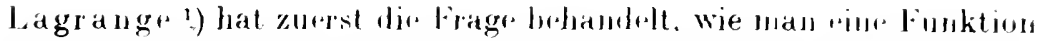

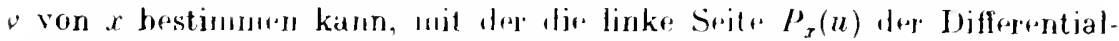

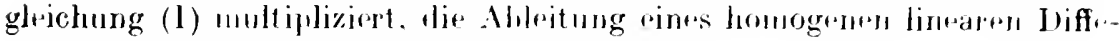
rentialausedrueks rerster Orelmung wird, also

$$
H_{x}(u)=d_{d x}^{d}\left(u(x) \frac{d u}{d x}+\beta(x) u\right) .
$$

Die Wichtigkeit rimer solchen Funktion. dir man rinen Multaplikator von (I) mennt, liegt anf dor Hand. Drent ist rin solcher Multi-

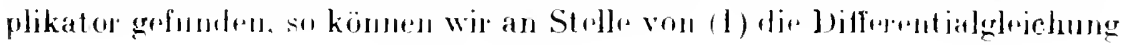

coler intregrimt

$$
d_{d r}^{d}\left(a(r) d_{d r}^{d u}+f(x) u\right)=0 \text {. }
$$

$$
u(x) \frac{d u}{d x}+\beta(x) u=r \text { orist. }
$$

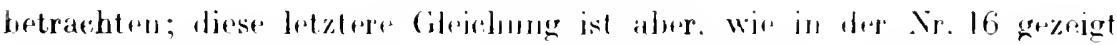
wurde, stets dureh Quadraturent zu integrienen. Dir Kanutnis rians

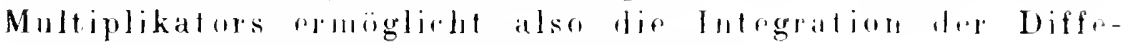

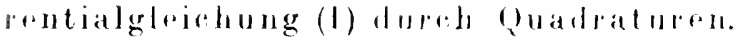

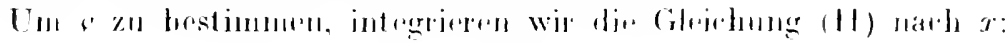

$$
\dot{f o p}_{x}(u) d x=a(x) \frac{d u}{d x}+\beta(x) u \text {. }
$$

und untedsuchen mun das linkeshand steherudr Integral

$$
f^{\prime} l_{x}(u) d x=f^{\prime}\left(p u^{\prime \prime}+q u^{\prime}-r u\right) d x
$$

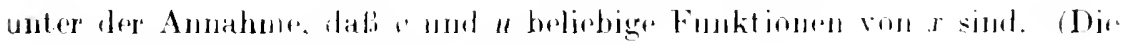

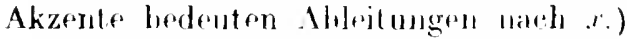

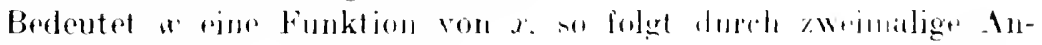
wendurg der pardichlen Integration

$$
\int u^{\prime} u^{\prime \prime} d r=\left\|u^{\prime}-i u^{\prime} w^{\prime} d x=\right\| u^{\prime}-u^{\prime} u+f u w^{\prime} d x .
$$

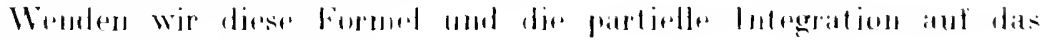
atigge Intrerral all, so wirt

$$
\begin{aligned}
& f\left({ }^{\prime} x(u) d x=f(v)\right) u^{\prime \prime} d x+f(u) u^{\prime} d x+f(x) u d x
\end{aligned}
$$

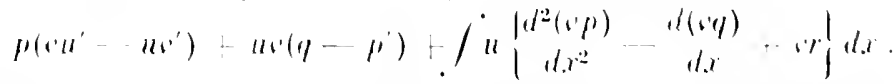

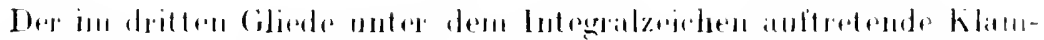

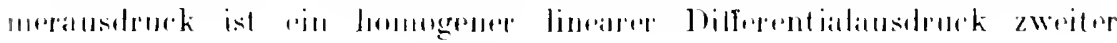

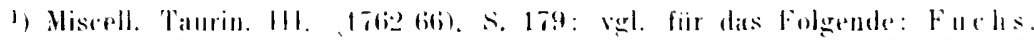

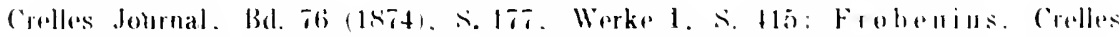

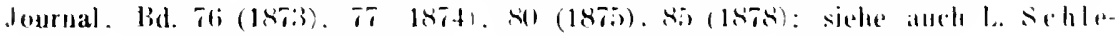

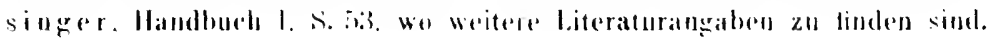




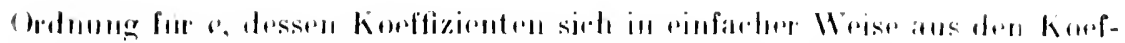
dirienten von $P^{5}(u)$ hilden lassen; wir sotzen

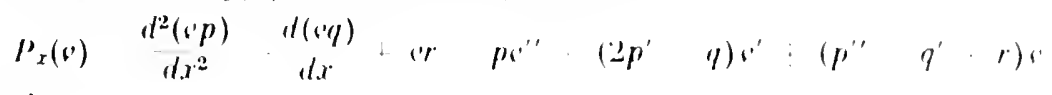

innil

$$
p\left(c^{\prime \prime \prime} \quad \| \prime^{\prime}\right):\left(q \quad p^{\prime}\right) \| c^{\prime} \quad P_{r}^{\prime}\left(u, c^{\prime}\right) \text {, }
$$

tamn lautet dir obige fileichumg

oder diflerentijert

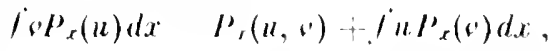

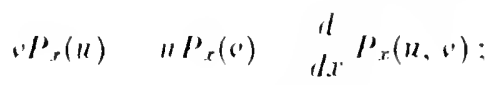

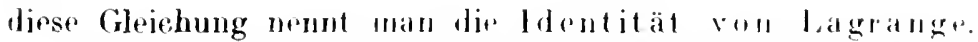

Der Ausdruck $P_{x}(u, r)$ ist in brzug anf $u$ rin hontugerser lintarer Differentialauselruck rester Orolnung: " wind also rin Multiplikator von (1) sein, wenn

$$
P_{x}\left(v^{\prime}\right)-0
$$

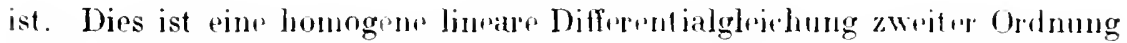
für ", die man nach Fuchs die adjungierl" von (I) nennt. Auch heift $P_{x}(v)$ der zu $P_{x}(u)$ aujungielte Differentialausdrurk. und $P_{x}(u, v)$. das sowohl in bozug aut $\|$ als auch in bezug anf a homogen, lineau

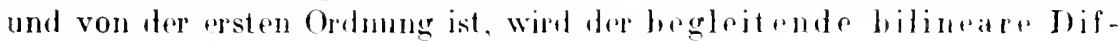
ferentialausdruck gemant (Frobenius).

Also ist 'pin Multiplikator von (I)

$$
P_{x}(r)=0 \text {, }
$$

wenn "eine Lösung der adjungierten Differentialgleichung ist: ungekefut zeigt die Lagrangesehr Identität sofort, daf " rin Multiplikator von

$$
t_{x}(0)=0
$$

ist, wemn $u$ der Differentialglejohung (1) grnügt. Jic Beziehung zwischen einer Differentialgleichung und ihrer adjungierten ist also rine grgenseitige, was ja ïbrigens sehon aus der Symmetrie der Lagrangeschen Identität erhellt.

Das Vorstehende genügt fïr di" \%werke, die wir augenblicklich verfolgen; an späterer Stolle ( $\mathrm{Nu} .75)$ kommen wir anf diess Betrachtungen noch einmal zuriick. 


\section{Integration der (a a uschen Differentialgleichung durch bestimmte Integrale. Vertauschune von Parameter und Iroument. Eulersche Transformierte.}

Wie beroits hemerkt, hat Euler ') die Jösungen rinfe Gandisthen Differentialglejohung in der Form vor bestimmton Integralen darenstallt. dir zwischen konstanten Grenzen resterekt die mabhängige Variahle, als Parameter enthalten. Wenn wir dirse Darstallong im Gebiete der komplexen Variabeln verwerten wollen, so missen wir rin solches Integral statt \%wischen festen Grenzen, längs rime geschlossenen hurre r. strecken; dann haben dir hire in bentracht konmmolon lutregrald die. Gestalt:

$$
f_{i}(x)(z)(z)=1, t z
$$

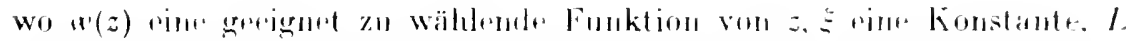

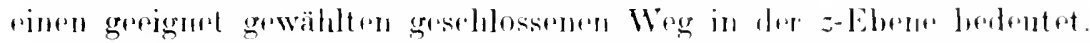

Diese Art von Julegralen birted rine in dir Angen fallemb. Analogi.

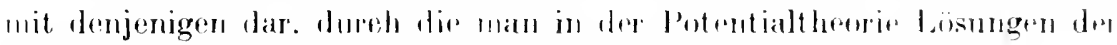

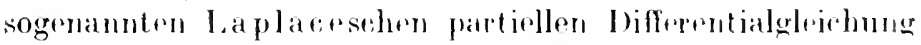

$$
\partial^{2} u+\frac{\partial^{2} u}{\partial \eta^{2}}+\frac{\partial^{2} u}{\partial_{-}^{-2}}-0
$$

darzustellen pthegt. Hat man z. l3, rine äber ninen gewisson Ranm r verteilte Masse, die nach dem Newt onsehen Gesetze anf einru and rerhalh dieses Rammes gelegenen Massempunkt mit dor Masse 1 mol den Koor-

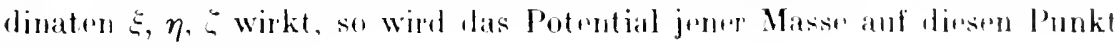
dureh das lntegral

$$
\prod_{(i j}\left[(x-\xi)^{2}-(y-\eta)^{2} \quad(z-\xi)^{2}\right]^{1} d x d y d=
$$

dargestellt, wo dann w(x,y,z) die Dichtigkeit der Masse in Junkt.. $(x, y, z)$ fes Rammes $C$ berloutot. Wir haben also anch hier oine Funktion "des orles, anf den sich die Integration bezinht. multiplizient mit pine lentenz (der ( 1)ten) des Abstandes jemes Optes von dell ..Aufpunkte"

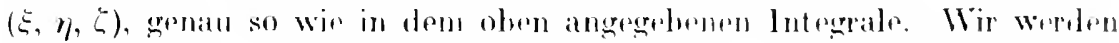

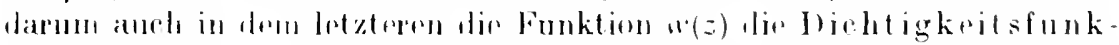

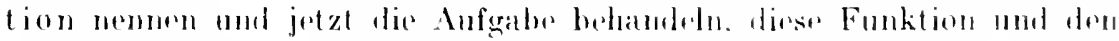

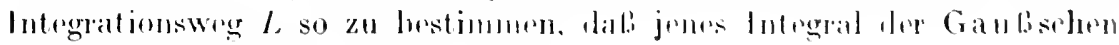
Differentialegleiohmeg Genïge leisted.

1) L. Euler. Institutiones calculi integralis 11,1769 ). ('ap. Xl. Opera. ser. I.

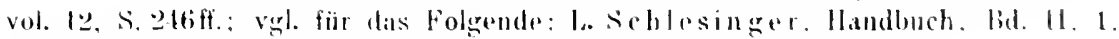
lll. Absehnitt. Wo anch die ensehlägige Literatur zusitumengestellt ist. 


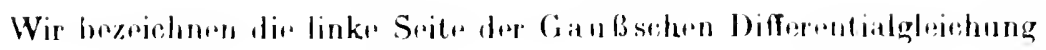
mit

$$
D_{x}(u) \quad r(1 \quad r) \frac{d^{2} u}{d x^{2}}+|\gamma-(\alpha+\beta+1) x| \frac{d u}{d x}-\alpha \beta u
$$

und setzen mun hieriu

$$
" l_{l} w(z)(z-r)^{\ddagger} 1,1 z .
$$

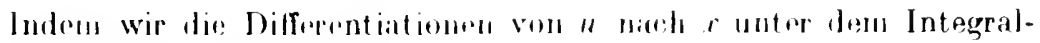
zeichen vollzirhen, finllen wir

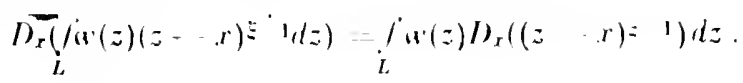

Wir formen zunächst den Ausdruck

$$
\begin{gathered}
D_{x}\left((z-x)^{z}-1\right)=x(1-x)(\xi-1)(\xi-2)(z-x)^{\xi-3} \\
-[\gamma-(a+\beta+1) x](\xi-1)(z-x)^{\xi}-\alpha \beta(z-x)^{\xi-1}
\end{gathered}
$$

um, indem wir seine von $x$ abhängigen Koefizienten nach Potenzen von $z-x$ entwickeln. Es ist:

$$
\begin{gathered}
x(1-x)=z\left(1 \quad z \quad(1-2 z)(z-x)-(z-x)^{2} .\right. \\
\gamma-(\alpha+\beta+1) x=\gamma-(\alpha+\beta+1) z+(\alpha+\beta+1)(z-x):
\end{gathered}
$$

dies eingesetzt und nach Potenzen von $(z-1)$ geordnet gibt

$$
\begin{aligned}
& \left.D_{x}\left((z-x)^{\xi-1}\right)=1 \xi \quad 1\right)(\xi-2)(z-x)^{\xi}-3\left(z-z^{2}\right. \text { । } \\
& \text { - } \left.(\xi-1)(z-x)^{\xi} 2\left[\begin{array}{ll}
-(\xi & 2
\end{array}\right)(1-2 z)-\gamma+(\alpha+\beta+1) z\right] \\
& -(z-x)^{5} 1[-(\xi-1)(\xi \quad 2)-(\xi-1)(\alpha+\beta+1)-\alpha \beta] \text {. }
\end{aligned}
$$

Nun ist :

$$
\begin{gathered}
(\xi-1)(\xi-2)(z-x)^{t}-3=\frac{d^{2}(z-x)^{5} 1}{d z^{2}} . \\
(\xi-1)(z-x)^{\xi-2}={ }^{d(z-x)^{5}:}
\end{gathered} .
$$

setzen wir also

$$
\begin{gathered}
\boldsymbol{I}_{z}(v)=z(1-z) \frac{d^{2} v}{d z^{2}} \\
+[-(\xi-2)(1-2 z)-\gamma+(\alpha+\beta+1) z] \frac{d v}{d z} \\
-[(\xi-1)(\xi-2)+(\xi-1)(\alpha+\beta+1)+\alpha \beta] v .
\end{gathered}
$$

so besteht die identische Gleichung

$$
D_{x}\left((z-x)^{\xi-1}\right)=\boldsymbol{t}_{z}\left((z-x)^{\xi-1}\right) .
$$

Der Ausdruck $\boldsymbol{I}_{z}(0)$ ist selbst in homogener linearer Differentialausdruck zweiter Ordnung für $\bullet$ als Funktion von $z$ uit in z rationalen Koeffizienten; die Differentialgleichung

$$
\boldsymbol{d}_{z}(\nu)=0
$$

ist zwar keine Gaußsche, aber sie gehört zum Fuchsschen Typus und ihre singulären Punkte sind 0,1, 0 . Dje Identität (20) nennen wir den 
Satz von der Vertauschung des Parameters mit dem Argumente, auf der linken Seite ist nämlich z der Parameter und $x$ das Argument, auf der rechten Seite dagegen $x$ der Paramerer und $z$ das Argument.

Nach Anwendung des Vertauschungssatzes auf die rechte Seit der Glejohung (18) lautot diese Gloichung:

$$
D_{x}\left(f_{L}(x)(z-x)^{\xi} 1, d z\right)=\int_{L}\left(w(z) \Delta_{z}\left((z-x)^{\xi-1}\right) d z\right.
$$

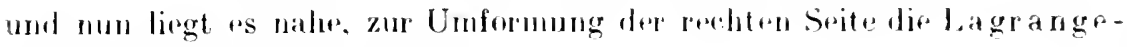
sche Identitiit homanzoiohen.

Bezeichnen wir mit $\boldsymbol{J}_{z}(\mathrm{a})$ den adjungierten Difforentialausdruck vont $f_{z}(v)$ und mit $f_{z}(a, a)$ den beglutenden bilimearen Ausdrurk. so ist

$$
w I_{z}(v)-v I_{i}(w)=\frac{d}{d z} I_{i}(v, w) \text {. }
$$

Nehumen wir hitrin

$$
r^{\prime}=(z-x) \leq-1,
$$

so könnel wir (22) in Her Form schreiben:

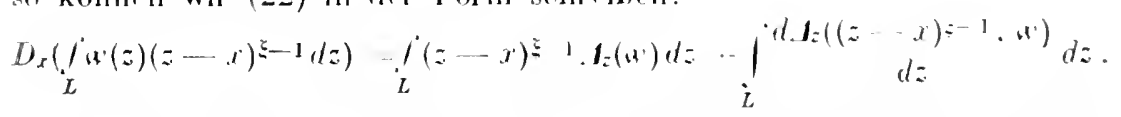

Wir wollten das Integral (17) so finriohten, dali ws der Gaufschen Differentialgleichung

$$
D_{x}(u)=0
$$

geniigt; wir werden also w(z) se wählen, dak

$$
\boldsymbol{t}_{z}(w)=0 \text {. }
$$

und $L$ so, dali.

$$
\int_{i}^{d \cdot d z\left((z-x)^{\xi}-1, w\right)} d z=0
$$

ist. Dir Gleichung (23) ist rine homogene lineare Differentialglejehune zweiter Ordmung för w, und zwar die arljungirete von (21): 11m (24) zu forfïlen, mule $L$ so gewählt werden. dali dor Austrurk

$$
\left.\varphi(z)=I_{z}(z-r)=1 . w\right)
$$

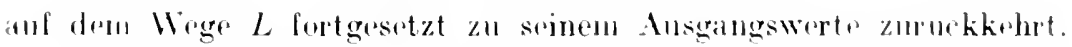

Dit Glevhung (23). dio die Dielutigkritsfunktion w(z) bestimunt. nemen wir die Eulersehe Transtermierte der Gauliselen Differentialglojeloumen.

\section{Bestimmung der Dichtigkeitsfunktion unt des Integrations- weres.}

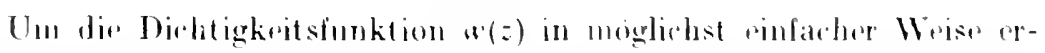
halten za könmen, disponierrn wir über die konstante E derant. dab in 
der Differentialgleichung (21) der Korfizirnt von r versehwimdut. Wii erhalten nach (19) durch diese Forderung fiur 5 dir Gluchung

\section{deren Lösungen}

$$
\text { (E-1)(E-2+a!Fi 1), ap } 0 \text {. }
$$

$$
1-a, 1 p
$$

sind. Da $\alpha, \beta$ in der Gaulisehron Ifffrentialgleichung ganz symmetrised: auftreten, ist es gleichgiiltig. Wolche dieser briden lö̈ungen wir wählen; is sei

Dann lautet der Ausdruck (1!)

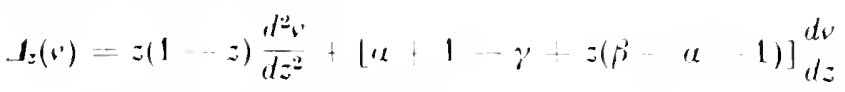

und nach den Formeln der Nr. 56 argeben sich für den adjungierten und den begleitenden bilinearen Differentialausdruck dif Werte

$$
\begin{aligned}
& \boldsymbol{I}_{\mathbf{z}}(w)=z(1-z) \frac{d^{2} w}{d z^{2}}+[1-2 z-u+\gamma-(\beta-a+1) z] \frac{d w}{d z} \\
& -(\beta-\alpha+1) w \\
& =\frac{d}{d z \mid z(1-z)} d z \quad|\alpha-\gamma+(\beta-\alpha+1) z| w \mid
\end{aligned}
$$

und

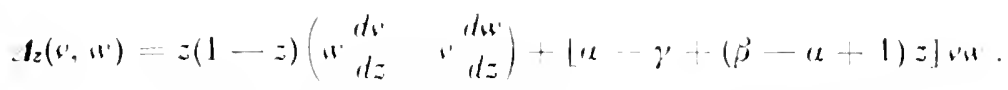

Die linke Srite $\boldsymbol{t}_{z}(w)$ der Eulerschen Transformierten ist jetzt ein vollständiger Differentialquotient: da es uns nur auf rine partikulär * Lösung ankommt, wählen wir die nach der ersten Integration auftretend willkürliche Konstante gleich Null. bestimmen also $w(z)$ aus der Gleichung

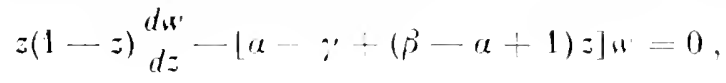

woraus sich

und weiter

$$
\begin{aligned}
& d \log \alpha=\alpha-\gamma+(\beta-\alpha+1) z \\
& d z=z(1-z)
\end{aligned}
$$

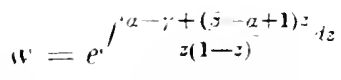

ergibt. Das im Exponenten auftretende lntegral läßt sich nach elmentaren Regeln ausführen:

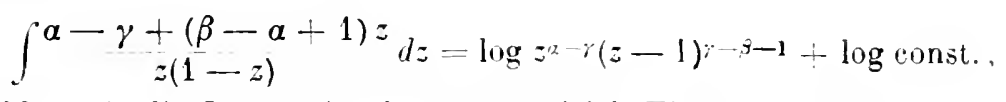

wählen wir die Integrationskonstante gleich Eins. so ist also

$$
w=z-r(z-1) r-s-1
$$

die Dichtigkeitsfunktion. 
Für die Funktion $q(z)$, deren wir zur Fixiernng des Integrationweges $L$ bediurfen, "rgiht sich nach Einsetzen der Werte von $\xi$ und a durch einfache Rechnung:

$$
\begin{aligned}
& I_{z}\left((z-x)^{-1 x} \cdot z^{\prime \prime-r(z-1) i--1}\right) \\
& =a z^{\alpha} r+i(z-1) x-j(z-x)^{-1-1}=q(z) \text {. }
\end{aligned}
$$

mollich nimmot das Integral (17) sellhst die Form an

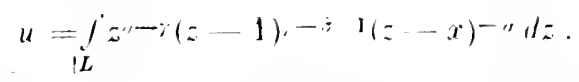

Wir wenden uns nun zur Bestimumung des Integrationsweges $L$.

Schliefen wir ans der z-Ehene die Punkte

$$
==0.1 . x \text {. o }
$$

durch unentlich kleine Kurven aus mnl legen vou 0. 1. $r$ aus nach dem Unendlichen hin die Querschnitte $l_{0} . l_{1} . l_{\text {. so }}$ ist in der so entstehendert einfach zusammenhängenden Fläche $T$ sowohl $q(z)$ als auch lipe unter dem Integralzeichon stehende Funktion

$$
\psi(z)=z^{\alpha} \because(z-1),-\beta 1(z-x)
$$

eindeutig und endlich. Der Integrationsweg $L$ ist so zu wählell, dabi auf diesen Wege fortgesetzt $\varphi(z)$ zu seinem Ausgangswerte zuriirkkehrt: diws würde jedenfalls frfolgen, wem wir $L$ als einen ganz innerhalb $T$ r.m. laufenden geschlossener Weg annähmen: lann wirde aber

$$
\int_{\boldsymbol{L}}^{\prime} y^{\prime}(z) d z=0
$$

sein, so dab ein solcher Weg unbrauchbar ist.

Wir verfahren daher folgendermaben. Es sei $z=-\approx$ an belimbige ron

$$
0,1, x, x
$$

versehiedener bunkt der $z$-Ebene: wir gehen von $z=;$ ans. inmerhalh $T$ bis dicht an einen dor Punkte $0.1, x, \infty$ heran, umkersen dann den be-

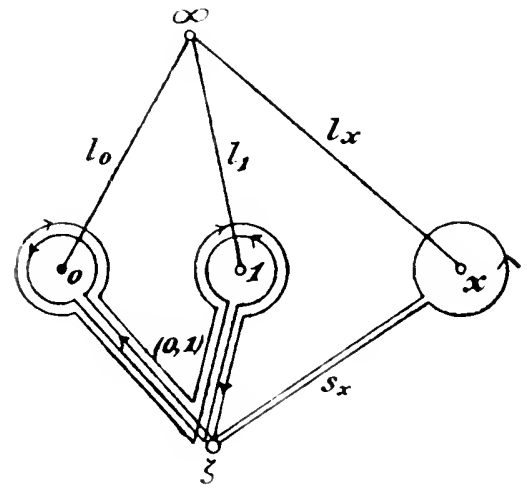

ing. 3. treffenden Punkt in positiven Sime (entgegengesetzt der Rirhturng nines in dem Punkt, beferstigt gedachten Uhrzeigers) und keluren wicher innerhalb $T$ (ntwa anf demselben llegre. anf desn wir gokommen sind) nach

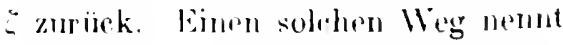
man eine voll ; ans nach dent breIreffenten der prunkte 0. 1, $x, x$ hin gelegte Sehloife (siohe die lige 3). Wir bezoichnem diese sohloifen der Reihe nath durch

$$
s_{0}, s_{1}, s_{x},,_{2}
$$




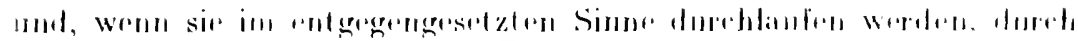

$$
s_{11}, s_{1}^{1}, s_{x}^{-1}, s_{x}^{1} \text {. }
$$

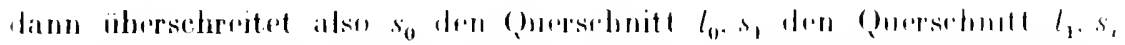

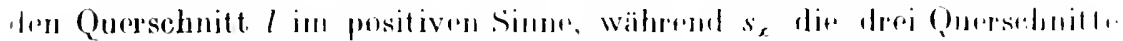

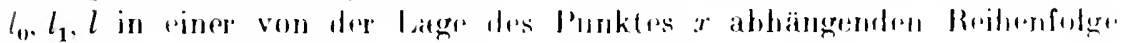
nacheinander in megativen Simme dorehoumel.

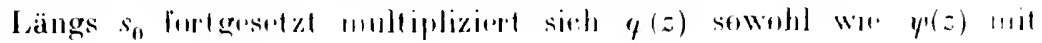
'lelli Faktor

$$
\text { iz } 11(1,-i)
$$

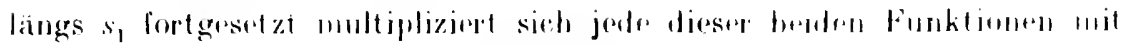
lings $s_{x}$ fortgesetzt mil

$$
p^{2}=\pi(\pi ;-\pi)
$$

$$
\text { p } \because \mathbf{a}+1,
$$

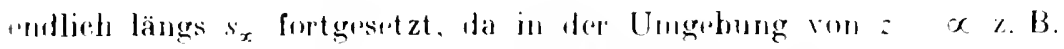

Ist, IIIit

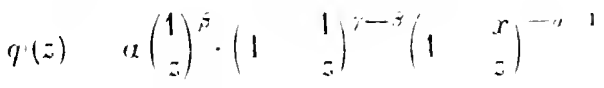

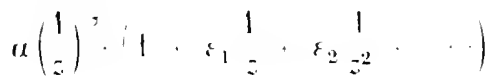

$$
\text { ㄴ.21: }
$$

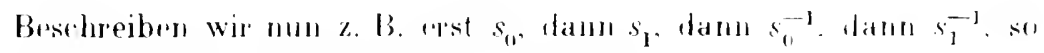
kehert $q(z)$, auf diessur Wrge (siche die. Fig. 3)

$$
\text { (0. 1) } \quad s_{0} s_{1} s_{1} s^{1}
$$

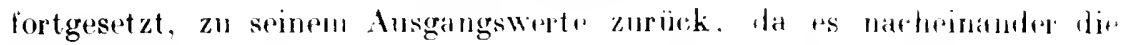
Fiktorell

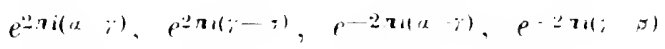

angenommen hat, dieser Mig liefort also in allgemeimen winen branthbaren Integrationsweg $L$.

Gleichermaken stellt $\left.{ }^{1}\right)$ allgemein gesprochen

$$
(i, k)-s_{i} s_{k} s_{i}^{-1} s_{k}^{-1}
$$$$
(1, k=0.1 . x \infty: i \neq k)
$$

einen brauchbaren Integrationsweg far, man nennt eimen solchen Wry eine un die Punkte $i, k$ hromgrelegtr Doppelschleifu; "s gibt derwn offenbar

$$
\begin{aligned}
& 1 \cdot 3 \\
& 1 \cdot 2=6
\end{aligned}
$$

roneinander verschiedrue, und indent wir für $L$ der Reihe nats diese sechs Doppelschleifen wählen, trhaltru wir sechs Integrale der Gauli-

1) C. Jordan. Cours dAnalyse 111 (188T, (189ti) S. 242. und L. Pochhammer, Mathematische Annalen Bd. 35 (1890). S. 470. 
schen differentialgleichumg. Wir werdon sehen, daß diese sechs Integrale, abgessben ron konstanten Faktoren, nit

iibereinstimm+n.

$$
\left\|_{011} \cdot\right\|_{02} \cdot\left\|_{11} \cdot\right\|_{12} \cdot\left\|_{x_{1}},\right\|_{x_{2}}
$$

59. Darstellung der (i a ubschen Reibe $r(\alpha .3 . \gamma . x)$ durch ein bestimmtes Integral.

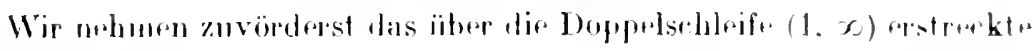
Integral

$$
\begin{aligned}
& u_{1}=\underset{(1, \infty)}{=i} i(z-1) ; \quad r 1(z-x)^{-x} d z \\
& =i z^{\alpha-r}(z-1) y+1 z-x(1-z)^{-\alpha} d z
\end{aligned}
$$

Fiilıen wir dureh die Gileichumgens

$$
z=1 \cdot d z=-\frac{1}{t^{2}} d t
$$

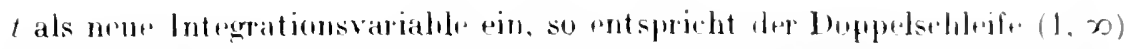

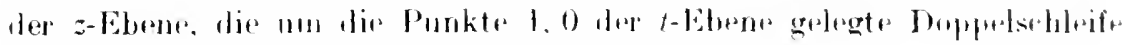
$(1,0)$. wir rerhaltin also

$$
\|_{1}-\int_{(1,11} t t^{x-1}(1-t)^{r}, x-1(1-x t)-u d t .
$$

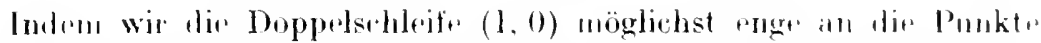

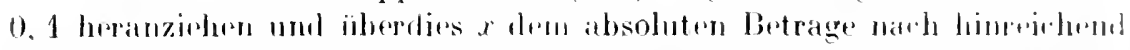

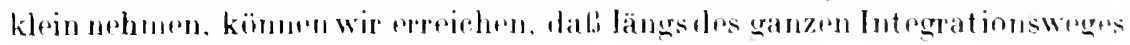

$$
\mid \text { it } \mid<1
$$

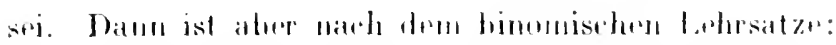

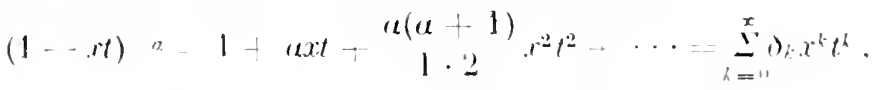

$$
\begin{aligned}
& \delta_{0} \quad 1 . \delta_{k}=\begin{array}{c}
a(u+1) \cdots(u+k-1) . \\
1 \cdot 2 \cdots k
\end{array} \quad(k-12:),
\end{aligned}
$$

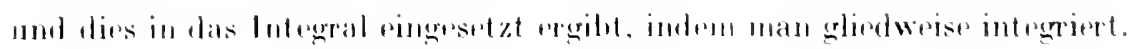

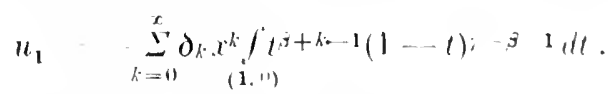

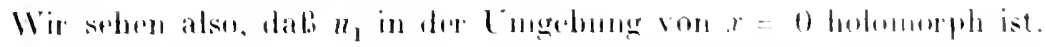

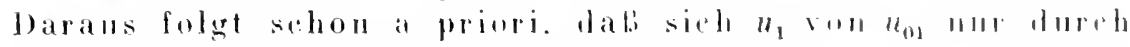

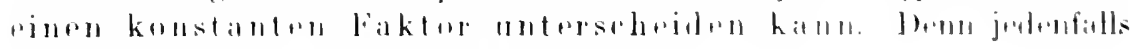
ist (fïr unbestimunt" $u, \rho, \gamma$ ) $n_{1}$ in der Form

$$
n_{1} \quad{ }_{1} \|_{111} \cdot r_{2} n_{02}
$$

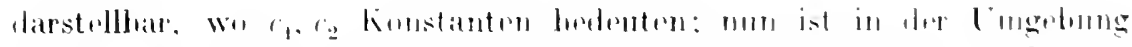

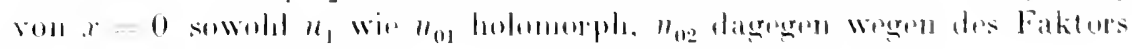

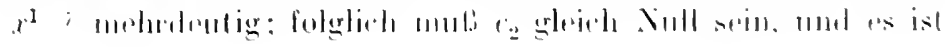


(25)

$$
u_{1} \quad r_{1} 1_{111}
$$

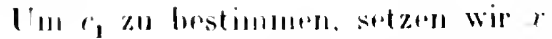

1). diann ist

$$
\begin{array}{ll}
\lim _{x \rightarrow 0} u_{1} & f t^{-1}(1 \quad t), \quad 1 d t, \\
\lim _{x \rightarrow 1} u_{n} & 1.0
\end{array}
$$

wir finden also:

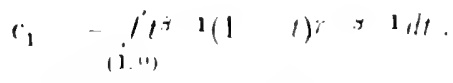

Wir heteachten allgemoin rin Integral ven dar Form

$$
f_{\text {(i. } 0)}^{p-1}(1-t)^{n-1, l l}
$$

wo $p, q$ bediebige Konstanten bedenten. Wir kommen uns dir Iboperelsehleife $(1,0)$ ganz dicht an das dire Punkte $t=0$ morl $t=1$ verbindende Stïek der reellen 1-A.list hreangezogen denken, dann bestrht diess Doppelschleife. Wenn wir ihwn Ausgangspunkt dicht bein Punkt. $t=0$ nehmen, aus folgemben Trilen. Dem geradlinigen Wege von 0 manh 1. einer kleineru, den Punkt 1 im positiven Simne unschlielienden Kurre $C_{1}$. dem geradlinigen Wege von 1 nach 0 . rimer kleinen. den Punkt 0 im positiven Sinne musehließendren Kurv (', abremals dem geradlinigen Wrge von 0 rach 1, der in entgegengesetzten Sime durchlauferen Kurve

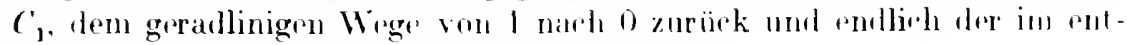
graengesetzten Sime durchlaufenen Kurve $C_{n}$.

Die Bildung des geradlinigers Integrals von 0 math 1 stoft auf Schwierigkriten, wron alas Interpral

$$
i^{\prime \prime}-1(1--t)^{7-1} d t
$$

fïr $t=0$ oder $t=1$ momblich wird. Cin dem vormbeugen. miissen wir vorausetzen. ala die ropllen Trild von $p$ und $q$ wesentlich positiv seien. dam wird nämlich die zu integrierende Funktion selbst für $t=0 . t=1$ von nirlrigerer als der erster Ordnung unendlich. das Integral bleibt infolge hessen mollich. Dann nähern sich aber auch die über die Kurven $C_{1}$. ${ }_{0}^{\prime}$ erstreckten Integrale der Null, wron wir die Dimensionen dieser Kurven ins Unundliche abnehmen lassen; wählen wir z. B. $C_{0}$ als Kreis mit dem Radius $r$. so ist. wenn

gesetzt wird.

$$
t=r e^{w}
$$

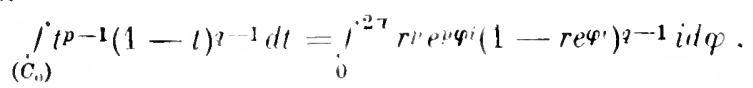

also in der Tat. da der reelle Tril rom positiv ist, für unendlich klejnes $r$ :

$$
\lim _{r \rightarrow 0} i_{\left(\dot{C}_{0}\right)} t^{p-1}(1-t)^{q-1} d t=\left.\lim _{r \rightarrow 0} r_{i}^{p}\right|^{2 \pi} e^{p r i}\left(1-r e^{q}\right)^{r-1} i d \phi=0 .
$$

Wir haben also, mit Rücksicht auf die multiplikativen Falitoren. die zu 


$$
t^{n-1}(1 \quad t)^{7 \cdot 1}
$$

beim Durchlaufen der Kurvon $C_{1} . C_{0}$ in positiven bezirhungsweise negativen Sinne hinzutreter:

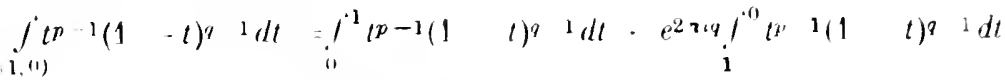

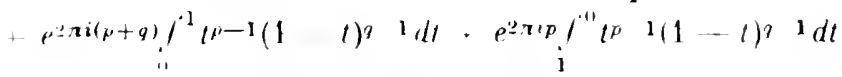

$$
\begin{aligned}
& \text { - (1 p2ncp)(1 p2riq)/ } /^{1} t p-1(1 \quad t)^{q-1}, l l \text {. }
\end{aligned}
$$

Man setzt gewöhnlich

$$
i_{i}^{i 1 p} 1(1 \quad t)^{q}{ }^{1} d t \quad B(p, q)
$$

und nennt dirsen Austruek ein Enlersches lutegral erster Gattung ofler eine Betafunktion. Durch diese Gleichung ist also die Betafunktion definiert, wenn die leellen Trilw von $p . q$ wesentlieh positiv sind: ist diese Bedingung nicht erfiillt. so definieren wir die Betafunktion durch die Gleichung

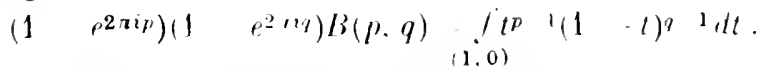

Mit Hilfe dirser Bezeichnung lantert die Gleichume (26) jetzt

$$
i_{1}-\left(1-e^{2+i ;}\right)\left(1-e^{27 i(-\gamma)}\right) B(\beta, \gamma-\beta) \text {. }
$$

und wemn wir unn unter

$$
u_{01} \ldots F\left(\alpha, p^{\prime} \cdot \gamma \cdot x\right)
$$

die aus der Gan lischen Reihe $F(\alpha, \beta, \gamma, x)$ dureh andytisehe Fortsetzung entspringende monogene Funktion verstehen. so ist nach (25)

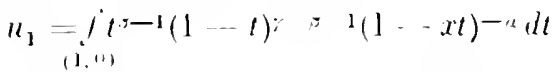

$$
\begin{aligned}
& \text { (1 } \left.e^{2 a i_{i}}\right)\left(1-p^{2 m a-r}\right) B(\beta, \gamma, \beta) F(\alpha, \beta, \gamma, l) \text {. }
\end{aligned}
$$

Damit ist also für das Integral $F(u, p, \gamma, x)$ der fian Bishen IDfferontalgleichung eine fïr alle endlichen Wertr von $x$ (nit Ausnahme von 0 nurl 1) giiltige einheitliche Darstellung gefunden.

Wrundie reellen Teile von $\beta$ und $\gamma-\beta$ wesentlirle posita sind. kann man das $u_{1}$ definierente. über dir Dopprlschleife (1. (1) "rstreckte Integral in ähmlicher Wrise nufornen. Wir wir es unt deut lutegrale (27) gotan haben. Es ergibt sich

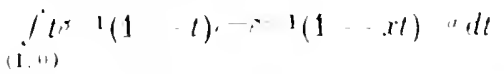

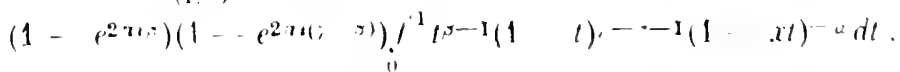

und folglieh haben wir moter der angegelonen Voraussetzung
(B) $\int_{0}^{1} t,-1 / 1$
l) $: 1(1$
.ll) id

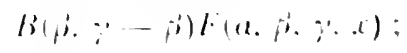


dies ist dio von Euler gegebone Darstellung dor fiunktun fir $\alpha, \beta, \gamma, x)$ durch ein bestimmtes lutrgral.

Setzen wir in diesur Gloiehung an dir Stello von (l al)" dir bureits oben aufgestolltr Rriho

$$
\sum_{k=1}^{\infty} a(u+1) \cdots(u \cdot k-1) x \cdot x^{k} \cdot 1
$$

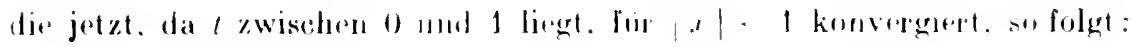

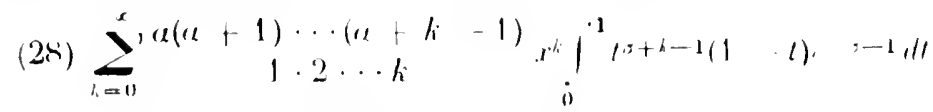

$$
\begin{aligned}
& -B\left(\beta^{\prime}, ; \quad-\beta\right) F(\alpha, \beta, \gamma, x) \text {. }
\end{aligned}
$$

Beachten wir mun, dali

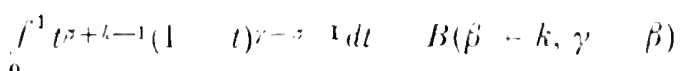

ıst, und vergleichen, inden wir uns für $F(\alpha, \beta, \gamma, x)$ die Gaulis sche Reih. gesetzt denken, in (28) heiderseits die Korffizienten gleich hoher Potonzen von $x$, so ergibt sich:

$$
B(\beta, k, \gamma-\beta)=\begin{aligned}
& \beta(\beta-1) \cdots(\beta-k-1) \\
& \gamma(\gamma+1) \cdots(\gamma-k-1)
\end{aligned} B(\beta, \gamma-\beta)
$$

oder indem wir $\beta=\gamma, \gamma-\beta=q$ setzen:

$$
\text { (29) } B(p-k, q)-(p+q)(p+q-1) \cdots(p+q+k-1) B(p \cdot q) ;
$$

dies ist eine oft grobrauchte Eigenschaft der Betafunktion. Setzen wir noch $p=1$, so ist

$$
B(1, q)=\int_{i}^{1}(1-t)^{q}+d t=\frac{1}{q}
$$

wir haben also die interessaute Gleirlung:

$$
B(k+1, q)=\begin{gathered}
1 \cdot 2 \cdots k \\
q(q+1) \cdots(q+k)
\end{gathered}
$$

Mit Hilfe der Gleichung (B) können wir jetzt auch den Wert von $F(\alpha, \beta, \gamma, x)$ für $x=1$ durch Betafunktionen darstellen; setzen wir nämlich $x=1$. so ist, vorausgesetzt, daß die reelles Teile von $\beta$ und $\gamma-\alpha-\beta$ wesent lich positiv sind,

$$
i_{i}^{1} t,-1(1-t) ; a-s d t=B(\beta, \gamma-\beta) f(\alpha, \beta, \gamma)
$$

oder indem wir das Integral aut der linken Seite durch die betreffende Betafunktion ersetzen:

$$
i(\alpha, \beta, \gamma)=\begin{gathered}
B(\beta \cdot \gamma-\alpha-\beta) \\
B(\beta, \gamma-\beta)
\end{gathered}
$$


dies ist di" in der Nr. 55) (5. 223) r withnte Darstrllung. Aus funktion+ntheoretisehen Gründen erhollt, dal.' die Giiltigkeit dieser Glrichung von der gemachten Voraussetzung iiber $\beta$ und $\gamma-\iota-\beta$ unabhängig ist.

In bezug auf die Betafunktion benurken wir nowh, lat. sirh ans (29) fiir $k=-1$ dir Glejchung

$$
B(p+1 . q) \quad p+q B(p, q)
$$

ergibt; vergleicht man diese mit der ans der Ta Torschen Entwichlung folgender

$$
B(p+1, q)-B(p, q)+\stackrel{d B(p, q)}{d p} \cdot \frac{1}{2 !} d^{2} B(p, q)+\cdots \text { in inf. }
$$

so erkennt man, dalis die Botafuntion der Gloichung

$$
q V+(p+q)\left\{\begin{array}{cc}
d l & 1 d^{2} l \\
d p & 2 ! d p^{2}
\end{array}+\cdots\right. \text { in inf. }
$$

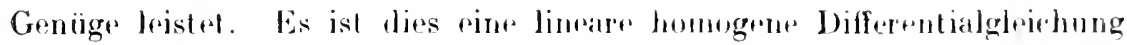
unendlich hoher Ordnung. Joir Theorir soleher Differentialglejehungen ist in neuerer Zeit erheblich gefördert worden ${ }^{3}$ ), sie stehen in engen Z.11sammenhang mit den Volterrasidrun Integralgleichnogen. Gesehichtlich interessant isl, dal.s sich schon linler ") mit ihmen befalit hat.

\section{Darstellumg des zweiten zu $x$ (" gehörigen kanonischen Integrals. Differentialgleichung fïr die Periodizitiatsmoduln des elliptischen Interrals erster Gattung.}

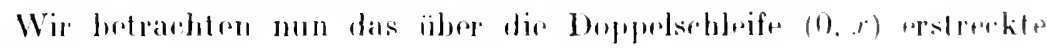
Integral

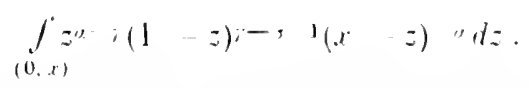

Unter der Vorausselzung. dal. die reedlen Trile von $a-y+1$ und $1-x$ wesentlich positiv sind, formen wir dirses Integral gloinh in ähnlicher Wrise um, wie oben das lulegral (27) ungeformt wurde. inden wir dip Doppesschleife $(0, x)$ dicht an dic gerallinign Verhimbugshine von 0 mil $r$ heranziehen. Fis ergibl sich so

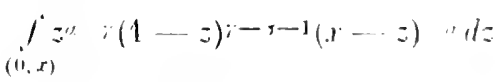

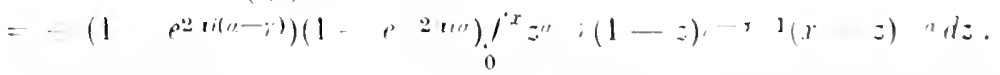

1) Siehe z. B. C. Bourlet. Annales de l'École Xormale (i) 14, 1897. \& 133. T. Lalesco. Lionvilles Joumal (i) 4. 1908. S. 127: E. Ililb. Mathem. Annalen Bd. S2. 1921. S. 1, Bid. s.t. 1921. S. 16 and 4i3.

2) L. Eutor. Inst. Eate. integrallis t. 11 (17699). art. 1195-1202t (1perat. ser. I. vol. 12. S. . 360 ft. 


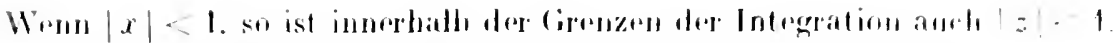

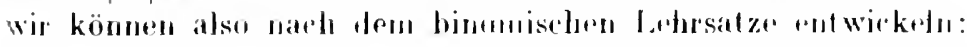

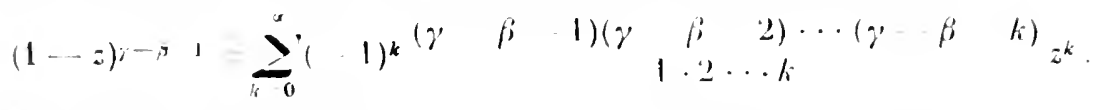

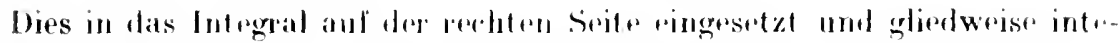
griert gilht:

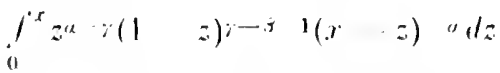

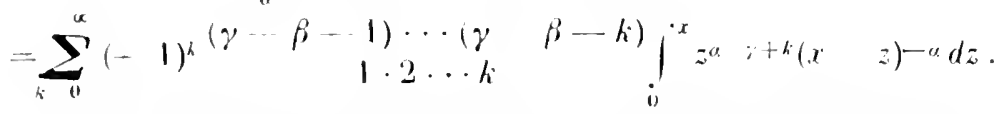

In dem unter dem Summenzoichen auftretemelen Integrale setzen wir

dann wird

$$
z-x t, d z=x d t
$$

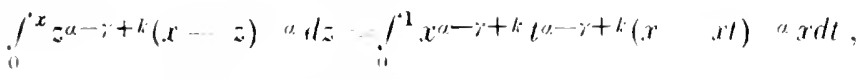

$$
\begin{aligned}
& =x^{1-i+k} B(\alpha-\gamma \cdots 1+k, 1 \quad a) \text {, }
\end{aligned}
$$

ader indron wir die in des rorigen Nummer entwirkelte Gleichung fïr $B(p+k, q)$ benutzen:

$$
\begin{gathered}
l_{i}^{x} z^{\prime \prime} ;+k(x-z-u l z \\
=(\alpha-\gamma+1) \cdots(\alpha-\gamma+k) \\
(2-\gamma)(2-\gamma+1) \cdots(2-\gamma+k-1)^{x^{1-\gamma+k} B(\alpha-\gamma+1,1-\alpha) .}
\end{gathered}
$$

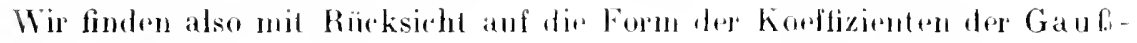
sehen Reihe

$$
\begin{gathered}
f^{x} z-\gamma(1-z)^{\gamma-\gamma-1}(x-z) \cdots d z \\
\left.=B(\alpha-\gamma+1,1-\alpha) x^{1-\gamma F(\alpha-\gamma+1} \gamma-\gamma+1,2-\gamma, x\right)
\end{gathered}
$$

und tiese, zunächst für $|x|<1$ abgeloitete Gleichung bleibt natürlich fïr alle $x$-Werte bestehen. Wir habon also auf diese Weise auch für $u_{02}$ eine allenthalben gïltige Darstellung durch ein bestimntes Integral.

In ähnlicher Weise kann man die Identität dor ïbrigen Doppedschleifenintegrale mit den Elementen der zu $x=1, x=\infty$ gehörigen kanonischen Fundamentalsysteme nachweisen. Wir gehen hierauf nicht weiter ein, bemerken aber, daß die Darstellung der Lösmngen der Gaulschen Differentialgleichung in aer Form von bestimmten Intrgralen sehr. geeignet ist zur Aufstellung der diesen Lösungen entsprechenden Fundamentalsubstitutionen. Diesclbe Methode, durch die wir hier tie Integration der Gaulischen Differentialgleichung durch bestimnte lntegrale geleistet haben, bleibt auch für oine beliebige linearo homogene Differentialgleichung mit rationalen Korftzienten anwendbar. Wir verweisen 
in broug anf dirse Fragen auf Bd. II. 1 ron L. Schlosingors Handbuch. wo auch dir einschlägige Lituratur zu finden ist.

Nur noch oines interessanten Sprzialfalles wollen wir Frwähnung tum. des Fallos nämlich. wo'

$$
\alpha=\frac{1}{2} \cdot \beta=\frac{1}{2} \cdot \gamma=1
$$

Hier ist $1-\gamma=0$. das zweite zu $x=0$ gehörige kanonisch. Integral wird also einen Logarithmus onthalton. Das Integral (17 $14,(5,230)$ lantet jetzt

$$
\int_{i} z(z-1)-(z-r)-d z \int_{i} \mid z(z-1)(z-r)
$$

und de Funktion of (z) ist (S. 290)

$$
q(z)=\frac{1}{2} z(z-1) \div(z-x)-\vdots
$$

Da sich dies. Funktion bein C̈berschreiten rines joden der Querschnitt $l_{0}, l_{1} . l$ mit den Faktor -1 multiphiziert, erhalten wir in diesen Falle brauchbare Integrationswege $L$. inden wir einfach gesthlossen Wege nehmen. die je zwei der Punkte

$$
z=0,1, x, \propto
$$

einschliefen. Bezeichnen wir einen geschlossenen Weg. der dir beiden Punkte $i, k$ in positiven Sinne einschließt, durch

$$
[i . k] \text {. }
$$

$(1, k=0.1 . x . x, \neq k)$

so stellen z. B. die Integrale

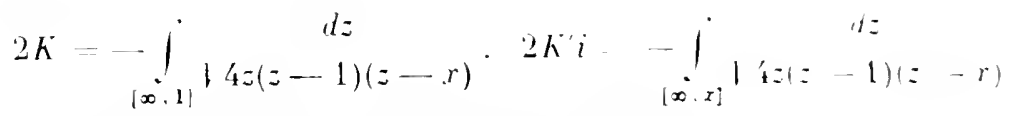

rin Fundamentalsysten der Differentialgleichung

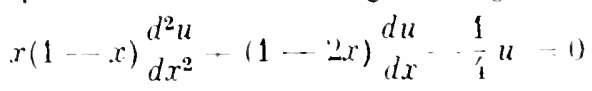

dar. die aus der GauBschen Differentialglechung fur du angegnhenen Werte der $\alpha, \beta, \gamma$ hervorgeht.

Das unbestinnte Integral

$$
\int_{14 z(z-1)(z-x)}^{d z}
$$

ist ein elliptisches lntegral erster Gattung: durch wa Substitution

$$
=-\frac{1}{t^{2}} \cdot d z \quad-2 t 3 d t
$$

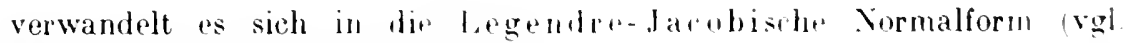
Nr.6. S. 25 und Nr. 29. S. 109) 
mit lem Modul

$$
\left.\int_{1} \quad \begin{array}{ll}
1 & t^{2}
\end{array}\right)\left(1+r t^{2}\right)
$$

$$
k=1 x .
$$

Die Gröbron $4 h$ und $2 h{ }^{\prime \prime} i$ sind nichts anderes, als dir sogenannten Periodizitätsmoduln dieses elliptisehen lutegrals: als Funktionm von $x=k^{2}$ aufgefalist, befriedigen diese alse die Differentialgleirhung (C). dir zuerst von loegendre') aufgestellt worten ist

Setzt man

$$
\begin{gathered}
k^{\prime \prime} i \\
k
\end{gathered}
$$

so hat diese Ciroble (der (Quotient der Elemente eines Fumbanentalsystems der Differentialglejehung (C)). wir man in der Theorir wer elliptischen Funktionen zrigt, die Eigensehaft. dat. ihr Koeffizient von $i$ stets. d. li für jeden von

$0,1, x$

rerschiedenen Wurt von $r$ wesentlinh positiv ist. Der alwolute Butrag von

$$
q-e^{r}{ }^{7}
$$

ist folglich stets kleiner als Eins, und dirser Lmstand lewirkt dir Konvergenz der von Jaeohi in dir Theorir der elliptischen Funktionen eingeführten Thetareihon ${ }^{2}$ ). Mit Hilfe dieser Reihen hat Jacobi ${ }^{3}$ ) dir. folgende Darstellung des Moduls $k$ dureh den Quotientmu $r$ grogeben:

$$
i x=\mid k=\begin{gathered}
2 q+2 q^{2}+2 q+\cdots \\
1-2 q+2 q^{2}+2 q^{9}+\cdots
\end{gathered}
$$

sie lehrt, dab $/ k$ und folglieh auch $x$ eine eindeutige, nur lur Wert. von $t$, deren Koeffzient von $i$ positiv ist, definierte Funktion von $t$ ist. Diese Funktion, die sogenannte olliptische Modulfunktion, entsteht also durch Inversion des Quotienten der Elemente eines Fundamentalsystems der Differentialgleichung $\left.(C)^{1}\right)$. Sie bildet aber nur das erste Glied in der Reihe viel allgemeinerer eindeutiger Funktionen, dir dureh Inversion des Quotienten der Elemente eines Fundamentalsystrus gewisser linearer Differentialgleichungen zweiter Ordnung des Fuchsschen Typus entstehen, und die. nachden $F_{11}$ hs zuerst auf dieselben hingewiesen hatte.

1) A. M. Legendre. Traité des fonctions elliptiques I (1525), S. 620ff.

2) C. G. J. Jacobi, Fundamenta nova theoriae functionum ellipticarum (18:9 Werke 1. (1881), S. 2o31. Gln. (1). (2).

3) ebenda. S. 236 . Gln. (10).

4) Über das Auftreten der Modulfunktion in den nachgelassenen Schriften von C. F. GauB vergleiche man die in Bd. X. 1 (1917) der GauBsclien Werke S. $173 \mathrm{ff}$. abgedruckten Fragmente "Zur Theorie des arithmetisch-geometrischen Mittels" und die zugehörigen Bemerkungen von L. Schlesinger. ebenda. S. 251 ff. 
von Poincaré und Klein zum Gegenstande winer ausgedehnten Throrm. der Theorie der antomorphon Funktionrn. genacht worden sind ${ }^{2}$ ).

Wir geben him nur noch dif Entwicklungen dar Elomanto des zu $x=0$ gehörigen kanorischen Fundamentalsystems der Differentialgleichung (C):

wosellbst

$$
\begin{aligned}
& u_{111}=F\left(\begin{array}{l}
1 \\
2
\end{array}, \frac{1}{2}, 1, x\right), \\
& u_{02}=F_{1}\left(\frac{1}{2}, \frac{1}{2}, 1, x\right)+F\left(\frac{1}{2}, \frac{1}{2}, 1, x\right) \log x .
\end{aligned}
$$

$$
\begin{aligned}
& F\left(\frac{1}{2}, \frac{3}{2}, 1, x\right)=1+\sum_{1}^{\infty}\left\{\begin{array}{lll}
1 & 3 \cdot 5 \cdots 2 v-1 \\
1 & 2 \cdot 4 \cdot 6 \cdot 2 y
\end{array}\right\}^{2} . \\
& F_{1}\left(\frac{1}{2}, \frac{1}{2}, 1, x\right)=4 \sum_{1}^{x}\left\{\begin{array}{l}
1 \cdot 3 \cdot 5 \cdots 21-1 \\
2 \cdot 4 \cdot 6 \cdot 2 \cdot 2
\end{array}\right\}^{2} \sum_{i}^{2}(-1)^{n-1} x^{2}
\end{aligned}
$$

\section{IDie Klassenbeziehung.}

Durch die an Anfang dre Nr. 50 angegebene Transformation (81) A'r abhängig"n. und "in linear" Transformation der unabhängigen Veränderliehen hatten wir das Riemannsehe Differentialsysten (E)

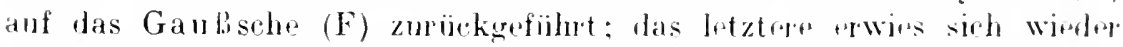
als mit der Gaulischen Differentialgh+jehung $(G)$ äquivalent. Wir können also durch die vollzogene Integration der Gaulis shen Differentialgleirhung anch dir Integration des allgemeinen Riemannschen Differentialsystents als vollzogen ansehen. Dasselbe gilt dann weiter von der allgemeinsten linearen Differentialgleichung zweiter Orduung des Furhsochen Typus

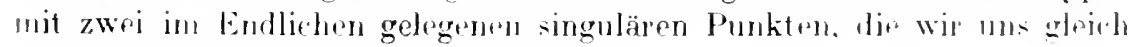
nach 0 und I verlegt denken kömen,

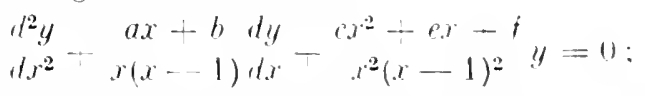

diese Differentialgleichung kann ibhigens durh pim ninfache Transfor-

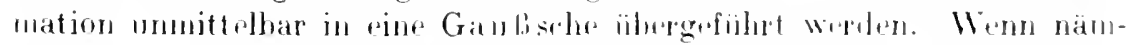
lich dir zu $x=0$ und $x=1$ gehörigen detuminitemene Funtanental-

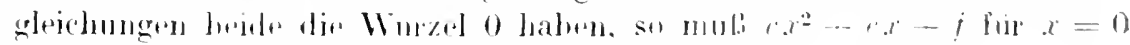
and für $x=1$ versehwindent, dann hat also (30) - when die form

$$
\left(\because(a) \quad x(x-1) \frac{d t^{2} y}{d r^{2}}-(a x+b) \frac{d y}{d x}-r y=0\right.
$$

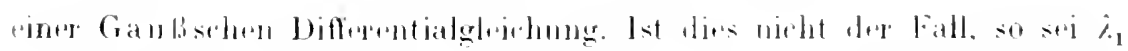

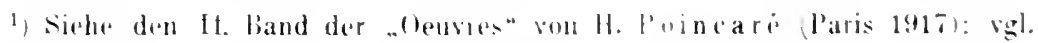
L. Schbesinger. Handbuch. BAl. Il. ‥ wo anch die einschliagige Lileratur angegeben ist. und friehe und fildin. Theorie der automorphen Funtionen J. 13:1\%: 11. 1911:2. 


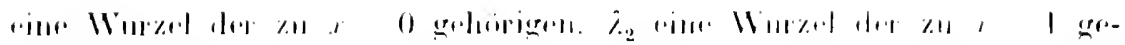

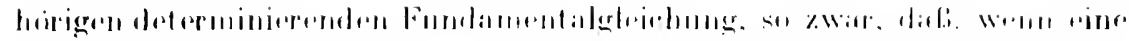

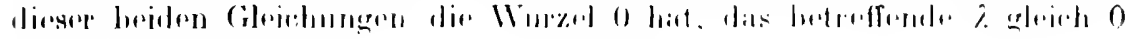

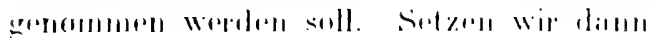

$$
\text { !l } \quad \cdots(x-1)^{i}:- \text {, }
$$

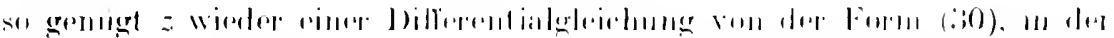

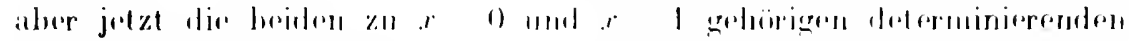

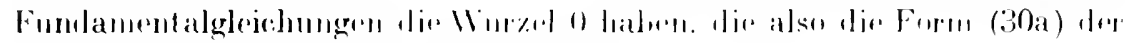

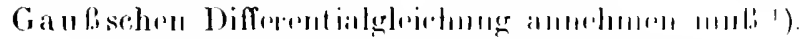

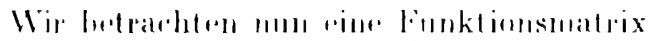

$$
1 .\left(\begin{array}{ll}
r_{11} & v_{12} \\
r_{21} & v_{22}
\end{array}\right) \text {. }
$$

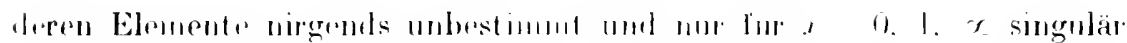

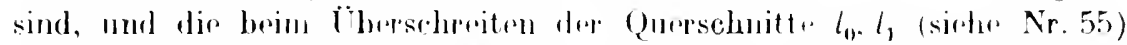
im positiven Sinne mit den Matrizen $\Omega_{0}, \Omega_{1}$ von links her komponiert

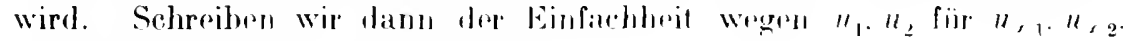
so wird also I mit lor Matrix

$$
\left(\begin{array}{l}
u_{1} u_{1}^{\prime} \\
u_{2} u_{2}^{\prime}
\end{array}\right.
$$

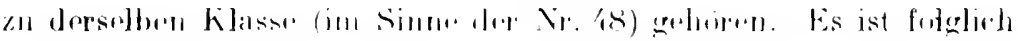

$$
1 \quad\left(\begin{array}{ll}
u_{1} & u_{1} \\
u_{2} & u_{2}
\end{array}\right) \theta_{i} .
$$

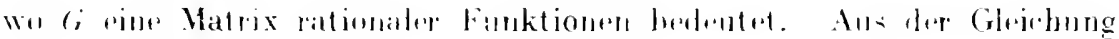

$$
\text { i) }\left(\begin{array}{ll}
u_{1} & u_{1}^{\prime} \\
u_{2} & u_{2}
\end{array}\right) \quad \text { i }
$$

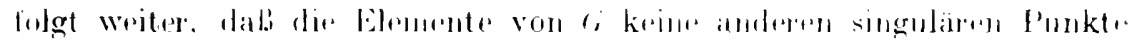

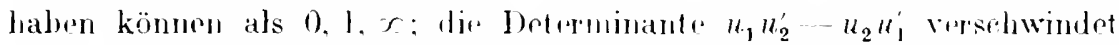

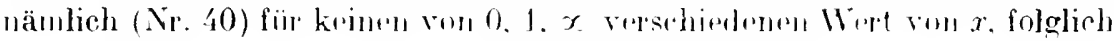

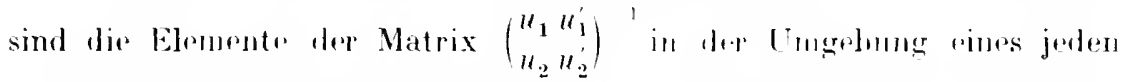
von diesen drei Punktin versehindement r-l'nnklos holomorph. Ungekehrt

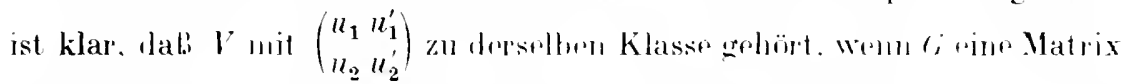
irgendwelcher rationaler Funktionem, Jeren Nomer hörhstens in $.=0,1$

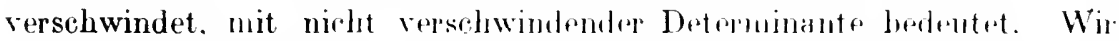

1: Durch die analoge Transformation wird eine Differentialgleichung zweiter Ordnung des Fuchssctien Typus mit den $\sigma$ singulären Punkten $a_{3} \ldots \ldots a_{\sigma}$ in dif Form

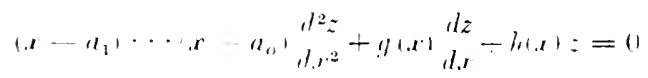

iibergeführt, wo $g(x), h(x)$ ganze rationale Funktionen von den liraden $\sigma-1$ bzu. $\sigma$. 2 bedeuten: vgl. I. Heffter. Dissertation. Berlin 1886. S. 5. 
sagen mun von den Funktionssystemen "11. '

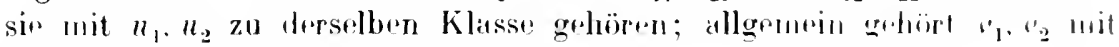
$u_{1}, n_{2}$ zu derselben Klasse, wenn

$$
\begin{array}{lll}
u_{1} & n_{1} g & -n_{1} h, \\
a_{2} & n_{2} g & \cdots
\end{array}
$$

ist, und $g, h$ rationale. Funktionen bestentern, die an kriner von 0, 1. so verschiedenen Stelle memllich werdern. Aus (31) folgt dann durele Jifferentiation und indem man $n_{1}^{\prime \prime}, n_{2}^{\prime \prime}$ vermöge der Differentialgleichune (G) duroh $n_{1}$. $u_{1}^{\prime}$ bzw. $u_{2}, u_{2}^{\prime}$ alusdrürkt:

$$
\begin{array}{ll}
v_{1}^{\prime} & n_{1} g_{1}+n_{1}^{\prime} h_{1} . \\
v_{2} g_{1}+n_{2}^{\prime} h_{1} .
\end{array}
$$

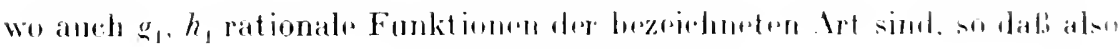

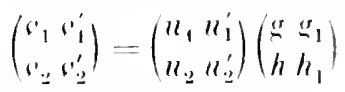

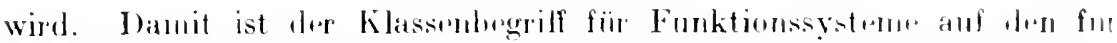
Matrizen zuriekgeführt. Durh melumalige Differmtiation ron (32) lolgt, dialis aturh

$$
\begin{aligned}
& { }_{1}^{\prime \prime}=u_{1} \omega_{2} \quad u_{1}^{\prime} h_{2} . \\
& \ddots_{2}=n_{2} g_{2}-n_{2}^{\prime} h_{2} .
\end{aligned}
$$

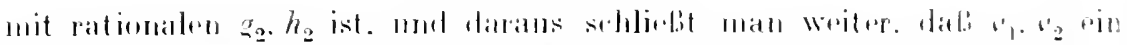

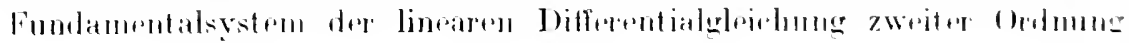

$$
\begin{array}{lll}
\because & g & h \\
\because & g_{1} & h_{1}=11 \\
\because & g_{2} & h_{2}
\end{array}
$$

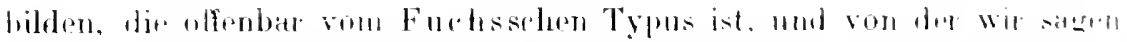

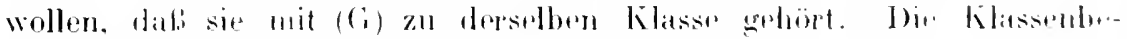

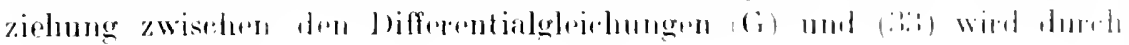
di. Gleirhung

$$
\therefore \quad n g-n+h
$$

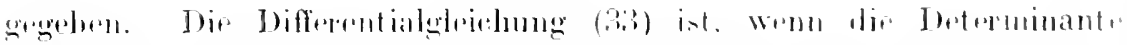

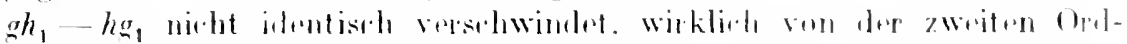
mung: ders ist im allgemeinen der Fall. Ware mämlinh identioh $g h_{1}-h_{-1} \quad$ (), 1 mil setzte tian

$$
\because \begin{gathered}
h_{1} \\
\because
\end{gathered}=i
$$

so wäı"

$$
n \sigma_{1}+n^{\prime} h_{1}=\hat{\lambda}(u g-n h) .
$$

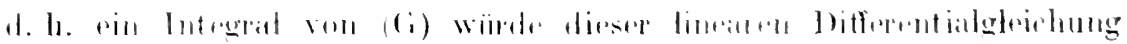

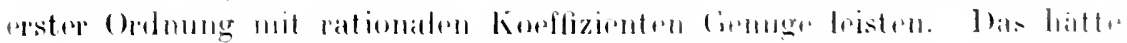

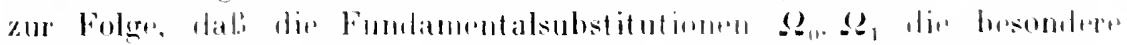

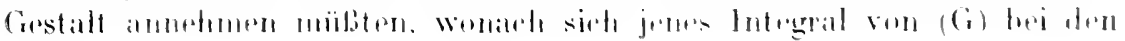




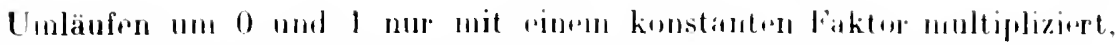

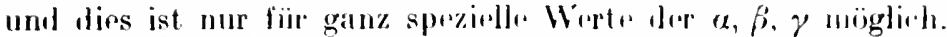

In diesem Fallo sagt man vou dre Diflerentialghojelumng (fi), dar. sie reduzibel sej ${ }^{1}$ ).

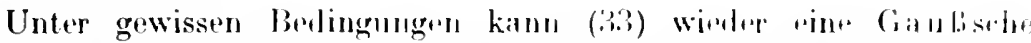
Differentialgleichung sein; Wenn dies der fall ist und die den Grölien $\alpha, \beta, \gamma$ entsprechenden Gë̈lien in dieser Gan lisehen Differentialgleielnung die Werte $\alpha^{\prime}, \beta^{\prime}, \gamma^{\prime}$ hahem. so muli julpnfalls

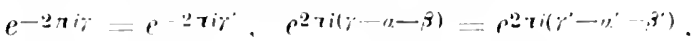

$$
\begin{aligned}
& e^{2 \pi i n}=e^{2 \pi a^{\prime}}, \quad p^{2 \pi i j}=e^{2 \pi i j}
\end{aligned}
$$

sein. Hieraus folgt aber, dal.i dir. Differenzen

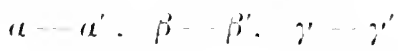

ganze \%ahlen simb. Die Gleichnngen (32) stellen dann bezirhungen dar, die sich in Rolationen zwischen gewissen Ganbschen Rejhen unsetzen lassen. Spezielle Fälle solehere Relationen hat Gialdi in stoner zitierten Abhandlung von 1812 als ..relationes inter functiones r.untiguas" aufgestellt.

\section{6.). Behandlung eines spezielleu Falles.}

Wir betrachten mun den besomeleren Fall. wo

$$
r_{1}=u_{1}^{(n-1)}, r_{2}-u_{2}^{(n-1}
$$

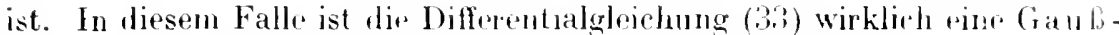
sche. Un dies einzusehen, bemrknll wir. dals (39) durch

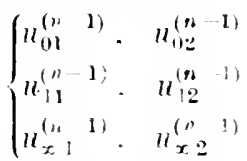

befriedigt wird, und dali diese Integralpaare. zufolge der allgemenen Theorie, die zu den singulären Punkten 0. 1. x gehörigen kanonischen Fundamentalsysteme der Differentialgleichung (.i3) darstellen. Im allgt* meinen kann die Differentialglejehung (33) aulier den singulären Punkten $0,1, \infty$ noch andere singuläro Punkte besitzen, diejenigon Punkt» näılich, für die der Zähler der rationalen Funktion

$$
g h_{1}-\lg _{1} \text {, }
$$

die in (33) den Koeffizienten von $g^{\prime \prime}$ hildet, versehwindet. Ha aber in

1) Der Reduzibilitätsbegriff wurde con Frobenius. Crelles Jourual Bd. Th (1873), S. $236 \mathrm{ff}$. in die Theorie der linearen Differentialgleichungen eingeführt. Auf Systeme wurde er von hoenigsberger (Lehrbuch. 1889. S. 15j) und Schlesinger (vgl. Vorlesnngen. 1908. S. 105) übertragen. Neuere wichtige I'ntersuchungen über diesen Gegenstand hat $A$. Loewy angestellt. 
der Ungehung solcher singuläres Punktr die Integrale $a_{1}$. $1_{2}$ und folglich alle Integrale von (39) holonorph sind. können es nur anberwesentlich singuläre Punkto sein. Sind soledere vorhandern. so ist zufrolge der Furhssehen Relation (Nr. 46. S. 194) die Summe der Wurzeln der zu

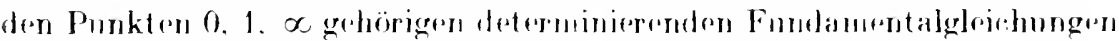
nirht wie hej der Gaubschen Differentialgleichung gleirhl Eins. sondern glejeh einer anderen ganzen Zahl.

Nun gohört abor das erste Paar der Integrale (34) zu den Exporm.n1,

$$
0.1 \quad \gamma \quad(n-1) \text {. }
$$

das zweite Paar zu den lexponenten

$$
0, \gamma \quad a-\beta \quad(n \quad 1) \text {. }
$$

das dritt. P'ala zu don Exponenten

$$
\text { " } " \text { 1. f " l } 1 \text { : }
$$

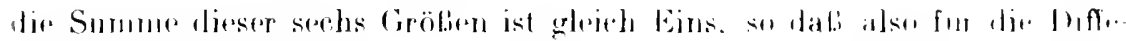
rentialgleichung, der

$$
\left.\left.u_{1}^{(1)} \quad 1\right), u_{2}^{(n} \quad 1\right)
$$

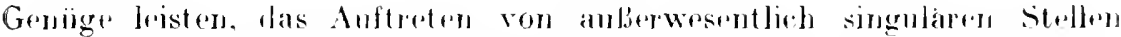

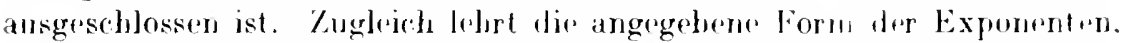

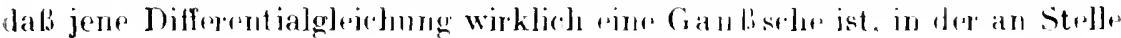
der

$$
a+n-1, p+n-1, \gamma+n-1
$$

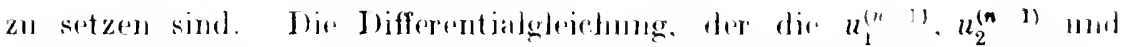
äberhaupt die $(n-1)$ ten Ableitungen der lösungen unserer ursprung.

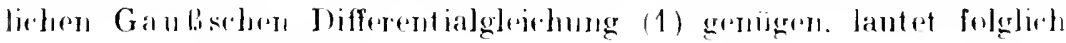

$$
\begin{aligned}
& x(1-r) d^{\left.d 2 u u^{n} 1\right)} d x^{2}+[\gamma+n-1-(u+p+2 n-1) x]^{d u^{\prime n}} d x \\
& \left.-(\alpha+n-1)(p) \cdot n-1) u^{(n} \quad 1\right)=0 \text {. }
\end{aligned}
$$

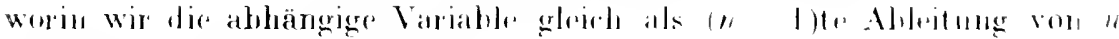
dureh $u^{(n-1)}$ bezeichnte halest.

Multiplizieren wir mun ') diexe. Gleichung mit

$$
x+n 2(1-x)^{a+r} ;+n 1 \text {. }
$$

so kinnen wir sir in der Form

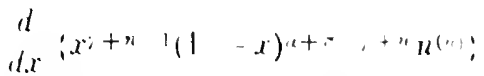

$$
\begin{aligned}
& (a+n-1)(f ;-n-1) x+n 2(1 \quad x), ;+u^{n}, 11
\end{aligned}
$$

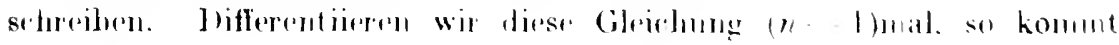

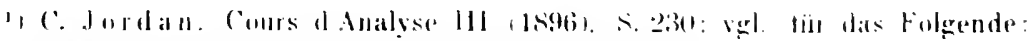

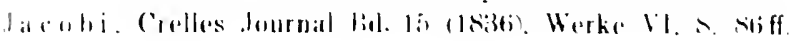




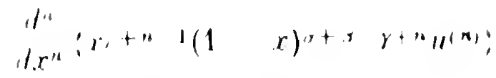

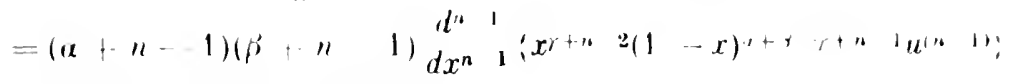

Setzen wir hierin der kidu. nath

$$
\text { n } \quad 1,2, \ldots .
$$

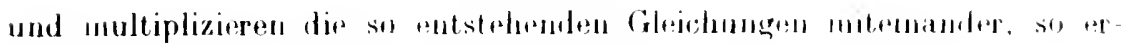
halten wir

$$
\begin{aligned}
& d d^{k} i x+1(1-x)^{\prime+1} ;+k u^{(k)} ; \\
& =\alpha(u+1) \cdots(u+k-1) \beta(\beta)+1) \cdots(\beta+k-1) r-1(1-x) \cdots r u
\end{aligned}
$$

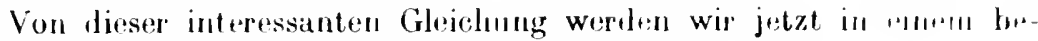
sonderen Falle eine wirhtige Anwendung zon machen haben

\section{bi3 Lege ndresche Polynome.}

Setzen wir in der Gianbschen linihe

$$
F(\alpha, \beta, \gamma, x)=\sum_{j=0}^{\infty}(\alpha(e+1) \cdots(u+v(1) \beta(\beta+1) \cdots(\beta+v-1) x
$$

an die Stedle von $\beta$ eine negative ganze Zahl $-k$, so bricht die lieihe mit den $(k+1)$ ten Gliede ab. sir stellt also eine ganze rationale runbtion $k$-ten Grades von $x$ lar ${ }^{1}$ ).

Offenbar ist alsdann :

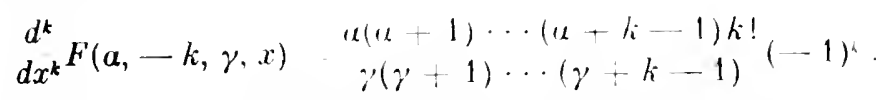

Setzen wir also in die an Sthhusse der vorigen Numuner abgeleitete Gleichung für $u$ diese abbrechende GauBsche Reihte eilı. so erhalten wir nach gehöriger Reduktion

$$
\begin{gathered}
1 \quad F(\alpha, k \cdot \gamma \cdot x) \\
=\gamma(\gamma+1) \cdots(\gamma+k-1) x^{\gamma-1}(1-x)^{\alpha} ; k d x^{k}\left(x^{\gamma+k-1}(1-x)^{\alpha-\gamma}\right) .
\end{gathered}
$$

und inden wir $\alpha+k$ an die Stelle von $\alpha$ setzen,

$$
\begin{gathered}
\quad F(\alpha+k, k, \gamma, x) \\
=\gamma(\gamma+1) \cdots(\gamma+k-1) x^{k}-1(1-x)^{\alpha-\gamma} d x^{k}\left\{x \gamma+k-1(1-x)^{1+k-\gamma} ;\right.
\end{gathered}
$$

Nehmen wir hierin $a=1, \gamma=1$. so ergibt sich

$$
F(k+1,-k, 1, x)=\begin{gathered}
1 d^{k} \\
k ! d x^{k}
\end{gathered}\left[x^{k}(1-x)^{k}\right] .
$$

1) Wir sehen hier einen Fall.| w" die konvergenz der Reihe iibel den Bereich $|x|<1$ hinausreicht. 
Diese spezielle ganze rationale Funktion k-ten Grades geningt rinm saubschen Differentialgleichung. in der

$$
a=k+1 . \beta=-k . \quad \gamma=1
$$

ist. die also die Form hat:

$$
x(1-x) \frac{d^{2} u}{d x^{2}}-(1-2 x) \frac{d u}{d x}-k(k-1) u=0 .
$$

Setzen wir hierin

$$
x=\frac{1}{2}^{-1}, \quad 1=1-2 x,
$$

$\therefore 0$ ist

und allgemein

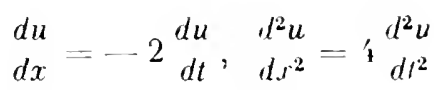

$$
d x^{n}=(-2)^{n} \frac{d^{n} u}{d t^{n}} .
$$

dif Differentialgluichung nimut also die Form an:

$$
\left(1-t^{2}\right) \frac{d^{2} u}{d t^{2}-2 t} d t-k(k-1) u=0 .
$$

und ihr ganzes rationales Integral lautet:

$$
\begin{aligned}
F(k+1 .-k, 1,1-t) & \left.=\frac{1}{2-(-2)^{k}} d d^{k} !\left(\begin{array}{c}
1-t \\
2
\end{array}\right)^{k}\left(\begin{array}{c}
1+t \\
2
\end{array}\right)^{k}\right\} \\
& =\frac{1}{k ! 2 d t^{k}}\left(t^{2}-1\right)^{k} .
\end{aligned}
$$

Man buzedonet diese ganze Funktion k-ten Grades von / gewöhnlich dureh

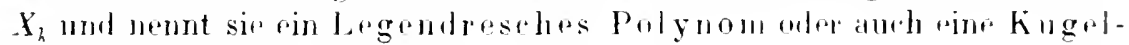
funktion k-ter Ordnung').

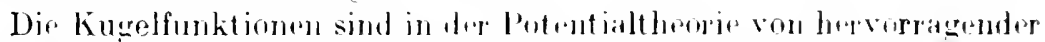
Wirhtigheit; von iliren zahlreichen interessanten Eigunsehaften woilen

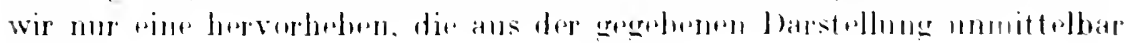
folgl.

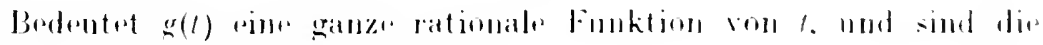
sänutlichorn Wurzeln dè Gleirlunng

$$
\text { (2) } 11)
$$

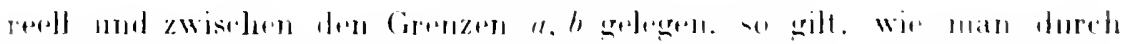

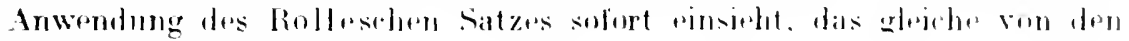
Wurzeln der Gloichung

$$
g^{\prime}(1) \quad(1)
$$

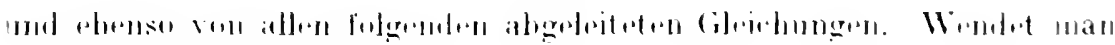
diess Bemorkung anf

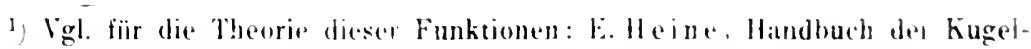
tunktionen Bd. I. "2. Infl. 1sist. we aluch literaturangaben zu finden sind. 


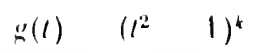

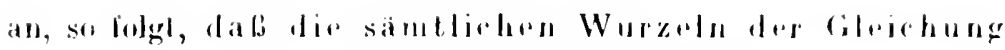

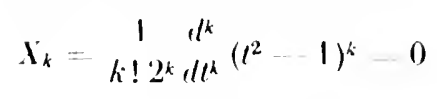

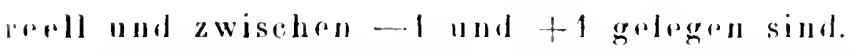

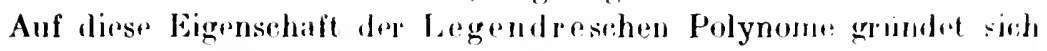
aine wichtige Anwendung derselbers in der 1 ahre von der angenähreter Berechnung bestimuter Intregrale, wofïr man Gaub' Abhandlung , Nothodus nova integralium valores per approximationem inveniendi" vergleichen mag ').

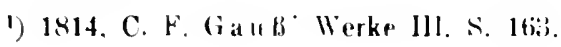


Avhtes hapitil.

\section{Untersuchung dor Integrale in der Umaebung eines Punktes der Unbestimmtheit.}

\section{Differentialsysteme vom Range Eins. Normalreihen.}

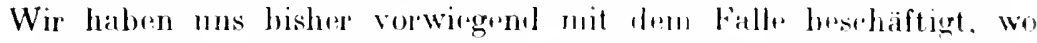
die Integralı einer Differentialgloivhung ondre rines Differrntialsystoms entweder in ror ganzen Ebere krimen Punkt lor Unbestimmtheit lusitzen. oder loch in einem singulären Punkte. anf dessen Tugetnng sich dann die Untersuchung lessehänktr, neht unbestimmt worlen. Dir rnt-

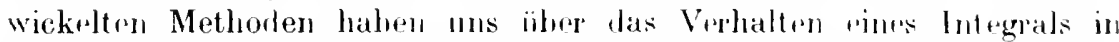
der ganzen Ungebung riness solehen singulären Punktes exshöpfenter

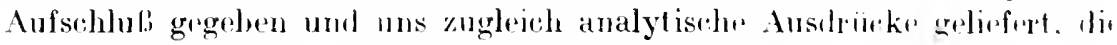

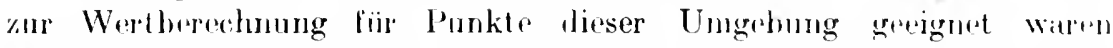

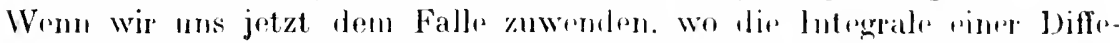
rentialgleithung in pinem singuläran Punkt" mubrstimmt simul. so mussen

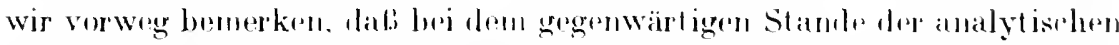

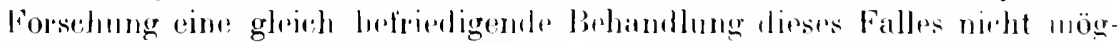

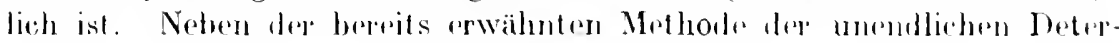

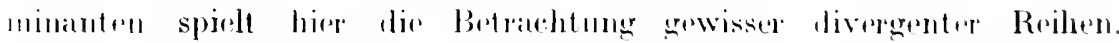

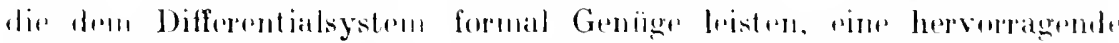

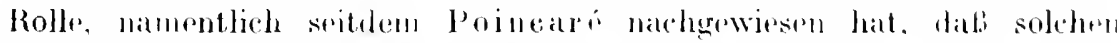

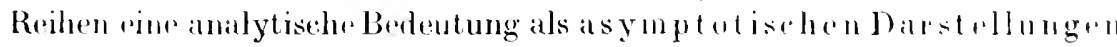

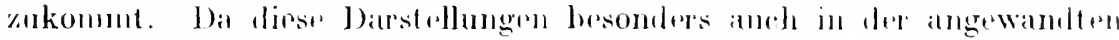

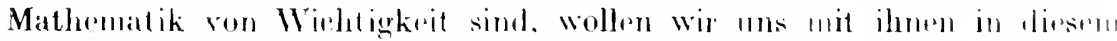

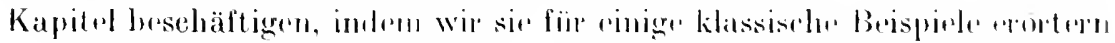

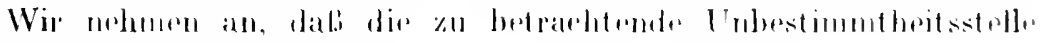
les linearon Jifferentialsystoms

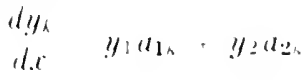

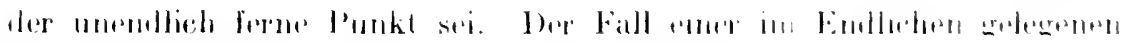

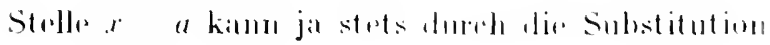




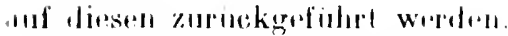

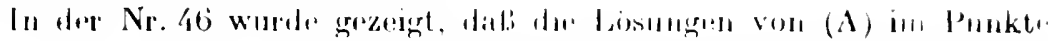

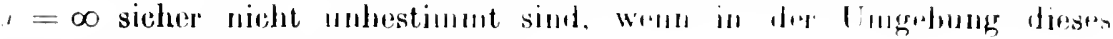
lunktes dir Entwicklungen grellen:

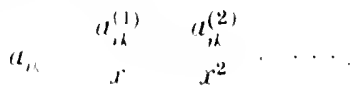

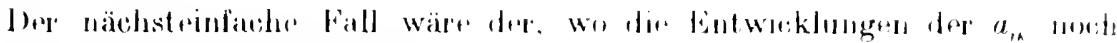
pin konstantes Gilind haben, also

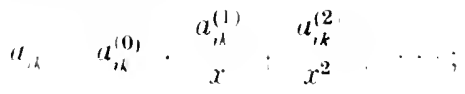

man sagt danm, das System (A) sei fïr $x=\infty$ vom Kang " linn. Fiir die linnare Differentialglejehumer arster Ordnung

$$
d y \quad y\left(a^{(1)}+\begin{array}{c}
a^{(1)} \\
d x
\end{array} \quad \begin{array}{c}
a^{(2)} \\
\left.x^{2}+\cdots\right)
\end{array}\right.
$$

ergäbe sich die läsung

$$
y \quad e^{\prime \prime(0)} x x^{\left(a_{1}\right.}\left(c_{0} \cdot{ }^{\prime}+\frac{c_{2}}{c^{2}}+\cdots\right)
$$

und zwar wäre dir Reilı" $\mathcal{L}_{x^{k}}^{c_{k}}$ in derselben Umgebung von $r=x$ knnvergent, wo die Reihe $\sum^{a_{k}}$ konvergiert. Wir versuchen nun das Systen (A) durch die analog gabildeten Ausilruiskr

$$
y_{1 k} \ldots e^{\prime 2 i^{\prime}} x^{0} \cdot\left(\varepsilon_{, k}^{(1)} \cdot \varepsilon_{, h}^{(1)}\right.
$$

zll befriedigen. Setzen wir

$$
u_{1 k}=x_{i}^{v_{i}}\left(\varepsilon_{i k}^{(0)}+\varepsilon_{i h}^{(1)}+\cdots\right) .
$$

so regibt sich. Wenn wir dir Austritek (2) in (A) rinselzen, nach I)jvision IIIit $p^{a_{i} x}$ :

$$
\begin{aligned}
& \frac{d u_{i 1}}{d x}=u_{1}\left(u_{11}-u_{2}\right)+u_{2} a_{21} . \\
& d u_{i 2}=u_{1} a_{12}+u_{2}\left(u_{22}-u_{i}\right) . \\
& d x
\end{aligned}
$$

Wir wählen nun $\alpha_{1}, \alpha_{2}$ als Wurzeln der sogenannten charaktr. ristischen Gleichung

$$
\left|A^{10}-I \alpha\right|=0
$$

und versuchen. das System (4) durch Auseliäcke ron der Form (3) zu 
befriedigen. Vum dir Brerehnmmg der $\varrho, \varepsilon_{, k}^{(2)}$ möglichst rinfarh zn gestalten. wenden wir dir folgende Transformation an.

Bereshnesı wir ans den Gleichungen

$$
\left.p_{i 1}\left(a_{1 k}^{(0)}-\delta_{1 k} a_{i}\right)+p_{, 2}\left(u_{2 k}^{(0)}-\delta_{2 k} a_{i}\right)-1\right)
$$

die vier Grölen $P_{\text {ih }}$ so ist ihre Determinante $|P|$ von Null versehieden.

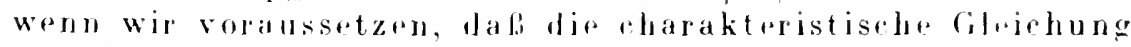
(5) zwei verschielene Wurzaln $\alpha_{1} \cdot \alpha_{2}$ hat. Lis ist dam

In (A) setgent wir IIIII

$$
P(0) \rho, \quad\left(\begin{array}{ll}
\mu_{1} & 0 \\
0 & \alpha_{2}
\end{array}\right) \text {. }
$$

$$
y_{h}=-\pi p_{1 k}+-z 2 p_{2 h},
$$

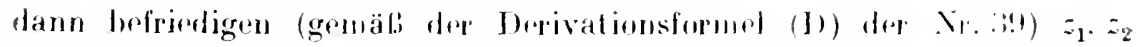
ein Differentialsystem mit We Konffizientenmatrix $P A P$. Setzen wir also

$$
P A(\nu) P=B^{(\nu)}
$$

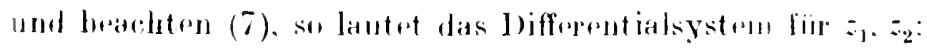

$$
\text { (A') } \quad d_{z_{k}}=a_{k} z_{k}+z_{1}\left(\begin{array}{c}
b_{1 k}^{(1)} \\
x
\end{array}+\cdots\right)+z_{2}\left(\begin{array}{c}
b_{2 k k}^{(1)} \\
x
\end{array}+\cdots\right) . \quad(k=12)
$$

Hiprin uarhen wir nun (2) und (ii) entspeederend den Ansat\%

$$
z_{i k}=e^{\alpha_{i} x} v_{i k}, \quad v_{i k}=x^{i}\left(c_{i k}^{(i)}+c_{i k}^{(1)}+\cdots\right),
$$

wo dann gemäl.i den Transformationsformeln (8)

$$
Y=Z P, \quad U=V P, \quad E^{(\nu)}=C(*) P
$$

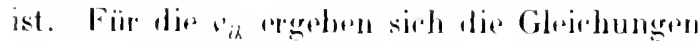

$$
d v_{i k}=c_{1 k}\left(a_{k} \quad a_{i}\right)+v_{i 1}\left(\begin{array}{c}
h_{1 k}^{(2)} \\
x
\end{array}+\cdots\right)+c_{12}\left(\begin{array}{c}
b_{2 k}^{1)} \\
x
\end{array}+\cdots\right) .
$$

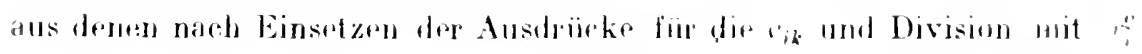

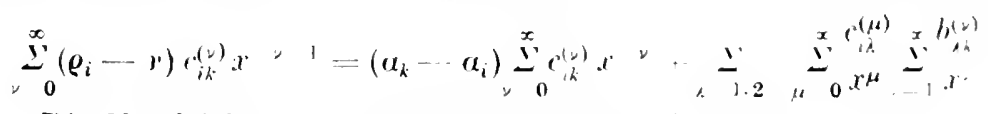

polgt. Die Verghriehung der absoluten Glieder liefort dann

$$
\left(\alpha_{k}-u_{i}\right) c_{i k}^{(0)}=0 \text {. }
$$

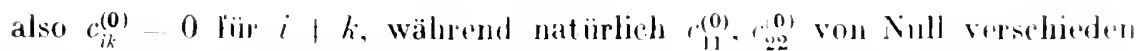

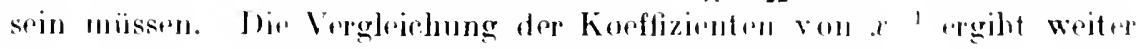
also fiir $k$ i

$$
\varrho_{i} c_{i k}^{(0)}=\left(a_{k}-a_{1}\right) c_{i k}^{(1)}+c_{i 1}^{(0)} b_{1 k}^{(1)}-c_{i 2}^{(0)} h_{2 k}^{(1)} \text {. }
$$

$$
\varphi_{i}=b_{11}^{(1)} \text {. }
$$

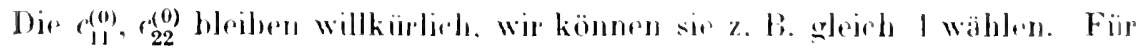
$\iota \mid k$ finden wir inshesondere 


$$
\text { (1) } \quad b_{i k}^{(1)} \quad\left(\ell_{i}-\ell_{k}\right.
$$

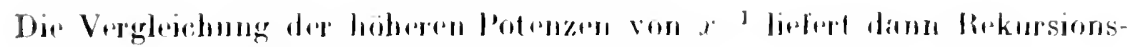

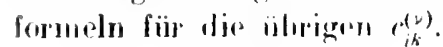

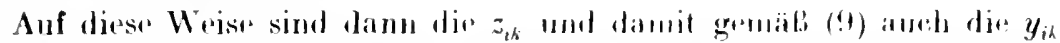
so bestimmt, da! dir Ansiitzr (2) drom Difforentialsystem (1) formal

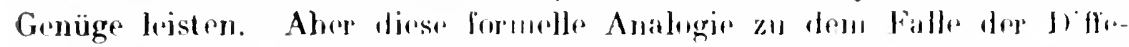
rentialgleichung erster Oplnumg (*) versagt mach der quantitativen Seitu.

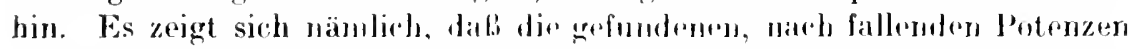

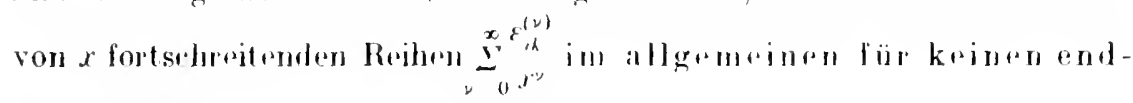
lichen Wret von $x$ konvergieren.

Un das anzusehen, betrachten wir als Beispiol die Differentialgleichung ${ }^{1}$ )

dip den System

$$
\frac{d^{2} y}{d x^{2}}+\left(\alpha_{1}+\frac{\alpha_{2}}{x}\right) d y+\frac{\beta_{0}}{d x}+\frac{x^{2} y-0}{}
$$

$$
\begin{aligned}
& d y_{1} \\
& d x \quad y_{2} \text {, } \\
& \left.\begin{array}{lll}
d y_{2} & f_{0} \\
d x & x^{2} y_{1} & \left(\alpha_{1}+\alpha_{2}\right. \\
x
\end{array}\right) y_{2}
\end{aligned}
$$

äquivalent ist. Sotzen wir in $(10)$

$$
y-\varepsilon_{0}+\frac{\varepsilon_{1}}{x}+\frac{\varepsilon_{2}}{x^{2}}+\cdots,
$$

so finden wir die Rokursionsformml

$$
\varepsilon_{\nu}\left(v(v+1) \cdots\left(\alpha_{2}+\beta_{0}\right)-(v+1) \alpha_{1} \varepsilon_{\nu+1}=0, \quad(\nu-0,1, \ldots)\right.
$$

der Gliederquotient Aer Reihr (11) lantet also

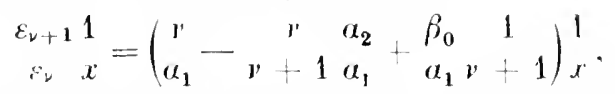

er wächst für jeden endlichen Wert von $x$ mit $v$ ins Inembliche, die Reihe (11) ist also beständig divergent.

Analoge Entwicklungen, wie wir sie für den Rarı Eins, l. h. für die Form (1) der Koeffizienten $a_{i k}$ gefunden haben, gelten aurh, wenn die $a_{i k}$ in der Ungebung von $x=\infty$ die allgemeine Form

$$
a_{i k}=a_{i k}^{(-p)} x^{p}+\cdots+a_{i k}^{(0)}+a_{i k}^{(1)}+\cdots \text { in inf. }
$$

besitzen. Man sagt danm narh l'oincalé, das Systen (A) sei für $l=\infty$ vom Range $p+1$. Läßt man sich wieder von den lür pine Differentialgleichung erster Ordnung

1) Vgl. Picard, Traité 111 (18!!(i). S. 280. 


$$
\left.d y \quad y(a-p) y^{p}+\cdots+a^{0}+a^{\prime \prime} \cdot \cdots \text { in inf. }\right)
$$

sich unmittelbar darbietenden Ergebnissen leitran. so kommt man zu deIII Ansatz

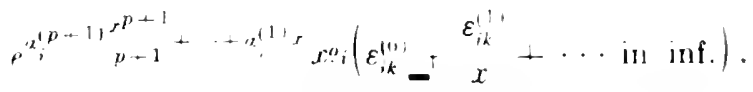

Man findet zunächst. 'daß $\alpha_{1}^{(p+1)} \cdot \alpha_{2}^{(p+1)}$ der charakteristischen Gleichung

$$
A \rightarrow-I_{1}:=0
$$

genügen miissern. und wenn diese Gloichnng verschirdene Wurzeln hat. so ergiht sich eine vollkommene Bestimmung der ïbrigen in (13) auftretrenden Gröben durch die Forderung. dal die Ausdrück (1:i) dem System (A) formell genügen sollent. Aher anch hier sind die Reihen $\sum_{0}^{x_{k}}$ im allgemeinen divargent. Man mennt die Ausdrücke (13) nach 1.. W Thomé ${ }^{1}$ ) Normalroihen. und in den hesonderen Fällen. wo sie konvergieren. Normalintegrale vou Range $p+1$. Wenn die rhalakt... ristische Gleichmeg eine doppelt. Wurzel hat. so treten an dif Stelle ron (13) Ausilriicke anderer Art. indem nänlich in Exponernten von statl der ganzen. grebrochene Potenzen von $x$, und an Stelle der nach fallemin Potenzen von $i$ fortschreitenden Reihen. ganze rationale Funktionen von $\log x$ auftrutrin, deren Koeffizienten nach fallenden Potenzen von $x$ fortschreiten. Man spricht daun mach Faliry²) von anormalen Reihen b7w. von logarithmisehen Normalreihen.

\section{Differentialeleichung zweiter Ordnung. Ricentische Differentialsleichungr.}

Eine lineare Differentialgleichung awrites Ordnung, deren Konffizienten sich in l'nukle $x=\propto$ wir rationat. linnktionen verhalten, hat die For'm

$$
\left.D(u)-x^{2} u^{\prime \prime}+x p_{1} u^{\prime}-p_{2} u \quad 1\right),
$$

wo in der Jungedung von $x=\infty$

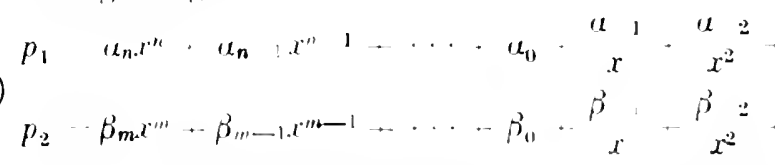

ist. Fur $m=n=0$ haben dir Integrale in $x=x$ keinen Punht der Unbestimutheit. Jur nächsteinfache Fall wäre der. wo nin partikuläres

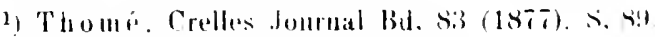

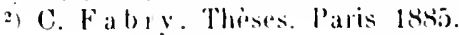




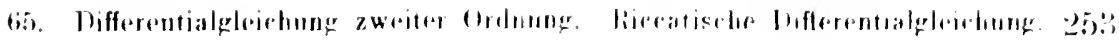

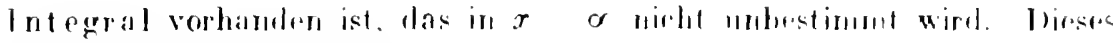
mïBte dann in leg form

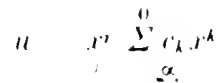

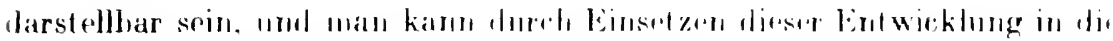

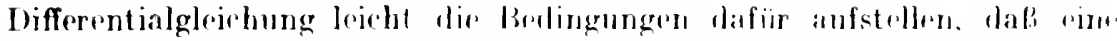
solche, dor Differentialgloichurge formal genügeredr Entwirklung rexist irert

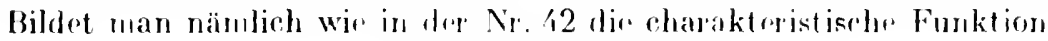

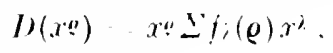

so rhät man dir Rekursionsiomuel

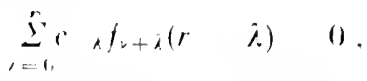

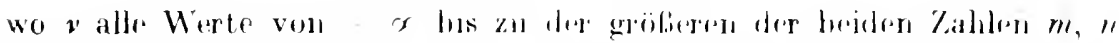

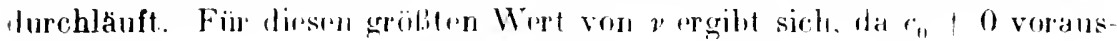
alssetzen ist.

$$
\begin{aligned}
& \text { wenn } m \cdot n \text { ist. } f_{m}(r) \quad \beta_{m} \quad 0 \text {. } \\
& \text { 'und wenul } n \quad m \text { ist. } i_{n}(r) \quad r k_{n}+\beta_{n} \quad 0 \text { : }
\end{aligned}
$$

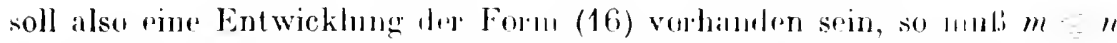

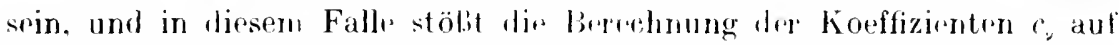
keine weiteren Schwierigkeitur. Nure dir so formal hergestelle Rrihr. (16) ist nicht notwendig komvergent; das zoigt das Breispiel (10) der Nr. 64. Die Aufstellung der Bedingungen für dir Konvergenz ist recht umständlich ${ }^{1}$ ), wir grhen nicht laranf ein und wenden uss zu deut Fallo $m>n$. Man kann dann²)

$$
n-p-1, m-2 p+2
$$

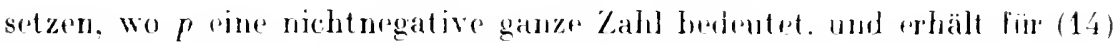
die Form

$$
u^{\prime \prime}+\left(q_{\mu}(x)-\eta_{1}\right) u^{\prime}+\left(q_{2 p}(x) \cdots y_{2}\right) u=0 .
$$

wo $\varphi_{p}(x), \varphi_{\overline{2 p}}(x)$ ganzo rationale Funktionen vom $p$-ten bzw. $2 p$-ten Grade in $x, q_{1}, q_{2}$ in der Ungebung von $r=\infty$ holomorph sind und füı $x=\infty$ verschwinden.

Setzt Iltan

$$
y_{1}-u \cdot y_{2}-i p u \text {. }
$$

so ergibt sich das Differmtialsystrul:

$$
\begin{aligned}
& d y_{1}=x^{p} y_{2}, \\
& \left.d x=-\left(\varphi_{1}+\frac{p}{i}-q_{1}\right) y_{2}-x p_{2}=-q_{2}+q_{2}\right) y_{1} .
\end{aligned}
$$

1) Von neueren Untersuchungen iaber diese Frage nennen wir (1. Perron Mathem. Annalen 70. 1911. S. 1: E. Hjilb. ebenda 82. 19:1. S. 40.

2) V'gl. P'oincaré. Amer. Journal of Math. Vll (1885). S. 2uz tt. 
das für $x=x$ vo!n Rangr $p+1$ ist. Wir sagen dam auch wn dter Differentialgleichung (17), sie sei für $x=-$ wour Range $p-1$.

Von dieser Differentialgleichung gehen wir ${ }^{1}$ ) durch die Substitution:

$$
y=x-p d \log n . \quad n=p^{i} x^{p} y d x
$$

in bekannter Wrise zu einer Riccatischen Differentialglechung für y über. Diese lautet nach Division durch $x^{2 p}$

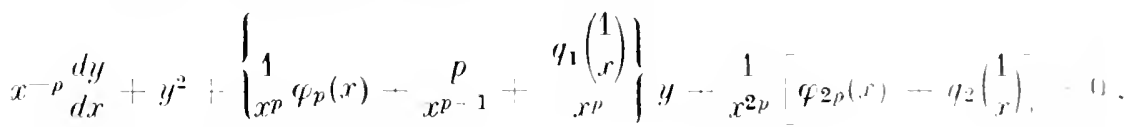

wir schreiben sie in tler Form

$$
\begin{aligned}
& x-p d y+y^{2}-y\left(\partial_{0} \cdots \partial_{1}{ }_{1}^{1}+\partial_{2} \frac{1}{x^{2}}-\cdots\right. \\
& \left(\varepsilon_{0}+r_{1}^{1} r_{2} e^{2}\right.
\end{aligned}
$$

wo also $\delta_{0}, \varepsilon_{0}$ die Koeffizienten der höchsten $x$-Potenzen in $q_{p}(x)$. $q_{2 p}(r)$ sind und die in Klammern stehenden Reiben in einer grwissen Cugebung von $x=x$ konvergieren. Un auch die analoge Form der Riceat ischen Gleichung in der Umgebung anes int Endlichen gelegentu singulärm Punktes a vor Augen zu habers. setzen wir

$$
x=\xi-a,
$$

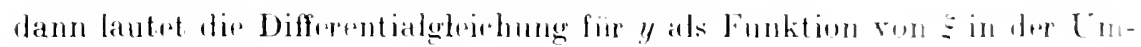
gebung voll $E=a$ :

$$
\begin{aligned}
& (\Sigma-a)^{p-2} d y=-y^{2} \cdots y\left(\delta_{0},-\delta_{1}(\xi-a)\right. \\
& -\varepsilon_{11}+\varepsilon_{1}(\xi-a) \cdots \cdots
\end{aligned}
$$

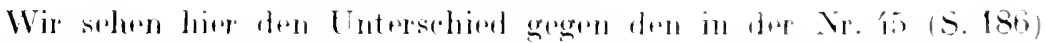
hehandelten Fall der Riceatischen Gleielunger in dem Exponenten den mit der Ableitung der nnbekannten Funktion unltiplizierten Potonz ron $\varepsilon-a$; dort war dieser Exponent ghtereh Eins. hier ist er. da $p=0$ ist.

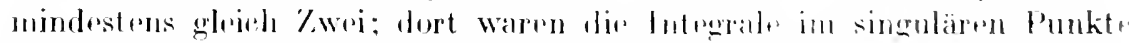
$\xi=$ a nieht unbestimmt, hier ist dagregen $\Xi$ atots rine stolle der

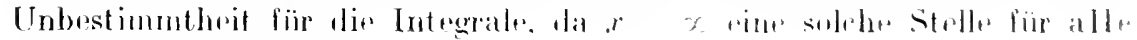
Integrale von (17) ist.

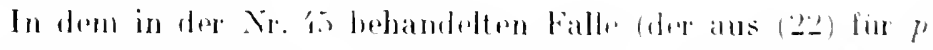

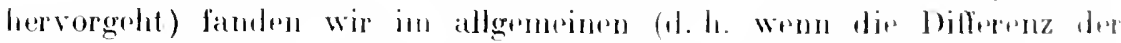

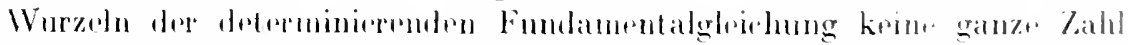
war) zwei in dor Jungebung von E a hohomorphe Integrale. Soher.

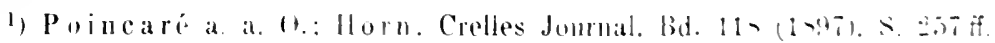




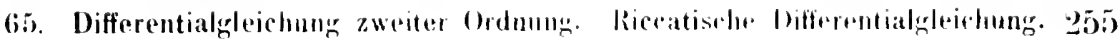

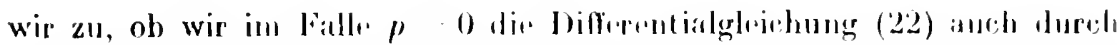
rine nach positiven ganzen loten\%en von 5 a, also dir Difforential

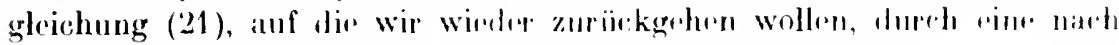
positiven ganzon Potrnzen von $x$ l fortsuhroitrma Roihr.

$$
y \quad \gamma_{11}+\gamma_{1}^{1}+\gamma_{2}+r^{2}+\cdots
$$

formal hefriedigen können.

Sotzen wir diese Rrilur in (21) rin, so kmmm!

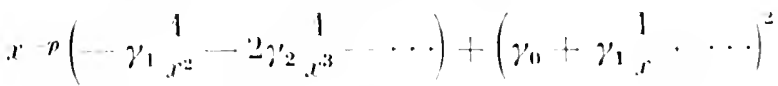

$$
\begin{aligned}
& =\left(\gamma_{0}+\gamma_{1}^{1}+\ldots\right)\left(r_{0}-i_{1}^{1} \ldots\right)+i_{0}+\varepsilon_{1}^{1}+\ldots n \text {. }
\end{aligned}
$$

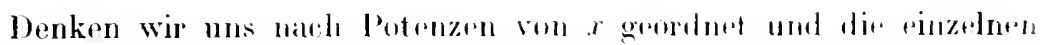
Koeffizienten gleich Null gesotzt, so finden wir als erstes Ciliod der zur Bestimmung der $\gamma_{k}$ dimenden Rokursionsformel:

$$
\gamma_{0}^{\prime 2} \cdot \gamma_{0} \delta_{0} \cdot \varepsilon_{0}=0 \text {. }
$$

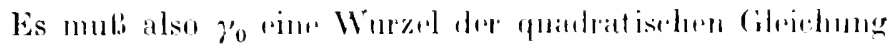

$$
\varrho^{2}+\delta_{01} \varrho: \varepsilon_{0}=-11
$$

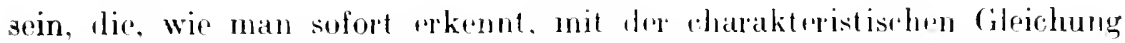
(13a) für das Differentialsystem (19) ïbeleinstimmt. und doren Wurzeln wir durch $c_{1}$, $c_{2}$ bezeichurn wollen. Nls zweites Glienl der Rekursionsformel prgibt sich

woraus

$$
2 \gamma_{0} \gamma_{1}-\gamma_{0} \partial_{1}-\gamma_{1} \partial_{0}-\varepsilon_{1}=0 .
$$

folgt, wenn

$$
\gamma_{1}=-\begin{aligned}
& \gamma_{0} \partial_{1}+\varepsilon_{1} \\
& 2 \gamma_{0}+\partial_{0}
\end{aligned}
$$

$$
2 \gamma_{11}+\theta_{11}+10
$$

ist. Das letztere ist stets der Fall. wonn $r_{1} \mid r_{2}$ ist; wir setzen anch hier voraus, dals dies pintritt, d. h. da fi dir beiden Wurzeln der eharakteristischen Gleichnng voneinander versuhioden sind.

Unter dieser Voraussetzung ist auch die Berechnung dre folgenden $\gamma_{k}$ ohne Schwierigkeit möglich; wir whalten also, entsprehend den beiden Wurzeln $c_{1}, c_{2}$, zwei Reihen

$$
\left\{\begin{array}{l}
y_{1}=c_{1}+\gamma_{11} \frac{1}{x}+\gamma_{12} x^{2} \cdots \cdots, \\
y_{2}=c_{2}+\gamma_{21} x_{x}+\gamma_{22} x^{2} \cdots \cdots
\end{array}\right.
$$

die der Rircatischen Differentialgleichung (21) formal Geniige Ieisten. 


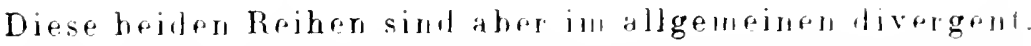

Wir bemerem. dak analoge betrarbtungen anch fior "in bolimbig. Differentialgleichung "rster Ordumg wom der Form

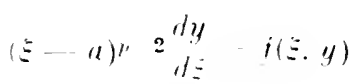

$n>0$,

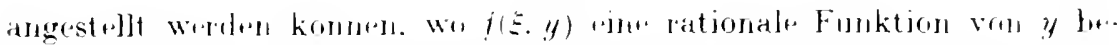

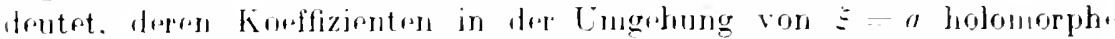

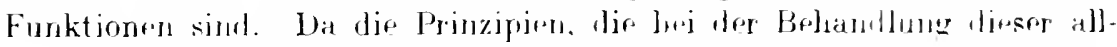
gemeinen Diffrentialgleirhung zur Anwendung kommen. im wesmitlichen dieselhen sind, wie bej ler Untersuchung ler Piecatisehru Diffe. rentialgleirhung (22). so wejelen wir uns darauf beschränken. dirse Prinzipien an dem Fallo dor Rjuratischren Differentialgleirhung za "rlän.

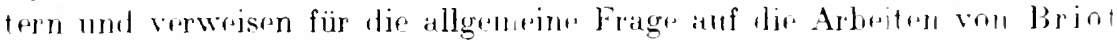
(and Bouquet ${ }^{1}$ ). Poincard ${ }^{2}$ ). Furhs ${ }^{3}$ ) und Horm ${ }^{4}$ ).

Von den Reihen (25) këmnen wir durch dit Substitutinn 201) zus

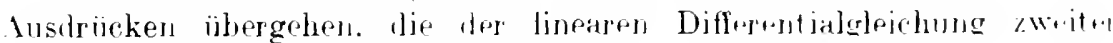
rrolnung (17) formal Gruige lejsten.

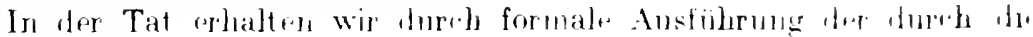
GleirhungeI

$$
\text { 11, } \quad \rho^{i{ }^{2} p_{4} ; "}
$$$$
14=1 \because
$$

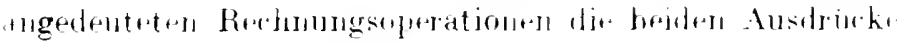

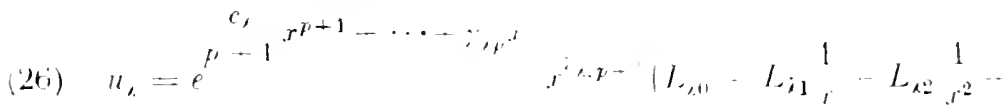

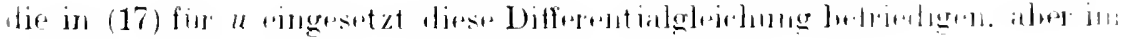

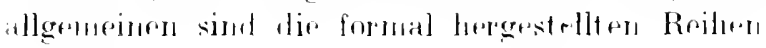

$$
l_{,, 1}+L_{, 1, r}^{1}-l_{-i 2}{ }_{x^{2}} \quad \ldots
$$

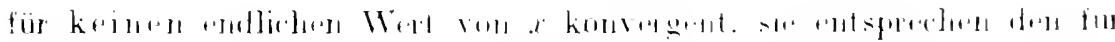

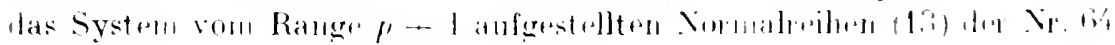

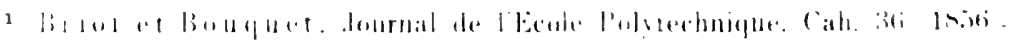

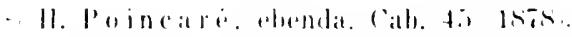

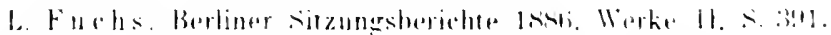

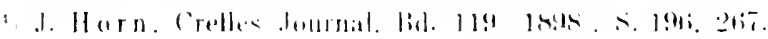




\section{tit. Begrifl der assmptotischen Darstellung. Different ialsleirhungen vom Range Kins. Angen̈̈herte Differontialarleichmgen.}

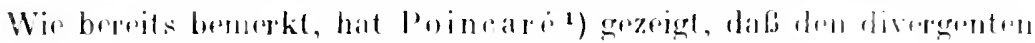

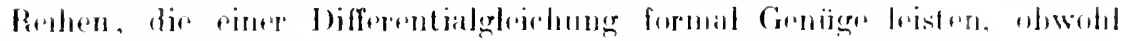

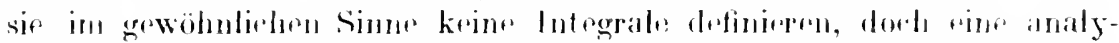

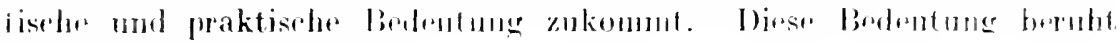
anl dem lolgenten bregrilfe.

Es sfi

$$
a_{0}+a_{3} x^{1}-a_{2} x^{2}+\cdots \text { in int. }
$$

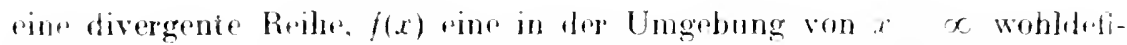

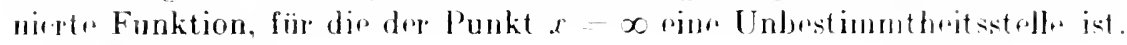

Wir setzen

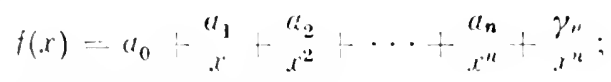

dann nöge fïr jerles positive ganzzahlige "

$$
\lim \gamma_{n}=0
$$

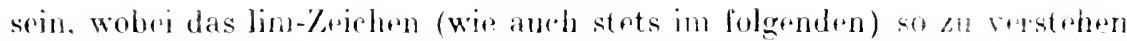
ist, dab $x$ als reolle positive Gröbe dom Unendlichen zusteret. Man sagt dann nach Poincarí, dafi jene divergente Roihe die Funktion $f(x)$ asyonptotisch darstellt, mind selureibt dies:

$$
f(x)-a_{0}+a_{1} x^{i}+a_{2} x^{2}+\cdots .
$$

Es bestehen dann dir mondlich violen Glrichungen:

$$
\begin{aligned}
& \lim \left(f(x)-a_{0}\right)=0, \\
& \lim x\left(j(x)-a_{0}-a_{1}\right)=0 \text {. } \\
& \lim x^{n}\left(f(x)-a_{0}-{ }^{i_{1}} \ldots \cdots x^{a_{n}}\right)=0,
\end{aligned}
$$

die zeigen. in welchen Sinne die Partialsummen dre divergenten Reihe zur angenäherten Berechnung ron $j(x)$ für grofe positive Wento von $i$ bemutzt werden können.

1) H. Poincaré. American Journal. Bu. III (1SS5). Actal lath matica. Bd. VIll (1886): diese beiden Abhandlungen. sowie die Arbeiten von j itorn. Mathematische Annalen. Bd. 49 (1897). S. 453, Bd. 50 1898). S. 52.; biden dit Grundlage der folgenden Untersuchungen (his Nr. 71 einschlieblich). 
Eine derartige analytische Drutung gowisser drepgenter Reihen findet sich auch sehon in älteren Untressehungen. Z. B. tritt int \& st. 29 der oft erwähnten Gaulischen Abhandlung iiber dir narh ihm benannte Reibe'), fire solehr divergerste Rrihr auf. deren Korffizient"rn die sogenannten Ber nomllischen Zahlen sind. und die mit der von Gaub dureb $\Pi(z)$ hezeichaten Funtion (von der weiter nutrin noch die Rede sein wird) in Beziehung gesetzt wird. Ga u L sagt daselbst über die Anwendung soleher divergenter Reilen: ..Ceterum negari nequit. theoriam talium serierum divergentium allue quibusdan difficultatibus premi, de quibus forsan alia occasione pluriluus commentabimur." Gauf ist auf dirse Frage wader in den von ihn veröffentlichten, noch in seinen nachg"lassenen Arbeiten zurückgekomman; die oben wiedergegebene Poincar $\therefore$. Definition der asymptotischen Darstellung diurfte aber wohl die von Gauli, gefiihlten Selwirrigheitun in dre Theorir joner divergenter Reihen heseitigt haben.

In bezug anf die Roihen (25) ergibs sivh num ans den Intersuchungen von Poincaré das folgende Resultat:

Wrin $x$ in einer bestimmten Richtung ins Unmdliche geht. so gibt $\because$ stets ain Integal der Ricratiselen Differentialgleirhung (21), das durch rint dirser Reihen asyuptutisch olargestellt wird. Andert man jene Richtung. so stellt die betreffende Reihe im allgemeinen inmer ein anderes Intregral asymptotiseh dar. In ähnlicher Weise stellen dip Reihen (27) grwisser. durch die Ausitrioter

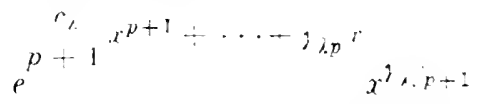

dividerte Integrale der Differentialglewhung zwpiter Ordnung (17) asym.-

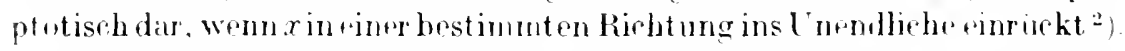

Wir wroden diesen Satz nicht allgemein. sondern nur an pinem Brispiete beweisen: der Gane der Untersuchung wird aber an diesen berspiele derselbe soin wie der. Aen Poinear is im allgemeimen Falle befolgt biat

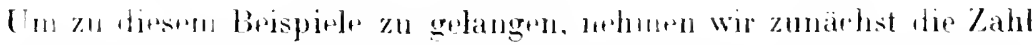

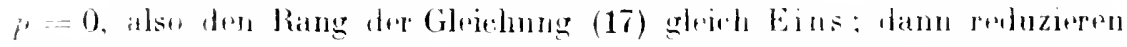

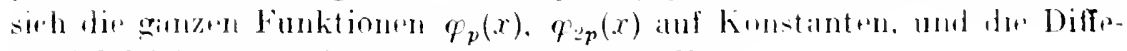

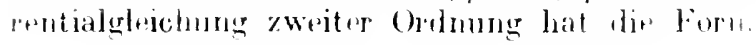

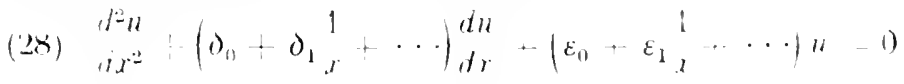

$$
\begin{aligned}
& \text { i) (ia a us Werke Bd. 111. ‥ 16.2 } \\
& \Rightarrow \text { In bezug auf die Bedeutung der formalen lusdrucke (2li) selbst vel due }
\end{aligned}
$$

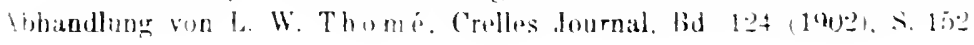


Dif formalen Ausdruckr (2ti) liuturn joutat

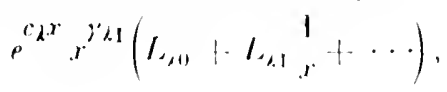

und nach (24) ist

$$
\because 11 \quad r_{\lambda} \delta_{1}+\varepsilon_{1} .
$$

Die Bedeutung des l'oinearésehen Satzes für die Anwendungen läbt sich an diesem Beispiele deutlich machen. Wïrde man durch eine physikalisehe Aufgabe auf die Cileichung (28) geführt werden und handelte es sich un die Bestimunung der lösungen dieser Gleichung fïr sehr grobe Werte von $x$, so wïrde der Physiker, nachderm er erkannt hat, dab ihm die exakte Integration dieser Differentialgleichung wesentliche Schwierigkeiten bereitet, etwa folgendermaßen sehlieben. Wenn $x$ sehr go ob ist, so ist $\frac{1}{x}$, und un so methe eine höhere Potenz von $\frac{1}{x}$ sehr klein, man kann also die mit Potenzen dieser Größe multiplizierten Glieder vernachlässigen und erhält dadurch dir , angenäherte Differentialgleichung"

$$
d x^{2}+\partial_{0} \frac{d u}{d x}+\varepsilon_{0} l l=0 ;
$$

diese hat konstante Korfizirntrn, ihre .,charakteristisehe Gleichung"

$$
\varrho^{2}+\varphi \delta_{0}+\varepsilon_{0}=0
$$

hat die Wurzeln $c_{1}, c_{2}$. also lantre das allgemeine Integral

$$
\gamma_{1}^{\rho c_{1} x}+\gamma_{2^{p: x}} \text {. }
$$

wo $\gamma_{1}, \gamma_{2}$ willkürliche Konstanten bedeuten. Dir leösungen $e^{c_{1} x}, e^{c_{3} x}$ der angenäherten Differentialgleichung sind also hier die Exponentialfaktoren in den formalen Ausdriieken (26a), die der ursprïnglichen Gleichung (28) genïgen. Nun ist man daran gewöhnt, ohne weiteres anzunehmen, daß eine bösnng einer angenäherten Differentialgleichung, die man aus einer gegebrnen Differentialgleichung durch Vernachlässigen gewisser höherer Potenzen des Inkrenments in deren Korffizienten erhalten hat, auch rine Anrihherung an die dureh dieselben Anfangswerte bestimmte Lösmng jener gegrbenen Differentialgleichung darstellt. Dies ist auch in der Tat der Fall, wemn die Lösungen der gegebenen Differentialgleichung in ler lingebung des Punktes, auf den sich das Inkrement bezieht, in konvergente Potenzreihen entwickelbar sind. also stets, wenn dieser Punkt krin singulärer oder wenn er eine Singularität ist, wo die Integrale nicht unhestimmnt werden $\left.{ }^{1}\right)$. Ganz anders liegt die Sache aber in den Falle, wo der hetreffende Punkt, wie in dem hier betrachteten Beispiele der Punkt $x=\infty$, ein Punkt der Unbestimntheit für die Integrale ist. Dann könnt'n allerdings die Lösungen der ange-

1) Vgl. für reelle Werte der Veränderlichen die Nummern is und 5 
näherten Differentialgleichung nath dem Poinearisehws Satze an h angenäherte Werte der Lösungen der ursprünglichen Differentialgleichung linfern. aber angenäherte Werte anderer und anderese solehere Lösungen. je nach der Richtunge in der die unabhängige Variable in den betreffouden Punkt oinsiokt.

\section{6i. I ap lace sche IDifferentialgleichung. Integration Inrch hestimmte Interrale.}

In die Klasse det Differentialgleichungen roun Rang Fins guhin! dir Differentialgleirhung

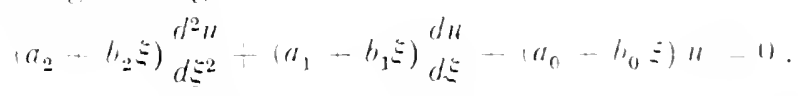

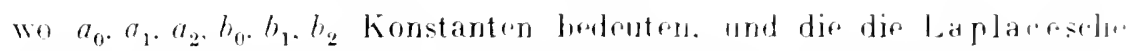
Gleichung genannt wird "). I'm sie direkt als spezirllen fall dele Gleichung (28) ersehrinen zu lassen, setzen wir

$$
a_{2}+b_{2} \xi=r \text {. }
$$

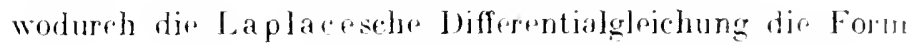

$$
J_{r}(u)=x^{d^{2} u} d r^{2}=\left(\partial_{r r} r+\partial_{1}\right)_{d u}^{d u}-\left(\varepsilon_{n} r-\varepsilon_{1}\right) u=0 .
$$

oder nach bivision durch $r$ die Form!

$$
d^{2} u+\left(\partial_{0}+\frac{\partial_{1}}{x}\right)_{d r}^{d u}-\left(\varepsilon_{0}-r_{1}\right) u=0
$$

annimmt, wo $\delta_{0} . \delta_{1}, \varepsilon_{0}, \varepsilon_{1}$ Konstanten bedenten. Es int dies also gleichsam Jer einfachste Fall der Cilejehung (28) nächst dem Fallo dor Differentialqleichung (29) mit kenstanters Kenfizienten. An dieso Gleichung (an) wollen wir unsewe writeren Brtrathtungen anknüpfon.

Die singulären Punkte der Differentialglejehung (rio) sind $r-0$

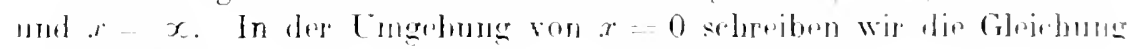
in der Form

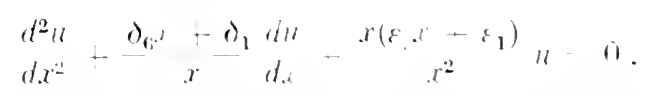

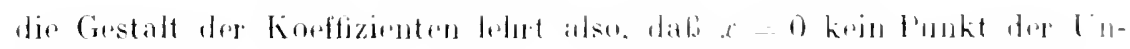
bestimnthest liir die Integrate ist. Die deterniniegende Fundamentalglejehung

$$
0(0-1) \cdot(2), 1)
$$

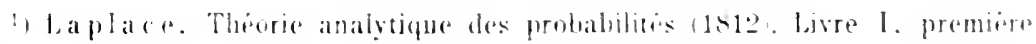

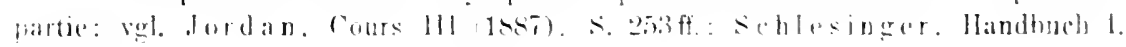
$\therefore$. 1191 it. 


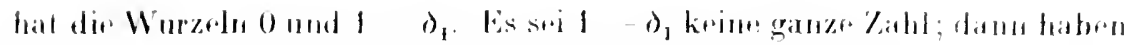

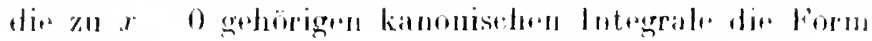

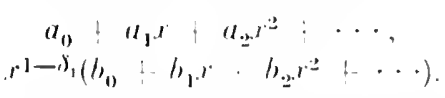

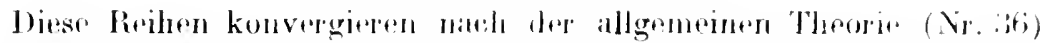
innerhalb riner sich bis zum näthsten singulärn Punkte hin erstrukerden Ungebung von $x=0$; dirser närhstr. Punkt ist aber $x=\sigma$, dir Reihrn konvergieren also beständig. d. h. fiir jeden endlichen Wert von $x$. Tha rine Integral ist demnach rime ganz" transzendente Funktion von $x$, das andere one ebensolehr Funktion, multipliziert mit riner Potenz. von $x$. Dir Korfizienten dieser Reihen sind mit Hilf" der allgemein anfgestellten Rekursionsformel leicht $\%$ armittedn, die Integration der Differontialgleichung (30) kann also als vollzogen angesehen werden.

Aber abgesehen davon, dab uns diese Differentialgleichung als Paradigma für die Untersuchung der Integrale in der Näh' von $x=c$ dienen soll, sprechen auch noch praktische Gründe dafür, bei der erlangten J)arstellung eines Fundamentalsystems nicht stehen zu bleiben. Differentialgleichungen von der Form (30) komminn in den Anwendungen sohr häufig vor und in der Regel handelt es sich un dir Untersuchung ihrer lösungen für sehr grobe Werter von $x$. Für solche Werte konvergieren die aulgestellten Reihen aber sehr schlecht, d. h. man mub sehr viel. Glieder nehmen, un einen vingermabrn angenäherten Wert zu mhalten. Jarum hat schon Laplace selbst eine Darstellung der Lösungen der nach ihm benannten Differentialgleichung durch bestimnte Integrals von der Form

$$
f_{i} w(z) e^{z r} d z
$$

gegehen und wir wollen jetzt zu dieser Darstellung zu gelangen surhen durch Anwendung einer Methode. die der in don Nummern 57-60 fiir die Gaubsche Differentialgleichung entwickelten analog ist, und gleich dieser auf beliebige lineare Differentialgleichungen mit rationalen Koelfizienten übertragen werden kann.

Wir setzen das Integral (31), wo w(z) eine noch zu bestimmende Funktion von $z$ (Dichtigkeitsfunktion), $L$ einen ebenfalls geeignet zu bestimmenden geschlossenen Integrationsweg in der z-Ebene bedeutet ${ }^{1}$ ). in die linke Seite $D_{x}(u)$ der Laplaceschen Differentialgleichung ein, dann ist

$$
D_{x}\left(\int_{L} w(z) e^{z x} d z\right)=\dot{f}_{L} w(z) D_{x}\left(e^{z x}\right) d z
$$

1) Wir bemerken übrigens. daß das Integral (31) durch einen einfachen Grenzübergang aus dem Integrale (1i) der Nr. 57 (S. 2.27) erhalten werden kann. 
Der Ausdruck

$$
D_{x}\left(e^{x}\right)=x^{2} e^{: x}+\left(\delta_{0} x+\delta_{1}\right) z e^{i x}-\left(\varepsilon_{1}:+\varepsilon_{1}\right) e^{i x}
$$

bann in folgender Wrise ungeformt warden:

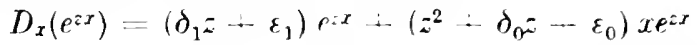

$$
\begin{aligned}
& =\left(\delta_{1} z-\varepsilon_{1}\right) \text { p.s } \ldots\left(z^{2}-\delta_{0}=-\varepsilon_{0}\right) \frac{d e^{i x}}{d z}
\end{aligned}
$$

Setzen wir nun den homogenen lincarer J Jifferentialausdruck erster Ordnung mit der unabhängigen Variabeln :

$$
\left(z^{2}+\delta_{0} z+\varepsilon_{0}\right) \frac{d v}{d z}+\left(\delta_{1} z-\varepsilon_{1}\right):=J_{1}(v) .
$$

so haben wir die der Glejwhung (20) der Nr. 57 (S. 227) analog Gleirhung

wodurch (32) in

$$
D_{r}\left(e^{: x}\right)=A_{z}\left(e^{i x}\right) \text {. }
$$

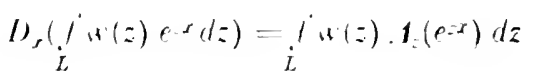

übergeht.

Wir fassen nun $I_{-}(0)$ als homogentul linearen Differentialausdruck zweiter Ordnung auf. in dem rer Koeffizient der zweiten Ableitung gleich Null ist. Dann Jautr.t mach derl Regeln der Yr. 56 der zu $\boldsymbol{J}_{i}(6)$ adjungierte Differentialausdruck:

$$
I_{i}(w)=-\frac{d(q w)}{d z} \therefore r w .
$$

wo

$$
q=z^{2}+\partial_{(\tilde{r}}+\varepsilon_{0} \cdot r=\partial_{1} z+\varepsilon_{1}
$$

zu nehmen ist, und der begleiternde bilineare Differentialausdruch ist einfach

$$
f(\omega, w)=q \cdot s .
$$

Durch Anwendung der Lagrangeschen Identitat

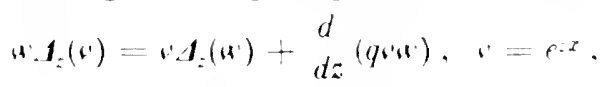

auf den auf der rechten Sojte der Glcichung (33) unter dent Integralzeichen stehenden Ausdruck. verwandelt sich dies, Gleichung in

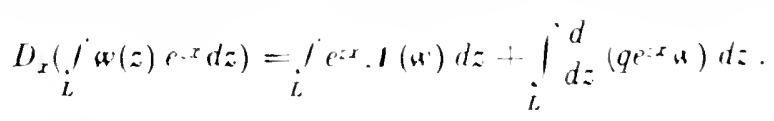

Das Integral (31) wird folglieh eine Lösung der Differentialgleirhung (30) oder

$$
D_{x}(u)=0
$$

darstellen, wenn $w(z)$ d ds looung der Gleichung

$$
J_{i}(w)=0
$$




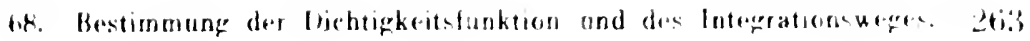

und $L$ so grwahlt wird, dab

$$
\int_{i}^{d} d z(g+a+1) d=0
$$

\section{fix. Bestimmung der Dichtigkeitsfunktion und des Integrationsweges.}

Dir Integration der Differentialelojehung (:3/), dir Poincaré die

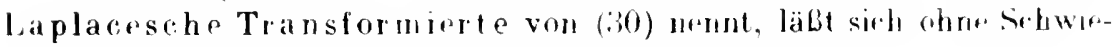
rigkeit vollzichen. Dìse Gleichung lautet nämblich

$$
\begin{gathered}
d(q w) \\
d z
\end{gathered}
$$

woraus sich

$$
\begin{array}{ll}
d \log (q w) & r \\
d z & =
\end{array}
$$

also durch Integration

$$
\therefore \text { - coust. } \frac{1}{q} i^{i r} q^{d}
$$

ergibt. Dir singulären Punkte der Differentialgleirhung (34) sind die Wurzeln $c_{1}$, $c_{2}$ der Glejchung

$$
\eta=z^{2}+\partial_{0}=+r_{0}=0
$$

wir setzen wie iul allgemeinen falle voraus, dab c / / sei. Denken wir uns

in Partialbrüche zerlegt:

$$
\begin{aligned}
& r=f_{1}+\partial_{1}= \\
& q=\left(z-c_{1}\right)\left(z-c_{2}\right)
\end{aligned}
$$

80 ist

$$
r^{r}=-r_{1} r^{-t}=-u_{2}
$$

oder, da

$$
u_{1}=\begin{gathered}
\varepsilon_{1}+\partial_{1} \gamma_{1} \\
\iota_{1}-\iota_{2}
\end{gathered}, \alpha_{2}=\frac{\varepsilon_{1}+\partial_{1} c_{2}}{c_{2}-c_{1}},
$$

$$
c_{1}+c_{2}=-\delta_{0}, c_{1}-c_{2}-\delta_{0}-\partial_{2}, c_{2}-c_{1}=-\delta_{0}-2 c_{1}
$$

ist, mit Rücksicht auf die Glpirhung (24a) (S. 259)

(36)

$$
\alpha_{1}=-\gamma_{11}, \alpha_{2}=-\gamma_{21} \text {. }
$$

Wir haben also bei geeignoter $\mathrm{Wahl}$ der Integrationskonstanten

$$
w=\frac{1}{q}\left(z-c_{1}\right)^{\alpha_{1}}\left(z-c_{2}\right)^{\alpha_{2}}=\left(z-c_{1}\right)^{\alpha_{1}-1}\left(z-c_{2}\right) c^{-1}
$$

und folglich

$$
\left.A_{i}\left(e^{x}, \omega\right)=e^{x} q u=(x) \cdots c_{1}\right)^{\alpha_{1}}\left(z-c_{2}\right)^{\alpha x} .
$$




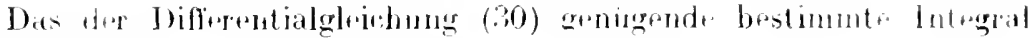
lautet deminteh

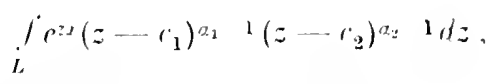

und der Integrationsweg $L$, ist so zu wahtem, dal.s

$$
\int_{i} d z\left[e^{x}\left(z-r_{1}\right)^{a_{1}}\left(z-c_{2}\right)^{x_{z}}\right] d z=0
$$

$\therefore+i$.

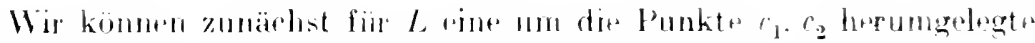

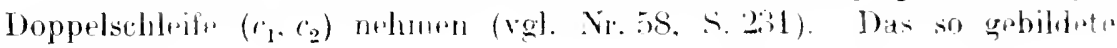
Integral

$$
n_{33}=i_{\left(c_{1} e_{1}\right)} e^{x}\left(z-c_{1}\right)^{\alpha_{1}} 1\left(z-c_{2}\right) x-1 d z
$$

lälit sich, womn wir liir $e^{2 x}$ seine Entwirklung

$$
\rho: r=\sum_{k=0}^{\infty} z^{k} k !
$$

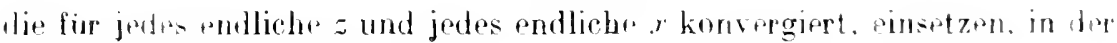
Form

$$
u_{3}-\sum_{k=1}^{x} d_{k !}^{d^{k}} j z^{k}\left(z-c_{1}\right)^{\alpha_{1}-1}\left(z-c_{2}\right)^{n z} \quad 1 d z
$$

darsteflen; "s ist also eine ganze transzendente Funktion und kann silh folglieh von irm oben grefundenen Intrgrale

$$
a_{0}+a_{1} x+a_{2} x^{2}+\cdots
$$

nur dureh einen konstanten Faktor unterseheden. Dhe korftizmenten

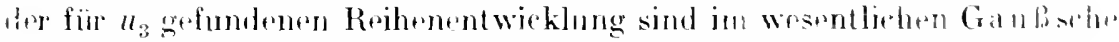
Reihen mit konstantem viertem Element.

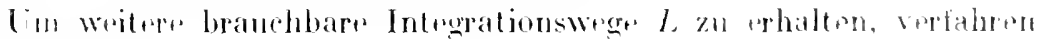
wir wir lolet :

Der berdingung (bisa) wird offenbar gentiggt. Henn wir $L$ so wahlen. dab in Anfungs- und Endpunkte dieses Weges der Austruek

versehwindet. Nun ist

$$
\rho: x\left(z-c_{1}\right)^{a_{1}}\left(z-c_{2}\right)^{\alpha}
$$

$$
\lim _{z \rightarrow \infty} e^{x z}=0 \text {. }
$$

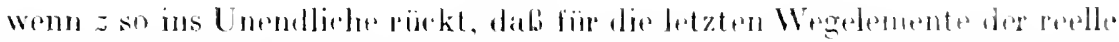
Teil von zer negativ ist. J) nken wir uns alse $L$ so grewäht. dab z in ten

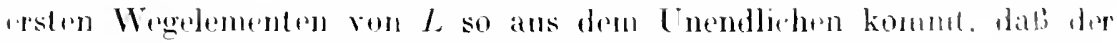
repelle Tril ron $z$ megativ ist. dab dann $L$ e einen im Endlehen golegenen - Wert im positiven simme unschliebt und in derselben Wetise. wie es ans deme Unendlichen gekommen ist, auch wieder dahin zortutkelurt. 


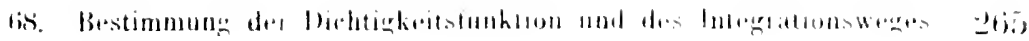

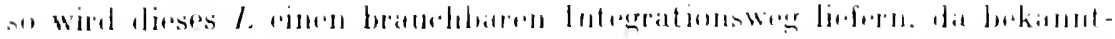

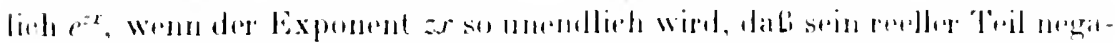

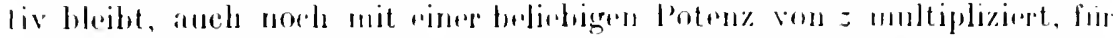

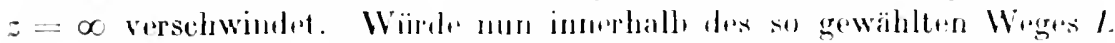

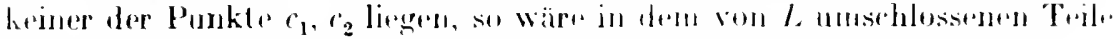

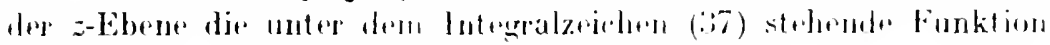

$$
e^{2 x}\left(z-c_{1}\right)^{x_{1}-1}\left(z-c_{2}\right)^{x_{3}-1}
$$

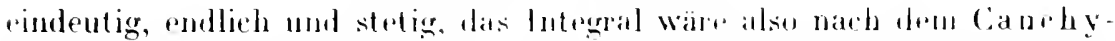
schen Integralsatze gleich Xill. I'm dies zu vermeilen, werden wir $l$.

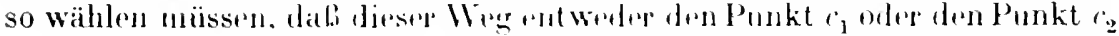
unschliebt. Wir ephalten anf liest Weise zwoi Wege, die wir durch $l_{1}, l_{2}$ bezedehnen wollen, und die wir als rinfache, vou lenendlichen aus um

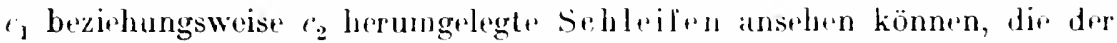
bedingung za genügen luaben, dab in ihren unendlich fremen lbegelementen ler reelle Teil von $z$ negativ sri. Dir so rntstehenden lösungen

$$
\begin{aligned}
& u_{1}=f_{i_{1}} p: x\left(z-c_{1}\right)^{a_{1}-1}\left(z-c_{2}\right)^{a-1} d z, \\
& u_{2}=i_{i_{1}} p x\left(z-c_{1}\right)^{\alpha_{1}-1}\left(z-c_{2}\right)^{\alpha \cdot-1} d z
\end{aligned}
$$

der Differentialgleichung $(30)$ stellen offenbar molutentige runktionen von $x$ dar; da nämlich die Richtung der uneodlich fernen Wegelementw

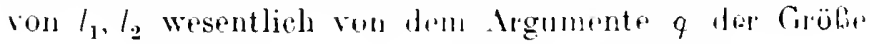

$$
r \text { remi. } r=|x|,
$$

abhängt, so modifizieren sieh die hutegrationswege $l_{1}$. $l_{2}$, Wrun $x$ einfn geschlossenen Cmlauf um den l'unkt $x=0$ vollzieht. die Integralr $u_{1}, u_{2}$ releiden also in allgemeinen eine Wertänderung.

Wir haben jetzt im ganzen drei Lösungen der Diflerentialgleichung (:30) gefunden, es ergibt sich aher sofort die lineare Beziehung. die dies. drei Integrale

$$
u_{1}, u_{2} \cdot u_{;}
$$

miteinander verknüpft. Zunächst ist nämblich klas. dab wir zur Herstellung der Doppelschleife $\left(c_{1}, c_{2}\right)$ die rinfachen Schleifen $l_{1}, l_{2}$ benutzen dürfen. Wenn wir in gewohnter Weise die ins entgegengesetzten sinnt

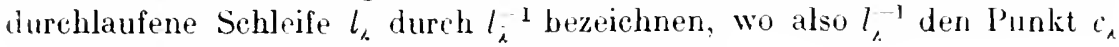
für $\lambda=1,2$ im negativen Sinne umschlieft. so ist

Beachten wir ferner. dab

$$
\left(c_{1}, c_{2}\right)=l_{1} l_{2} l_{1}^{-1} l_{2}^{-1} \text {. }
$$

$$
\left(z-c_{2}\right)^{\alpha_{2}^{-1}} \text {. }
$$

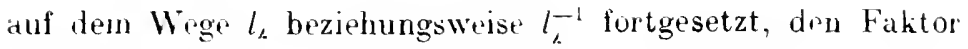

annimut, so folgt

$$
e^{27 i a i} \text { beriehungsweise } e^{\text {-riak }}
$$




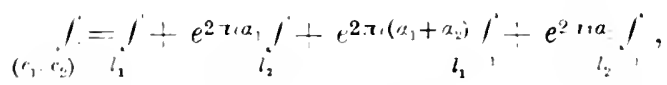

wo wir der kinzeren Schruibwejse weren das unter den Intrgralzeirhen auftretende

$$
\left.e^{z r}\left(z-r_{1}\right)^{a}, 1 z-r_{2}\right)^{a} \cdot{ }^{1} d z
$$

weggelassen haben. Nun ist aber offenbar las Resultat der Interation auf den hintereinander durrhlaufenen Wegen $l_{\lambda}, l_{i}^{-1}$ gleirh Null: wir haben also

und folglich

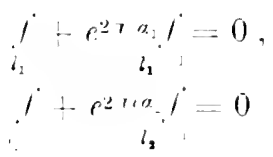

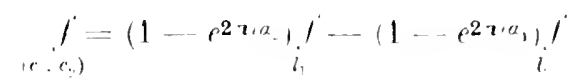

oder

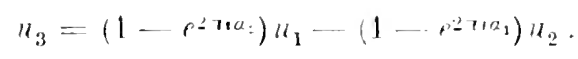

\section{i.4. Reellpositive Werte der unabhïngigen Verinderlichen. Reihenentwicklnng der Integrale. Gammafunktion.}

I'u einen bestimmiten Fall vor Augen zu labem, und auch um zu Formeln zu gelangen, die für die Anwendungen unmittolbar brauchbar sind, setzen wir uns vor, das Verhalten der Inte, ale $u_{1}$. $u_{2}$ fur sohr grobe replle positive Werte der mahhängigen Verämlerlichen $x$ zu erforschen ${ }^{\mathrm{T}}$ ).

Der Bedingung, dab der rende Teil ron $x$ z negatis sei. wirl geningt, wenn wir die Integrationsschleifen $l_{1} . l_{2}$ so wählen. hall sie parallel

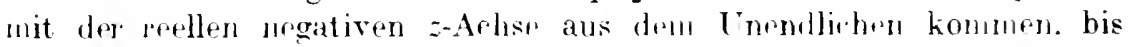
dicht an die Punkte $c_{1}$ respektive $c_{2}$ derangrehen. diese otwat in der Form von kleinen hreisen in positiven sinne umschlielien und dann wieder parallel nit der negation Richtung der reellen z-Achse sich ins Enemdliche entfernen. Wir hetrachton dinn zuvörlerst das längs des so fixierten Wiages / genomment Integral

und setzrn hiprin

$$
u_{1}=i_{l_{1}} e^{x}\left(z-r_{1}\right)^{a_{2}} 1\left(z-t_{2}\right)^{u-1} d z
$$

1) Dab die folgenden Intersuchungen auch äber das lerbalten in der ganzen lmgebung von $r=\frown$ Aufschlub geben kïmen. hat Horn a. a. O. und Acta Mathem. 23 (1s99). S. 171 und auf anderem Wegre W. Jacobsthal in seiner Inauguraldissertation (Strabburg 1899, und Mathem. Annalen it (1902) . S. 1299

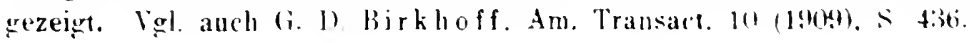


Der der Sibleifo lo entsprechende Integrationsweg $k_{1}$ der $t$-Ebene kommt längs des negativen Teiles der lerellen $t$-Achs" aus dem Unendlichen bis dicht an den Puntit $t$ O heran, umsehliekt diesen in Form eines kleinen Kreises un positiven Sinne und kehrt wieder längs der negativen

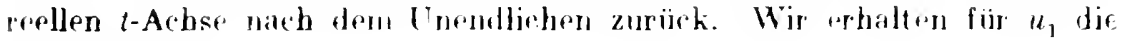
Darstellung

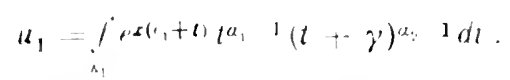

Wenn $|t| \cdots|\gamma|$ ist. so gilt die Entwirklung:

$$
\begin{aligned}
& (t+\gamma)^{\alpha \cdot 1}-\underline{\Sigma}_{k}^{x} j_{k} t^{k}
\end{aligned}
$$

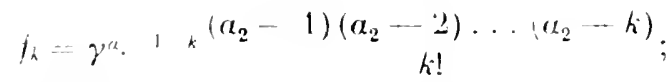

nun ist aber die Bedingung $|l|<\gamma$ offenbar nucht längs des ganzen Integ ationsweges $k_{1}$ erfuillt, wir liirfen also die angegebene Entwicklung nicht in das Integral $u_{1}$ einsetzen. $W^{*}$ nehuren darun nur die $(n+1)$ rrsten Glieder derselben und fügen ein Restglied hinzu:

$$
(t+\gamma)^{a_{2}-1}=j_{0}+j_{1} t+\cdots+i_{n} t^{\prime}+K_{n}(t),
$$

von dell dann

$$
\lim _{n, x} R_{n}(t)=0
$$

gilt. Dies eingesetzt gibt

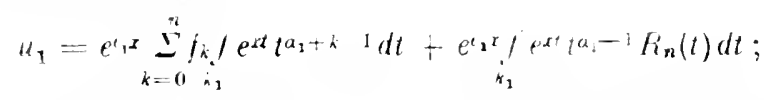

wi»formen zunächst dasunter dem Summenzeichen duftretende Integral um.

Führen wir darin durch die Gleichung

$$
x t=-1
$$

die neue Integrationsvariable $t$ ein, so entspricht der Schleife $k_{1}$ eine Schleife $\lambda$ in der $\boldsymbol{x}$-Ebene, die (da $x$ reell positiv ist) längs der positiven reellen $\boldsymbol{t}$-Achse aus dem Unendlichen herankommt, den Punkt $\tau=0 \mathrm{im}$ positiven Sinne umkreist und sich wieder längs der positiven reellen $\boldsymbol{r}$-Achse nach den Unendlichen entfernt. Es wird

also

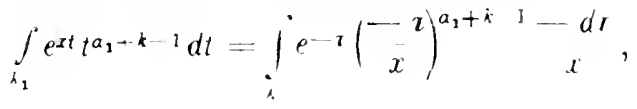

$$
\begin{aligned}
& \int_{k_{1}} e^{x t} t^{a_{3}+k-1} d t=(-1)^{a_{1}+k} x-a_{1}-k j e^{-t} t^{a_{1}+i-1} d t .
\end{aligned}
$$

$$
\begin{aligned}
& u_{1}=e^{c_{1} x} x^{-a_{1}} \sum_{k}^{\infty}(-1)^{a_{1}+k} j_{k} x^{-k} j_{\dot{z}} e^{-l} t_{t_{2}+k}^{a_{2}} d t \\
& +e^{t,{ }_{1}} \int_{i_{1}} e^{\text {st }} t^{a_{1}}{ }_{l}^{l} R_{n}(t) d t .
\end{aligned}
$$


Setzen wir in dem iaber die schleife $\lambda$ ersterekten Integrale $p$ tn die Stelle von $\alpha_{1}+l$, so sehen wir, dab das Integral

$$
\text { ie } \boldsymbol{c}^{p}{ }^{1} \mathrm{~d} \boldsymbol{t}
$$

hier eine ähnliche Rolle spielt, wie bei den analogen Betrachtungen in the Theorie der (iaubschen Differentialgleichung ( Vr.59) Aas Euler.h. Integral erster (iattung. Unter der Voraussetzung, dal der reell T.il ron $p$ wesentlich positiv ist, bleibt das Integral fïr $t=0$ und $t=50$ endlich, wir kömmen es also, indem wir d'n den Punkt $t=0$ umg -

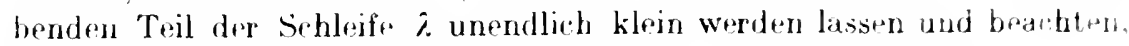
dah $t^{p-1}$ bei positiver Umkreisung des Nullpuntetes den Faktor

$$
e^{2 * t i p}
$$

annimut, in der Form

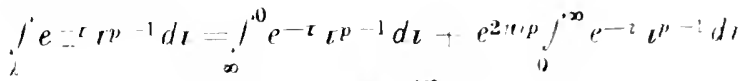

$$
\begin{aligned}
& =\left(e^{2 x i p}-1\right) j_{0}^{\infty} e t^{t} t^{1} d t
\end{aligned}
$$

shlureiben. Man setzt gewöhnlich

$$
\int_{0}^{\infty} e^{-\tau} I^{p-1} d \boldsymbol{\tau}=I^{\prime}(p)
$$

und nennt dies das Eulersehe Integral zweiter Guttung bupr dr. Gammafunktion; Gau B bezeichnet diese Funktion abwrichnd dureh lie Charakteristik $\Pi$, ps ist nach Gaub:

$$
\Gamma(p)=I I(p-1) .
$$

fiü Werte von $p$, deren reeller Teil nicht positiv ist, gilt als leafinition lor Gammatunktion die Gleichung

$$
i e^{-\tau} t^{p-1} d r=\left(e^{2 * t \eta}-1\right) \Gamma(p)
$$

liu negative ganzzahlige Werte con $p$ ist $Y(p)$ unendleh. Wie bodiurten riniger rinfacher Eigensehaften dieser Funktion. dir wir hier ableitan wollen.

Setzen wir

(n) wird

$$
\boldsymbol{\iota}=(g+1) \sigma
$$

$$
\begin{array}{r}
\Gamma(p)=\int_{0}^{\infty} c^{-(g+1) \omega}(g-1)^{p} \sigma^{p} \cdot d \sigma \\
-(g-1)^{p} j_{0}^{\infty}(g-1) \cdot \sigma^{\nu} d \sigma .
\end{array}
$$

oler

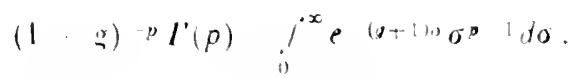

When $|g|<1$ ist, kann $(1+g)^{-p}$ Dach dem binomisehen Lebersatze ent. 


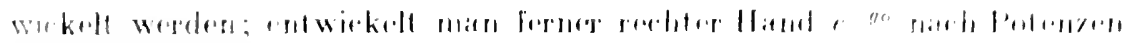
von go, so kommit

$$
\begin{aligned}
& \sum_{k}^{\infty} g^{n}(1)^{k} p(p+1) \ldots(p \cdot k \quad 1) /(p)
\end{aligned}
$$

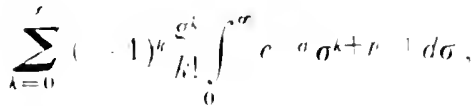

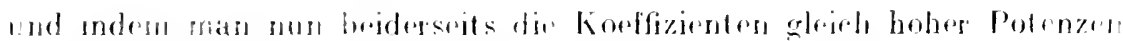
ion 8 rergleicht.

(ai) $p(p+1) \ldots\left(p: k \quad\right.$ 1) $\Gamma(p) \quad j_{0}^{\infty} \rho " \sigma^{k+\mu}: d \sigma \quad \Gamma(p \quad \vdots$ l). wo he rine bolinhige positive ganze hahl bedeuted.

Setzen wir $p=1$ nud beachten. daf.

$$
r(1)-i_{0}^{\infty x} \rho d t=1
$$

Ist, so folgt aus (19)

$$
\Gamma(k+1)=k !
$$

Miesc Gleichung kann als Definition der Gammafunktion für positive ganzzahlige Worte von $k$ dienen; einige ältere Analysten waren bestrebt, aus lieser Definition auch dir Wrethestimmung der Gamnafunktion lür brlisbige Werte von $k$ abzuleiten; iiber diese Art von Untersuehungen vergrloche man die Einleitung zu Woierstra Ablandlung iiber die Throrie ier analytischen Fakultäten ${ }^{i}$ ).

Auf Grund der. Gleichung (43) ist nun

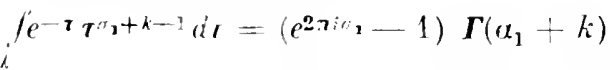

$$
\begin{aligned}
& =\left(e^{2} \cdots_{1}-1\right) \mu_{1}\left(\alpha_{1}+1\right) \ldots\left(\alpha_{1}+l-1\right) \Gamma\left(\alpha_{1}\right) \text {. }
\end{aligned}
$$

dius in ('41) ringesetzt gibt:

$$
\begin{aligned}
& u_{1}=e_{2} x-r_{1}\left(e^{2 r_{1}}-1\right) \Gamma\left(\alpha_{1}\right)(-1) n^{\prime \prime} . \\
& \sum_{k}^{n}(-1)^{k} f_{k} \alpha_{1}\left(a_{1}+1\right) \ldots\left(\alpha_{1}+k-1\right) x^{-k}+f_{1} x j_{i} e^{\text {tt }} t_{1}-1 / l_{n}(t) d t .
\end{aligned}
$$

Wonn wir hier $n$ ins Unondliche warhsen lassen und $R_{n}(t)$ vernachlässigen. so erhalten wir auf der rechten Seite eine (im allgemeinen) divergent" Reihe, die mit Rücksicht darauf, daf nach (36) (S.263)

$$
\alpha_{1}=-\gamma_{11}
$$

1st, mit der ersten der Normalreihen (26a) (S. 259) der Form narh vollkommen ühereinstimmt. In ähnlicher Beziehung steht $u_{2} \mathrm{zu} d \mathrm{de}$ anderen dieser Normalreihen, wi* man durch analog geführte Rechming sofort erkennt.

1) K. Wejerstrab. Crelles Journal, 51 (1856). Werke I, S. 13 . 
Wir werden nun beweisen, daß, wenn $x$ als positive replls Grobe ins Unendliche rückt, für jeden Wert von n

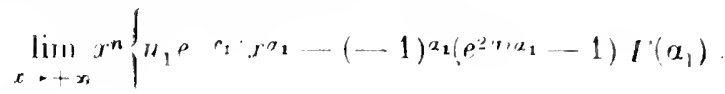

$$
\begin{aligned}
& \sum_{k}^{n}(-1)^{k} l_{k} \mu_{1}\left(a_{1}+1\right) \ldots\left(a_{1}-k-1\right) r k \mid=0
\end{aligned}
$$

ist, damit wird in Sinne der Poincaréschen Definition ( Nr. grf) grzeigt sein, daf die divergente Reihe

$$
(-1)^{a_{1}}\left(e^{2 \pi / \alpha_{1}}-1\right) \Gamma\left(a_{1}\right) \sum_{k}^{\infty}(-1)^{k} f_{k} a_{1}\left(a_{1}+1\right) \ldots\left(a_{1}-k-1\right) k^{-k}
$$

die Funktion

$$
u_{1} e^{c_{1}^{r}} x^{a_{1}}
$$

asymptotisch darstellt, falls $x$ als positive replle Grobe ins Uneudliebe ruckt.

\section{Beweis der asymptotischen Darstellung durch Untersuchung des Restgliedes.}

Mit Rücksicht auf die gefundrnt Darstellung von " bonn die zu beweisende Gleichung (4/4) auch so schreiben:

$$
\left.\lim x^{n+a_{1}} l^{\prime} e^{r t} t^{\prime \prime n_{2}-1} \operatorname{lin}_{n}(t) d t=0^{1}\right)
$$

Die Schteife $k_{1}$ denken wir uns folgendermaben. Von $t=-\infty$ anigehend läuft sie längs der negativen reellen $t$-Achse bis zun Punkte $t=\cdots p$. wo $p$ einen positiven reellen Wert bedeutet, dann in eineis $u n . i=0$ als Mittelpunkt mit dem Radius $p$ heschriehenen heise $C$ im positiven sinne um $l=0$ herum, dam von $t=-p$ wirder längs der nogattren perllen Achse nach $t=-\infty$ zurük. Es ist dann

$$
\begin{aligned}
& x^{n+x_{2}} f_{p+t} t^{2}+h_{n}(t) d t
\end{aligned}
$$

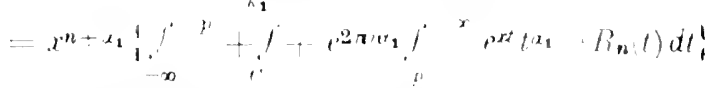

wo der Integrand in dem beiden ersten hetegralen reehter hamd ineselbe ist. wite in dem Integrate auf der linken Seite, und in dem lntegranden des dritten Integrals auf der rechten Seite $R_{n}(t)$ den Wert bedented. den $h(t)$ amninme, nachdem / die Kurve $C$ durehlaufen hat.

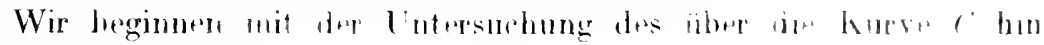
erstreckten Integrals

1) Das lim-Zeichen ist him und im folgenden wioder so zu rerstehen. dab $r$ als reelle positive Gröbe nith linendlich strebt. 


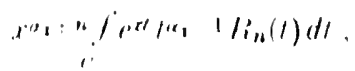

worin wir dureh

$$
r t=-\quad,
$$

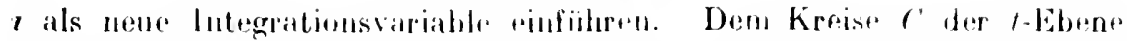
entspricht der um t 0 als Mittulpunkt mit deu Radius p.r beschrimbent $k$ reis $k$ der $t$-Ebente. Inser Integral $(46)$ lanted darum

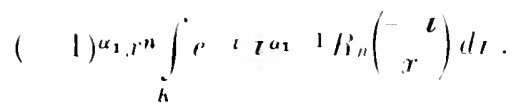

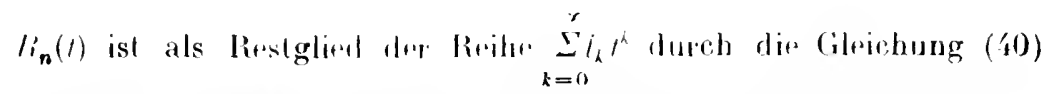
(S. 267) definiert. Wenn diest. Reihe konvergirent, so giht rs brekanntlich stets zwei positive Gröbren $M$ und $a$ von der Besshaffenheit, dal

$$
\left|f_{k}\right|<\begin{aligned}
& N \\
& a^{k}
\end{aligned} .
$$$$
(k=1, \geq \ldots, n)
$$

also ist in diesen Falle für $|t| \cdot\{a$

$$
\begin{aligned}
& |\operatorname{Rn}(l)|=\left|\sum_{n+1}^{\infty} f_{k}\right|+\sum_{n+1}^{\infty}\left|f_{k} t^{k}\right| \sum_{k+1}^{\infty} \begin{array}{c}
\boldsymbol{M} t^{k} \\
a
\end{array},
\end{aligned}
$$

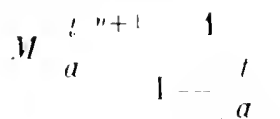

Wir finden demnach für den ahsoluten Betrag museres Integrals die Ungleichung

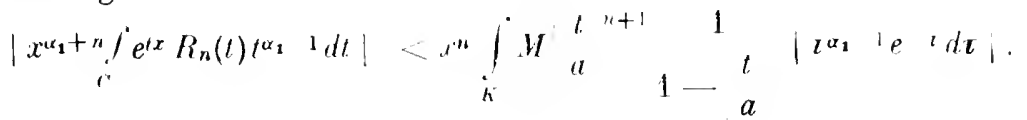

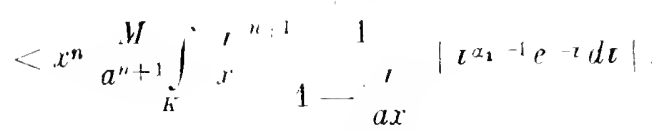

Hierbei ist vorausgesetzt, dab lie lingleirhung $\mid t<a$ anf der Peripherie des Kreises $C$ besteht. d. h. daß $p<a$ sei. Wenu $t$ inmerhalb oder auf der Peripherie von $C$ lifgt, d. h. wenn $t \mid \leq p$ ist. hat man $|t| \Rightarrow p . c$, also

$$
1-\quad 1 \quad 1 \quad p
$$

und folglich

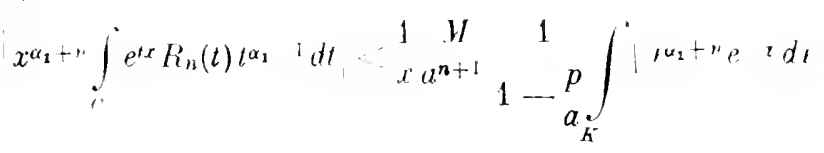




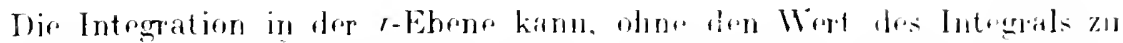

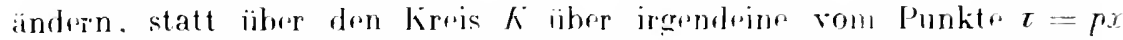
ausgehende und den Punkt $\tau=0$ rinschliefernde geschlessme Kurve erstreckt werden, da ja die unter dem Integralzeidhen stehendr liunk-

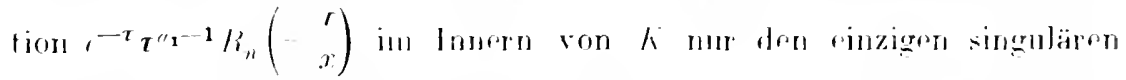
Punkt $r=0$ besitzt. Wir resetzen also $f$ dureh winr ron $!=0$ pr ausgrehende un $\tau=0$ herumgelegte Sehleifu he dir von $r=p r$ läugs der reellen positiven $t$-Arhse bis dieht an,$\quad n$ hrranguht. diesen Punkt in rinem unendlich kleinen Kreise im positiven Sinne muschlieft und wieder längs der reellen $T$-Arhse nach $p x$ zuriickkehrt. Dir durchgeführten Absehätzungen bleiben auch fïr diesen Integrationswegr li giiltig. Nehmen wir $n$ so groh, dafo der reelle Teil rom

$$
a_{1} \div n \div 1
$$

positiv ist, so bleibt das Integral

$$
\int x_{n}+1+n e^{-t} d t
$$

im Punkte $\boldsymbol{\tau}=0$ endlich und verschwindet denmach, wenn man es über den unendlich kleinen kreis um $r=0$ erstreekt: wir haben folglieh

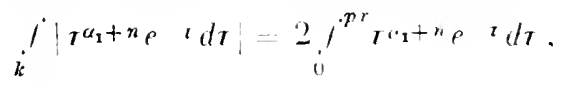

also lautet unsere T'ngleichung

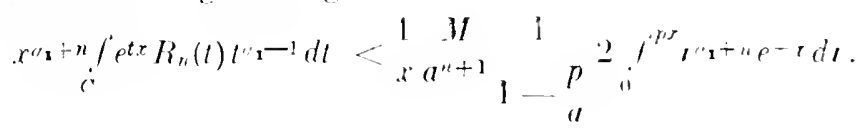

Wir untersuchen nun den Grenzwert der rechten Seite. whom $x$ als positive roell. Gröbe ins Enendliehe geht. Yunirhst ist

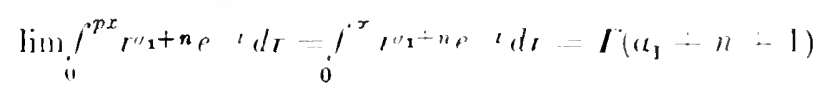

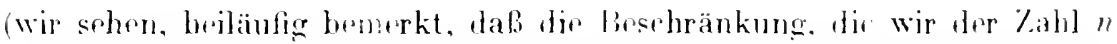
aluferlegt haben, unwesentlich ist, sie wurde nur arnacht. um den Girnziibergang bequemer vollziehen zu können): die iibrigen lionstanten Fah-

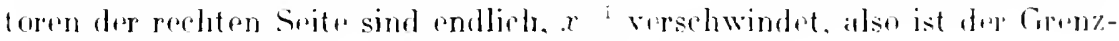
wert gleich Null, 1. l. wir hahen als erstes Resultat

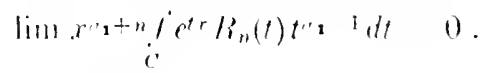

Es folgt nun die Contersuelomer der anf dir geraullinigen Teile von $h_{1}$ berä̈glichen Integrale anf der reshten seite von (45). Für diese grilt

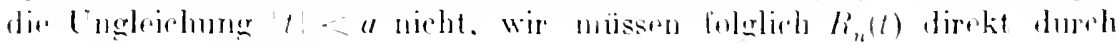

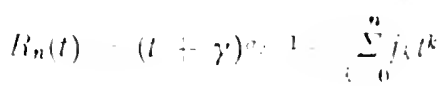




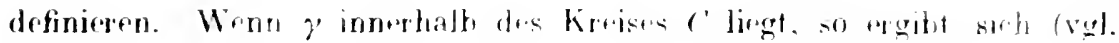
S. 27(1)

$$
i_{n}(t) \quad, 2 \cdots a:(t \cdot \gamma)^{\prime \prime} \quad 1-\sum_{k=0}^{n} j_{k} t^{k}
$$

wir haben also. abgesehern won kunstanten Faktorrn, die briden Jntregale

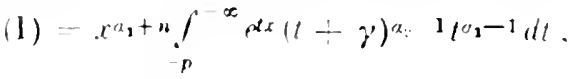

$$
\begin{aligned}
& \text { II) }=\sum_{k=0}^{n} f_{k} x^{\alpha_{1}+n} !^{-x} t^{0}+k-1 e^{t x} d t
\end{aligned}
$$

zu nntersuchesu. Wir beginnen mit (1).

Fïr hinreichend groBe Werte von $t$ ist offerbal

$$
|\log t|<|t|, \quad|\log (t+\gamma), \cdots| t,
$$

man kann folglich pine positive Gröbe $h$ stets so angeberı, dab

$$
\left|\log t^{a_{1}-1}(t+\gamma)^{a_{2}-1}\right|<h|t| \text {. }
$$

also

$$
\left|t^{\alpha_{1}-1}(t+\gamma)^{a_{2}-1}\right|<e^{t_{t} t}
$$

ist. In dem Integrale (I) ist $t$ während des Verlaufs der Integration jeell negativ, also

und folglich

$$
\left|t_{1} 1(t+\gamma) \cdot 1:-1\right|<e^{-h t}
$$

$$
\begin{aligned}
& |(\mathrm{I})|<x^{u_{1}+n} \stackrel{!}{-\infty}^{-\infty} e^{t(x-h)} d t \\
& \left.-x_{1}^{\prime n_{1}+n} \mid\left[\frac{e^{t(x-h)}}{x-h}\right]_{t}-\infty \quad x-h e^{-\mu(x-h)}\right\} .
\end{aligned}
$$

Für ein hinreichend grobes positiv reelles $x$ ist $x-h$ positiv. also $\lim _{x \rightarrow-\infty} e^{t(x-4)}=0$

ebenso ist

$$
\lim x^{a_{1}+n} \frac{e^{-r(x-h)}}{x-h}=0
$$

und folglich

$$
\lim (I)=0 \text {. }
$$

Bei der Untersuchung von (II) nehmen wir das unter dom Summenzeichen stehende Integral

$$
x^{a_{1}+n} !_{-t^{\prime}}^{\infty} a_{1}+k-1 e^{t x} d t
$$

und führen darin wieder

$$
-r=t x
$$

als neur Integrationsvariable ein. Das Integral wird dann gleich

$$
x^{n-i}(-1)^{a_{1}+i} j^{+\infty} c-t l^{a_{1}+k-1} d t \text {; }
$$

$\mathbf{s c h l e s i n g e r , ~ D i f j e r e n t i a l g ] e i c h n n g e n . ~}$ 
riwekt $x$ als positive Gröbe ins Unendliche, so fält die untere Grenze px mit der oberen $+\infty$ zusamonen, das Introgral reduziont sirh also auf den Girenzwert seines Elementes fïr $, p x, x=+\infty$, und wir erhalten:

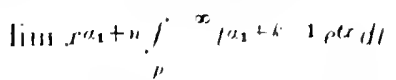

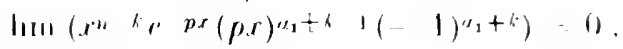

daralls folge abere dalis anch

$$
\text { (149) } \quad \lim (11)-0
$$

isi.

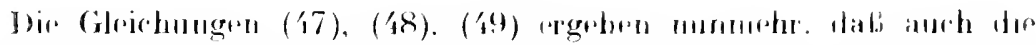

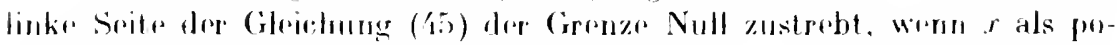

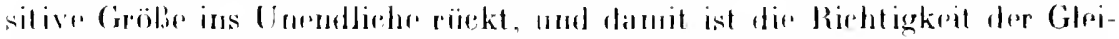

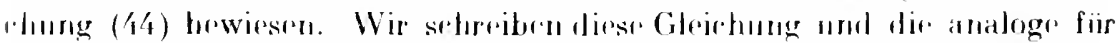

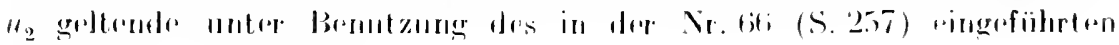
Yaicherlss in der liorm

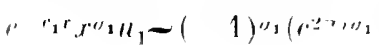

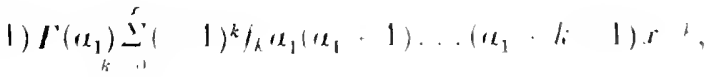

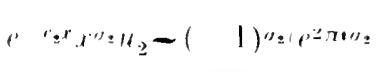

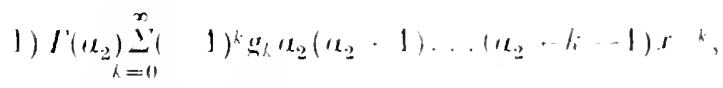

Wo dieg die korftizinten der lintwirklung von

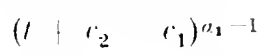

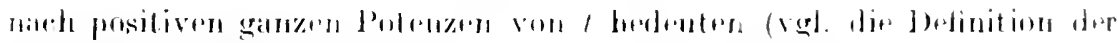
1., (ibrichnng (39), S. 26iT).

\section{Die Besselsche Differentialglei(chung ${ }^{2}$ )}

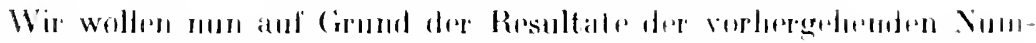

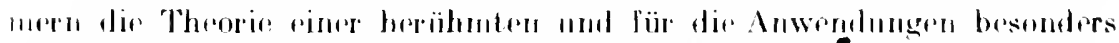

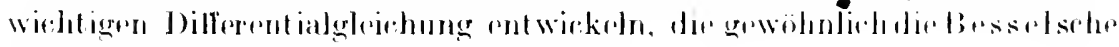
gernamet wirel und die folgende forme hat:

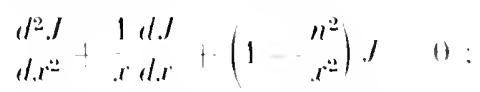

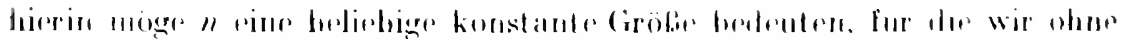

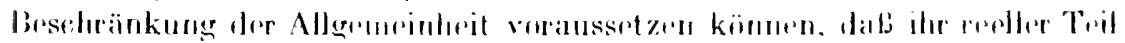
nieht negativ ist.

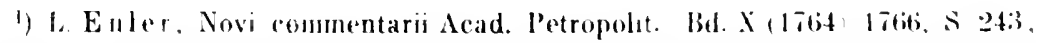
J Fonrier. Thécie de lat chaleur (1820). S. 369: Fr. W Bessel. Mhiandl. der

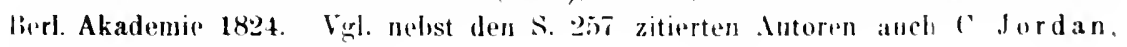
('ours III (1887). S. 255) ff 
Sitzen wir

$$
\text { I rn" }
$$

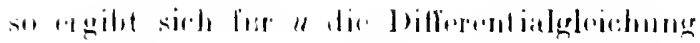

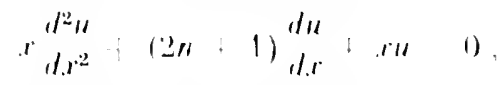

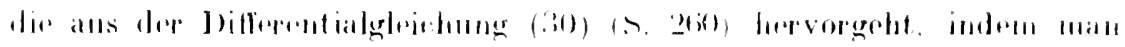
dascilbst

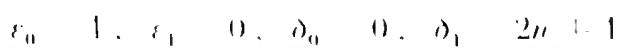

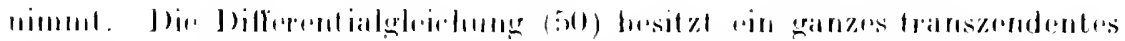

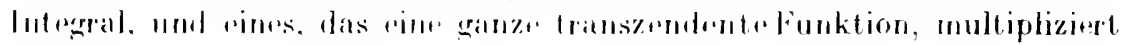
init

$$
\text { is } x_{1} \quad x \stackrel{n}{2} .
$$

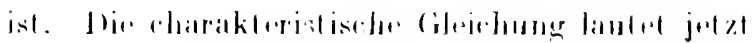

wir habull alsi

$$
Q^{2}+1-0 \text {. }
$$

III)

$$
\begin{array}{ccc}
2 \prime \prime & -1 \\
2 & u_{1}-1 & 2 n+1 \\
2
\end{array}
$$

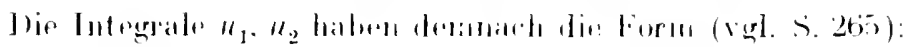

$$
\begin{aligned}
& \left.n_{1} \int_{i}^{\operatorname{sen}(z-1} 1\right)^{2 n} d z \\
& u_{2}=\left.\right|_{i} ^{e r r}\left(z^{2} \ldots 1\right)^{2 n}=d z
\end{aligned}
$$

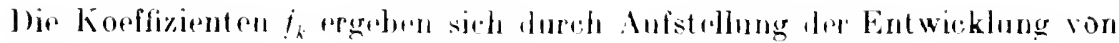

$$
\begin{aligned}
& 2 n-1 \\
& (t+\gamma)^{\prime \prime 2}-1=(t+2 i)^{2}
\end{aligned}
$$

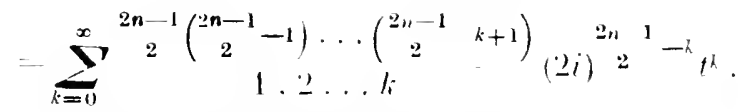

$$
\begin{aligned}
& j_{k}=(2 n-1)(2 n-i) \ldots(2 n-2 k i+1)(2 i)^{2 n-1}-k \text {, }
\end{aligned}
$$

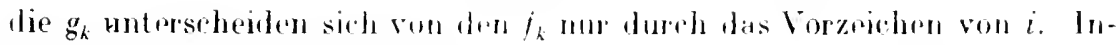
dem wir noch beachten. dal.i

$$
\begin{aligned}
& e^{2 \pi i a_{1}}-1=e^{2(2 n+1)}-1=-\left(1 ; e^{2 r n}\right) \text {, } \\
& -1=\rho^{2} . \quad i=e^{2} .
\end{aligned}
$$

ist, finden wir dit asymptotische Darstellung

$$
x \stackrel{2 n+1}{2} e^{-x i} u_{1} \sim e^{-2^{\pi i}\left(n-\frac{1}{2}\right)}\left(1+e^{2 \pi i n}\right) 2^{n-\frac{1}{2}} \Gamma\left(n+\frac{1}{2}\right) .
$$




$$
\sum_{k=0}^{\infty}(-1)^{4} n^{2}-1 \cdot{ }_{4}^{4 n^{2}-9} \ldots{ }_{4}^{4 n^{2}-(2 k-1)^{2}} \cdot \underset{4}{12 i x)^{-k}} \cdot 2 \ldots k
$$

und analog :

$$
\begin{aligned}
& x^{2 n+1} e^{x i} u_{2} \sim e^{2 n}\left(n-\frac{1}{2}\right)\left(1+e^{2 n+n)} 2^{n} \div r\left(n+\frac{1}{2}\right) .\right. \\
& \sum_{k=0}^{\infty}(-1)^{k}{ }_{4}^{4 n^{2}-1} \cdot{ }_{4}^{4 n^{2}-9} \ldots{ }_{4}^{4}-(2 k-1)^{2}+\underset{4}{(-2 i x)} 1.2 \ldots k
\end{aligned}
$$

Da der reelle Teil von $n$ richt negativ, also der romb Tril von $2 n+1$
2 jedenfalls wesentlich negativ ist, so baben wir

$$
\lim _{x \rightarrow+\infty} e^{2 n+x i}{ }^{2 n+1}=0
$$

und können folglich die beiden vorstehenden asymptotischen Ausdriucke im Sinne der Definition der asymptotischen Darstellung ( $\mathrm{N}_{\mathrm{r}}$. 66. S. 257) auch in der Form schreiben:

$$
\begin{aligned}
& u_{1}-x^{-2 n+1} e^{x i} e^{-n^{n !}\left(n \frac{1}{2}\right)}\left(1+\rho^{2 a i n}\right) 2^{n} \frac{1}{2} \Gamma\left(n+\frac{1}{2}\right) . \\
& \sum_{k=0}^{\infty}(-1)^{k}{ }_{4}^{4 n^{2}-1} \cdot{ }_{4}^{4 n^{2}-9} \ldots{ }_{4}^{4 n^{2}-(2 k-1)^{2}} \cdot \underset{4}{1}{ }_{4}^{(2 i x)^{-k}} \\
& u_{2}-x \stackrel{2 n+1}{2} \text { e xi } e^{\cdot 2}\left(\frac{1}{2}\right)\left(1+e^{2 \pi n}\right) 2^{n} \stackrel{\frac{1}{2}}{2} I\left(n+\frac{1}{2}\right) . \\
& \sum_{k=0}^{\infty}(-1)^{k}{ }_{4}^{4 n^{2}-1} \cdot{ }_{4}^{4 n^{2}-9} \ldots{ }_{4}^{4 n^{2}-(2 k-1)^{2}} \cdot \begin{array}{c}
(-2 i r)^{-k} \\
1.2 \ldots k
\end{array}
\end{aligned}
$$

Wenn $n$ die Hälfte einer ungeraden Lahl ist, so brechen die hipr auftretenden Reihen ab; die im allgemeinen divergenten Ausdrïcke stellm also in diesen Falle wirkliche Lösungen der Differentialgleichung (50) dar.

Nun ergibt sich, nach Gleichung (38) (S. 266). das ganze transzendente Integral $u_{3}$ in der Form

$$
u_{3}=\left(1-e^{2 n_{1}^{2 n+1}} 2\right)\left(u_{1}-u_{2}\right)=\left(1+e^{2 x^{2 n}}\right)\left(u_{1}-u_{2}\right) .
$$

so daß sich aus den asymptotischen Darstellungen von $u_{1}, u_{2}$ sofort auch die asyumpt tische Darstellung von $u_{3}$ für positive reelle sehr große Werte von $x$ angeben läßt. Wir stellen vorerst noch dir heständig konvirgente Entwicklung von $u_{3}$ nach positiven ganzen Potenzen von $x$ ituf.

Wir haben (vgl. S. 264)

$$
n_{3}=\int_{(2 .}^{j} e^{2 x}\left(z^{2}+1\right)^{2 n-1} d=\quad \sum_{k=0}^{\infty} x_{(1, j)}^{x^{k}} j z^{k}(z-1)^{n} \quad d=
$$

Bezeichnen wir dureh (i). (- i) einfache von rimm beliehigen nieht

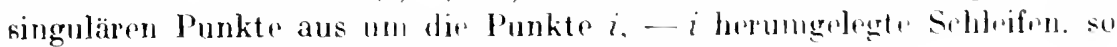
ist (vgl. z. B. dir analoge Umformung s. 265. 266) 


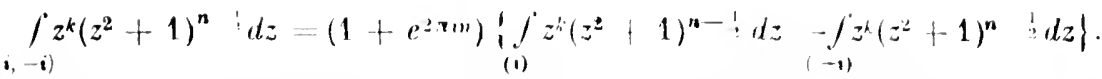

Setzt man in dem längs $(-i)$ erstreckten Intrgrale - $z$ an dir Stelle von $z$, so ist für ein gerades $k$

$$
\int_{(-1)}^{i} z^{k}\left(z^{2}+1\right)^{n} \frac{1}{2} d z=\int_{(0)}^{j} z^{k}\left(z^{2}+1\right)^{n} \frac{1}{2} d z
$$

und für ein ungerades $k$

$$
\int_{(i)}^{l} z^{k}\left(z^{2}+1\right)^{n} \leq d z-f_{(i)} z^{k}\left(z^{2}+1\right)^{n} \cdot \frac{1}{2} d z
$$

Es bleiben also in dem Ausdrucke für $u_{3}$ nur die den gerarlen Werten von $k$ entsprechenden Glieder stehen, d.h. wir erhalten

$$
u_{3}=\left(1+e^{2 \pi i n}\right) \sum_{k=1}^{\infty} \frac{x^{2 k}}{(2 k) !} !_{(i)}^{2} z^{2 k}\left(z^{2}+1\right)^{n-1} d z .
$$

Machen wir in dem unter dem Summenzeichen auftretenden Inte. grale die Substitution

so wird

$$
z=i \boldsymbol{t}^{\prime}
$$

$$
\underset{\text { (i) }}{2 f^{2} z^{2 k}}\left(z^{2}+1\right)^{n-1} d z=(-1)^{k} \underset{\text { (1) }}{\text { if }} \boldsymbol{t}^{k-1}(1-\tau)^{n-\frac{1}{2} d \boldsymbol{t}},
$$

wo (1) eine un den Punkt $t=1$ herumgelegte einfache Schleife bedeutet. Nehmen wir zum Ausgangspunkte dieser Schleife den Punkt $t=0$ und setzen voraus, daß der reelle Teil von $n+\frac{1}{2}$ wesentlich positiv ist, so können wir das über die Schleife (1) erstreckte Integral in bekannter Weise umformen:

$$
\begin{aligned}
& \int_{(1)} z^{k-\frac{1}{2}}(1-\tau)^{n-\frac{1}{2}} d t=!_{0}^{.1}+e^{2 \cdot r i\left(n-\frac{1}{2}\right)} j^{.0}
\end{aligned}
$$

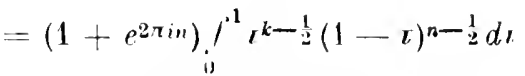

$$
\begin{aligned}
& =\left(1+e^{2 \text { Tin }}\right) B\left(k+\frac{1}{2}, n+\frac{1}{2}\right)
\end{aligned}
$$

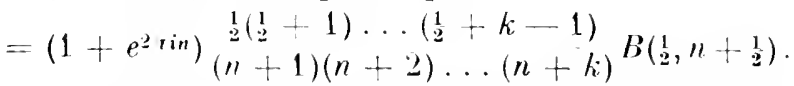

Wir benutzen nun eine Formel, die die Betafunktion durch Gamnafunktionen auszudrücken lehrt und deren Beweis man in jeden Lehrbuche der Integralrechnung findet:

Hiernach ist:

$$
B(p, q)=\begin{aligned}
& \Gamma(p) I^{\prime}(q) \\
& l^{\prime}(p+q)
\end{aligned}
$$

$$
B\left(\frac{1}{2}, n+\frac{1}{2}\right)=\frac{I^{\prime}\left(\frac{1}{2}\right) \Gamma\left(n+\frac{1}{2}\right)}{I^{\prime}(n+1)},
$$

oder, da nach einer bekannten Formel $\left.{ }^{2}\right) \quad I\left(\frac{1}{2}\right)=l \pi$ ist,

1) Vgl. Gaub a. a. O.. Werke III, S. 148. 


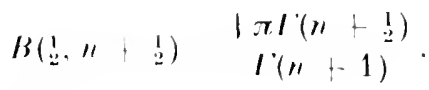

Beachtern wir fermer. da!

$l(n+1) \cdot(n+1)(n+2) \ldots(n+k)-l(1)-l i-1), I(k-1) t i$

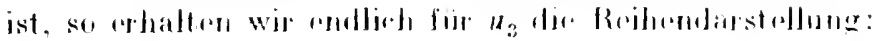

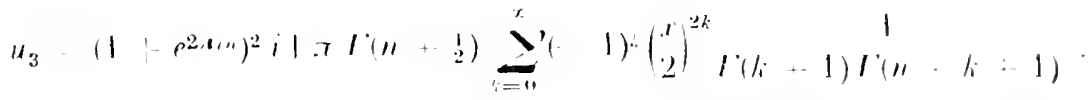

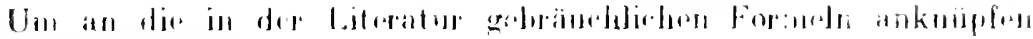
zul könners, setzon wil:

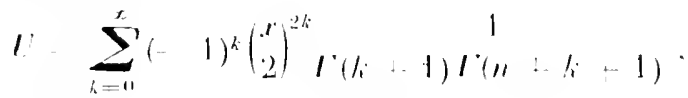

dann ist marh (51)

$$
\begin{aligned}
& H_{1} \quad H_{2}
\end{aligned}
$$

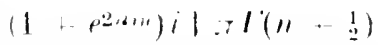

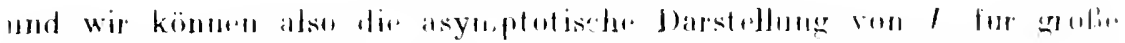

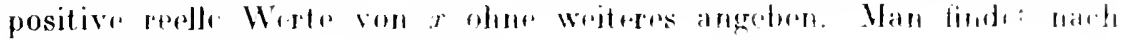
rinigen rinfartion timformungun

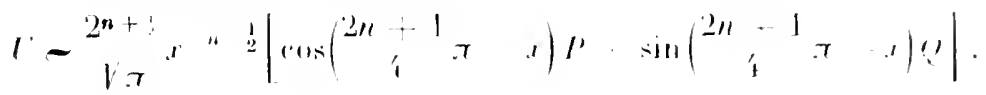

wo $\mu$, die divergentren Railen

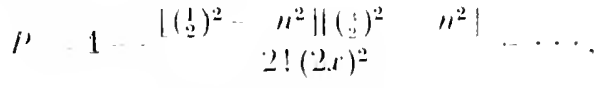

$$
\begin{aligned}
& \text { () } \\
& \left(\begin{array}{l}
1 \\
2
\end{array}\right)^{2}-11^{2} \\
& 21
\end{aligned}
$$

bedeutert.

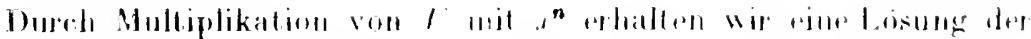

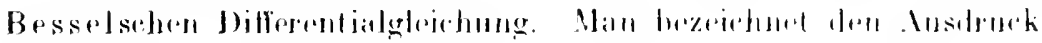

$$
I_{n}(x) \quad \begin{aligned}
& l \cdot x^{n} \\
& 2^{n}
\end{aligned} \sum_{k=0}^{x}(1)^{k} l(l+1) l_{i}\left(l_{i}+n-1\right)\left(\begin{array}{l}
x \\
2
\end{array}\right)^{2 k+n} .
$$

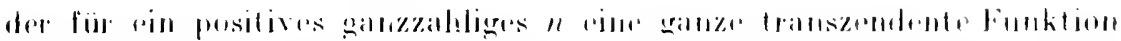

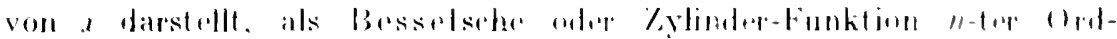

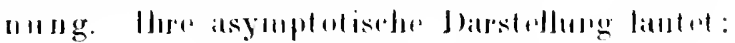

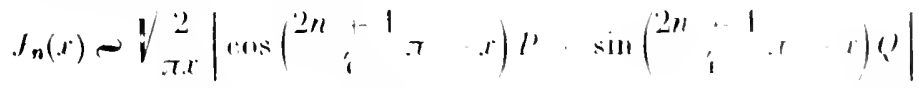

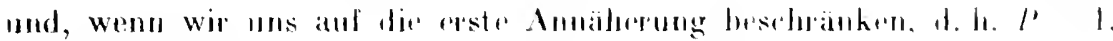
$Q=0$ nehmm!n, 


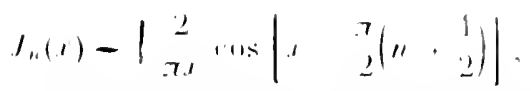

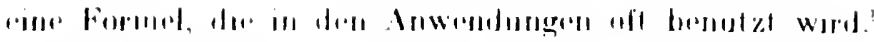

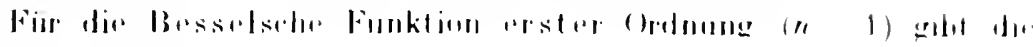
Darstillumg (a)

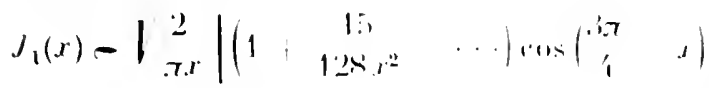

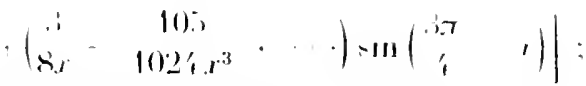

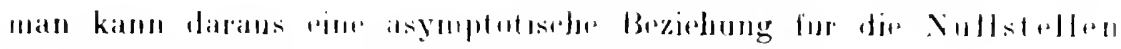
von $J_{1}(x)$ gewimmen. Lus $J_{1}(\lambda)$ 1) fulgt mämlicl

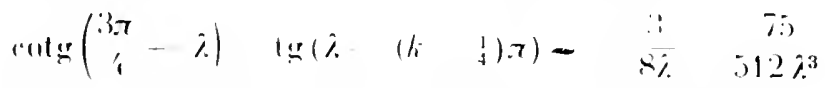

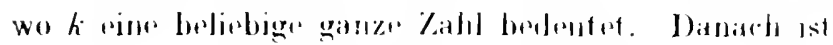

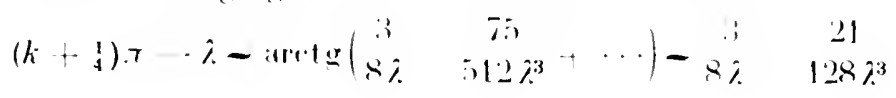

diso

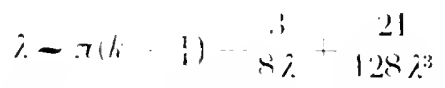

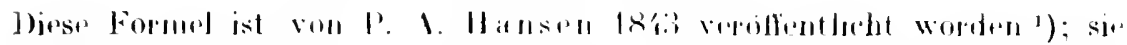

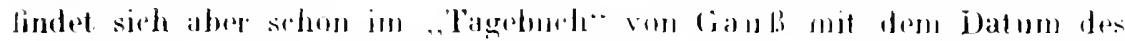

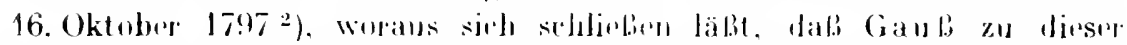
heit dir asymptotisch. Darstellung von $J_{1}(x)$ gekannt hat. Zum ersten Male bekanntgenateht hat die asymptotisehe l)arstellung tiar $f_{1}(x)$ wohl II ansen a. a. (). lur $J_{0}(x)$ linder sie sich 182:3 hei S. J). Poisson $\left.{ }^{3}\right)$.

Für $x=x$ rohuziert sich die Diflerentialgleichong (50) ant

$$
d d^{2} u-\|-0 \text {. }
$$

deren allgemeines lutegral dureh resin $\left(a+c_{2}\right)$ mit dan willkiulichen Konstanten $r_{1}, c_{2}$ gegeben wird. Mir sohen ans den abgeleiteten asymptotischrn Darstellungen von $u_{1}$. $u_{2}$, da fim im Simm dor Auseinandersetzungen an Sehlub der Nr. bti die loosungen rom (50) in eler Tat fïr grobe Werte von $x$ asymptotisch durch dir lösumgen von (54) dargestellt werden. In einer Abhandlung aus dem Jaher 1913 hat l'. Buntroux ${ }^{4}$ ) aus diesem asymptotischen Vinlalten der Bessolsohen Funktion liefgehende FulS. 115.116.

1) P. A. Il ansen. Ermittlung der absoluten stïrungen etc, Gotha 1843. S. 388,389 .

2) C. F. GauB. Werke. Bd. $X_{1}$ (1917), S. 525. vgl. ehenda die Anmerkungen

3) S. I). Poisson. Journal de 1 Ecole Polyt. 19 (1523), S. 249.

4) P. Boutroux. Annales de l'École Normale supérieure. $30.5 .255 \mathrm{ff}$. 
gerungen gezogen, die un so bemerkenswerter sind, als sie sich auf allgemeinere Funktionsklassen erweitern lassen. Wir kommen auf diese Fragestellungen in folgenden Kapitel (Nr. 80) zurück.

In bezug auf die Besselsche Differentialgleichung bemerken wir noch, daB, da diese Gleichung ungeändert bleibt, wenn man - $n$ an die Stelle von $n$ setzt, auch $J_{-n}(x)$ eine Lösung von ihr darstellt. Wenn $n$ krine ganze Zahl ist, so bilden

$$
J_{n}(x), \quad J_{-n}(r)
$$

ein Fundamentalsysten; ragegen unterscheiden sich diese beiden Intrgrale in Falle pines ganzzahligen $n$ nur durch den konstanten Faktor $(-1)^{n}$. Die Wurzeln der zu $x=0$ gehörigen determinierenden Fundamentalgleichung der Besselschen Differmtialgleichung sind $n$ unt $-n$, der Fall, wo $n$ einr ganze Zahl ist, zeigt also nach der allgemeinen Theorie an, dab nur zu dem Exponenten $|n|$ rin in Reihenform darstellbares Integral. nänlich $J_{n}(x)$ gehört, währemd das zweite Element des kanonischern Fundamentalsystems pinen Logarithmus enthält. Man vergleiche Näheres uber dirses zweite Integral z. 13. bei E. Hoine ${ }^{1}$ ). C. Neumann ${ }^{2}$ ) und N. Nielsen ${ }^{3}$ ).

\section{7.. Darstellung der Lösungen der Laplace schen Differential- gleichung durch Fakultitenreihen.}

Firr dir Lösungen der Laplareschen bifferentialgleichung (30), $\mathrm{N}_{\text {r. }} 67$ haber wir mit Hilfe ihrer Austrieke durch dir Laplacesehen Integrale die nach fallenden Poterizen ron $x$ fortsel eitenden, divergenten Entwieklungen hergestellt and nachgewiesent. dali diese Entwieklunger eine asyuptotische Darstellung jener Lösungen liefern. Es ist aber nöglich. wir $110 \mathrm{r}^{4}$ ) allgemein gezrigt hat. an die Strlle der divergenten Potenzreihen, die linearen l)ifferentialgleichungen genügen, konvergente. sogenannte Fatultatenreihen treten zu lassen, d. h. Reihen von Her form

$$
a_{13}+{ }^{a_{1}}+\frac{a_{2}}{x}+x(x+1)+x(x+1)(x+2)+\cdots \text { in } \|^{2} .
$$

wo dir $a_{0}, a_{1}, a_{2}, \ldots$ konstante Konffizienten bedentern. Eine soluhe lieshe hat die bemerkenswerte Eigenschaft, dabir. Wenn sie fir $r=x_{0}$ kon-

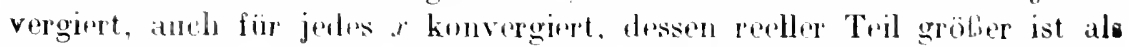
der reelle Teil von $x_{0}$. d. h. dab ihr Konvergenzgebiet stets rine Halb-

b) E. Hoine, llandbuch der kugelfunktionen 1 (15is). S 193 ft.

2) C. Naumann. Theorie der Besselschen F aktionen (1569).

") N. Nielsen. Handbuch der Theorie der /ylinderfunktionen. Leipzig 1904.

y) J. Horn, Mathem. A aten Bd. B1 (1911). S. jlo: vgl. Watson. Rendironti del Circe mut. di Palermo it (1910). St. fl. 


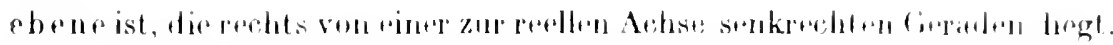
Wir werden in folgenden') niehts ans der Theorio der Fakultatenreiben als bekannt voraussetzen, knïpfen vielmehe unmittnlbar in di. Intregralalarstellung

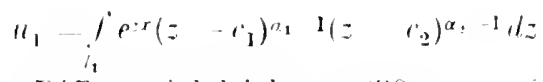

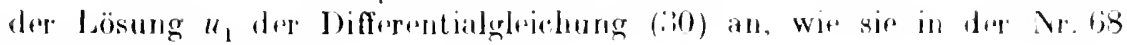
gergeben worden ist.

Wir stellen uns dir Anfgabr, firr dir Funktion

$$
\text { "1 (z) } \quad\left(z-c_{1}\right)^{\alpha_{1}-1}\left(z-r_{2}\right)^{\alpha_{2}-1}
$$

rine Reihomentwicklung zu linden, die fiur den ganz+s Integrationsweg $l_{1}$ konvergiert. Dabei denkent wir uns $l_{1}$ jotzt in folgender Wrise gelegt. Von dem Punkte cr der z-liherno aus sei rin llalbstrahl s nath dem Unendlichen gelegt, dre nit dor positiven renllon Achs' den Winkel 0 bildet, und der nicht durch r hindurchgeht. Der Wog $l_{1}$ durchläuft dann vom Unendlichen komment den llalbstrahl $s$ bis dicht vor $c_{1}$. unkreist $r_{1}$ im positiven Sinne und kehrt längs s werder nach dern Unendlichen zurück. Das Integral (55) hat dann (sieh. Nr. 68, S. 265) pintn Sinn und stellt eine Lösung der Differentialgleichung (30) dar. wrmn fïr hinreichend grobe Werte von z $\mathfrak{H}(\cdots)<0^{2}$ ) ist, d. h. also wenth

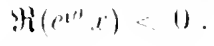

Wir denken uns mun num den llalbstrahl $s$ und seine Verlängerung als Mittellinie einen Parallelstreifon grelegt, der den Punkt r. nicht enthält (Fig. 4); dies wirl erreicht, wenn dir halbe Breite dieses Streifrns - die wir $\gamma$ nemnen wollen - kleiner gewählt wird als $\Im\left(\left(c_{2}-c_{1}\right) e^{-t \theta}\right)$. Durch die Funktion

$$
z-c_{1}=k \log t, \quad k=-\gamma_{\pi}^{\gamma} \rho^{H_{1}} .
$$

wird unser Parallelstreifen auf die längs der negativen reellen 1 -Achse aufgeschnittene $\iota$-Ebene abgebildet. dem Punkte $z=c_{1}$ entspricht $t=1$,

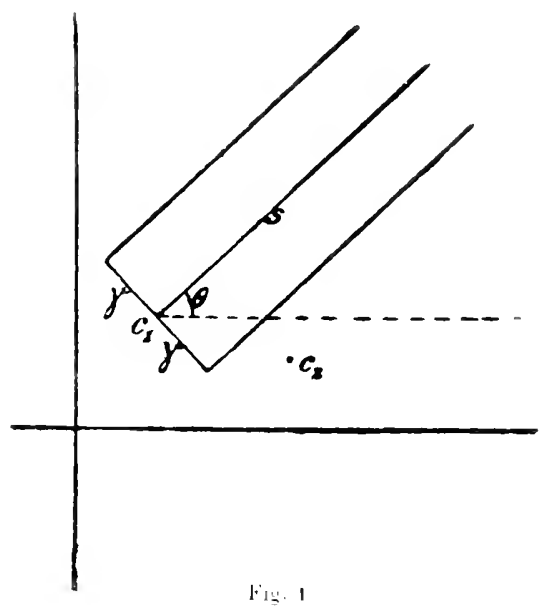

1) Die folgende Varstellung schliebt sich an Horn. Math. Zeitschrift $8(1920)$. S. $100 \mathrm{ff}$. und F. Nevanlinna. Zur Theorie der asymptotischen Potenzreihen. Dissertation, Helsingfors 1918 an: vgl. auch die z. Z. im Druck befindliche Arbeit von J. Hor n . Zur Theorie der nichtlinearen Differentialgleichungen. Matb. Zeitschrift 13 (1922).

$\left.{ }^{2}\right) \mathfrak{h}(a)$ bedeutet den reellen Teil. $\mathfrak{J}(a)$ den Koeffizienten von $i$ der komplexen Giröbe $a$. 


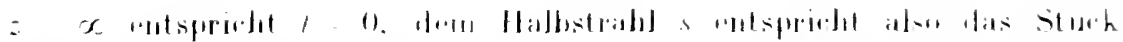

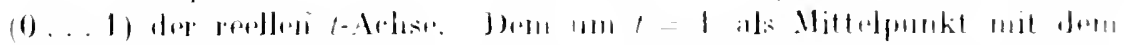

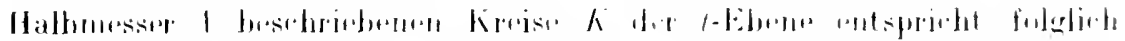

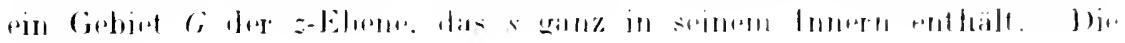
Finklinill

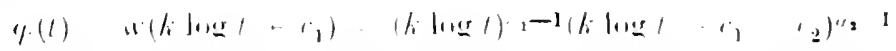

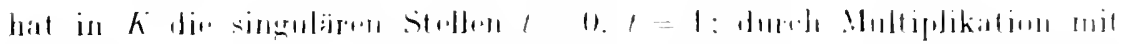

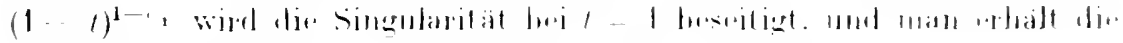

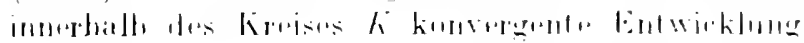

$$
y(1)(1-1)^{1-\cdots}-\sum_{,=0}^{\infty} b,(1 \quad 1) \text {. }
$$

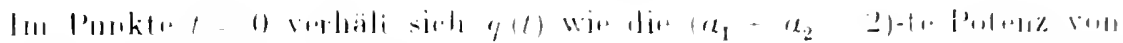

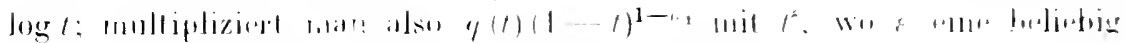

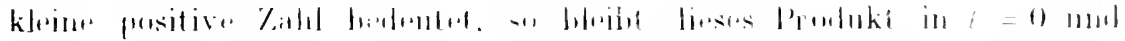

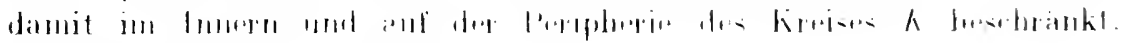

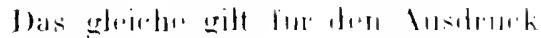

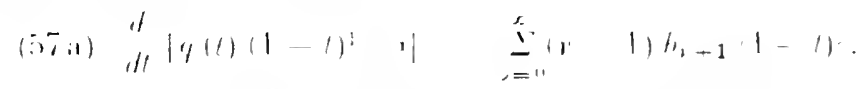

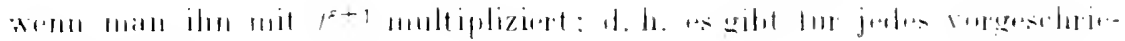

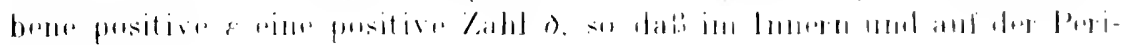
pherin virk

$$
\left.\frac{d}{d 1} \mid 4(1)+1--1\right)^{1-1 \mid+\cdots 1} \quad \text { ) }
$$

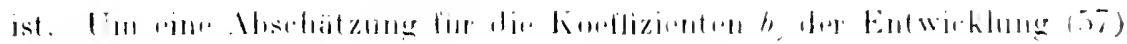

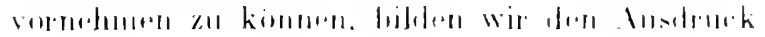

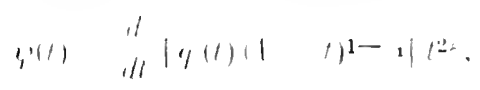

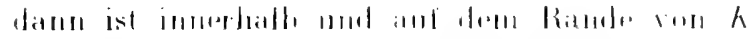

$$
y(t)=0,-1
$$

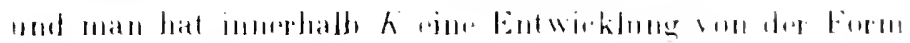

$$
y^{\prime \prime \prime)} \quad \sum_{x=0}^{\infty} l,(1 \quad 1) \text {. }
$$

I): IIIII

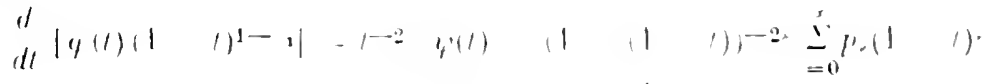

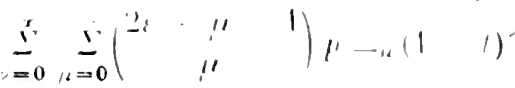

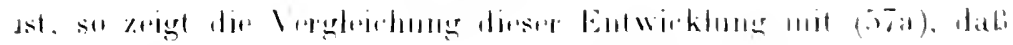

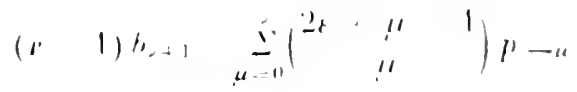




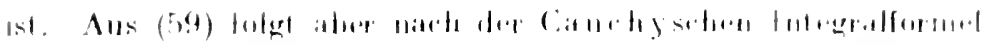

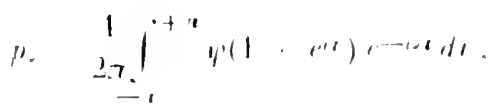

also, dis nach (5is)

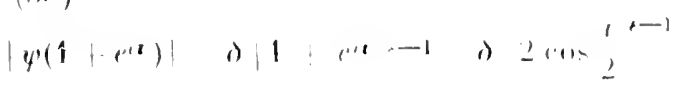

IsI.

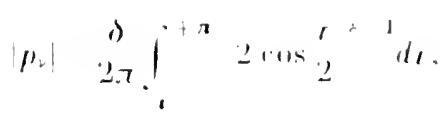

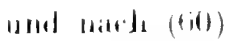

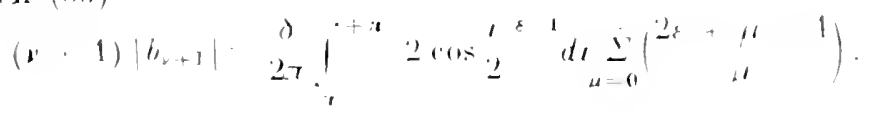

Sitzoll wir

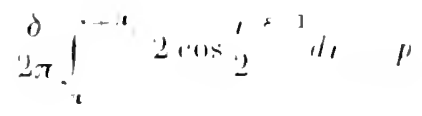

Ind bearhtere, dati

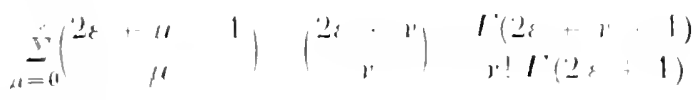

Ist. Sol baken wh abou

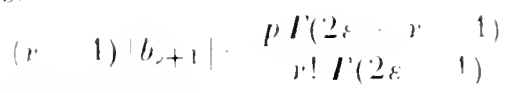

und damit fin b, dir Mashatzumb

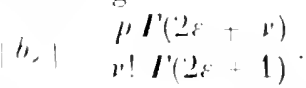

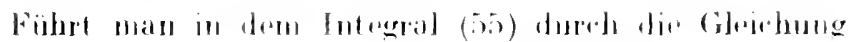

$$
=r_{1}-l i \log t
$$

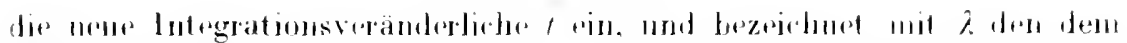

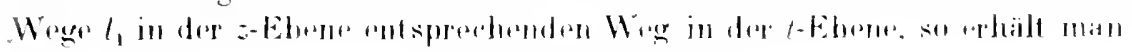

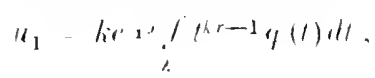

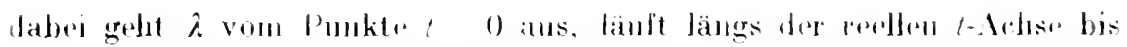
kurz vor den Punkt / a... 1. mukpesist diusen im positiven Simme und kelut wioder längs der reollen t-luhse nach $t=0$ zuriuck. Wir können also

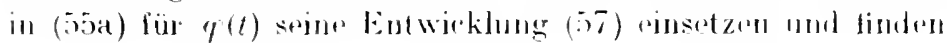

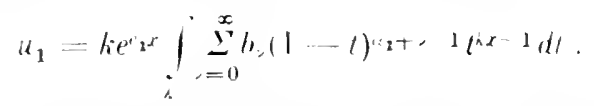

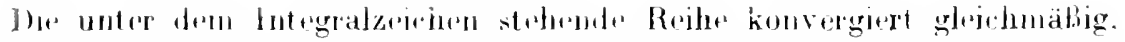

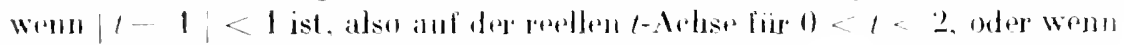

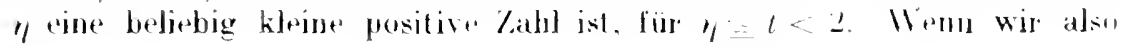


den Integrationsweg, statt in Punkte $t=0$, im Punkte $t=\eta_{\text {ansetzen }}$ und endigen lassen, kann die Integration gliedweise ausgefiihrt werden. Den so abgeänderten Integrationsweg bezeichnen wir mit $\lambda_{\eta \eta}$, daun ist also

$$
\text { (63) } \int_{\lambda_{\eta}} \sum_{\nu}^{\infty} b_{0},(1-t)^{a_{1}+\nu-1} t^{k x} 1 d t=\sum_{,=0}^{\infty} b_{j} \int_{\lambda_{\eta}}(1-t)^{\mu_{2}+}{ }^{2} t^{k r-1} d t \text {. }
$$

Nun hatten wir vorausgesetzt (Gl. (56)), daL $\Re\left(e^{\prime \phi} x\right)<0$ ist: daraus folgt $\mathfrak{A}(k x)>0$. Wir können ferner $n$ so groß wähler, daß $\mathscr{H}\left(\alpha_{1}: n\right)>0$ ist, dann wird (vgl. Nr. 59, S. 234) für $v=n$

$$
\text { (64) } f_{i_{\eta}}(1-t)^{\alpha_{1}+\nu-1 / k_{x}-1} d t=\left(1-e^{2 \pi i x_{1}}\right) f_{\eta}^{1}(1-t)^{\alpha_{1}+\nu-1} t^{k x-1} d t \text {. }
$$

Lis ist aber

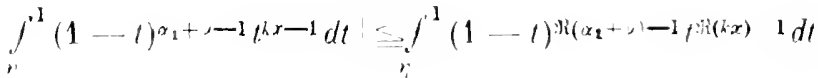

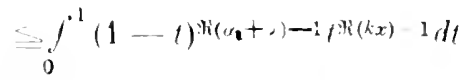

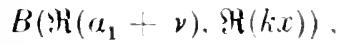

also mit Rücksicht auf (61) und (64)

$$
b_{\nu} \int_{i_{\eta}}(1-t)^{\alpha_{1}+,-1 / k x-1} d t=\begin{aligned}
& C p I(2 \varepsilon+v) \\
& v ! l(2 \varepsilon+1)
\end{aligned} B\left(\Re\left(\alpha_{1}-v\right) . \mathfrak{R}(k x)\right),
$$

wo $C$ eine lionstante bedeutet, für die $1-e^{2 \pi a_{2}}=($. und went wir die Be afunktion durch Ganmafunktionen darstellen (...71. \$. 277).

$$
\begin{aligned}
& b \cdot \int_{i_{\eta}}(1-t)^{\alpha_{2}+-1} t^{k x-1} d t
\end{aligned}
$$

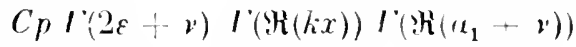

Nun ist nach rimer bekannten formel ${ }^{1}$ )

$$
I(z)=\lim _{n \rightarrow \infty} z(z+1) \cdots(z+n)
$$

oder mit Rücksicht auf Gleichung (43) (S. 269)

$$
\begin{aligned}
r(z+n+1) \\
n ! n:
\end{aligned}=1+; \ln _{n \rightarrow n}=0 \text {; }
$$

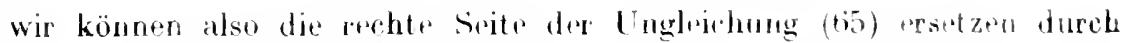

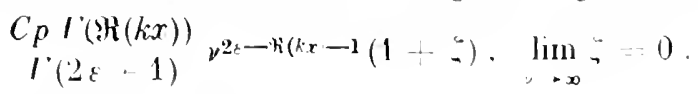

Daraus folgt, dab die Reihe

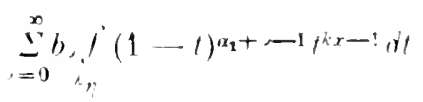

f) Vigl (ia ab b, a d. O). Werke III, S 145. 
unbedingt and gleichuabig kemsergert fiur alle Mirte von $\%$ dir dem Intervall $0 \cdot \eta \cdot 1$ angehören, wenn

$$
2 \varepsilon-? h(h x) \cdot 0
$$

ist. Dir letztere Bedingung ist aber, da $\varepsilon$ belirhig kloin sajn sollte, wougen $\mathfrak{R}(k x)>0$ sicher erfüllt. Dir lioilere (6ti) stellt demnarh rine in dem Intervall $0 \supseteq \eta \cdot 1$ stetige Funktion von $\eta$ dar, diw Gleichung (6i3) gilt folglich auch nech für $\eta=0$, d. h. wonn die Integrale über den Wog $\lambda$ erstreckt werden. Wir können also in (62) ghedweise integrieren und erhalten dann, nach oft angewandtron Unformungen, liur $u_{1}$ die Reihe

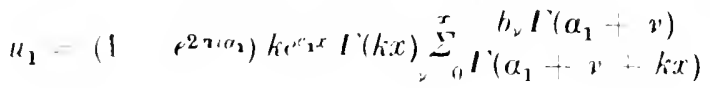

oder

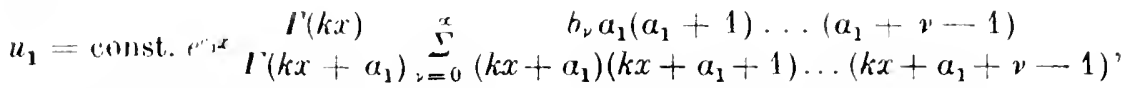
also eine Fakultätenrejhe, von der feststeht, dals sie fiur $\Re\left(e^{i \theta} x\right)<0$ unbedingt konvergiert.

Eine analoge Darstellung kann natürlich anch fïr $u_{2}$ angegeber werden. 


\section{Vinntrs Kapil \\ Verallgeneinerungen. Parmetrale Probleme}

\section{7:. Differentialsysteme fiir n Unbekamte. Intezrodifferential sleichungen.}

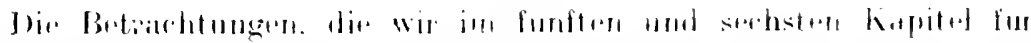

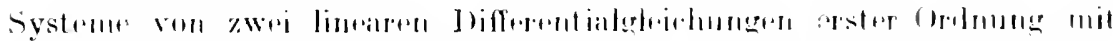

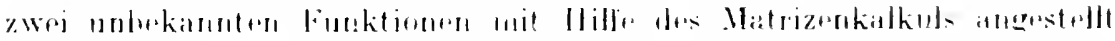

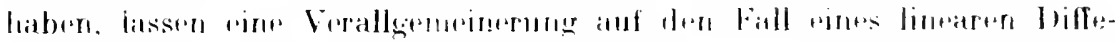

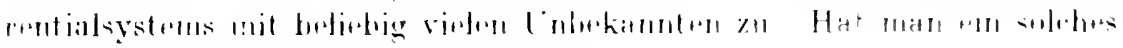

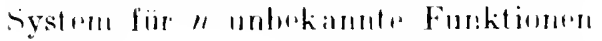

$$
\text { dy. } \quad \sum_{i=1}^{n} y_{k} a_{i k} . \quad \quad i=1 \Omega n
$$

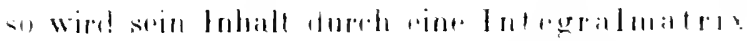

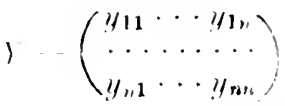

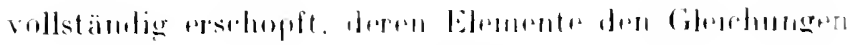

$$
\text { dy,k } \quad \stackrel{n}{n}, \cdots, n_{n}
$$

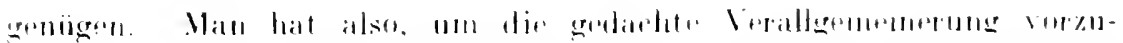

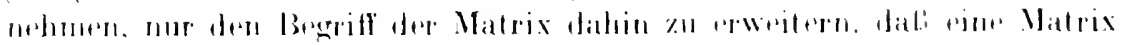

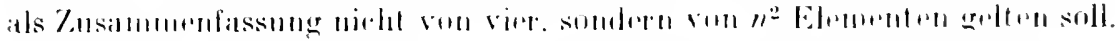

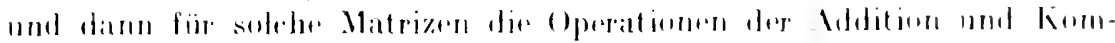

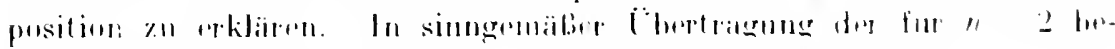

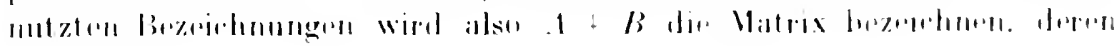
Elominte

$$
a_{l k}+b_{i k} \quad 1, i \quad 12 \ldots, 1
$$

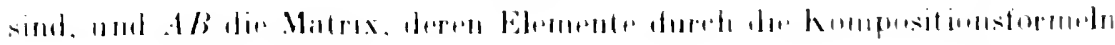

$$
\sum_{i=1}^{n} a_{1} b_{i} \quad \quad(0, x=12 \ldots n
$$

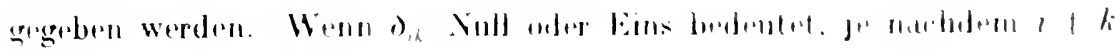




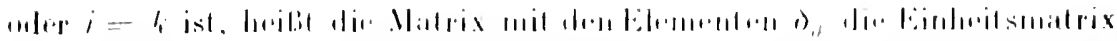

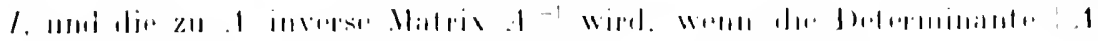

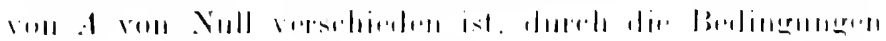

$$
11-1 \quad 1-1 \quad 1 \quad 1
$$

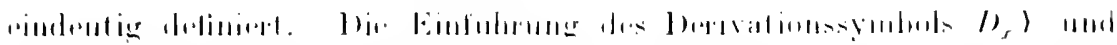

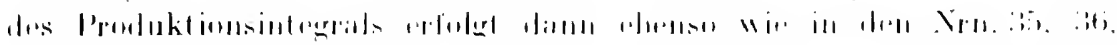

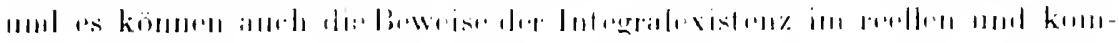

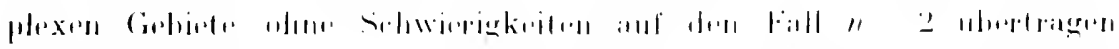

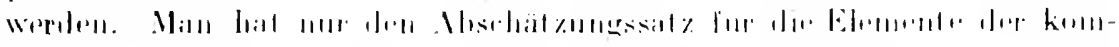

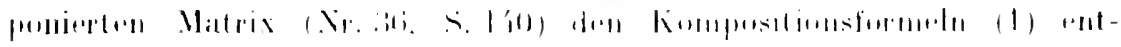

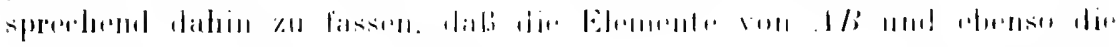

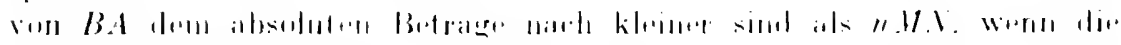

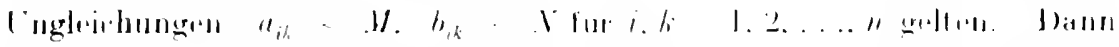

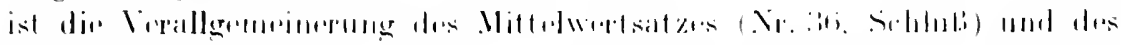

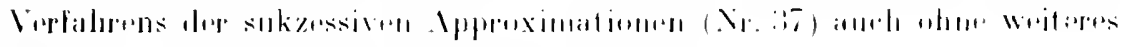

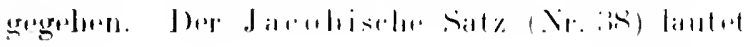

$$
\gamma \quad \gamma_{1+1}, !^{\prime \prime} \sum_{k=1}^{n} k k^{d r} .
$$

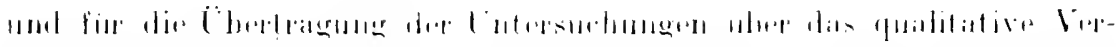

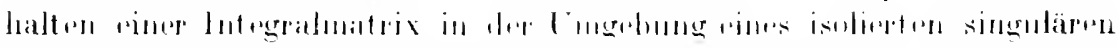

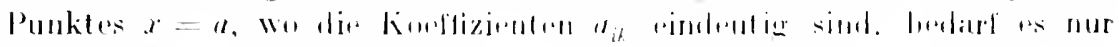

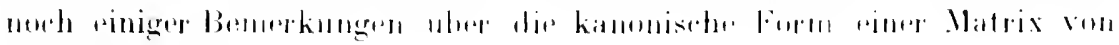

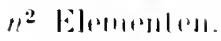

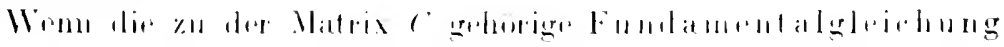

(2) ('- lo $=11$

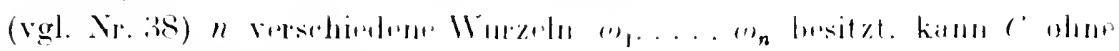
Selwierigkeit in sin linm

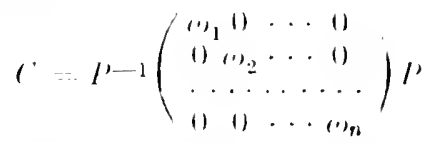

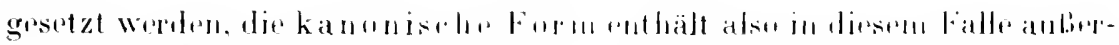

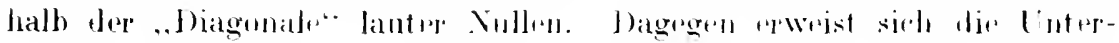

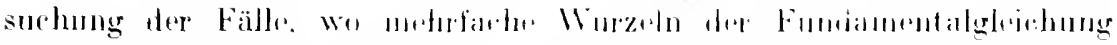

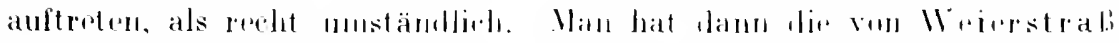

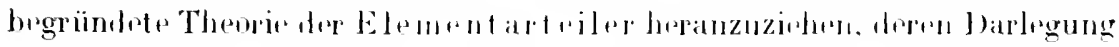

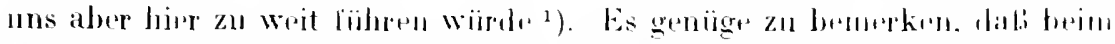

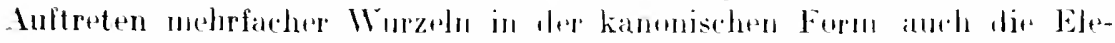

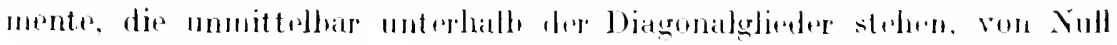

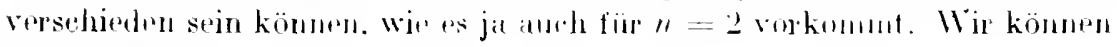

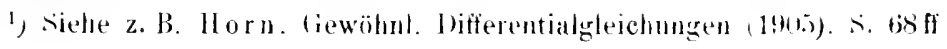


hier un so rher anf die ausfïhrliche Entwirklung der Elmomtartrilertheorio verzichten. als dir Integration dre Canohysoben Systems (rgl. Nr. 39)

$$
d x=\sum_{1}^{n} n_{2} n_{r}+\cdots a
$$

auch fur rin bedicbiges $n$ ganz allgemein durch das syumbul

$$
?=(x-a)^{R}
$$

gegehen wird. und die kanonisehe Form rine .Hatrix $f^{\prime}$ nur in Butracht kommt. Wennes sich darum handelt. $R$ so zu bestimmon. dab diw Fileirhung

$$
e^{2 \cdot 1}=C^{2}=
$$

refült wird. Mir können diese Brstimmung olun writrese durrhführen. wenn die Glejchung (2) laut'r verschiedene Wurzeln hat, wir haben fiir $n=2$ auch dir Untersehiedo vor Augen. die las AuftedeI mehrfacher Wurzeln mit sich bringt, und bregnïgen uns im allgemrimen Falle mit du Angabe, dab die Inversion der Matrizenfunktion e $e^{2 \pi i L}$ stets mögliols ist.

Jann verläuft alles Weitere ebenso wie fïr $n=2$. anch die Lntersuchung der Stellen dre Bestimmthrit, der kanonisehen Systeme. endlich dje des Fuchsschen Typus und der schlechthin kanonischrn Systemm erfordert kejne weiteren Frläuterungen. Nur in bezug anf die Form dur Koeffizienten ejner lintaren Differentialgleichung $n$-t+r Orinung in der Ungebung einer Str]le $x=a$. wo die Integrale nicht unbestimnt worden. wollen wir benerken. dab sich durch rollständige Induktion (Schlul.' von $n$ auf $n+1)$ ergibt. dab die notwendige und hinrejchende Form allgemrin so lautet $\left.{ }^{1}\right)$ :

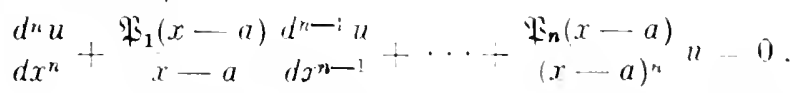

wo $\mathfrak{F}_{1}, \mathfrak{L}_{2}, \ldots, L_{n}$ in der Ungehnng von $x=a$ hulomorphe Funtionen bedeuten.

Man kann die eben angedentete Verallgenoinerung now weiterfuhren, indern man nicht bei sinem endlichen $n$ stohenhleibt. somdern den Grenzubergang $n \rightarrow \infty$ ausfihrt. J)as kam mon in zwefache

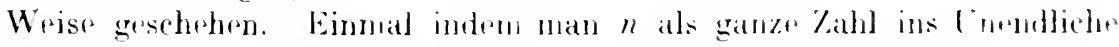

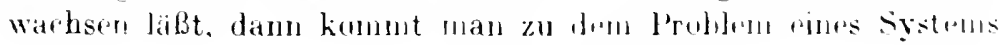

$$
d y_{i}=\sum_{i}^{3} y_{1}^{3} a_{/ \mu}
$$$$
(i=1,2 \quad, x)
$$

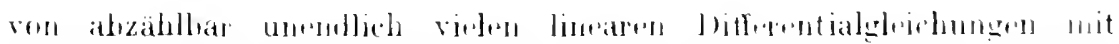

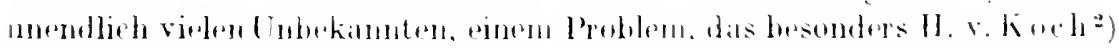

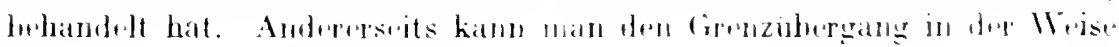

1) Fuehs. 1s6is. Werte. 1. S. 21:.

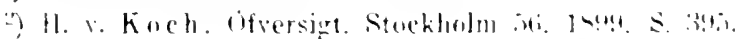




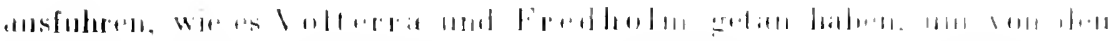

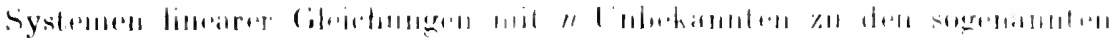

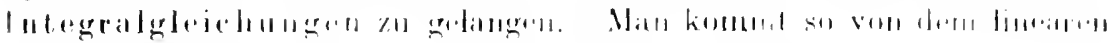

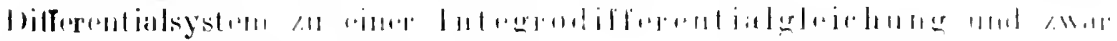

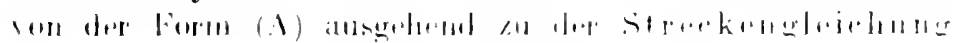

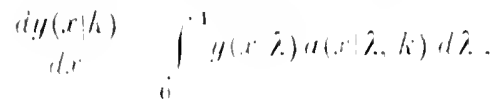

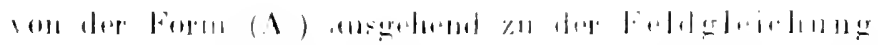

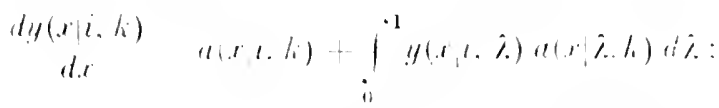

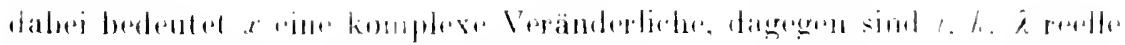

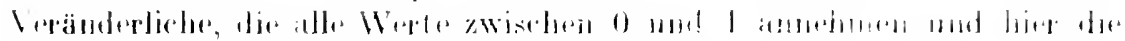

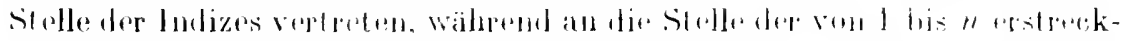

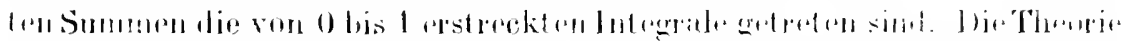

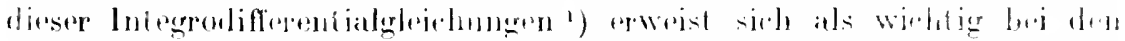

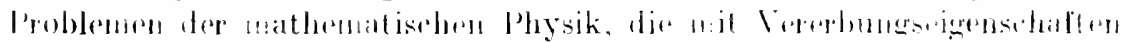

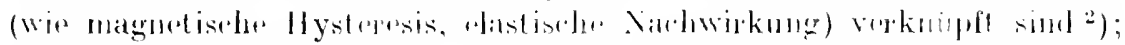

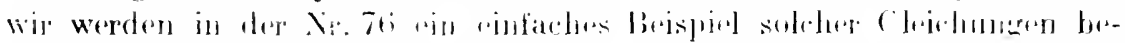

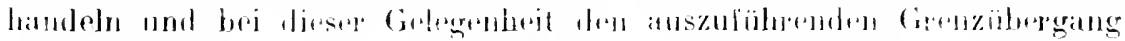

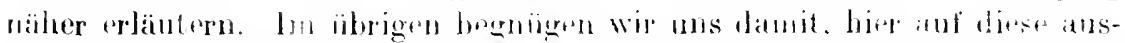

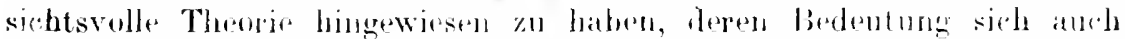

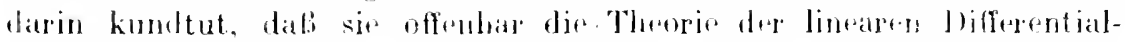

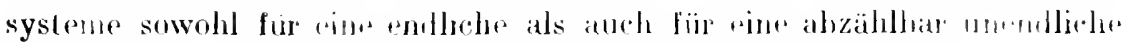
Anzahl rou unbekannten Finntionen als Sonderläle umfatir.

\section{Die Artheziehung. Aljungierte Systeme.}

Dagegen wollen wir eine andere Verallgenfinerung des Differentialsystems $\left(A^{\prime}\right)$ besprechen, zu der tian gefülurt wirl, wenn radn an den Begriff der Art bzw. der Klasse (Nr. 48) anknïpft. Es sei l pine Intw sralmatrix des Systens (A), von den wir voraussetzen wollen, dafs es zum Fuchsschen Typus gehört, und es bedeute $Z$ pine mit l' zu duselben Art gehörige Matrix, so dafi also

$$
Z=Y \%
$$

1) Siehe V. Volterra, Lecons sur les fonetions de ligues. Paris 1913. Xlli. Kap. S. $189 \mathrm{ff}$, und Drei Vorlesungen über neuere Fortschritte der math. Physik. Leipzig und Berlin 1914, :3. Vorl.. S. 166ff.: I. Schlesinger. Jabreshericht der D. M.-Y. 24 (1915). S. 84: E. Hilb, Mathem. Annalen 7T. 1916. ‥ ilt: T. II. II il d e brandt. Transactions of the Am. Math. Soc. 18 (1917), S. 73 und 19 (1918). S.97.

2) Siehe Voiterra.a. a. (). sehlesinger, Differentialgleichungen. 


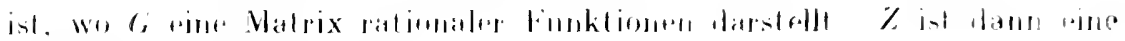

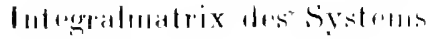

$$
a_{i=k} \quad \sum_{i=1}^{n}=1, \ldots
$$

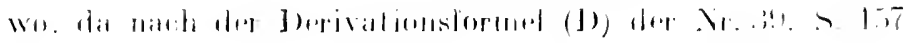

$$
D, \%-(; 1 \cdot 1), 1 \cdot(;-1), 1 ;
$$

isi.

$$
\text { I; } \quad 1 ;-1,11 ;: 11,1 ;
$$

anfumber willi.

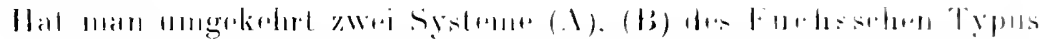

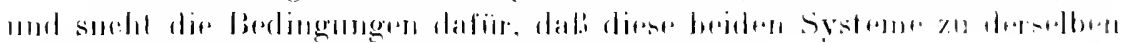

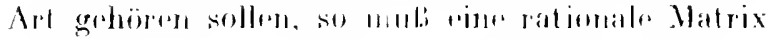

$$
\text { (i) }>1 \%
$$

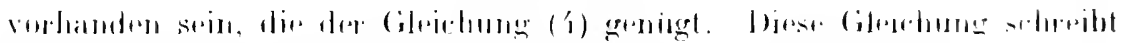

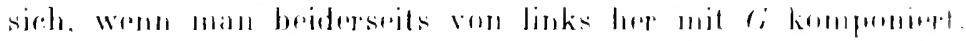

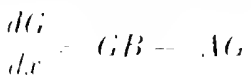

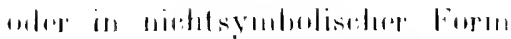

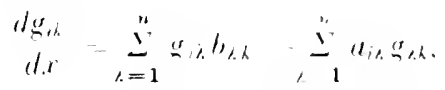$$
1 \cdot-12 n
$$

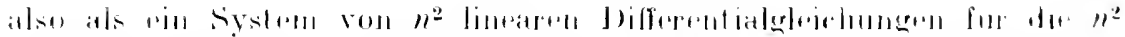

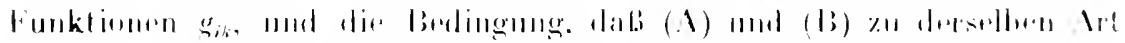

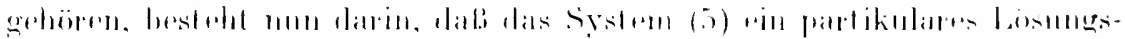

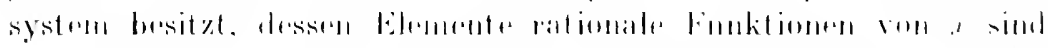

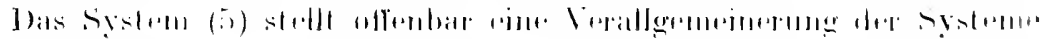

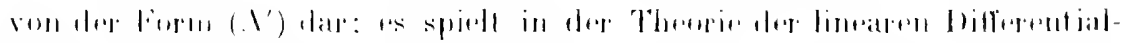

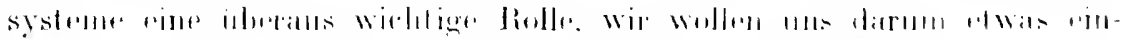

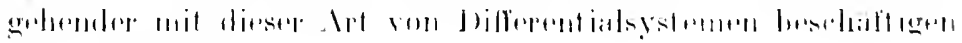

\section{Sidzl 111all}

$$
\ddot{2} \quad y_{i} ; z k \text {. }
$$

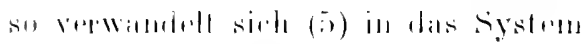

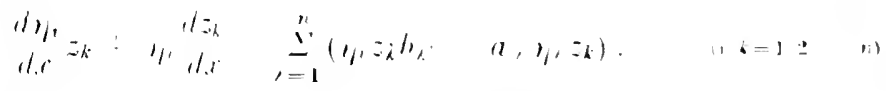

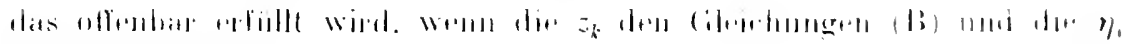

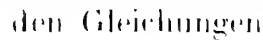

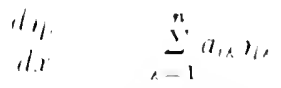

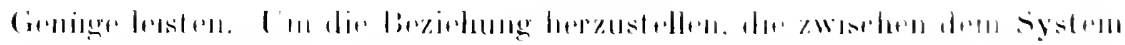

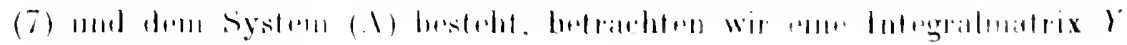
ind (.1) 1110 sil\%, 
(B)

$$
111 .
$$

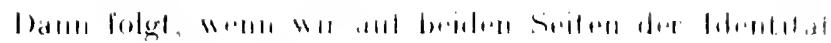

$$
\text { II) I }
$$

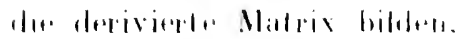

$$
\left.\gamma-i \cdot\left|x_{x} / 1 \cdot\right\rangle+11,\right\rangle \quad 11,
$$

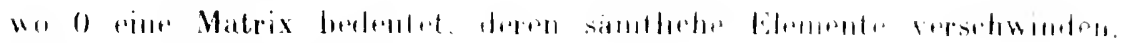
meter mit Rireksicht ant (Á)

$$
\text { dII } 1 /
$$

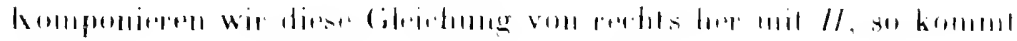

$$
\begin{array}{lll}
d 1 / & 1 / 1
\end{array}
$$

inlor

$$
\text { ii. } d x \text { एँ } a, x, 1 / k \text {, }
$$

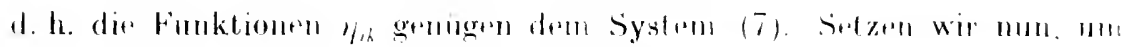

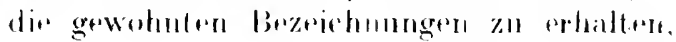

$$
1,-n_{k}, \quad a, k-a_{h} .
$$

sil verwatedelt sieh (9) in

$$
\text { da. } \quad \therefore b_{k}, a_{n}
$$

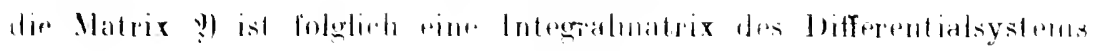

$$
\text { dik } \quad \sum_{i=1}^{n} 1, a, \ldots .2 \ldots n
$$

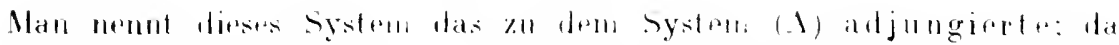

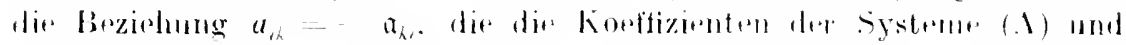

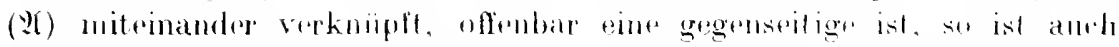

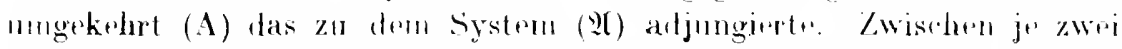
Integralmatrizen eines Systems (1) und seines adjungierten (20) hesteht

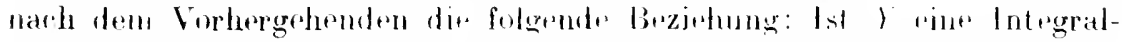

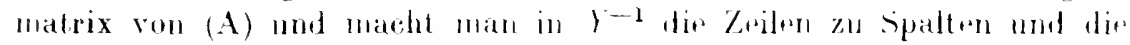

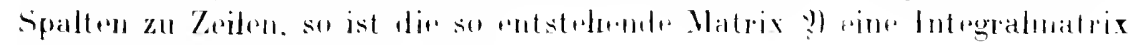
des adjungierten systems (i)

Wir können mun ohme Sehwirrigkrit dir allgrateint lösumg des Differentialsystems (5) angeben. Bodputef $/ /$ rint Integradmatrix von (7) und $Z$ eine Integraluatrix von (bi). so geniigen die $n^{2}$ Funktionen

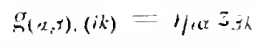

dem System (5). Ebenso bilden aber der Elemente der Matrix

$$
\text { C: }=111 \%
$$

wo I' eine beliebige konstante Matrix mit nicht versehwindender beter- 


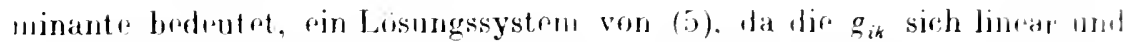

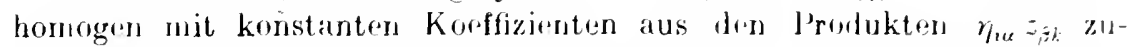
sammensetzen. Wir belatupten aber. dab in der Form (10) das allgw. ureinste Lösungssystem von (5) rnthalten ist. Unu dies narhzuweisru. nehmen wir an, daf dir gik irgendein Lösungseystem von (5) berdeutem.

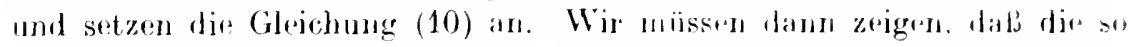
W.dinierten $\gamma_{i k}$ Konstanten sind. In der Tat folgt durch bifferentiation roII (10)

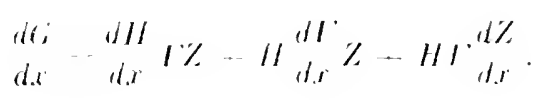

L)a abm

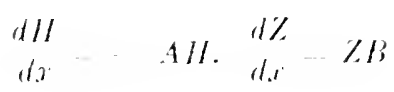

ist, so folgt atus (11)

$$
d x-A 111 \%-\| \frac{d}{d x} \%-H 1 \% B
$$

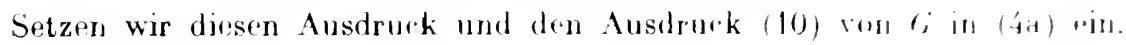
so erhalten wir

$$
11 \frac{d 1}{d x} \%=0
$$

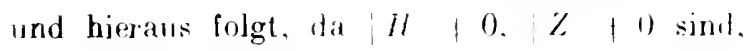

$$
\text { di } 16
$$

was zu beweisen war.

\section{Theorie der adjungierten systeme. Integration des roll- stïndigen linearen Differentialsystems.}

1)ab die in der vorigral Nummer gegebene Definition der adjungirerten Systeme die der adjungierten Differentialgledelumg zwejter Ordnung, wje sie in der $\mathrm{Nr}$. sf eingeführt wurde, in sich lergroift. sieht man solort, wemu man für das der linearen bifferentialgleirhumg

$$
u^{\prime \prime}+q u^{\prime}+1 n=0
$$

aquivalente system

das arljungierte Siystenl

$$
\begin{aligned}
& \frac{d y_{1}}{d r}-y_{2} . \\
& d y_{2} \quad r l_{1} \quad q ! \cdot{ }^{2}
\end{aligned}
$$

$$
\begin{array}{ll}
d n_{1} & n_{2} . \\
d x & n_{2} \cdot \\
d x & n_{1} \cdot q n_{2}
\end{array}
$$




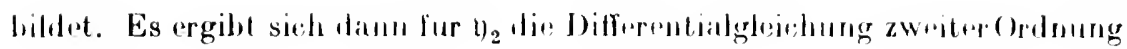

$$
\begin{array}{ccc}
\left(d^{2}\right)_{2} & & \left.d(g)_{2}\right) \\
d x^{2} & r u_{2} & d x
\end{array} .
$$

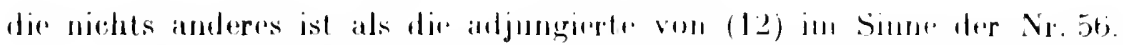

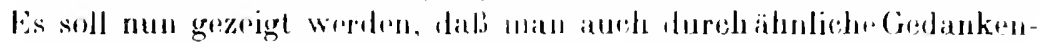
gängre, wie dir in der Xr. 56 lofolgten, zal den adjungierten Systemen gelangen kann. Wir solmerben dazu das System (A) in der form

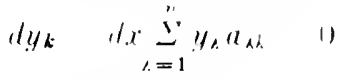$$
(k-1 \div \ldots
$$

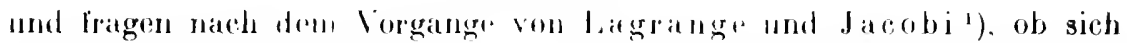

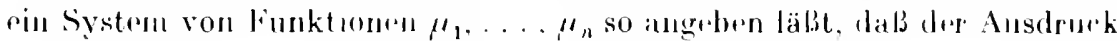

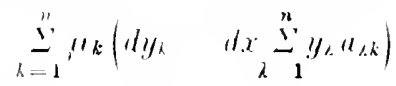

das vollständige Differrntial einer l'unktion $z$ der unablängigen Veränderlichen $x, y_{1}, \ldots y_{n}$ wirl. Falls solch. Funktionen $\mu_{k}$ vorhanden sind, werden wir sie als Multiplikatoren des Systems (A) zu bezeichnen liabril.

Wrom man in der identischen Girrohung

$$
\| \sum_{k=1}^{n} \mu_{k} y_{k}=\sum_{k}^{\prime}\left(\mu_{k} / y_{k} \rightarrow y_{k} d \mu_{k}\right)
$$

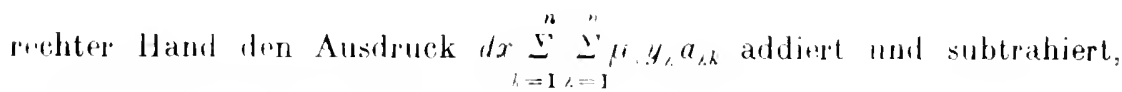
su rhält man dis Gloichung

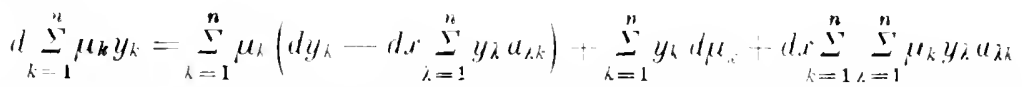

orler, inden man in der letzten Doppelsumme die Imdizes h, $\lambda$. miteinander vertauscht.

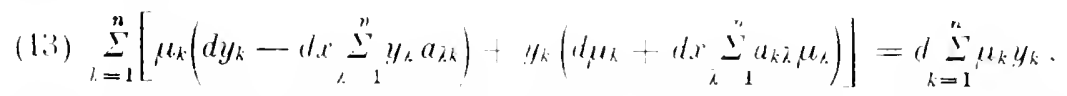

die wir als Identität ron langrange brzeichuen wollen. Wählen wil diu $\mu_{k}$ so, daß

$$
d \mu_{k}+d_{x} \underset{n=1}{\left.\stackrel{M}{\prime} a_{k} \mu_{1} \mu_{k}=1\right)}
$$

$$
(k=1,2 \ldots, n
$$

ist. dann ergibt (13)

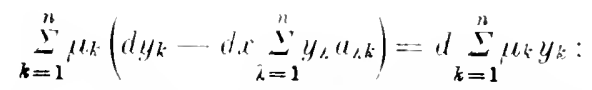

dit so gewählten $\mu_{1}, \ldots, \mu_{n}$ sind also Multiplikatoren und befriedigen, wie die Gleichungen (14) zeigen, das adjungierte Differentialsysten (ㄴ).

1) C. G. J. Jacobi. Werke IV, s. 23ti. $319 \mathrm{ft}$. 


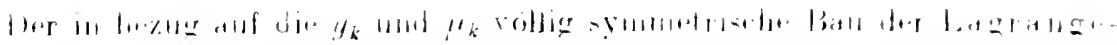

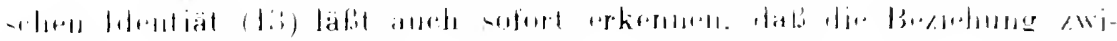

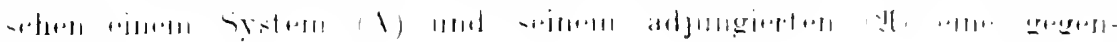
-ritign ist.

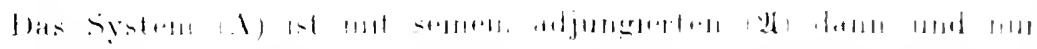

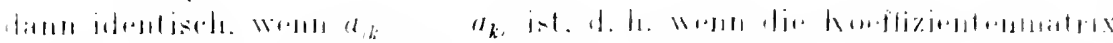

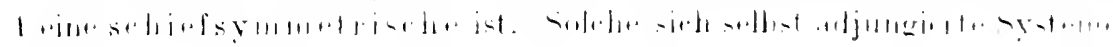

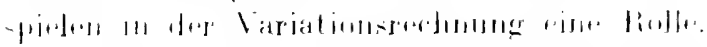

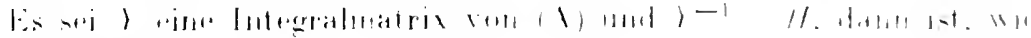

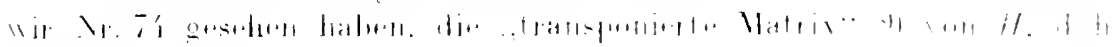

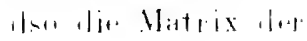

$$
i, k-1, \cdot
$$

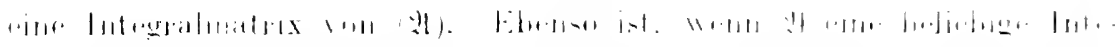

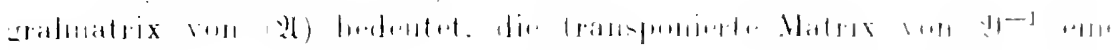

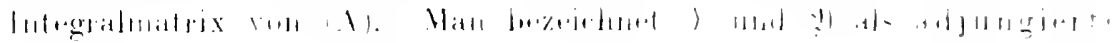

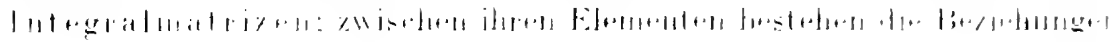

$$
\begin{aligned}
& \stackrel{\because}{1} 4,10 k \pi \\
& \text { In, yik o. } \\
& \stackrel{n=n, n \quad \theta k}{=1}
\end{aligned}
$$

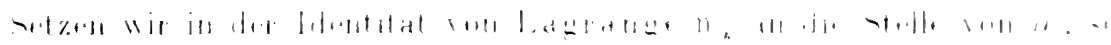
Poil

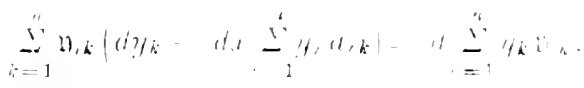

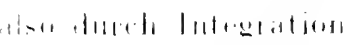

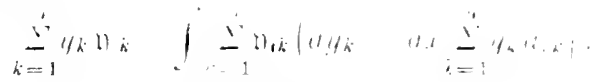

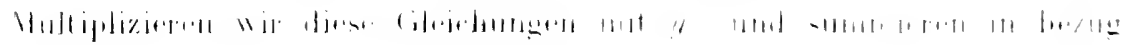

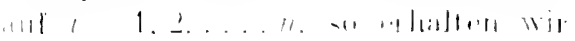

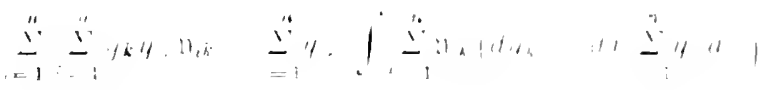

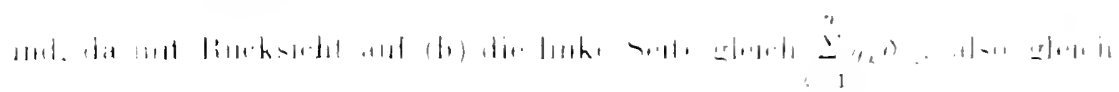
1. 1 . 1 .

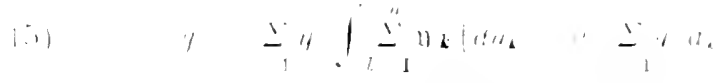

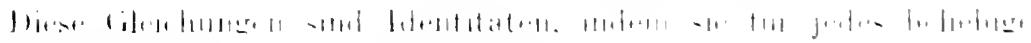

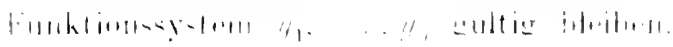




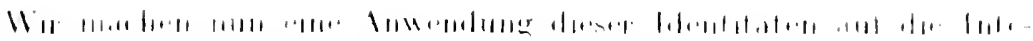

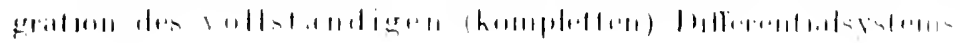

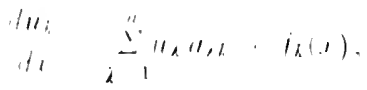

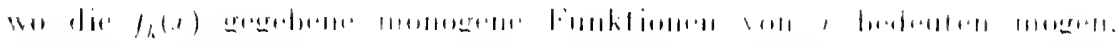

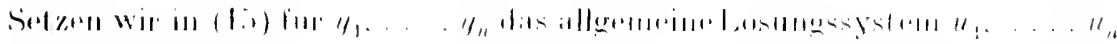

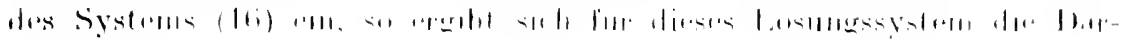
slollong

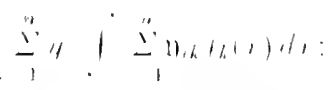

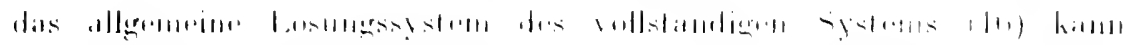

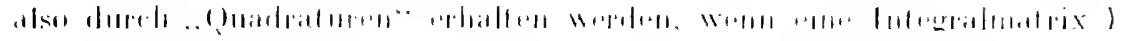

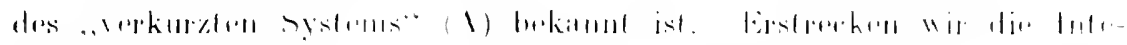

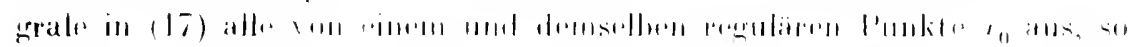

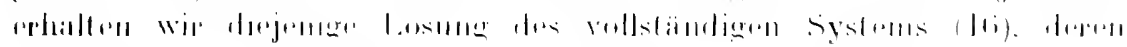

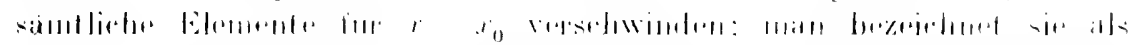

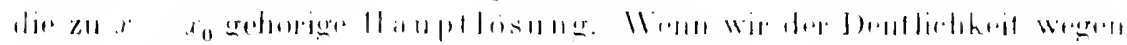

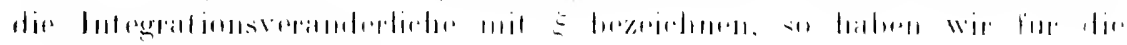

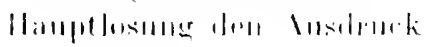

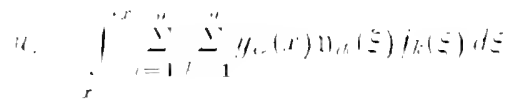

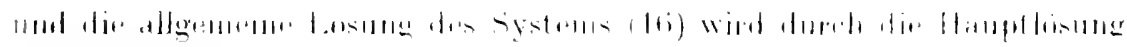
In dire lintu

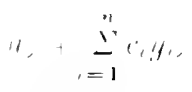

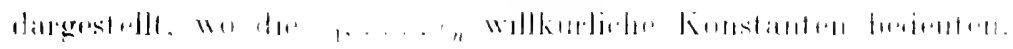

\section{7t. Beispiel ainer linearen Interrodifferentialogleirhme.}

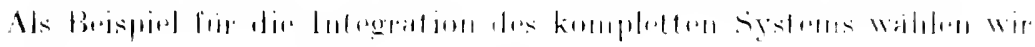

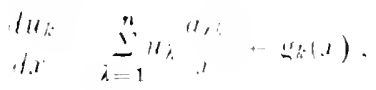$$
\therefore=12 \text {. }
$$

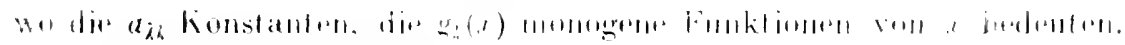

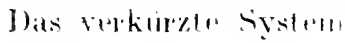

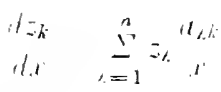

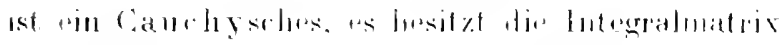

$$
\chi=1.1 \text {, }
$$

und "wnll wir "

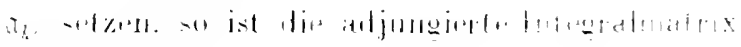

$$
\begin{aligned}
& \text { i) }
\end{aligned}
$$




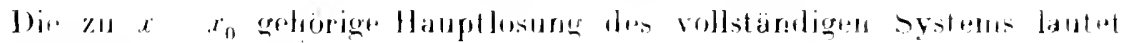
lininate

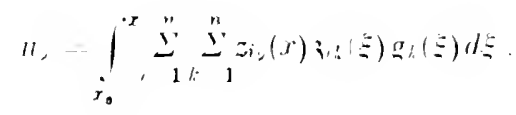

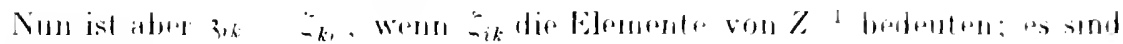
folglieh

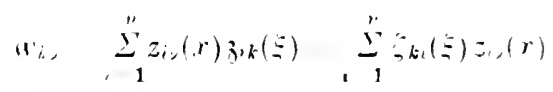

die Elemente dre Matrix

$$
11=Z(\xi)^{-1} \%(x) \quad\left(\begin{array}{l}
i \\
\xi
\end{array}\right)^{1}
$$

and wir finders

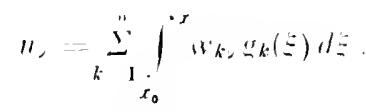

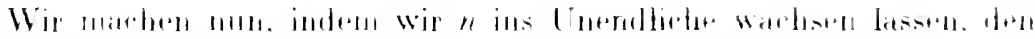

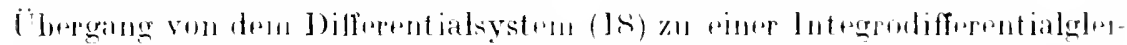

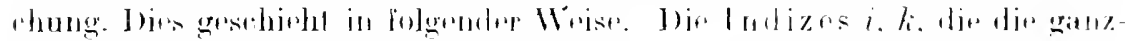

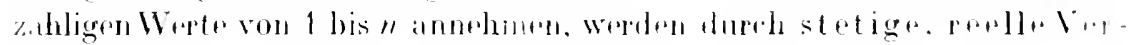

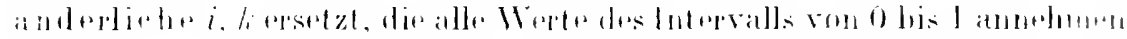

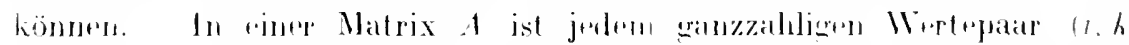

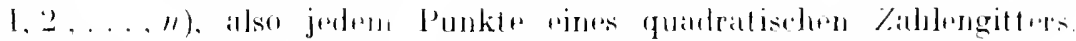

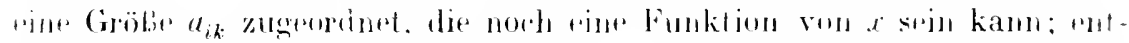

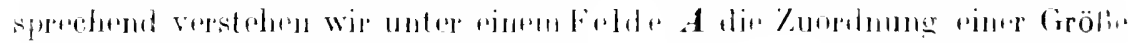

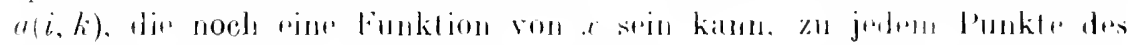

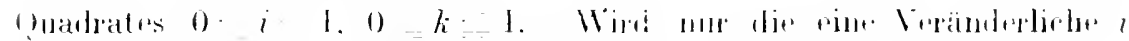

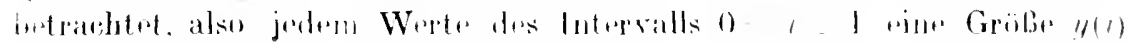

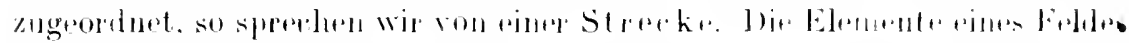

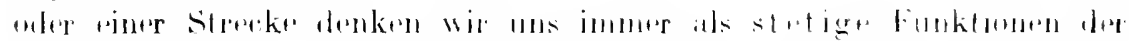

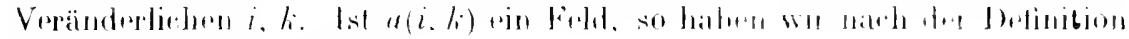
des bustimmlen lutegrals

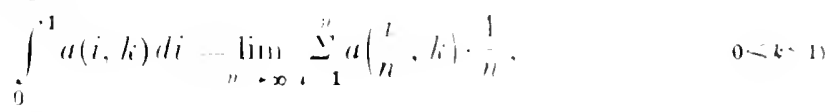

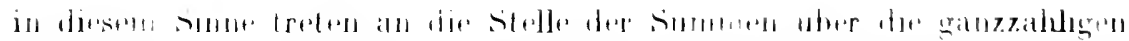

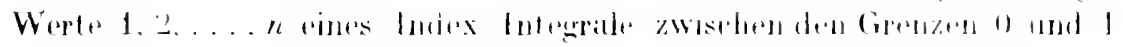

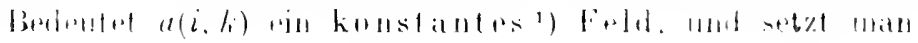

$$
{ }_{n} a\left(\begin{array}{cc}
i & k \\
n & n
\end{array}\right) \quad u_{1} k
$$

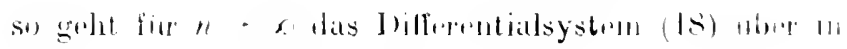

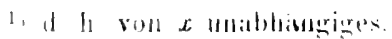


$(18,1)$

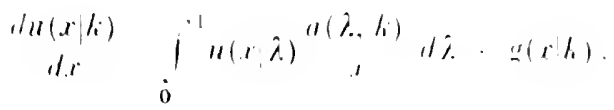$$
\text { (1) }+1 \text {, }
$$

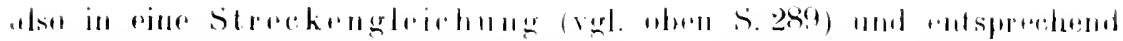

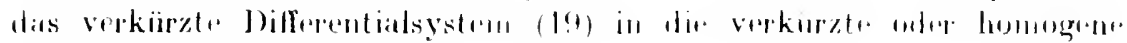
streckeng]nichung

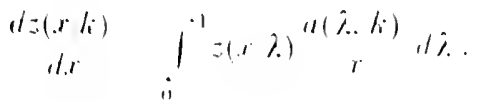$$
\text { (0) } 1 \text { 1) }
$$

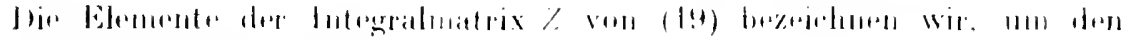
(ipenzübergang vormehmon zu kïnurn. mit

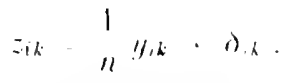

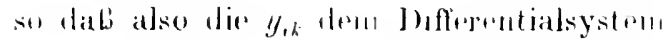

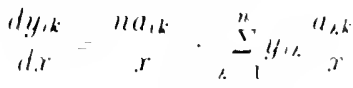

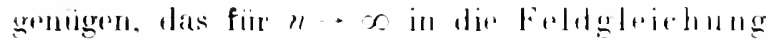

$$
d y(x \mid i, k) a(i, k)=\int_{i}^{1} y(x, i . j)^{a(j . k)} d i
$$

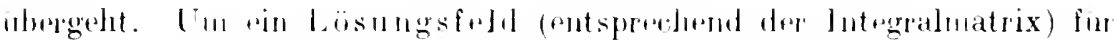

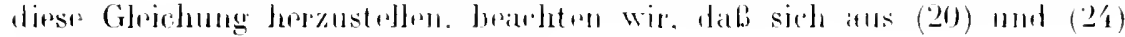
fär llir Matrix \& dir J)atrstellung

$$
\gamma=n .1 \log x+\frac{1}{11}(n .1)^{2}(\log x)^{2}-\frac{1}{2 !}-\frac{(n-1)^{3}(\log x)^{3}}{3 !}
$$

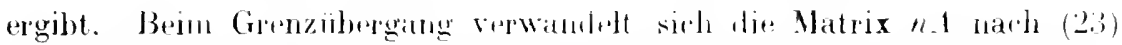
in das Feld $\boldsymbol{A}$ der $a(i, k)$. Firmer sind nach der hompositionsglendung (l)

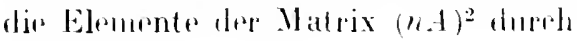

$$
\stackrel{2}{2} n a_{21, n} n a_{j k}
$$

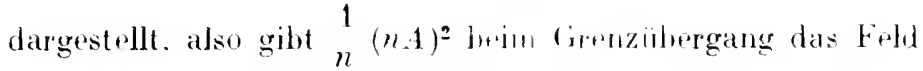

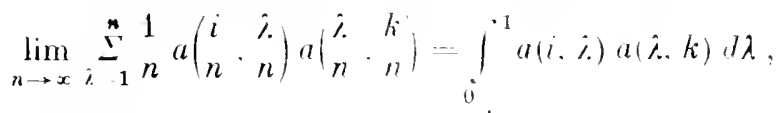

das wir mit $\boldsymbol{A}^{2}$ bezeinduen; weiter wird $\frac{1}{n^{2}}(n-4)^{3}$ zu droll Felde

$$
\boldsymbol{A}^{3}=\int_{i}^{1} \int_{i}^{1} u(i, \lambda) u(\lambda . u) a(\mu, k) d \hat{\lambda} d u
$$

usw. - Wir erhalten also fur das Lüsungsfeld $\boldsymbol{Y}$ der Feldgleichung (19b) die Darstellung

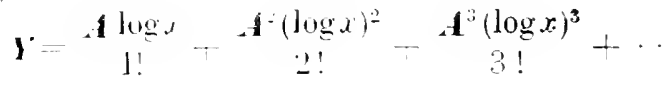


lias lind

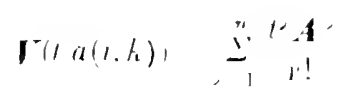

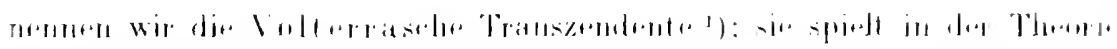

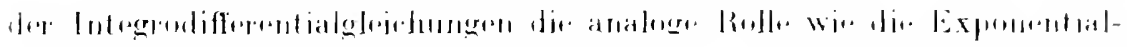

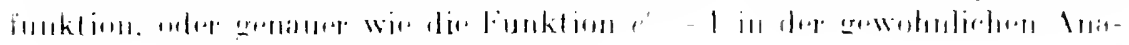

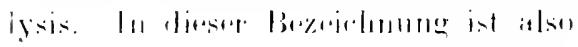

$$
\text { Ir } \quad r(\log x a(i . t)):
$$

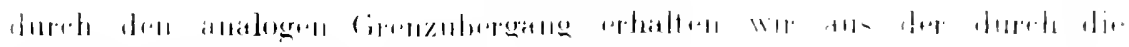

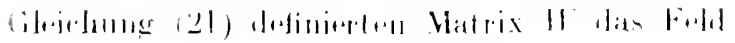

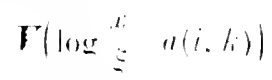

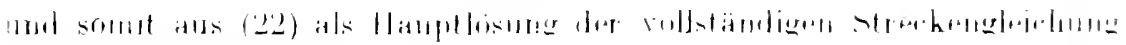

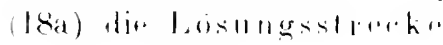

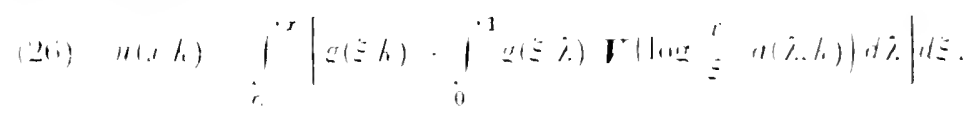

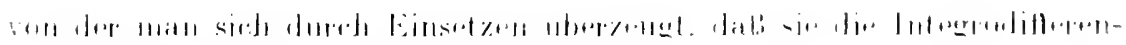

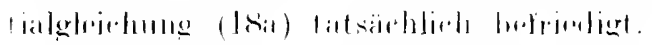

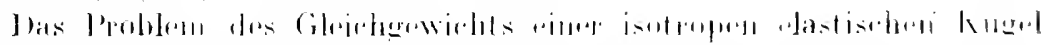

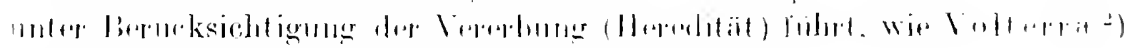

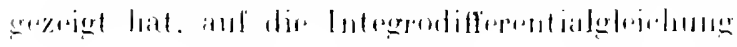

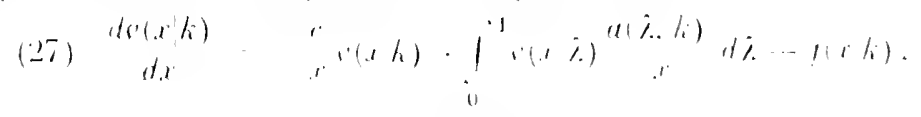

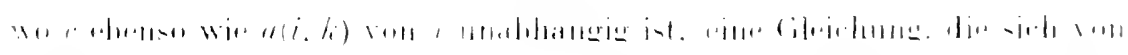

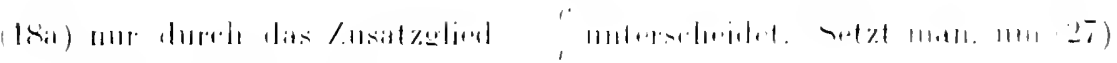

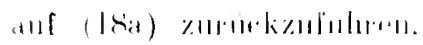

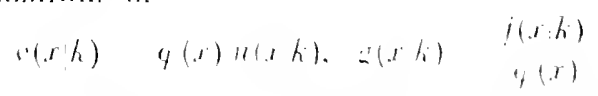

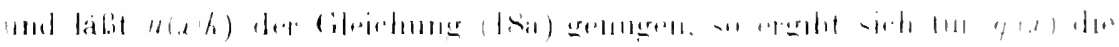
(i) eivflumer

$$
\begin{aligned}
& \begin{array}{ll}
\operatorname{lo}(x) \\
1, i
\end{array} \quad, q(x)
\end{aligned}
$$

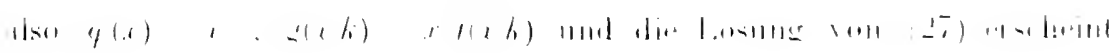




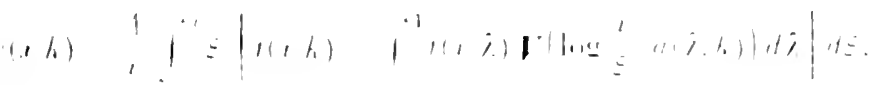

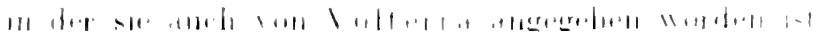

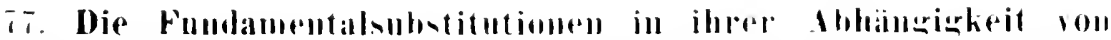

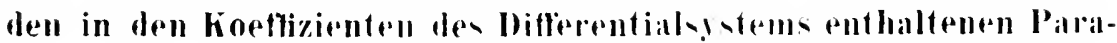 metern. Ilas Riemannsehe Problem.}

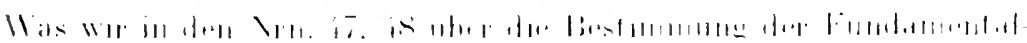

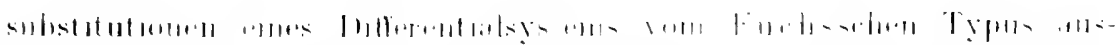

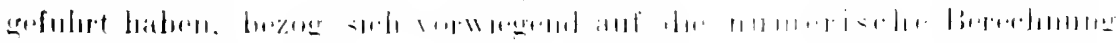

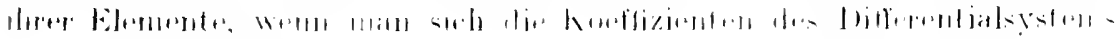

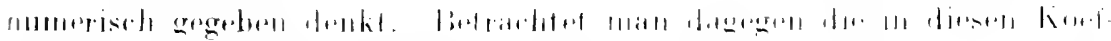

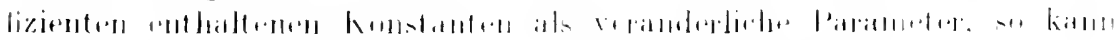

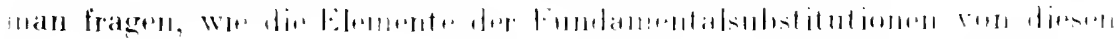

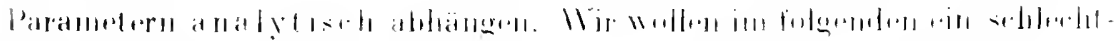

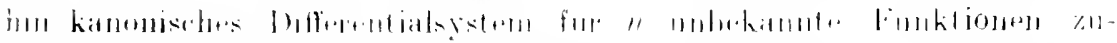

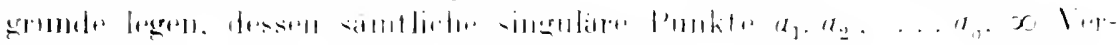

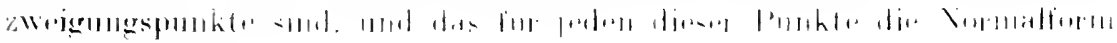

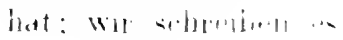

$$
\text { the } \quad \therefore 1, a, k
$$

W1

\section{(2)}

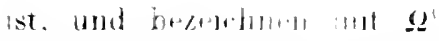

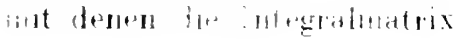

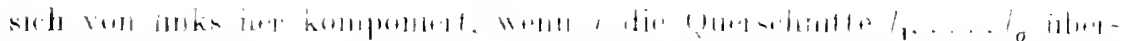

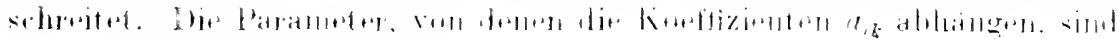

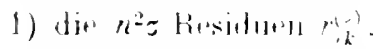

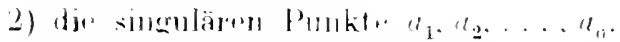

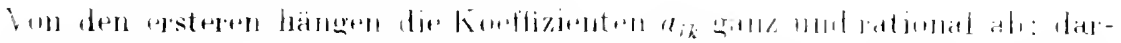

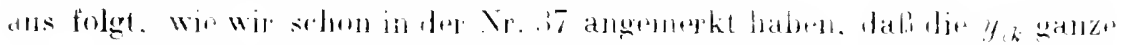

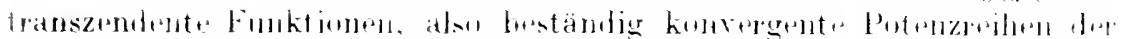

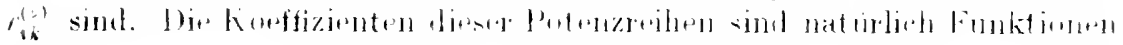

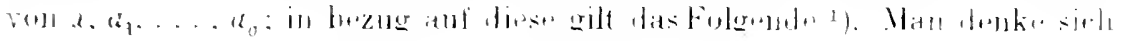

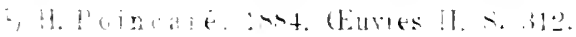




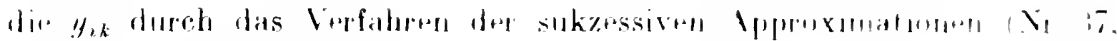
s. 14:i) largestellt; alsw

$$
\begin{aligned}
& y=I+I(1) \cdot I(2), \ldots+111 \mathrm{inf} .
\end{aligned}
$$

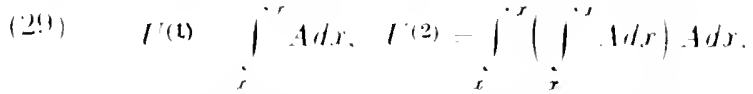

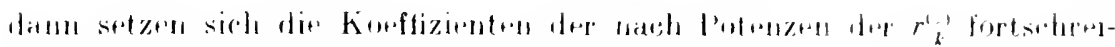

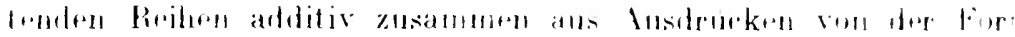

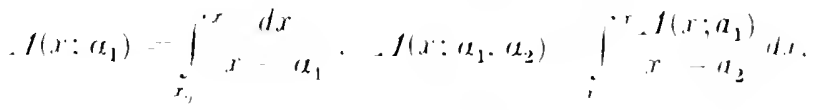

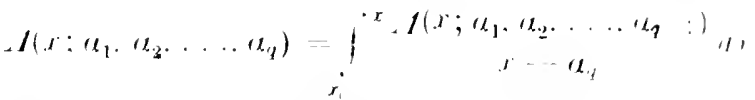

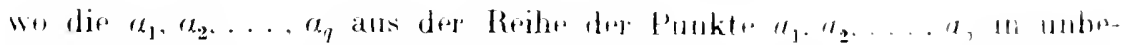

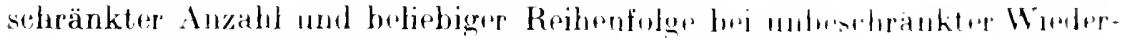

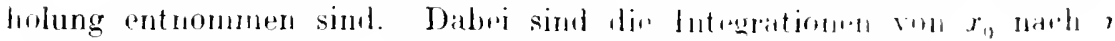

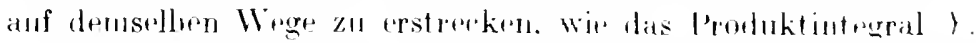

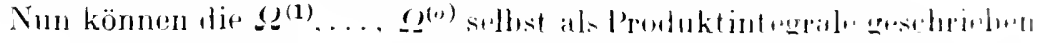

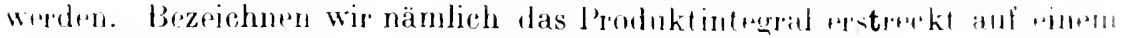

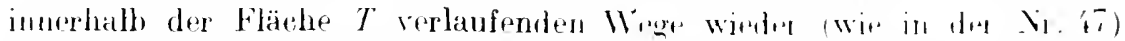

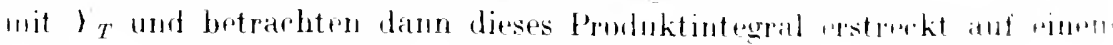

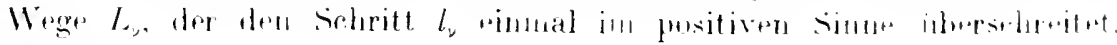
sol ist

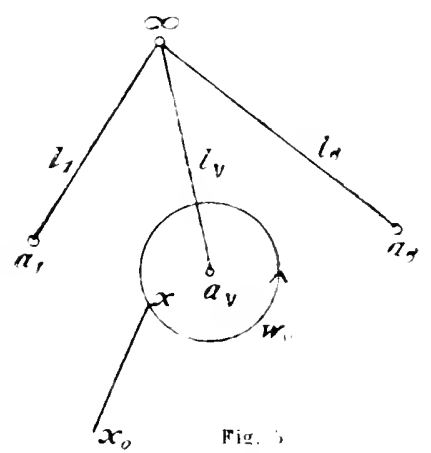

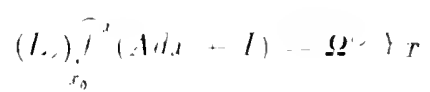

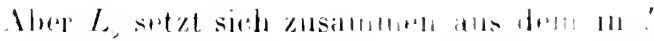

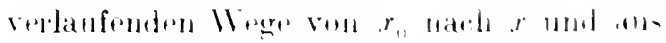

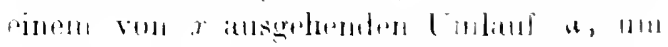

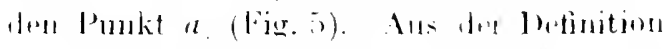

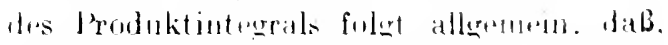

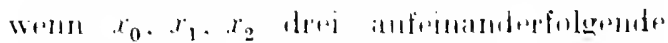

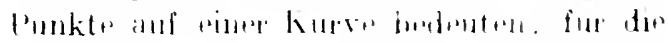

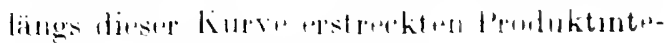

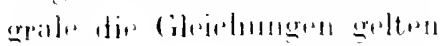

$$
\begin{aligned}
& \hat{i}^{r}(1 d x-l)=\hat{i}^{x}(1 d x+l) \hat{i}^{x}(1, x \cdot l) \\
& f_{x}^{x_{1}}(1, x \cdot l)=\left(i x^{x_{0}}(1,1, x+l)\right.
\end{aligned}
$$

Wir rrhalten alst

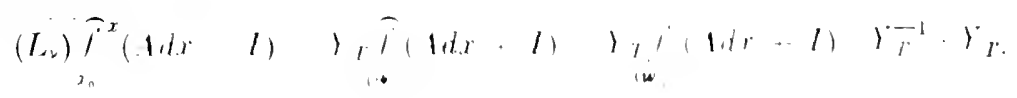


Es ist aber

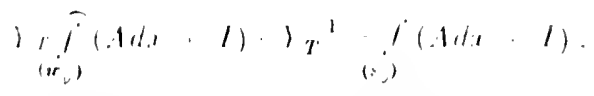

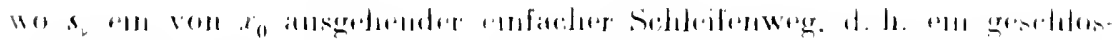

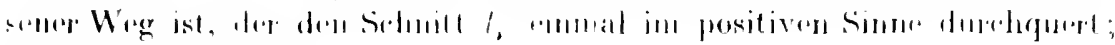

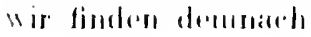

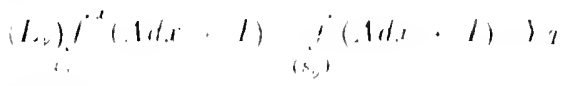

und folgheth mal Rurksichl anf (:i1)

$$
\text { I } \quad \therefore \quad i(1 d x \cdot 1) \text {. }
$$

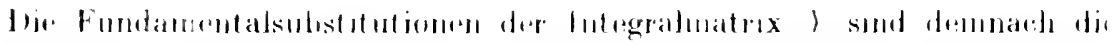

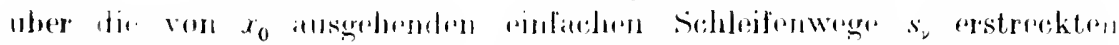
Produktintegrale. Minn wir also in (29) dir Integrationen iblure diese

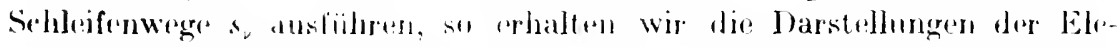

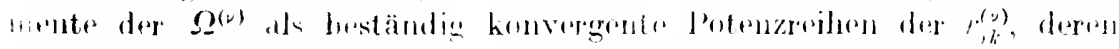

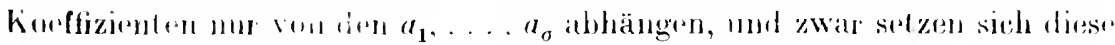

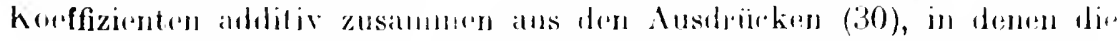

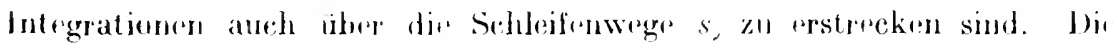

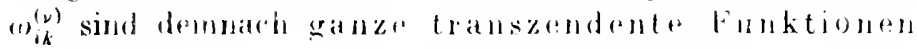

$$
(1))_{i k}^{(u)}=e_{i k}^{(w)}\left(r_{11}^{(1)} \ldots \ldots r_{n i t}^{(n)}\right)
$$

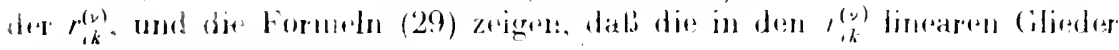
der ex dis form habers:

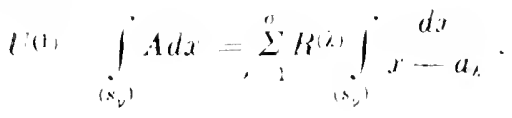

Nun ist aher

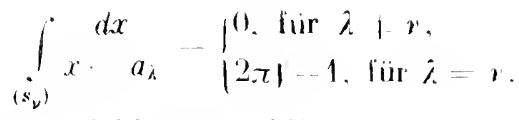

folghich lauten ar Entwicklungen $(32)$

$$
()_{i k}^{b)}-\partial_{k}+2 \tau \mid-1 r_{i k}
$$
wo die durch Punkte angedeuteten Glieder in den $r_{2 k}$ vom zweiten und höheren Grade sind.

Bilden wir also die Funktionaldeterminante 1 der $n^{2} \sigma$ ganzen transzendenten Funktionen $e_{l}^{(v)}$ wach den $n^{2} \sigma$ Residuen $r^{(v)}$, so hat $t$ fiir die Stolle

$$
r_{11}^{11}=0 \ldots r_{11}^{\prime \prime \prime}=0
$$

den Wert

$$
J_{0}=(2-2 \mid-1)^{n^{2} i s}
$$

$\Delta$ ist also jedenfalls nicht identisch gleich Null. 


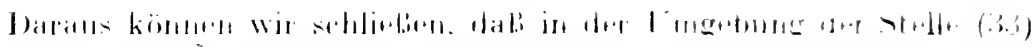

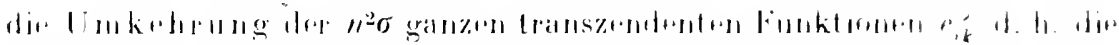

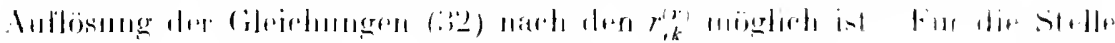

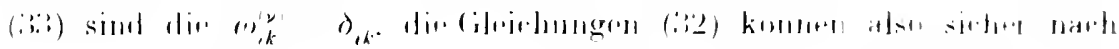

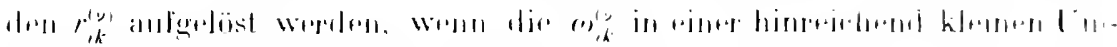

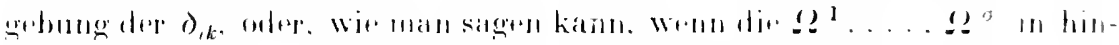

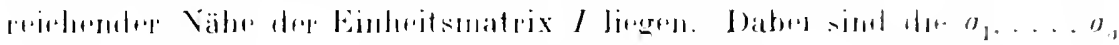

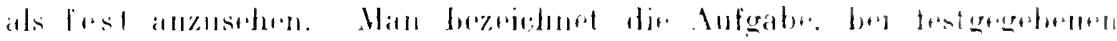

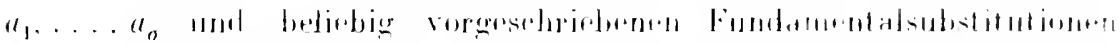

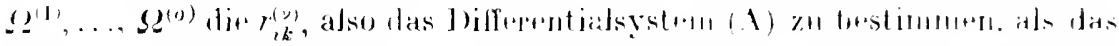

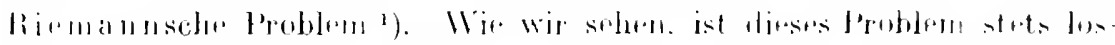

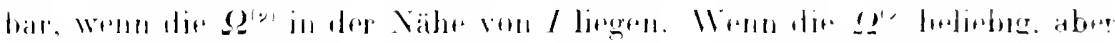

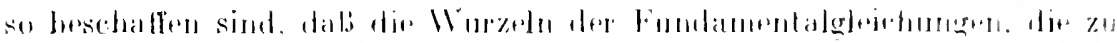

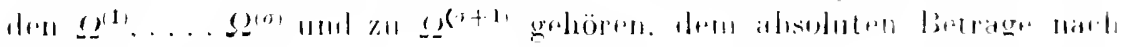

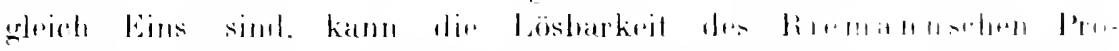

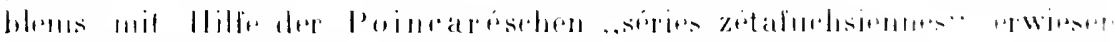

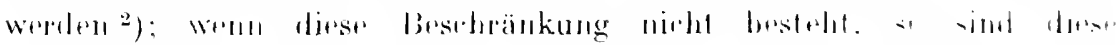

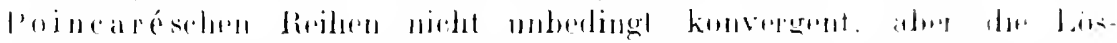

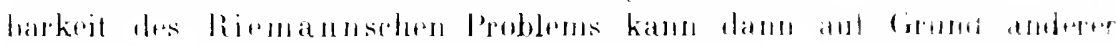

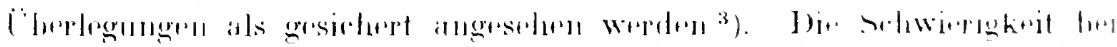

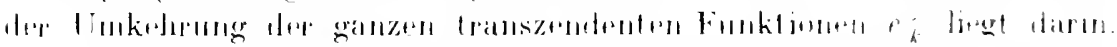

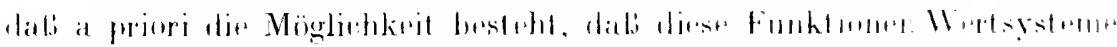

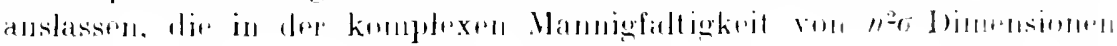

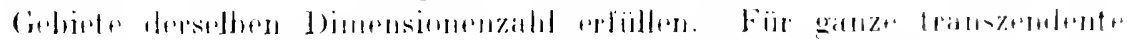

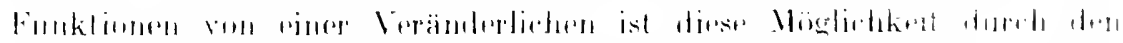

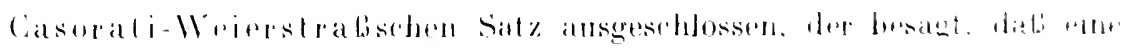

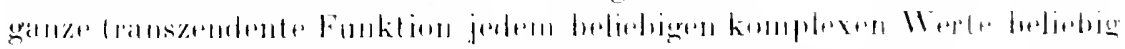

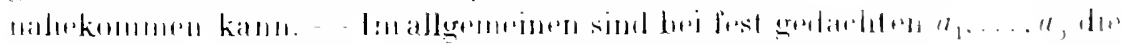

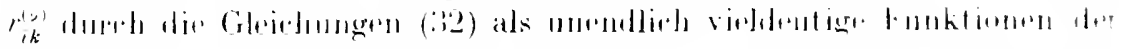

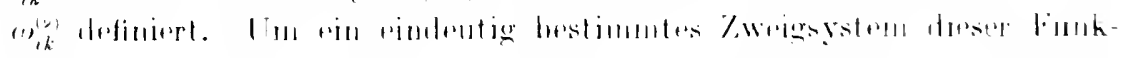

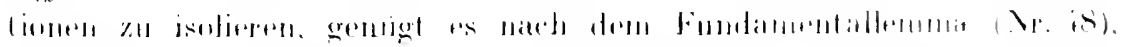

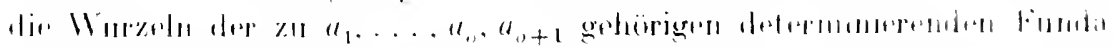

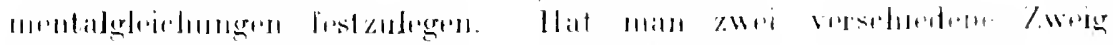

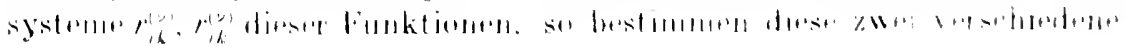

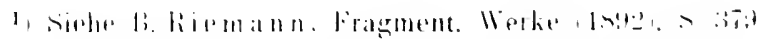

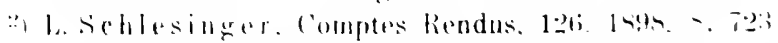

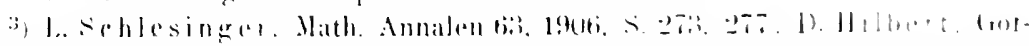

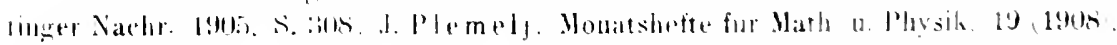

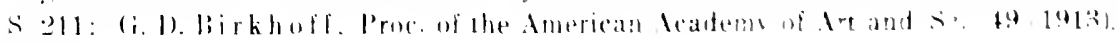
(5) 


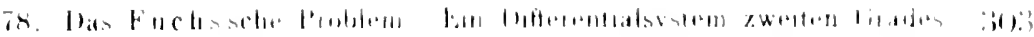

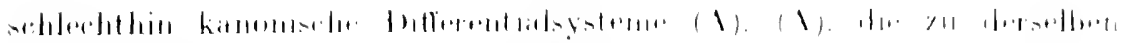

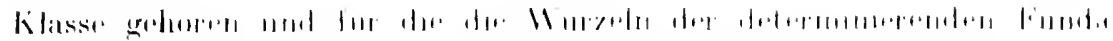

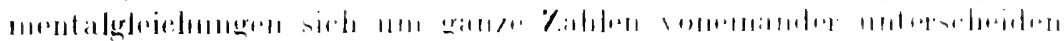

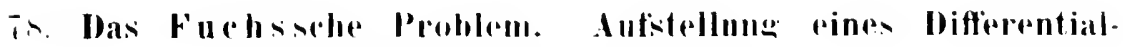 sytems zweiten tirades.}

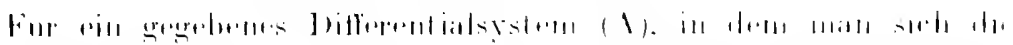

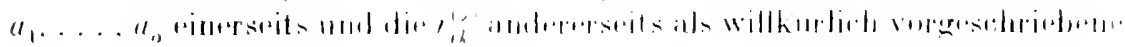

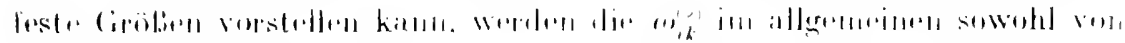

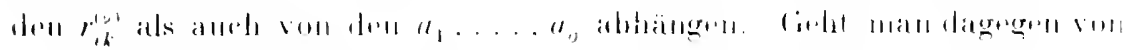

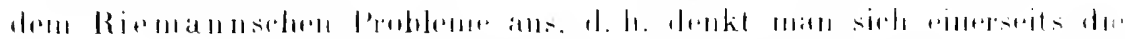

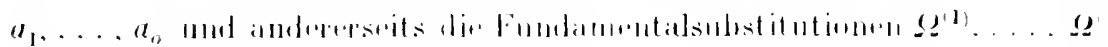

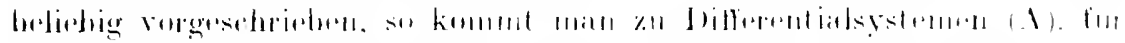

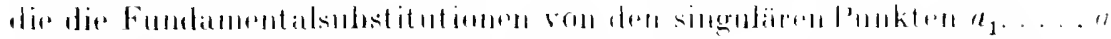

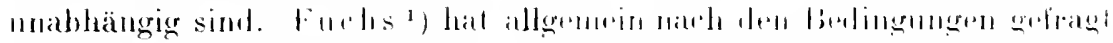

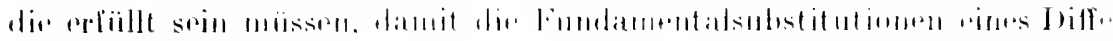

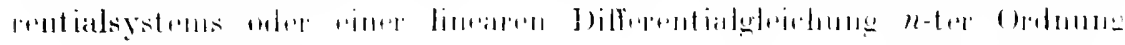

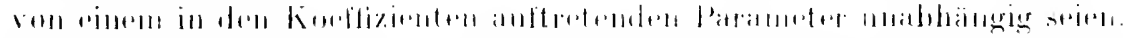

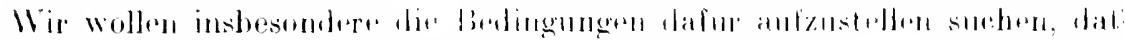

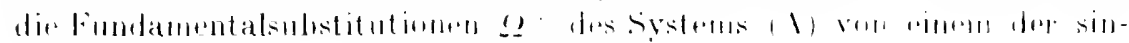

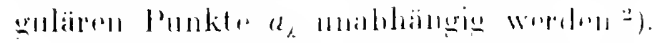

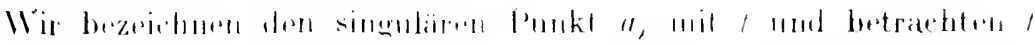

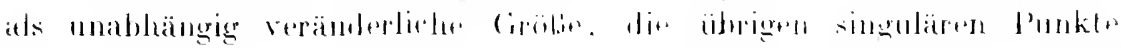

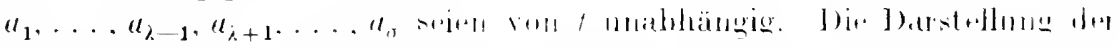

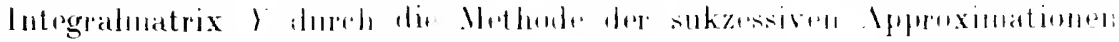

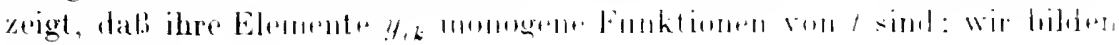

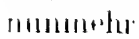

$$
1,\rangle \quad \gamma-1+r
$$

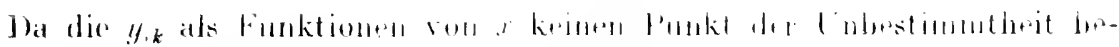

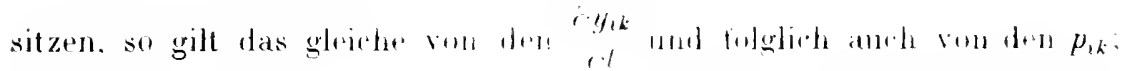

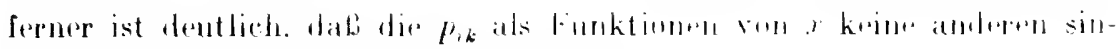
gulären stellen haben kïnnell als die !nk.

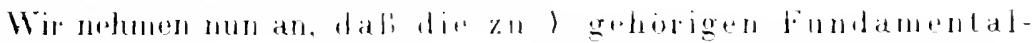

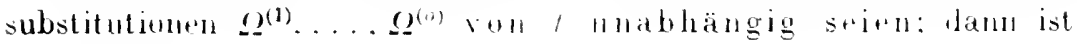

1, L. Fuchs. 1856. siehe Herke 111. $\therefore 117 \mathrm{ff}$.

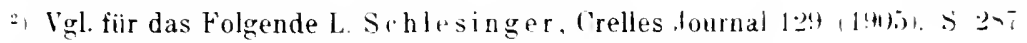
Vorlesungen 1905. s. 31\%. Crelles Journal $1+1$ (1912). ‥ 9 if 


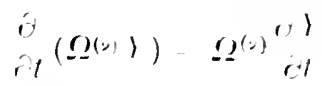

ind folglich

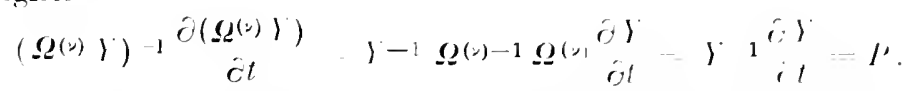

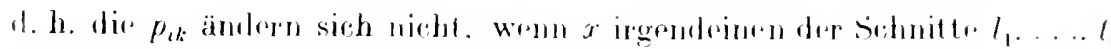

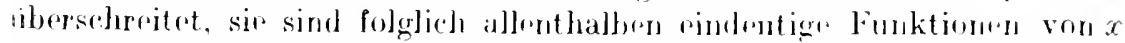
und, la sie nur rine ondliche Anzahl singulärer Strden dre limstimuthrit haben könuen, rationale funktionen von $x$.

Es sei nun in der limgroung voll $r=a, y+\%$

$$
\gamma=C^{(\nu)}\left(x-a_{\nu}\right)^{(\infty)} \Phi^{(\infty)} \text {. }
$$

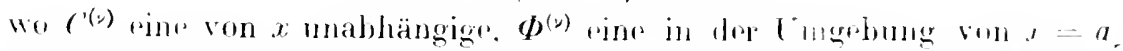

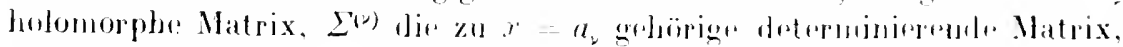

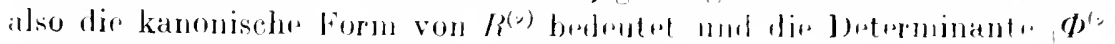
firr $l=a_{\nu}$ nirht vestelswindel. J) it

$$
\left.e^{2}+r i \div\right)
$$

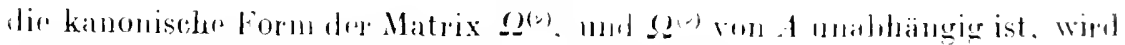

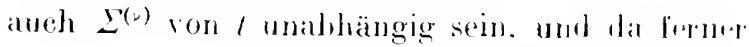

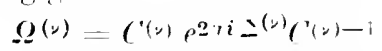

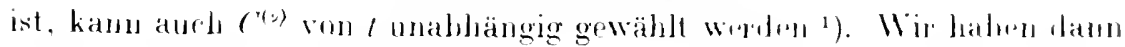

und folglich

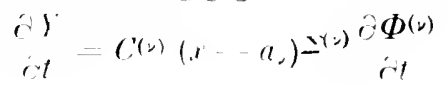

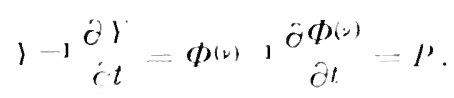

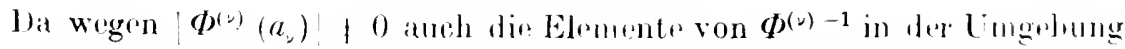
von $r-a_{2}$ holomorph sind, gilt das gleiche fïr die klenente von $l$. Jiese sind also in der Umgebung lor voll t lnablängigun

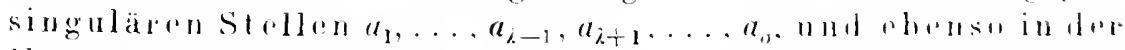

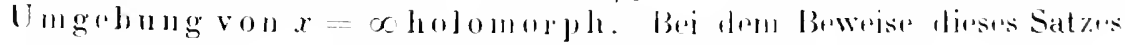
worden in beyog auf $t$ nur die Eigenschaften henutzt, dali die $y_{i k}$ mor-

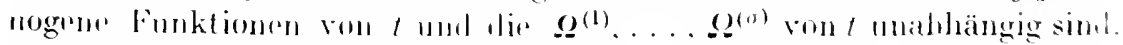

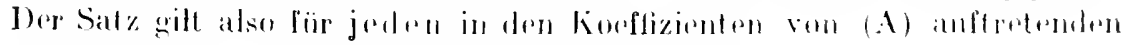

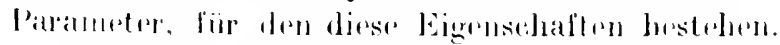

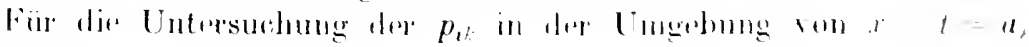
$\therefore\left(2011+i^{2}\right)$

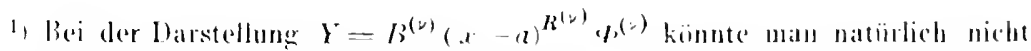
schlieben. dab $R^{(\nu)}$ und $S^{(2)}$ von $/$ mabbingrig sind. deshath mubten wir hien die determinierendo Matrix $Z^{(2)}$ selbst zur ladstollung bon Y benutzene

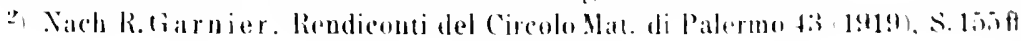




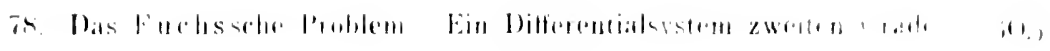

datin wird

$.11 \times 0$ hatwn 111

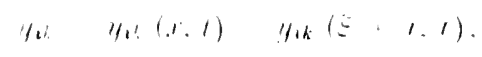

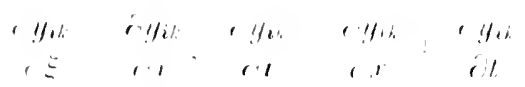

IIII) folgrliclı

$$
\begin{aligned}
& \begin{array}{r}
y> \\
y=-1 .
\end{array} \\
& 1101.11
\end{aligned}
$$

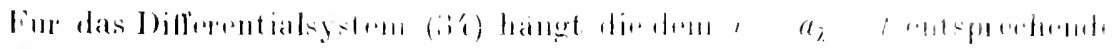

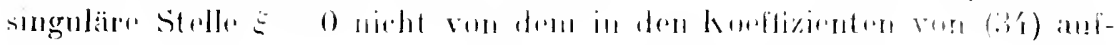

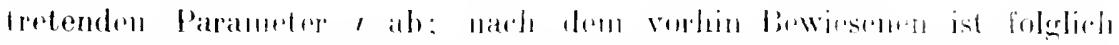

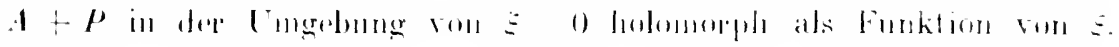

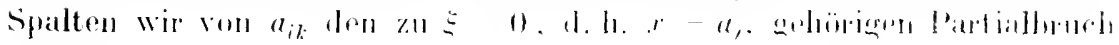
$r_{i k}^{(i)}$ i $a_{i}$

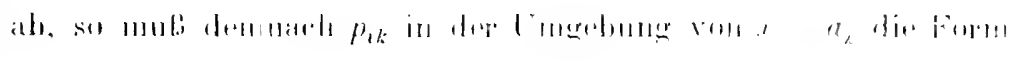
hahen:

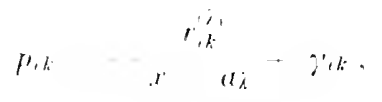

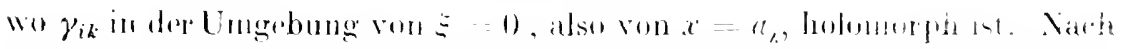

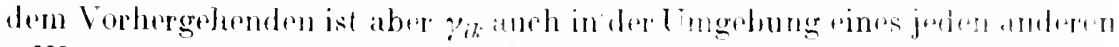

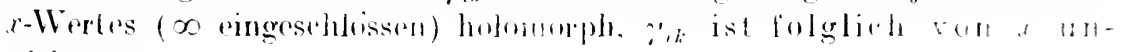
ablı̈̈ngig.

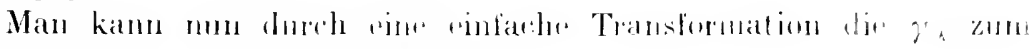
llogfall bringen. Sotzen wij nämblich

$$
r_{-}=C^{\prime} \text {, }
$$

wo dir Elementr ron ('vm a mabhängig sind, su ist

$$
\begin{aligned}
& \hat{\partial} Z=Z C^{\prime} .1 C^{\prime} \cdot
\end{aligned}
$$

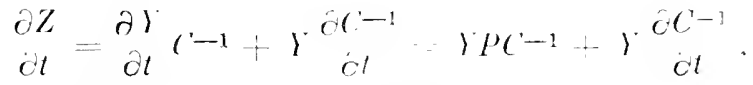

Ninn folgt aus ( $\left(^{-1}=I\right.$ ilureh Differentiation narh $/$

$$
\partial C^{C-1}+C^{c-1}=0 \text { : }
$$

wir können alsel (iig) in der Form schreibru:

$$
\frac{\partial Z}{c t}=r P(-1-)\left(-1 \frac{\partial C}{C^{\prime}} C^{-1}\right. \text {. }
$$

Setzen wir nunmehr

schlcsinger, Differentialgleichungen. 
str folgt at1s (:i9:1) 11011 (:iT)

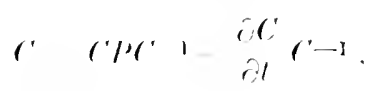

$$
\ddot{\partial}=Z c^{\prime}
$$

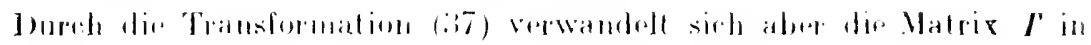

$$
r \text { rir } 1 \text { r. } r i
$$

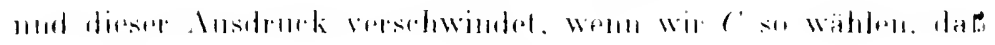

$$
\text { ar } 1 \% \text {. }
$$

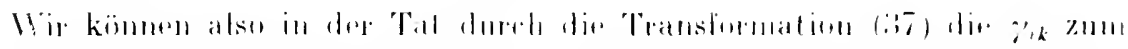

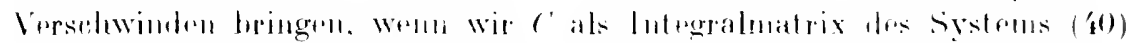

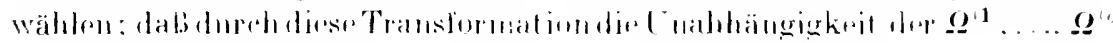

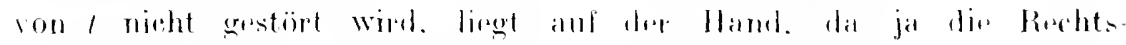

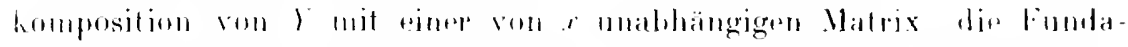

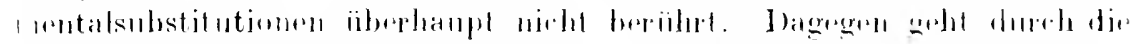

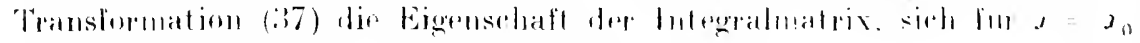

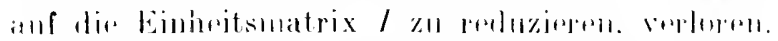

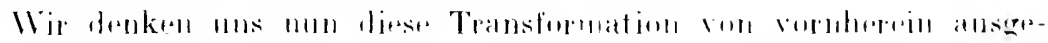

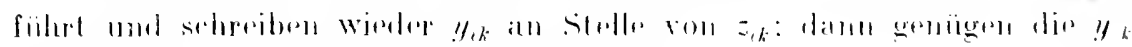

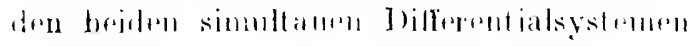

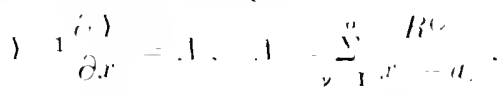

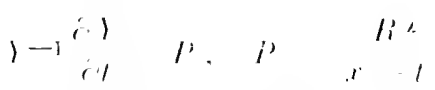

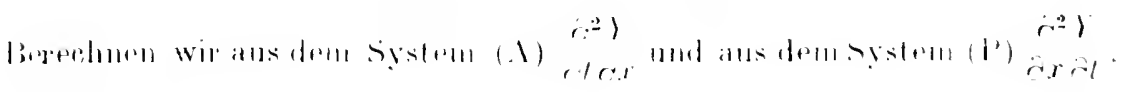
su findoll wil

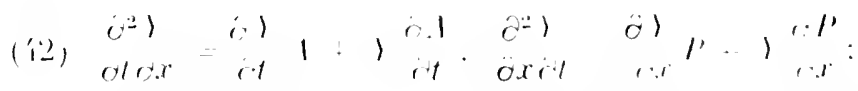

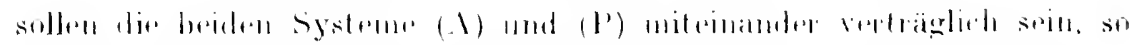

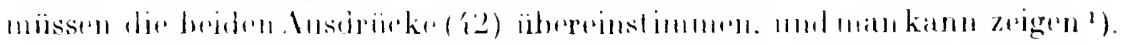

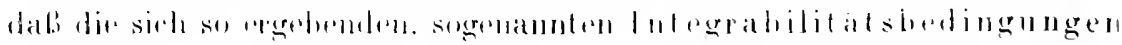

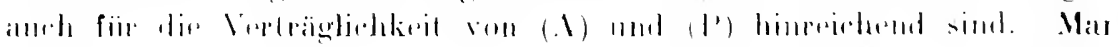

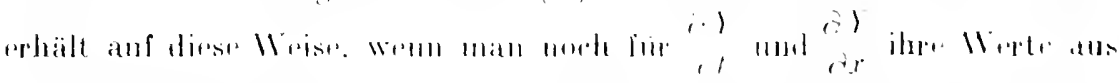

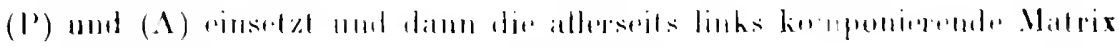

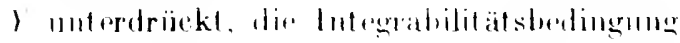

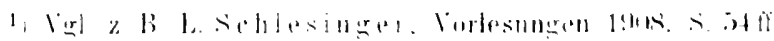




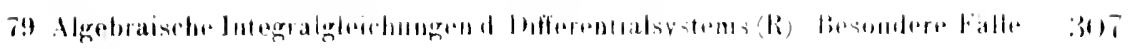

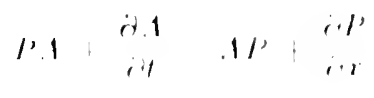

mler ausfuturlirber

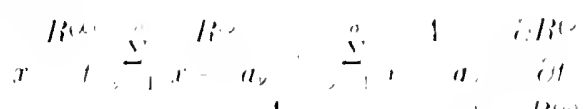

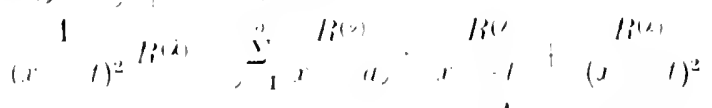

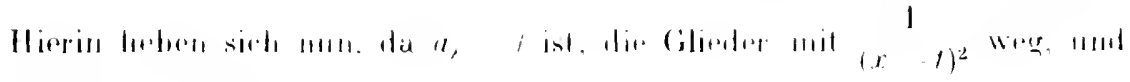
wrint IIIII IIorh

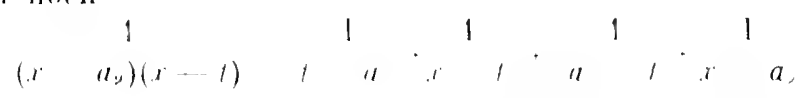

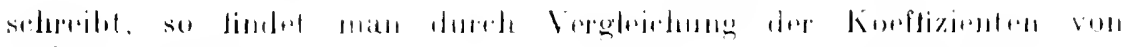

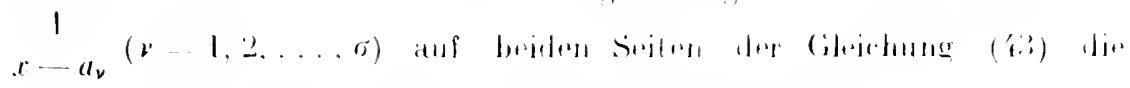

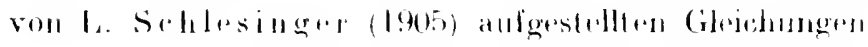

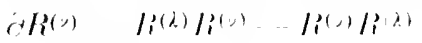

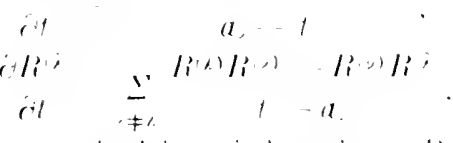

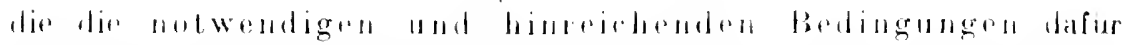

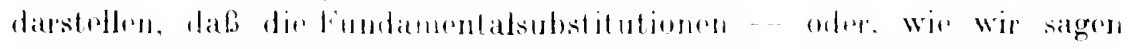

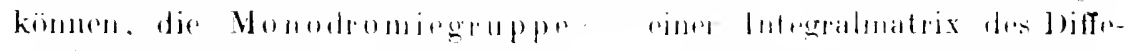

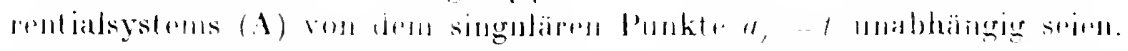

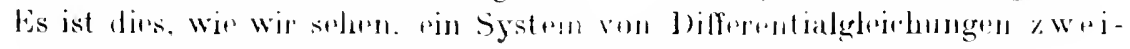

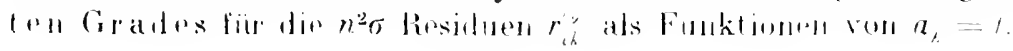

\section{i!. Algebraische Interralgleichungen des bifferentialsystems ( $\mathbf{h}$ ). Besondere Faille.}

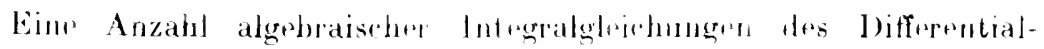
systems (R) läbt sich sofort angendon.

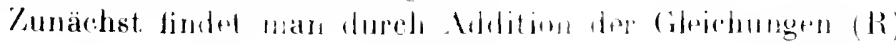

also

$$
\therefore \text { ili }
$$

$$
\stackrel{2}{2} t=
$$

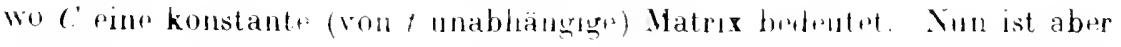
$\sum_{\nu=1}^{0} R^{n}=-R^{(0+1)}$ aje zunt Punklr $x=\infty$ gehorign Residuenmatrix ates 


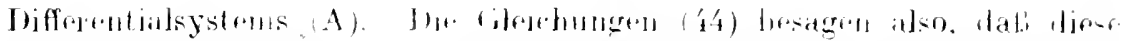

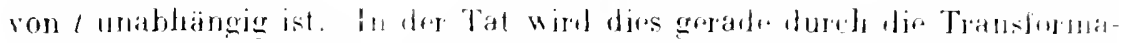

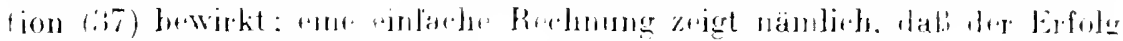

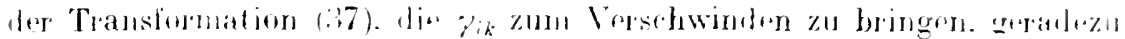

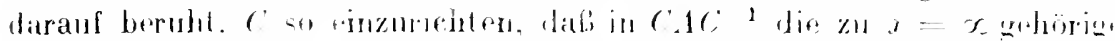

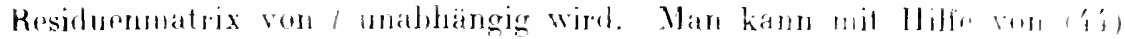

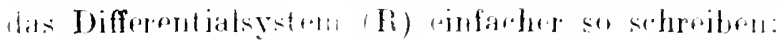

$$
\begin{aligned}
& \text { d } \\
& 11 \quad 0,1
\end{aligned}
$$

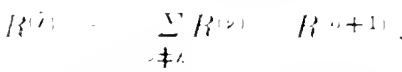

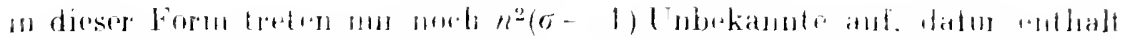

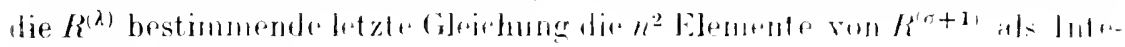
grationskonstant"m.

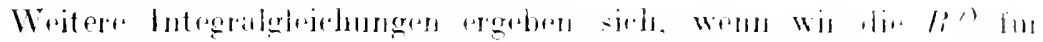

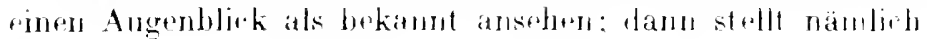

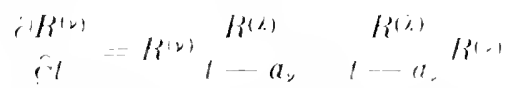

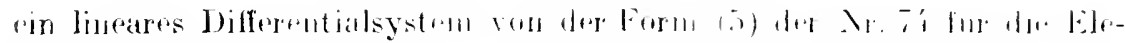

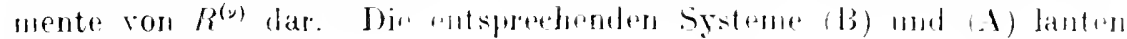
hier iibereinstimmend

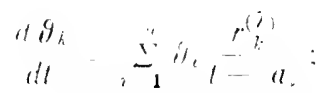

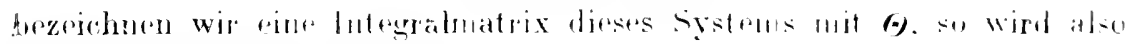
(sinter Cil. (10) der Xr. Th)

$$
h(x)=(-1 \pm(*)
$$

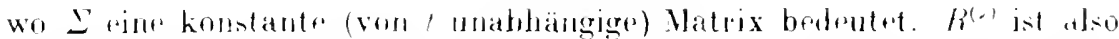

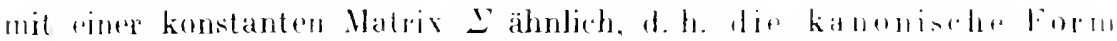

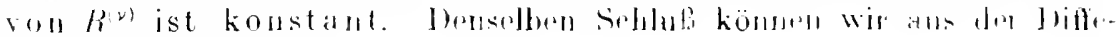
rentialdeleirlunter

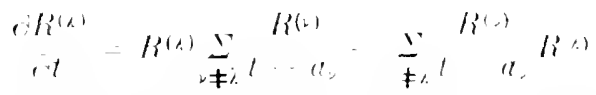

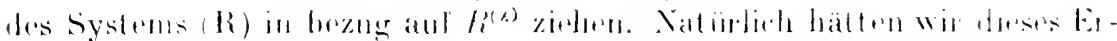

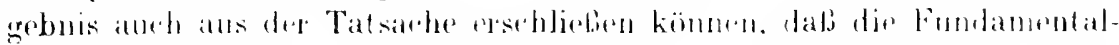

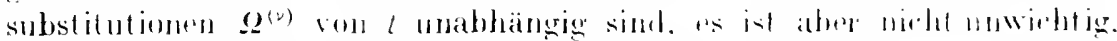

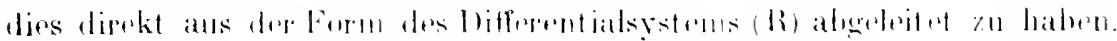

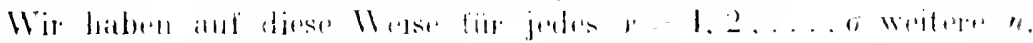

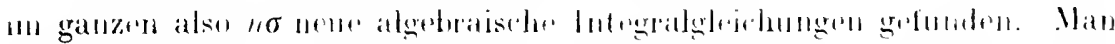

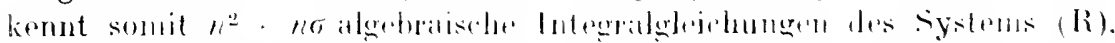

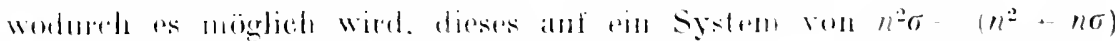

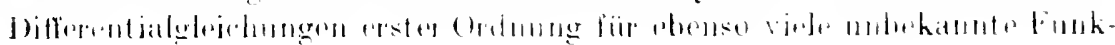




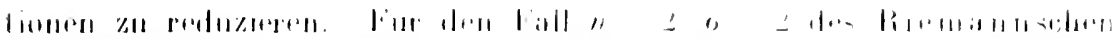

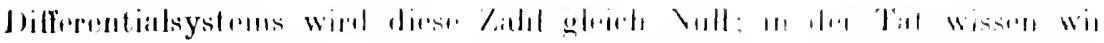

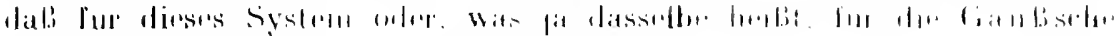

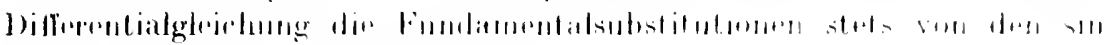

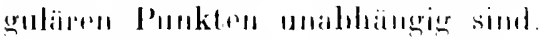

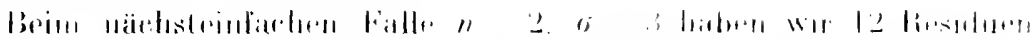

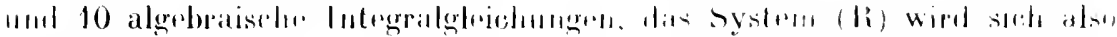

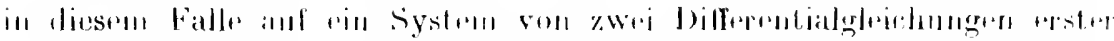

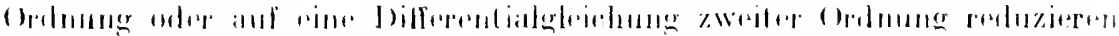

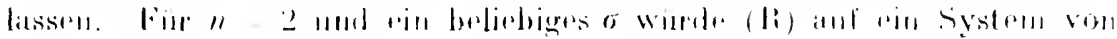

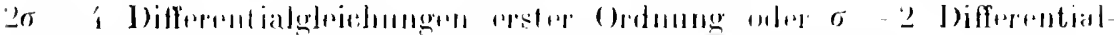

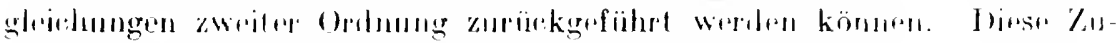

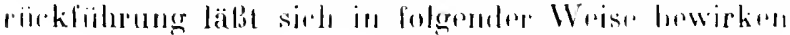

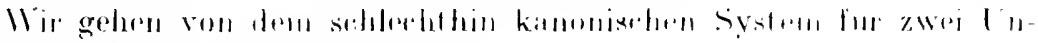

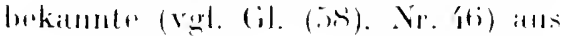

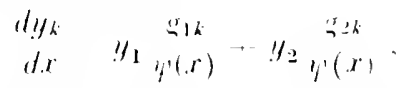

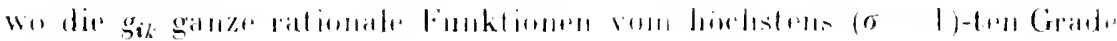
fwitrutin 11111

$$
y(r) \quad\left(r \quad a_{1}\right) \ldots\left(\begin{array}{ll}
y^{\prime} & \left.a_{i}\right)
\end{array}\right.
$$

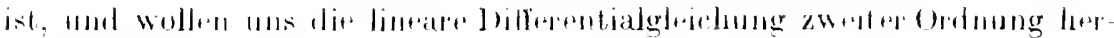
getstellt denkm, der

$$
=\quad l_{1} h_{1}+t_{2} / h_{2}
$$

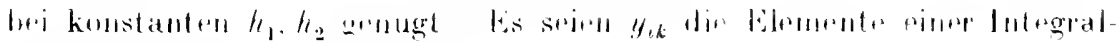
matlix von (份). dann bitden

$$
\Rightarrow-y_{11} h_{1}+y_{1-2} h_{2}
$$$$
11=1, \underline{2}
$$

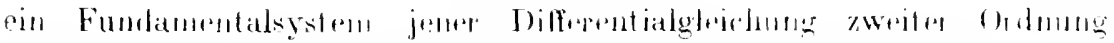

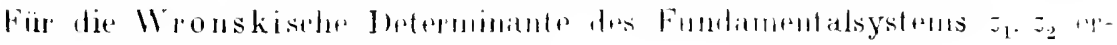

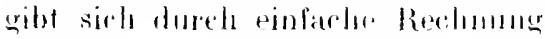

$$
\begin{aligned}
& z_{1} z_{1}^{\prime} \quad y_{11} h_{12} \quad h_{1} \frac{g_{11}}{y} h_{1}+\frac{s_{12}}{y_{f}} h_{2} \\
& z_{2} z_{2}^{\prime} \quad l_{21} l_{22} \quad h_{2} \quad \frac{g_{21}}{\eta_{1}} h_{1}+\frac{g_{22}}{y^{\prime}} h_{2}
\end{aligned}
$$

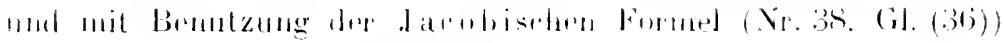

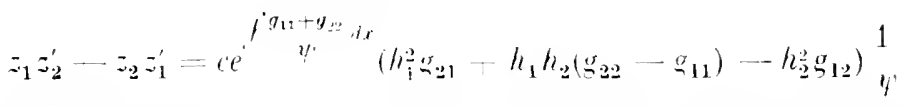

Wir sohen darans, dab. die Jollerentialghtohung zweiter Orthumg fur z

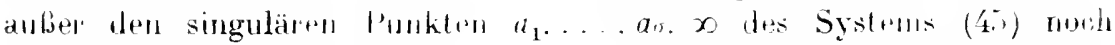
anlierwesentlich singular" Punkt" besitzl. an den stellen nan. linh. Wu

$$
h_{1}^{2} n_{21} \cdot h_{1} h_{2}\left(n_{22}-n_{11}\right)-h_{2} n_{12}=1
$$




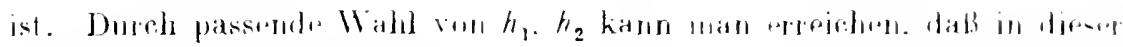

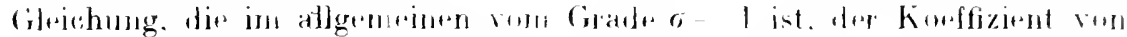

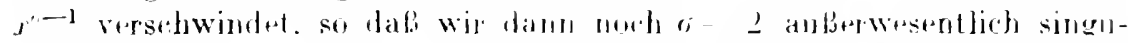

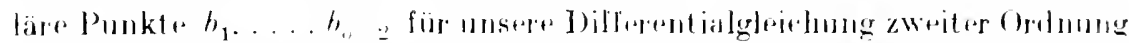

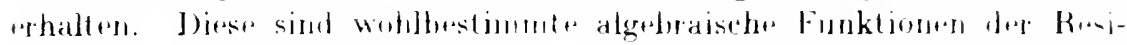

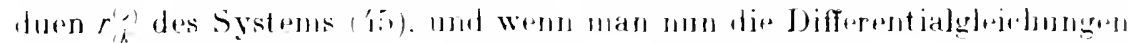

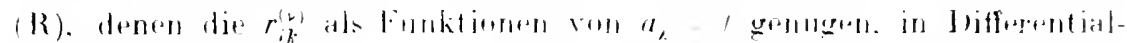

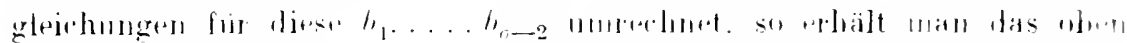

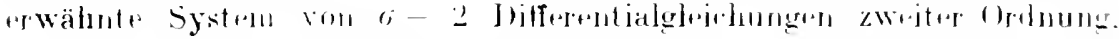

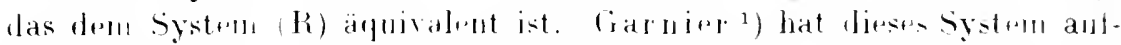

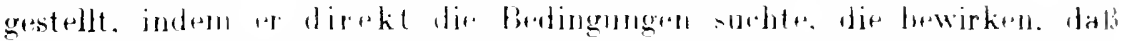

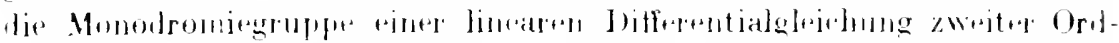

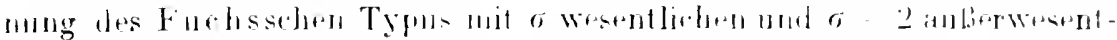

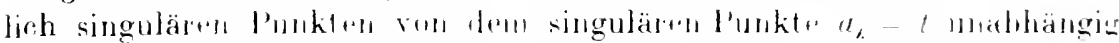

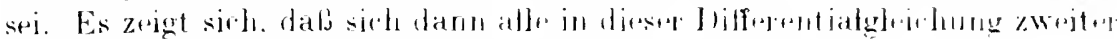

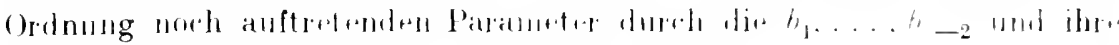

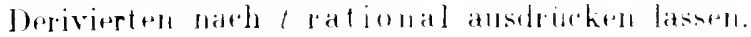

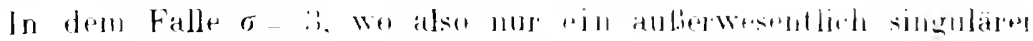

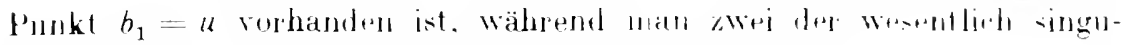

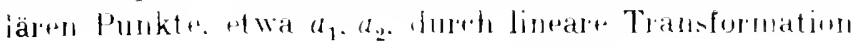

$$
\Xi=r-a_{1}
$$

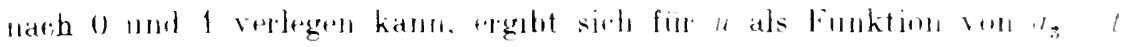

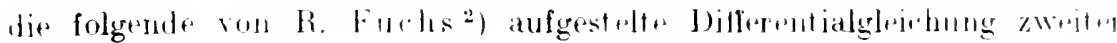
(r)dnurig:

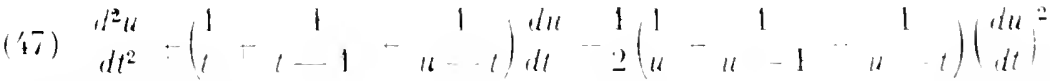

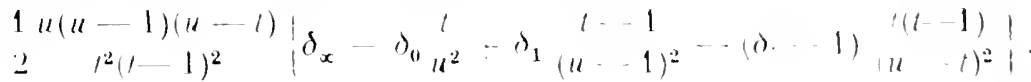

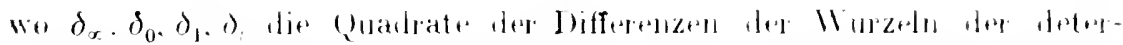

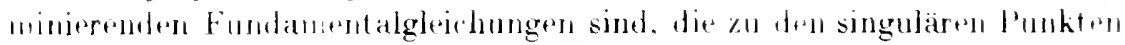

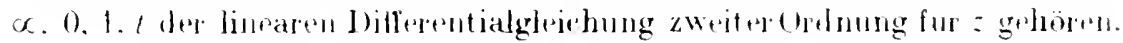

Es hat sich mon das mberrasehemele Ergehois herausgestellt ). dati

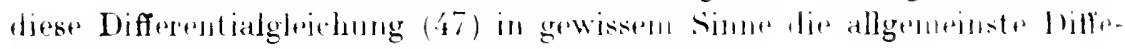

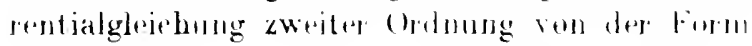

$$
d^{2} u=\operatorname{li}\left(\begin{array}{l}
d u \\
d t
\end{array}, u, t\right)
$$

1) R. liarnier. Thises. l'aris 1!111. S. his.

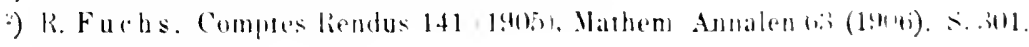
) Vul. R. (iarnipl. a. a. (1). 


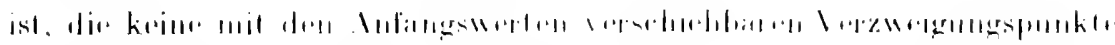

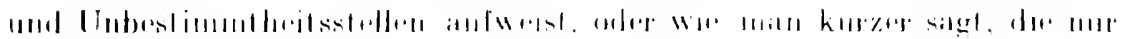

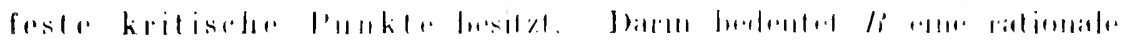

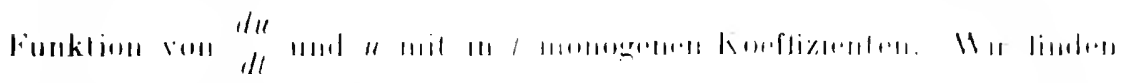

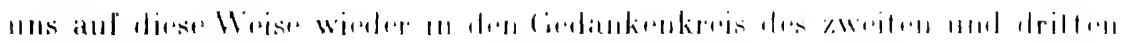

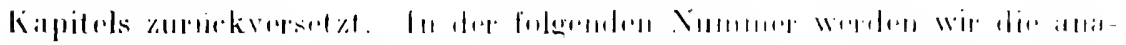

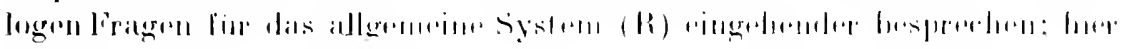

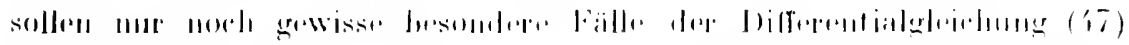

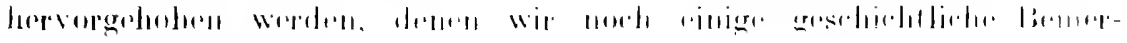
killgan vorillstillon.

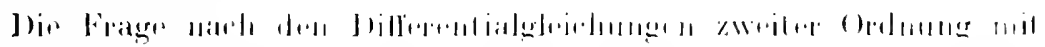

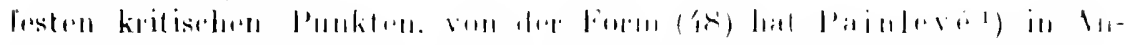

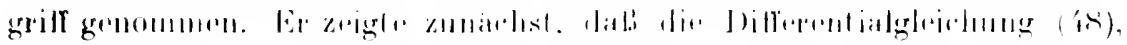

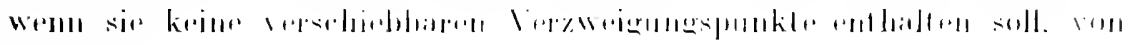
der Form

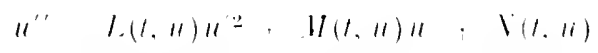

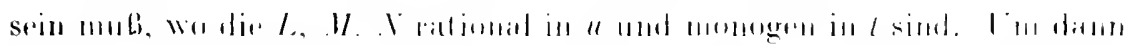

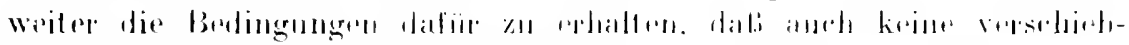

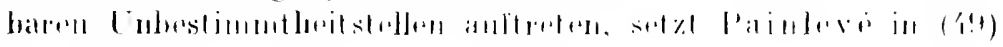

$$
1-t_{0} \cdot 20
$$

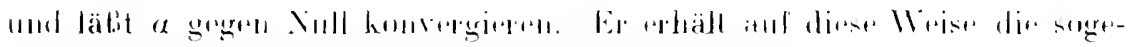

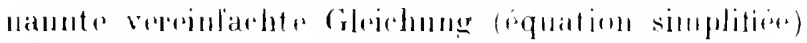

$$
\left\|" \quad L\left(l_{11} .11\right)\right\|^{\prime 2}-(11) \|^{\prime 2} \text {. }
$$

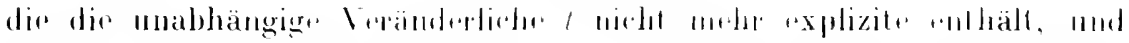

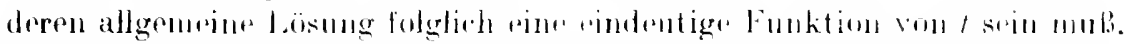

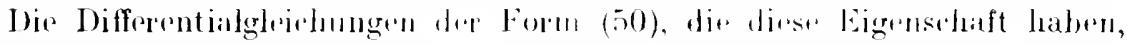

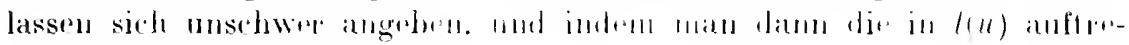

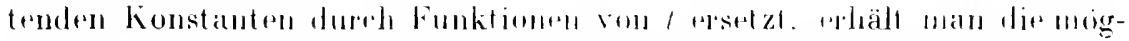

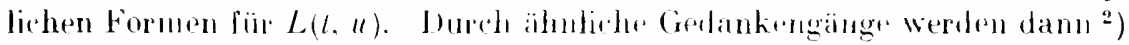

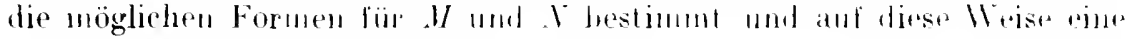

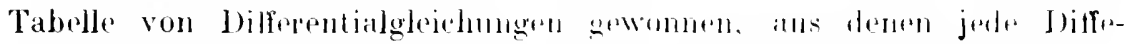

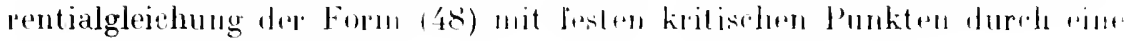
Transformation del Form

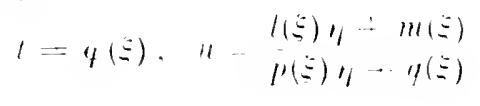

1) P. Painleve. bulletin de la soc. Mathém. de France os (a!he). S. 2011: Acta mathem. 25 (19(1) ). S. $1 \mathrm{ff}$. Vgl. fïr das Folgende den Enzyklopädieartikel II l. 6 von E. Hilb.

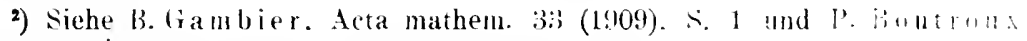

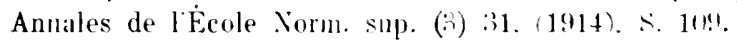




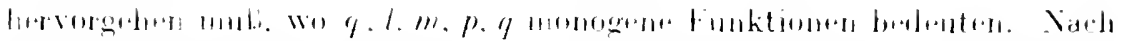

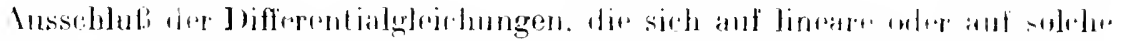

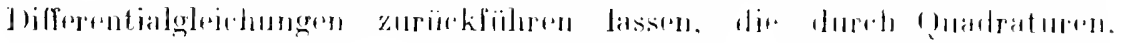

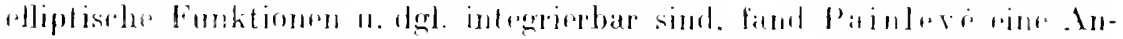

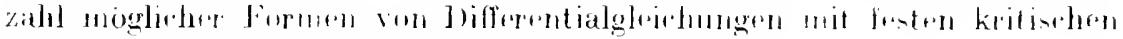

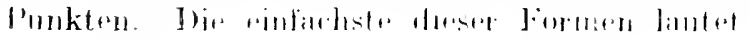

$$
\text { (5i) } \quad \|^{\prime \prime}-\left(i^{2}+1\right. \text {. }
$$

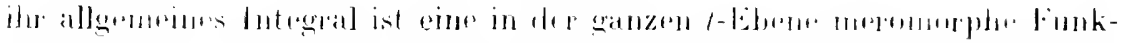

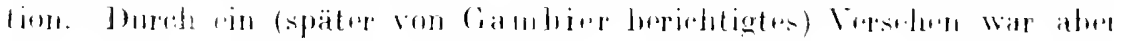

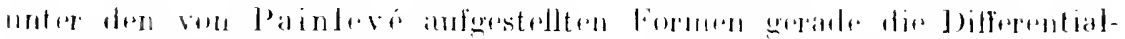

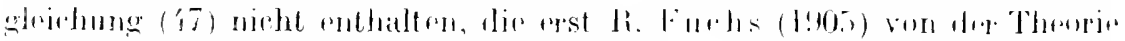

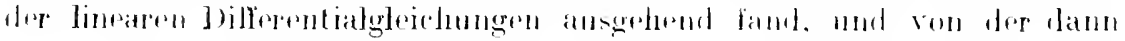

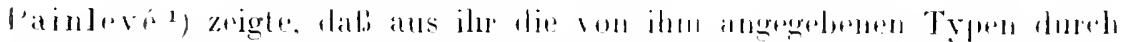

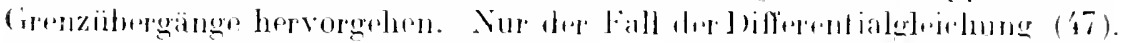

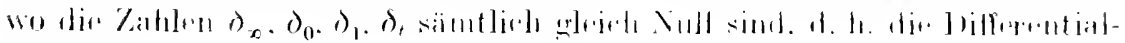

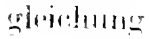

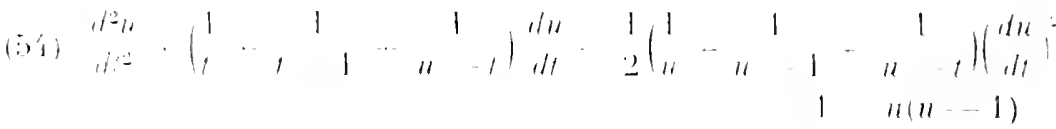

$$
\begin{aligned}
& 24 t-1)(11-1)
\end{aligned}
$$

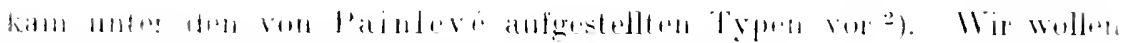

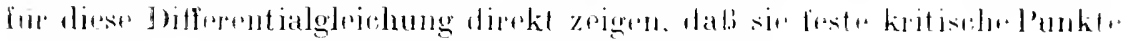

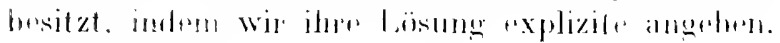

Sill ?!111

$(x)$

$$
\int_{\infty}^{\infty} 111(11 \quad 11)(11 \quad-1) .
$$

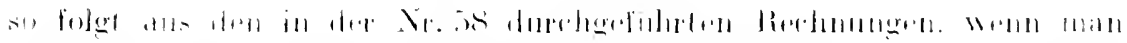

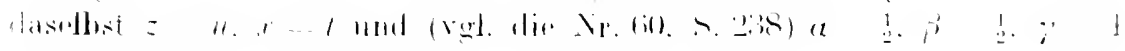

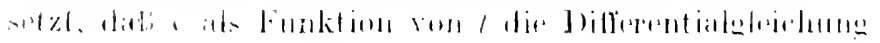

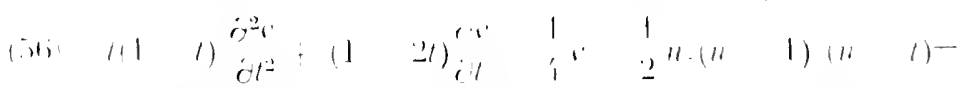

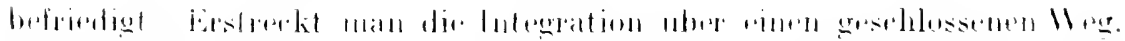

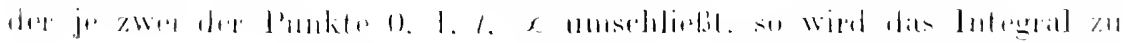

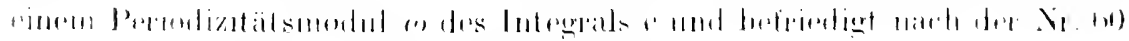

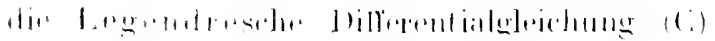

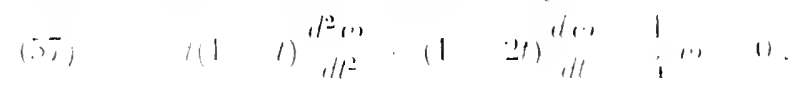

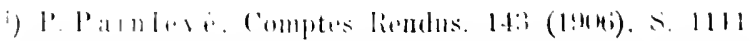

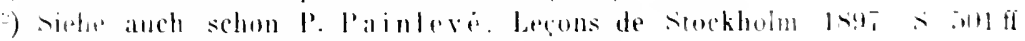

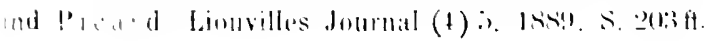




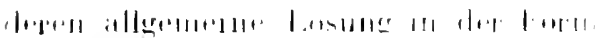

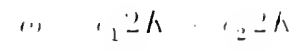

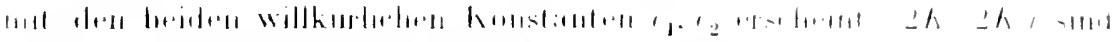

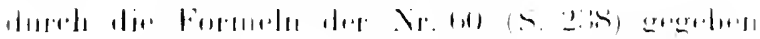

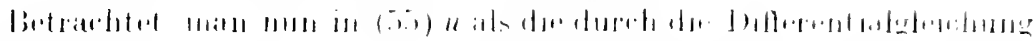

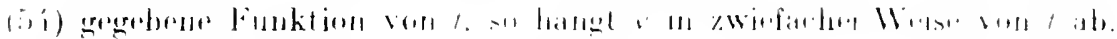

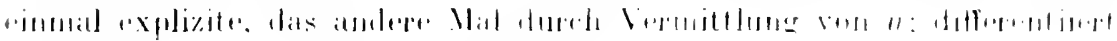

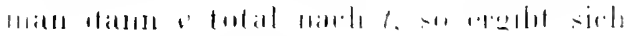

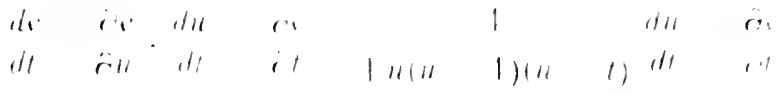

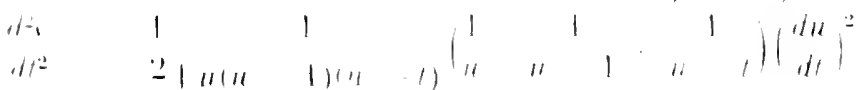

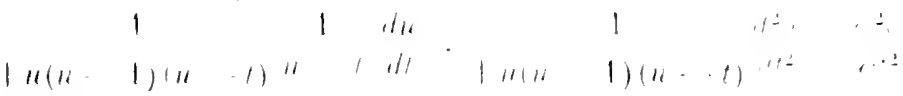

Man eliäl atsu

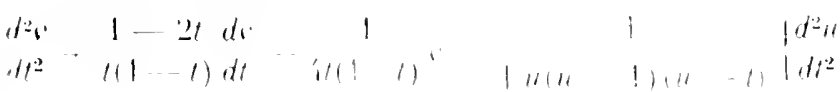

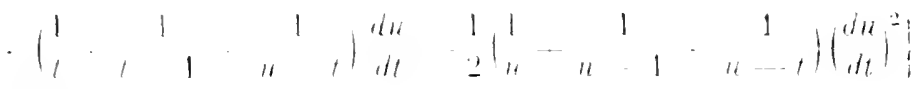

$$
\begin{aligned}
& \begin{array}{ccccc}
2+1 & 1 & 2 & 1 & 1 \\
t^{2} & 111 & 11 & 1 & +11
\end{array}
\end{aligned}
$$

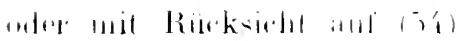

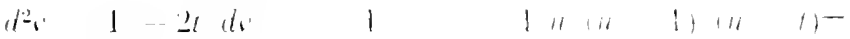

$$
\begin{aligned}
& \left.\left.d t^{2} \cdots(1-1) d l^{-2} \text { it } 1101\right)^{\prime-2} \quad 11-1\right)
\end{aligned}
$$

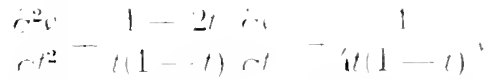

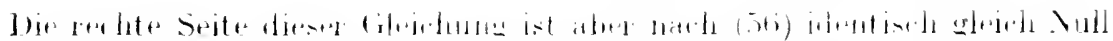

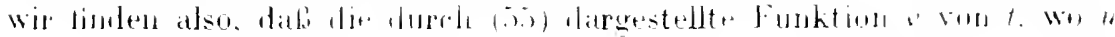

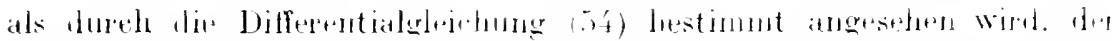

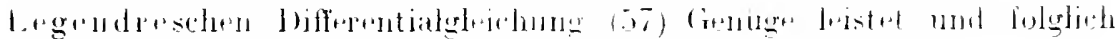

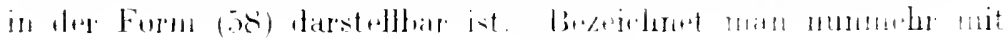

$$
11-i(1)
$$

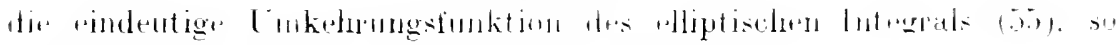

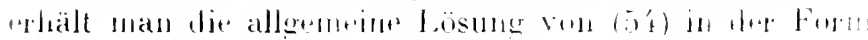

$$
\|-i\left(r_{1}-k-i_{2}-2 h i\right) \text {. }
$$

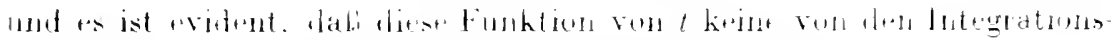

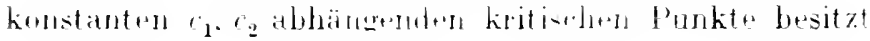




\section{Integration des Differentialsystems $(\mathbf{R})$. Bericht ïher nenere Untersuchungen.}

Mir stellen was numbehr dir Anfgabe. das J)ifferentialsystem (R).

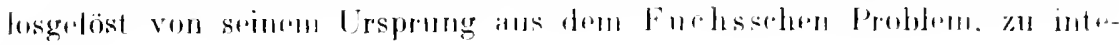
grinill.

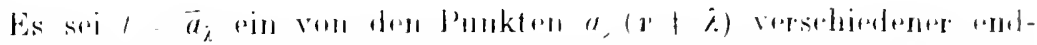

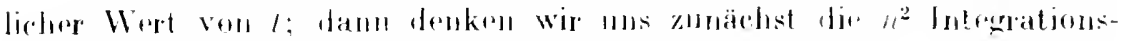

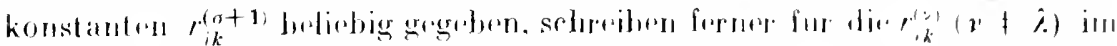

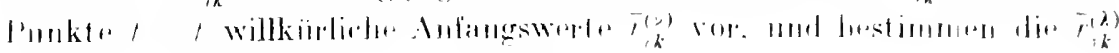
dureh die Ciluiehume

$$
R^{(\lambda)}=-\underset{+\infty}{N}(R(x) \quad R(n+1) .
$$

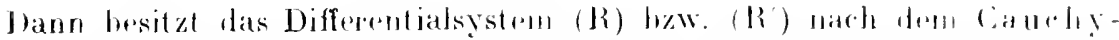

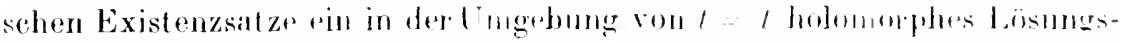

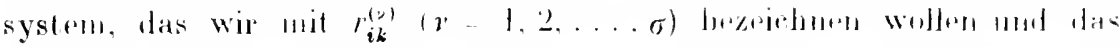

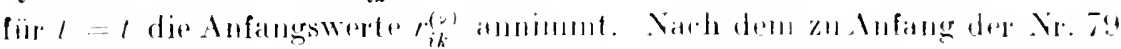

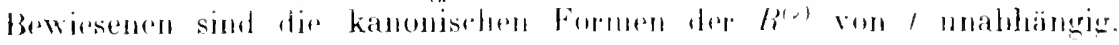

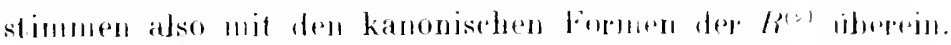

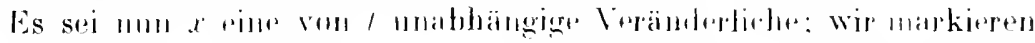

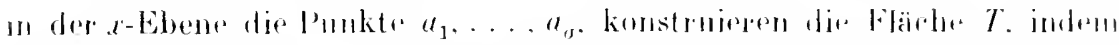

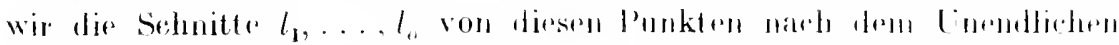

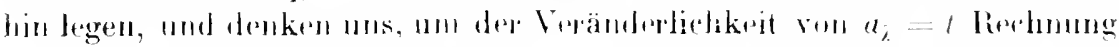

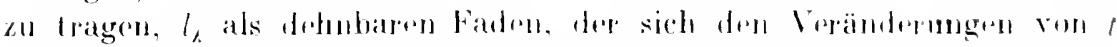
anzupassen vermag. Fïr $l-l-\bar{a}_{7}$ lezeichnen wir $l ;$ mit $l$, mod $T$ mit $T$.

Wir setzen dam

bilden das Jifferentialsystem

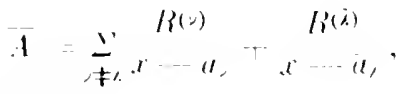

mal die lolegralmatrix

$$
d_{y_{k}}={\stackrel{n}{n} y_{u} u_{u k}}_{\mu=1}
$$

$$
y \quad \int_{i}^{x}(1 d x+1) .
$$

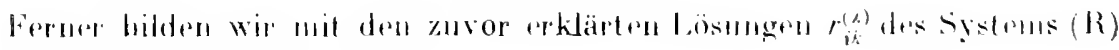
dats doifrerentialsystem in

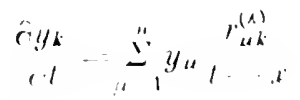

omd diejenige lntegralmatrix

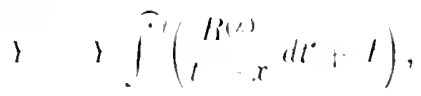




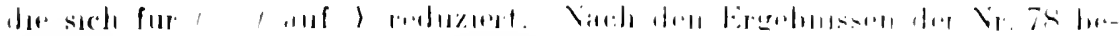

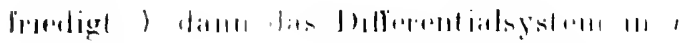

$$
\text { , th } \quad \stackrel{n}{1} 11,11, n
$$

"11

$$
1 \stackrel{3}{1} \underbrace{l+1}
$$

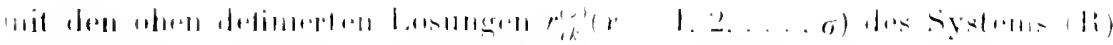

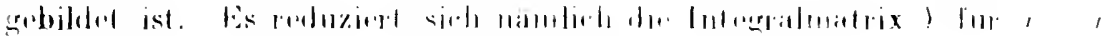

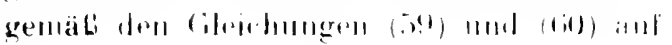

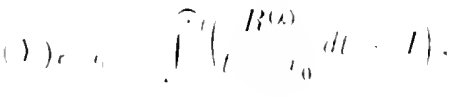

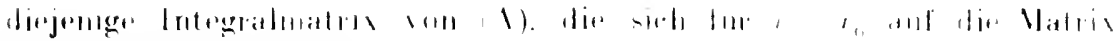
(61) penduziert, ist ablil

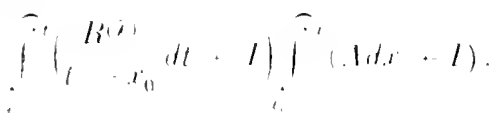

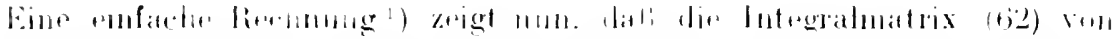

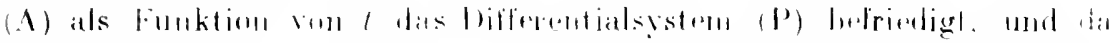

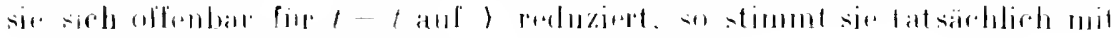

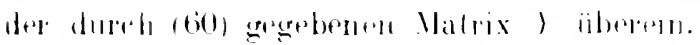

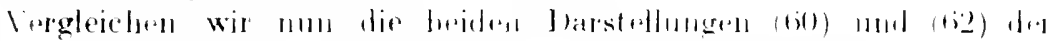

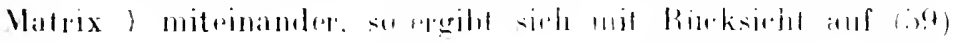

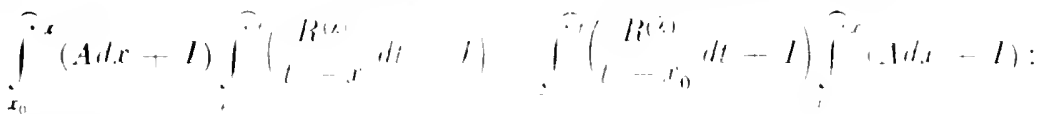

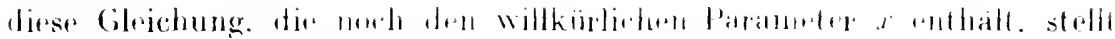

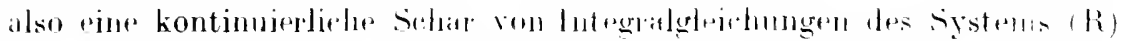

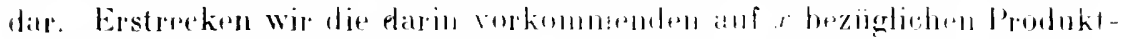

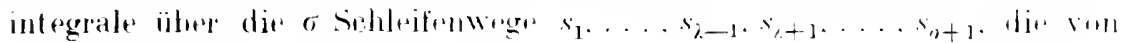

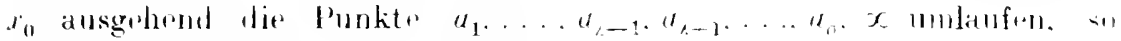
rhä̈t de den llert $r_{0}$ und wir limlın

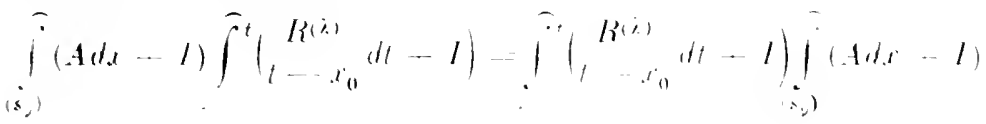

arter

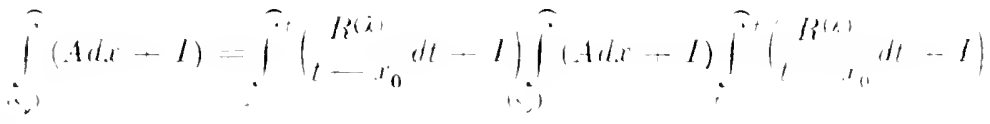

logl. L. Schlesinger. Crelles Joumal. 141,1412$)$. S. 96. 1. Teil. 22. 


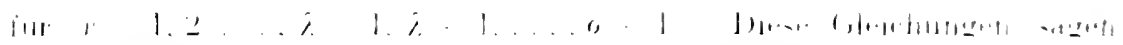

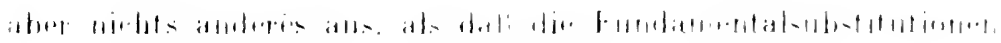

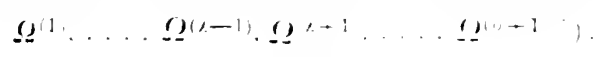

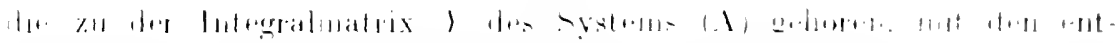

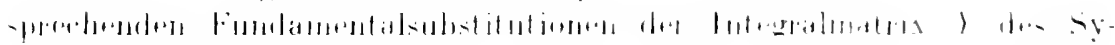

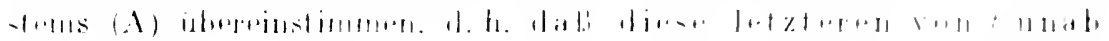

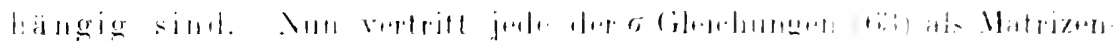

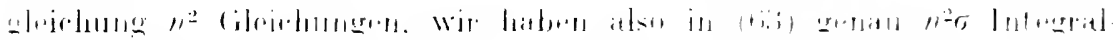

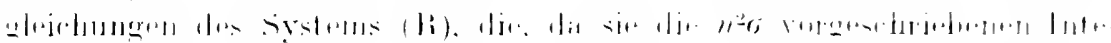

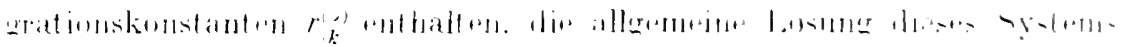

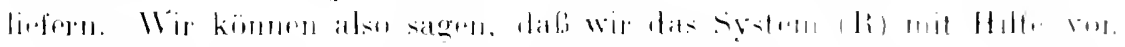

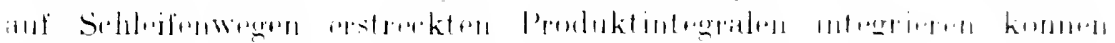

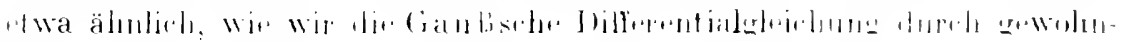

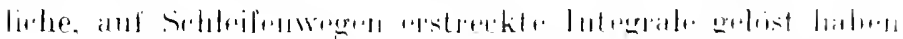

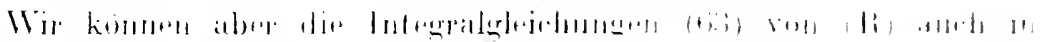

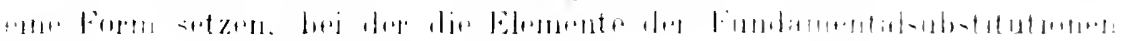

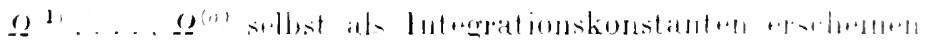

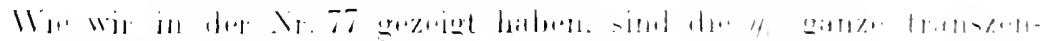

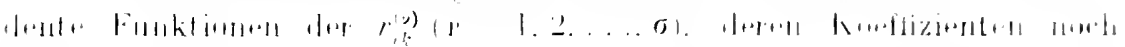

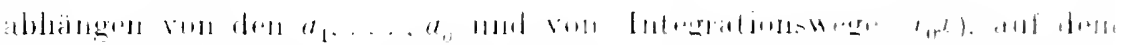

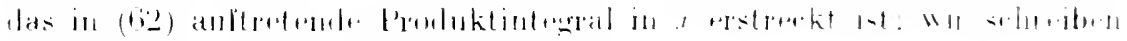

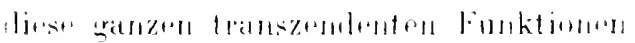

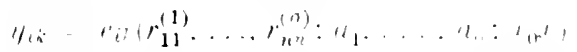

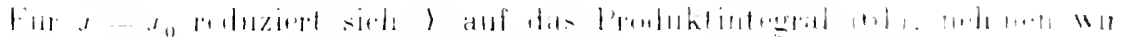

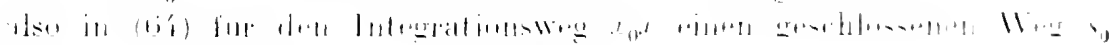

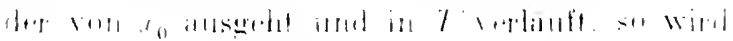

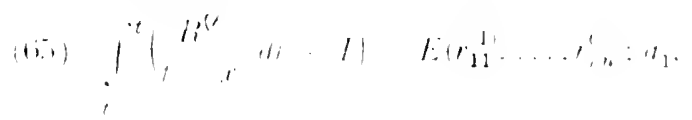

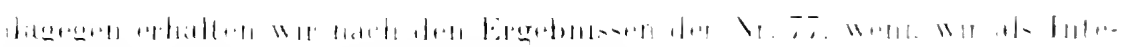

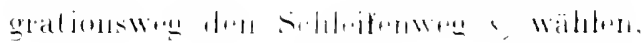

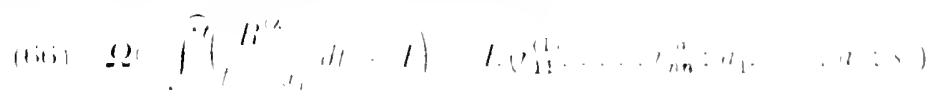

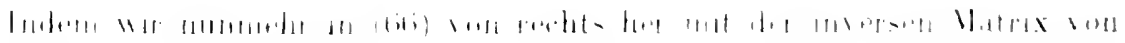

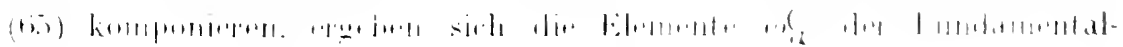

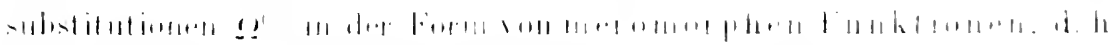

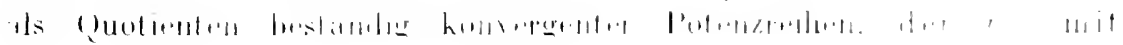
(i11111

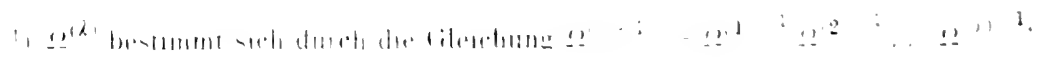




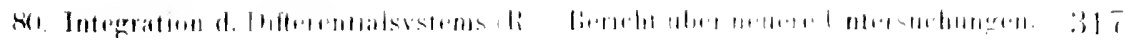

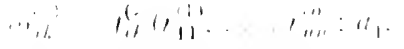

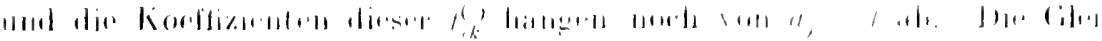

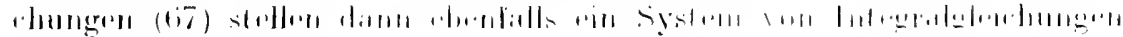

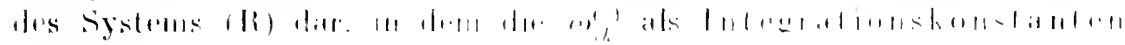
inugiemen.

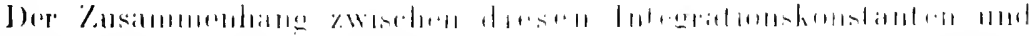

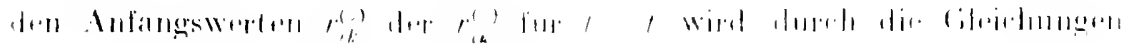

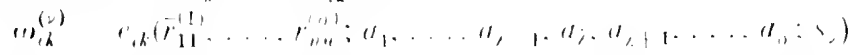

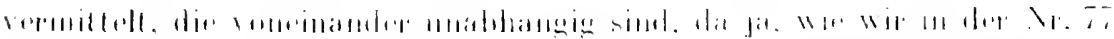

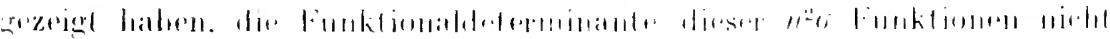
mbritiseh verseliwindel.

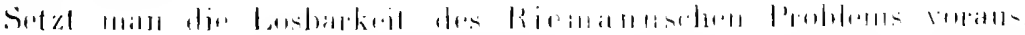

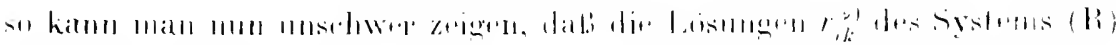

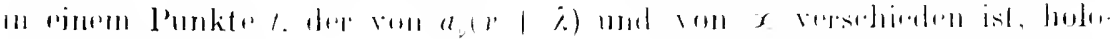

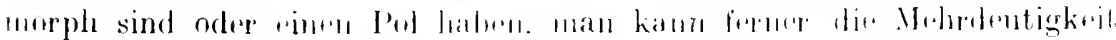

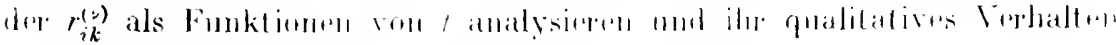

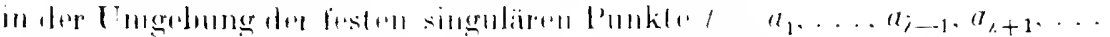

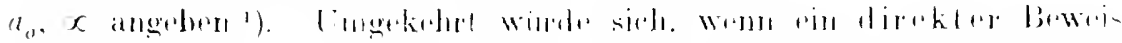

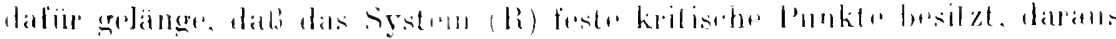

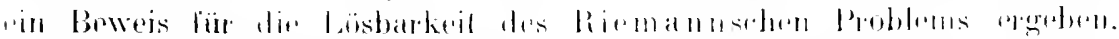

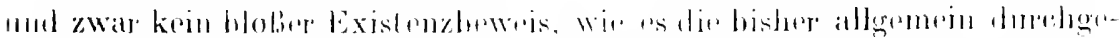

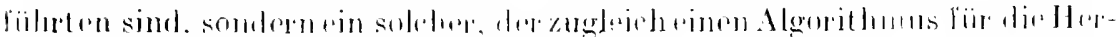

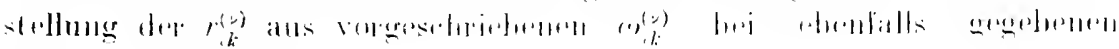
$t_{1} \ldots . . . a_{0}$ lindirte.

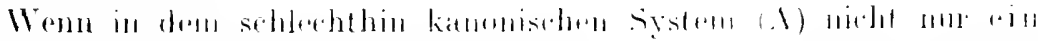

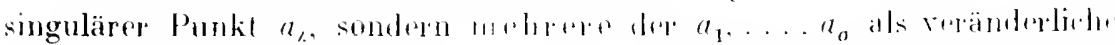

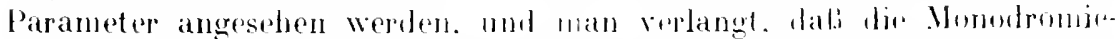

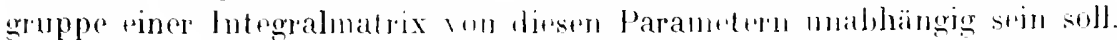
so haben die Residuen entspredend moheren Systemen von der Forsu (R) zu genügen. die dann als simmlane Sirsteme partioller Differential-

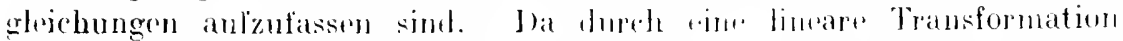

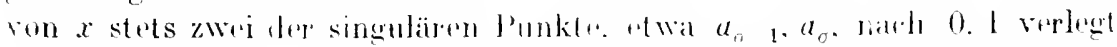

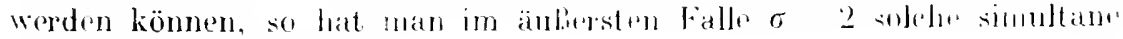
systeme zu betrathtell.

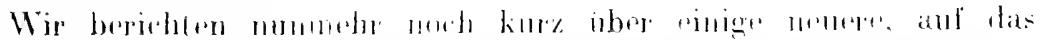
system (R) bezigliche Unhrsuchungen französischer Mathematiker.

Will man nach der Nothole von l'ainleve (siehe Yr. 79, s. 3ll nachzuweisen suchen. dal.s das Systen (R) jeste klotische P'unkte besitzt.

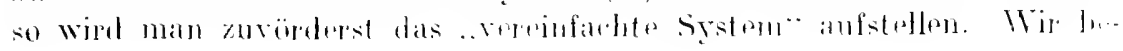

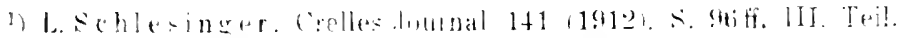




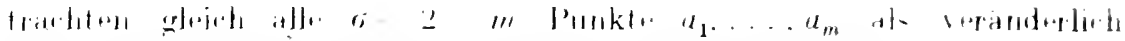

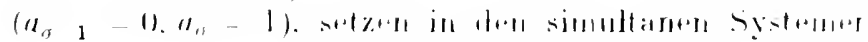

(li)

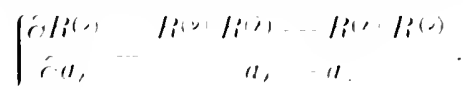

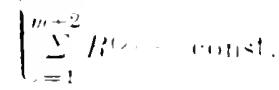

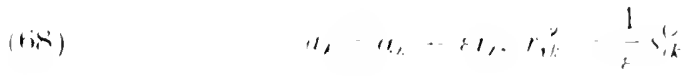

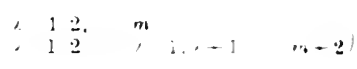

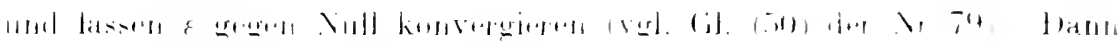

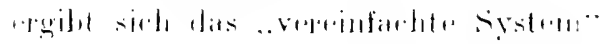

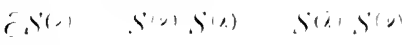

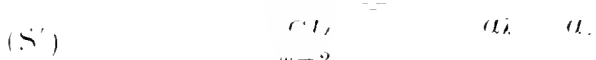

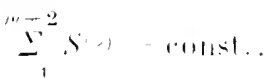

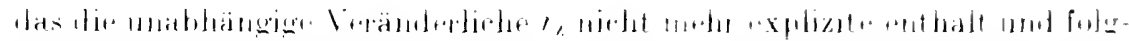

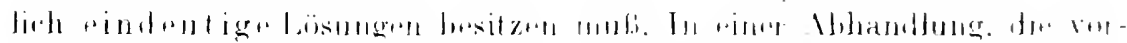

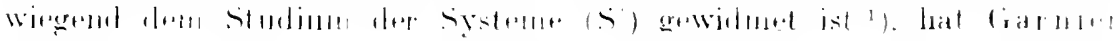

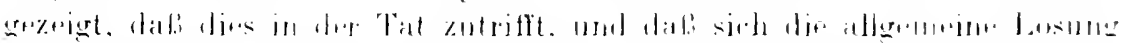

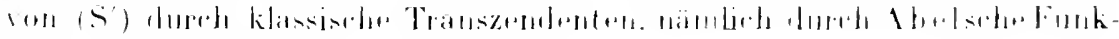

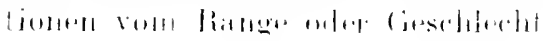

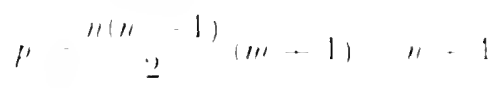

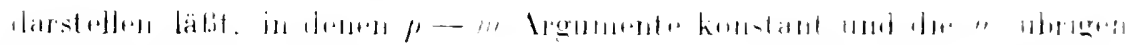

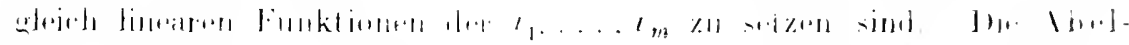

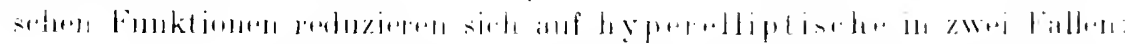

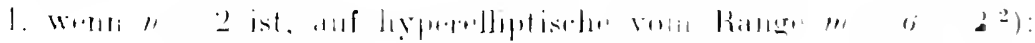

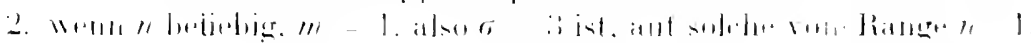

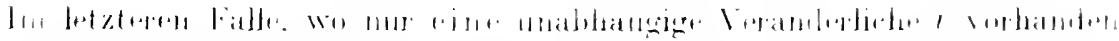

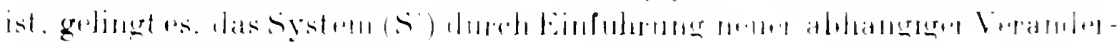

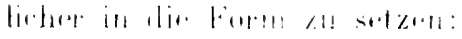

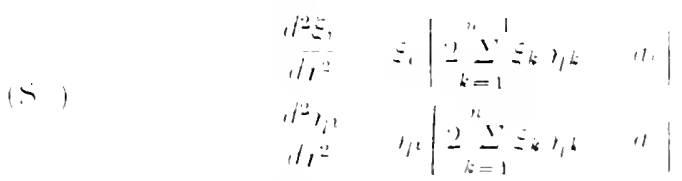

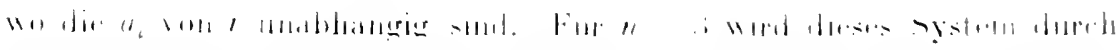

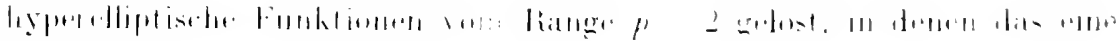

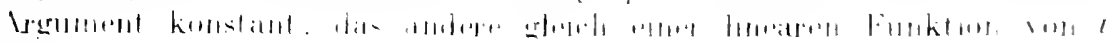

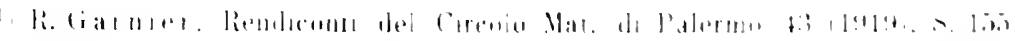

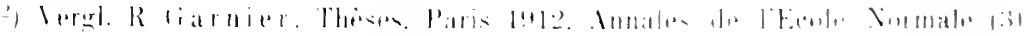
-4. $\therefore 1$ 


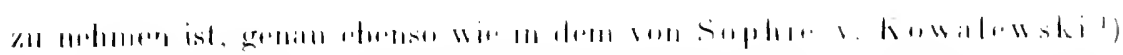

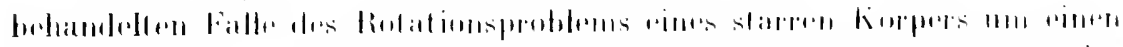

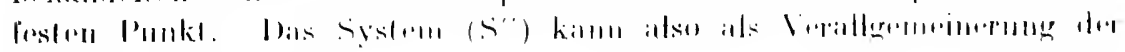

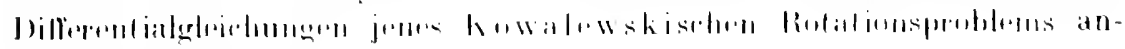

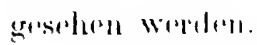

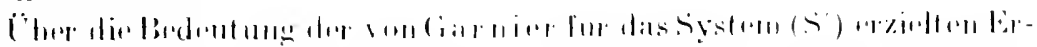

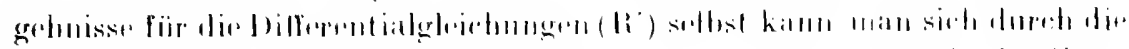

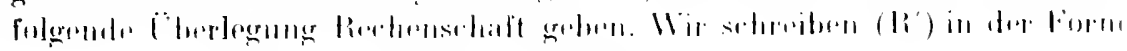

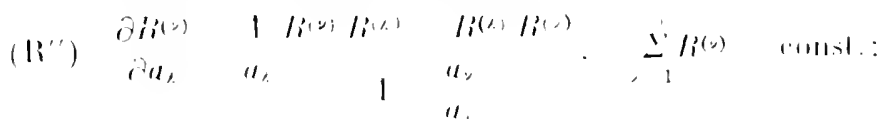

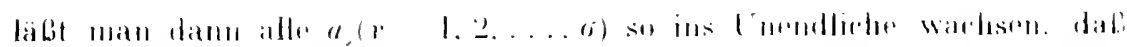

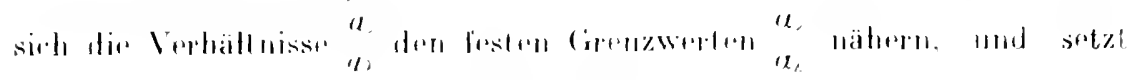
$u_{1} \operatorname{lng} \|_{2} \quad l_{2}$.

so verwanlelt sich $\left(l^{\prime \prime}\right)$ in

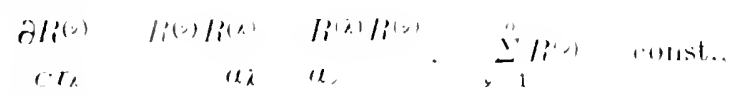

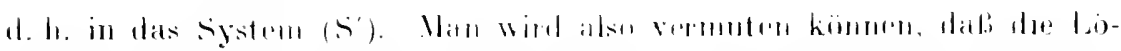

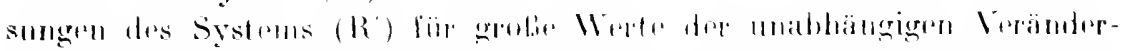

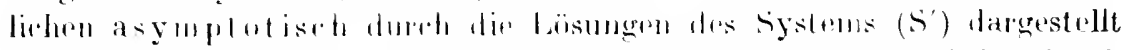

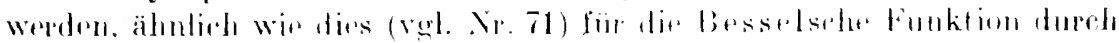

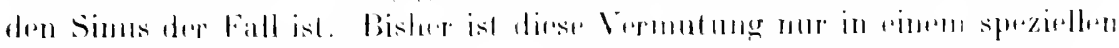

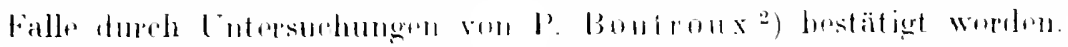

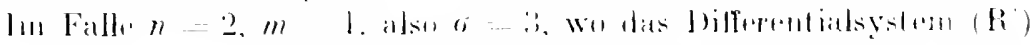

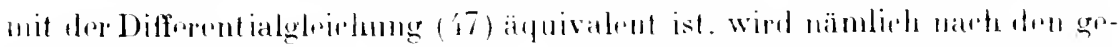

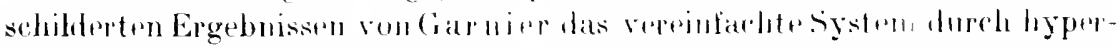
olliptische Funktimm rom Ratrge $p=m=1$. alsu hureh nliptiseh.

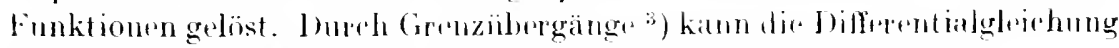

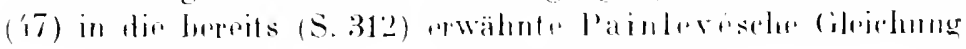

$$
\text { din } n=t i t^{2}+t
$$

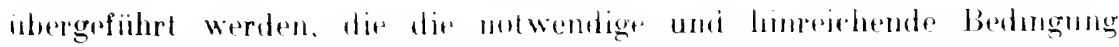
dafür darstellt, dab die Monodromicegruppeder linearen I)ifferentialghejehung zweiter Ordmung

1) S. r. Kowalew ski. Arta mathematica 12) (16s9). S. 17 T

2) P. Bontroux. Annales de l'École Nom. Sup. (i3) 30. 191:3. i 25.; 31 1914. S. $\$ 9$.

3) Siehe R. liarnier. Thèses. is toff.: P'Boutroux a. a. 1). 31, 1914 S. 1 iff. 


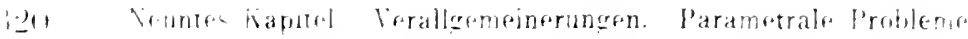

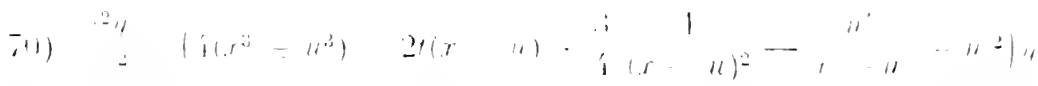

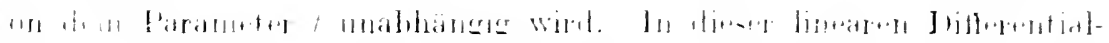

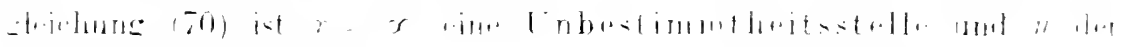

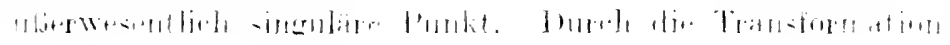

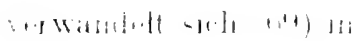

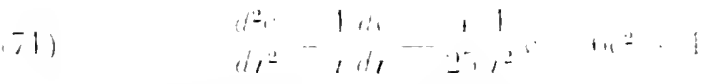

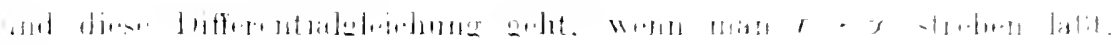
uining ir:

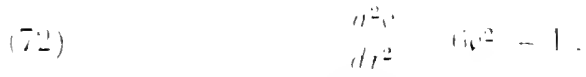

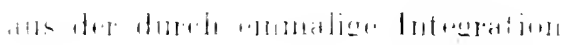

$$
(7,)
$$

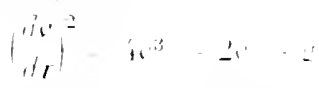

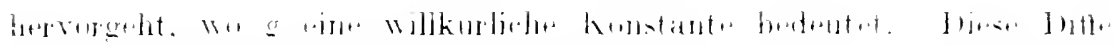

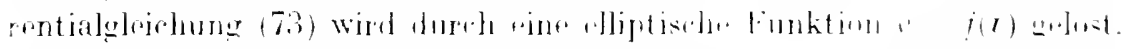

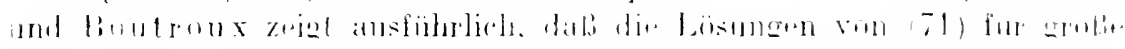

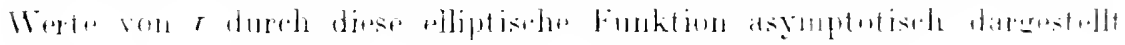

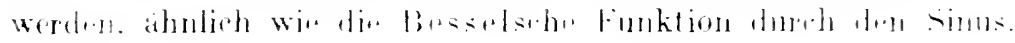

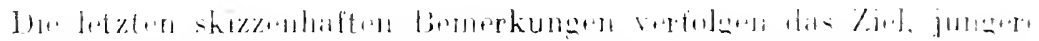

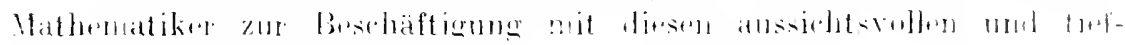

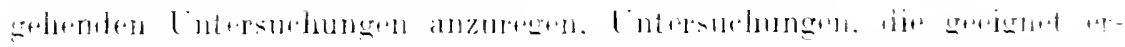

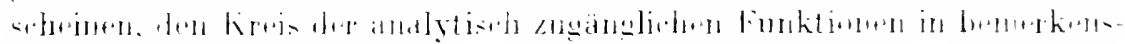

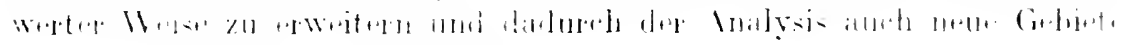

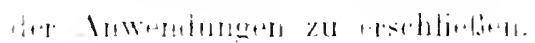




\section{Sach- und Namenverzeichnis.}

Abrl, \. 11, 26, 129

Abelsthe Funklionen !- : ils (ileichung liil.

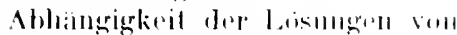
den Antaneswerten $15,8 \mathrm{~m}$.

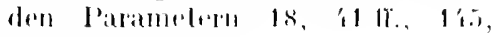
$2991 \%$.

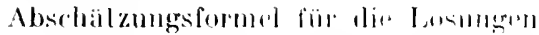

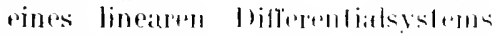
141.

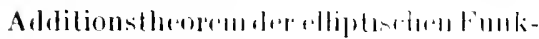
tionen 11:.

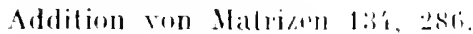

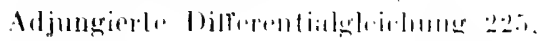
$29 \%$.

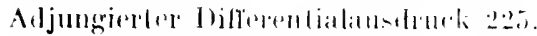

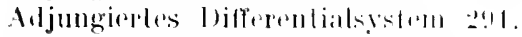

Adjmogierte Inlegralmalrizen :2"?'.

Ahuliches Inatrizen lis.

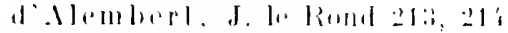

Algebraiseher Vorzorigungepmet as.

Agebraisehe lisemellichlicitsolelle ill.

Algebroial ill.

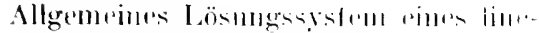
anen Dillerentialsistems l:3:3.

Analylisedue Forlselzumg iti.

Anfangsberlingrungen

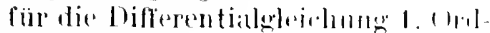
nunge

fïr ein Diflegentialsysten 19. 1:3:

- Pür die Differmbialerlejelumar $"$. Ordmung 19.

Anlangsmatrix 13:.

Aufangswerte, regulare is. , singuläre 35.

Anormale Reihen 25:2.

1ppell, P. 65, 76, 47, 49.

Art bei lotherentialsislemen liti, 201. 289.

- bei Matrizen 18:2, 201.

schlesinger, Ditlerentiaglechuncers.

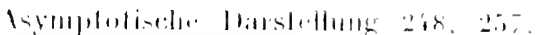
$271111 ., 319,3211$

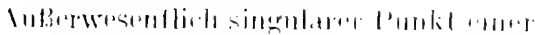

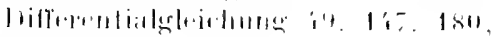
$2012,: 311910$.

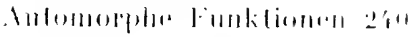

f11 1011110, 1. 12.

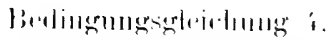

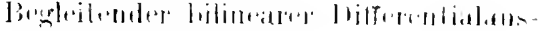
drork 2.2 .5$.

lindroulli, 11, sti.

.1. bil.

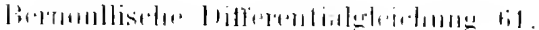

Tramsformation bí.

Barle. 1.9\%

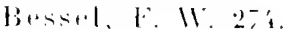

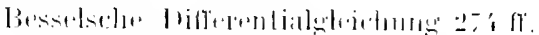

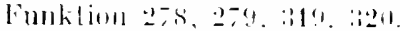

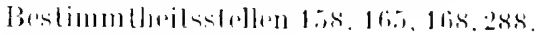

Belafmklion 2:ita m.

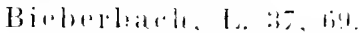

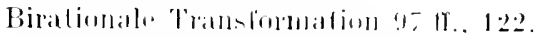

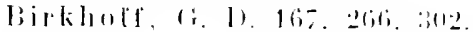

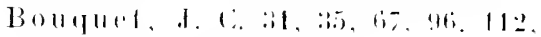
12:121, 1s. 189, 25ti.

Bowrlal, 1\%, 2:3i,

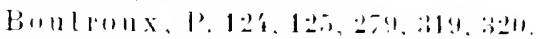

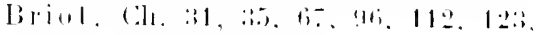

$124,185,159,250$

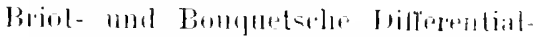

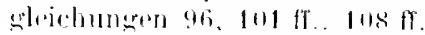

Calloul des limites 301. 1'a. 1'1.

liantor, M. 89 .

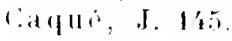

liasorati, F. :318.

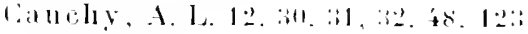

145.5 
Canchy-lipschitzshes Verdahen la.

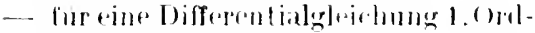
nuner \& IT.

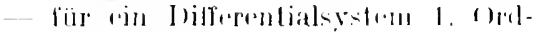
millye $18 \mathrm{ll}$.

- liar ein limeares homoseme Dif-

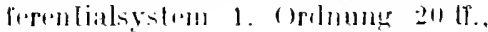
$1: 57$ II. 1'il.

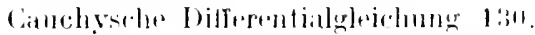
$1,5$.

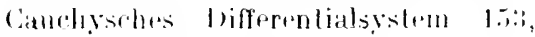
208.295.

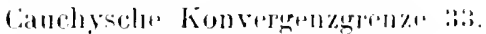

ciayley, .1. 1010.

Cesiro, E. 19s.

Charakterististhe (ileiglumng

- riner Mafrix l's!.

riner bitferentialgheichnog mil konstantern korftizienten 18.).

rines bitrementialsystrme vom Ranü 1 2't!!

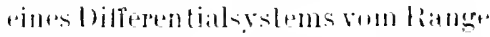
l, 1252.

Chatakleristisehe lnvaluntern einte Systems ahnlicher Matring lis If.

Clairaut. A. C. 89.

Clatirantsche Differentialdeleichumer !!u. C:lobsh F. 1. 9:

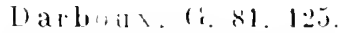

Derivationsformol 15:

Derivierla Matrix 1:36.

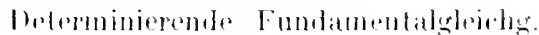

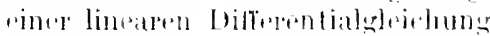
170.181.

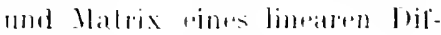
lemblialsystem, 182.

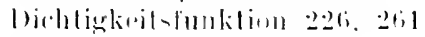

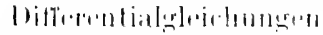

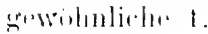

piatlindle. $=$.

lipad vinl 2 .

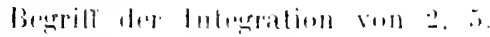
syy-lom vill it.

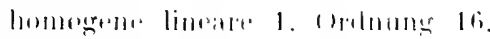

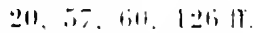

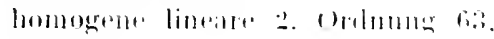
$15981 \%$.

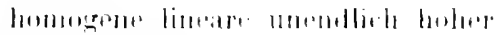
(1)lmmer 236 .

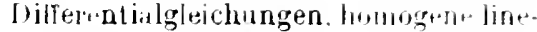
ar. mit kombtantor kogefizienten 175.

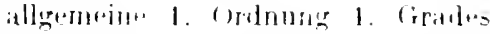
li. 27 ff.

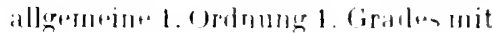
lesten Verzwrigung-junktun 5.j fr.

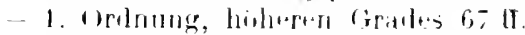

- 1. Ordnung. holmeren Gidelm, mit fwiten Verzwrigung-puntetro $93 \mathrm{ff}$

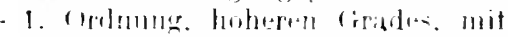

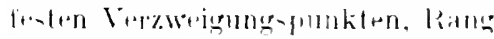
Vill fiill it.

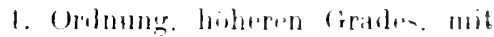
lexten Virzwignneren. Radng Eimloift.

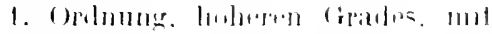

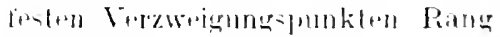
Zwei 1 ! $5 \mathrm{~m}$.

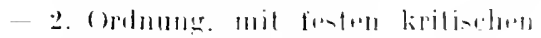
louklen 12.; : : 119 IT.

Jitregentialoriom i. 1?.

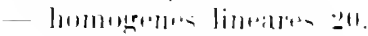

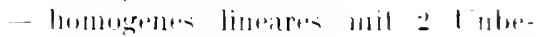
kimuten bi: 1:12 ft.

- bumogenes limeares mil "Inbekanntin 20). Zstiofi.

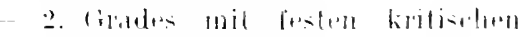
l'mklen :31, tr.

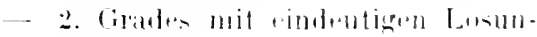
and 318.

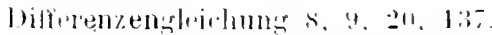

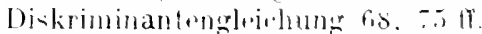

Diskriminantenkurw st.

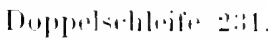

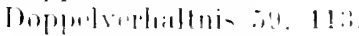

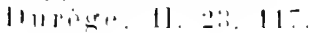

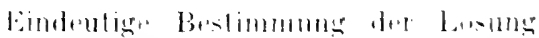

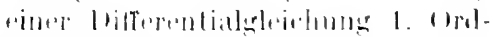
mung $1: 3$

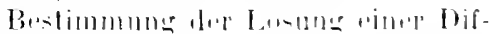

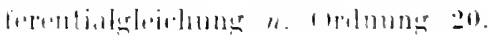

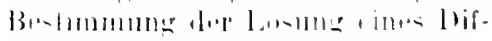

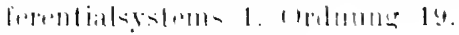

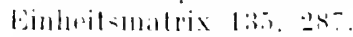

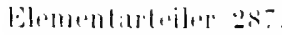

Elliptish he fonktiomen 25, 1111, 116 IT. $: \because 211$.

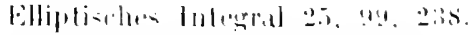

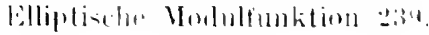




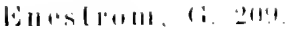

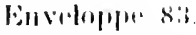

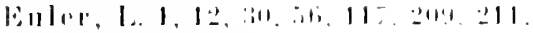
$223,226,2: 3,230,2=1$

bulersidese lnterrial

1. (iallanir 2:isi.

2. I Rallumer zlis.

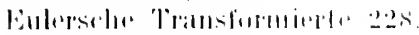

Fixistumberis

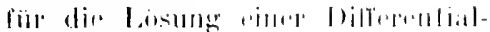

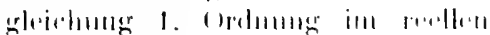
(inbiul. s ll.

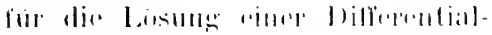

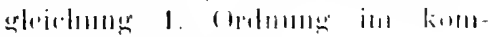

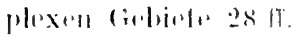

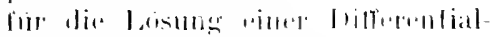

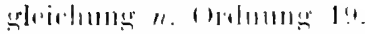

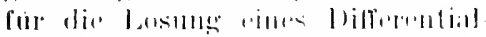

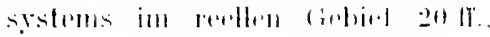
$1: 17$ 1\%.

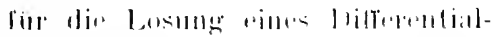

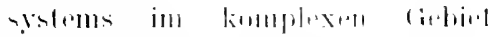
$13901 \%$ 1 $431 \%$

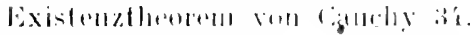

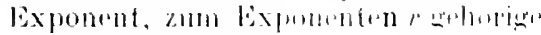
lounklion $1: 28$

Exponentialfunkliun rin.i. Matix isis. rilles findere 2us.

Fabry, ( : 202.

Fakultatementhen zost.

Fojer, L. 19s.

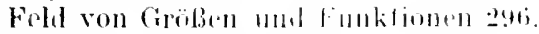

Feldgleichmeng 289, 29:-

Forsyth, A. R. 6., ss.

Fourier. J. B. 2-í.

Fouriersche Reily 198.

Frellholm. I. 289.

Fricke, R. 220.

Frobenius, (i. 1tis. 169. 21:2. 224. $225,243$.

Fuchs, L. 6t, 53, 54, 55, 5t. 6-, -1, $88,93,94,100,123,128,145,158$. $161,168,170,185,198.212,221$, $225,239,256,288$. 341:

- Satz von 168.

Fuchs, R. 311, 312.

Fuchssche Bedingungen liu Ditrerentialgleichungen mit festen Vip. zweigungspunkten 9f.

Finlissche Relation 19'. 195.

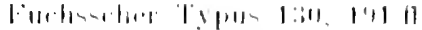

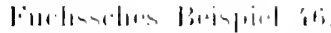

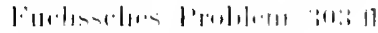

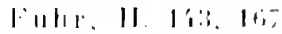

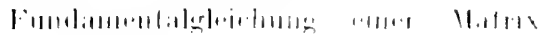
$1,11,28-$.

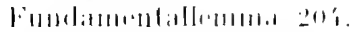

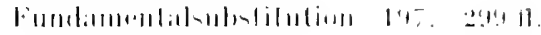

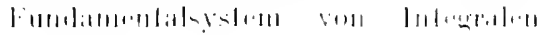
$|i| \mid$

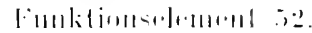

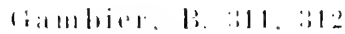

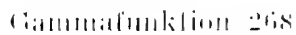

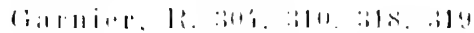

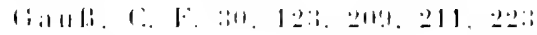

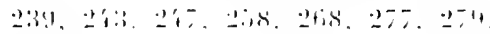
28 i.

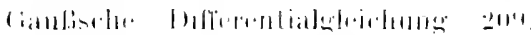
21011 .

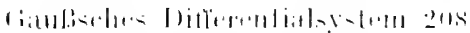

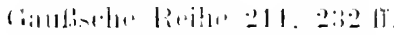

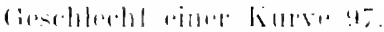

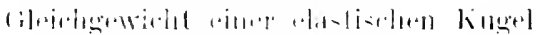

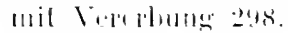

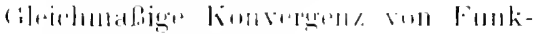
limblatolgen 11 .

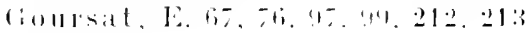

limllar. l'. 1'is.

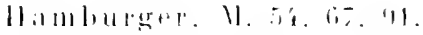

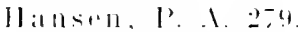

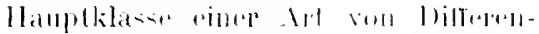
tialivitumeth 2112 .

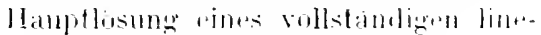

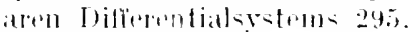

Inaptwet 38. 19s.

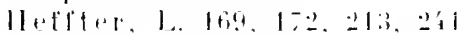

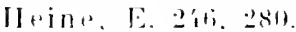

Hensel. K. Hi, y?

Hermite. (ih. 100. 111, 12:i.

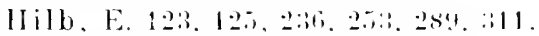

llilbrit, D, :302.

II ildablandt. T. IJ. 284.

Hill, II. I. M. H'.

Holonomph 竞.

Ilolomopphitherejull l's.

Iouke. R. I:s.

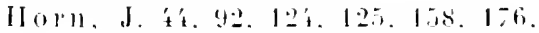
185. 254.254 .25 .266 .284 .281$. 28, 


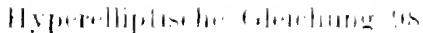

Fouklinotill : 1 s.

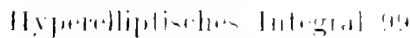

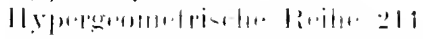

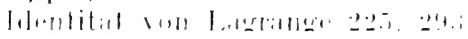

Implate limblem it:

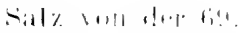

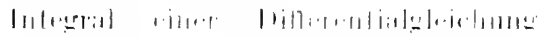

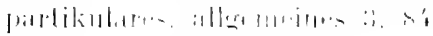

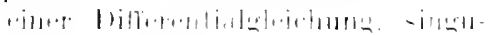

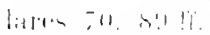

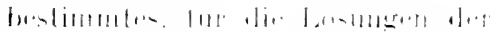

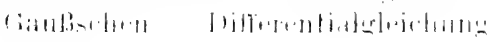

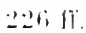

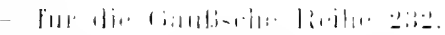

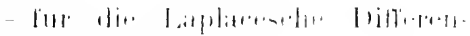

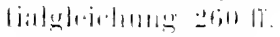

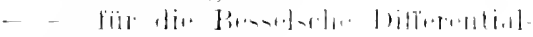

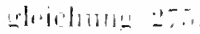

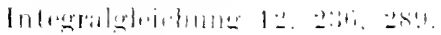

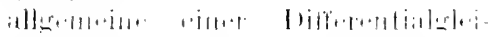
dilutig sto

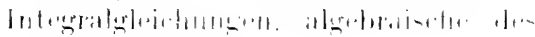

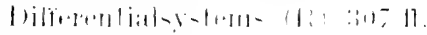

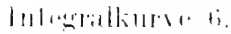

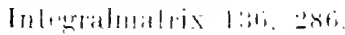

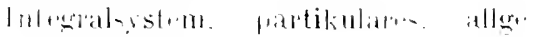
mitrinte is

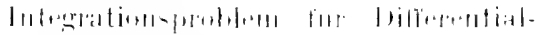

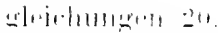

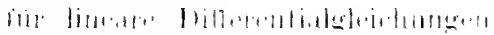

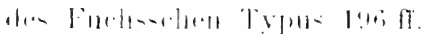

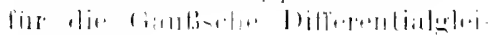
hisme zo:?.

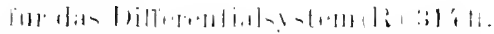

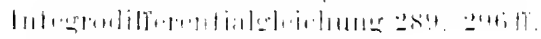

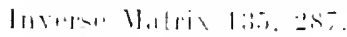

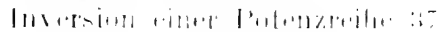

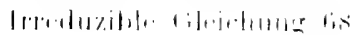

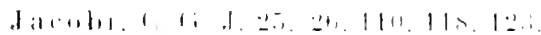

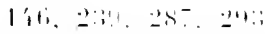

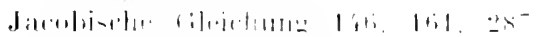

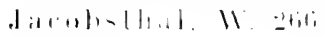

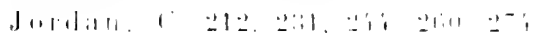

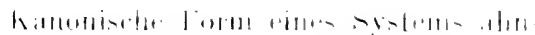

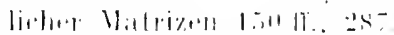

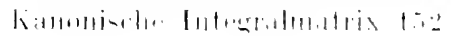

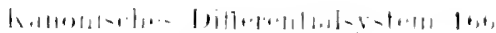

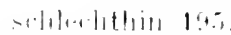

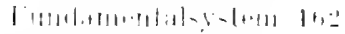

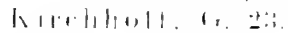

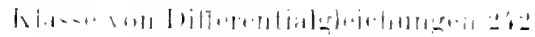

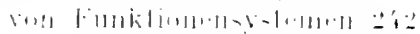

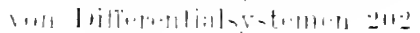

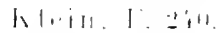

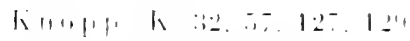

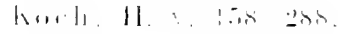

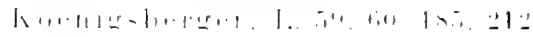
ㄴit.

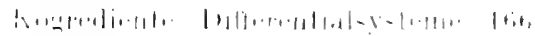
ㅍi11

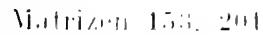

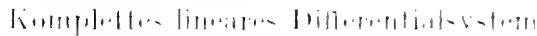
in. 2015

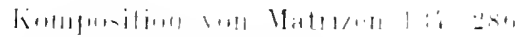

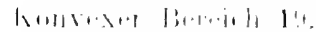

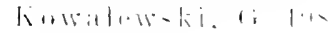

- mphin. r. : :1:"

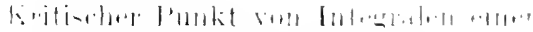

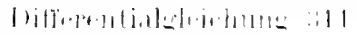

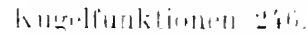

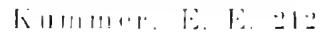

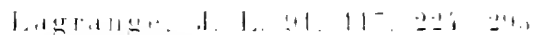

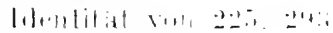

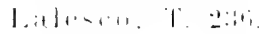

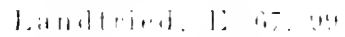

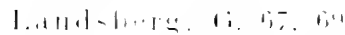

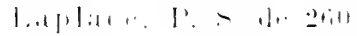

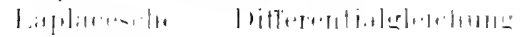

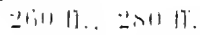

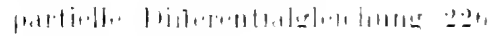

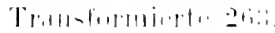

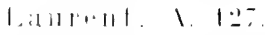

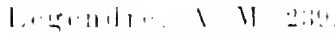

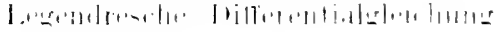

ais. $\quad$ : 11

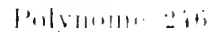

1.i. - i.

I.i. 1.111,1111. i! 1-"1

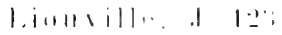

l,iphlit/. 1: 1:

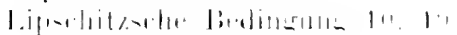

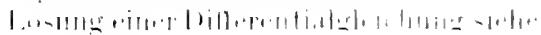
latrential.

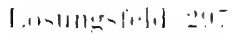

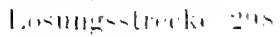

| $11.11191 .:$ 


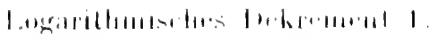

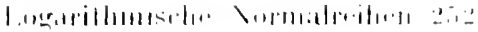

Maljurantl. : :

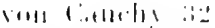

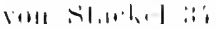

11.11111111-1. 1. 1:1. 12,

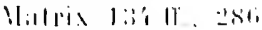

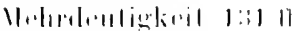

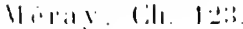

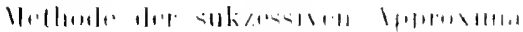
limiln 1 i:i.

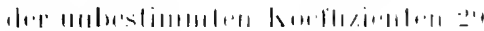

H11 I

llillowl-ill\% lil lis,

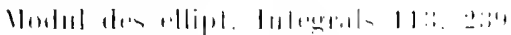

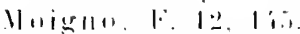

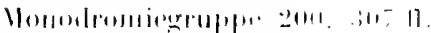

Monngene liunlilime 2-.

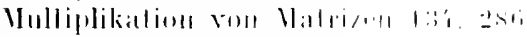

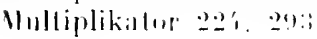

Veraliver simn $1:: 1$

\1411111111. \& 280

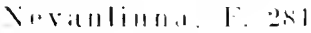

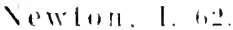

$\backslash$ inlsill, \. 2811

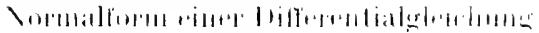
lii!.

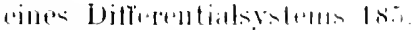

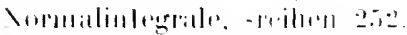

15goud. 11. I: 11, is. :

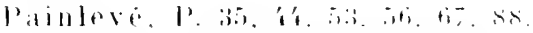
1166, 114, 118, 121, 129, 12: :11. $\because 12,37$

Sial\% vint is

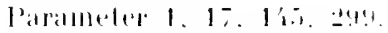

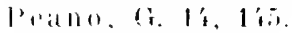

Peanosehes Beispitel 1's

prinlel 2:3. 1:5.

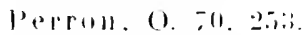

piali. I. I\%, 211.

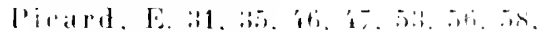

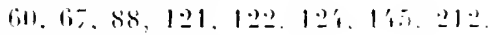
$220,251,312$.

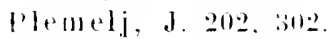

l'lessller, .1. 5:3.

Purhhammer. 1. 22:1.

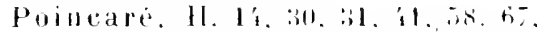
100. 106. 11', 121, 12:8.126. 125.

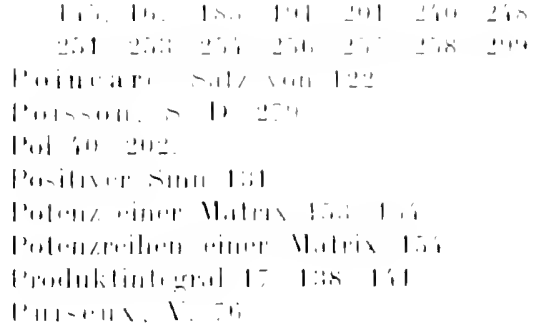

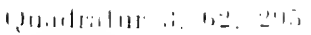

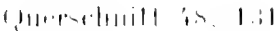

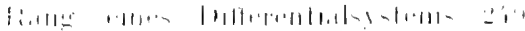
2.1.

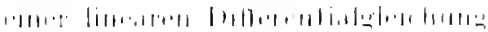
$2+1$,

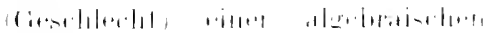

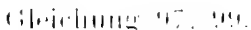

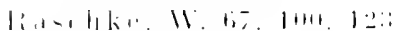

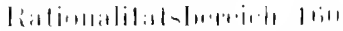

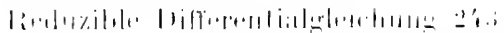

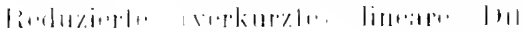

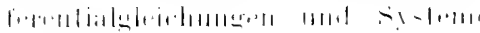
lill, 2us

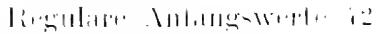

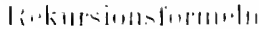

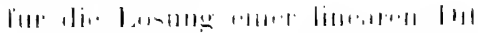

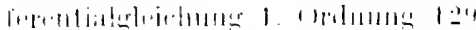

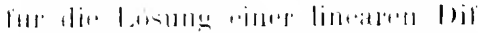

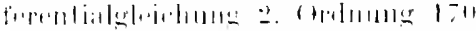

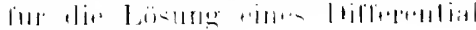
$\therefore 101111-1: 11.2511$

limill11111 J2:!

lidialli. I. iti.

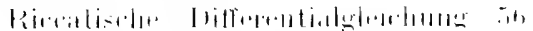

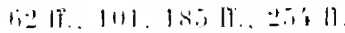

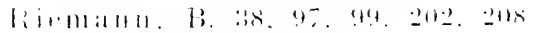
2019.212 .220 , 202.

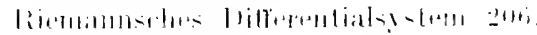

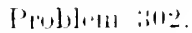

kith. F, 158

Ritll. II, 1:

Rouckliwhlumkl sil

liunge, i.. 1:.

siattrlpulukt 1 is.

iallvare, L. 16,

xal\% vinl

$\therefore \cdot 11+[1+2]=1 ; .62$

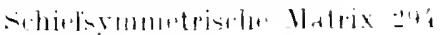


Seluleshthin kanonisches Difmentialsystem 195, 299. kegrediente Matrizen 201.

sirhleifonwerg rinfacher 1:31, 2:30, 265. doppeller $2: 39$

sirhlosinger, L. 125, 1:59. 165, 169. $198,223,224,226,238,239.244$. $24: 3,260,289, \quad 302.303,2306,2307$. :315, $31 \%$

Sichnitt $: 8, \quad 1: 31$.

sichwankung rime fumklion s. 10.

sichwingungen, kloine rines Punkles 176 if.

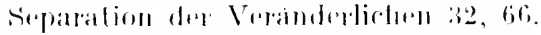
simultane farlinlle lofferentialsystrme. $306,: 318$.

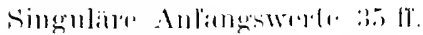

Singuläres Inleglat $70,81 \mathrm{lt}$.

Singuliare sterllen

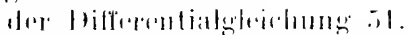

der homogenen linearen biflerente

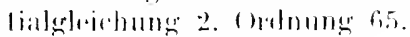

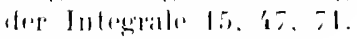

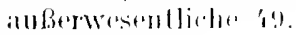

feste 51 . 71 .

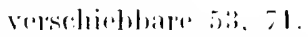

Jer Bestimmlfuil 1:28. 1.; 17.

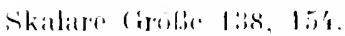

spannungszostand ainel kingllathensehale tos.

stiokel, P. : 's.

slabl. II. ti:.

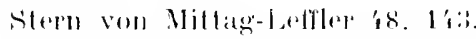

streke ron Giobsen mol Funktionen 296 .

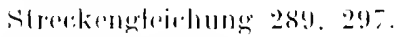

substilution 1/\%.

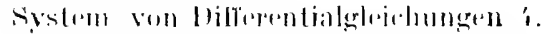
von linearen homogenen Differen-

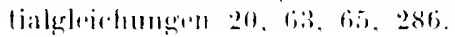

Taylur. B. 2- 89.

Thetaribren von larobi zas.

Thom

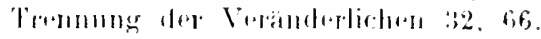

forrgangesnbslitulim 199, 220.

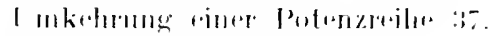

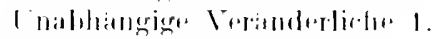

lobestimmtheilstull. 128.

t nikmestlimror lon.

liniliat is. 1!00.

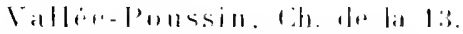

Vorbergungen reo liugel 1-9.

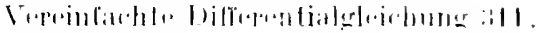
$: 31$.

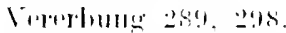

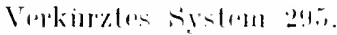

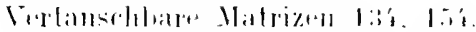

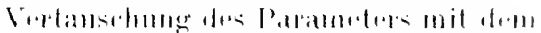

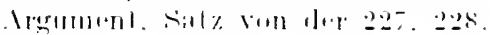
$26 i 2$.

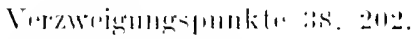

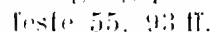

Vissiol, F. 6.2.

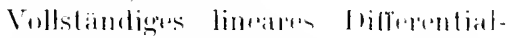
system 60, 20y5.

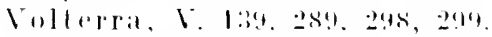

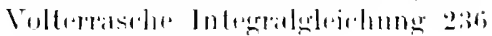
Transandente 29 s.

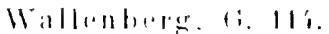

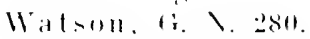

WricktraB. K. 287.3112.

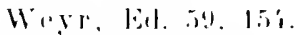

Wibler, II, I:s.

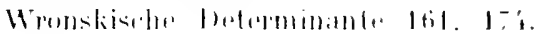

\%yklus -is.

Crliuderunktion 2:-s. 


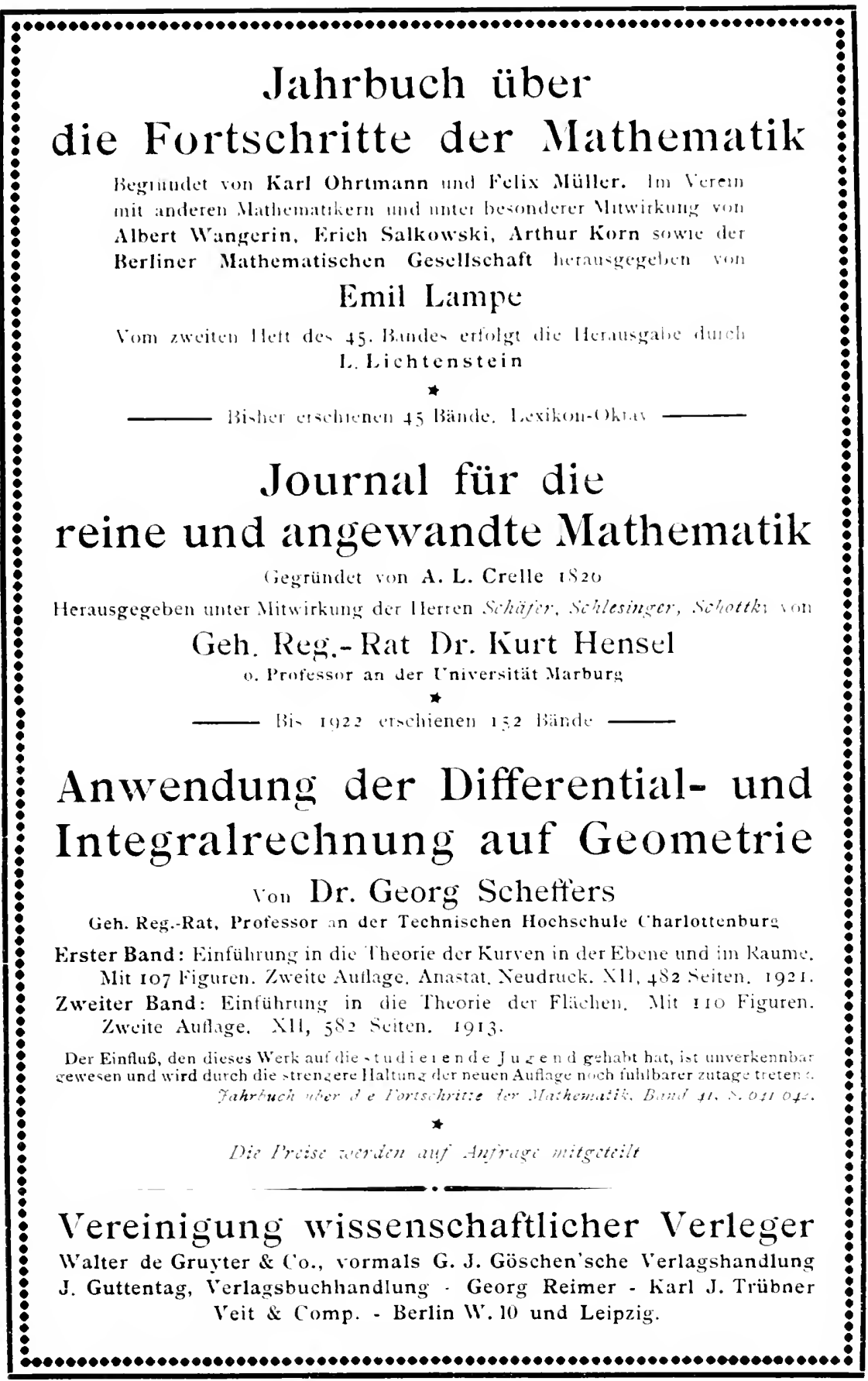




\section{Geschichte der Elementar-Mathematik}

ion I) r. Johannes Tropfke

Iirchtor der kirschner-Oherrealschule zu Berlin

Lweitc Autiage. I.ex.-(Ihtas.

Erster Band: Kehnen. 111, 177 -eitell. 1921. Zweiter Band: Allgemeinc Irsthmeih. IV. 221 aciten. 1921./Dritter Band: Proportionen. Gleichungen. IV. 151 - eiten. 1022 .

lle weiteren Bande -ind in Vorbencuturg

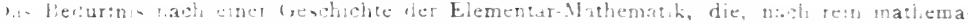

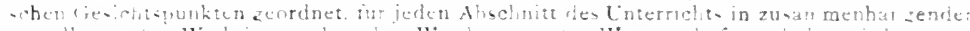

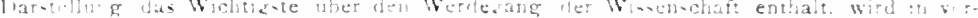

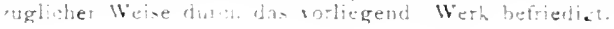

L.ehrbuch der Differentialgleichungen

ron Dr. Heinrich Liebmann

“. Professor an der Lniversität Münchon

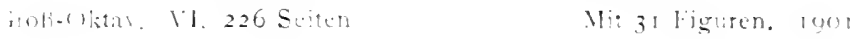

G öschens Lehrbücherei

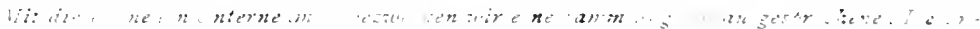

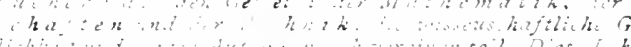

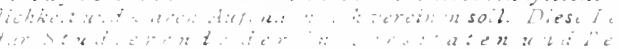

tor fi....t?

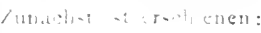

Gruppe I: Reine Mathematik

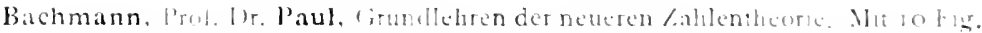

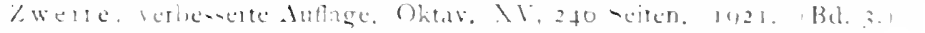

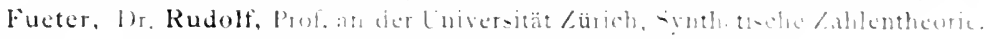

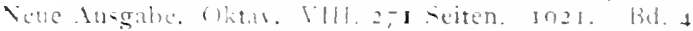

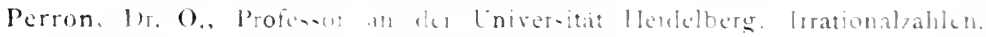

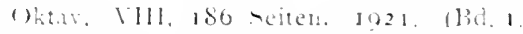

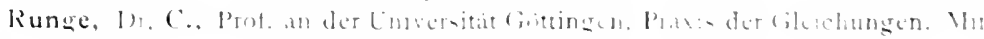

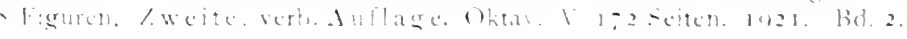

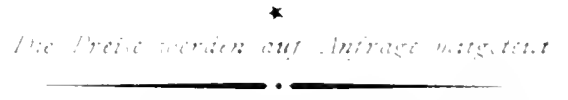

Vereinigung wissensehaftlicher Verleger Walter de Gruyter \& (a.. vormals G. J. Göschen'sche Verlagshandlung J. Ciutentag, Verlagshochandlung - Georg Reimer - karl i. Trürner Veit is comp. - Berlin $W^{\circ}, 10$ und lecipzis. 


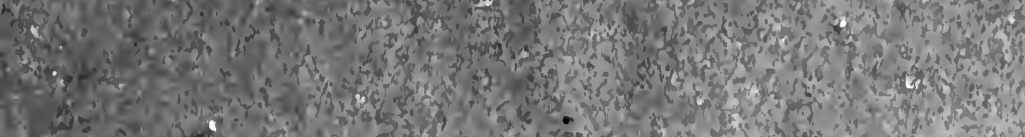

W.

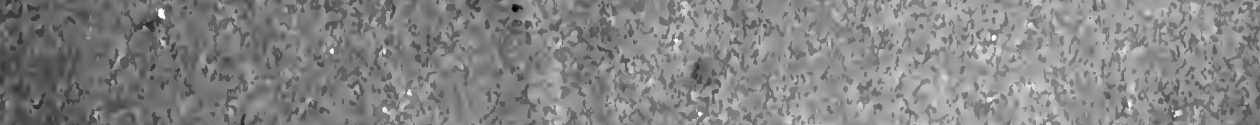

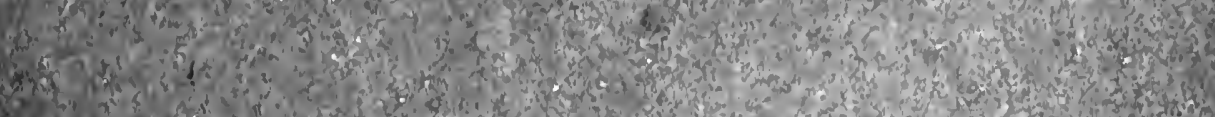

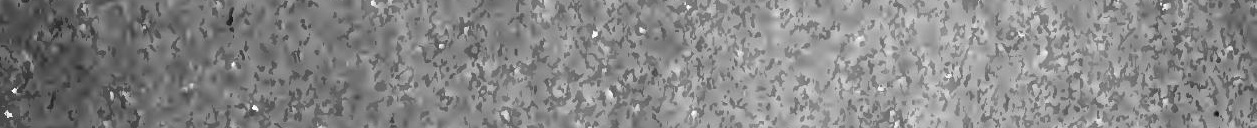

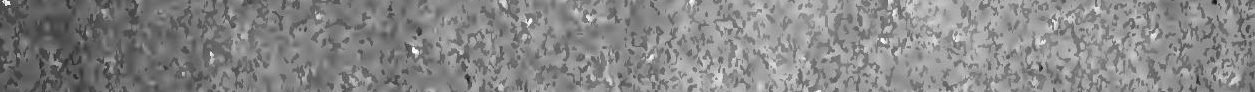

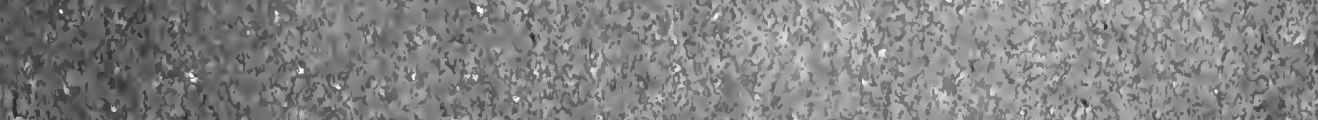

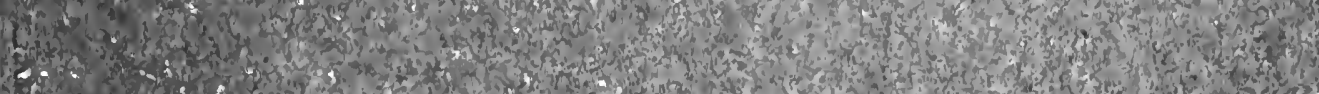

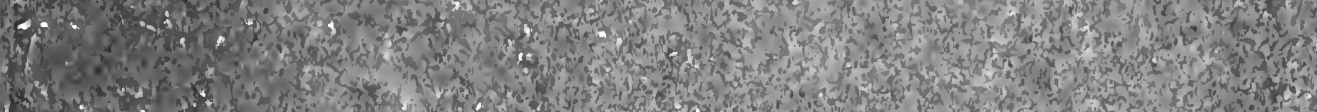

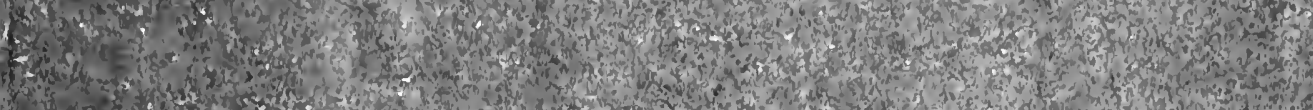
6.5.

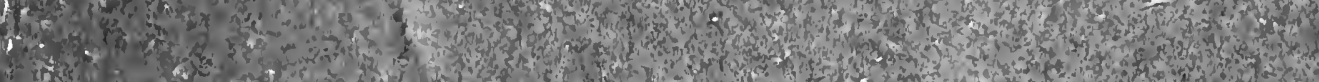

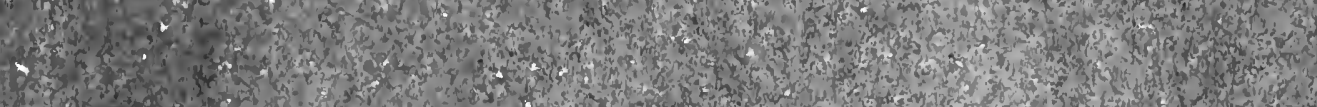

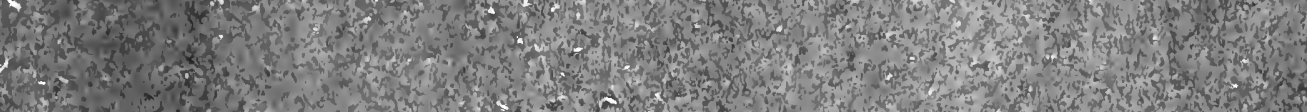

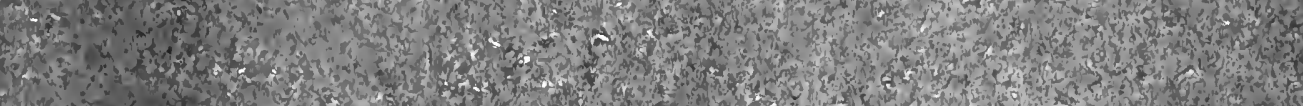

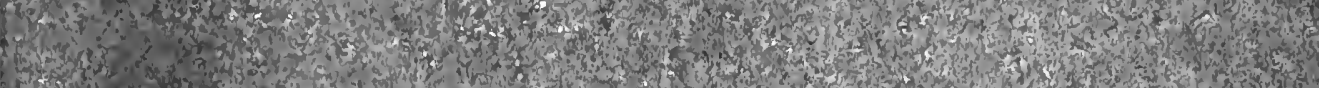
6.7.

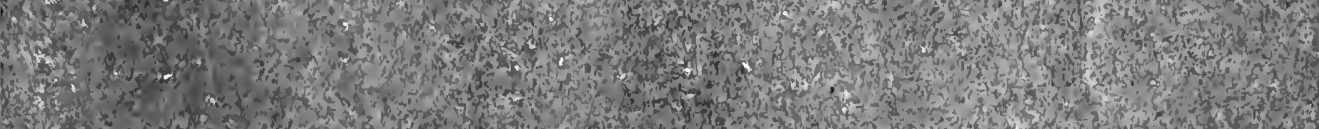
T. Pow

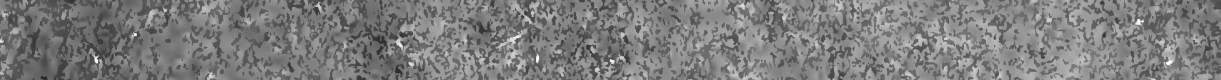

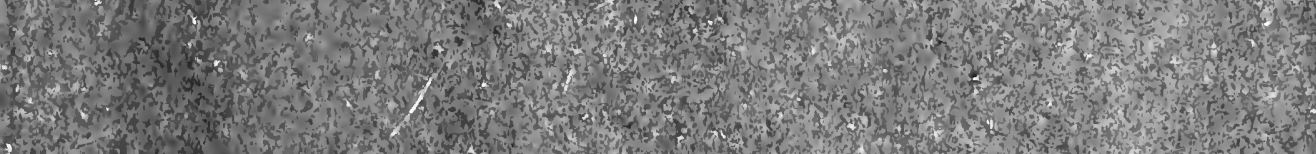

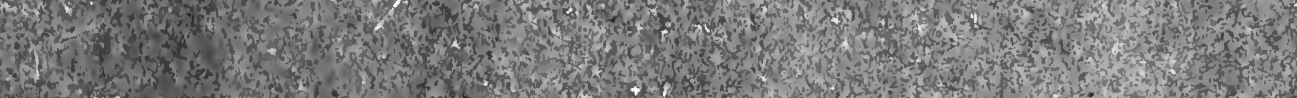
(2)

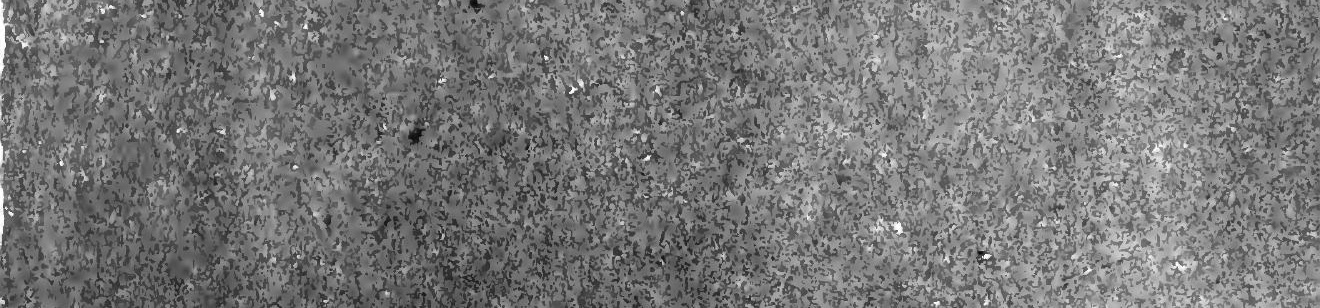


17r:

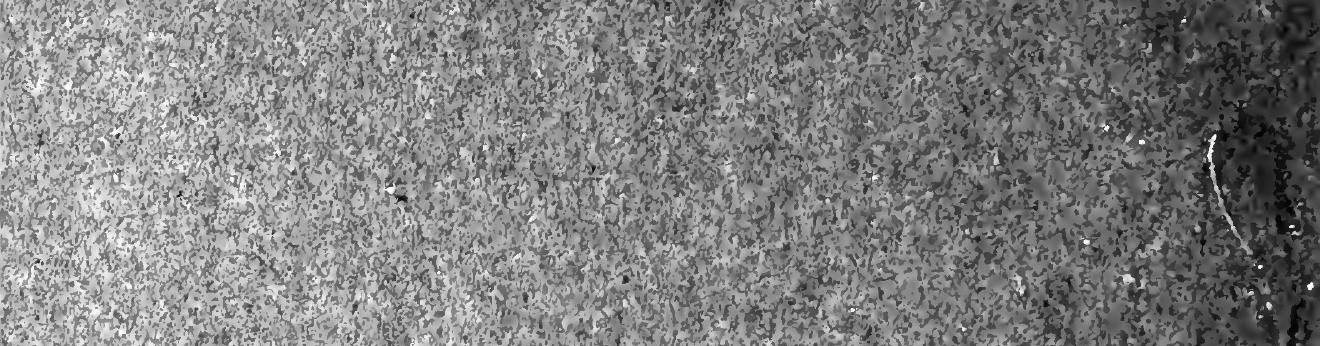

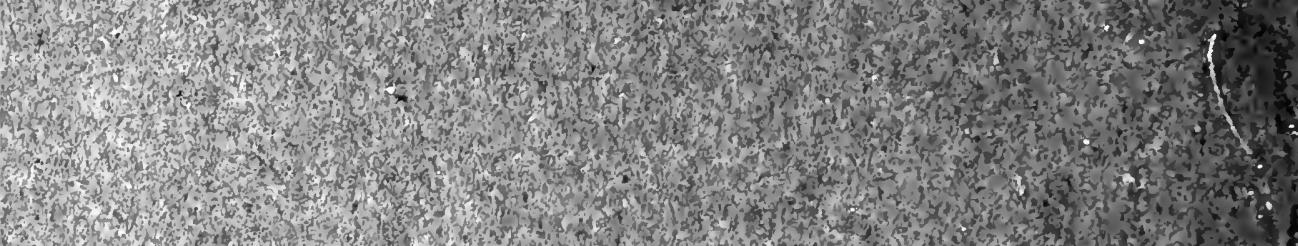
1.5. 3
3

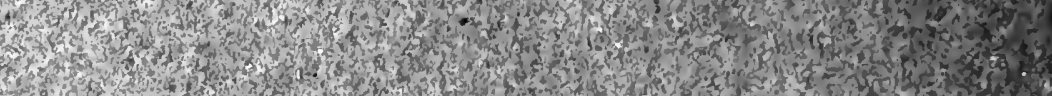

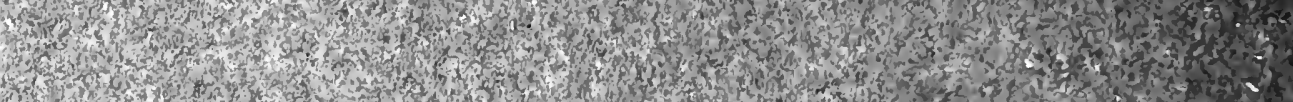

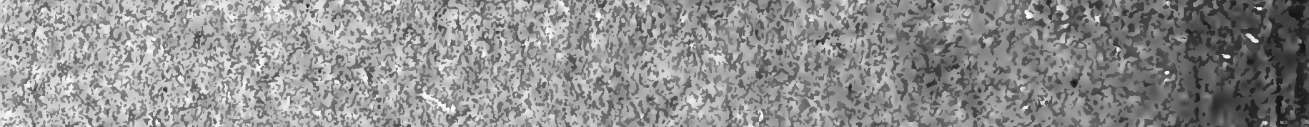

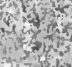
(2) Th -

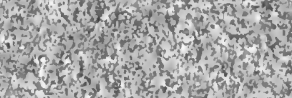


QA Schlesinfer, Luduis

372 Tinfihrung in die Thenre

S349 der gewölichen Jifferential-

1922

lejchungen auf funktionentheoretischer crundlare

3. neubearh. tufl.

PLEASE DO NOT REMOVE CARDS OR SLIPS FROM THIS POCKET

UNIVERSITY OF TORONTO LIBRARY 
